
fang. 

KAIATONSERA

TEIERIWAKWATHA 


\section{IMPRIMATUR :}

4 Eduardus.Car., Arch. Marianopolitanus. 


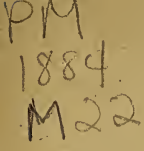

1890

$\operatorname{SiA}$

KAIATON

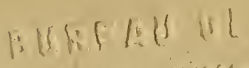

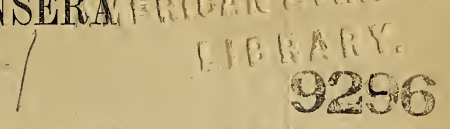

TEIERIWAKWATHA 6

$j+\tan x+2$

, ONKWEONWENEHA

$-$

in gronguois!

Tsini kahaseres Iokarenre oni, nok tsi naiontkaristiotasi,

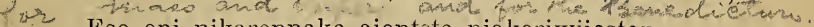
Eso oni nikarennake aiontste niakoriwiioston

Qum mar Ononsatokentike nok akononskon.

Akwesasne karennaroron.

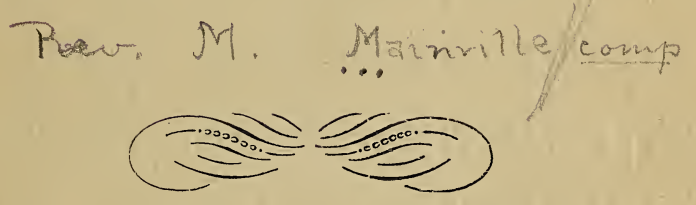

\section{TIOHTLAKE}

Tehonaristorarakon E. SEnekar NOK RoIEnHA Pingen Genteal and su 1890 


\begin{tabular}{|c|c|c|c|c|c|c|c|c|}
\hline 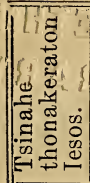 & $\mid \begin{array}{l}l n \\
1 \\
0 \\
0 \\
0 \\
0 \\
\vdots \\
\vdots \\
z\end{array}$ & 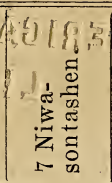 & 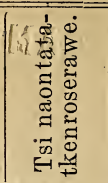 & 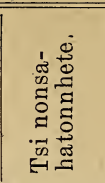 & 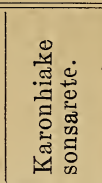 & 焉离 & $\left|\begin{array}{c|}5 \\
0 \\
0 \\
0 \\
0 \\
0 \\
3 \\
4 \\
4\end{array}\right|$ & 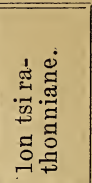 \\
\hline $\begin{array}{l}1891 \\
1892 \\
1893 \\
1894 \\
1895\end{array}$ & $\begin{array}{c}d \\
c \mathrm{~b} \\
\mathrm{~A} \\
\mathrm{~g} \\
\mathrm{f}\end{array}$ & $\begin{array}{ll}25 & \text { Jan. } \\
14 & \text { Febr. } \\
29 & \text { Jan. } \\
21 & \text { Jan. } \\
10 & \text { Febr. }\end{array}$ & $\begin{aligned} 11 & \text { Febr. } \\
2 & \text { Mar. } \\
15 & \text { Febr. } \\
7 & \text { Febr. } \\
27 & \text { Febr. }\end{aligned}$ & $\begin{array}{l}29 \text { Mar. } \\
17 \text { Apr. } \\
2 \text { Apr. } \\
25 \text { Mar. } \\
14 \text { Apr. }\end{array}$ & $\begin{aligned} 7 & \text { Mai. } \\
26 & \text { Mai. } \\
11 & \text { Mai. } \\
3 & \text { Mai. } \\
23 & \text { Mai. }\end{aligned}$ & $\begin{aligned} 28 & \text { Mai. } \\
16 & \text { Jun. } \\
1 & \text { Jun. } \\
24 & \text { Mai. } \\
13 & \text { Jun. }\end{aligned}$ & $\begin{array}{l}27 \\
24 \\
27 \\
28 \\
25\end{array}$ & $\begin{array}{rl}29 & \mathrm{Nov} \\
27 & \mathrm{Nov} \\
3 \mathrm{Dec} \\
2 \mathrm{Dec} \\
1 \mathrm{Dec}\end{array}$ \\
\hline $\begin{array}{l}1896 \\
1897 \\
1898 \\
1899\end{array}$ & $\begin{array}{l}\mathrm{c} \\
\mathrm{b} \\
\mathrm{A} \\
\mathrm{g}\end{array}$ & $\mid \begin{aligned} 2 & \text { Febr. } \\
14 & \text { Febr. } \\
6 & \text { Febr. } \\
29 & \text { Jan. } \\
11 & \text { Febr. }\end{aligned}$ & $\begin{aligned} 19 & \text { Febr. } \\
3 & \text { Mar. } \\
23 & \text { Febr. } \\
15 & \text { Febr. } \\
28 & \text { Febr. }\end{aligned}$ & $\begin{array}{r}5 \text { Apr. } \\
18 \text { Apr. } \\
10 \text { Apr. } \\
2 \text { Apr. } \\
15 \text { Apr. }\end{array}$ & $\begin{array}{l}14 \text { Mai. } \\
27 \text { Mai. } \\
19 \text { Mai. } \\
11 \text { Mai. } \\
\text { 24 Mai. }\end{array}$ & $\begin{array}{r}4 \text { Jun. } \\
17 \text { Jun. } \\
9 \text { Jun. } \\
1 \text { Jun. } \\
14 \text { Jun. }\end{array}$ & $\begin{array}{l}26 \\
24 \\
25 \\
27 \\
25\end{array}$ & $\begin{array}{rl}27 & \mathrm{No} \\
3 \mathrm{Dec} \\
2 \mathrm{De}\end{array}$ \\
\hline $\begin{array}{l}03 \\
04 \\
05\end{array}$ & $\begin{array}{c}f \\
e \\
d \\
c b \\
A\end{array}$ & $\begin{aligned} 3 & \text { Febr. } \\
26 & \text { Jan. } \\
8 & \text { Febr. } \\
31 & \text { Jan. } \\
19 & \text { Febr. }\end{aligned}$ & $\begin{aligned} 20 & \text { Febr. } \\
12 & \text { Febr. } \\
25 & \text { Febr. } \\
17 & \text { Febr. } \\
8 & \text { Mar. }\end{aligned}$ & $\begin{array}{r}7 \text { Apr. } \\
30 \text { Mar. } \\
12 \text { Apr. } \\
3 \text { Apr. } \\
23 \text { Apr. }\end{array}$ & $\begin{array}{r}16 \text { Mai. } \\
8 \text { Mai. } \\
21 \text { Mai. } \\
12 \text { Mai. } \\
1 \text { Jun. }\end{array}$ & $\begin{array}{r}6 \text { Jun. } \\
29 \text { Mai. } \\
11 \text { Jun. } \\
2 \text { Jun. } \\
22 \text { Jun. }\end{array}$ & $\begin{array}{l}26 \\
27 \\
25 \\
26 \\
24\end{array}$ & \\
\hline $\begin{array}{l}8 \\
9 \\
0\end{array}$ & $\begin{array}{c}\mathrm{g} \\
\mathrm{f} \\
\mathrm{e} d \\
\mathrm{c} \\
\mathrm{b}\end{array}$ & $\begin{aligned} & 11 \text { Febr. } \\
& 27 \text { Jan. } \\
& 16 \text { Febr. } \\
& 7 \text { Febr. } \\
& 23 \text { Jan. }\end{aligned}$ & $\begin{aligned} 28 & \text { Febr. } \\
13 & \text { Febr. } \\
4 & \text { Mar. } \\
24 & \text { Febr. } \\
9 & \text { Febr. }\end{aligned}$ & $\begin{array}{l}15 \text { Apr. } \\
31 \text { Mar. } \\
19 \text { Apr. } \\
11 \text { Apr. } \\
27 \text { Mar. }\end{array}$ & $\begin{array}{l}24 \text { Mai. } \\
9 \text { Mai. } \\
28 \text { Mai. } \\
20 \text { Mai. } \\
5 \text { Mai. }\end{array}$ & $\begin{array}{l}14 \text { Jun. } \\
30 \text { Mai. } \\
18 \text { Jun. } \\
10 \text { Jun. } \\
26 \text { Mai. }\end{array}$ & $\begin{array}{l}25 \\
27 \\
24 \\
25 \\
27\end{array}$ & \\
\hline & $\begin{array}{l}\text { A } \\
\text { g f } \\
\text { e } \\
\text { d } \\
c\end{array}$ & $\begin{aligned} 12 & \text { Febr. } \\
4 & \text { Febr. } \\
19 & \text { Jan. } \\
8 & \text { Febr. } \\
31 & \text { Jan. }\end{aligned}$ & $\begin{aligned} 1 & \text { Mar. } \\
21 & \text { Febr. } \\
5 & \text { Febr. } \\
25 & \text { Febr. } \\
17 & \text { Febr. }\end{aligned}$ & $\begin{array}{r}16 \mathrm{Apr} . \\
7 \mathrm{Apr} . \\
23 \mathrm{Mar} \\
12 \mathrm{Apr} . \\
4 \mathrm{Apr} .\end{array}$ & $\begin{aligned} 25 & \text { Mai. } \\
16 & \text { Mai. } \\
1 & \text { Mai. } \\
21 & \text { Mai. } \\
13 & \text { Mai. }\end{aligned}$ & $\begin{array}{l}15 \text { Jun. } \\
6 \text { Jun. } \\
22 \text { Mai. } \\
11 \text { Jun. } \\
3 \text { Jun. }\end{array}$ & \begin{tabular}{l|}
25 \\
26 \\
28 \\
27 \\
26
\end{tabular} & \\
\hline & $\begin{array}{c}\mathrm{b} \mathrm{A} \\
\mathrm{g} \\
\mathrm{f} \\
\mathrm{e} \\
\mathrm{d} \mathrm{c}\end{array}$ & $\begin{aligned} 20 & \text { Febr. } \\
4 & \text { Febr. } \\
27 & \text { Jan. } \\
16 & \text { Febr. } \\
1 & \text { Febr. }\end{aligned}$ & $\mid \begin{array}{cc}8 & \text { Mar. } \\
21 & \text { Febr. } \\
13 & \text { Febr. } \\
5 & \text { Mar. } \\
18 & \text { Febr. }\end{array}$ & $\begin{array}{r}23 \text { Apr. } \\
8 \text { Apr. } \\
31 \text { Mar. } \\
20 \text { Apr. } \\
4 \text { Apr. }\end{array}$ & $\begin{aligned} 17 & \text { Mai. } \\
9 & \text { Mai. } \\
29 & \text { Mai. } \\
13 & \text { Mai. }\end{aligned}$ & $\begin{array}{l}22 \text { Jun. } \\
7 \text { Jun. } \\
30 \text { Mai. } \\
19 \text { Jun. } \\
3 \text { Jun. }\end{array}$ & $\begin{array}{l}24 \\
26 \\
27 \\
24 \\
26\end{array}$ & \\
\hline & $\begin{array}{r}\mathrm{b} \\
\mathrm{A} \\
\mathrm{g} \\
\mathrm{f} \text { e } \\
\mathrm{d}\end{array}$ & $\begin{aligned} 23 & \text { Jan. } \\
12 & \text { Febr. } \\
28 & \text { Jan. } \\
17 & \text { Febr. } \\
8 & \text { Febr. }\end{aligned}$ & $\begin{aligned} 9 & \text { Febr. } \\
1 & \text { Mar. } \\
14 & \text { Febr. } \\
5 & \text { Mar. } \\
25 & \text { Febr. }\end{aligned}$ & $\begin{array}{r}27 \text { Mar. } \\
16 \text { Apr. } \\
1 \text { Apr. } \\
20 \text { Apr. } \\
12 \text { Apr. }\end{array}$ & 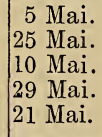 & $\begin{array}{l}26 \text { Mai. } \\
15 \text { Jun. } \\
31 \text { Mai. } \\
19 \text { Jun. } \\
11 \text { Jun. }\end{array}$ & $\begin{array}{l}27 \\
24\end{array}$ & \\
\hline $\begin{array}{l}28 \\
23 \\
30\end{array}$ & $\begin{array}{c}\mathrm{c} \\
\mathrm{b} \\
\mathrm{Ag} \\
\mathrm{f} \\
\mathrm{e}\end{array}$ & $\begin{array}{ll}31 & \text { Jan. } \\
13 & \text { Febr. } \\
5 & \text { Febr. } \\
27 & \text { Jan. } \\
16 & \text { Febr. }\end{array}$ & $\begin{aligned} 17 & \text { Febr. } \\
2 & \text { Mar. } \\
22 & \text { Febr } \\
13 & \text { Febr. } \\
5 & \text { Mar. }\end{aligned}$ & $\begin{array}{r}4 \text { Apr. } \\
17 \text { Apr. } \\
8 \text { Apr. } \\
31 \text { Mar. } \\
20 \text { Apr. }\end{array}$ & $\begin{array}{rl}26 & \mathrm{M} \\
17 & \mathrm{M} \\
9 & \mathrm{M} \\
29 & \mathrm{M}\end{array}$ & $\begin{array}{l}3 \text { Jun. } \\
16 \text { Jun. } \\
7 \text { Jun. } \\
30 \text { Mai. } \\
19 \text { Jun. }\end{array}$ & $\mid \begin{array}{l}26 \\
27\end{array}$ & 1 \\
\hline
\end{tabular}




\section{IERENNISAKSTHA.}

Iontatiatataas tha ............... lakowentaon akohasera....... Takwentenr ....

Eksa okon kenha iontatiata. Iákòwentaon akorenna....... Tsinatenhahnekenton we ..... Takw. Rotiiatatokentison.... " Wari aorenna............. "Awentokon. ............... " Iakawentontietha....... " Ion tatakenroserastha " Kowa........................ " Orennakaion .............. " Awennenhawikeha ... “ Anen ......................... " Dumontneha .............. " Awentokon sh........... Awentokon iokaren re........... Niio ise Sew enniio ............... Rosennaiens A wentokon ion terennaien... "، Ienen rinekenstha ....... "Tekaronhianekenneha

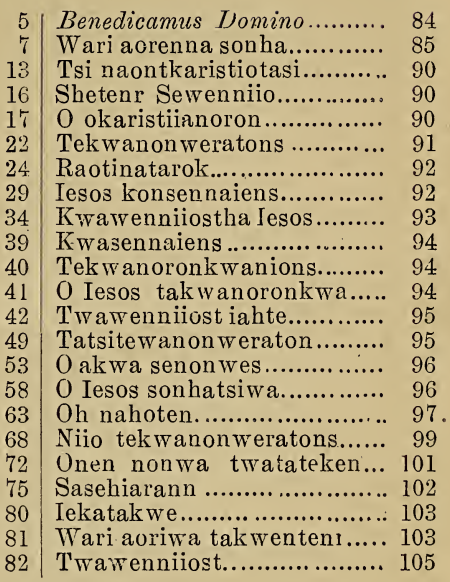

II

Aietikonnienstake ............ 233 Aionkwanikonhranonwaks.. 163 Akwekon iokenrat............. 19 Akwekon tetwariwak......... 11! Akwa Iesos..................... 141 Akwa ioriwatsanit.............. 230 Akwa tsini ionwesen.......... 137 Akwa wakeronhiaken......... 194 Antre saiatatokenti........... 228 Aonton ken..................... 193 Arekho te....................... 143 Areriia, areriia................. 123 Areriia. Ken wenniserison.. 174 Asiatarake ..................... 160 Asonthenne ..................... 113 Atonwesentsera.................. 149 Atsitwasennaien................ 170 Hetsitew anehrakwas........... 185 Hetsitwasennaien ............... 174
Hetsitwaw enniiost ........... 179

Iah nonwenton................ 206

Iahte sewatiaktanion.......... 123

Iak otaskat ...................... 145

Iesennakeraton ................. 210

Iesos akatonnhetston......... 182

Iesos karo kase ................. 110

Iesos ken tsiteron.............. 189

Iesos kwawenniiostha........ 196

Iesos oriwiio................... 146

Iesos rakeni akwa............. 186

Iesos rakeni sanikonh......... 153

Iesos raoriwa takwentenr... 235

Iesos rawer... ise............... 199

lesos rawer... rawer........... 197

Iesos Sewenniio................. 186

Iesos tekon... niawen......... 131

Iesos tekon... tesaton.......... 165

Iesos tekwanonweraton ...... 185 
Iesos tekwanonweratons...... 196

Iesos wahateseuni.............. 172

Iesos W. S. kawat .............. 224

Iesos W. S. Soasen............... 225

Ii nonwen tsiake................ 151

Ikehre akesennaien............ 206

Ikehre Wari..................... 216

Ionehrakwat ionwesen........ 178

Ionehrakwat o Sonkw......... 191

Ionehrakwat sonkwano....... 235

Ionkwanistensen................

Ionkwataskat.

Ionwesen konwaienha.........

Ionwesen ne......................

Ionwesen tatsi...................

Ioriwanehrakwat ...............

Iotsennonni Wari...............

Ise Iesos Sewenniio............

Iseke Wari......................

Ise Niio hetsienba

Ise o kwennakeraton ...........

Ise sewaien okonha.............

Ise sewaronhiakehronon......

Ise swaron hiakehronon.......

Ise Waria

Ise Wari Watere.................

Itewe twatateken...............

Kakwiriie........................

Kanatiio .........................

Ka nitewes ......................

Karo kase Iesos.................

Karo kase karo..................

Karonhiake.......................

Kasene ............................

Katke onte ..................... 141

Kenheion ....................... 18

Ken ire.......................... 179

Ken kaien....................... 182

Kenni karihoten................ 159

Ken renteron..................... 184

Kento........................... 183

Koniennitentase ................ 220

Konsennaiens................... 196

Kwasennaiens Saksarie....... 229

Kwasennaiens o Wari......... 217

Kwasennaiens Wari........... 218

Kweh sanonsanoron........... 200

Kweh swawatsiranoron...... 220

Ne nonwa twatatek........... 228

Ne nonwa wennis.............. 171

Ne ok ieskenha ................. 162

Niio hetsienha kanis............ 218

Niio hetsienha senis........... 202
Niio hiatatien

Niio Sewenniio................. 188

Nonwa ken wente.............. 194

Ohni kwaieren................... 169

O iawenniseranehrakwat.... 114

O Iesos konsennaiens.......... 185

O Iesos tekwanoronkwanions 197

0 ise ne ast.................... 18

$\mathrm{O}$ ise swakwekon.............. 124

Okaristiianoron ................ 190

Okti............................. 115

Onen ietinonteratie............ 127

Onen nonwa rawentawen.... 230

Onen nonwa swatorisen...... 227

Onen onkwe .................. 112

Onen swakatatrewaton....... 150

Onen wahniakenne ............ 143

Onen watennitoktane ........ 222

O Niio kenwente................ 134

Onka onte.................... 119

Onkwe rotonhon N. R. Ii..... 117

Onkwe rotonhon $\mathrm{N}$. R. Ne.... 125

Onkwe rotonhon N.R. Tew. 121

Onwari ionkwaienha.......... 215

Onwentsiakon ................. 21

Oriwakon ....................... 157

O swanatiio ..................... 146

Otsiratokenti ................... 178

O Wari kwenmakeraton...... 207

O Wari, o Wari................ 220

O Wari senistenseriio.......... 208

Rakiakisáks .................... 148

Raonekwensatokenti........... 164

Raswe........................... 229

Ratasetha ....................... 190

Rawenniio hetsiswaien........ 211

Rawenniio sewatenro......... 153

Rawenniio sonkwari........... 140

Riiatisaks ........................ 187

Rinonwes ......................... 128

Risentenni .................... 163

Ronatsennonni. ............... 204

Ronnbek Iesrs ionnhek....... 164

Ronnhek Iesos taonken. ‘.... 129

Ronnhek sonkwa .............. 136

Rotonui N. R.................. 121

Sahatiken ....................... 128

Saonkinekwahestanion........ 157

Sasakoiatisa .................... 223

Sasehiarann Sew ............... 133

Satkon Niio.................... 175

Satkon Sti. kwennitha........ 175

Satkon Sti. satekat .......... 177 
Satkon Sti. sheiatienhas..... 233

Satkon Sti. takwa............. 177

Satkon takwatekwaten....... 176

Satsteniaron..................... 110

Seniaken......................... 127

Senistenseriio .................. 201

Sennakerat .................... 118

Sewariwiioston nahoten...... 161

Sewariwiioston okon........... 180

Sewaronhiakehronon.......... 120

Sewatka nohneka.............. 226

Sewatka onen ne............... 217

Sewenniio akwa............... 178

Sewenniio ken... ne........... 144

Sew enniio ken... tsi.......... 145

Sewenniio kwanonwes........ 138

Sewenniio onen................. 147

Sewenniio takwatsen......... 190

Sewenniio takwentenr.......... 150

Sewenniio tsi iakionnhe...... 160

Sewenniio tsi konnhe......... 146

Sewenniio tsinahe.............. 149

Shetenr Sewenniio.............. 234

Shotonnheton Iesos............ 173

Sirisare sewaienha.............. 167

Sirisare waeriwihewe ........ 167

Sonhaa konniahesen........... 212

Sonhaa Sewenniio.............. 150

Sose roiatatokenti.............. 224

Sose tekonnonweratons....... 223

Sose tekwanonweronnions... 224

Sotsi notiake.................. 151

Sotsi wakataskat.............. 187

Swakwekon ..................... 191

Swanikon hrarak ................. 137

Swannhohatokenti .............. 174

Swaronhiakehronon sotsi..... 113

Swaronhiakehronon tetsi..... 143

Swaskon tako.................... 151

Swatatiesatanions............... 142

Swaton tek...................... 114

Taetew ataten on wehak......... 168

Takitenr o Iesos.................. 167

Taùitenr Wari.................. 215

Takwaien okon................. 213

Takwaien okonha.............. 203

Takwahrori . ..................... 125

Takwehiarak .................... 179

Takwentenr..................... 219

Tesatsnent..................... 179

Taswatotek...................... 192

Teiakokwatasehonnes .......... 227

Tekonnoronkwanions .......... 201
Tekwanonweraton............ 170

Tekwanonweronnions......... 133

Tekwanoronkwanion.......... 166

Teswaiatoretak ................ 20

Tetewaiena..................... 216

Tetewariwak tionkwe........ 132

Tetewariwak tsini............. 181

Tetewariwakwas .............. 231

Tetewasen totak................ 135

Tewakwekon ................... 141

Tewanenton ne Kateri........ 232

Tewatsennonni ................. 231

Thone.......................... 115

Tiotkon ........................ 152

Toni iotsennonniat.............. 189

Tosa tsiron.................... 150

Tsiatahonsatat keuto......... 19

Tsiatahonsatat tsionkwe...... 198

Tsiatanoron ...................... 219

Tsiaton tek...................... 158

Tsini ionkwaiatawens ........ 169

Tsini seniseriio................ 139

Tsi nonwa....................... 234

Tsionkwe Niio.................. 122

Tsionkwe Rawenniio.......... 134

Tsionkwe tosa................. 142

Tsi rohasen.................... 181

Tsitenr nisa..................... 200

'Twakwekon tetewari.......... 118

Twakwekon tewatis............ 183

Twanikonbrarak............... 184

Twasennaien ................... 221

Twatateken tetewa............. 137

Twatateken tetsitewa......... 225

Twateken ........................ 134

Twatenro........................ 115

Twatiaken...................... 188

Twatonnharen ne ionkwa... 171

Twatonnharen tetwariwak... 130

Twaton tat...................... 120

Twatsennonni nonwa.......... 179

Twatsennonni twatateken... 170

Twatsteniaron.................. 118

Wahatonni..................... 112

Wahonwateweton.............. 126

Wakatsennonni................. 17

Wakenaktaien................... 202

Wakenistensen.................. 202

Wari seniienha................. 228

Wari tekonnoronkwanion.... 208

Watstarha..................... 147

Wenniseranoronson ............ 117

Wennitanoron kowa.......... 209 



\section{TEIERIW A K W ATHA}

\section{IONTATIATATAASTHA}

Tokat Sewexniro.-Sewenniio satahonsatat tsi tewakhenretha kanikonhraksenserakon, " etho akewennatirhene tsi tsiteron.

Satahonsatatsek tsi enkaterennaien, * enkoniemnitentaseheke.

* Tokat, Sewenniio, sotsi askahenhion tsi ionkwariwaneren, * onka, Sewenniio, aiekweni iahtaiakohteronseke taietane tsi tsiteron?

Nok tenhnon sananon natanitenrasera, wakonniahese, ${ }^{*}$ aseken saianerensera ionkwannhas akwaniahesenhake.

Ne sawennatokenti ionikonhrahniraton nakwatonnhetston, * akweriasakwekon wahiniahese ne Rawenniio.

Orhonkehne aiontasawen eniokarawe nieioha, ${ }^{*}$ ahonwaniahese ne Rawenniio sakoien okonha Israerronon.

Aseken iahtaontontsahate raotanitenrasera, * okti hawenniio naonsasakoskontako.

Raonha ensesakoiatokewe ne sakoien okonha Israerronon tsinikon ronwanikonhraksaton.

Asheion, Sewenniio, atorisenserakont, * nok teioswathekowa tiotkon taiakoswateke.

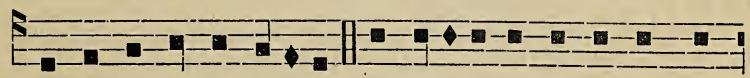

Enkontonnharen Niioke. Sewenniio Isi iakionnhe kon-

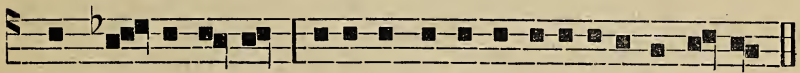

hien-ni - ten-ta-se * takitenr ni-ha tsini sataniten-ra-skon.

Iahtaontaieriwasaate tsini sanikonhriio, * ne se karihonnis sekon nonwa konniahesen.

Akwa tokenske wakeriwanerahakskon, * sasanikonhren niha tsini konnikonhraksaton.

Onen keriwaienteritsihon tsini wakeriwannhikon, *iahte watons skennen aonsakennontonnion. 
Iahte sanekherens tsini wakeriwaneren; "iken tiotkon teskanere othenon nikatieranions.

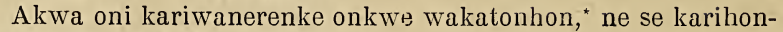
nis asteson katatkenhronnis.

Takitenr, Sewenniio, askiatohare niha, * nok enkkenni noniete tsi nenkiatakenratane.

Heren niha asatkarenrako, * eso tsi ionewarat tsini konkwetaksen.

Satsianerenst niha akwatonnhetston kenha, * tsini saierenne saonknekoserawe.

Tosa nonwenton takiatonti : * tiotkon tenhnon takwatsterist.

Enseskionweskwaten, Sewenniio : * akenikonhrake enseriwahnirate kariwiio.

Ethone akwa skennen enskennontonnion, * aseken iahtenseskiataswenseke.

Skariwat ok iesanikonhraiewentotha, * tsini iakonikonhranonwaks tsini iakoriwanerer.

Takitenr kati, Sewenniio, * ne tiotkon katstarakwa tsini konnikonhraksaton.

Akwa takatonnhakarias nonwentsiake, "toha onte orit aontokten tsi konnhe.

Aonhaa wakeriwiios tsi wakeriwaneren, * ketsanis tiotkon enskat eh naonsonkiatawen.

Ionehrakwat tsina tkaterientakari, * okse nonnakiere aonsakonnikonhraseronni.

Ne kati karihonnis tiotkon konhiatisaks, ' iahte katorisens tsi konhiennitensase.

Tiotkon katiken, Sewenniio, enskewennonti : * iah ken taonton askwatkatho ne nostonha oni.

Kariwaneren iotiesaton tsi konnhekwe, * karonhiakenhon ok nonwa tsiotatenron.

Are sane akwa sewakiewentahonne, * nok kennaheha sonkrane ne karonhiakenhon.

Satontek kati, Sewenniio, tsina tekasenthos, * takitenr konhiennitentase, tontakwatontats niha. Asheion...
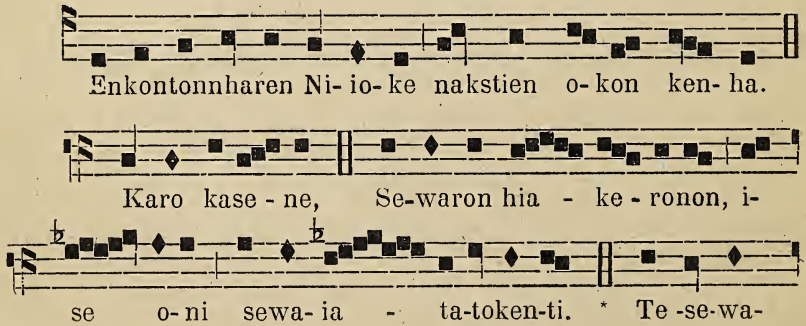


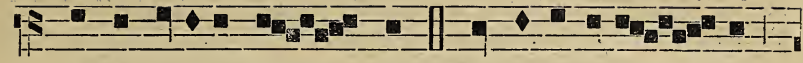

iatak raotonnhets - ton * Het si sewawi - ra

F-二 -

karon-hia-ke ne lia - wenni-io. Ashe-ten-re.

If

Ie - sos, sa-nekwen-sa seskontakwaton nok tsi tsi-

彭-

teron karonhia-ke a-shena - ktari - kwa - se.

S-1

Tese- waiatak raotonnhet - ston Asheion

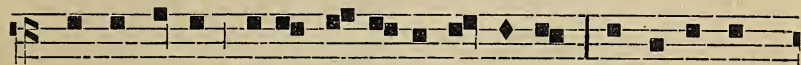

Sewen-ni-io ato - ri - sense-ra-konte nok te-io-swa-

S

the kowa ta - iako - swathe- ke. Hetsi- se-wa...

\section{IAKOWENTAON AKOHASERA.}

IONT. -2

T 6.

-

-

Sashetenr Se - wen - niio ia-kotiak-ta-ni

S2-10 -

tsi tie - seronniatha iakorı wa-neren ok sa ok sa-

Se-

sheia - ton-ta-ko she-ia-ti - niont karonhia - ke. 
$s$

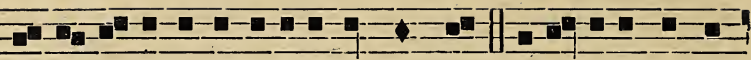

$\mathrm{Ni}$ - io i - se Sewenniio tsi iakionnhe Aie - sasen-na-ien

E2 -

karonhia - ke Takwatontats ni-sa ne kwen-ni - ta,

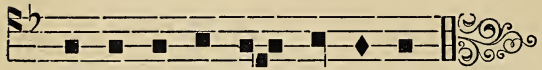

sonhat-si- wa kwa-nia-he-sen. Sashelenr...

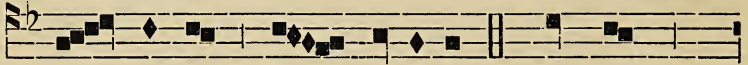

Ta - kwentenr Se - wenni-io. III. Kris-tos

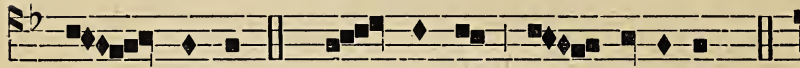

ta - kwentenr. III. Ta - kwentenr Se - wenni-io. II.

S2-二

Takwentenr Se - wen-ni- io.

T. 2. Se- wenni-io Ie - - sos she - tenr ni -

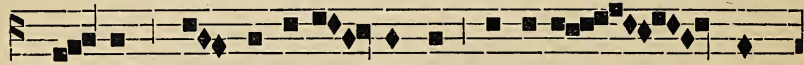

- sa ia - ko-riwi - io-ston ne ieron - - hia-

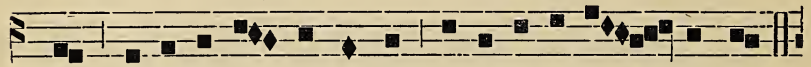

ken tsi tetiese - ron- nia-tha tsi iako-ri-wa - ne-ren.

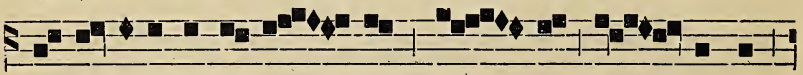

Karon-hiake sheia-ti - niont e - tho tsi . nenwe

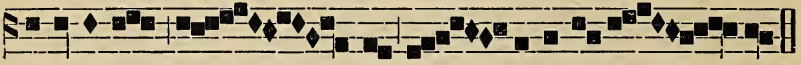
sheiatsennon-nia - ten sa - taniten-ra - - skon. 
T 8. \$二 Sasa-ni - konrhen, Sewenniio o thenon iako-

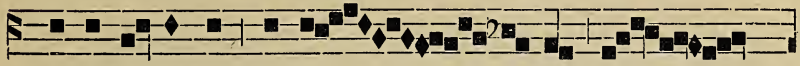

ri-wane- rakton ne ia $\quad$ - $\quad k w a-t a$

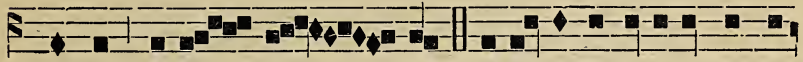
teken okon ken - - ha. leia - tarak rotitiohkwato-

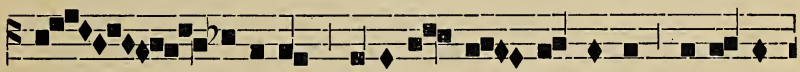
ken - - like ratiron - hia - keli-ronon ne as- te-

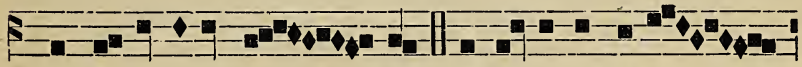
son a - iesasen-na - ien. Asheion iahte ka

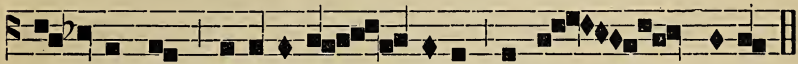
konte a-to-risen - se-ra karon - hiake.

T 1.-2.

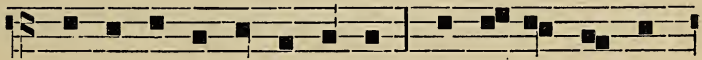

Ne iawen-ni - se-ra -tsanit Tenhontia - to - reIe sos ten-ha- ia - to- re-te Ka-ri-wi - io ka-

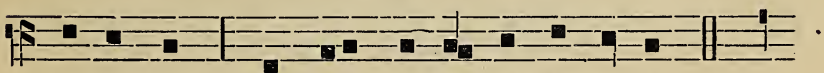

te nonkwe

Tsi-nèn - swaton wenlsia-ri-si.

ri-wak-sen

$\mathrm{Ne}$ tsi - ni ia - ko-ie - ranion.

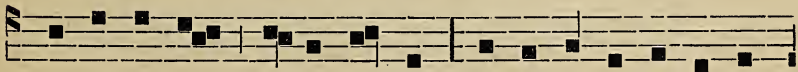

Enhon-tewen - na-ienton - we Ne ra- ti- ronhia- kehro-

Ok sa ok tsi en ion ta - ti Akwekson ia-ko-wenta-

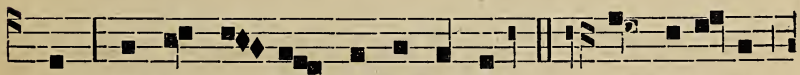
non Enhon- ni - ron
satsiatonnhet.
Enhro-ta- si

on Tenieta-ne tsi ithres le-sos.

Én-hatro-ri 


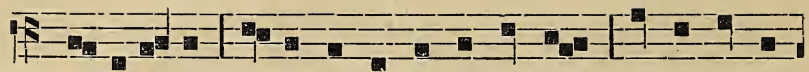

tsi kahiaton Ne akwekon en-watro-ri Tsi-ni ia- koakwa tsi ni Ka-ie-rha ka-ia-ton-se-ra lah enskat ta-

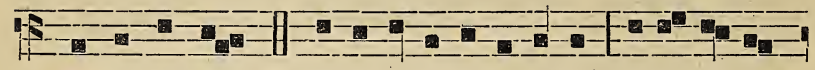

ieren nonkwe. Tokat wake-ri-waneren Etho-ne enho-ri wenre. Enwa-ki tenta-ne onwe Iah onka ton-
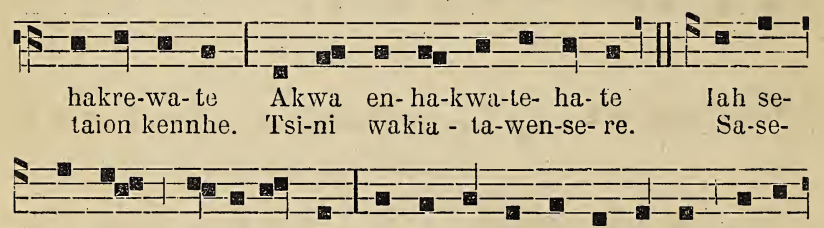

ta-te - tio-tatemne Iahta ion-te-rì - wa-te-ko Enionhiarann i-se Ie-sos Ne tsi-ni sa-ronhiakenhon Takia.

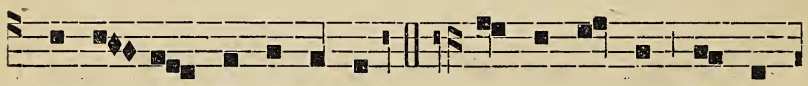

tsente a - kwekon nonkwe. To-sa wahton ta-kita kennhen ken si-seskwe. Seskwakta-ton nonwen-

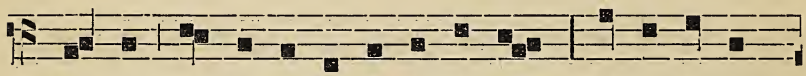

ten-ron E - tho-ne saskwenhe-ia-se Ne te-ka-ien-

tsiakon Iah-te kakon-te tsinen-we Ta-onkwatonn-

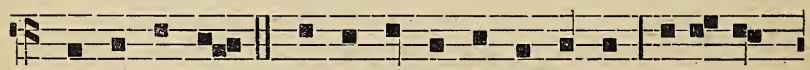

tannha-re-ke. Karon-hia-ke ienskia-te-we Ne en-seha-ka-ri-hon. lo-nehrakwal ni-kia-to-ten E-so wa-

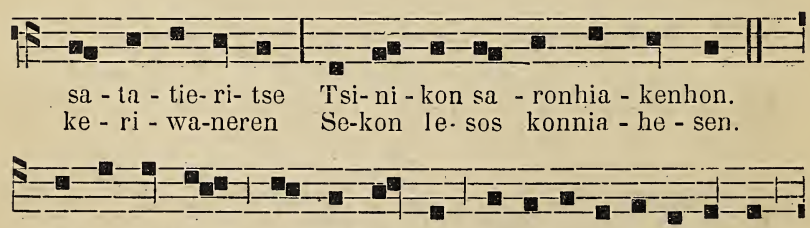

To-sa notkon - se-raksenskwa Naon-ta-tewen-ni-io-ste

To-sa ni-sa ia-kwa-ri-sko Ne rononwentsiakonronon 

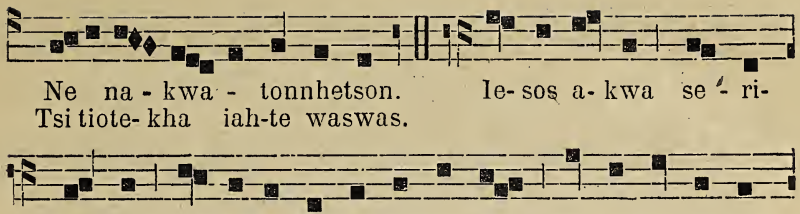

waswens A-kwekon tsi-ni wa-kie-ren 'Ta-ki-tenr Isi nen-

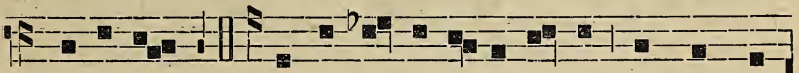

ki-he-ie. Ne ia-wen-ni-se-ratsa-nit Tsi nentsion-

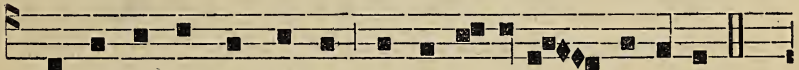

tonnhe-te nonkwe kenha Te-sa-ko-ia-to - re-ta-ne.

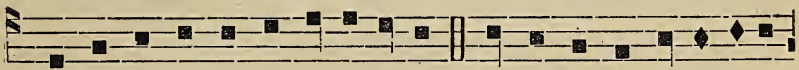

Iahten tesa - ko-ten-ra-ne Iesos. Shetenr Iesos Sewenniio,

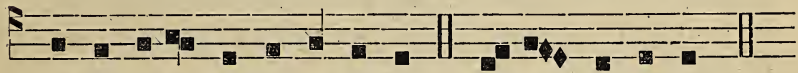

Iakwa-ta-te-ken okon kenha. E- tho na-ia-wen.

T 2.

Se-wenni-io, lesos Kristos, ie - senna - ke - raton,

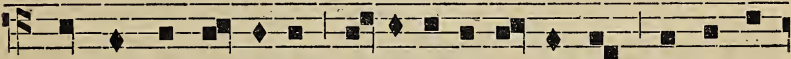

shetenr ka-ronhiake ia-ko-ri-wi-ioston-ne wahonte-

S-

tsi-ra - te o - tsira - ke iotsi-ra - tsa- nit, se-ra-kef

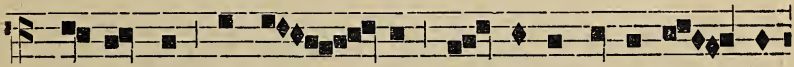

io-tak-sen ne io - ra-nen-taken a-ko-tonn - he-

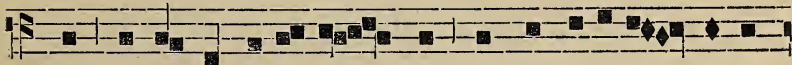

tston; saton-tek teionsen - thos liotkon iesaia - tisaks 
F=-

tsini iesa - nonwes, iien - hre a - sakwaken

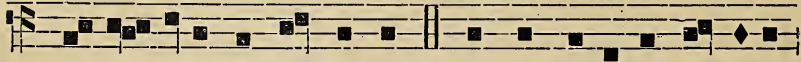

Ra-werı-ni-io tsi ia-kionnhe. * Shetenr ka-ti Sewen-niio

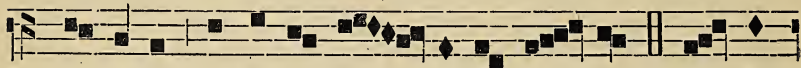

ok - sa-ok sashe-ia - ton - ta-ko ni - sa. Son - ha-

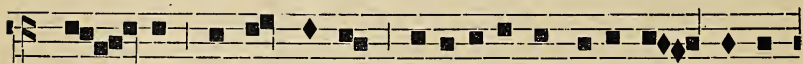

tsi - wa Sewen-ni-io. iesaniahesen ne shehre - watha

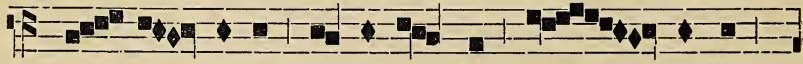

son - ha - tsiwa ie - sa non-wes, ne - sa-ne

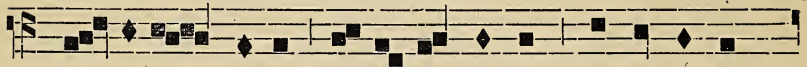

io - tsanit tsi-ni ia-ko-ia - tawens tsi io- te-kha

F=

ne i - se sa-te - ka-toll. She...

S-口-

Saia- ta-token -ti. II. Sa-ia- ta-token-ti Sewenni-io tsi

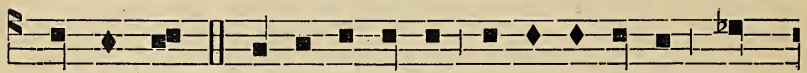

iakion-nhe. Karonhiakwekon onwentsiakwekon sa-

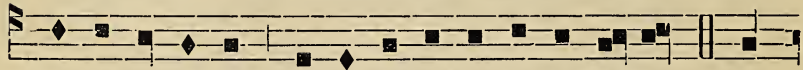

ia-ta-nehrakwat ie-sa-sen-naien karon-hia-ke. Ron-

E- -

wanenton ne thare ra-sen-nakon Rawenni-io ronwa-

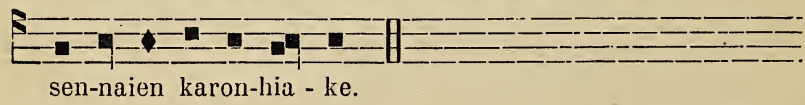




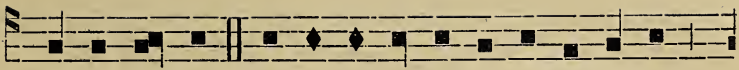

Ie-sos Kristos se-ri-wahtontha ka-ri-wa-neren

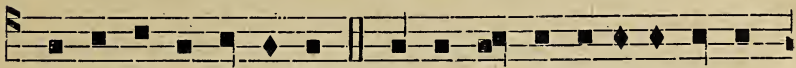

shetenr ia-kowen-ta- on. II. Ie-sos Kristos se-ri-wahtontha

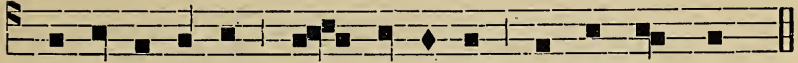

ka-ri-wa-neren she - ia-tiniont ka-ron- hia - ke.

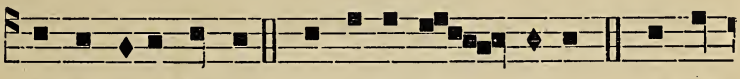

Sewen-niio shetenr ne iakowen - ta-on * A-iont

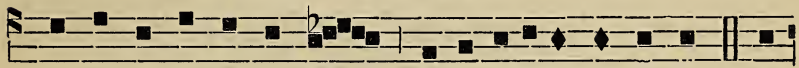

sennonni iahte kakont tsini sataniten- raskon. Ia-

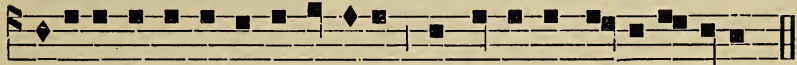

kiia-te-rennaien-ni Sewenniio takwatontats asheion nisa.A...

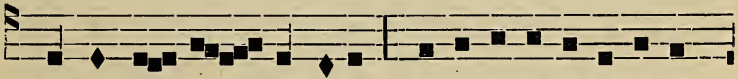

Takwentenr Se . - wenniio ia-ki-ia-teren-naien-ni

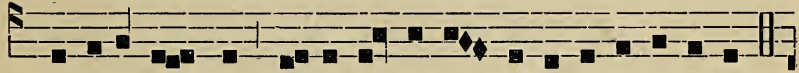
ionkiia-ton - tion, ia- kotia - ktani tsi te-tie-seronniatha

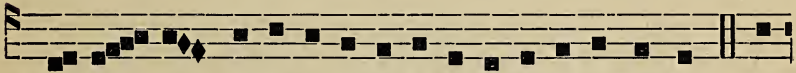

* Sashe - tenr sasheia-tonta-ko iakwata-teken kenha * A-

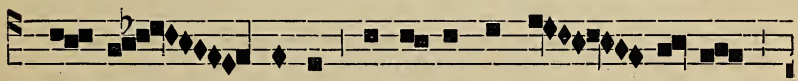

she-ia ra-ne tsi i - se sheia - - ti - son

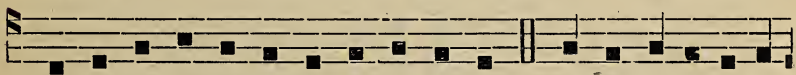

ia- kotent nonwa sheien okon kenha. Akwa ie-sa-ia-ti- 


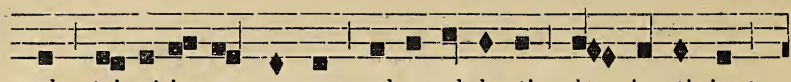

saks tsi-ni ie-sa-nonwes ok sa ok ka-ti shie ia-tiniont

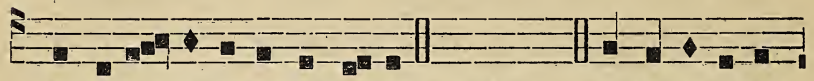

tsi tesi - teron karonhia- ke. " Sashe...' Teska-nerak tsi-

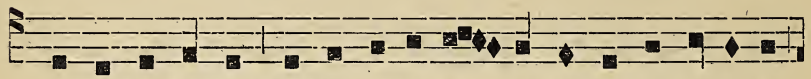

ni ie-ronhiakens shetenr nisa a - satka-we shehrewatha

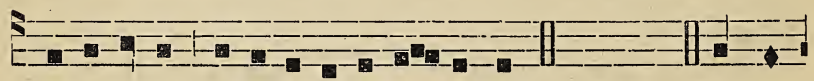

ie-sahtoron karonhiake iahte kakont. * Asheia... Kwania-

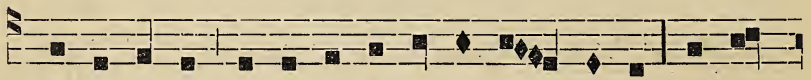

hesen ka- ti hetsa-ton-tats Iesos n'Hetsien-ha iakwa-

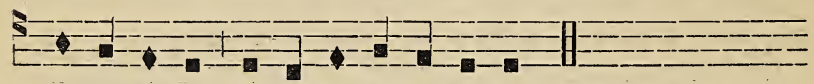

wennakwekon tsi- ni kwenniten-ta-se. Takwentenr...

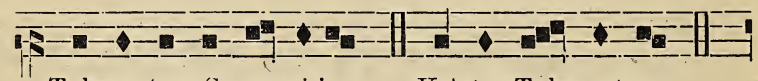

Takwentenr Sewenni-io. Kristos Takwentenr.

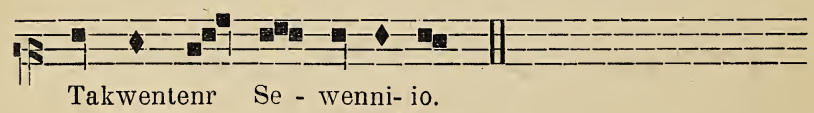

[...in lenlationem]. Sed libera nos a malo.

[...inferi.] Erue Domine animam ejus (tokal tohka) animas eorum.

[...in pace. $]$ Amen.

[...orationem meam.] Et clamor meus ad te veniat.

[...vobiscum.] Et cum spiritu tuo. ...Amen.

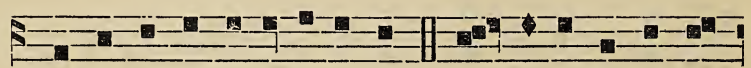

Karonhia-ke iesa-iaten-ha ra - tiron-hia-kehro- 


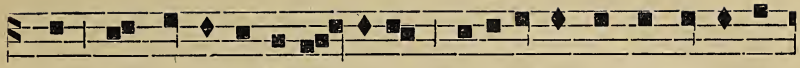

non nok ta-iesa-te-ra-tana kari-wiioston ronwa-tirioh-

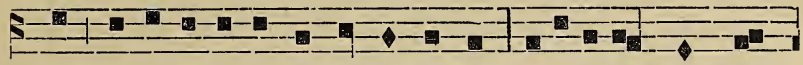

ton asat-katho ne ionwesen kowa, rao-ti-tiohkwa-kwe-

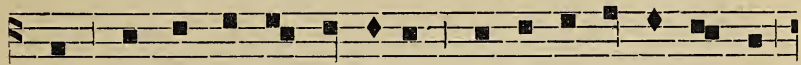

kon ron-watkathos ne Niio a-ie-sannhotonkwa- se

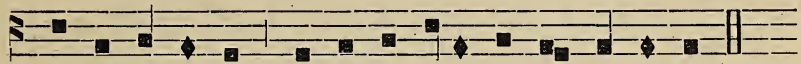

nok iahte kakont tsinenwe aeswa-kwe-konha-ke

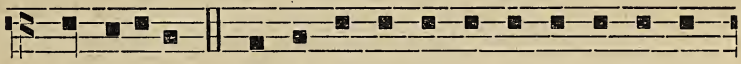

Akwa i-i Tetsi-te-wanonwe-raton ne Rawen-

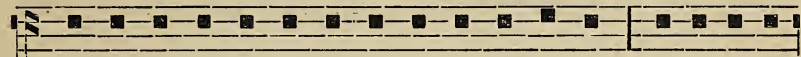

ni - io ne ronwasen-naiens I-sra-er-ro-non: A-seken ta-

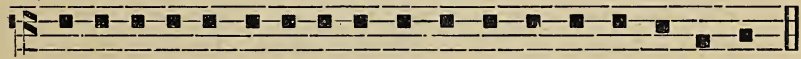

sako-ia-torenne ra- otiokwlıa naon- sa- sa-koskon- ta-ko.

Raonnilıatie tsi nontawe naetewatsennonni, * raononskon Tafit raonkweta tioiakenhon.

Tsini thotati naonnise, " ronwawennakarataton ne rotitokensehakwe rotiiatatokenti tsionkilırori.

Ensesonkwaiatatako, ne ionkinaskwaientakwe ne ionkiswens.

Naonsasakonatonhase raotanilenrasera ne ietini okon kenha, * aseken iahte shonikonrhenhon tsini sakorharatstenni.

Akwa roriwahniraton rowennentase hetsitewanikenha $A$ bra. ham, * tsi ienwawe ensonkwentenre.

Aontiesenhake iataonsaietitsaniseke ne ionkikenrenseronnihakwe * skennen tenhnon aonsatsitewaseronnienniheke.

Aontaionkwaierike aiakwaiatatokentonhake, * atsitewehiaraseke niate wenniserake.

Ok nise, seksaa, liawenniio rowennenhawe eniesanatonkwe, * aseken ise enshente ne Sonkwawenniio enthianonteratie natshahaseronnien ne raonha. 
Nok asherihonnien ne raotiohkwa tsinaieiere ne karonhiake aiontsennonni * nentsioterakewen nakoriwanerahaksera.

Ne raotanitenraserakowa ne Sonkwawenniio * tsi ehneken nitháwenon nasonkionwentsiorenne.

Tasakoswateten nasatakon iakonnhe, iawet kenheiatne ientskwahere * nasonkwaharate tsini ieiothahinon ne skennen konwaiats. Asheion...

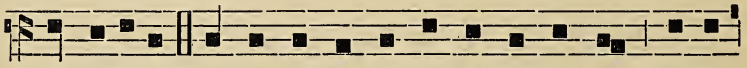

Akwa i-i kheionnhetha nok sekheionnhe-tha, netia.

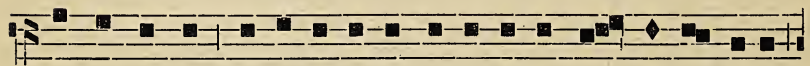

kawehtakon noko-ni a-ia-kawenheion en - iakonnheke,

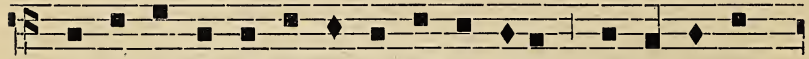

ne iakonnhe nok tiaka- wehtakon iike iahtiaontkon-

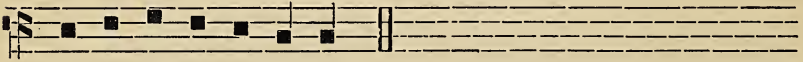

takwe nen-ia- i- he - ie.

[...eleison.] Christe eleison.

[...tionem $]$ Sed libera nos a malo.

[...ei (eis) Domine.] Et lux perpetua luceat ei (eis).

[...in pace.] Amen.

\section{EKSA OKON KENHA IONTATIATATAHANE.}

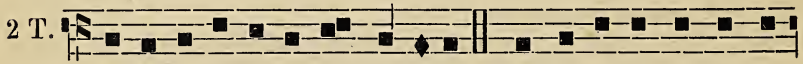

Ahonwasennaien Rawen niio Swaksa okon kenha te-

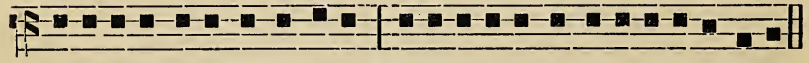

tsisewanonweraton ne Niio * raonhatsiwa tsini hoiatatoken-ti.

Nonwa iahte kakonte oni * ahonwasennanoronkwake raosennatokenti.

Onwentsiakwekon ienakerenion nonkwe * tahonwanonweraton ne Rawenniio. 
Aseken raonhatsiwa ieiotohetston tsini haiatoten * onwentsiakwekon karonhiakwekon oni.

Onka satehniiatoten ne Niio * Sonkwawennilo oktiwakwekon raiatananon.

Raonha ieiesas sakotsennonniatennis * saheto okenhrake ionteseres senha ok sakoriwakwatakwennis.

Wasakosennowanate ne iakotentonne * iesennowanenonton raotiohkwatokentike. A honwasennaien...

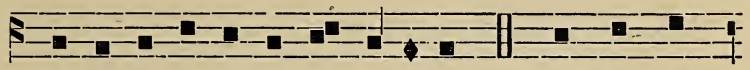

Ahon-wa-sen-na-ien lia-wenni-io

non- wa nok

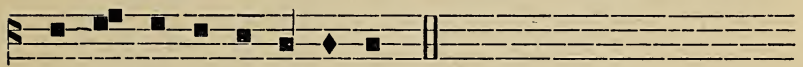

tsinen- we iahte ka kon-te.
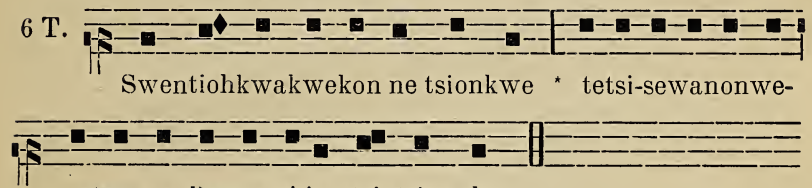

raton ne Rawenni-io tsi tsionnhe.

Aseken asteron sonkwentenranions * nok oni enwaton tsini sonkwarharatstenni. Ahonwasennaien...

\section{IAKOWENTAON AKORENNA SONHA.}

Wakatsennonni, toha akatkatho lahothenon tonsakaiotate Tsinonni henteron Rawenniio Akwa ahinoronkwake onwe. Iah othenon taonsakakweni Ostonha aonsonkenikonhraksen. Wakerhare ne nonwa katon[sen
[Akwa iah othenon sateionwe- Wakerhare tsi wakatiaktani Naiontkatho tsini kanaktiio Wakerhare toha enwaterisi $\{$ Ne karonhiake, iahtekakonte Ne nonwa wakenikonhrotakwas. [onwe

Etho akwa eniontonwesenseke. Onen kati wakatewentete Onweritsiake aoriwa sonha, Etho kati enhiienterhane onwe Nok enskat enskenikonhrarake, Rawenniio roiatanehrakwat Enwakati ne kieronke kenha. 
$\left\{\begin{array}{l}\text { Kenheion ne iotsanit kowa; } \\ \text { Tesewaiatoret kati nonwa. }\end{array}\right.$

Tesewakaneré nonwa

Ne ken iaiheionsere

Tiakotarihenseraris

leiatakwekon iowisto.

Sewatkatho tsi neniawen Onen sewatonnhoktane Akwa iahte kakonte Tsi eh nensewaiatawen.

Tsiniiot nakatsitsienne Akwa aionawateton: $\mathrm{Ne}$ teskiateriwatieren Kenheion konwanatonkwa

Kats, sariwanerahaksk on Satateniahesen kowa ; Tontasatkens tsi ieiati Ne iienskwe nonwentsiake.

Onen karontotserakon Raiatat tsiaten'o kenha Onen watetsiatekhasi
$\mathrm{Ne}$ satelsiatonwesonskwe. Iahtaionnise saiotken Nene raieronke kenha Tetsiatoret kati nonwa Ne sariwanerahakskon.

Atonwesentsera kenha Onen roiatontion onwe; Onen kati wahorane $\mathrm{Ne}$ ratennowentakwe.

'Tsiaken kati, nise sekon Sarıhonte onwentsiake; Ne nasatatiatakennha Naonsasatatrewate.

Ise iawet sathonrohon Kariwanerakserake Sotsi sakonnienstonhatie Ne waskaneks ne tsieronke.

Oh nensaiatawen nonwa Ahiatewentete Niio ? Iah ken onwentsiakon hronon Tahontatewenniioste?

3

Oh ! ise ne ase tsi tsionnhe, sewaiatahniron kowa, Tosa sewatateniahesen, aseken enwatohetste, Ensewatieren ok ohnaa, enswarane ne kenheion, Iahten taonseswakwenieseke, naeswatatiatakennha.

Iah sekon teioriwatoken, ne tsi tsionnhe onwentsiake. Ne ok enskat swawenniio, nonwa iahten eniorhene: 'Tosa kati sewatiesat, tsi nonwa sewarihonte, Sotsi kanoron onwentsiakon, ienswaiatanise?ene.

Tosa kati skennen sewentaf kariwanerakserakon, Aseken ne sotsi iotteron, iah nonwenton tensewaie, Iahten tensewanaktotake, nene sewaserentakon; Ise ensewatatkaronni, sewariwakowa enwaton.

Oh kenheion! sotsi satsanit, sekenrenseronnis, Iah othenon te setenrase, iahte satanitenraskon, Isehre : aionketsanike, aionkenikonhrarake.

Aiontatiatakennhaseke tsiakotatrewatonhatie. 
Ise, Sewenniio, takwentenr, takwaiatanonstat nonwa, Tosa aionkwasennieseke nene kenheionseraksen : Karo onsaiakwatatrewate, kariwiiose okonha Onsakwanikonhraseronniate aiakwenheiontiiohak.

Akwekon iokenrat Onowen nok iohetskat Tsinikon tewatkathos 'Tsi ietewes nonwentsiake ; Ionkwaiatahtontha, Itewehre ne ioianere Onwentsiake, ok eken kati $\mathrm{Ne}$ ok iotohetstonhatie.

Tsiniiot notsitsia Onwar wakatsitsionten, Tsi kati ni iosnore Nok iotsitsientaonhatie : Etho kati niiot onwentsiake Othenon sonha tewanonwes ; Ken ni kariwes Akwekon iohtonhonhatie.

Oriwakon kati

Iontateronhiakentha

$\mathrm{Ne}$ ok iakawesakon

Aiontonwesonniheke ;

Ok eken se tiotkon isinison

Tiakotoktani tsi ionwesen,

Iah se nonwenton

Taiakonikonhraierinn.

Niio Rawenniio,

Raiatakweniio

$\mathrm{Ne}$ tsini iawenserons

Tsi itewes nonwentsiake :

Enskat enthatati, nok ok saok Eniontsente onwe niakotsanit, Otsinonwa se

Ne tsini haiatanoron.

Tsiatahonsatat kento iakwatstarha Tsi tetieseronniatha;

Seskwaiatanikonhrenhon,

Takwaterennaienhas.

$\{$ Twatatenonkwe, onkwatenro

$\{$ Takwannhe iakwatiatatsas.
Oh onte neniawen

Entewatonnhoktane,

'J'sini ionkwatieniton

Ne kariwaksenskwe okon?

$\Lambda$ kaonha oni onwentsiake

Akwekon aienwenniioks,

Eniaiheie

Nahoten onte eniontste?

Tenhnon isewehre lse sewaskennhase,

$\mathrm{Ne}$ ok sewanontonnion

Kakatensera, kasenna,

Sewatenninonta sewatonnhetst,

Sewanoronkwa ne iokenrat,

Iawet sewentas

'Tsi nenswenheie enswaie.

Oriwakon oni

Iakonikonhrowanen,

Nok ne iesennowanen,

Nok iakoiatanehrakwat

Iahte iakokwenion onwentsiake

Aiakoriwiiostonhake;

Iahten tsi kati

Taiakoiatakennhaste.

Taonken iakotent

Iakonikonhranentaks

Ne kento nonwentsiake

Iah se ken taiontsennonni

Akwa raonha Rawenniio

Roiatanehrakwat, roianere :

Raonha kati

Atsitewanonwehake.

\section{5}

Sehiarak tsi ise takienha, Iontstarha ne sheienha;

Takitenr, aseken sanekwensa

Nok ii akenekwensa.

Takwannhe tsini ionkwaronhiaken Tiakohenretha tsiatatekenha, - 
Eso wasatstaren tsi wakiheie, Ok sa ok tsi sanikonrhenhon.

Tiatenro, tetiatatenoronkwakwe Wahonnise nonwentsiake; Satsienterenn tsi waktare, Katon takaterennajenhas.

Akwekon natakwennia Skonhiawi nonen sakiheie Runton hianiha nok sanistenha, Hianoha nok swatatenonkwe.

Ionkwaronhiaken nonwa,
Eso tsi ionkwakwisron

Atakwennia ionkwaiena

Ne iakiien okon akowenk.

Otsire nonkwanaktake Akwekon ne ionkwahere ; Ne iakionnhekon notsire, Iotarihen tsi iakwatonries.

Tsiatkatho tsini ionkwaronhiaken Tiotkon ionkwarhare naontkawe; Takwentenr, takwaterennaienhas Tsiniiot nenionswathe nonkwatontihets

6

Teswaiatoretak sewakwekon ne tsionkwe Sewasennowanens, ise oni sewentent Sewatkatho tsini wakiatawens, Ise oni etho nensewaiatawen.

Onen wakiheie sekon ase tsi konnhe, Iahte wakattoken to akwa nikonnhoten ; Kerhakwe se, sekon enionnise Tenhakiatoretane ne Rawenniio.

Tokenske wakatatenikonhratanionni $\mathrm{Ne}$ tsi wakatatewenniioston tsi konnhe Raonha se ne sonkwaiatison Akwa Rawenniio te iakonnhe nonkwe

Okstentsi kennhatens nonwa tsini wakieren Tsi wakatsteriston iontonweskwatha sonha Sase tiotkon ionkehretsiaronskwe ; Oriwakon nonwa ii akeriwa.

Wakatiesatanion noriwatokentison, Kheiatentoriaton ne tiakoriwaieri ; Onen nonwa iahte tsionkwatkathos Nakoiatatokenti okonha.

Otsinonwa sonha onen enkontekwisa Ne kieronke kenha ne sotsi kenonweskwe; Onentoha iahte skaieronni 'Tsini kiatolenne, okenra enskaton.

Heren sasewete, swatenroseraksenskwa, Ne takwanikonhrotakwen skennen tsi ikes ; Ise wahi akwa sewariwa

Tsi enhakiatonti nonwa Rawenniio.

Aonha kanoron tsini wakatontaton Tsinikon takwanikonhraten ne iotaksen : 
Onen nonwa tsiononwakte kowa

Akenikonhrakwekon tsi wakatontaton.

Aiawens tokenske aonkateweienton

Tsinahe sikeskwe ne skennen nonwentsiake

Watiesen naonkatsennonnihon

Nok nonwa tokat enkatkaronni.

Sewateweienton ne sekon skennen tsionnhe,

Tosa sewanisko tsi nensewatonnhokten,

Tosa enskwanakeraniheke,

Aseken ise oni enswatkaronni.

Onwentsiakon ne tiotekha

Iotsiratsanit onwe;

Etho iakwatetsiratha

Tsini ionkwaiatoron.

Akwa(2)iahte kakwen

Tsi nate iakwasenthos.

Ise twatateken kenha, Takwentenr, takwatkatho, Takwaiatakennha nisa. Akwa (2) ise ensewakweni Takwaterennaienhas.

Naonsaskwaskontako.
Tosa tesewanonhianik Sewanikonhriiohak Sewaterennaien oni, Ietsitenr ne iakotent.
Niakwatetsiratakwa.

Niio, satkatho n'Hetsienha

Tsi roien raieronke, $\mathrm{Ne}$ onsaskwaskontakwate $\mathrm{Ne}$ ok tsiakwaniahesen. Akwa (2) aionkieiaraseke $\mathrm{Ne}$ tsi iehasentakwa.

6

Swatatiesatanions, swariwanerahakskon Sewennontonnion ken wenniseranorons Nonwentsiake tsionnhekwen nioserakeha Sonkwawi ne Niio sonkwatenientense.

Iah se tetsiakawenniseraien noneshon, Aseken iakotenniseratiesaton :

Sakawenniserawine ne Rawenniio, Tiakokaras onwe iakotetsiraton.

Onen wahonnise onwentsiake tsisewes, lahta tekontitas ne sewennisera, Ionatentionhatie akwekon tsi sewentas, Akwa iah nonwenton taontakonkete.

Sewentorhakowa tsi sewariwiioston, Iah othenon te ken sewariwaneraks, Iahte sewatsanis naesewatkaronni, Oh kati naiawen naeswatsennonni? 
Ne awennisera atanitenrasera, Nonwentsiake tionnhe, tosa tewatiesat, Iosnore enwawe ne kahrewatatsera, Iahtiiaiehewe ensewententane.

Tewateweienton onwentsiake tsi tionnhe Iahta sonkwenniserawi Rawenniio, Iah nohnaken taionkwateweientonke, lahte kakont onwe noneshon wetewe.

\section{TSINATENHANEKENTONNWE TSINI IOSERES.}

T 7.
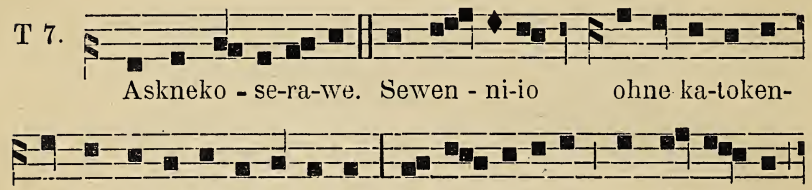

ti nok a-kiatakenra- la-ne. A-skia- tohare nok enken - ni

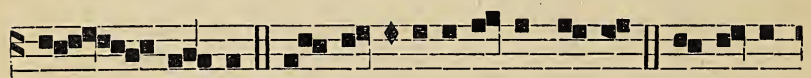

o - - nie-te. Se - wenniio taki-tenr ni-sa tsi-ni a-

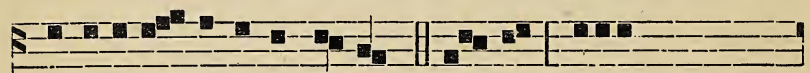

kwa satani- tenraskon ko-wa. A - hon - wasenaiien Roniha

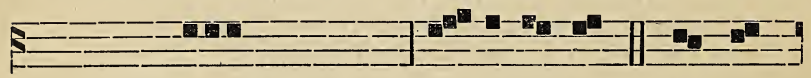

nok Roienka nok Rotkon Roia- ta - to-ken-ti.

Tsi- ni

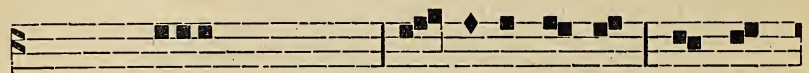

iohtonne tsinahe ethonaioh- ton - hake nonwa, tiot-kon

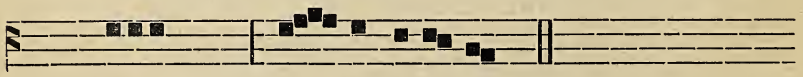

oni tsinenwe e - tho naia-wen.

...tuam (sh.) alleluia. R. Et salutare tumm da nobis (sh.) alleluia. ...meam. R. Et clamor meus ad te veniat. 
TSI NONSAHATONHETE.

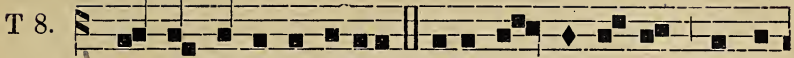

Wa-katkatho ohneka ioiaken - honha-tie onons-

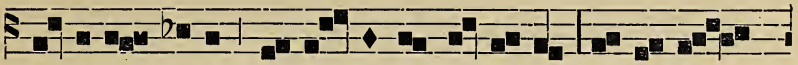

a-token - ti-ke tsi ka - weien-teh-takon a-re ri -

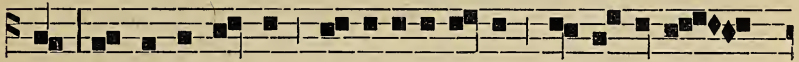

ia, nok tsini iakon ia-kotnekoserhon ne ken ohne -

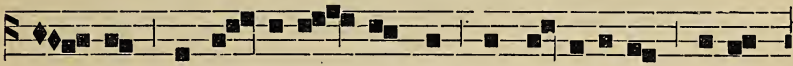

ka tsiako- tialon - ta-kwen nok en- iairon a-re-

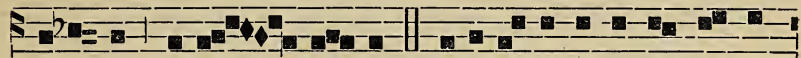

ri - ia. are - ri - ia. Atsitewahtonron ne Rawen-

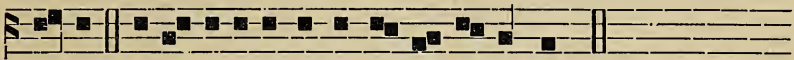

ni-io tsini hotanitenraskon iahte kakont. Ahonwasennaien.

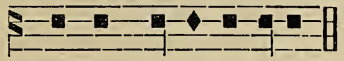

Et cum spi-ri-tu tuo.

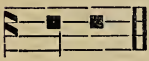

Amen.

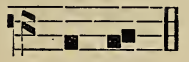

Amen.

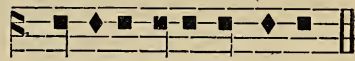

Glo-ri-a tibi Do-mi-ne.
(Wentanoron.)

Ha-be - mus ad Do-minum.

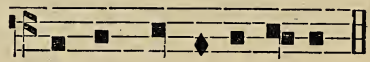

El cum spi-ri- tu tu- o.

\section{et justum est.
(Awentokon)}

Dignum et justum est.

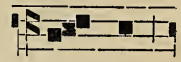

Dignum 


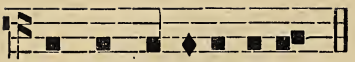

Et cum spi-ri-tu tu-o.
(Iokarenre.)

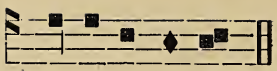

De-o gra-ti-as.

\section{TAKWENTENR ROTIIATATOKENTISON}

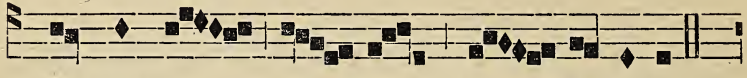

Takwentenr

Se - wen-ni-io. III.

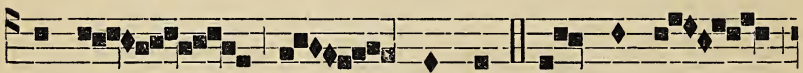

Kristos

ta - - kwentenr. III.Ta-kwentenr

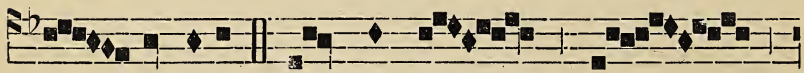

Se - wenniio. Il. Ta - kwentenr

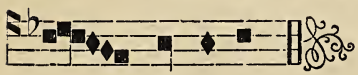

Se - - wenni-io.

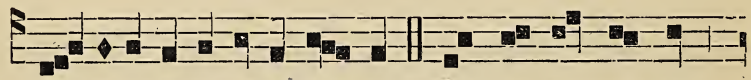

Glo-ri-a in ex-cel-sis De - o. Nok on-wen-tsia- ke

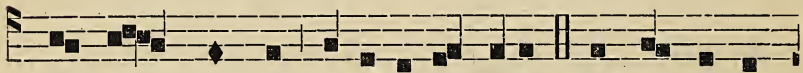

skennen nonkwe ia-ko-nikon-hriio. Tekwa - nonwe-

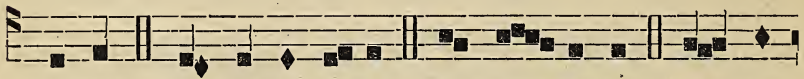

ratons. Kwatsennonnia se. Kwasen - naiens. Kwa-ron-

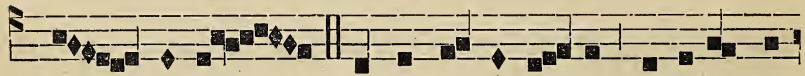

$$
\text { hia - ientons. Kwatonra - seron - se tsini akwa }
$$

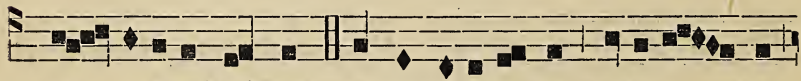

sa - iatanehrakwat. Sewen-ni-io ie-sen - nakara - ton 


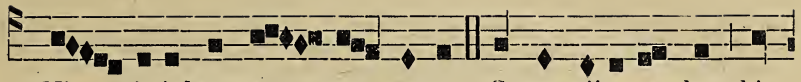

$\mathrm{Ni}$ - io iahte sa - no - ronse. Sewenniio son- ha hia-

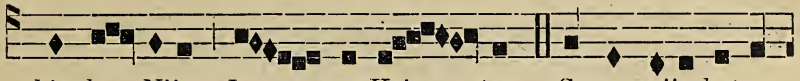

hienha Niio Ie - sos Kris - tos. Se-wenniio hetse-

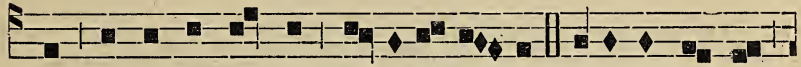

ni - konhraie- wenthos hia-niha $\mathrm{Ni}$ - io. Seriwahton - tha

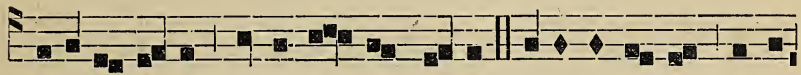

kariwa-neren ka.takwentenr ni-sa. Seriwahtontha ka-ri-

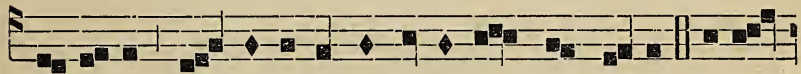

wa-neren Sa - tontat onen-nonwa ne kwenni-tha. Ne sa-

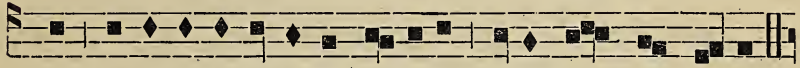

tiens tsi raweientehtakon hianiha katakwen-tenr ni-sa.

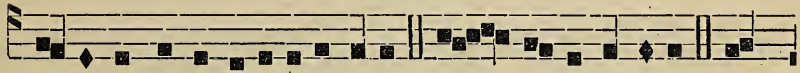

A- seken sonha saiata-tokenti. Son - ha Sewenniio. Son-

E二-1- -

hatsiatanoron Ie - sos Kris - tos. Rotkon-ro - iata.

E-

to - kenti Niio Roniha ietsisen - naien E - - tho naiawen.

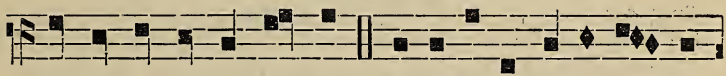

Cre-do in unum De-um. Ro-ni-ha îahte ho-no-ron-

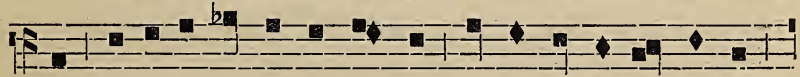

se roson karonhia nonwentsia Akwekon tsi-ni iekens 


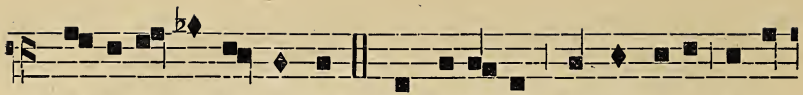

nok tsini iahte ickens. Nok saia- tat Rowenni-io Iesos

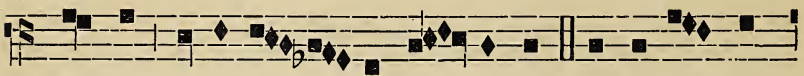

Kristos raon-lıa $\mathrm{Ni}-$ io $\mathrm{Ro}$ - ienha. Ok tsi hia - ta-

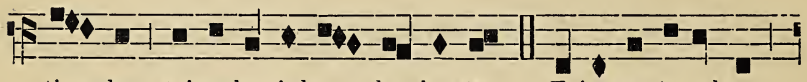

tien-ha tsi-nahe iahte ka-konte. Teioswa-te-takwa

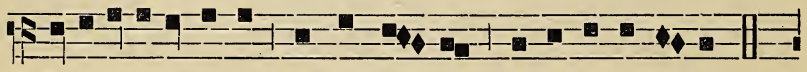

teioswatetakwake akwa $\mathrm{Ni}$ - io raonha-ke $\mathrm{Ni}$ - io.

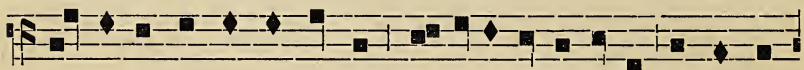

Roienha iahte honwason sahniiatat Roniha Akwekon

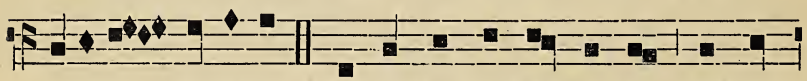

rotewe - iennison. Ii tionkwe sonkwaniente sonkwa-

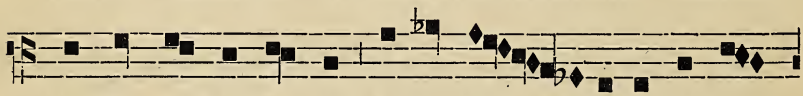

tsennonnia - ten-ni - re ka-ron-hia - - ke tha-we-non-

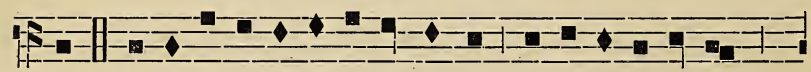

ton. Raonniaton raieron-ke Rotkon Roia-ta-to-kenti

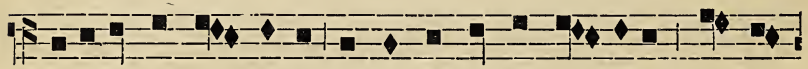

a-onekwensa Wari iabte kanakwaien - teri nok on-

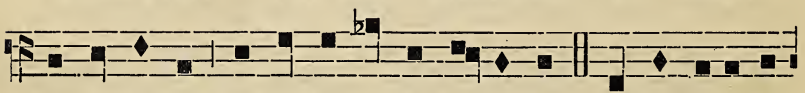

kwe roton-hon, nok onkwe, onkwe rotonhon. Onkwariwa ron-

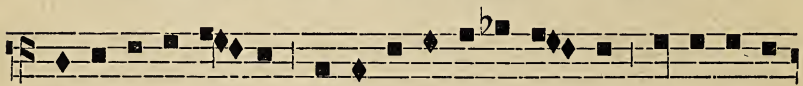

wa-ia-tanen-takton Pilat ro- te-rihon-ta - kwe rawenheion 
F-

ronwaia-taten. Nokslıotoninheton asen watontha tsi-ni

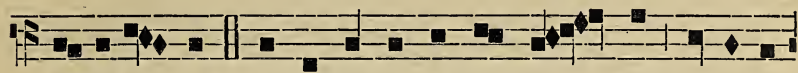

ka-hiatonkwe. Karonhia-ke shawenon - ton tsi ra-we-

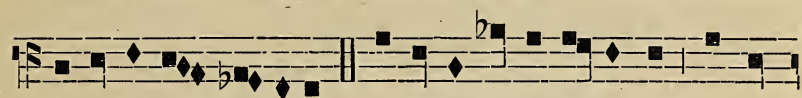

ientehtakon Ro- niha. Sékon tentre enthonehra - kwaton-

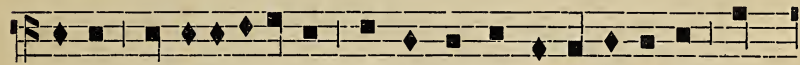

hatie tensakoia-toret iakonnhe nok ia-kowentahon, tio-

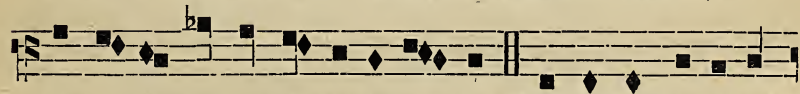

tkon, tiotkon enhonwenna-ke-ra - tse. Nok Rotkon Ro-ia-ta-

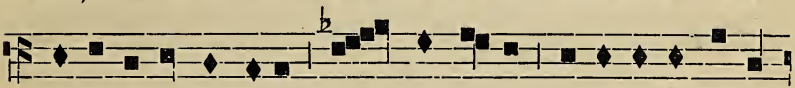

token-li lawen-ni-io son-kionnheton rononhake Ro-ni-

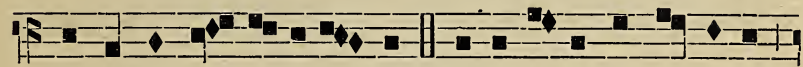

ha Roien-ha - thoienta - kon. Ne Roni- ha nok Ro-ienha

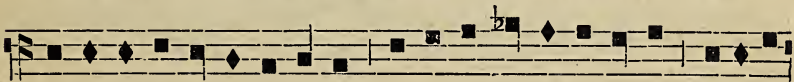

sa-tehonwa-tiwenniiostha sa-te-honwatisen-naieus Ro-ti-to-

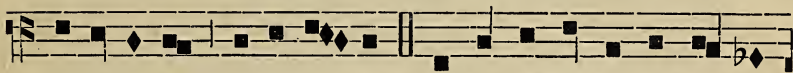

kensehakwe ronwatro - ri. Enskal ok Kentiohkwato-ken-

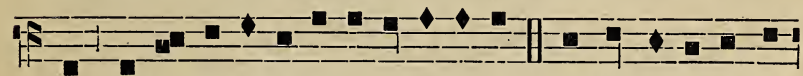

ti Ra-li-kwekonne te-ho-na-renihon. Twakehtakon enskat

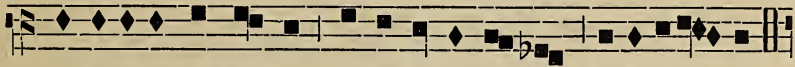

ionta-te-nekwahestha swate-ra-kewa - tha kariwane-ren. 


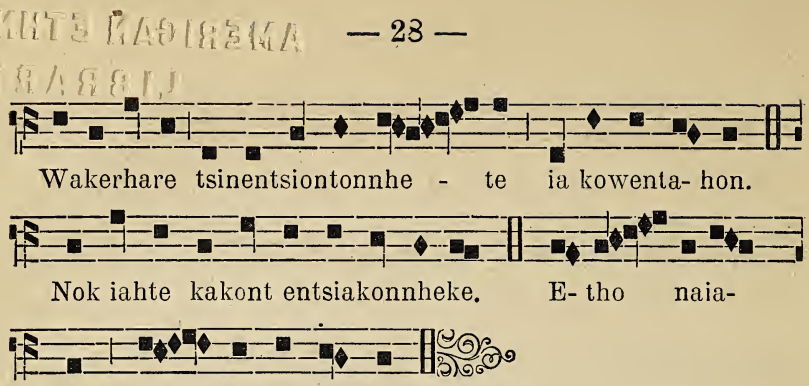

wen. $\mathrm{E}$ - tho na-ia-wen.

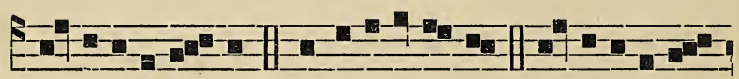

Sa-iala-token - ti, Sa-iata-token-ti, Sa-ia-ta-token-

5-1二-10 -

ti, Sewenniio tsi ia - kionnhe. Karonhiakwekon onwentsiaE二 二

kwekon sa-ia-ta-nehra - kwat ie-sa - sennaien, ka - ron-

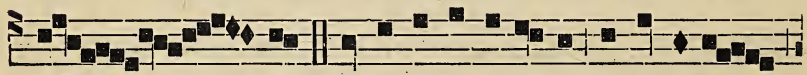

hia - - ke. Ronwanenton ne thare rasennakon

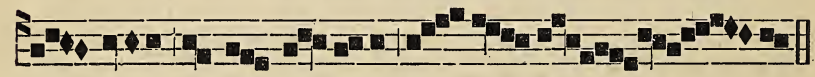

Ra-wenniio ronwa - sennaien ka - ron - hia _ _ ke.

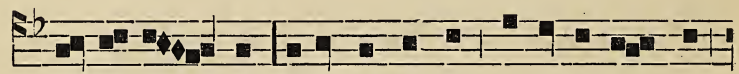

le-sos Kri - stos seri-wahtontha ka-ri-wa-ne - ren,

E- E- -

ka-takwen- tenr ni - $\quad$ sa. Iesos Kristos se-riwahtontha

E-

ka-ri-wa - ne-ren, katakwen-tenr ni - - sa. Ie- 
S2010

sos Kri - stos se-ri-walitontha ka-ri-wa-ne - ren

E-

Takwentenr ta - kion sken - nen.

TAKWENTENR WARI AORENNA

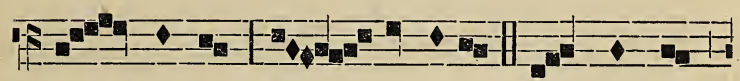

Ta - kwentenr Se - wen-ni-io. Ta - kwentenr

ra

Se - wenniio. Ta - kwentenr Se - - wenniio.

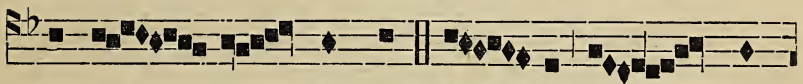

Kristos ta - kwentenr. Kris - tos ta - - kwen-

S-2

tenr. Kristos ta - kwentenr. Ta-kwentenr

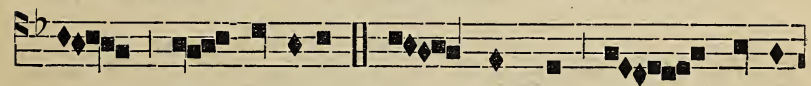

Se - wınni-io. Ta - kwentenr Se - - wenni-

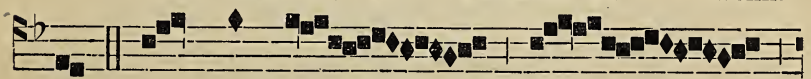

io. $\mathrm{Ta}-\mathrm{kwentenr}$

Se -

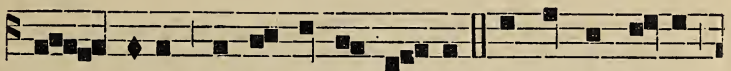

Glo - ri-a in ex-cel-sis De - o. Nok nonwentsiake 


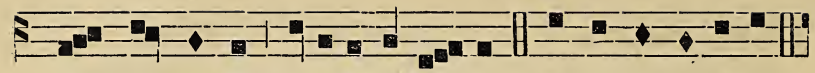

skennen nonkwe iakonikonhri - io. Tekwanouweratons.

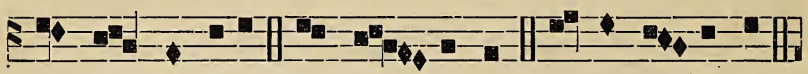

Kwalsen-nonniase. Kwasen - naiens. Kwaronhia-hientons.

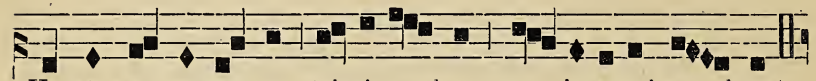

Kwatonra-se-ron-se tsi-ni a - kwa sa - ia-tanehra - kwat.

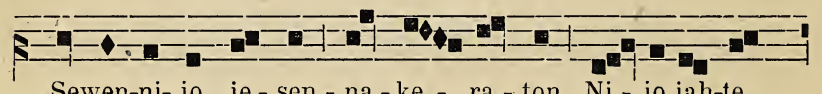

Sewen-ni- io ie-sen - na-ke - ra-ton $\mathrm{Ni}$ - io iah-te

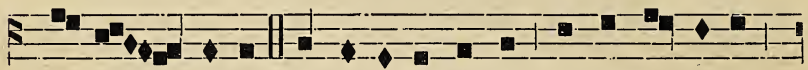

sa-no - ronse. Sewenni- io sonha hiaienha Niio

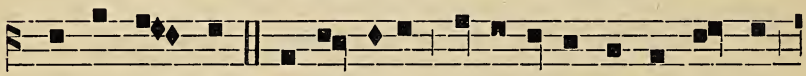

Ie-sos Kris- tos. Sewen-niio hetse-nikonlıra-ie-wentlos

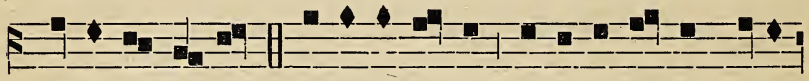

Hianiha $\mathrm{Ni}$-io. Se-riwahtontha ka-ri-wane-ren kala-

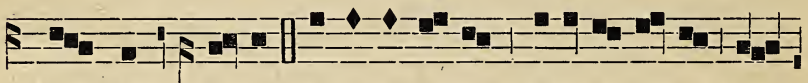

kwentenr ni-sa. Se-ri-watontha kariwa-ne-ren sa-

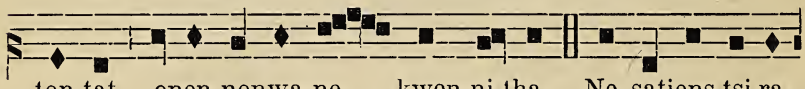

ton-tat onen nonwa ne kwen-ni-tha. Ne satiens tsi ra-

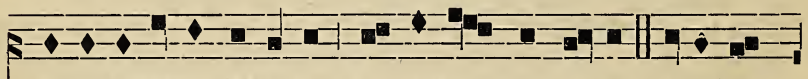

weientehtakon Hianiha ka-takwentenr ni-sa. Ase-ken

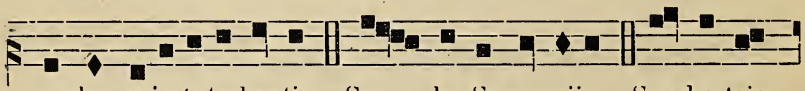

sonha saia-tato-kenti. Son - ha Sewenniio. Son-ha tsia- 

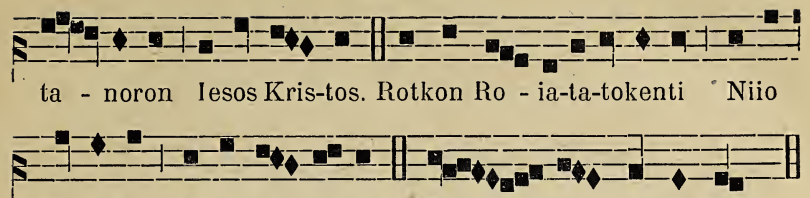

Ro-ni-ha ietsi-sen-naiens. E - tho na-ia-wen.

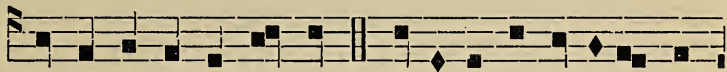

Cre-do in unum De-um. Ro-ni-ha iahte hono - ron-

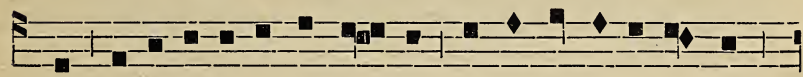

se roson karonhia nonwentsia $\Lambda$ kwekon tsi-ni ie- kens

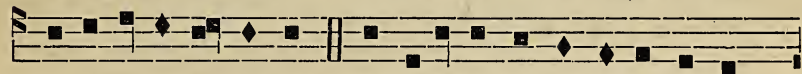

nok tsini iahte iekens. Nok saiatal Rawen-ni-io le-sos

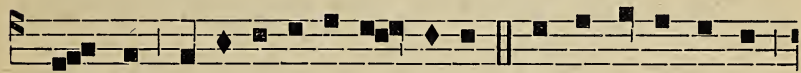

Kris - tos ra-on-ha Niio Ro - ienha. Ok tsi hiata-tienha

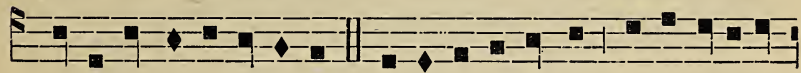

tsi-na-he iahte kakonte. Teioswa-te-takwa teios-wate-ta-

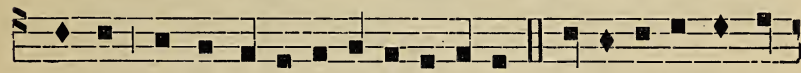

kwake akwa $\mathrm{Nl-io}$ ra-onha-ke Ni-io. Roienha iahte hon-

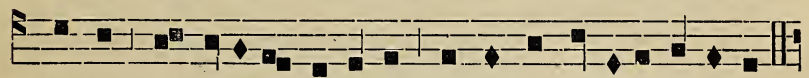

wason sahniia-tat Roni-ha akwekon ro- teweiennison.

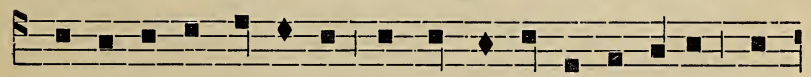

Ii tionkwe sonkwaniente sonkwatsennonnia-ten-ni-re ka-

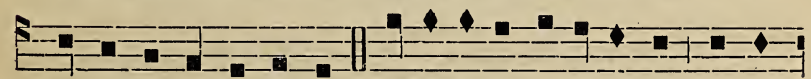

ronhiake thawenonton. Raonniaton ra-ie-ronke Rotkon 


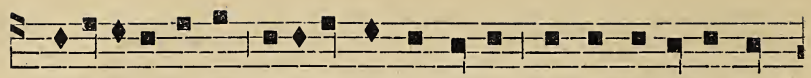

Roia-tatoken-ti a-o-nekwensa Wari iah-le kanakwaien-

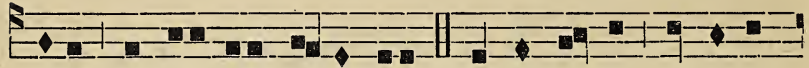

te-ri nok on-kwe ro-ton-lıon. Onkwari-wa ron-waia-

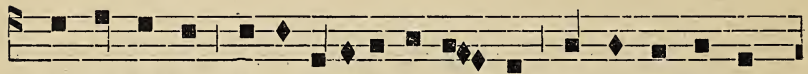

tanen-takton Pilat rote-rihon-la - kwe rawenhe-ion ron-

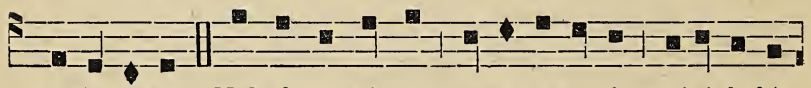

wa-ia-taten. Nok shotonnheton asen watontha tsini kahia-

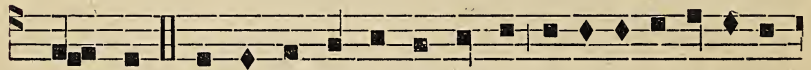

ton - kwe. Karonhia-ke shawenonton tsi raweienteh-takon

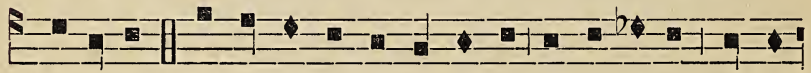

Ro-ni-ha. Se-konten-tre entho-nehrakwatonhatie tens a-

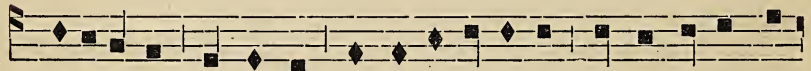

koia-toret jakonnhe nok iakowentaon tiolkon enhonwen-

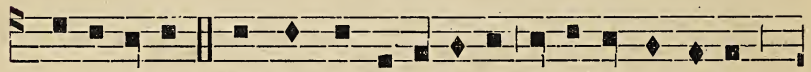

na-ke-ratse. Nok Rotkon Ro-ia-ta-to-kenti Rawen-ni-io.

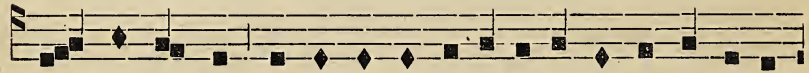

Son-kionnhe-tha ronon-ha-ke Roni-ha, Roien-ha thoien-ta-

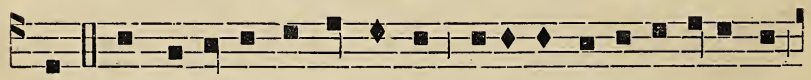

kon. Ne Ro-niha nok Ro-ien-ha sate honwatiwen-niiostha

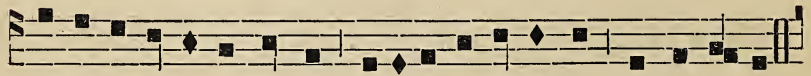

sa-tehonwatisen-naiens Roti-tokensehakwe ronwatro-ri. 


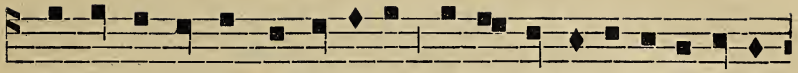

Enskatok kentiohkwa-token-ti Ra-ti-kwekonne te-ho-na-re-

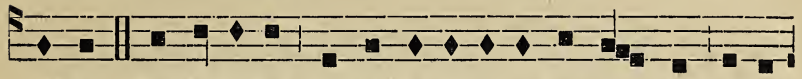
nihon. Twakehtakon enskat ionta-te- nekwahe - stha swate-

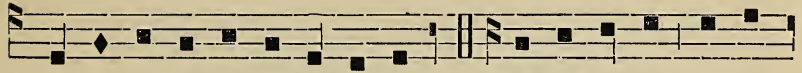

ra-ke-watha ka-ri-wa-ne-ren. Wakerha-re tsinen-

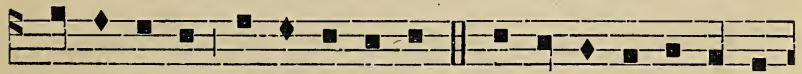

tsiontonnhe-te nia-kowen-ta-on. Nok iah-te kakont entsia-

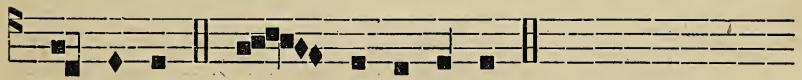

kon- he- he. E - - tho na- iawen.

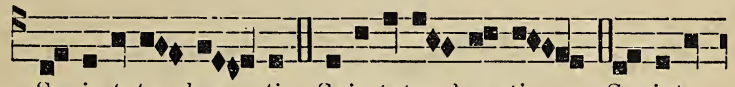

Sa-ia-tato - ken - ti, Saia-tato - ken-ti, Sa-iata-

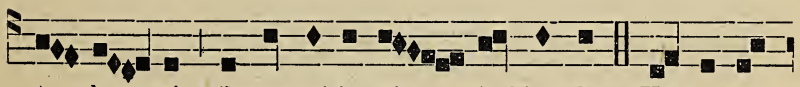

to - ken - ti Sewen-ni-io tsi ia-kionnhe. Ka-ronhia-

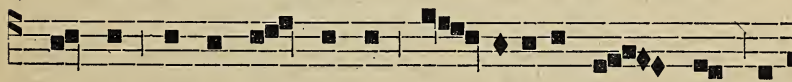

kwekon onwentsia-kwekon sa - iata-ne-hra - kwat ie-

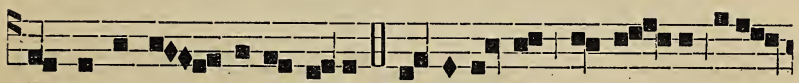

sa-sennaien karonhiake. Ronwanenton ne tha - re rasen-

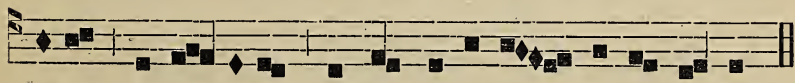

nakon Rawen-niio ronwa-sennaien karon-hia-ke.

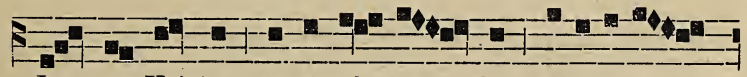

Ie - sos Kristos se- riwah-ton - tha kariwane - 


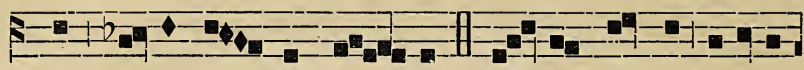

ren ka-ta-kwen-tenr ni - sa. II. Ie - sos Kristos seriwah-

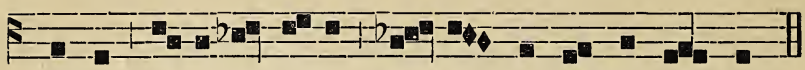

tontha ka-ri-wa-ne-ren ta-kwen-tenr ta-kion skennen.

\section{TAKWENTENR AWENTOKON}

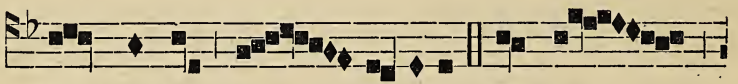

'Ta - kwentenr Se - - wenniio.ıi.Kristos

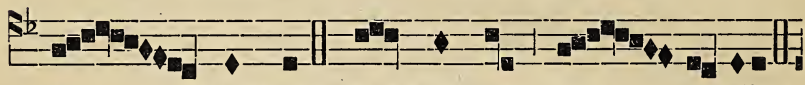

ta - - kwentenr. II. Ta- kwentenr Se - - wenniio.ı. St

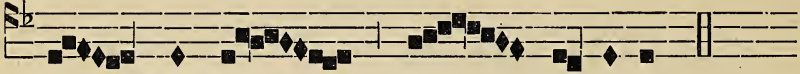

Ta - kwentenr

Se - wen-ni-io.

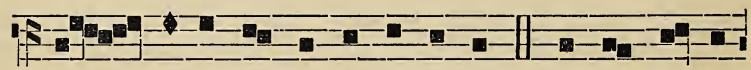

Glo - ri-a in ex-cel-sis De-o. Nok non-wentsia-

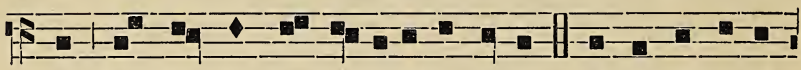

ke skennen nonkwe ia-ko-nikonhriio. Tekwanonwera-

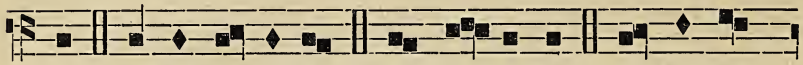

tons. Kwatsennonniase. Kwasen- naiens. Kwaronhia-

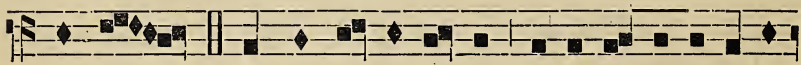
ientons. Kwatonra-se-ron-se tsini a- kwa sa-ia-ta-

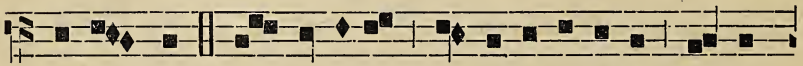
nehra-kwat. Se-wenniio iesen-na-ke-raton $\mathrm{Ni}$-io 


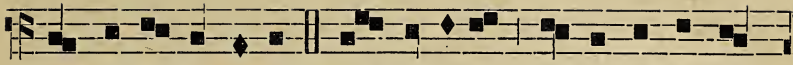

iah- te sa-noron-se. Se- wen-ni-jo son-ha Hiaien-ha

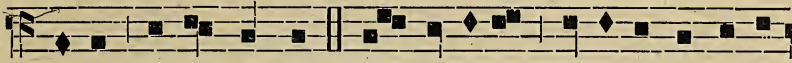

Ni-io le-sos Kristos • Se - wenniio hetse-ni-konhra-ie-

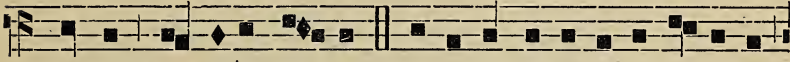

wenthos, Hia-niha $\mathrm{Ni}$ - io. Se-riwahtontha ka-ri-waneren,

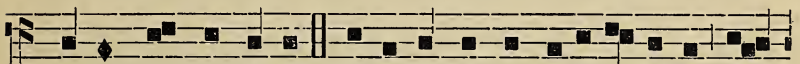

ka-takwenten: nisa. Se-riwahtontha kariwa-neren sa-

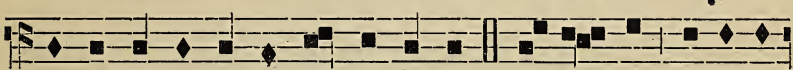

tontat onen nonwa ne kwennitha. Ne sa-tiens tsi rawe-

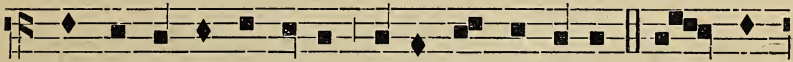

ienteh-ta-kon Hiani-ha ka-takwentenrni-sa. A - se-

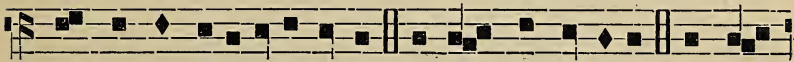

ken son-ha saia-ta-token-ti. Sonha Sewenniio. Sonlia

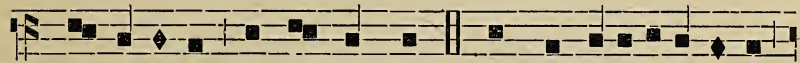

tsia-ta-noron le-sos Kristos. Rotkon Roia-tatokenti

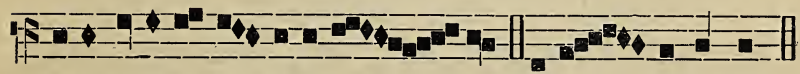

Niio Roniha ie - tsisenna - - iens. Etho naiawen.

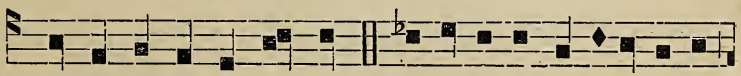

Cre-do in unum De-um. Roienha iah-te honoronse

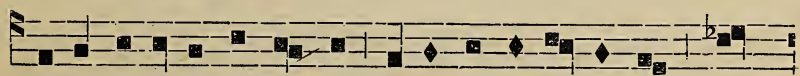

roson karonhía nonwentsia akwekon tsi-ni iekens, nok 


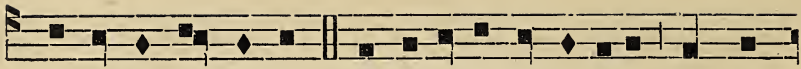

tsi-ni iah-te iekens. Nok sa-iatat Rawen-niio le - sos

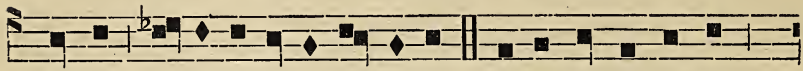

Kristos ra-onha Niio Ro-ien-ha. Ok tsi hia-ta-tienha

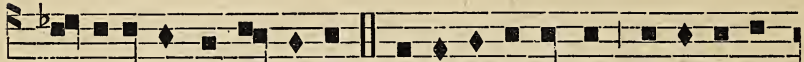

tsi-nahe iahte ka-kon-te. Teioswa-te-takwa teios-wa-te-

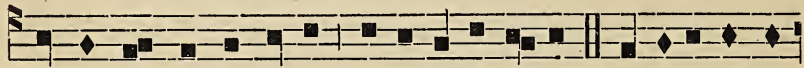

takwake akwa $\mathrm{Ni}-\mathrm{io}$ raon-hake $\mathrm{Ni}$-io. Roienha iahte

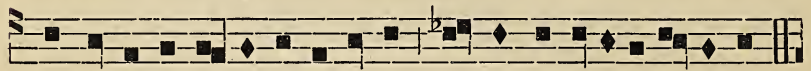

honwason sahni-iatat Ro-ni-ha akwekon roteweiennison.

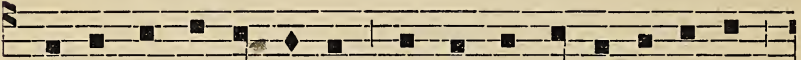

Ii tionkwe sonkwaniente, sonkwatsen-non-nia-tenni- re

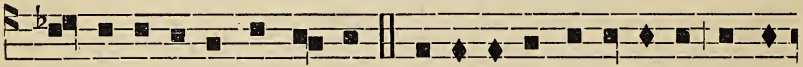

ka- ronhiake thawenon-ton. Raonniaton ra-ieronke Rotkon

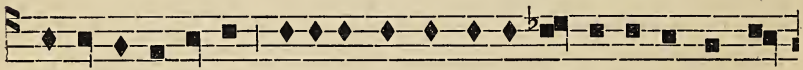

Ro-ia-ta-to-kenti a-o-nekwensa Wari iah-te kanakwaien-

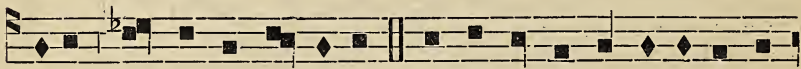

te-ri nok onkwe ro-tonhon. Onkwa-ri-wa ronwaia-tanen-

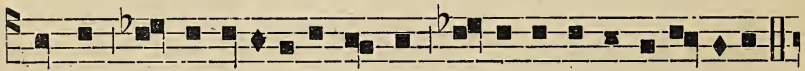

takton Pi - lal Roterihonta-kwe Rawenlıeion ronwaia-taten.

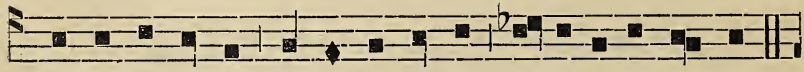

Nok shotonnheton a-senwahtontha tsi-ni ka-ia-tonkwe. 


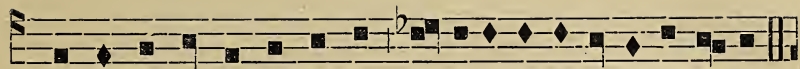

Karonhia-ke shawenonton tsi raweientelitakon Roni- ha.

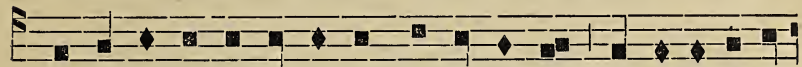

Se-kon tentre en-tho-nehra- kwaton-ha- tie tensa-ko-ia-to-

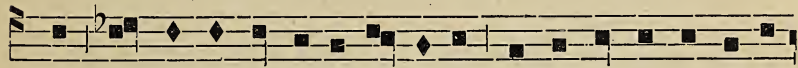

ret ia. konnhe nok iakowen-ta-on tiotkon enhonwenna-ke-

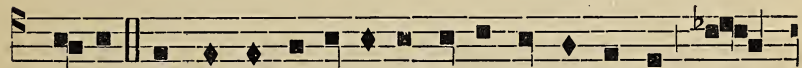

ra-tse. Nok Rotkon Roia- ta-to-kenti Rawen-ni- io son-

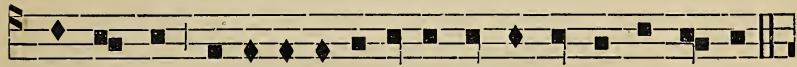

kionnhetha rononha-ke Roni-ha Roien-ha thoien- takon.

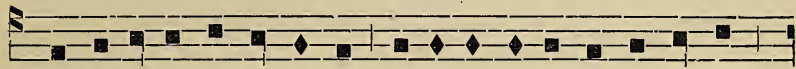

Nok Roniha nok Roien-ha sa-tehonwa-tiwen- ni-iostha,

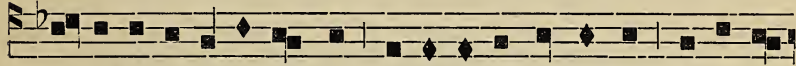

sa-tehonwati-sen-naiens Ro-ti-token-se-hakwe ronwatro-

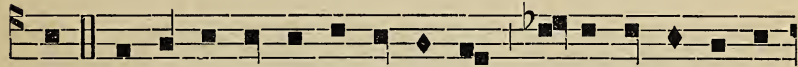

ri. Enskat ok Kentiohkwatokenti Ra-ti-kwekonne te-

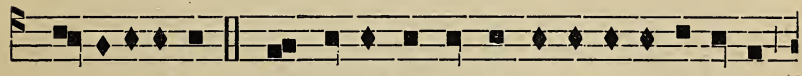

ho-narenihon. Twakehtakon enskat ionta-te-nekwahestha

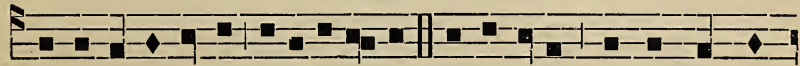

swate-ra-kewatha kariwaneren. Wakerhare tsi nentsionton-

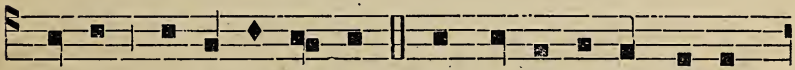

nhete nia-kowen-ta-hon. Nok ialıte kakont entsia- 


\section{5-}

konnhe-ke. E - tho na- iawen.

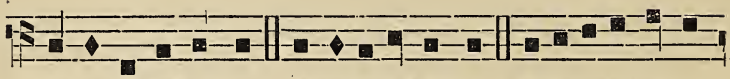

Sa-ia- ta-token- ti， Sa-iata-token- ti, Saiata-token-ti

F-日

Se - wenniio tsi ia - kionnhe. Karonhiakwe-kon,

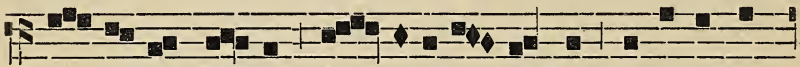

on-wentsiakwekon sa - ia-tane - hrakwat, ie-sasen-na-

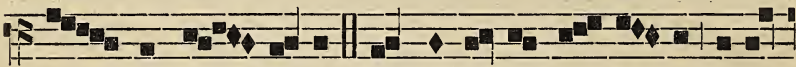

ien ka-ron - hiake. Ronwanenton ne tha-re rasen-

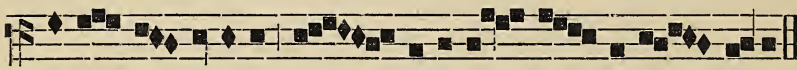

nakon Ra- wenniio, ron - wasenna - ien karon - hiake.

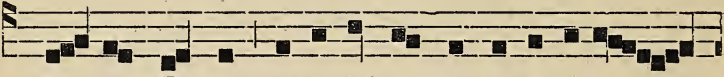

Ie-sos Kristos se-ri-wahton-tha ka-ri-wane -

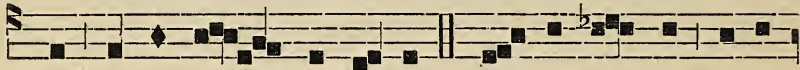

ren, ka-takwen - tenr ni - sa. le - sos Kri - stos se-ri

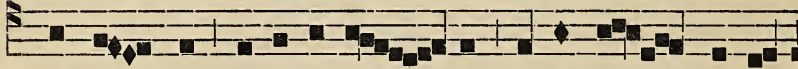

wahton - tha ka-ri-wane - ren, ka-takwen - tenr ni-

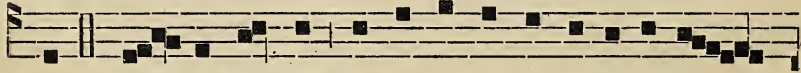

sa. Ie - sos Kristos se- ri-wahtontha ka-ri-wa-ne -

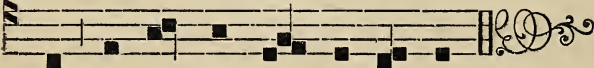

rell, takwentenr ta - kion skennen. 
TSI RA'THONNIANE NOK IAKAWENTONTIETHAKOWA TAKWENTENR

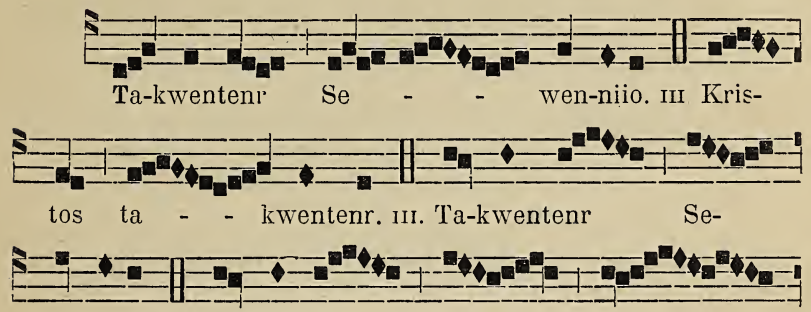

wenniio. II. 'Takwentenr

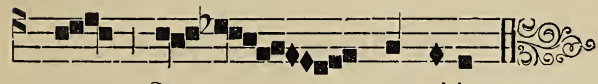

Se - - wen-ni-io.

Tewakehtakon awentokon.

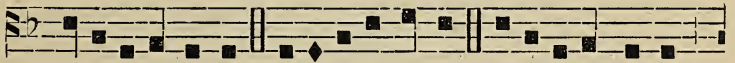

Saia-ta-tokenti, Saia-la-tokenti, Saiata-to-kenti,

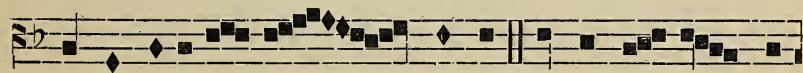

Se-wenni-io tsi ia - - kionnhe. Karonhia-kwe - kon

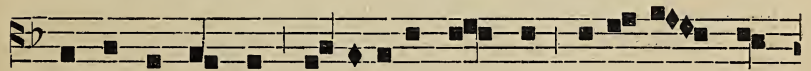

onwentsiakwekon sa-ia-tane-hrakwat, ie-sa-sen - na-

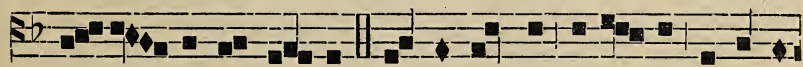
ien karon-hia-ke. Ronwanenton ne tha- re rasenna-

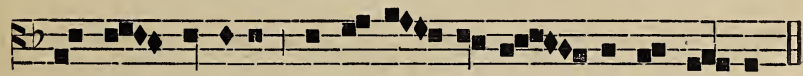

kon Ra - wenniio, ronwasen-na-ien karon-hia-ke. 


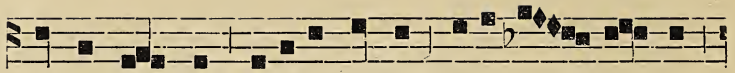

Ie-sos Kris- tos seriwahtontha kari-wa - ne-ren,

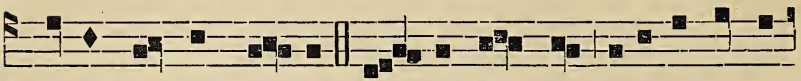

ka-ta-kwentenr ni - sa. Ie - sos Kris- tos, seriwahtontha

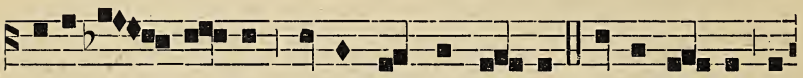

kari-wa - ne- ren, katakwentenr ni - sa. Iesos Kristos, se-

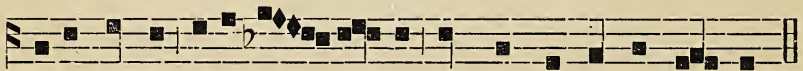

riwahtontha kariwa - ne- ren, takwentenr takion skennen.

\section{ION'CATAKENROSERASTHA TAKWENTENR}

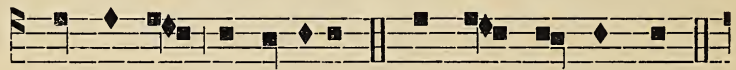

Takwentenr Sewenni-io. JI. Kristos takwentenr. III.

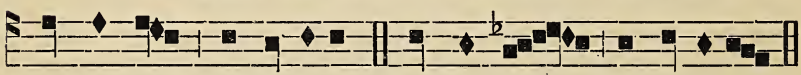

Takwentenr Sewenni-io. II. Taawentenr Sewenniio.

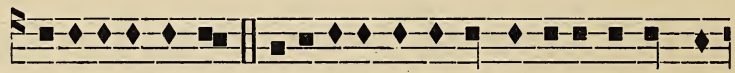

Sa-ia-ta-tokenti. II. Saia-tatoken-li Sewenniio tsî iakion-

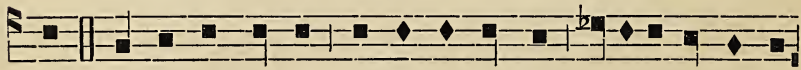

nhe. Karonhiakwekon onwentsiakwekon sa-iatanehrakwat,

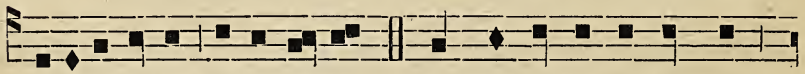

iesasennaien karonhia-ke. Ronwa-nenton ne tha-re

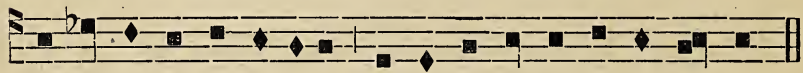

rasen-nakon Rawonni-io, ronwasen-naiens karon-hia-ke. 


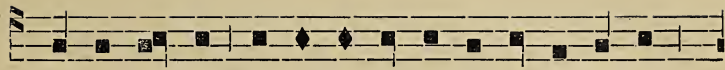

le-sos Kristos se-riwah-tın-tha ka-ri- wa-neren,

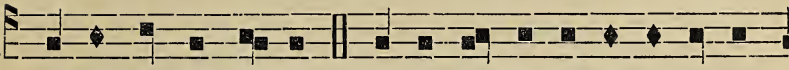

Ka-takwentenr ni-sa. 11. Ie-sos Kristos se-ri-wahtontha

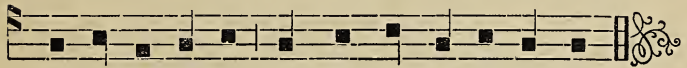

ka- rl- wa-ne-ren takwentenr lakion skennen.

\section{IENENRINEKENSTHA TAKWENTENR}

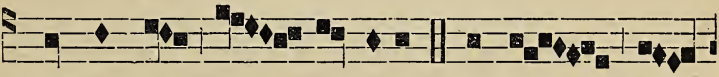

Takwentenr Su - wenniio. III.Kristos Ta-

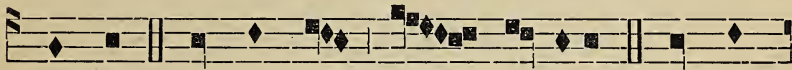

kwentenr. II. Takwentenr Se - - wenniio. ir. Takwen-

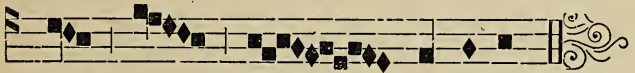

tenr Se - _ -

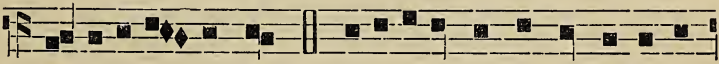

sa- ia-talo-ken-ti I1. Saia-la-lokon- ti Sewenni-io

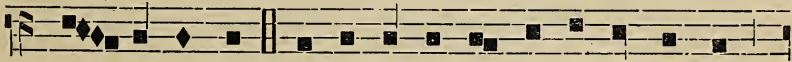

tsi iakionnhe. Karonhiakwekon onwentsiakwekon

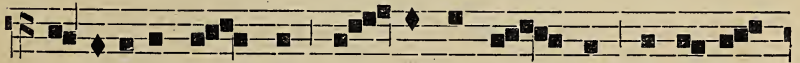

sa-iatane-hra - kwat ie - sa-sen-na - ien karonhia-

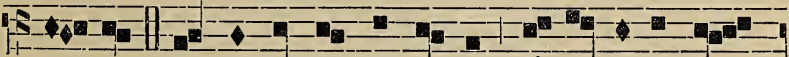

ke. Ron-wanen-ton ne tha-re ra-sen-nakon Ra- 


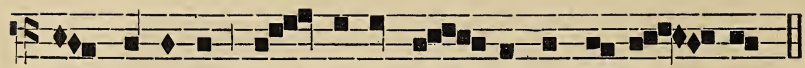

wenni-io ron - wasenna - ien ka-ronhia - ke.

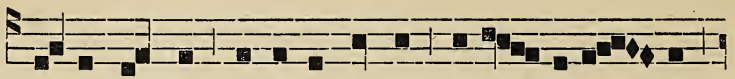

le-sos Kristos se-riwahtontha kari - wane - ren

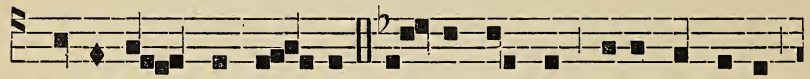
ka-takwen-tenr ni - sa. Ie-sos Kristos, seri-wahtontha

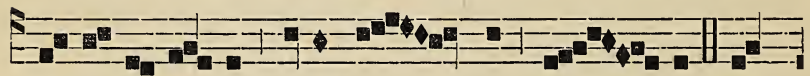

ka-ri-wa-ne-ren katakwen - tenr ni - - sa. Ie-

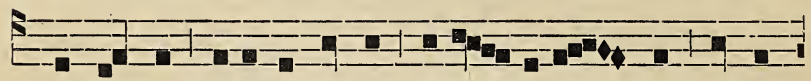

sos Kristos seriwahtontha kari - wane - ren takwen-

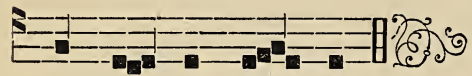

tenr ta - kion sken - nen.

TAKWENTENR KOWA

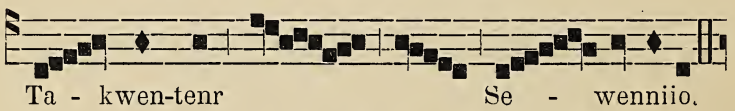

5.

Takwentenr

Se - wen-

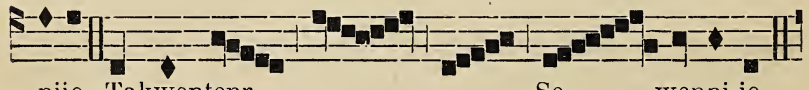

niio. 'Takwentenr

Se - wenni-io.

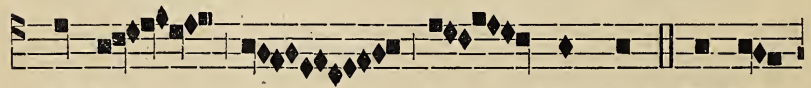

Kristos

ta - kwentenr. Kristos 


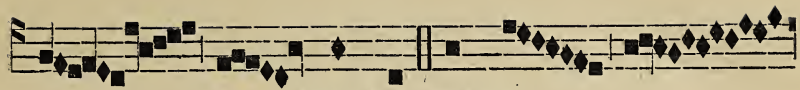

ta - kwentenr. Kristos

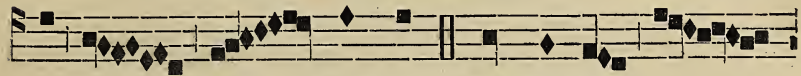

ta - kwentenr. Takwentenr

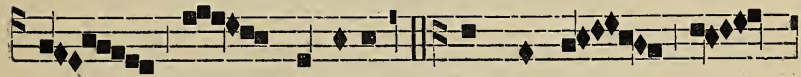

Se - wen-niio. Takwentenr

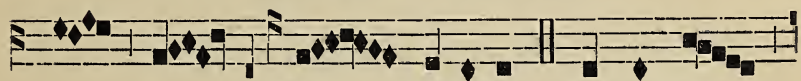

Se - wenni-io. Takwentenr

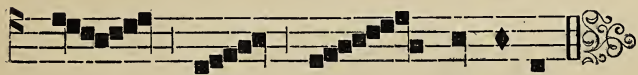

Se - - wenni-io.

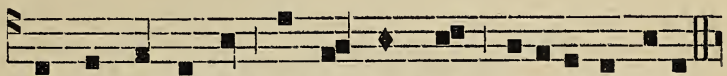

Nok nonwentsiake skennen nonkwe iakonikonhri-io.

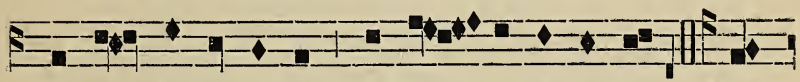

Tekwa - non-we-ratons, Tekwa - non-we- ratons. Kwa-

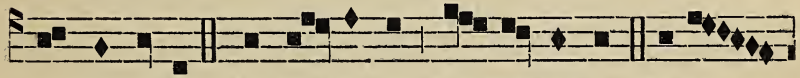

tsennon-niase. Kwasennaiens, kwasen-naiens. Kwaron-

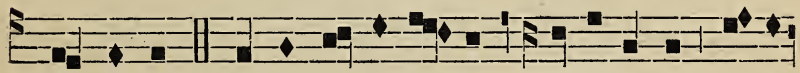

hia-ientons. Kwatonra-se-ron se tsi-ni akwa sa - ia-

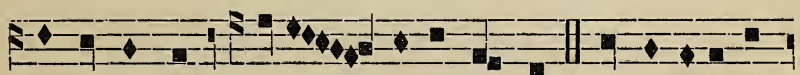

ta-nehrakwat, sa-ia - ta-nehrakwat. Sewenniio ie-

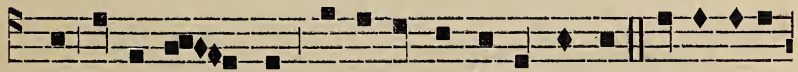

sen-na-ke-ra - ton Ni-io iahte sa-no-ron-se. Sewenniio 


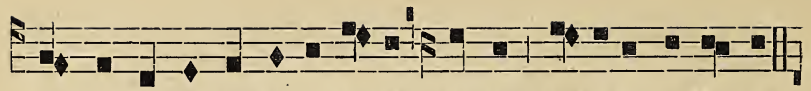

sonha Hiaien-ha Ni-io Ie-sos Kristos Iesos, Iesos Kristos.

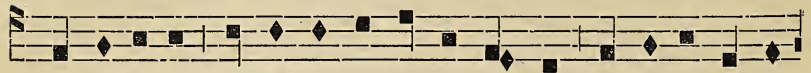

Sewenni-io hetse-ni-konhra-ie- wenthos Hiani-ha, Hiani-

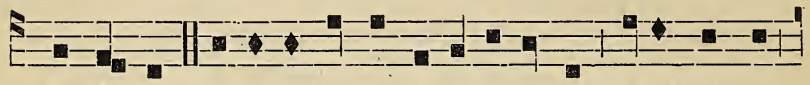

ha Ni-io. Se-riwahtontha ka-ri-wane-ren katakwentenr,

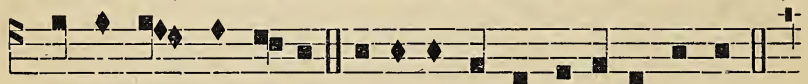

ka- takwentenr ni-sa. Se-riwahtontha ka-ri-wa-ne-ren

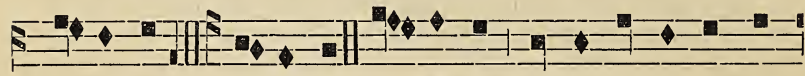

sa-tontat, sa-ton-tat, sa-tontat onen nonwa ne kwen-

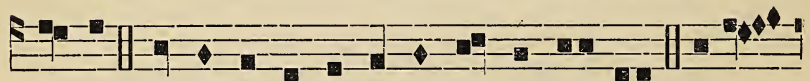

ni-tha. Sa-ton-tat onen nonwa ne kwenni-tha. Ne sa-

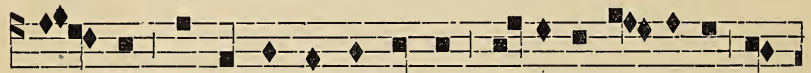

tiens tsi ra-weien- tehta-kon Hianiha, Hia-ni-ha ka-

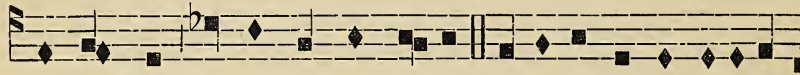

takwentenr, ka-takwentenr ni-sa $\mathrm{A}$-seken sonlıa saia-ta-

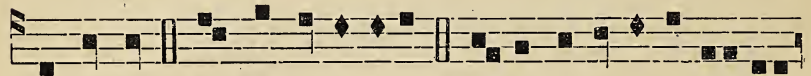

to-ken-ti. Son- ha Sewenniio. Sonha tsiatanoron le-sos

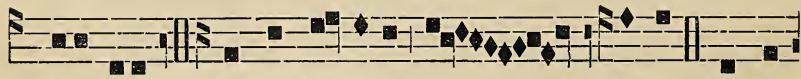

Kris- tos. Rotkon Ro-iata - to _ _ kenti, Rotkon

E-

Roia-ta-to-kenti Niio Roni-ha ie- tsi - sennaiens. 


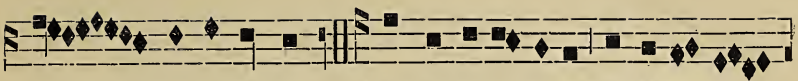

E - - tho naiawen Rotkon Roia-ta-to-ken-ti Ni- io

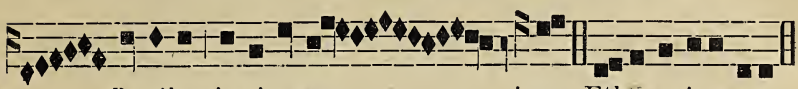

Roniha ietsisenna - - iens. Etho naia- wen.

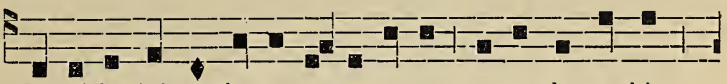

lio-ni-ha jah-te hono-ron-se roson, roson ka-ronhia

E二-二-日十 nonwentsia akwekon tsi-ni iekens nok tsini iahte iekens.

E-

Nok sa-iatat Rawen-ni-io Ie-sos Kristos Raonha Ni-io

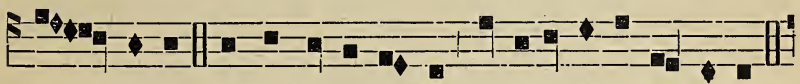

Ro - ienha. Ok tsi Hiata-tienha tsinahe iahte ka-kon-te-

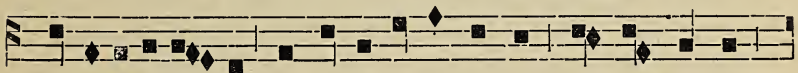

Teioswateta -kwa. te- ioswa-te-takwa-ke a-kwa $\mathrm{Ni}$-io,

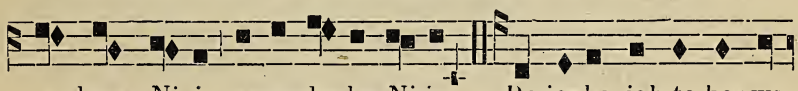

a-kwa $\mathrm{Ni}$-io ra-on-ha-ke $\mathrm{Ni-io}$. Ro-ienha iah-te honwa-

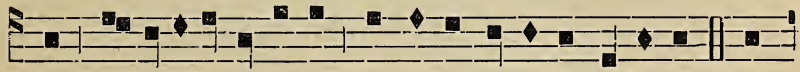

soll sa-niiatat Ro-niha akwekon rote-we-ien-nison. Ii

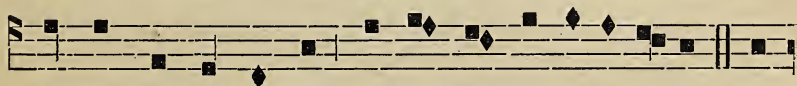

tionkwe sonkwanien-te sonkwatsen-non-niatenni-re. Ka-

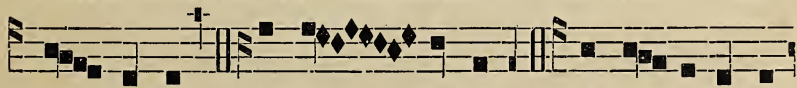

ron - hia-ke, karon - - hiake, ka-ron-hiake tha- 


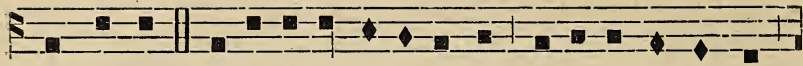

wennonton. Raonniaton ra-ieron-ke ra-ieron-ke Rotkon

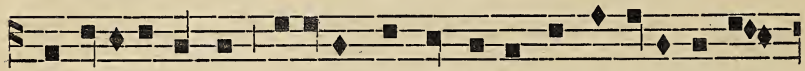

Ro-ia-tatoken-ti a-()-nekwensa Wari iah-te kanakwaien-

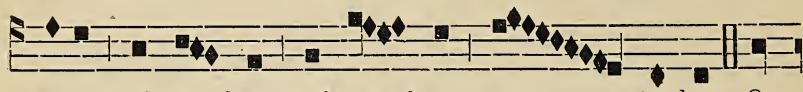

te-ri nok on - kwe nok on - kwe ro - tonhon. On-

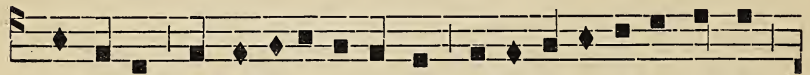

kwari-wa ronwa-iatanentakton Pilat ro-te-ri-hontakwe

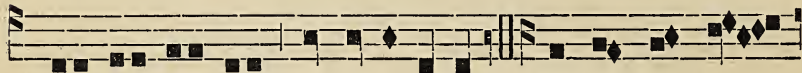

ra-wen-he-ion ron-waia-taten. Nok sho-ton-he-

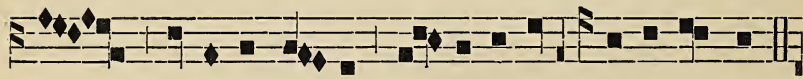

ton a-sen waton-tha tsi - ni, tsi-ni ka-hiatonkwe.

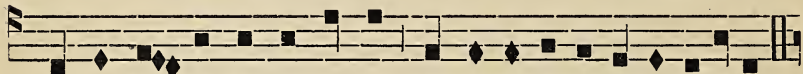

Karon-hia-ke shawe-nonton tsi raweientehtakon Roniha.

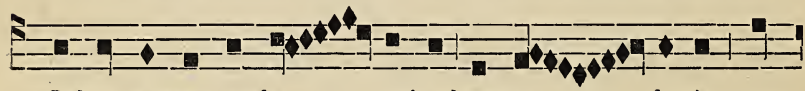

Sekon tentre entho - - nehrakwaton - hatie ten-

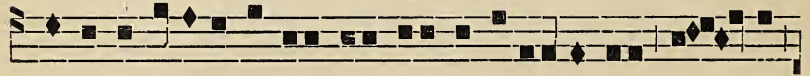

sa-ko-ia-to-rete iakon-nhe nok ia-kowen-ta-on tio - tkon

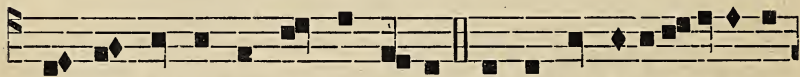

tiotkon enhonwenna-ke- ra-tse. Nok Rotkon Roiatatokenti

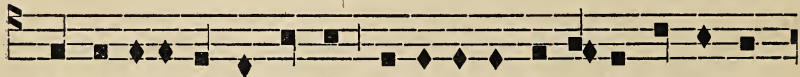

Rawenni-iosonkionnhetha rononhake Roni-ha Roienha 


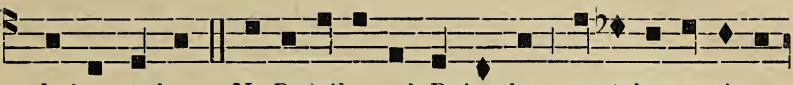

thoien-ta-kon. Ne Ro-niha nok Roien-ha sa-tehonwatiwen-

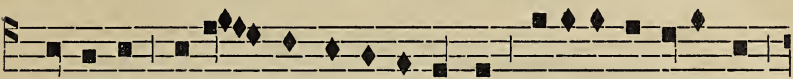

niiostha sate - hon-wa-ti-sennaiens Ro-titoken-sehakwe

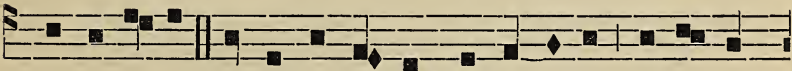

ronwatro- ri. Enskat ok kentiohkwa- token- ti Ra-ti-kwe-

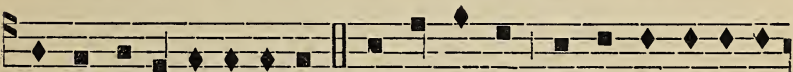

kon-ne te-ho-na-re-nihon. Twakehtakon enskat ion-ta-tene- .

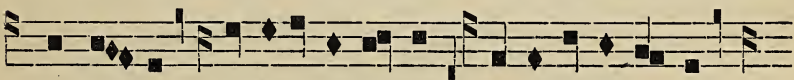

kwahestha swalera-ke-wa-tha, swate-ra-kewa-tha

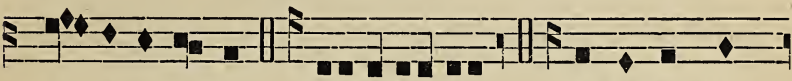

ka - riwa-ne- ren. Wakerha - re. Tsi nentsionton-

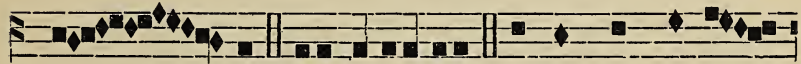

nhe - - te. Wa-kerha - re tsi nentsiontonnhe-

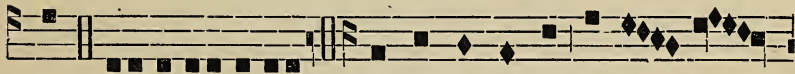

te ia-kowenta-on. Nok iahte kakont entsia - kon-

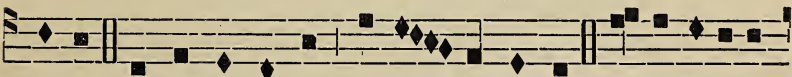

heke, nok iahte kakont entsia - konnheke. Etho naiawen,

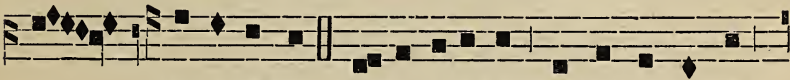

e - - tho naiawen, e- tho naiawen, nok iahte kakont,

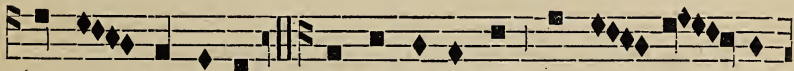

entsia - konnheke, nok iahte kakont entsia - kon - nhe- 


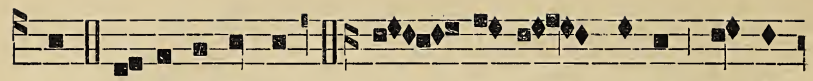
ke e-tho naiawen, e - tho na - iawen, e - tho

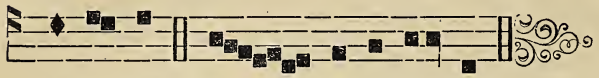

na-ia-wen, e - - tho na-ia-wen.

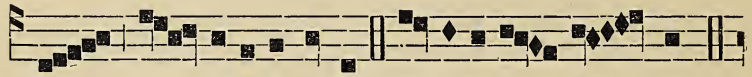

Sa - ia-ta-tokenti, Sa-ia-ta-to - ken - ti,

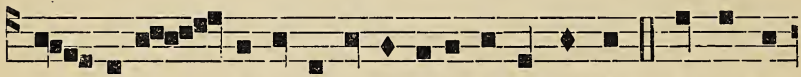

Sa - ia-ta - token-ti Sewenniio tsi iakionnhe. Karonhia-

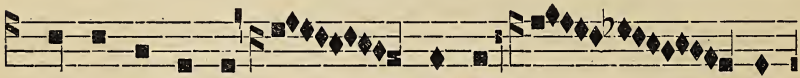

kwekon onwentsia - _ kwekon sa - _

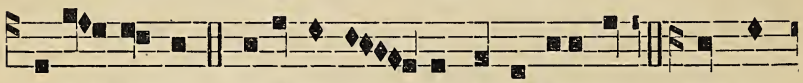

ta-nehrakwat, iesasen-na - ien karonhia-ke. Ronwa-

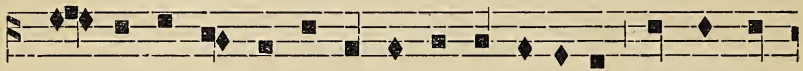

nen-ton ne tha - re ra-sen-nakon Rawenni-io ron-wa-nen-

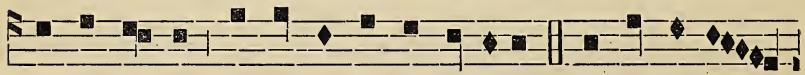

ton ne tha-re ra-sen-nakon Rawenniio :on-wasenna-

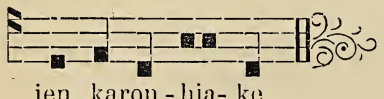

ien karon-lia-ke.

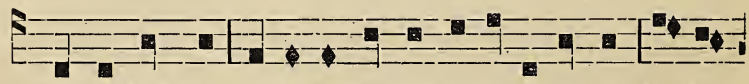

lesos Kristos se-riwahlontha ka-ri-wa-neren, ka- ta-

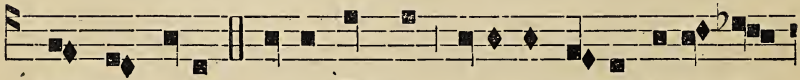

kwentenr nısa. Iesos Kristos se-riwahtontha kari - wa- 


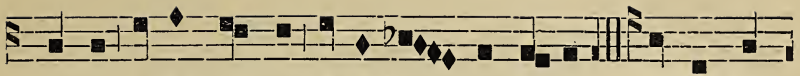

ne-ren, katakwentenr, ka-takwen-tenr ni- sa. Iesos Kris-

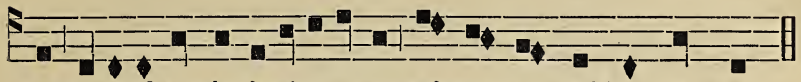

tos seriwahtontha kariwaneren,takwentenr takion skennen.

TAKWENTENR ORENNAKAION
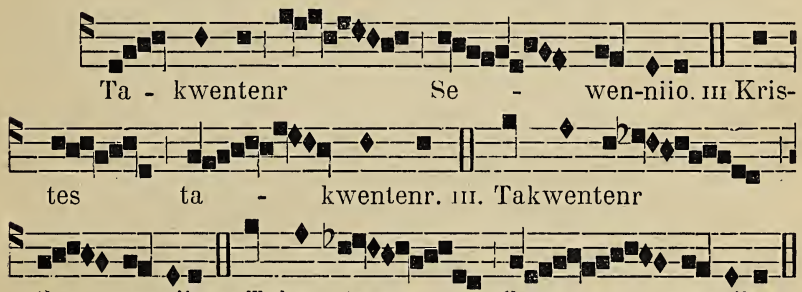

Se - wenniio. Ir.Takwentenr Se - wenniio.

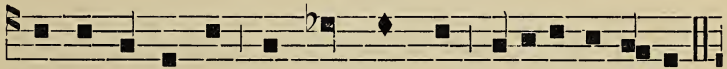

Nok nonwentsiake skennen nonkwe iakonikonhri- io.

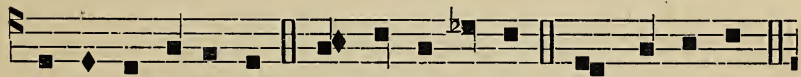

Tekwanonweratons. Kwatsennonniase. Kwa-sen-naiens.

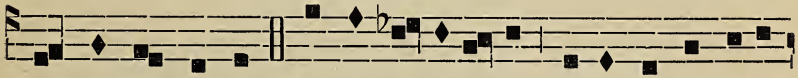

Kwaronhiahientons. Kwaton-ra-seronse tsi-ni akwa saia-

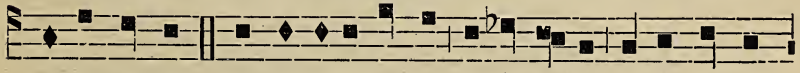

tanehrakwat. Sewenni-io iesen-na-ke-ralon $\mathrm{Ni}$-io iahte

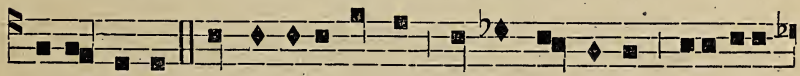

sa-noron-se. Sewenniio sonha IIiaien-lıa Niio Ie-sos 


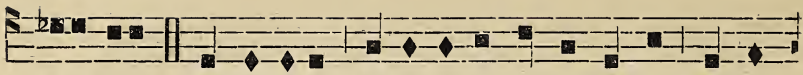

Kristos. Sewenniio hetseniikon-hra-iewenthos Hiani-

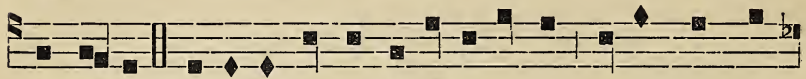

ha $\mathrm{Ni}$-io. Se-riwahton-tha ka-ri-waneren, ka-takwen-tenr

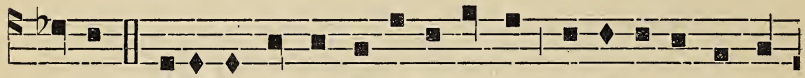

ni-sa. Seriwahtontha ka-ri-waneren, satontat onen non-

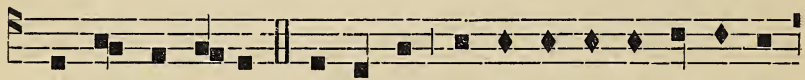

wa ne kwennitha. Ne satiens tsi ra-we-ien-teh-takon Hia-

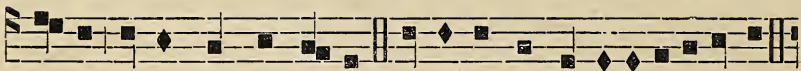

ni-ha katakwentenr ni-sa. A-seken sonha sa-iata-tokenti.

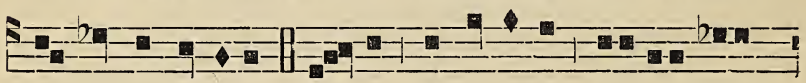

Son-ha Sewenniio. Son-ha tsia-tanoron Ie-sos Kris-

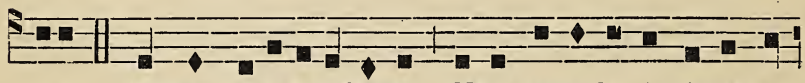

tos. Rotkon Roia-tato-ken-ti Ni-io Roni-ha ietsi-sen-na-

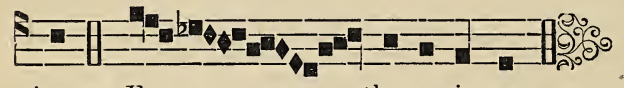

iens. $\mathrm{E}$ - $\quad$ tho na-iawen.

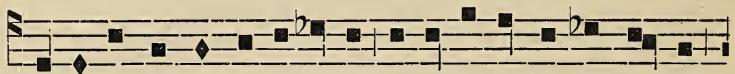

Ro-ni-ha iahte ho-noronse, roson karonhia nonwentsia,

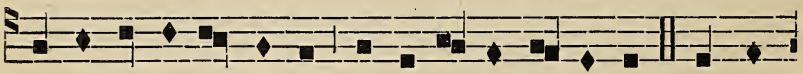

akwekon tsi-ni iekens nok tsi-ni iah-te iekens. Nok sha-

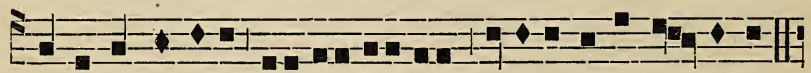
iatal Rawenniio le-sos Kristos raonha $\mathrm{Ni}$-io $\mathrm{lio}$ - ienha. 


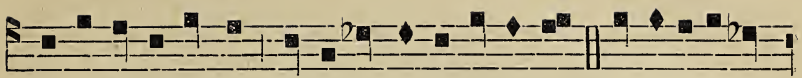

Ok si Hiatatienha tsi-na-he iahte kakonte. Teioswate-ta-

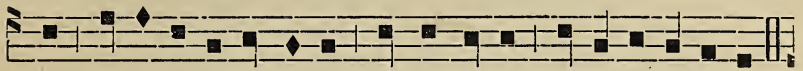

kwa teioswa-te-takwake aliwa Niio, ra-on-ha-ke Niio.

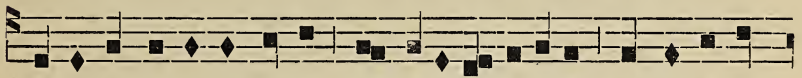

Ro-niha iahte honwason sahni-iatat Roniha akwekon ro-

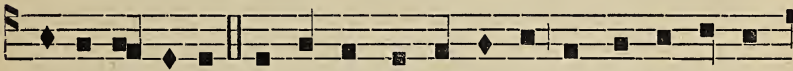

te-weien - nisen. Ii tionkwe sonkwoniente sonkwatsennonnia-

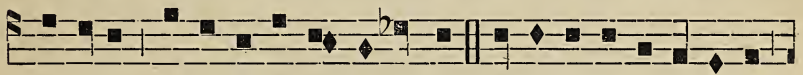

tennire karonhiake thawenonton. Raonniaton raieron-ke

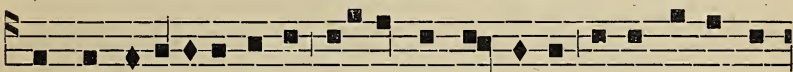

Rotkon Roia-tatokenti aonekwen-sa wa-ri iahte kanakwa-

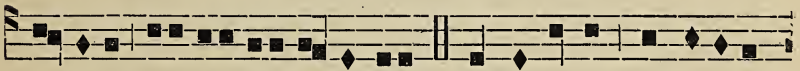

ien-te-ri nok on-kwe ro-tonhon. Onkwa-riwa ronwaia-ta-

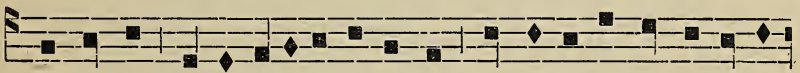

nentakton Pilat rote-ri-hontakwe liawenheion ronwa-ia-ta-

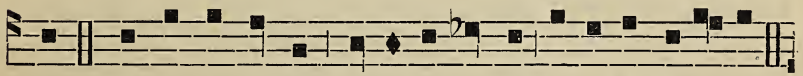

ten. Nok shotonnheton asen watontha tsi-ni ka-iatonkwe.

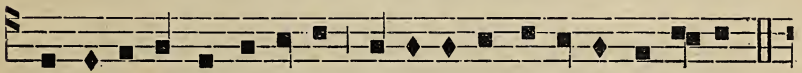

Karonhiake shawenonton tsi raweienteh-takon Roni- ha.

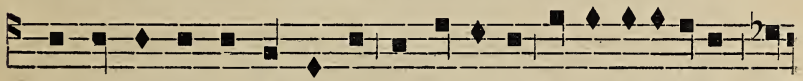

Sekon tentre en-tho-nehrakwatonhatie tensa-koia-toret ia- 


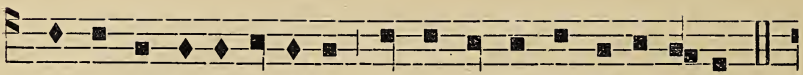

konnhe nok iakowentaon, tiotkon enhonwennakera - tse.

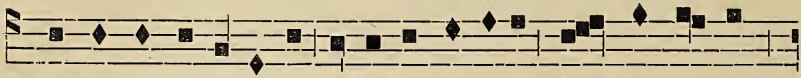

Nok Rotkon Roia-ta-to-ken-ti Rawenniio son- kionnhe-tha

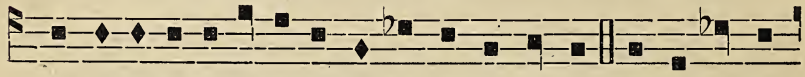

rononha-ke Ro-niha Ro-ien-ha thoien-takon. Ne Ro-ni-ha

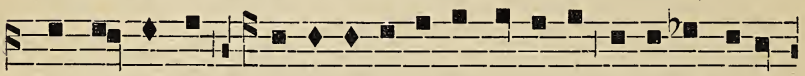

nok Roien-ha sa-tehonwa-tiwen-ni-iostha sa-tehonwati-

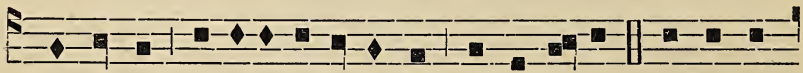

sen- naiens ro-ti-tokense-hakwe ronwatro-ri. Enskat ok

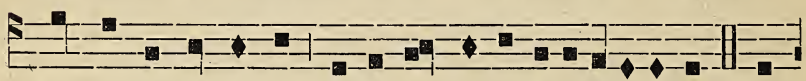

kentiohkwato-ken-ti Ra-tıkwe-konıe tehonarenihon. Twa-

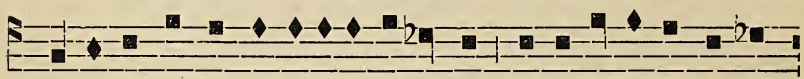

kehtakon enskat iontatenekwahestha swater.1-ke-watha ka-

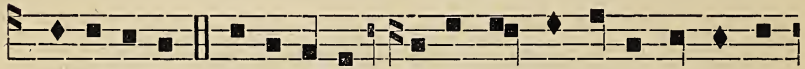

riwa-neren. Wakerhare tsi nentsiontonnhete niakowen-

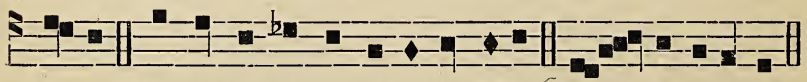

ta-hon. Nok iahte kakont entsiakonnheke. E - tho naiawen.

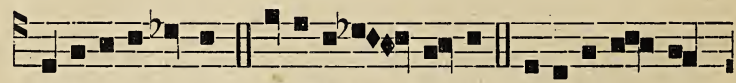

Saia-ta-to-kenti, Saia-ta-to - ken-ti, Saia-ta-to - ken-

E-1-10-

ti Sewenni-io tsi iakionnhe. Karonhiakwekon onwentsiakwe- 


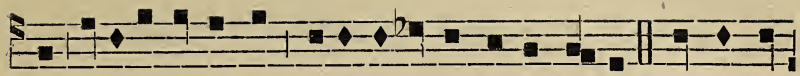

kon saiatanehrakwat, iesasennaien karonhia-ke. Ronwanen-

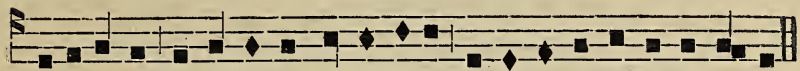

ton ne thare rasennakon Rawenniio ronwasennaien karonhiake.

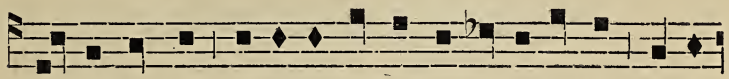

le- sos Kristos se-riwahtontha ka-ri-waneren, ka-ta-

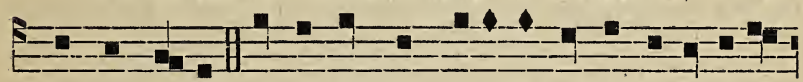

kwentenr ni-sa. Iesos Kristos seriwahtontha ka-ri wane-

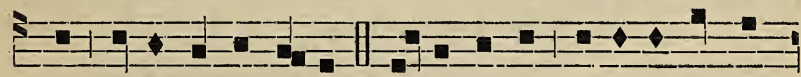

ren. katakwentenr ni-sa Ie-sos Kristos se-riwahton- tha

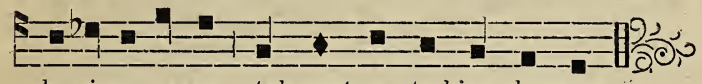

ka-ri-wa-neren takwentenr ta-kion skennen.

\section{TAKWENTENR AWENNENHAWIKEHA}

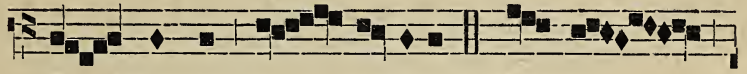

Ta - kwentenr Se - wenniio. III. Kris-tos

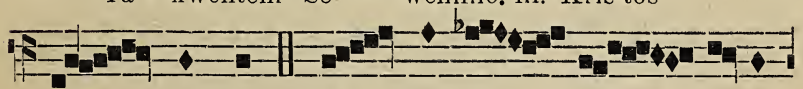

ta - kwentenr. III. Ta - kwentenr Se - wenni-

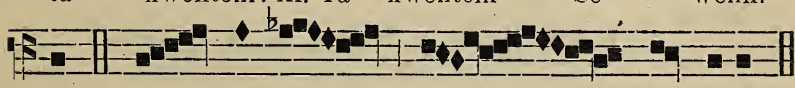

io. נI. Ta - kwentenr Se - _ wen-ni-io.

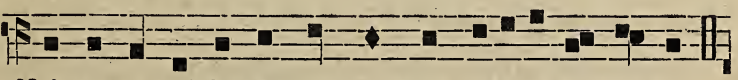

Noknonwentsiake skennen nonkwe iako-ni-konhri-io. 


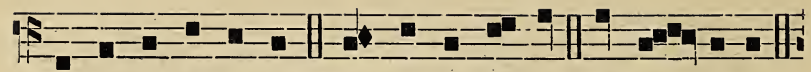

Tekwanon-we-ratons. Kwatsennonnia- se. Kwasen- naiens.

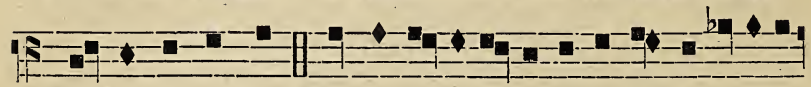

Kwaronhiahientons. Kwatonra-seron-se tsini a-kwa saia-ta-

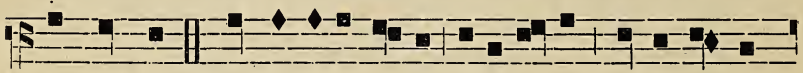

nehrakwat. Sewenniio ie-sen-nakera-ton $\mathrm{Ni}-\mathrm{io}$ iah-te

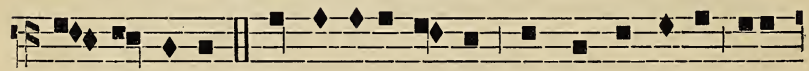

sa-no-ron-se. Sewenniio son-ha Hia-ien-ha Ni-io Ie-

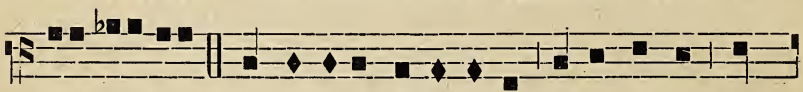

sos Kristos. Se-wenni-io hetse-nikonlıra-ie-wenthos Hia-

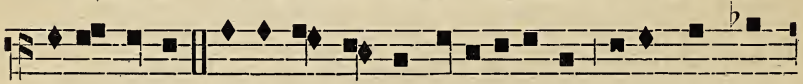

niha Niio.. Se-ri-wah-tontha kariwaneren katakwentenr

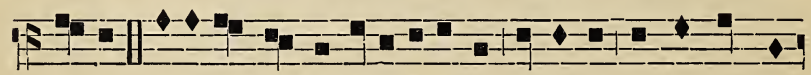

ni-sa. Seriwahtontha kariwaneren satontat o-nen non-wa

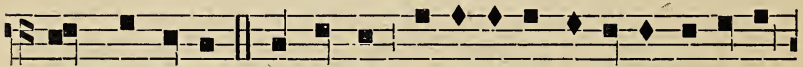

ne kwennitha. Ne satiens tsi raweien-teh-takon Hianiha

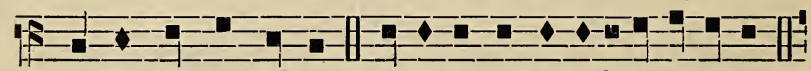

ka-takwentenr ni-sa. $\Lambda$-seken sonha saia-la-to-kenti.

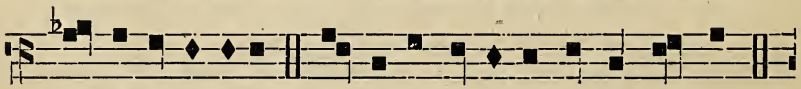

Sonha Sewenniio. Son-ha tsiatanoron Ie-sos Kristos.

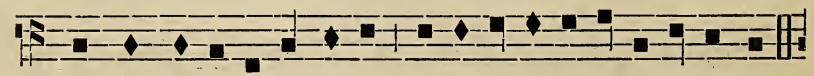

Rotkon Ro-ia-ta-to-ken-ti Ni-io Ro-ni-ha ietsisennaiens. 


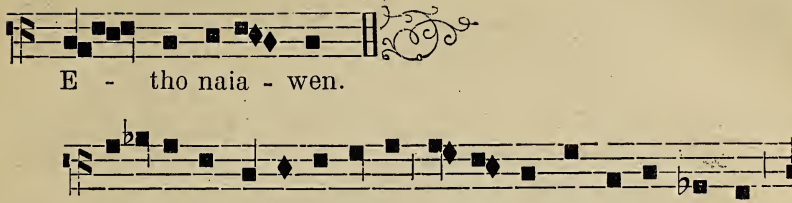

lioniha iahte ho-noron-se ro-son ka-ronhia onwentsia

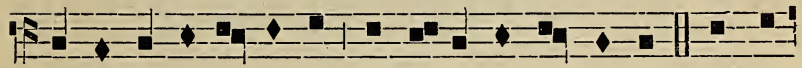

a-kwekon tsi-ni iekens nok tsi-ni iah-te iekens. Nok sha-

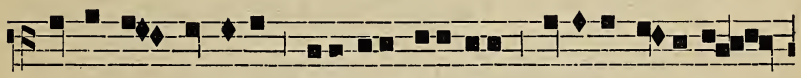

iatat $\mathrm{Ra}-$ wenniio It-sos Kristos raonha Niio Ro-

H-

ienha. Ok si Hiatatien- ha tsi-na-he iah-le kakonte. 'Te-

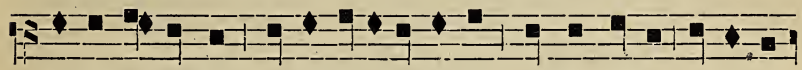

ioswate-takwa teioswatetakwake akwa $\mathrm{Ni-io}$ ra-onha-

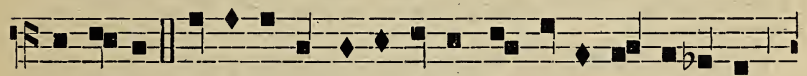

ke Ni-io. Roienha iahte honwason sahni-ia-tat Roni-ha

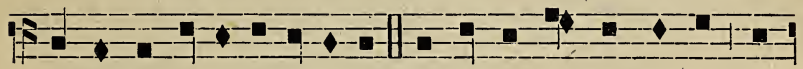

akwekon ro-te-weiennison. li tionkwe sonkwaniente son-

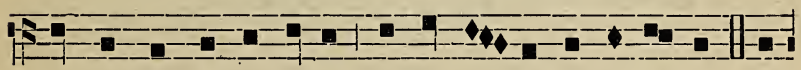

kwatsennon-niaten- ni-re ka-ron-hia - ke thawenonton. Ra-

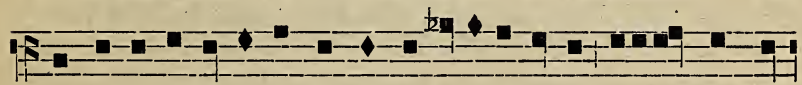

on-niaton ra-ieronke Rotkon Roia-ta-token-ti ao-nekwen-sa

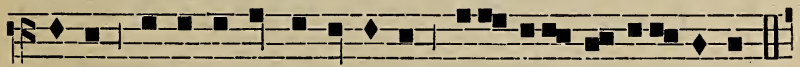

wa-ri iahte ka-nakwaiente-ri nok on - kwe ro - tonhon. 


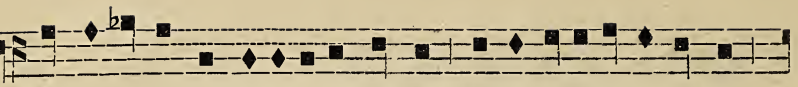

Onkwa-riwa ronwaiata-nentakton Pi-lat ro-te-rilionlakwe

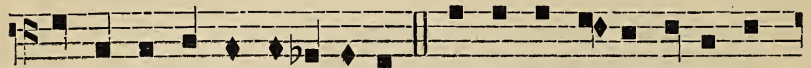

ronwenhe-ion ronwa-ia-ta-ten. Nok shotonnheton a-sen wa-

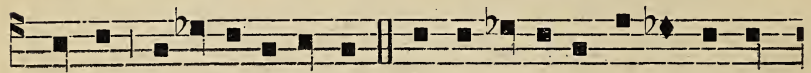

tontha tsi-ni ka-iatonkwe. Ka-ronhia-ke shawenonton tsì

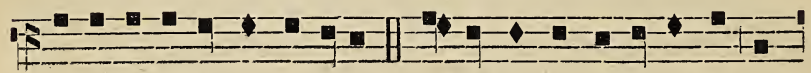

raweienteh-takon Ro-niha. Sekon tentre enthonehra-kwa-

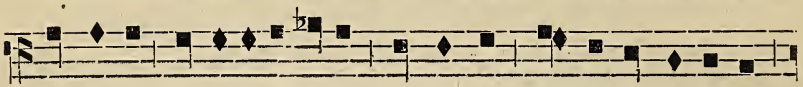

tonhatie tensakoia-toret iakonnhe nok ia-kowentahon

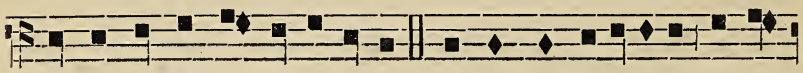

tiotkon enhonwen-na-ke-ratse. Nok Rotkon Roia-tato-ken-ti

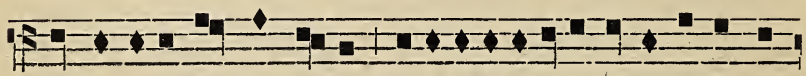

Rawenniio sonkionnhetha raonhake Roniha Roienha thoien-

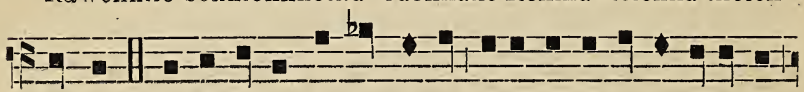

takon. Ne Ro-ni-ha nok Roienha satehonwa-tiwenniiostha

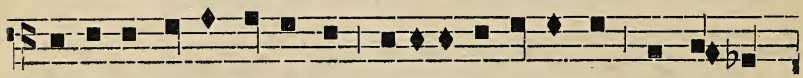

sa-tehon-wati-sennaiens ro-ti-token-sehakwe ronwa-tro-

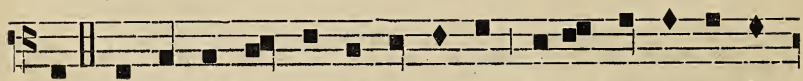

ri. Enskat ok kentiohkwa-to-kenti Rati-kwekonne te-

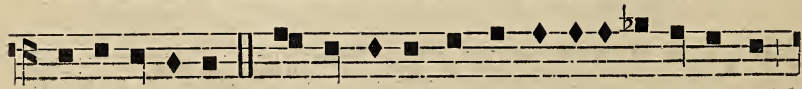

hö-na-re-nihon. Twakehtak on enskat ionta-tenekwahestha 


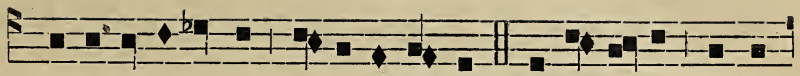

swatera-ke-watha ka-ri.wa-ne-ren. Wakerha-re tsi nen-

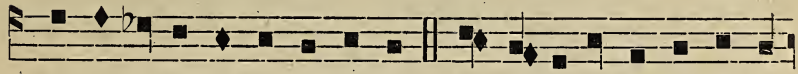

tsiontonnhete niakowen-ta-hon. Nok iahte kakont entsiakon-

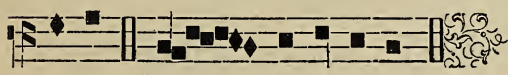

heke. E - tho naiawen.

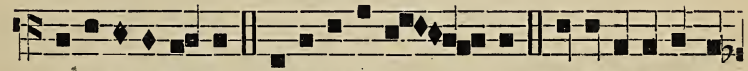

Säiata-tokenti, Saiatatoken - ti, Saiatatokenti

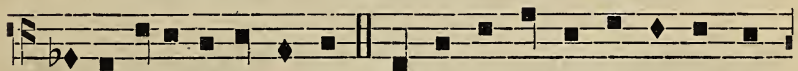

Sewenniio tsi iakionnhe. Karonhiakwekon onwentsiakwe-

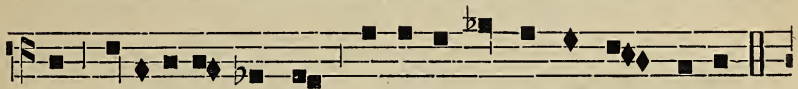

kon saia-ta-nehrakwat iesasen-naien ka-ron-hia-ke.

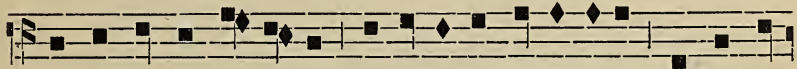

Ronwanenton ne thare rasennakon Rawenniio ronwa-sen-

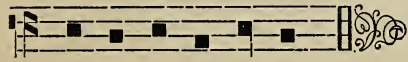

naien ka-ronhia-ke.

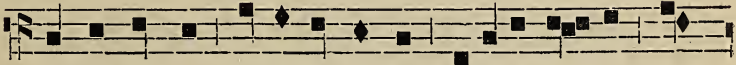

Ie-sos Kristos se-riwahtontha kariwa-ne-ren, ka-

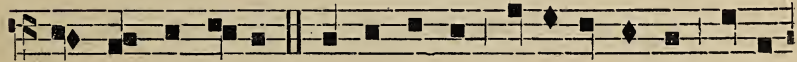

ta-kwentenr ni-sa. Iesoś Kristos se-riwahtontha ka-ri- 


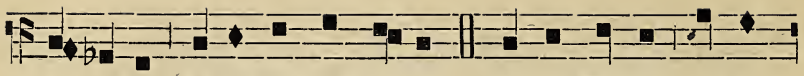

wa-neren, ka-takwentenr ni-sa. Iesos Kristos se-ri-

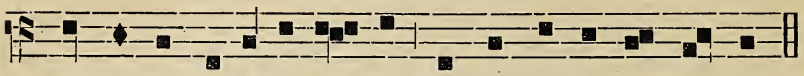

wahtontha ka-ri-wa-ne-ren, takwentenr takion skennen.

\section{TAKWENTENR TFKARONHIANEKENNEHA}

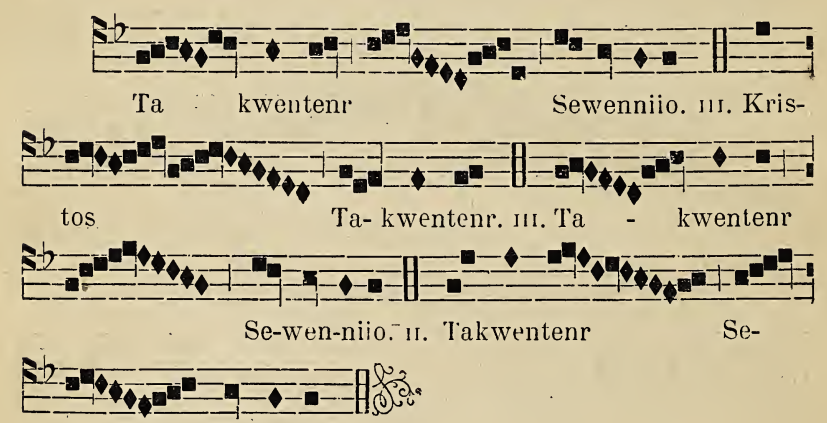

wenni- io.
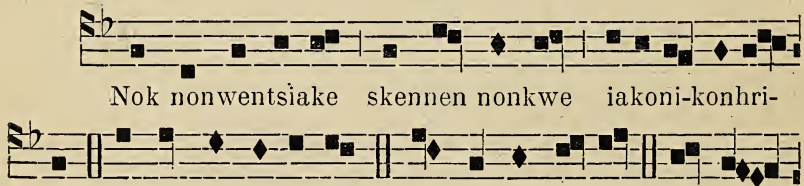

io. Tekwanonwe-ratons. Kwatsennonniase. Kwasen-

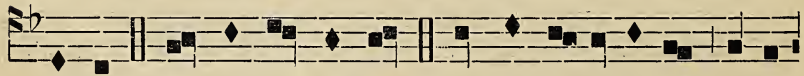

naiens. Kwaronhia-lientons. Kwalon-ra-seron-se tsi-ni-

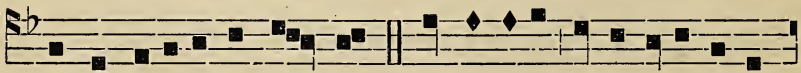

akwa saia-la-:ehra-kwat. Sewenniio iesenna-ke-raton 
E2

Niio iahte sa-no-ron-se. Sewen-niio sonha Hia-ien-ha

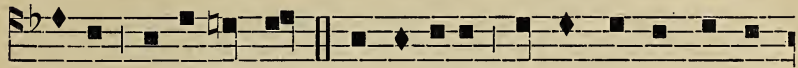

$\mathrm{Ni}$-io Iosos. Kristos Sewenni-io hetse - ni-kon-hra-ie-

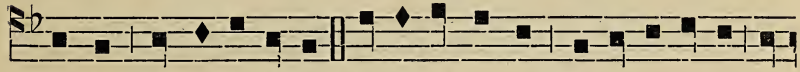

wenthos Hianiho Niio. Se-riwahtontha ka-ri- waneren ka-

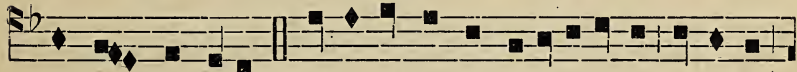

takwentenr ni-sa. Se-riwahtontha kariwaneren satontat

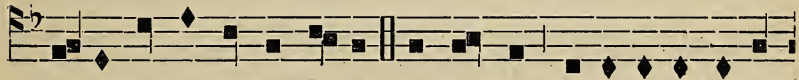

o-nen nonwa ne kwennilha. Ne sa-tiens tsi rawe-ienteh-ta-

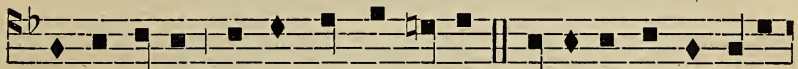

kon Hianiha ka-takwentenr ni-sa. A-seken son-ha sa-ia-

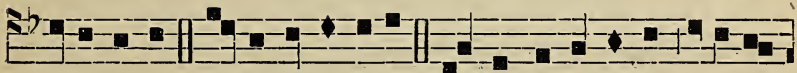

tatoken-ti. Sonha Sewenniio. Sonha tsiatanoron le-sos

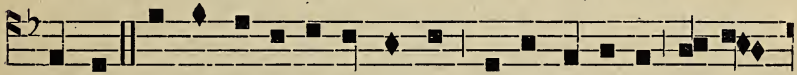

Kristos. Rotkon Ro-ia-ta-to-ken-ti Ni-io Ro-ni-ha ie-tsi-

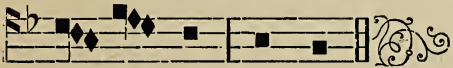

sen - na - iens. Amen.

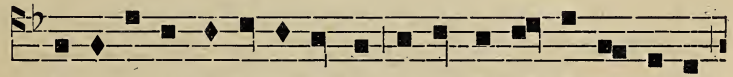

Ro-ni-ha iah-te honoron-se roson karonhia nonwentsia

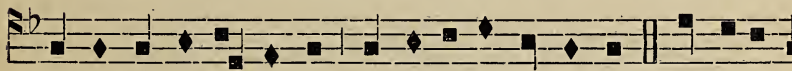

akwe-kon tsi-ni iekens nok tsi-ni iah-te iekens. Nok saia- 


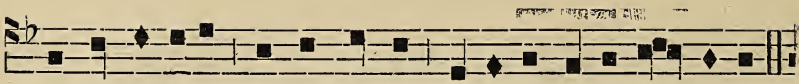

tat Rawenniio Iesos Kristos ra-onha $\mathrm{Ni}$-io $\mathrm{Ro}$ - ienha.

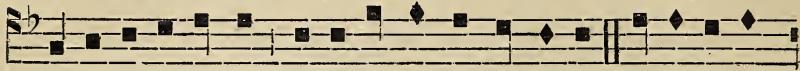

Ok si Hiatatienha tsina-he iahte ka-konte. 'Teioswa-te-

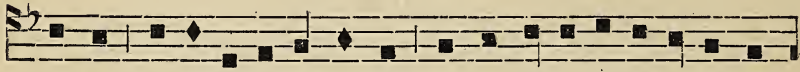

takwa teioswa-te-takwake akwa Niio raon-ha-ke Ni-

E-

io. Roienha iahte honwason sah-niiatat Roniha akwe-

E-

kon roteweiennison. Ii tionkwe sonkwaniente son-kwaisen-

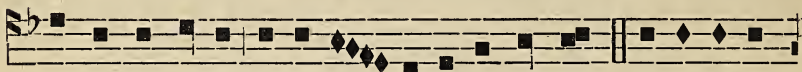

nonniaten- ni-re karonhia - ke thawenonton. Raonniaton

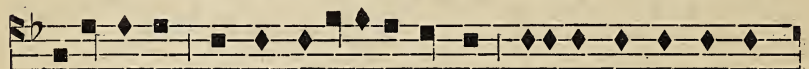

ra-ieronke Rotkon Roiatatoken- ti a-onekwensa Wa-ri-

52

iahte ka-nakwaien-te-ri nok on-kwe ro-tonhon. Onkwa-

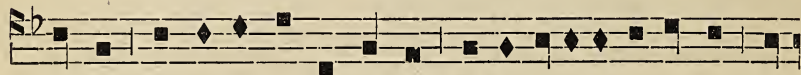

ri-wa ronwa-ia- ta-nentakton $\mathrm{Pi}$-lat rote-rihontakwe ra-

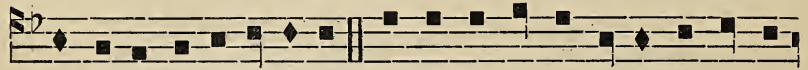

wenheion ronwaia-taten. Nok shotonnheton asen watontha

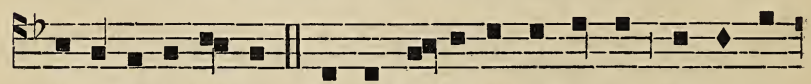

tsi-ni ka-ia-tonkwe. Karon-hiake shawenonton tsi ra-we- 


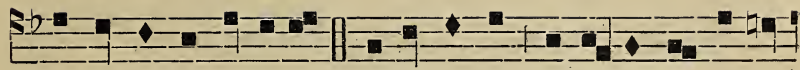
ienteh-ta-kon Roni-ha. Sekon tentre entho-nehrakwa-ton-

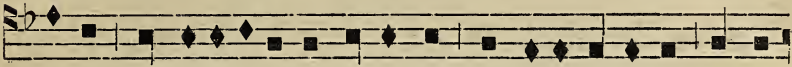
hátie tensako-ia-to-ret iakonnhe nok iakowentaon tiotkon E- $=-1=0$ enhonwenna-ke-ratse. Nok Rotkon Roiatato-ken-ti Rawen-

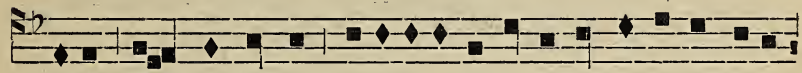

ni-io son-kionnhetha raonhake Ro-ni-ha Roienha thoienta-

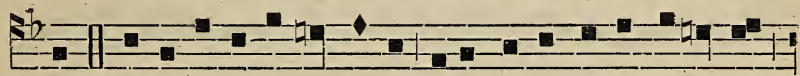

kon. Ne Roniha nok Roienha sa-tehonwa- tiwen-ni-iostha

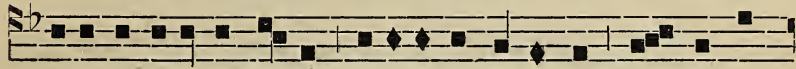
satehonwa-tisenna-iens ro-ti-tokense-hakwe ron- wa-tro-

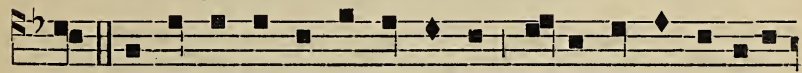

ri. Enskat ok kentiohkwatoken-tl Ra- tikwekon-ne teho-

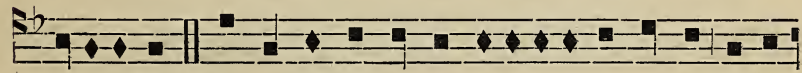

narenihon. Twakehtakon enskat iontatenekwahestha swate-

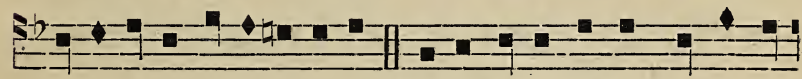

ra-kewatha ka-ri-waneren. Waker-hare tsi nentsiontonnhe-

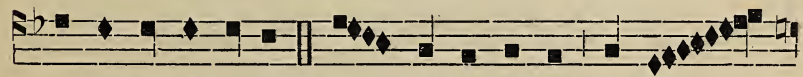

te nia-kowen-ta-on. Nok iah-te kakont en-tsia-

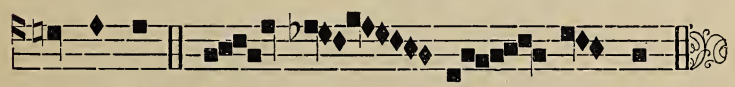

konnheke. $\mathrm{E}$ - tho na - ia - wen. 


\section{2-10 -}

Sa - ia - ta - token- ti, Sá - ia - ta-to- ken-ti,

s2-

Sa - ia - ta - token-ti Se-wenniio tsi ia - kionnhe.

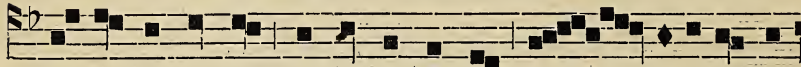

Ka-ronhiakwekon onwentsiakwekon sa - ia-ta-nehra-

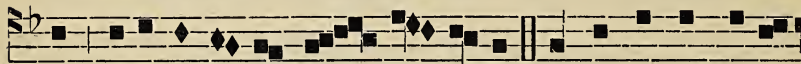

kwat iesa-senna-ien ka - ron-lia-ke. Ronwanenton ne tha-

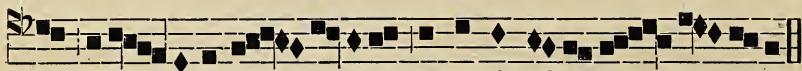

re rasen-nakon Ra-wenniio ronwasennaien ka - ron-hia-ke.

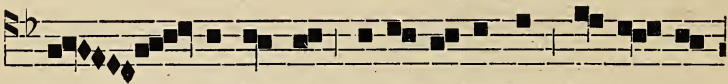

Ie - sos Kristos seri-wahtontha ka-ri - wa-

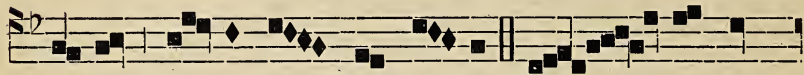

ne-ren ka - takwen - tenr ni - sa. le - sos Kris-

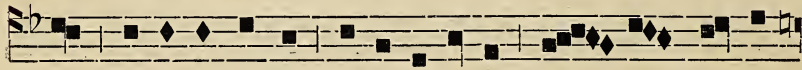

tos se-riwahtontha ka-ri-wa-neren, ka - ta-kwentenr

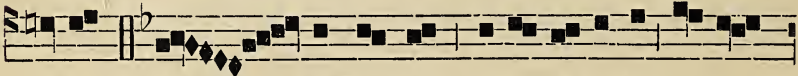

ni sa. Ie - sos Kristos se-riwahtontha ka- ri-

S2-10 -

wa-ne-ren, takw־n-tenr ta - kion skennnen. 


\section{TAKWENTENR ANEN}
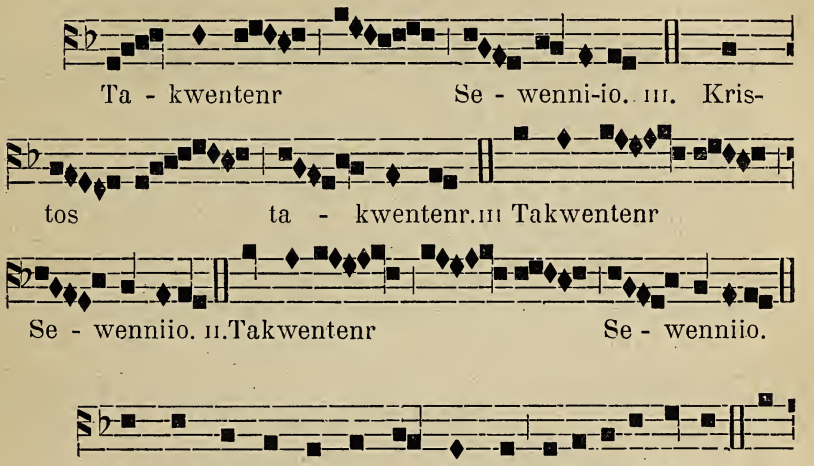

Nok nonwentsiake skennen nonkwe iakonikonhriio. Te-

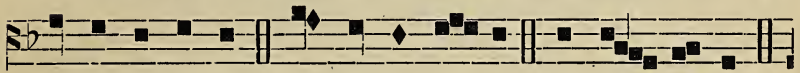

kwanonweratons. Kwatsennonnia-se. Kwasen - na-iens.

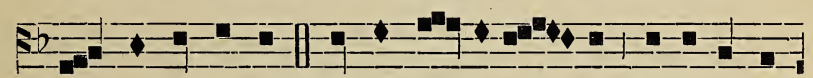

Kwa-ronhiaientons. Kwatonra - seron - se tsi-ni akwa

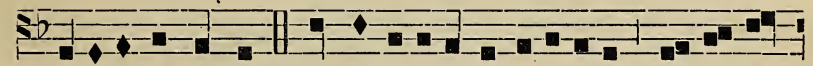

sa-iatanehrakwat. Sewenniio iesennakeraton $\mathrm{Ni}$-io iah-

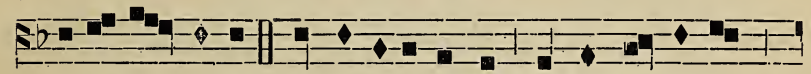

te sa-no - ronse. Sewenni-io sonha Hiaien-ha Ni-io

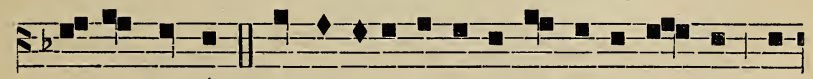

Ie-sos Kristos. Sewenni-io hetseni-konhra-ie-wenthos Hia-

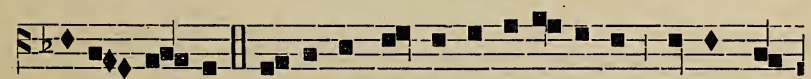

ni-ha $\mathrm{Ni}$ - io. Se-riwahton-tha ka-riwa-neren ka-takwen- 
S工二二A

tenr ni - sa. Se-riwahtontha kariwa-ne-ren salontat onen

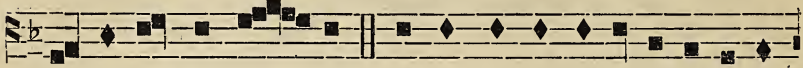

non-wa ne kwenni - tha. Ne satiens tsi ra-weienteh-takon

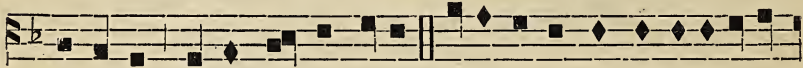

Hia-niha katakwentenr nisa. Aseken sonha sa-iata-token-

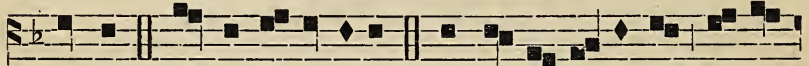

ken-ti. Sonha Sewenniio. Son-ha tsiata-noron te- sos

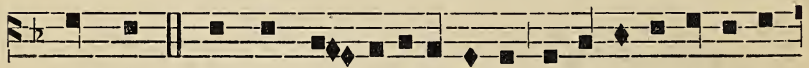

Kristos. Fotkon $\mathrm{Ro}$-ia-tato-kenti Ni-io Ro-ni-ha ietsi-

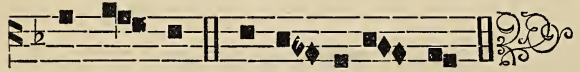

sen-na - iens. E-tho na-ia - wen.

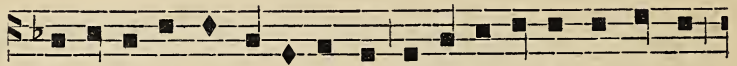

Ro-ni-ha ialite ho-noron-se roson karonhia nonwentsia

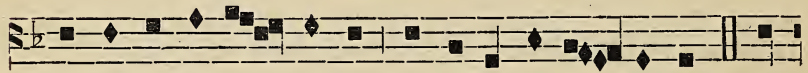

akwe-kon tsi-ni iekens. Nok tsi-ni iahte iekens. Nok

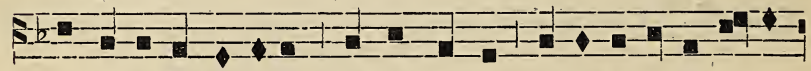

sha-iatat Rawenniio le-sos Kristos ra-onha Ni-io Roien-

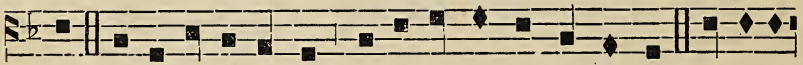

ha. Ok si Hiatatienha tsi-na-he iahte kakon-te. Teioswa-

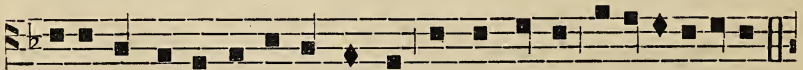

tetakwa teioswa-te-ta-kwake akwa Niio raonhake Niio. 


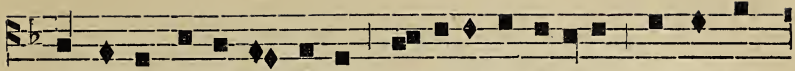

Roien-ha iahte honwason sah-niiatat Poniha akwe-kon

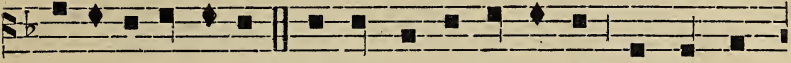

ro-te-weiennisnn. Ii tionkwe sonkwaniente sonkwatsen-

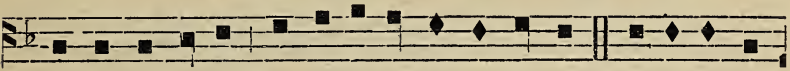

nonnia-ten- ni-re karonhia.ke thawenonton. liaonnia-ton

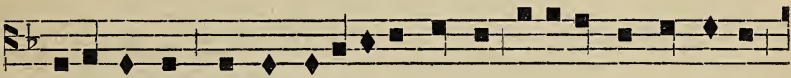

raie-ron-ke liotkon roia-tato-ken- ti a-o nekwensa wa-ri

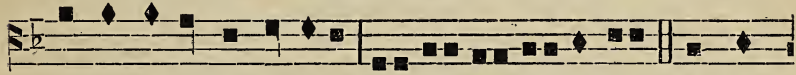

iahte kanakwaien-teri nok on-kwe ro-tonhon. Onkwa-

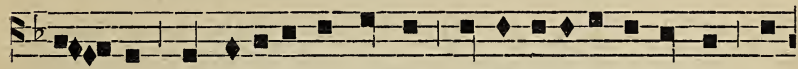

ri - wa ronwaia-tanen-takton Pilat rote-ri-hontakwe ra-

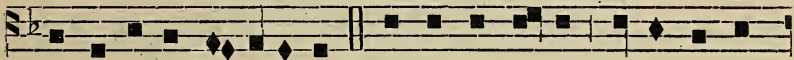

wenheion ronwa-ia-taten. Nok shotonnheton asen wahton-

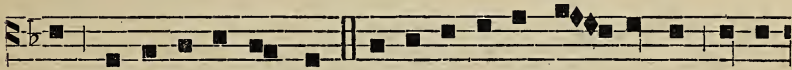

tha tsi-ni kahiaton-kwe. Karonhiake thawe - nonton tsi ra-

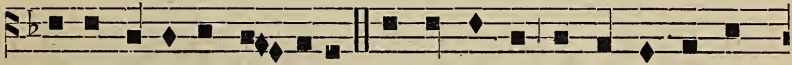

weien-teh-takon Ro-niha. Sekon tentre en-tho-nehrakwa-

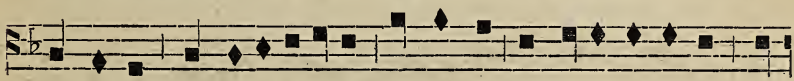

ton-ha-tie tensa-ko-ia-toret iakonnhe nok iakowentahon tio-

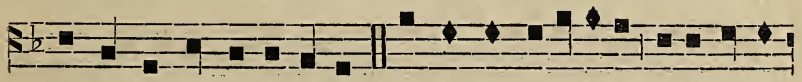

tkon enhonwenna-ke-ratse. Nok Rotkon Roiatatokenti Rawen- 
St-1-1

ni-io son-kionnhetha-ro-nonhake Roni-ha Roienha thoien-

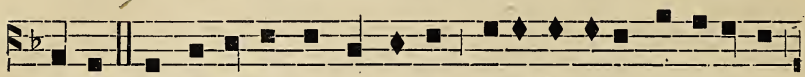

takon. Ne Roni-ha nok Roienha satehonwatiwenniiostha

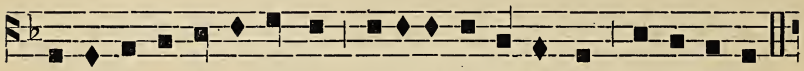

sa-tehonwa-tisennaiens rotitoken-se-hakwe ronwatrori.

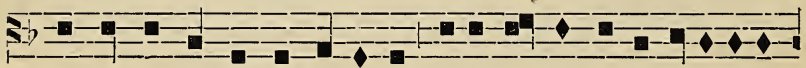

Enskat ok kentiohkwatokenti Ratikwekonne te-hona-reni-

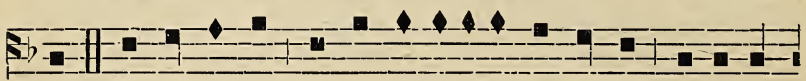

hon. Twakehtakon enskationta-tene-kwahestha swate-ra-

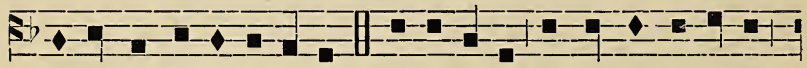

kewa-tha ka-ri-waneren. Wakerhare tsinentsiontonnhete

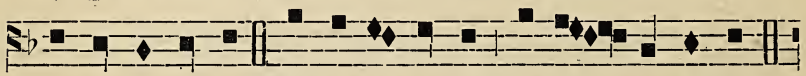

nia-kowen-ta-hon. Nok iahte kakont entsia - konnheke.
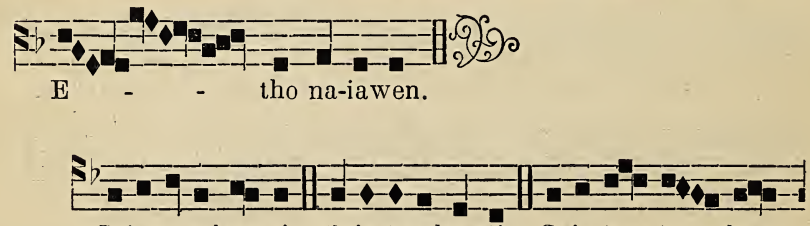
Saia-ta-token-ti, Saia-tatoken-ti, Saia-ta - to - ken-

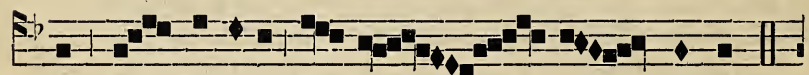

ti Se - wenni-io tsi ia - _ _ - kionnhe.

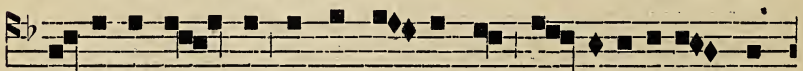

Ka-ronhiakwe-kon nonwentsiakwekon sa-ia-ta-nehrakwat 


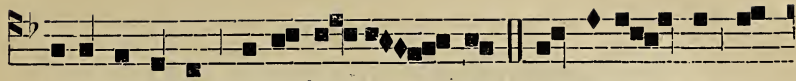
ie-sasennaien karonhia .. ke. Ronwanen-ton ne Si)

tha - re rasen-nakon Rawenniio ronwa-sen-naien kaSh-

ron - hia - ke.

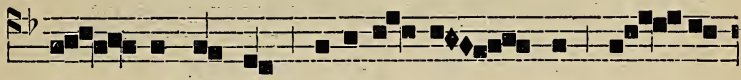
Ie - sos Kristos seriwah- ton - tha ka - ri 5) wane- ren ka- takwentenr ni - sa. Ie - sos

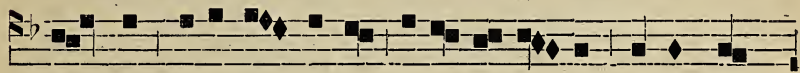

Kris-tos se-riwah-tontha ka ri-wa-ne - ren ka-takwen-

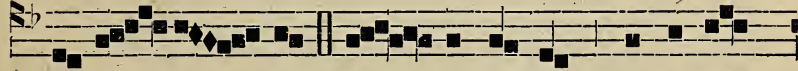
tenr ni - sa. Ie - sos Kristos se - riwah 5) ton - - tha ka - ri-wane - ren takwentenr ta-kion Si) =

sken - - nen. 


\section{TAKWENTENR DUMONTNEHA}

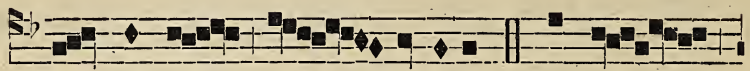

Ta-kwentenr Se - wenniio. m. Kristos

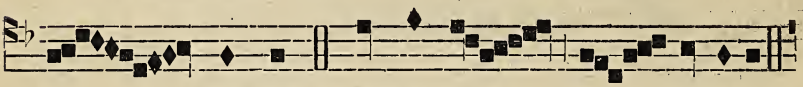

ta - kwentenr. m. Takwentenr Se - wenniio. I.
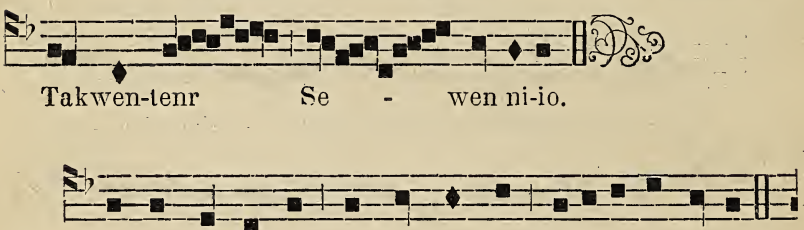

Nok nonwentsiake skennen nonkwe iako-nikonhri-io

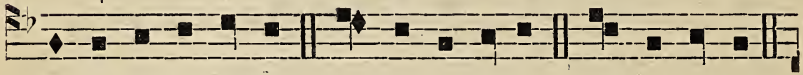

'Tekwanon-we-ratons. Kwatsennonniase. Kwasennaiens.

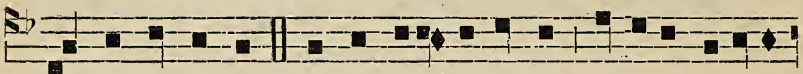

Kwaronhiaientons. Kwatonra - seron-se tsini akwa saia-

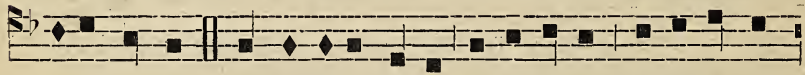

tanehrakwat. Sewenniio iesen-na-ke-raton Niio iahte

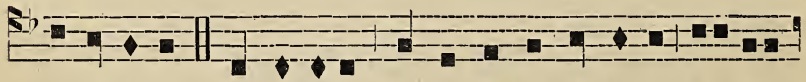

sa-noronse. Sewenniio sonha Hiaien-ha $\mathrm{Ni}-\mathrm{io} \mathrm{Ie}-\mathrm{sos}$

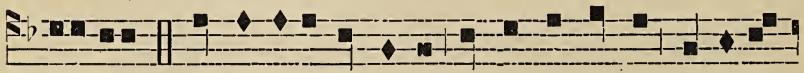

Kris-tos. Sewenniio hetse-ni-kon-hraiewenthos Hiani-ha 


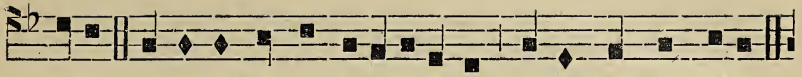

Niio. Se-ri-wahtontha kariwaneren katakwentenr nisa.

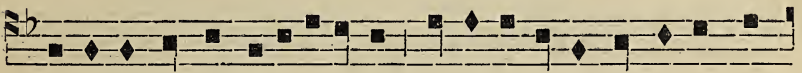

Se-riwahtontha kariwaneren satontat onen nonwa ne kwen-

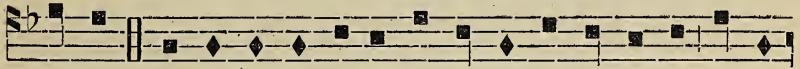

ni-tha. Ne satiens tsi ra-we-ienteh-ta-kon Hiani- ha ka-ta-

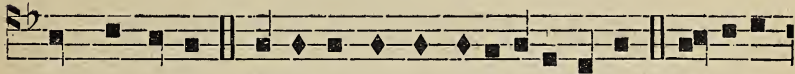

kwentenr ni-sa. A-seken sonha sa-iata-tokenti. Sonha Se-

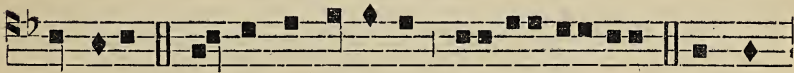

wenni-io. Son-ha tsiata-noron le-sos Kristos. liotkon

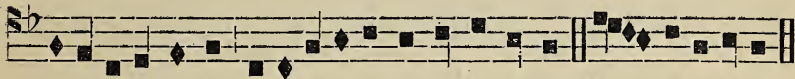

Roiata-tokenti Niio Roniha ietsisennaiens. E - tho naiawen.

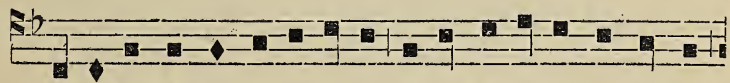

Ro-ni-ha iah-te honoron-se roson karonhia nonwentsia

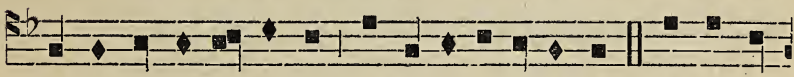

akwekon tsi-ni iekens nok tsini iahte iekens. Nok shaia-

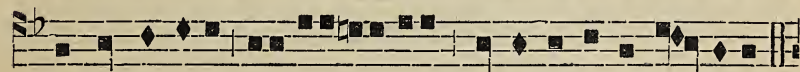

tat Rawenniio Ie-sos Kristos ra-on-ha Ni-io Ro-ienha.

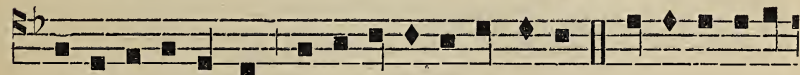

Ok si Hialatien-ha tsi-nahe iahte ka-konte. Teioswa-te-ta-

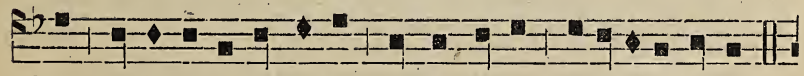

kwa teioswa-te-lakwake akwa $\mathrm{Ni}-\mathrm{io}$ raonhake $\mathrm{Ni}$-io. 


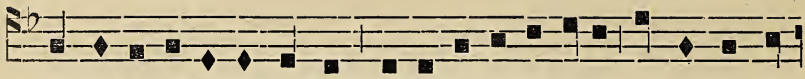

Roienha iahte honwason sahni-ia-tat Roniha akwekon ro-

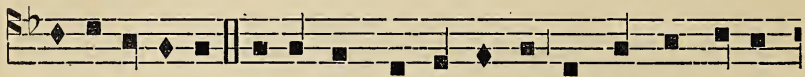

te-weiennison. Ii tionkwe sonkwaniente sonkwatsennonnia-

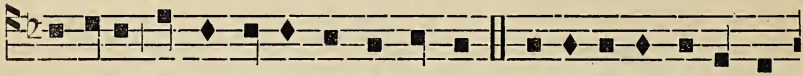

ten-nire karon-hiake tha-wenonton. Raonniaton Ra-ie-ron-

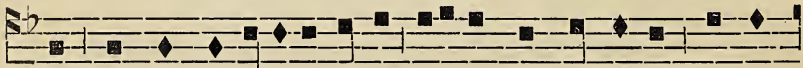

ke Rotkon Ro-iata-tokenti aonekwen-sa Wari iahte

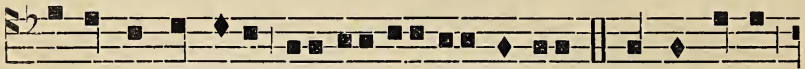

ka-nakwaien-te-ri nok onkwe ro - tonhon. Onkwa-ri-wa

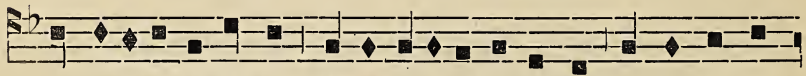

ronwaiatanentakton Pilat rote-rihon-takwe rawen-he-ion

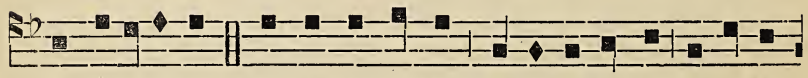

ronwa-iataten. Nok shotonnheton asen watontha tsinika-

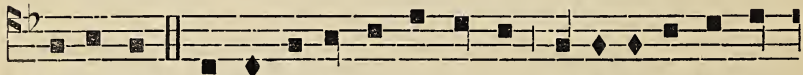

hiatonkwe. Karonhia-ke shawenonton tsi rawe-ienteh- ta-

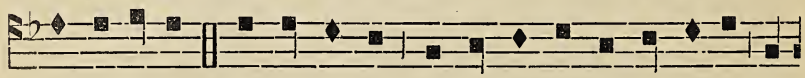

kon Roni-ha. Se-kon tentre en-tho-nehrakwaton-hatie ten-

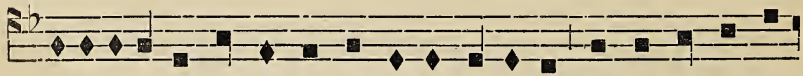

sako-iato-ret iakonnhe nok ia-kowentahon tiotkon enhonwen-

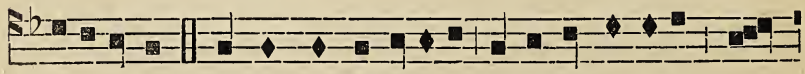

nake-ratse. Nok Rotkon Roia-ta-to-kenti Rawenniio son- 


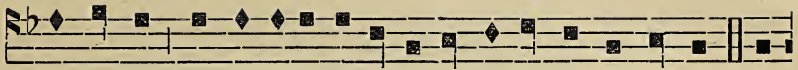

kionnhetha ro-nonhake Roni-ha Roien-ha thoien-takon. Ne

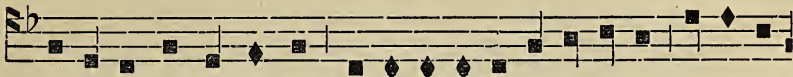

Roni-ha nok Roien-ha sa-tehonwa-tiwenni-iostha satehon-

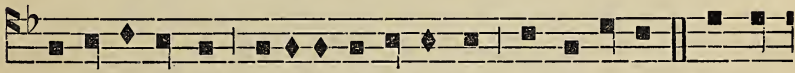

wa-tisen-naiens ro-ti-token-se-hakwe ronwatro-ri. Enskat

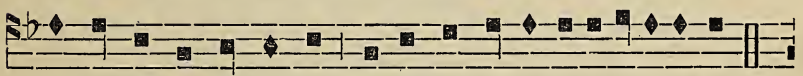

ok kentiohkwatoken-ti Ra-tikwekonne teho-narenihon.

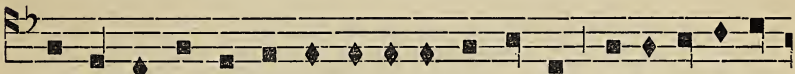

Twakehtakon enskat ion-ta-te- nekwahestha swatera-kewa-

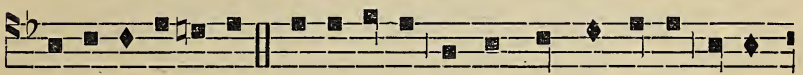

tha ka-riwaneren. Wakerhare tsi nentsiontonnhete niako-

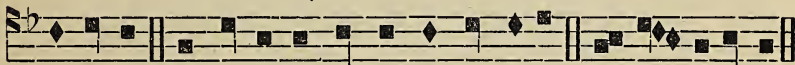

wentahon. Nok iahte kakont entsiak onnheke. E - tho naiawen.

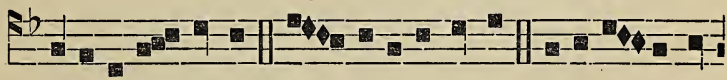

Saia-ta-to - kenti, Sa - ia-tato-kenti, Saia-ta - token-

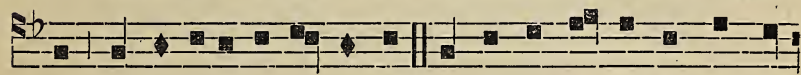

ti Sewenni-io tsi ia-kionnhe. Karonhiakwekon nonwentsia-

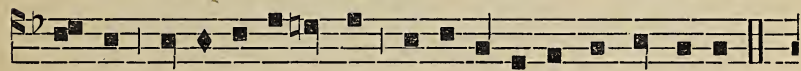

kwekon sa-ia-tanehrakwat ie-sasen-naien ka-ronhia-ke.

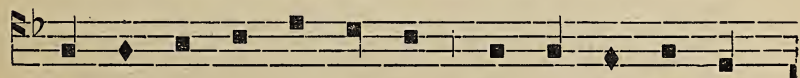

Ron-wa-nen-ton ne tha-re ra-sen-na-kon Ra- 
52

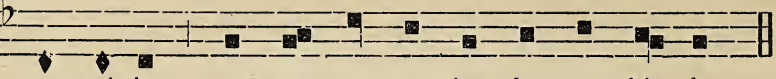

wen-ni-io, ron-wa-ser- na-ien ka-ron-hia-ke.
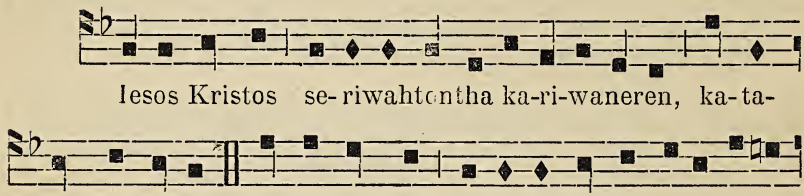

kwentenr ni-sa. Ie-sos Kristos se-riwahtontha ka-ri-wa-ne-

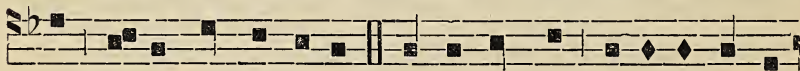

ren ka-takwentenr ni-sa. Ie-sos Kristos se-riwahtontha

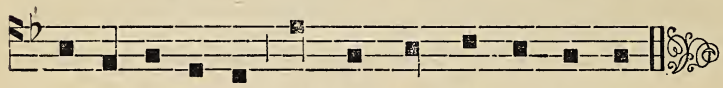

ka-ri- wa-neren ta-kwentenr takion skennen.

\section{AWENTOKON SHOTONNHETSTON}

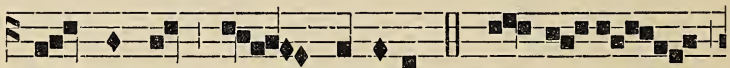

Ta-kwentenr Se - wenniio. III. Kris-tos

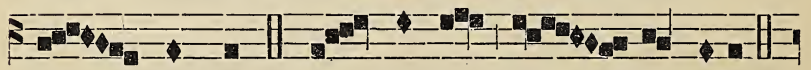

ta - kwentenr. II. Ta - kwentenr Se - - wen-niio. Ir.

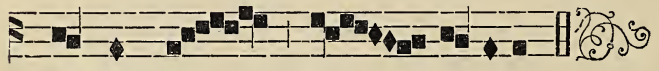

Ta-kwentenr Se - - wenniio.

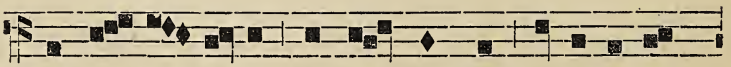

Nok non-wentsia-ke skennen nonkwe ia-ko-ni-kon- 


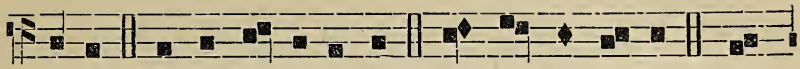

hri-io. Tekwanonwe-ratons. Kwatsen nonnia-se. Kwa-

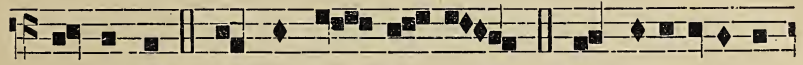

sen-naiens. Kwaronhia - ien- tons. Kwatonraseronse

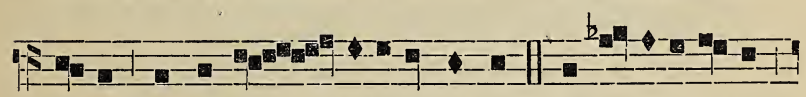

tsi-ni akwa sa - - iatanehrakwat. Sewenniio ie-sen-

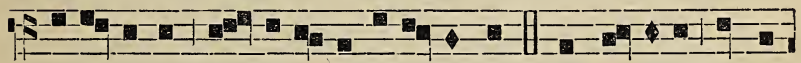

nake-ra-ton $\mathrm{Ni}$ - io iahte sano-ron-se. Sewen-ni-io sonha

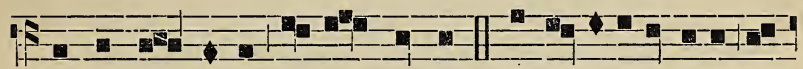

Hiaien-ha Ni-io Ie-sos Kristos. Sewen-niio hetsenikon-

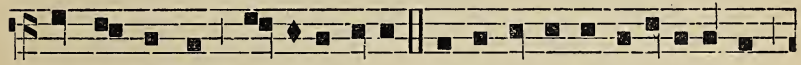

hra-iewenthos Hianiha Niio. Seriwahtontha kariwaneren

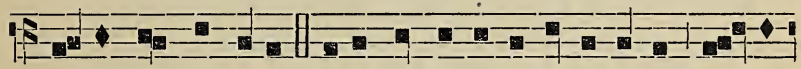

ka-takwentenr nisa. Seriwahtontha ka-riwa-neren sa-ton-

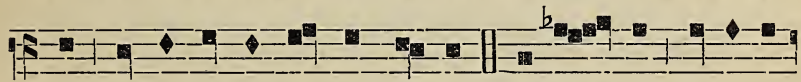

tat onen nonwa ne kwenni-tha. Ne sa-tiens tsi rawe-

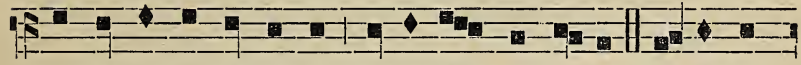

ien-teh-lakon Hia-ni-ha ka-lakwentenr ni-sa. A-se-ken

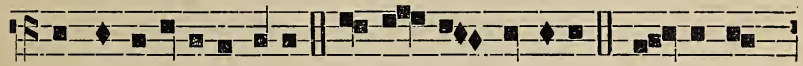

son-ha sa-iatatokenti. Sonha Se - wenniio. Son-ha tsia-

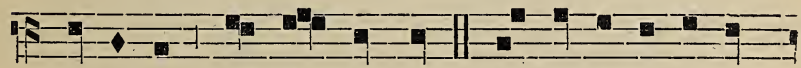

ta-no-ron Ie-sos Kristos. Rotkon Ro-ia-ta-to- 


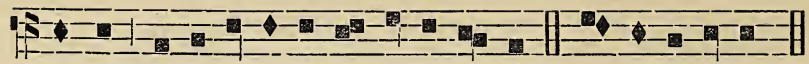

ken-ti Ni-io Roni-ha ie - tsisennaiens. E- tho na-iawen.

Twakehtakon: Anen aorenna (sk 69).

ST-

Saia-tatoken-ti, Saia-tato-kenti, Sa-iata-tokenti

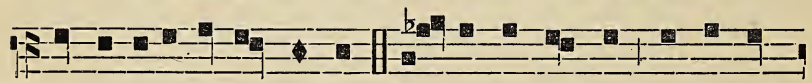

Sewenniio tsi iakionnhe. Ka- ron-hiakwekon onwentsia-

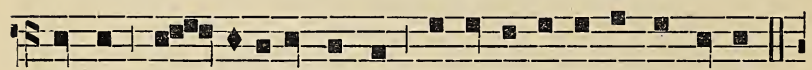

kweken sa - iatanehrakwat ie- sasennaien karon-hiake.

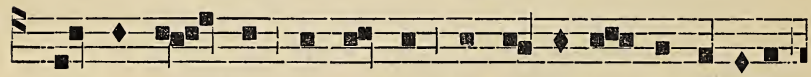

Ronwa-nen - ton ne tha-re Fa-sen-nakon Rawenniio

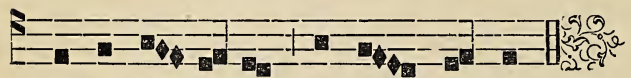

ronwa-sen- na-ien ka-ron - hia-ke.

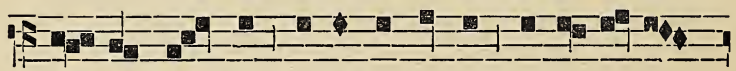

Ie - sos Kris-tos, se-ri-wahtontha ka-ri-wa-ne-

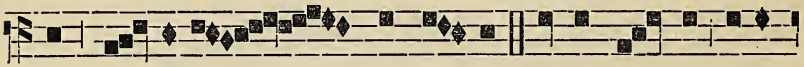

ren, ka-takwen - tenr ni - sa. Ie-sos Kristos se-ri-

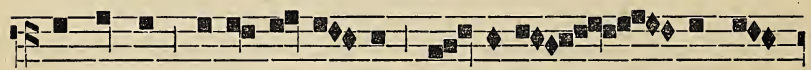

wahtontha ka-ri- wa-ne-ren, ka-takwen - tenr ni-

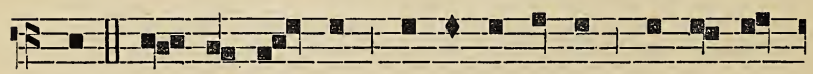

sa. Ie - sos Kris-tos se- riwah-tontha ka-ri - wa- 


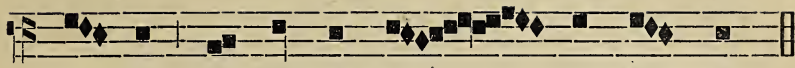

ne - ren, ta-kwentenr ta - - kion sken - nen.

\section{AWENTOKON IOKARENRE}

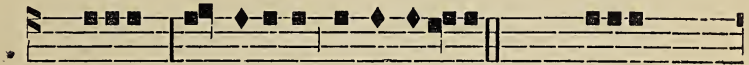

Niio askenikonhrarake, askienawase, Sewenniio sastoron

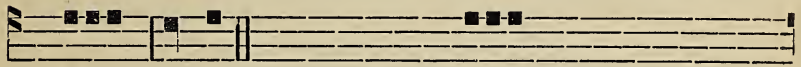

askiatakennha. Aonwasennaien Roniha nok Roienha nok Tsiniiotonnhe tsinahe, ethonaiohtonhake Tiotkon oni tsinenwe etho na

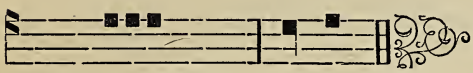

Rotkon Roiatato - ken-ti. nonwa.

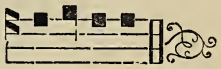

A-re-ri-a. iawen.

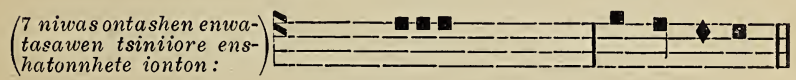

Aiesasennaien Niio Sewen-ni-io.

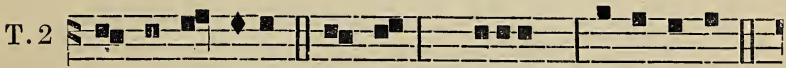

Ne Rawenni-io. Satkon Niio saia - ta token-ti, *

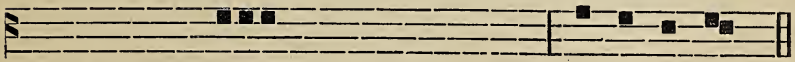

askenikonhrahnirate tsi wake - - ri-wi-ioston.

Tewakehtakon enskat ok Niio * tiotkon tsi hanakere iahte kakonte enhanakereke.

Otkon nihaiatoten * iahtiiaiehewe tsini hoianere.

Raonhaa tsini hattokha kowa * raonhaa tsini thoriwaieri. 
Raonhaa tsini hoiatatokenti * raonhaa tsini hotanitenraskon.

Akwekon roteweiennison, iah othenon te honoron * oktihawenniio othenon neniawen, nok oni tosa etho naiawen.

Akwekon raienteri tsini iawenhon tsinahe * tsini iawens nonwa nok tsini iawensere.

Oktiwakwekon tenteron, akwekon tehakanere * othenon oni sotsi ioleriwasehton nonkwanikonhrakon.

Sakononwes nok sakotsennonniatennis iakoianere * sakoswens nok sakohrewalha ialste iakoianere.

Askitenre. Sewenniio, tiotkon akateweienton * etho naiawen iah nonwenton taskswenseke.

Ahonwasennaien...

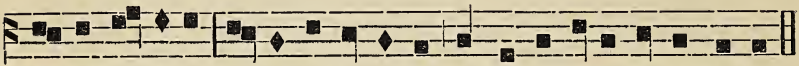

Ne Rawenniio rawenni Roienha: satien tsi keweientehtakon.

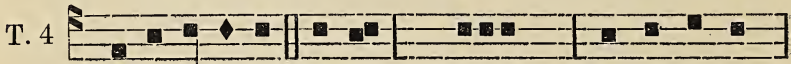

Tkariwa-ie-ren. Ni-io Sewenniio tsi iakionnhe,

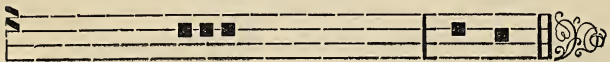

sotsi wakitent tsi wakeriwa - neren.

Okti wakiatisonkwa tsini konsentenni * iah seken tiiaiehewe tsini konwennontion.

Ne wakiatakennhaon satanitenrasera kowa * tsi iahten teskiatont-on noneshon.

Akwa wakerientaksens tsini kariwaksen tsini wakieren * ne tsi iahtiiaiehewe tsini saianere nok tsini seriwaswens ne kariwaneren.

Wakaterientason enwaksnienontie saianerenstakwa * iah nonwenton taonsakeriwanerake.

Rakeni, wakeniseriio kowa " tosa seriwarako tsini konsentenni.

Ne askitenrate tsini satanitenraskon * nok tsini sonkwatsennonniatenni Iesos Kristos.

Swaronhiakehronon nok swaiatatokentison, eh sewatiskwentare tsi renteron $\mathrm{Niio}^{*}$ ietsisewennitentase sekon aonsahakitenre.

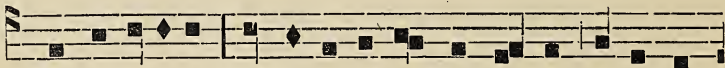

Tka-ri-wa-ieri nok io-te-ri-wa-kwa-ri - sion (sini ion-

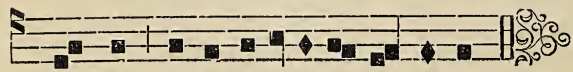

wannhas Niio ra-oia-ne-r'en- se-ra. 

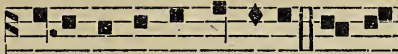

Rao-senna Rawenniio. Sewenniio ise iateskahraientas na-
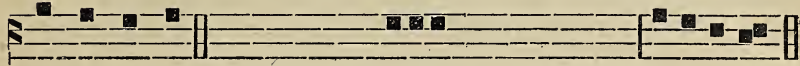

kwatonnhetston, nok oni tsienteritsihon akwekon tsini katieranions.

Akwekon iesienteri tsini kennontonnionkwa nok arekho tsi wakeron * ise takwatsiatani tsinenwe tsi enkalohetstanionheke.

'Tsiatasnore tsi teskanere tsi wennontonnionkwa nakwatonnhetston * arekho tetewakewenninekenhon, onen ok wesanikonhraientane.

Tsienteri tsinahe nok oni tsi neniawensere * ise takiatison tiotkon teskenentsawakonhatie.

Ionehrakwat iahtiiaiehewe tsini tsiatananon * kennikonhroktha tsini saiatanehrakwat.

Ka nonwe iake, iahtaonton akatasehtake, tsini sanikonhrowanen, * ka nonwe akateko, iah othenon te iothrenhoston tsi tesatkahtonnions.

Tsina tenteskiatakwe, Sewenniio, enskenentsine tsi ienskiteron * takitenr tenhnon tosa takrewat.

Ise, Sewenniio akweriasakwekon * ise takiatison takonnhetonhatie.

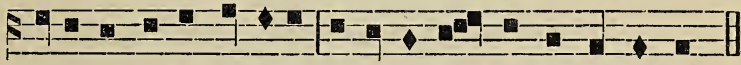

Rao-senna Rawenniio akonwanen-ton iah-te kakont.

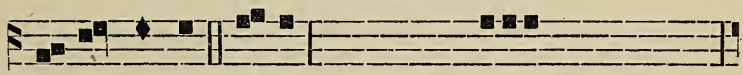

Ii tsi tionnhe Tekwakehtakon akwatokenske otkon non-

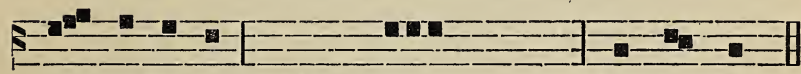

kwatonnhetston, * iahtakenheie

nonwen - ton.

Iaiheions jakoiatoronhatie iaiheiatha * eniakoiatekhake iahte kakonte.

lakoiatoronhatie iahte iaiheiatha tsi tetieseronniatha tentsieriwaserako, * nok karonhiake eniente.

Nok iuh othenon te iakoiatoron, " oksa karonhiake eniontsennonni.

Iontatitenratha tsi tetieseronniatha iakoronhiaken " tsi iontaterennaiennis, nok senha naontathasenhase. 
Enwatonwentsiokten entsitewatomnhete * enskontikwekonhake nonkwatonnhetston ne tewaieronke.

. Tensonkwaiatoretakwentho Iesos Kristos * karonhiake eniente iakoriwiiostonne.

Sotsi tenhnon iakotentasere iakoriwanerahakskonne, * oneshon iahte kakonte eniontetsirate.

Askitenre Sewenniio * askiatanonstate nonen enkatonnhokten.

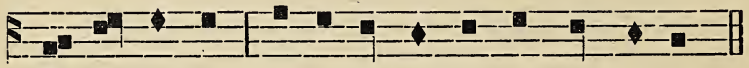

Ii tsi tionnhe hetsi-twanen-ton Ra-wen-ni-io.

Karenna sonha iokarenre leieriwakwalha.

Rotaskat onwe iahte honaktaien tsi rontkennisahatha ne rotiriwanerahakskon *iah oni te hatiens tsi ratitsienhaientakwa.

Rariwisaks tenhnon tsini honikonhroten ne Rawenniio * wenniseratatie asontakwekon oni ne tehoiatoretonhatie.

Teshiatieren kerhite kahionhakta ioniote " tiotkon ioianionte wahiakon tsini iohiari.

Iah oni te kaneratens, saheto watkaweratase * tsini iohrenion ioiaskats iah othenon teioiotatse.

Ethoniiot tiakoriwaieriton eniontsennonni * kariwiio okon tsini iakoieren nonwentsiake nok oni karonhiake enwatehiaron.

Iah eh taiawen ne iakoriwanerahakskonnhe * aiairon iah eh taiawen.

Etho enwaton nonwentsiake tsiniiot nosewaseri sonha * tekontikens tsi iahote iah kaneka te kaieronni tsini ieionenon.

Tiotkon sakonentsawakon ne tiakoriwaieriton * etho eniontahitha tsi rohahaieronniston.

Akwa iakotent ne iakoriwanerahakskonne * iahte kakonte tsi iotonwentsiokte ienieiatenne.

Tewenniseraien sane enshaie * tenhotskarawen tsinenhahronke tsini ioriwatsanit tsinenhariwisa no Rawenniio.

Eniorakarheke ne raontakon tsini ioriwatsanit enhahronke * aseken Isini kariwes kowa enhanakereke ne Niio, ethoni kariwes enhatkaronni.

SEwennilo to ok nioserashen teskwanaktiiostenni * eh kati nahe teskwennontonnionsehatie.

Arekho tsi sonwentsison nok oni arekho tsi ionontenions * onen ok si iakoterientare sonhaa Sewenniio. 
Ethone iah othenon te tiotoktaon * tsini iaweta kariwanoronson takwentenranion.

Niawen kati tsi iahte kakonte senakere * nok oni iahte kakonte ensenakereke.

Oieri tewenniawe nioserake tsi teskanere iawet sewenniserat * tokat kenniwasontesha ne skennen ti sanaktiio.

SEwennio iahte sanekherens tsini ionkionwentsioten nonwentsiakwekon * etho niate wenniserake iakwenheions kati.

Etho niiosnore tsi iakionnhe ne iakionkwe * tsiniiot orhonke ne takatsitsianekare, entie ontonnisa, iekahra wakatsitsientane.

Ok eken senha satsnoraton kenniskionnhetstonha * ne takwahrewatakon tsini ionkwariwanerahakskon.

Aseken teskanere oriwakon tsini iakwatierha * tikenha tsina teiakwakanere tsina teioswathe.

Iahte oriwatoken othenon ionkwariwaiote nok oni tsi tsi tionnhe * tsiniiot teionaharaton tsini iahte iohniron.

Serenkene tsiatak nioserashen iakonnhiio * aseken isi nonkwati onen ok niate karonhiakenserake tsineniakoronhiakentake.

Sekon takwentenron kowa tsi seron : kennikariwesha aiakonnheke * tiken ioriwatsanit takwahrewatakon.

Niawen, Sewenniio, takwatokenstenni * nok oni tsini iaweta takionnhetonhatie.

Tiotkon nisa skennen taskwakanerake * tontakwaswateten nii nok iakiien okonha.

Tewakehtakon asen nihati raonhakon Niio * Roniha, Roienha nok Rotkon Roiatatokenti.

Roniha Niio, Roienha Niio * Rotkon Roiatatokenti Niio.

Tiotkon tsi Hiatatienha arekho othenori teiotierenne * raonhake thoientakon Rotkon Roiatatokenti.

Tiotkon si hanakere Roniha, tiotkon si hanakere ne Roienha * tiotkon si hanakere Rotkon Roiatatokenti.

Iah othenon te honoronse Roniha, iah othenon te honoronse ne Roienha, * iah othenon te honoronse Rotkon Roiatatokenti.

Iah tenhnon ashen Niio teken * ashen nihati enskat ok Niio.

Eh niiot iah ashen tiotkon sahatinakereke * enskat ok tiotkon si hanakere.

Iah ashen niah othenon to hotinoronse, enskat ok iah othenon te honoronse * iah ashen ratiwenniio te ken, enskat ok Rawenniio.

Satehotiiatatokenti, satehatiiatanoron * satehonwatisennaiens, satehonwatiwenniiostha.

Tkariwaieri nok kariwanoron kowa ionkwarihonniennis Kentiohkwatokenti * aiontennowentake iah karonhiake taiente.

Aetewakwisron atsitewawennaierite ne Rawenniio * nok karonhiake akwa entsitewaienterhane. 
TEWakehtakon onkwe rotonhon Niio Roienha * kaiatakon ioiatatokenti Wari iahte kanakwaienteri.

Ratienni Rotkon Roialatokenti * nok aonekwensa roieraton ne raieronke.

Ronakeraton ronwawarakwetaron * nok Iesos wahonwanatonkwe.

Sonkwarihonnienni tsi naieiere karonhiake aiontsennonni * nok tsiatak ni sonkwariwisahani noriwatokenti kowa.

Iontatenekwahestha, Iontatkentsiokhas, Okaristia, Tsiontateronkwenni, Iontatiatokhas.

Iontaterennaiennis hetsihenstatsi waonton * nok Iontataterennaiennis wakoniakhe.

Ronwaiesaton nok rawenheion tekaiasontne * ne teshoriwaserakwaton ionkwariwaneren.

Ronwaiataten nok shotonnheton * nok karonhiake ieshawenonton.

Etho renteron tsi raweientehtakon Roniha * enwatonwentsiokten tensakoiatoretane akwekon nonkwe.

Senha konwanehrakwatha zaotanitenrasera * ne tsi rawenheiaton tsini sonkwanonwes, nok tsi rotasehton okaristiakon.

Askitenre, Sewenniio, * ne enskitenrate tsini sonkwatsennonniatenni n'Hetsienha.

Tewakihtakon Rotkon Roiatatokenti * sakotienni iakoriwiio stonne tsini hawen Iesos Kristos.

Etho nahe ratsteristonhatie Kentiohkwatokenti * liotkon enhatsteristake tsinenwe.

Skentiohkwat ok Kentiohkwatokenti, “ iahtaieiatarake iah karonhiake taiente.

Tsini sonkwariwisahani Iesos Kristos * akwekon rarihonte ne Kentiohkwatokenti.

Tewahketakon Niio ronistenha ioiatatokenti Wari * tiotkon ionkwaterennaiennis.

Kiatarakwas wakienha aonton * kennitha aonkwaterennaienhase nonwa nok enkiheie.

Kheiennitha ratironhiakehronon nok nakoiatatokentison * kenoronkwa tsi ratiiataronnion nok raotistien okon kenha.

Niawen, Sewenniio, kiatare Kentiohkwatokenti * askitenre ok iakiheie tiotkon akiatarake.

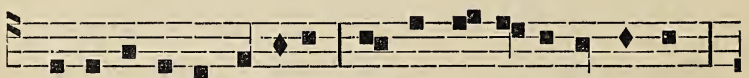

Ni-io i- se Sewen-niio Nonkwe o-kon tsi iakonnhe,

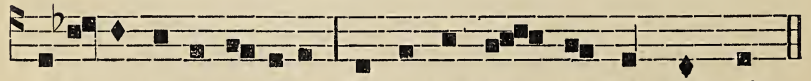

I- kehre tka-ri-wa-ie-ri T'si-ni-kon skwa-ri - hon-nien-ni. 
Nok ne ionkwarihonniennis A kwa Kentiohkwatokenti Aseken senikonhraten Tsi nise nisewenroten.

Tsini salanitenraskon Wakerhare enskitenre Aetenikwekonhake Karonhiake tsi tsiteron.

Ikehre naskitenrate Ne ionkwatsennonniatennis Ne raoronhiakensera Iesos Kristos ne hetsienha.

Niio tokenske konnonwes Ontakennikonhrasaat Askitenre ne Isinenwe Senha akonnonwehake.

Khenoronka nok khenonwes Ne nakwatateken okon Kaneka oni aionkswen Tsi nii ni katatenonwes.
Tekonnoronkwanions Niio Niawen tsini skitenranion Tsi takwatsteristonhatie Takiatanonstatonhatie.

Wakenikonhranonwaktha Tsini konnikonhraksaton Akwa wakaterientason 'T'si swakatatrewaton.

Konriwaienthose Niio Tsinenkennontonnionkwake Tsinenkatieranionheke Nok tsinenkatatiatake.

Konhiennitha Sewenniio Tiotkon akonnoronkwake Nok akonwennaierite Tsinenwe enkatonhokten.

Ahonwasennaien Niio Roniha nok ne Roienha Rotkon Ro:atatokenti Tiotkon ahonwasennaien.

Etho naiawen.

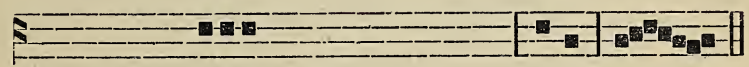

Sonhaa Sewenniio konwenniios - - tha.

Katewentetha noneshonoron nok aoriwa okon.

Awenlokon akennhake lsiniiore Rothomiane Rosennaiens iorennawelarhoseron.

Rosennaiens ne Rawenniio * nakenikonhra.

Iatiiaiehewe tsini wakatsennonni * ne tsini hakitenron ne Sonkwaskontakohe.

Rakiatarakwen iah othenon te wakerihontakwe * ne wahonni onkwe eniontonseke tsinenwe eniontonheke: iotsennonni.

Sotsi seken ioriwanehrakwatons tsini hakierase * iah othenon tahonoron nok raonhaa tsini hosennatokenti.

Tiotkon tsinahe iakononteratieskwe raotanitenrasera * ne ronwatsaniskwe akwekon nontatien okonha.

Tokenske iah othenon tahonoron * ok eken tahrehre nok sakohrewaton ienikonhraiehne.

Sakotskwarakwaskwe teiontaterensaronskwe * nok sakosennowanatakwe iontatkenhronnis.

Iakotentonne sakotsokowastakwe "nok iakotsokowannkenne ne saiakotentane. 
Onen tenhnon nonwa sahrehiarane raotanitenrasera * ne tsi wahawatsiranoronste Israel ne roseronniennis.

Tsini sakoierasehne ietini okon kenha * Abraham nok sakoien okon tsinenwe.

AWENTOKON IONTERENNAIENTAKOHE IOKARENRE RONWENNENNITENTASETHA NE NIJO.

1on. Wari Ioiali aorenna.

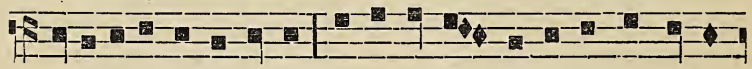

Saia-tatoken-ti Wari sheia-taken- nha te-iontonnhaka-

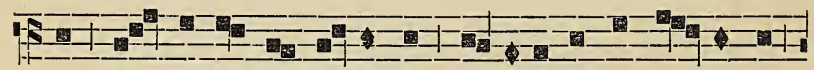

rias, she - nikon- hrahni-ratsek ia-ko-nikonhraksen-se-rons,

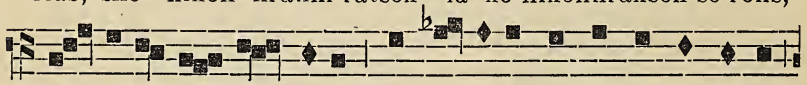

she- ia-te-ren - na- ienhas Ra-wenniio ronwawennenhawe

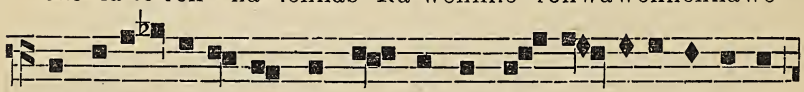

nok ie-iata-re tsi kentiohkwa- ienton ken-tiohkwatoken-tison

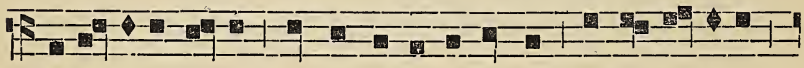

aiont-toke tsi-ni a-kweniatse-ro-wanen Rawenni-ioke

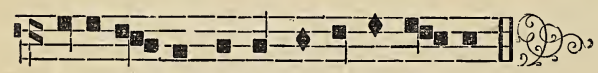

tsini-ia- kon ne ie-sen-niten-ta - se.

v. Takwaterennaienhas Saiatatokenti Niio Hetsienha.

k. Aiawen taiakwatensa tsini sonkwarharatstenni Kristos.

2on. Sose Rli.

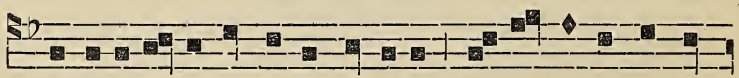

Thoriwaie- ri nok rattokha Sose Ro-ri -honte Rawen-

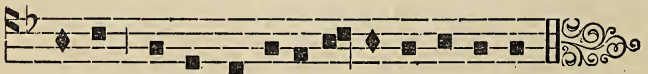

ni-io tahasnie rao-watsi-ra-token-ti. 
v. Niio roiatakweniioston Sose raowatsirake.

R. Nok akwekon raotsokowatsera okon.

3on. Tier Kor oni Rli.

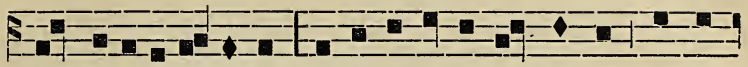

Tier Rola-ta-to-kenti Kor o-ni Ratikwe-kon-ne ion-ki-

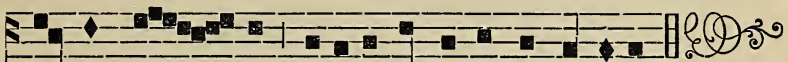

ri-honnien - ni sa-ia-neren-se-ra, Sewenni-io.

v. Tehonarenihon kariwiioston nonwentsiakwekon.

r. Nok tsiniiore iotonwentsiokte ronwatiwennahronken.

40n. Saksarie tokat Resis Rti.

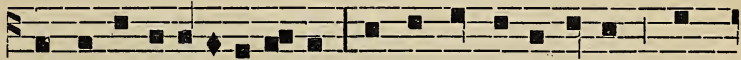

Saksarie saiata-token-ti ne ken-to sa-te- nata, kwen-
Re-sis

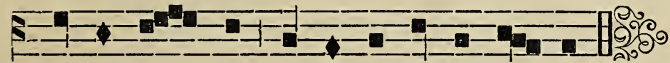

na-ke-ra - ton ta-kwate- renna-ien-has.

v. Rononwehon ne Rawenniio nok roiatanoronston.

r. Ronaktawi kanaktanoron karonhiake.

5on. Skennen akenliake.

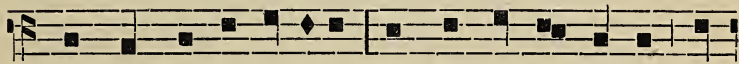

Takwentenr Sewen-niio skennen ta-ken-ha-ke, a-

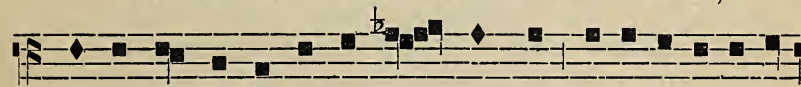

se-ken iah on-ka ten-te- son - kionnhe to-sa aion-ki-son-

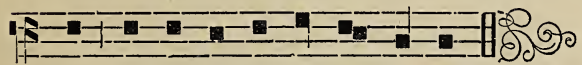

ni sonha- tsiwa kwania- he-sen.

v. Eren shawit, Sewenniio iontkaronniata okon.

R. Skennen takenhake nonwentsiakwekon. 
1on. Wentanoron.

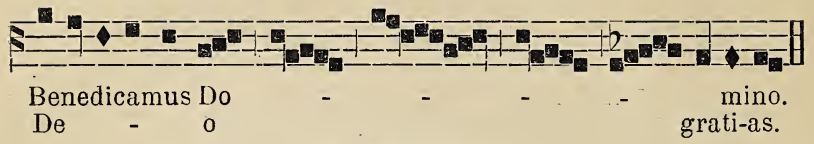

2on. Wentanoron.

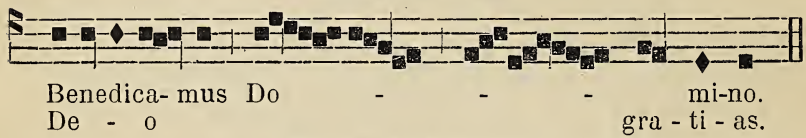

Wari nok okarislia.

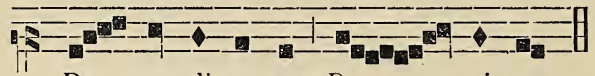

Be - ne-di-ca-rnus Do - - mi-no.

De - - o grati-as.

Awenlokon awenlatokenlike.

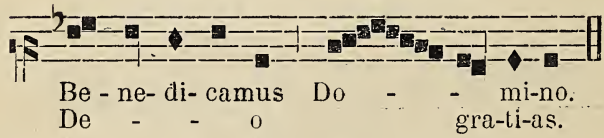

Sonsahatonnhele.

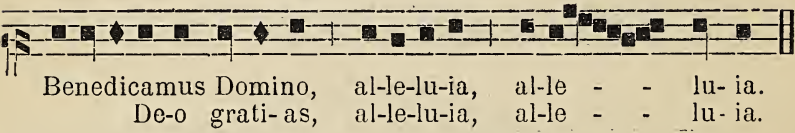

IONTSTA ONI TSINI KAHASERES TENHNON ARE OIA.

Tsi iakawenlontiella kowa nok lsi Rallonniane.

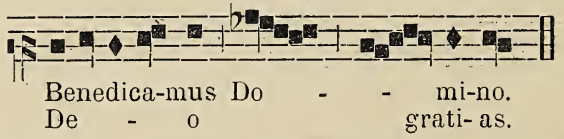

Awenlokon lsini ioioiakseres.

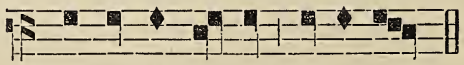

Be-ne-di - ca-mus Domino.

De - - o gra-ti- as. 
Tsikallawis Rathonniane tsiniiore enionlathaseron entakla.

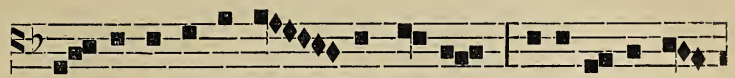

I Wa-ri hetsa-te-we - ton Ie-sos i- se karonhia-

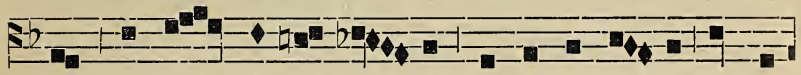

ke takwan - nho-ton-kwen- ni nok onwentsia-ke teskwa-

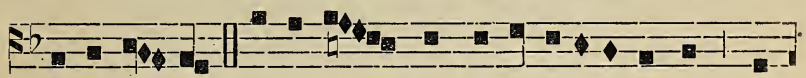

swate-ten - ni. Io-nehra - kwat tsi-ni sa-iatawenhon he-

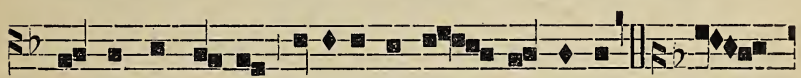

tsonnheton $\mathrm{Ni}$-io. raonha ionnhe - ton-ha-tie. Son-

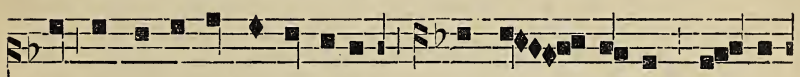
ha iah-te se-nakwaienteri, sonha - tsi-wa tsi - ni

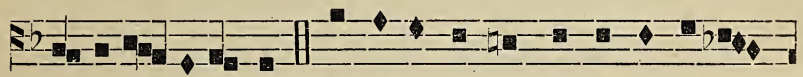

sa- ia-ta - te-ri- hon. Tehia-no-ronkwanion karonhiake-

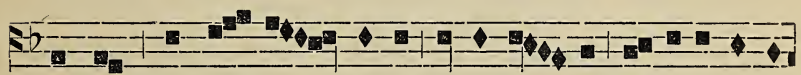

hronon hiahro-ri tsi-ni hianoron-kwa Ni-io ha-ia-ta-

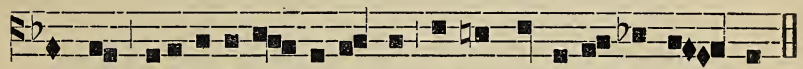

rakwen ise kati ta-kwaien-ha askwentenre ia-kwaie - sas.

v. Angelns Domini nuntiavit Mariæ.

1. Et concepit de Spiritu Sancto

T'si nahatonni lsiniore enionlalhaseron, ionlons :

v. Post partum virgo inviolata permansisti.

1i. Dei genitrix intercede pro nobis.

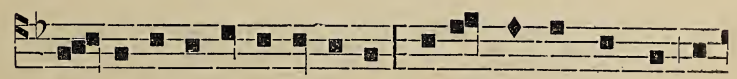

II Wa - ri hetsa-te-weton Iesos takwanrhotonkwenni ka- 


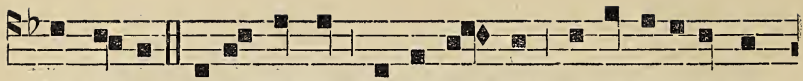
ron-hiake. Io-nehrakwat io-néhra-kwat tsi-ni sa-ia-tawen-

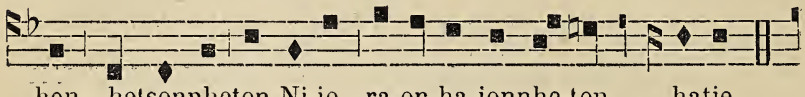

hon, hetsonnheton Ni-io ra-on-ha ionnhe-ton - hatie.

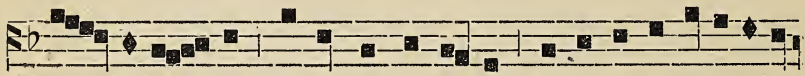

son - ha iah - te se-nakwaiente - ri sonha tsini sa-iatate-

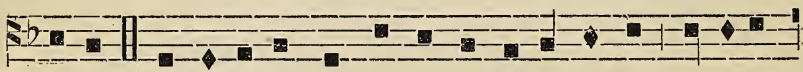

rihon. Te-hia-noronkwanion ra-ronhia-kehronon hiaro-ri

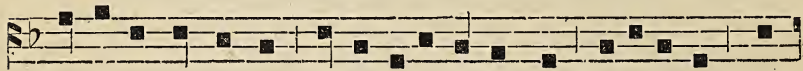

tsini hia-noronkwa $\mathrm{Ni-io} \mathrm{hiaiata-rakwen:} \mathrm{i-se} \mathrm{ka-ti} \mathrm{ta-}$

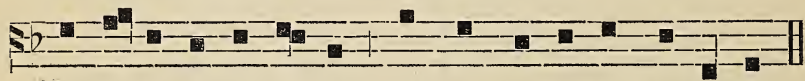

kwaienha askwenten-re askwen-ten-re ia-kwaie-sas.

Tsikahawis saonlathaseron lsiniiore enshatonnhele entakla.

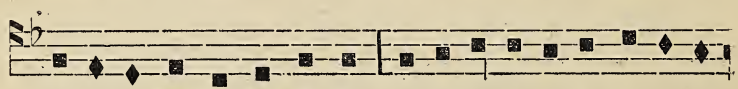

Wari tekwanon-we -ratons iesenna-ke-raton karonh a-

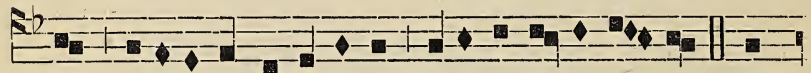

ke teiesawenna-ka-ne-re rati-ronhia-ke-ro-non. Kwa-

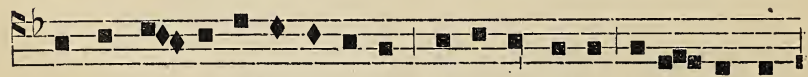

tsennonnia- se hetsa-te-weton $\mathrm{Ni}$-io Ro-ienha raon - ha son-

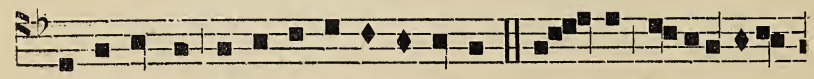

kwanatonni karonhiake iothahinon. Wa-ri sa-iatateri- 


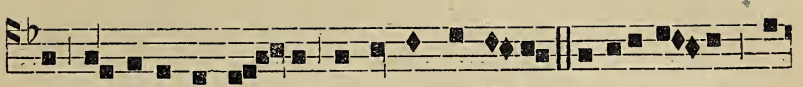

hon sonhatsiwa tsi - ni hianoronkwa Ni-io. Satanitenron kwen-

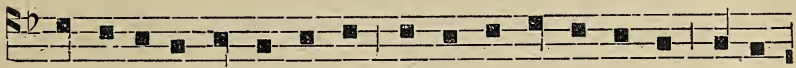

ni-ten-ta-se ta-kwaienha takwa-te-ren-na-ienhas ason

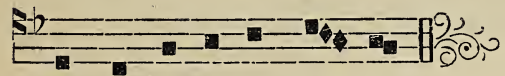

kwentenr Iesos n'Hetsien- ha.

v. Dignare me laudare te Virgo sacrata.

1. Da mihi virtutem contra hostes tuos.

Sonsahalonnhete ?ikahawis tsiniiore entakla Asenseralokenti.

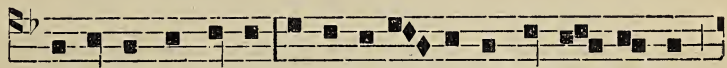

Wari ka-ronhia-ke iesenna-ke - raton satsen-non-ni

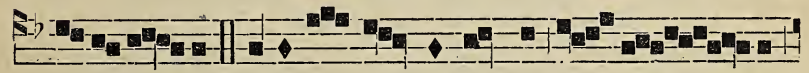

a-re-ri - a. $\Lambda$-seken Ie - sos Kristos He - tsien - ha

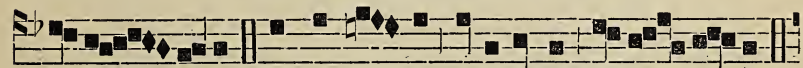

a-re - ri-ia. Shotonnhe-ton tsini hawen a-re - ri - ia.

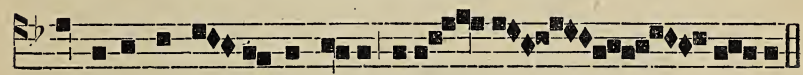

Takwaterenna-ienhas ni-sa, are $\quad$ - $\quad$ - $\quad$ - $\quad$ ri - ia.

v. Gaude el lætare Virgo Maria alleluia.

r. Quia surrexit Dominus vere alleluia.

Asenseratokenti nilhakawis tsiniiore Rallonniane entakta.

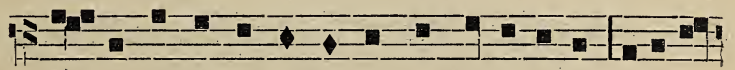

1 Wa-ri tekwanon-we-ratons kwennake-raton "i-se a- 


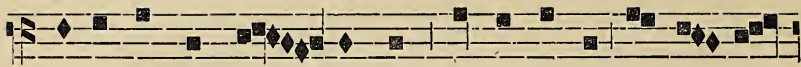

teson takwenten - ranions, takionnheton waskionwes-

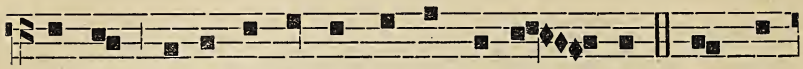

kwaten onen iah-te kakont kwaniahe - sen. Kwaron-

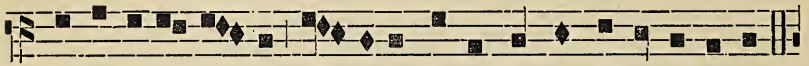

hienha iakwaie-sas ion- ki-katon-ni ionkwanistenha E-va.

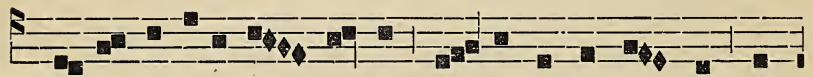

Sa-ton-tek teiakwa - sen-thos tiotkon ionkionsen- nha nok

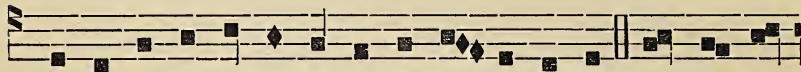

iakwatstarha tsi iakwes ken ionwentsiaksenke. Tsiaken ka-

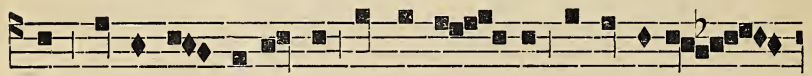

ti tontakwatste-ris-tak karonhia - ke kasatkaren-

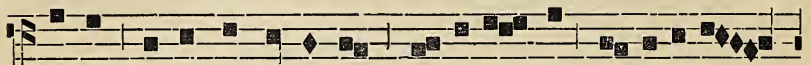

ra-ko tontakwa-ka-nerak tsi-ni a-kwa sa-ta-niten-

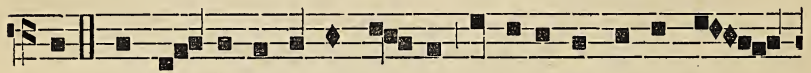

ron. Nok Ie - sos Ni-io Hetsien-ha setsenikonhra-ie-wen-

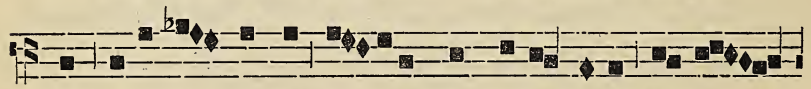

thos, a-ontíe - senhak a - sakwaken karonhiake tsi-nen-

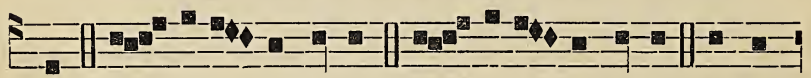

we. O sata - ni-tenron. O sami-kon-hri-io. sa-ton-

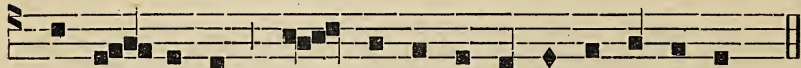

tat kwen-ni- tha IVa - ri iahle se-nakwaien-te-ri. 
v. Ora pro nobis Sancta Dei genitrix.

R. Ut digni efficiamur promissionibus Christi.

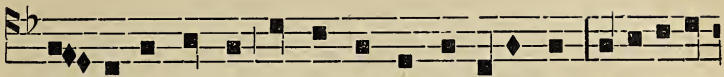

II Wa-ri tekwanonwe-ratons kwennakeraton, i-se aste-

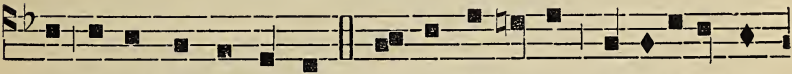

son ne takwentenranions. Ionwentsiananon ne sata-ni-ten-

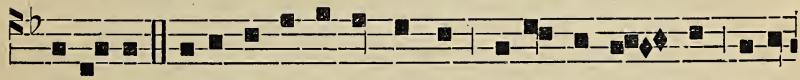

rase- ra. Satkatho tsini ionkwentent,teiakwa-sen - thos oka-

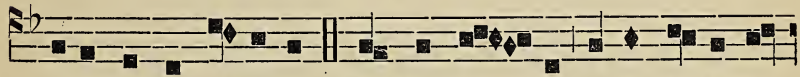

se-ri ionwentsiananon. Tsiaken ka - ti tontakwatste-ris-

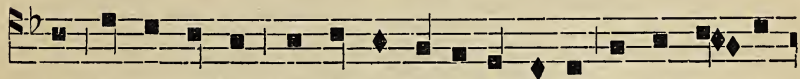

tak karonhia-ke ton-takwa-ka-nerak tsi-ni akwa sa - ta-

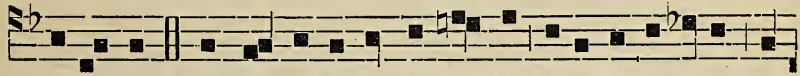

ni-tenron. Nok le- sos Ni-io Hetsienha a-on-ti-senhake a-

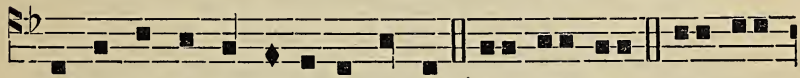

sakwaken ka-ron-hiake tsinenwe. O Wa-ri Takwen-

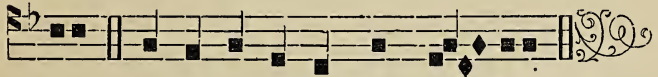

tenr, ton-takwatontats kwenni - tha.

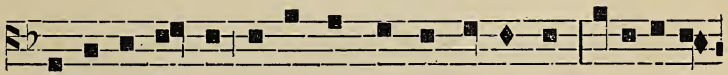

III Wari tekwanonweratons kwennake- raton, i-se aste-

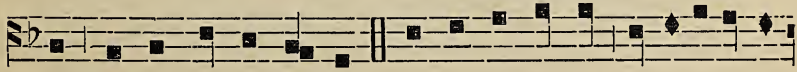

son ne takwentenra-nions. Ionwentsiananon ne sataniten- 


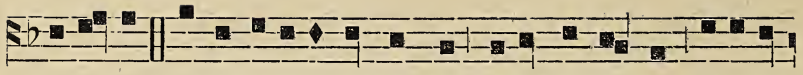

ra-se-ra. Satkatho tsini ionkwentent teiakwatsenthos oka-se-

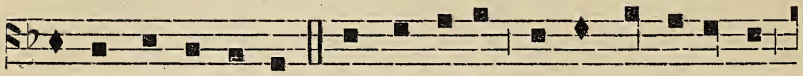

ri ionwentsia-nanon. Tsiaken kati ton-ta-kwatsteristak

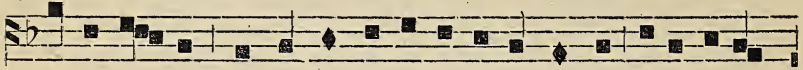

karonhia-ke ton-takwa-ka-nerak tsini akwa sa-ta-ni-ten-

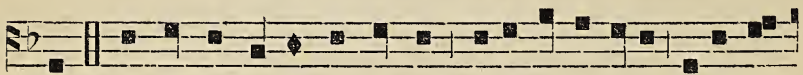

ron. Nok Iesos Ni-io hetsienha, aon-liesenhake a-sakwa-

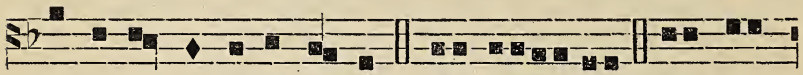

ken karon- hia-ke tsi-nenwe. $\quad \mathrm{O} \quad \mathrm{Wa} \quad$ - ri. Takwen-

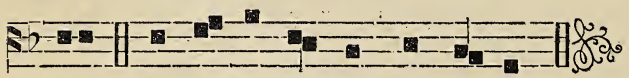

tenr. Ton-la-kwaton-tats kwenni- tha.

\section{TSINAONTKARISTIOTASI}

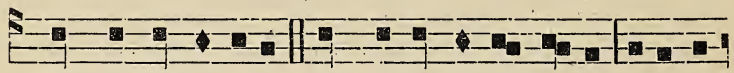

Shetenr Sewenniio. shetenr ne sheien o-kon, tosa iah-

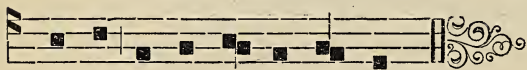

te kakont askwa-na-kwa- se.

I OKARISTIA.

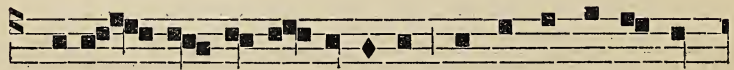

I $\mathrm{O}_{0} \mathrm{~A}_{\mathrm{k}} \mathrm{ka}$ - ri-sti - ia-noron, Karon-hiake ti - seHetsi - lwa - sen-na-ien Ni-io, Ro-ni- ha le-sos Ro- 


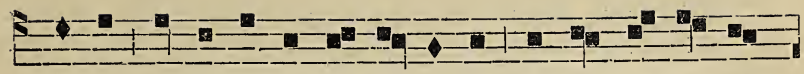

nonton, Askwani-konhrahni - ra-te, Askwa - ie-na- waien-ha, Rotkon Ro-ia - ta - to-ken-ti, A-ste - son he - tsi-

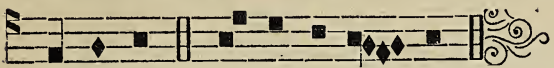

se-he-ke.

twanenton. E- tho na-ia - wen.

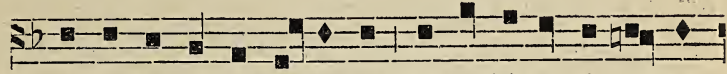

II O o-ka-ri-sti ia-no-ron, Karon-hiake ti-se-nonHetsi-wasen-na- ien Ni- io, Ro-ni- ha Ie-sos Ro-ien-

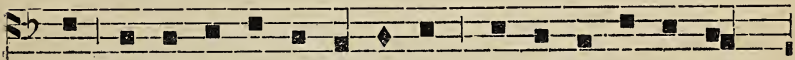

ton, Askwani-konhrahni- ra-te Askwa-ie-nawa- se-

ha, Rotkon lio-ia-ta-to-ken-ti, A-ste-son he-tsi-twa-

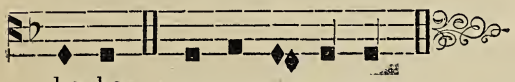

he- ke.

nenton. E- tho na- iawen.

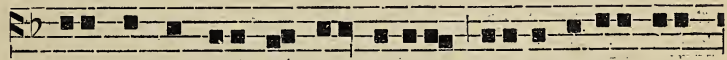

Te- kwanonwe-ra-tons Iesos, o-ka-ristia sa-

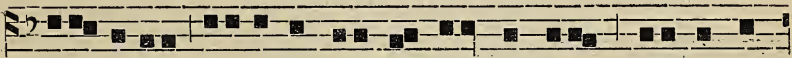

ta-tonni, ne senontens non-kwa-tonnhets, Se-ionnhet-

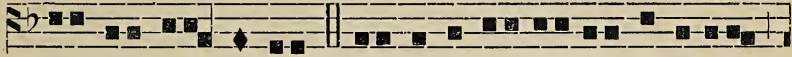

stha non-wen-tsiake. A-honwasen-na-ien Roni-ha,

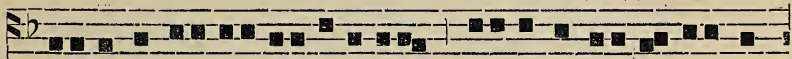

a- honwasen- na-ien Roienha a-honwasen-na- ien Rot-

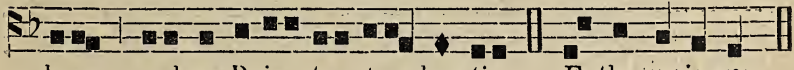

kon a-kwa Roia - ta - to - ken-ti. E- tho na-iawen. 


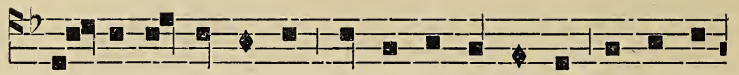

I Ra-o-ti - na-ta-rok e- ron-hia-kehronon, Nonwa ia$\mathrm{He}$ - tsi-twa-sen-na-ien ne Roien- ha Ni-io,Sonkwaten-

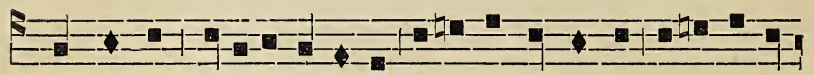

konnhekon iako-ri-wiioston,Tsinisonkwanonwes ne Roieriha nio- ta - se o-ka-ri-sti-iakon, A-ie- ti- senna-ien Ro-ni-ha nok

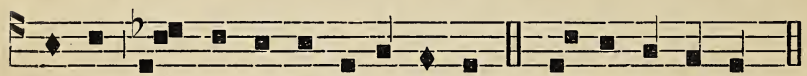

$\mathrm{Ni}$ - io Son- kwaien-ni rā-ie-ron-ke.

Rotkon Sa - te - ha-ti - ia-ta-no-ron. E- tho na-iawen.

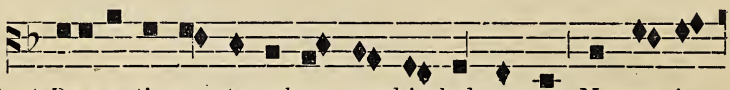

II * Ra-o- ti- na - ta-rok e - ronhia-keh-ronon, Nonwa ia-

* He-tsitwasen-naien ne Ro- ien- ha Ni-io Sonkwaten-

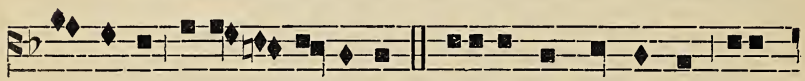

konnhekon iako- ri- wi-iostón. $2{ }^{*}$ Tsi-ni sonkwanonwes ne nio ta-se o-ka- ri-sti-iakon.2 * A-ie - ti - senna-ien Ro-

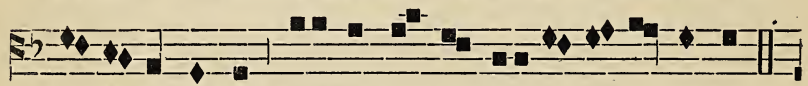

Ro-ien-ha Ni-io Sonkwa-son-kwa-ien-ni ra-ie-ronke. 2 ni-ha nok Rotkon, Sa - te - sa - te - ha- ti - ia - ta-noron. 2
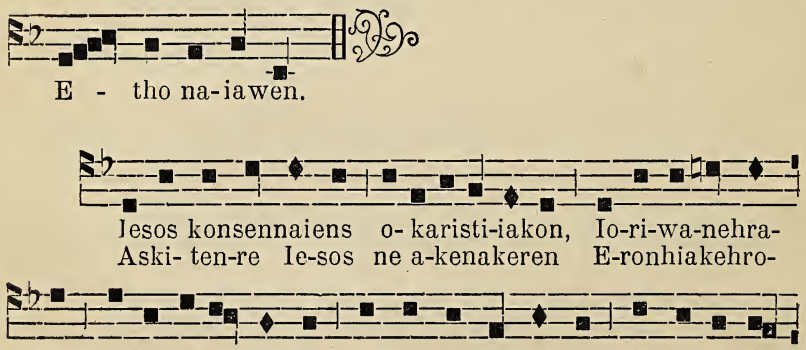

kwat e-tho sa-ta-sehton, senha io-nehrakwat iieks netsienon tsi-ni ie-sanonwes, nok en- ka-tonhokten e- tho en-ke- 


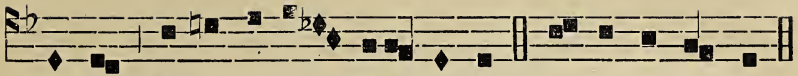

ron-ke, Akwekon o-ni ie - ie-sas.

ra-ne tsi tia-kotsennon-ni - ko-wa. E- tho na-ia-wen.

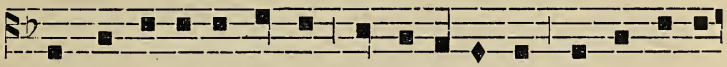

I Kwawenni-iostha Ie-sos ken sa-tasehton, O- ka- ri-stiTsi - ni jotkwakens sase tsi sa-taseh-ton Askwentenre

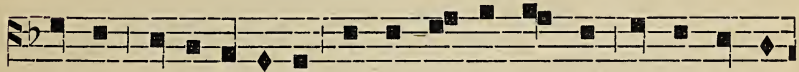

iakon sa-ta-ti-teron, sonha kwa-sen-na-iens sonha kwanon-

tsi-ni sa-ta-nitenron, a-ia-wen a-kwaken ne karon-hia-

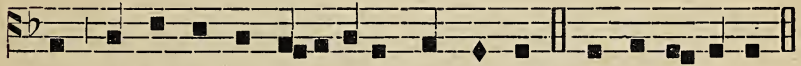

wes, A-seken son-ha- a o-ni kwaskwaneks.

ke, Takwa-ri-wakwase o-ni tsinenwe. Etho na-iawen.

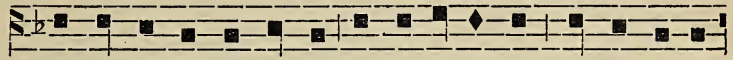

2 Kwawenni- iostha lesos ken sa-tasehton, O- ka- ri-sti-

Tsi-ni iotkwakens sa-se tsi sa-tasehton, Askwentenre

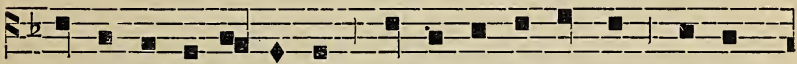

iakon sa- ta- ti - te-ron

tsi-ni sa- ta-ni-tenron
Sonha Kwasennaiens

A- ia-wen akwa-ken sonha ne ka-

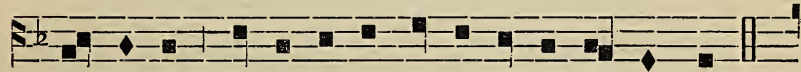

kwanonwes, A-se-ken son-ha- a 0- ni kwaskwaneks. ron-hia-ke, Tekwa-ri wakwa-se o-ni tsi-nen-we.

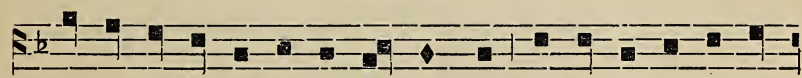

Ie-sos Ni- io tekwa-no-ronkwanions, Se-hiaron onkweria-

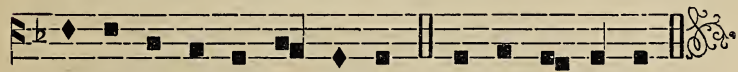

sakon ne tsi tionkwehtakon. E- tho na-iawen. 


\section{1}

3 Kwasennaiens Iesos ken sa-ta-sehton, O- ka-ri- stiIe-sos kwakens sa-se tsi sa-ta-sehton, Askwentenre

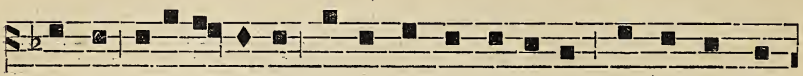

ia-kon sata-ti-teron Sonha kwawenniiostha sonha kwanontsi-ni sata-nitenron Iahte kakont akwaken ne ka-ron-hia-

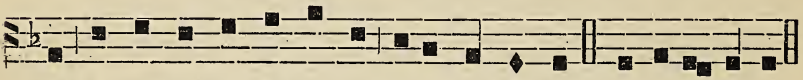

wes A-se-ken son-hatsi- wa oni kwaskaneks.

ke Nok takwa-riwakwa-se oni tsi-nen-we. Etho na-iawen.

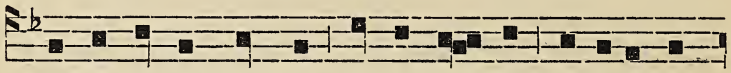

Tekwa-no-ronkwanions Iesos Kris-tos Waria ieE-

sa-ienha. Saron-hiakenhon swaskonta-kwaton tsieron-

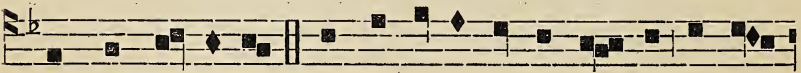

ke skionnhe-takon. Kwawenniiostha kwanon-wes onkwa-

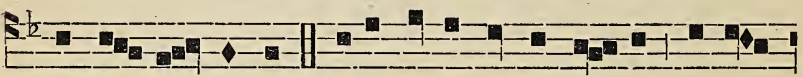

ni - konhra-kwekon. Aia-wen askwatienha - se o-nen

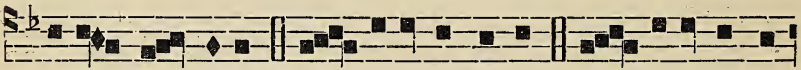
aia - kwen-heie. O sata-ni-tenron. 0 sanikonhri-

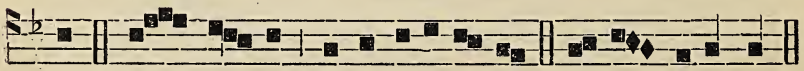
io. O Ie-sos Wari ie-saien-ha. E-tho naiawen.

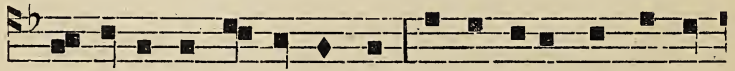

O Iesos takwa-no-ronkwa, takwa-iata-nonstats o- 
年-

thenon tenionkwatonharen-ron nok entiakwenhe-ion-se-

5-

re nok a- se-ia - tsen-nonniaten non-kwa-ton-nhetston. 2

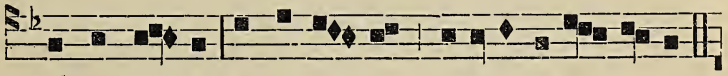

1 Twawenni- iost iahte ka- kont o-ka-ri- stiia - no-ron. 2

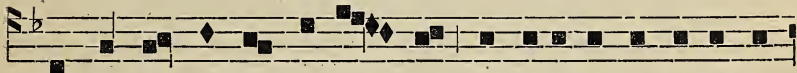

Swentiohkwakwekon ne tsion- kwe, thetsi-sewanon-we-raton

5-1-

Rawen-ni-io tsi tionnhe. A-seken as-teson sonkwentenraEnions nok oni enwaton tsi-ni sonkwarha-ratsten - ni. Twaw :

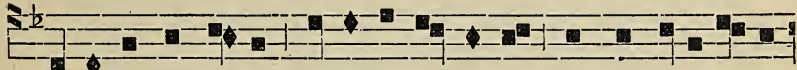

Ahonwasen-naien Roniha Ro-ien-ha, Rotkon Roia-ta-to-

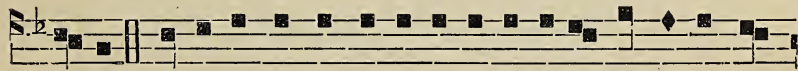

ken-ti. Tsini-iohton-ne tsina-he, etho-na-iohton-ha-ke non-

5

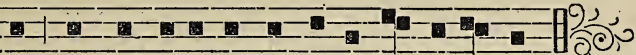

wa, tiotkon oni tsi-nenwe, e-tho na-ia-wen. 'Twaw:

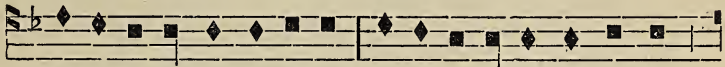

2 Tatsi- te-wanon-weraton, ta-tsi- tewononwe- raton

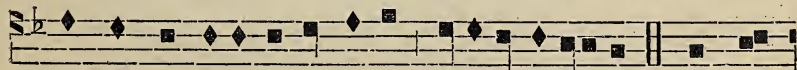

Sonkwawen-niio ken renteron oka-ri-stiia-kon. 2 Swentioh- 


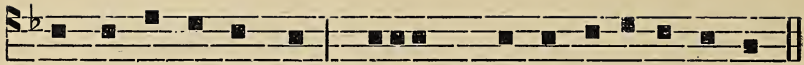

kwakwekon ne tsionkwe * thetsi... liawen-ni-io tsi tionnhe.

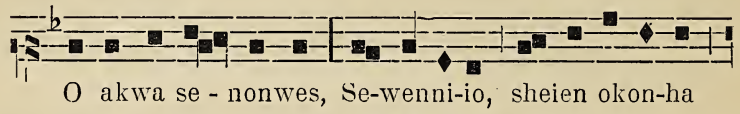

更-

ne shelokaten-ni-tha teshena-ta-renha-wi-tennis karon-

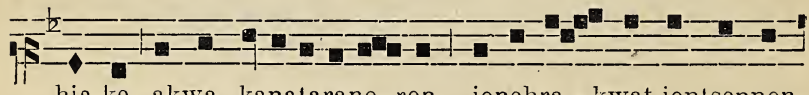

hia-ke, akwa kanatarano-ron ionehra-kwat iontsennon-

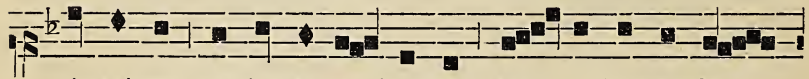

nia-takwa ne ion-te-we-ientons tsi - ni honwaska-

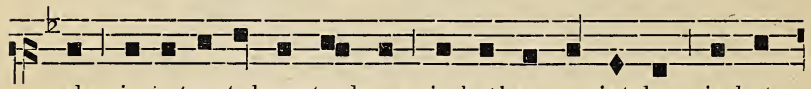

neks ia-koten-takwa tenhnon ia-kotkenronniatskon ia-kot-

so-kowa ia-kotenta-se - re.

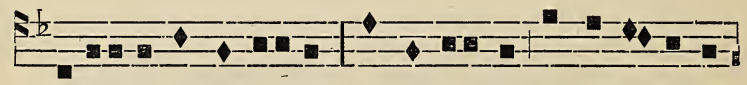

O Ie-sos sonha-tsi-wa takwaien- ha sonha sa-ni-kon-

E- 三 hri-io. 2. O Ie - - sos skwanonwes io-nehrakwal, 年-

takwawis tsia-ta-noron Iesos kwa-no-ronkwa Iesos kwa- 


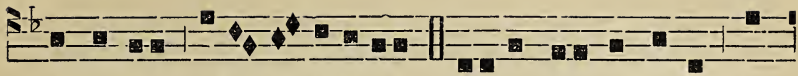
sen-naiens o-ka-ri-stiiakon. A-ion-kwa-nehra-ko tsi5-6-1 -

ni io-riwanehrakwat; akwa ka-ti twate-weienton; hetsi-

E-bor

twa - nenton, hetsi-twa - sen-na-ien Ie-sos

5t二-

ken sataseh-ton iah-te kakont iahte kakont $\mathrm{O}$ Ie-sos

E-

kwa-nonwes, o le-sos kwanonwes son- ha kwanon- we-

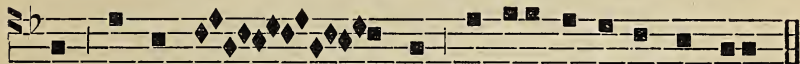

hak, iahte ka - - kont ne ta-kwariwakwa-se.

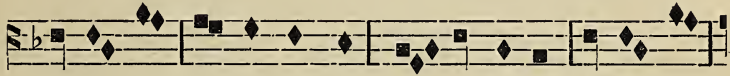

Oh na- ho- ten tenkonnon - we ra-tonte? oh na- ho-

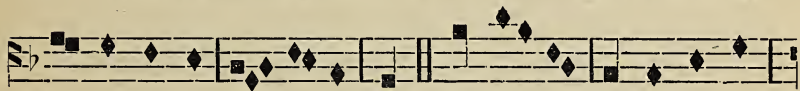

ten tenkonnon-we - ra-ton-te? oh na - ko - ten tonkonnon-

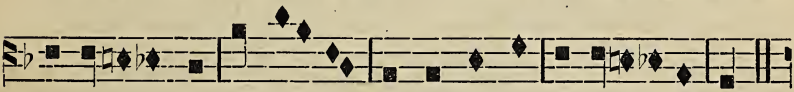

we- raton - te? oh na- ho - ten tenkonnonwe-ra - ton-te?

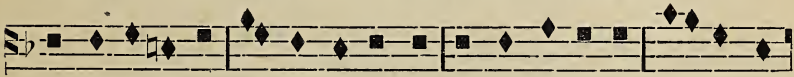

Sewnnni-io tsi-ni ski-tenranion, Sewenni-io tsi - ni skiten-

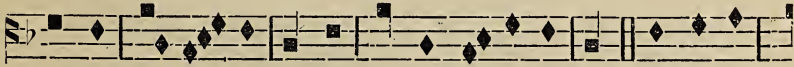

ranion, ah na-kolen ten!ionnonwe- raton-le? Sewenni- 
b圈-

io tsini ski-ten-ranion, tsini skiten-ranion. Te-sek onen

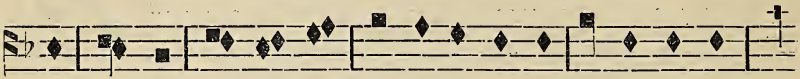

wa-konhion, wakonhion nakweri wakni-ra-ton wakni-

51 -

ra-ton enkonwen-na-rakwa-ke. Tesek o-nen wa-konhion

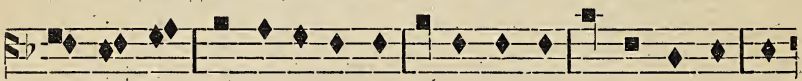

wakonhion nakwe-ri wakni-raton wakni- raton tiotkon en-

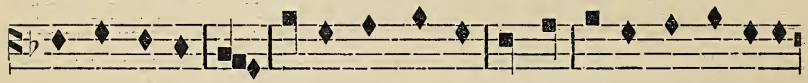

konwenna-ra- kwa-ke, enkonwenna- ra-kwake,enkorwwennara-

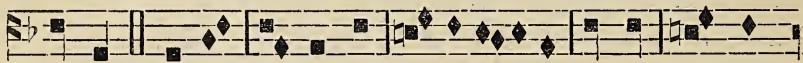

kwake. Oh no-ho- ten tenkonnonweraton-te, tenkon-non-

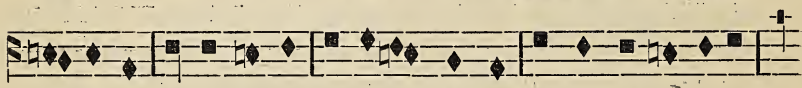

we-ra-ton-te Sewenni- io tsi-ni ski-ten-ranion Sewen-niio

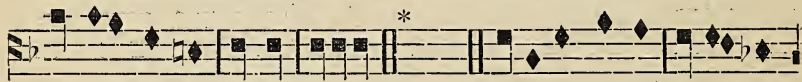

tsi-ni ski-ten-ra-ni- on. Tesek... I- se Sewenni- io takwas-

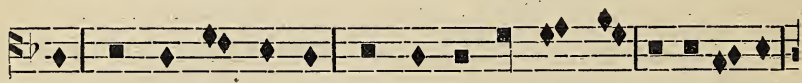

kon- takwen takwaskon- takwen wakonwen-ni - ioste na-ke-

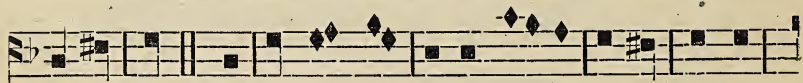

ni-konhra, wakonwen-ni - ioste na - ke-ni-konhra, oh

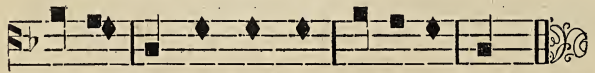

na-ho-ten ten-kon non- wera-ton- te ? T'esek... 


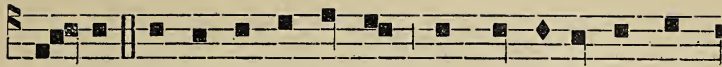

$\mathrm{Ni}$ - io tekwanonwe- ra-tons skwawenni-io kwasen-

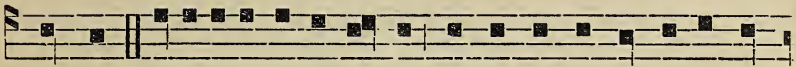

naiens. Iesaniha ok si sonnhe onwentsiakwekon iesanen-

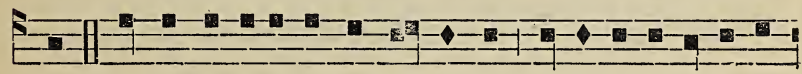

tons. Akwekon rati-ron-hia-kehronon asteson tie-sa-riwa-

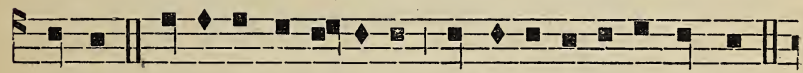

kwase. Kerubim et Se- rafim iahte kakonte rontonnions.

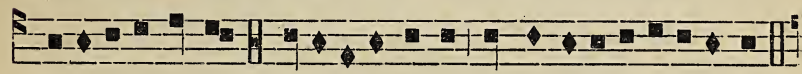

Saia-la-tokenti. 2. Saia-tatoken-tt, Sewenniio Niio Sabaoth.

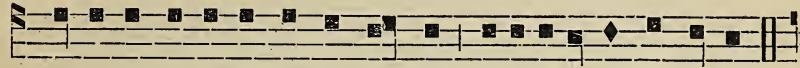

Kanaron karonhia nok nonwentsia saia-tanehrakwatse-ra.

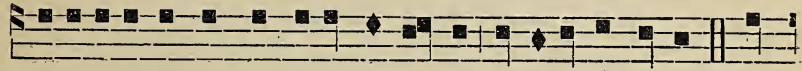

Teiesanoronkwanions ratikwe-konne ne rotitiohkwiio. Nok

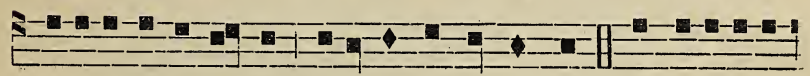

roti-tokense-ha-kwe ro-titiohkwanehrakwat. Nok oni kari-

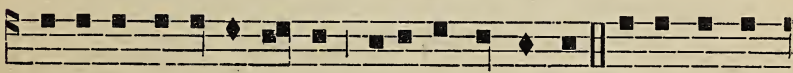

wiioston ronwati-riohton rotitiohkwanoron. Tsi ok nonwe

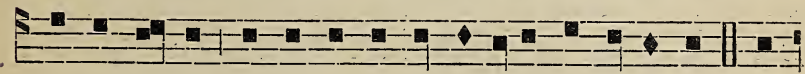

nonwentsiake sentiohkwatoken-ti iesawen-ni-iostha. 'Ie-

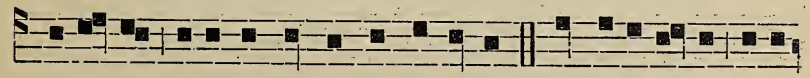

sa-ni- ha ok-tiwakwekon tsiata-nanon. Nok o-ni Ie-sos raon- 


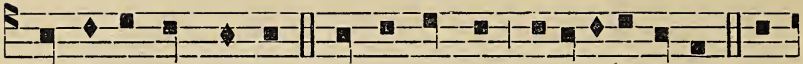

ha-tsiwa n'ketsienha. Nok ne Rotkon Roiatatoken-ti. Tie-

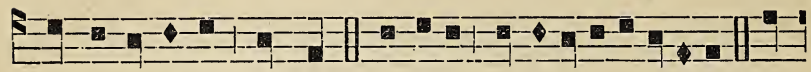

senna-ke-raton Kristos. Hianiha ok sa-te-seni-nakere. Ne

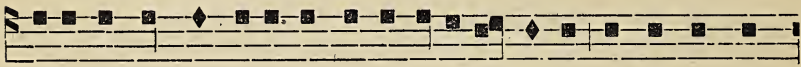

aonsaskwaskontako onkwe tsi sa-taton-niane, iahte saswen-

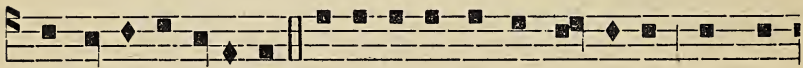

hon Wari ka-ia-takon. Onen tsi sesennion kenheion takwan-

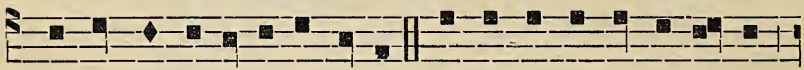

nhotonkwenni ne karonhiake. Satiens nonwa ka-ronhia-ke

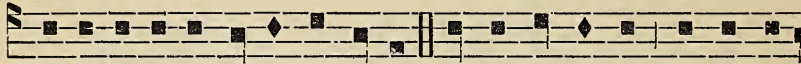

tsi raweienteh- takon Hiani-ha. Ne tionkwehtakon tenteskwa-

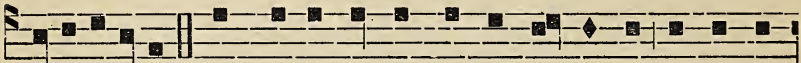

iato-letane. Kwenni-ta shetenr sheien o- kon-ha sesheskon-

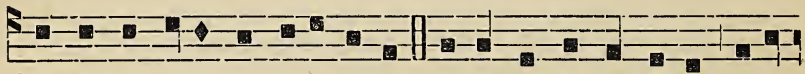

takwaton sanekwensatokenti. A-iawen ka-ronhiake a-ia-

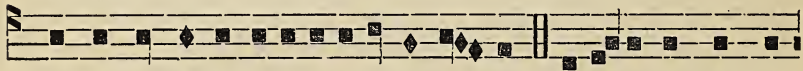

kwakwekonhake nakoiata-token-ti - son. Sheia-tanonstats sen-

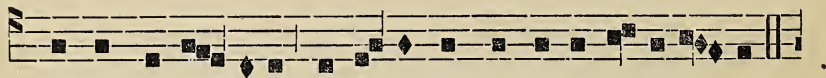

tiohkwa, Sewen- niio, sheia-ne-renstak ka-ri- wi-io son- ha.

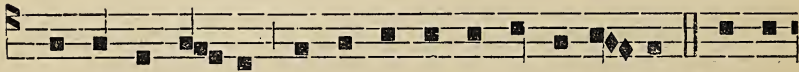

Nok sheiatste - rist a-iontsennon-ni iah-te ka-kont. Niate 


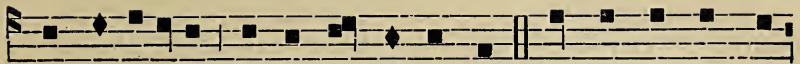

wenni-se-rake tekwu-no-ronkwanions. Nok tiotkon kwasen-

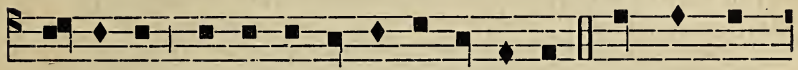

nanentons nok tsinen-we iahte kakon-te. Takwentenr

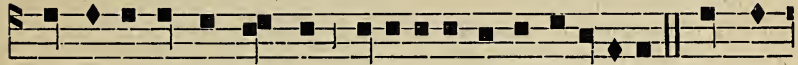

Sewenni-io ken wen-te tosa a-iakwariwane-rake. 'Takwen-

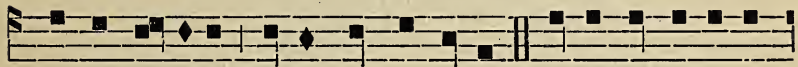

tenr Sewen-niio, ka-takwenterir ni-sa. Ka-sa-ten-na-ke-rat

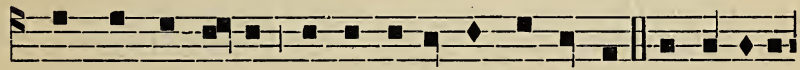

nenskweritenra-ne, akwa tsini kwa-niahesen. Sewenniio

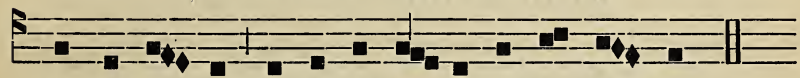

kon-niahe - sen iahta-skia-ton-ti iahte ka-kont.

v. Tetewariwak hetsitewasennaien nonwa nok oui tsinenwe (sh) areriia.

R. Niio Roniha nok Roienha nok Rotkon Roiatatokenti $(s / \iota)$ areriia.

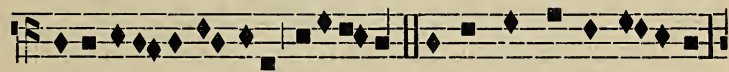

Are-riia a-re - riia, a-reri-ia. Onen nonwa twataleken,

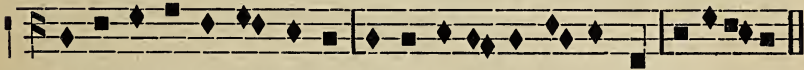

Tewatka ne tewatstarha Iken Iesos shotonnheton, areri-ia.

Orhonke iakotkenseron, lesos tsi thaiatatakwe, Ne kati iahte shaiatat.

Etho raronhiakehronon Otinnhetien sakohrori Rawen Iesos shotonnheton.
Ethone Tier nok San oni Oksa rotiriwakohon 'Tokenske iahte shaiatat.

Enskat ronatkennisonhon Ne Iesos Ratikwekonne Nok wasakotianeronkwen. 
Tonwa iahte haiatare; Ronwahrori : sonsharawe, Onen Iesos shotonnheton.

Iah kati te hotontaton, Wahenron: iah etho te iot, Enkatontate nenhiken.

Onen si shonatkennison Iesos ieshotaweiaton, Rawen : Tekwanoronkwanions. Iah sane te honwatkathos.

Ken wente ne wentanoron Thetsitewanonweraton, Ionehrakwat sonkwentenron.

Tesannhohonte. Onen wathonnhohonti Rotenniote Iesos. Ionehrakwat.
Takwatkatho, ise Tonwa, Satka ne satennowentha, 'Tokenske swakatonnheton.

Oksa ok shotatrewaton, Wahenron: Iahte kattokha, Ne Sewenniio tsi konnhe.

Senha iakoiatateris, $\mathrm{Ne}$ Iesos ronwatontatis,

\section{WARI AORENNA SONHA.}

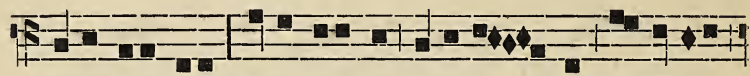

Sase-hia-rann, Sasehiarann, Sasehia - rann O Waria

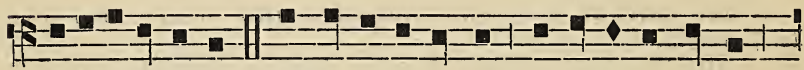

saia-ta-token-ti. 2. Iahte ia-ko-ronken ne ashe-ia-tontion

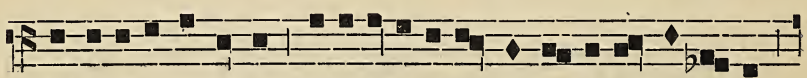

ashe-ia-tewentelon kaneka ne iesanonwes ie-sa- niahe-sen

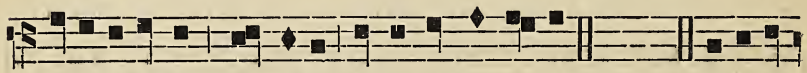

ie-saia-tisaks nok o-ni iesen-niten-ta-se. Sase... Ii kati

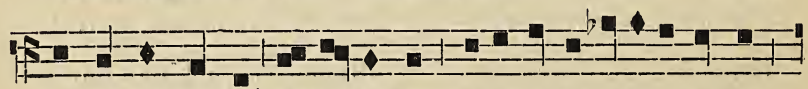

nonwa konniente, kon-ia-ti-saks askiten-re, satani-tenron,

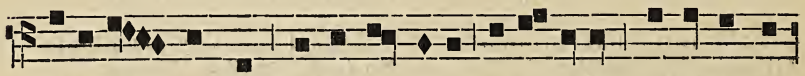

askiata - nonstats, askie-na-wase sa-si-takta wakatiskwen- 


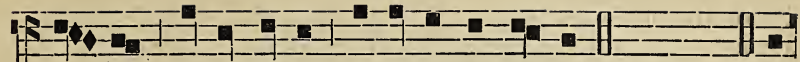

ta - ren, Wari, Wari, wa-katiskwenta-ren. Sase... To-

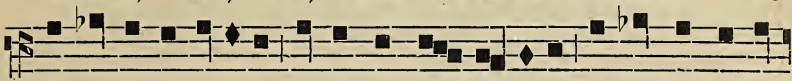

sa senisten-se-riio askewennon - tie-seke ne tenhnon aski-
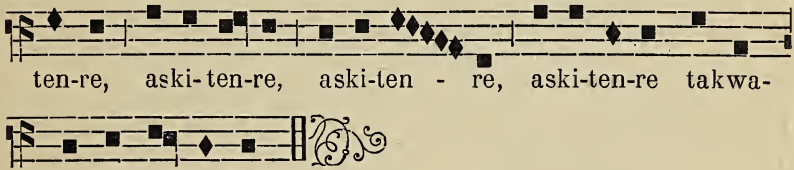

te-ren- na-ien-has. Sase...
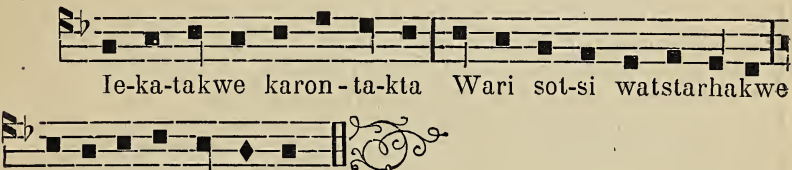

Ra-ia-ta-ni-iont Ie-sos.

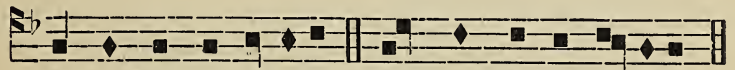

Takwentenr Sewenniio, Ta-kwentenr Sewenniio.

Kris - tos takwentenr Kris - tos takwentenr.

Takwentenr Sewenniio, Ta-kwentenr Sewenniio.

Kris - tos takwatontats, Kris-tos takwawennarak.

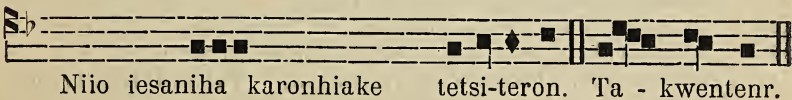

Niio iesaienha nonkwe sesheiatontakwen.

Niio Satkon saiatatokenti.

Asenseratokenti enskat ok Niio.

Wari saiatatokenti iah othenon te saia-

toronhon sasatiatata.

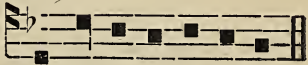

Takwaterennaienhas.

Ise ionistenseranehrakwat.

Ise sonhaa sateweton iah sane tesenakwaienteri.

Ise hetsienha ne Niio.

Ise hetsienha ne Iesos

Ise hetsienha sonkwaiatison.

Ise hetsienha sesonkwaskontakwen. 
Wari ise kanistenseriio.

Ise tsiati ioianerenstakwa.

lse iah othenon te saiatoronhon ne iotaksen.

Ise iah enskata te sariwannhikon.

Wari ise saiatanehrakwat.

lse akwa kanonsanoron.

lse akwa sanikonhratokenti.

Ise akwa sanikonhrowanen.

Ise akwa sanikonhriio.

Ise iah othenon te sanoronse.

Ise oriwakwekon tisariwaieriton.

Ise akwa aiesakonnienstake.

Ise aiesanakeren.

Ise aiesanatahnoton.

Wari ise satanitenraskon.

Ise tikionwesk watennis.

Ise tesentenhawitha.

Ise karonhiake takwannhotonkwennis.

Ise iesaniahesen tsiontonnhakatonnis.

Ise seshetsientha teiakokonhentonnions.

Ise shenikonhranirats ne iakonikonhranonwaks.

Ise shetenranions niakoriwiioston.

lse akwa tsiatanoron.

Ise iontekwasentakwa niakoriwanerahakskon.

Ise iesatsanis nonwentsiakonronon.

Ise iesennakeraton karonhiake.

Ise tiesawennakanere ratironhiakehronon.

Ise iesennakeraton ratiienterihne tsinontaiawensere.

Ise iesennakeraton Iesos ratikwekonne.

Ise iesennakeraton iaiheiatha kariwiioston.

Ise iesennakeraton ratirihowanatha kariwiioston.

Ise iesennakeraton Rawenniio ronwawennenhawe.

Ise iesennakeraton iahte ienakwaienteri.

Ise iesennakeraton akwekon akoiatatokentison.

Ise iesenuakeraton ne ierensoktha ne karensatokenti.

Iesos Kristos seriwahtontha kariwaneren, 10 Takwaiatokef Iesos. $2 \circ$ 'Takwatontats Iesos. $3^{\circ}$ 'Takwentenr Iesos.

Krìstos takwatontats. Kristos takwawennarak.

Iotsennonni Waria.

- Akwa tsiatiio Waria.

O tsiatanoron o saiat.
Wari, Wari, Wari.

O Wari kwennakeraton.

Tekwanoronkwanions.

v. Ora pro nobis sancta Dei genitrix $(s h)$ alleluia.

R. Ut digni efficiamur promissionibus Christi $(s / h)$ alleluia.

Thoriwaieri... skar. 82. v. Niio roiatakwenniioston... 
'TWAWENNIIOST

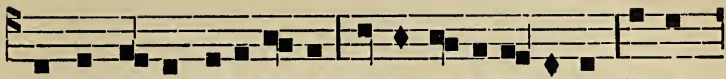

1 Twawenni-iost o-ka-ristia Etho ie- ho-ta-seton Iesos

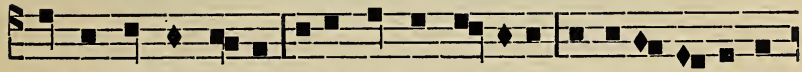

ne sonkwawenni-io Ae-twakwekonhake Tsiniiot na-tsi-te-

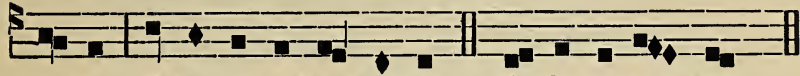

waken E-tho nitionkweh-takon. E-tho na-ia - wen.

Atsitwasennaien Niio, Roniha nok Roienha, Rotkon Roiatatokenti. Atsitwanentonseke, Nonwa nok oni tsinenwe Iahte kariwentane.

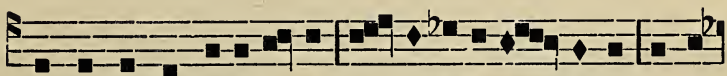

2 Twawenniiost o-ka-ristia $\mathbf{E}$ - tho ieho-ta - seton Iesos

St

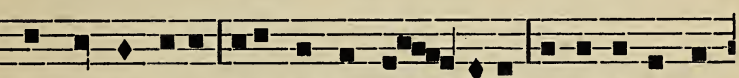

ne sonkwawenniio Ae-twakwekon - hake Tsiniiot na-tsi-

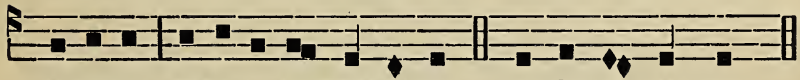

tewaken Etho-ni tionkwehtakon. E- tho na - ia-wen.

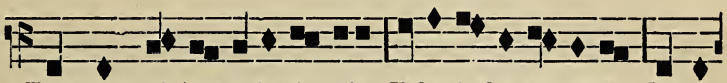

3 Twawen-ni-iost 0-kari - stia Etho ie-ho-ta-seton lesos

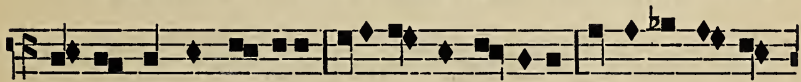

ne sonkwawenni - io Aetwakwekonhake. Tsiniiot na-tsi-

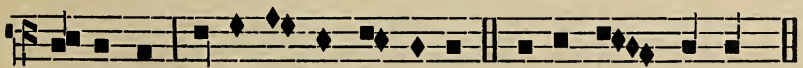

te- waken Etho nitionkwehtakon. Etho na - iawen. 
e.

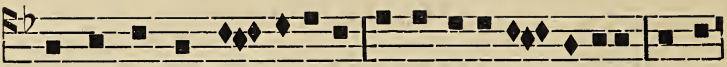

4 Twawenniiost 0 - karistia Etho iehota - se-ton Iesos

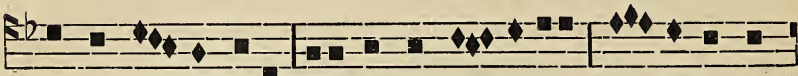

ne sonkwawen-niio Aetwakwekon-hake. (Tsi-ni-iot naE-1 -

tsi-lewaken 2) E-tho ni tionkwehtakon. E-tho na-iawen.

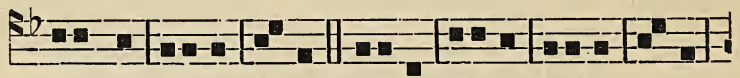

5 Twa-wen-ni-jost o- ka - ri- stia E-tho ie- ho-ta-se-

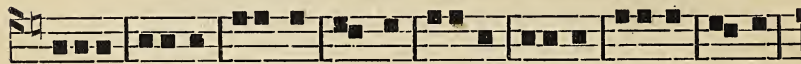
ton Ie-sos ne Sonkwawenni - io A - e-twakwekonha-

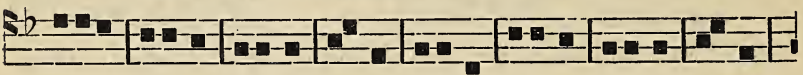

ke Tsi-ni-iot na-tsi-te-waken E- tho ni tionwehta${ }^{6}=-1$ -

kon. A - men, A - men.

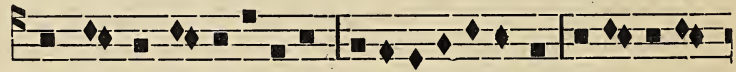

6 Twawenniiost o-karistia ethoiehotaseton Iesos ne son-

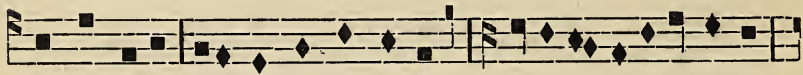

kwawenniio Aetwakwekon-hake. Tsiniiot natsitewaken

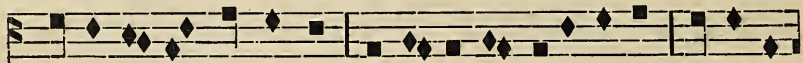

Etho ni-tionkwehtakon Tsi-ni-iot ra-tsitewaken E-thoni-

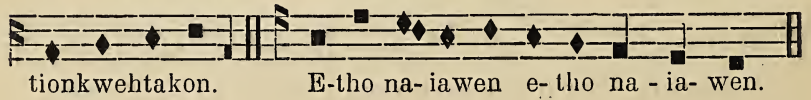




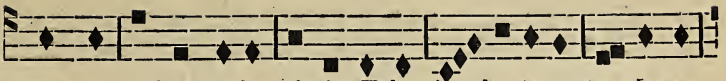

7 Twawenniiosi noka-ristia Etho-ie - ho-ta-se-ton Iesos

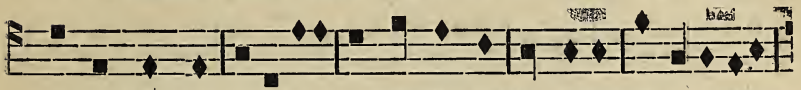

ne sonkwawen-niio Ae-twakwekonha-ke Tsini-iot natsitewa-

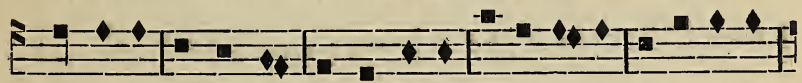

ken Etho ni tionkwehtakon Tsini- iot na-tsi-te- waken Etho-

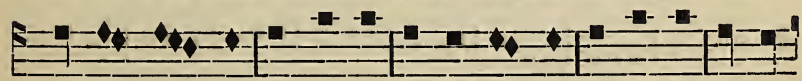

ni tionkweh-takon E- tho-ni tionkwehtakon E- tho-ni tion-

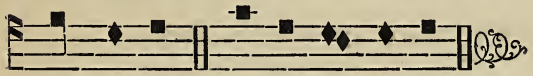

kwehta-kon. I- tho na - ia-wen.

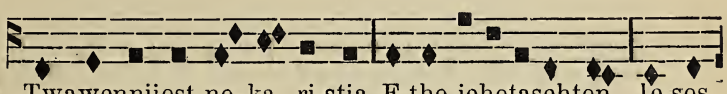

8 Twawenniiost no-ka-ri-stia E-tho iehotasehton 1e-sos

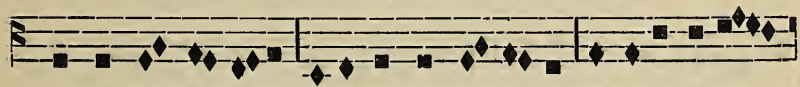

ne Sonkwawenni-io Naetwakwekonha- ke Tsiniiot natsi-

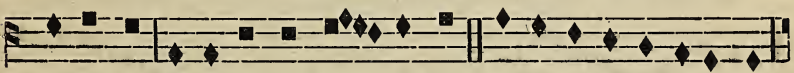

tewaken E-thoni tionkweh-takon Tsi-niiot na-tsi-tewaken

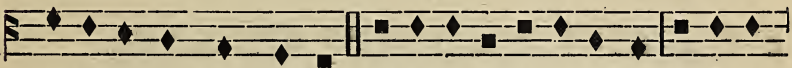

E-thoni tionkweh-takon Tsi-niiot natsi-te-waken E-thoni

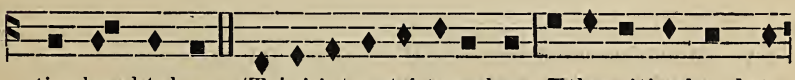

tionkwehtakon (Tsini-iot natsi-tewaken Etho-nitionkweh-ta-

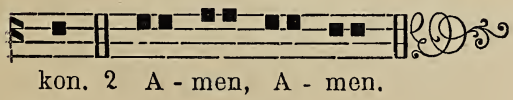




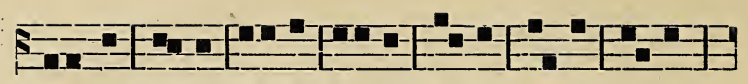

9 Twawen-niiost 0 - ka-ri-stia E-tho ie-ho-ta-se-

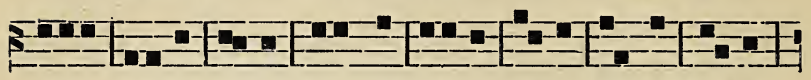

ton le-sos ne son-kwawen-ni- io A- e-twakwekonha-

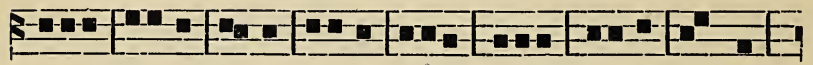

ke Tsi-ni-iot na-tsi-te-wa - ken E- tho-ni tion-

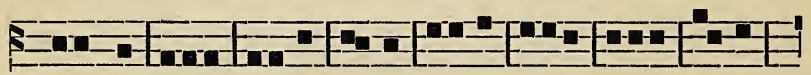

kweh-ta-kon tsi-ni-iot na-tsi-te-wa - ken E-tho-

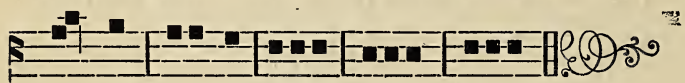

ni tionkweh-ta - kon. A - men.
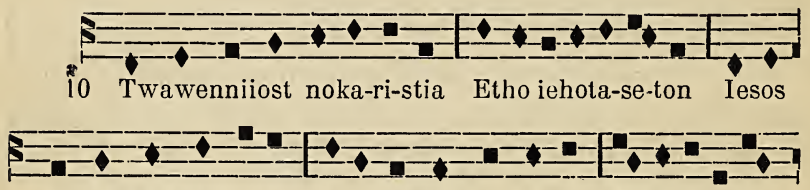

ne sonkwawenni-io Nae-twakwekon-hake. Tsiniiot natsi-

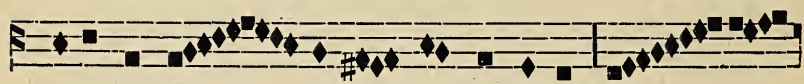

tewaken E - - - thoni tionkwehtakon E - -

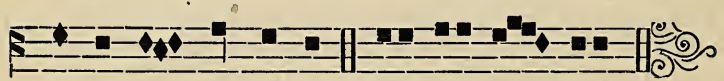

tho-ni tion-kwehtakon. A - men, A - men.

v. Panem de cœlo præstitisti eis $(s / \iota)$ alleluia.

R. Omne delectamentum in se habentem $(s h)$ alleluia.

Swentiohkwakwekon... 


\section{KARENNA SONHA}

Karo kase, Iesos, Ok sa askwennakeratse Onen ionkwarharenion Tsini sariwison.

Kasatsente karonhiake, Taesatsterihensere Askwaiatakennhasere ; Sonkwakarewatanion Ne notkon noneshonronon: Rakenrenseronnihon Ne nonkwatonnhetston.

Teskanerak tsi ionkwentent Asteson iakwatstaronnions Iah skennen tiakwennontonnion Ion kiriwaneraktenni Ne thonatierenton nonkwe ; Ise skwasentanionni Ionkwariwaneren.

Kasatskarenrako nisa
Akwekon kwennitentase Askwarihonniennire Ionkwaterientasonhon 'Tsi ok nenskwatenientense, Eniakwatontatseke Tsini sewennoten.

Tosa kati ensanisko Tsini sariwisonhatie Aontaskwennakeratire Teskwannhotonkwennire Aiakwakwen karonhiake ; Iakwehre : eh ieniakwe lakwakwekon nonkwe.

Aiawen nii akwatkatho Nonen eniakwatonnhokten! Akwa aontaskwarhoton; Ethone askwentenron Iakwakwekon karonhiake Naonsaskwaskontakwen Tsi tiotekha onwe.

Karonhiake senakere kasatsnente Karo kase, ionkwanaskwaien noneshonronon ; Takwannholonkwas ne karonhiake.

Akwa ionehrakwat ionkwentent

Onwentsiake tiakwanakere Askwentenre, askwaiatisakha, Ok sa askwennakeratse.

Ise wahi sariwison, seron Onkwe enkaton nonwentsiake : tosa kati ensanisko Tosa enkwaiatanikonrhen.

Tosa kati aetwatsaniseke Tosa oni aetwatstarhake, 'Tosa onen tsionkwanikonhraksensek' Wetewatsennonni sonkwatonniennire 
Ii onkwariwa ratonniane nonwa kahawis; Eksatanoron onen enhatiatotenste; Aetewatsteniaron niate wenniserake Aiokentonhak tsini tsitewanoronkwa.

Iesos karo kase

Saianere onwe

Akwaienterhane

Tasatsnent karonhiake

Onen ki Sewenniio

Takwatsennonniaten,

Tetsiatoret nisa

Saonkwententane;

Ionkwanaskwaientaties

Ne noneshonronon.

Niate tsionkwetotenson,

Swaiataseronni

Swatonnhetston okon

Swatonnhakanonni :

Swatennikonhrasaat

Tsi swatatrewatha.

Tokenske Swenniio

Sotsi kwasentenni

Tsi wakwakonnienste

Ne wahetken sonha;

Tsi satanitenraskon

Oh! takwentenr nisa

Oksa swatennhohonti

Ise, karonhiake,

Oksa tontahatsnent

Nene sakwarhare.
Asonk wennakeratse

Ne Niio Roienlıa

Oksa tontahatsnent

Ne Niio Roienha;

Sonkwaskontakohe,

Sonkwatonniennire

Raonhaa rakwenies

Nasonkwanerensi

Ok sa ok taontare

Ratetsientseriio :

Sesonkwatsientane

'Tsi ionkwerientaksens ;

Rariwahtontha kowa

$\mathrm{Ne}$ kariwaneren.

Niate tsionkwetotenson Skennen swennontonnion

Kwawennaieriton

Skennen swennontonnion Onwentsiake enrawe Iesos ne Riienha.

Iosa swatonnharenron, Onen se kwentenron ;

Onkwe ratonsere

N'hetsisewaiatisaks;

Enskat ok kwanekennis, Onon takweriason.

Satsteniaron Iesos, satsteniaron Ionkwarhare nontaskwentenrane.

Unen wahonnise Rotitokensehakwe Rotirihowanaton lesos onkwe enhaton Rotiiàtatokenti ahonatsennonnihake Thare sonkwannhotonkwennire karonhiake 
'Tosa ronnikonkriak rọtiriwanerahakskon Ne sesakoiatisakhe Iesos ratonniane Roiatanehrakwat, ronikonkriio onwe. Rotiesen kowa, onen ronwennitentase.

Ii sonkwaniente sonkwanentsatironten Sonkwaskontakohe aionkwatetsiraton Sewatsteniaron, tsionkwe, sewatsteniaron Sonkwanonwes, katsiswanonteratie.

Kakwiriie Nasareth ne wahahtenti Rahroriane Wari ne isi neniawen; Rariwenhawi nakwa kariwanoron: $\mathrm{Ne}$ ensateweton ne Niio Hiatatienha.

Wari wahotsanike ne Kakwiriie, Wakenron: Iah taonton ne satrori, Ne wahonni iahte kenakwaienteri, Niio rirharatstenni, nentkatatewennaierit.

Satka ne setsanis, ionsahenron, Iahten te holloronse rakennhaon; Rotkon akwa ne Roiatatokenti Enhiatiatahase nonwa ksatonniane.

Onen tontati Wari, iotontaton : Etho neniawen tsini sewennoten, Rakiatawenk raonha Rawenniio Ethoni hakierase tsini horiwison.

Iahten tehonisekwen Niio Rotkon Kaiatakon Wari ne raksatonni; Onen nonwa wasonkwennakeratse, Twakwekon tsi twanonkwe Niio Hiatatienha.

Wari saiatateri, satontaton, Akwekon nonkwe okon shetenranions Rawenniio hetsienha watonsere, Sonkwaianerenstha ne ionkwententonne.

Iahte sewanekherens se, twatateken, Tsini hanoronkwa Iesos ronistenha, Iah nonwenton thawennontion ne Wari Twakwekon twatsteniaron naetewaniahese.

Tsiaken kati, Wari ne takwaienha, Hetsenikonhraseronni tsiatatienha, K aronhiake nonen eniakwenheie, Iensakwaiatorenne sesonkwaskontakon. 
6

Wahatonni raksaa ne Niio Roienha Tsini hotanitenron, sonkwaskontakohe Ionkwariwanerahakskon ionkwatkaronnionne.

Onen twatatcken etho iaetewe Hetsitwanoronkwanion, tetwanikonhrasat, Raksatanoron kowa hetsitwanonwehak

Wari ne roteweton, okti ionehrakwat Ratironhiakehronon tehatiriwakwa Raksaa ronwanentonnions, rotirenniioson.

Ronton: Ronwasennaien Niio karonhiake Nok kento nonwentsiake skennen takenhake! Onen se wahatonni ne skennen ranonwes.

A tanitenrasera wenniserontakon : Ne hetsitwarhareke sonkwennakeratse, Kaianerensera rotiatontakonhatie.

A kwa roiatanonwet, roiatanehrakwat Onkwe nihaiatoten, iah se te honisen, Niio nihaiatoten, iahte honistensen.

Iesos sonkwaskontakwen, hetsitwanehrakwas Rotiatotenstonhatie tsini twaiatoten, Sonkwaiatanoronstha, sonkwentenronhatie.

Tiotkon hetsenonwehak sanikonhrakwek on lah ki sen tetsenonwes, iahte sarihonte, Satateriasatako, sotsi seriasaksen.

Naetwaiere kenha atwateweienton, Kariwaksenskwe okon oksa ionkwarihonti, $\mathrm{Ne}$ ok kariwiioston akwa twatsteristak.

Sewenniio tsi iakionnhe, takwaiatateni, Iahte tionkwarihonte askwaianerenstak, 'Tsini akwa senonwes takwaiatotenstak.

Onen onkwe rotonhon Iesos Niio Roienha. [sh: Onen tsionwesen Iesos shotonnheton] Tsitewanenton ken renteron okaristiiakon. Sonkwentenron, sonkwanonwes, sonkwawi raieronke. Ne sonkwaianerenstha, ne sonkwaiatakennhas, Ne oni sonkwarharatstenni, karonhiake ieniakwe, Etho ienionkwakwatse. Areriia, Arerlia. 
A sonthenne tsi iakotas, sonkwennakerati ne tionkwe Aonsasonkwaskontako, ronariskonhon ne katsenen

Raonha ne raksatiio akwekon nonkwe tesakononks A kwekon hetsitwawira tsi tionnle nasonkwentenrhẹke.

Tewatennikonlıranentakt tsi nonwe ni sonkwatonnienni Roiatanelırakwat kowa, akwa rotatitenstetenni.

lahte kanakwaienteri nonwa waowiraientane; Onkwe iakotewetonni naonsasakaonnhetstake.

Hetsitwawenniiostakwak tsi teionkwariwakwenhatie; Tetsitewanonweraton Niio sonkwawi ne Roienha

Twakwekonhak ne ken wente, onkweri atsitwaienhase, Atsitewanoronkwake nok atsitwakonnienstake.

Sonkwaiatisakonhatie taetsitewateratana; Iensonkwaiatenhawite tsini thawenon karonhiake.

Swaronhiakehronon Sotsi sewarenniio Onka akoriwa Teswariwak wenhatie. Asennon renteron Maria Sose. leresare ne ken Kanatakweniioke ! Akwa Wetreherke Iesos ronakeraton.

Tosa swakenraten Tsi roieshaonhatie ; Akwa Rawènniio Twakwekon ne tsi tionnhe.

Niawen sewatkon Ne sewaronhiakehronon, Askwaroriana, Niio sonkwentenrane

Twatenro twastoron letewet tsi thotonni Ii sonkwaniente Tsi sonkwaskontakohe
Ha! onen ken ranats Niio sonkwaiatison! Ionkwariwaneren, Shariwaserakohe.

Sotsi ionehrakwat Akwa Niio Roienha Oniataraa sonha Ronwawenonniaton

Atwateweienton 'Tetsitwanoronkwanion ; Hetsitwanonwehak, Hetsitwawenniiostak.

Sakatatrewate Wakeriwanerahakskon Onen sakatsterist Tsini honikonhroten

Ririwaienthose A kenikonhrakon Ne akatonnhetston Akwekon ne kieronke 
Nok oni ne Sose Aetsitewennite

Nikwekon Waria

Ronwatsteristonhatie
Takwaterennaienhas

Waria Sose oni

Nok ok iakiheie

A hiwennarakwake.
Swatontek ne twatatekenson Sewariwanehrakwasek

Niio raksaa rotonhon;

Sotsi seken sonkwanoronkwa Enaskwa rotonhon.

Saetho nonwentsiakwekon Nonwentsiakwekon raowenk.

Niio sakorharatstennihne $\mathrm{Ne}$ ietisot okon kenha;

Rotatewennaieriton, Nonwa rotonni ne Roienha

O ! ratatkenhronnis...

Asonthen Wari roteweton $\mathrm{Ne}$ raiatanoron kowa Akwa ioriwanehrakwat Niio takwatekenha onton 0 ! tsini haiesas...

Katsenen tsi iotinonsote Rentakere Rawenniio Oniataratseraksenskwa Tsi rohere ennekerake Nene rotent onwe...

Akwa sonkwanoronkwa Iesos
Ne rotatitenstetenni

Wahawistoske, ratstarha, Iah othenon te honoronse Toni hotentaon...

Etho ietewe, twatateken, Tetsitwawenniiostana

Ne sesonkwaskontakohe; $\mathrm{Ne}$ ok onkweriasa raskaneks Sonkwaiatisakhe...

Ionwesen tsitwariwakwase Ne akwa karenniioson Twakwekon twatonnharonnion, Iro sesonkwaskontakohe Sonkwaianerensionhe:

Twakwekon tetewariwak: Ne sonkwanoronkwa onwe

O Iesos! aiesasennaien O Iesos! aiesanenton Kannhotonkwe karonhiake; Onen takwannhotonkwennire, Waskwatsennonniaten

Twakwekon tetewariwak : Iesos aiesasennaien.

11

0 ! iawenniseranehrakwat Tsi sonkwatonnienni Iesos Oh! akwa naiakwaiere Tonsaiakwariwaserako Sewariwanerahakskon, sewaSewathi, Iesos aiakiatenroke

Nahoten sannhaon, o Iesos! T'si karonhiake tisenon? Ne tsi shenoronkwa nonkwe, Shenerensions ietsenenne.

Isehre: ne ensekheieren Tsi onkwe satíatotenstha : Watiesen ashetenrheke
'Tsi ne satiatontakwenhatie

Tokenske satateksatonni Ne naiesanoronkwake

[thi Nok tsini takwentenrane Aiontennikonhranentakte Isehre: akwa aionkwisron Aonsaionkierenhake Tosa tsiakoiataksatak T'sini kanakere iotaksen.

I esos waskenikonhrasenni Sonka akonnonwehake Onen wakatewentete Wakenikonhrotakwatakwe. 
Okti ionehrakwatons

Tsinahoiatawen

Rawenniio Roienha

Nonwa wasontate

Iawet watharaneken

$\mathrm{Ne}$ ken nonwentsiake

Nok oni ne karonhiake

Enskat aonsonton.

Raonka raiatison

$\mathrm{Ne}$ wahoteweton,

Raonha sakoienha

Roienha rotonhon;

Niio ne karonhiake

Onkwe rotatonni

Rotatitenstetenni

Ne ken onwentsiake
$\mathrm{Ne}$ teioswathe kowa

Tsini haiatoten,

Asatakon tarete

Tasonkwaswateten

Katsenen kontiteron

Eh wahatekwasen!

Sonkwarihonniennitha

Tosa ienaiehak

Takwentenr oni nii

Seksatatokenti

Takwanikonhrateni.

Aiakwattokhake,

Taiakiotarhoseke

Iotaksenskwe okon

Sonha akwanatonnien

Nonkwanikonhrakon.

13

Twatenro eh ietewe hetsitewatkensera Nonwa sonkwatonnienni ne Wari roteweton; Twatontat te sonkwennonkson tosa onkwaiatateri.

Kento ietiiatonti nonkwatsenen okonha, Ononha niha niare akontonnhisakseke ; Hetsitwatkensera tsi ranats nene Sonkwaiatison.

Iaweronhatien oni naiohahaksenhake Tosa ne ionkwaiotats senka ok tewahtenti; Nene roiatanonwet onwe hetsitwaiatisakha.

T'si sonkwatonniennire ne ken onkionwentsiake, Ok eken tetwatkenni natsitwaienterhane Aonsatsitewaieritse tsi wasonkwanoronkwe.

Oh etho tewenteron tsi nonwa hatekwasen Sonkwawire ne skennen karonhiake nontawe :

0 ! wasonkwanatanoronsten ne ken tewanakere.

Niio takwatonnienni Iesos takwaswateten, Tsi watkwatontsotase tsiena nonkwakaseri, Takwentenr aiakwatsennonni nonwa nok ok tsinenwe.

Thone sahennakerate Iesos Niio Roienha roriwison ne Roniha oksa wakarihowannha. Ne kati sakorori aheren thatihakerekwe, otsistok sakononkton asen ratisennowanenskwe. Oksa tho ronenonton tsi Iesos tahalonni. 
'Tsi ronahtentionhatieskwe, iotsanit tsi naawen, aonha tiotkon kahente otsistok ohahakeson. Onen sahontorisen, aonha ehnikaierha. nok tsi iakononweton, iahten tsi eren tewetha, onen tsinitiawenhon, iahte iakoiatontion.

To shonte niiononwetsion iehoti Sirisare tsi thonwennakeratonne Erot ne raserohen. Ethone tsiotatontaton otsistok ne konwakenne. Ronnerhe : kento onte rotonni ne sakwaiatisaks. $\mathrm{Ka}$ kati, rontonhakwe, Rawenniio tsi tionnhe?

Erot ne iasakononke, wasakoriwanonton : Ohni sewatierenhatiese ? Kanon tiswaientakon? Aheren'tionkwahtention tsini tkarakwinekenstha, ne sakwaiatisakhe ne nonwa ronakeraton; ken, iaken, ionwentsiate ronwennakeratane.

Erot ne sakowennisaks ne rotiksten okonha; ka, wahenron, thatonnihane sesonkwaskontakohe?. Akwa rotihtarakwen. Wetreerke, aonha ne watrori noiatonseratokenti, etho enthaiakenne sakotenrane nonkwe.

Ok sa shotiriwaronken tsini karihotenne, nok sonsahatiiakenne, Wetreerke wahonnete, wahontsennonni kowa otsistok sonsakonwaken, iakonatonnihatie kanon iontohetstakwa; ontien ne kanonsake tsi Iesos tahatonni.

Akwa wahotinehrako tsini kanonsaksenne, tsi wahonwaiatorenne ronwaiatisakhonne, ok sa wathontontsuten raiatakta ne raksaa thonwanonweronniontha, enskat iontatiatokastha, owista notsinekwar, nok iontionkwaronniatha.

Rawenne ne raserohen: Tenteskwaroriana tsi iensewarihorenne swariwisakonhatie. Oia kati niawenhon, iken raronhiakehronon rawen: Akte sewatohetst. Eso tsi ranikonhraksen, irerhe : ahirio raksaa Rawenniio.

Kanekhere, twatateken, rotiiataterihon Iesos ronwaienterhaon aheren thonahtention; senha kati twakwekon nonwa ionkwaiatateris, iken Iesos asteson atsitwaiatorenne, satsitwaiatisakha ononsatokentike.

Onen kati, Sewenniio, tekonnonweratonnions, tiotkon nokaristiakon tokenske sataseton ; iahte katennowentha, saheto iahten takonken, katiken sanowen, sonhaa sawennatokenti, iah kati takatkawe tenkonnonweratonne.

Kasene, kasene, sewariwiioston, hetsisewatkatha sonkwawenniio sonkwaiatisaks. Sonkwatiennire okaristiakon sonkwaiatisaks. Sonkwatiennire ionkwentent okon. Tsiatsennonni tsionkwe okon. Tosa sewakenhronnihek netsisewentenre, hetsisewawis tsini hoien. Onwa hetsitsion seweriasa. Ronikonhriio, rotiesen kowa, sesonkwaskontakwen. Sewatsiaken kati, sewanikonhriohak. 
16

Wenniseranoronson $\mathrm{Ne}$ ken wentatenion Onen tsi ronakeraton Iesos ne Sonkwawenniio Sonwaskontakwenhatie Nonwa wenniserate
Onen twateweienton Iesos ronakeraton Sonkwatsennonniatennire Tsini sonkwentenronhatie Aonsetwatatrewate Onen iahte kakonte.

Onkwe rotonhon Niio Roienha Ii twakwekon ne tionkwe sonkwatonniennire Twakwekon kati twatsteniaron Etho ietewe tsi thotonni.

Ionehrakwat ne sonkwanoronkwa Sase ionkwaiatoron kariwaksenskwa, Shariwaseronniane Rawenniio, Sonkwanikonhratahane kariwiio.

Tetwaiatoret tsini hoiatawens, Ok sa ionkwatokense tsini honikonhrawens Atatkenhronni rariwanoronkwa Nok rariwaswense aienaiehak.

Ratstarha Iesos tsi wahatonni Tsini hokararens ionkwatkaronni, Sekon kati otiake iakoieshon

Nonwentsiakon waakawenontonhatie.

Sakoiatisaks niakotent nonkwe lrerhe : karonhiake iakheiatewe ; Eniakoiatateri neniontontat Raoriwakwekon iontsteristha.

Twatsiaken kati, twatatekenson, Akwa twatsteristak kariwiioston, Aontiesenhake ken twanakere Iaetwakwekonhake karonliake.

Satontat Iesos tsini iakwaierha Ken iakwanakere nonwa skwaien okon Onen ionkwariwison iakwakwekon Akwa kwawenniiostha iahte kakont.

Ionkwentent Iesos, saterientare, Ii ionkwennakeraton kariwaksenke ; Askwaianerenstake Sewenniio Aiakwanonwehake kariwiio. 
Sennakerat akweriane

O Iesos Sewenniio !

Etho satiatakweniiost

Tsinenwe enkonnheke

Sonha ishawe ne skennen

Aionnontonnionheke

Tosa aonsaskiatonti

Tiotkon tenikwekonhak.

Sotsi seken kanaktaksen

Katsenen kontiteron, Satontat akweriasakon

Akonnaktarakwase ;

Senonwes tsiontatrewatha

Iakotonnhakanonni

Ethoniiot nakweriane

Eh iasatenaktonni.
Nok senha ieskanaktaksen

Ne nakonikonhrakon

Othenon iakoiatoron

Asheiatienniheke ;

Iawet se iesaiesatha

Niahte tsiontatrewatha

Senha ok kati iienhre

Ahakwataweiaten.

Seksatatokenti kowa

Ase tontakweriason

Ne naweriasatokenti

Naesanoronkwake ;

Oriwakon takwahroris

Iahte skwanentsawakon,

Iah se ken taiakwakweni

Onkionha akwasere.

Twatsteniaron twatatekenson Ionehrakwat sonkwanoronkwa

Niio sonkwawi Roienha

Ionkikwate nonwa

Ne ratironhiakehronon

Skennen sewennontonnionhek

Iesos onkwe rotonhon

Wetreherke ronakeraton

Kario tsi iotinonsole.

Ne Niio Roienha

Wasakotekwate notkon

Onwentsiakon ronnhoton

Aionkwatkaronnion

Onwentsiakon aionkwenonton ; Iesos sonkwaskontakohe

Raonha sonkwanerensionhe.

† Iwakwekon tewateweienton, twatonwesonnion ; onen ronakeraton ne Iesos onwentsiake * Ne raniente tsi rotonni skennen aonton onwentsiakwekon nok satekwatane notkonseraksen. Tetewariwak: * Ne... Ionehrakwat sonkwanoronkwa, sonkwannhotonkwennire ne karonhiake. "Ne... Akwa sane ne raksaa Niio nihaiatoten. † Twakwekon...

† Ken ranats ne Sonkwawenniio, akwa raiesas, taonken raksatiio, hetsitewanoronkwak. * Tewatsiaken tewakwekon, tewateweienton, hetsitewasennaien ne Niio Roienha onkwe rotonhon. Tetewariwak: * Tewatsiaken...Kariwiio sonkwatatiane, sonkwaiatiniontane ne karonhiake. " Tewatsiaken... Sahaiatat rotaseton ne okaristiiakon. + Ken...

† Okstentsi rotentaonhatie nok tsini sonkwanonwes, ethonihoiatawens. * Oksa kati tewakwekon etho ietewe tsi ronakeraton, - tewatiskwentaren tsi rentakere. Tetewariwak: " Oksa... Son- 
kwaniente sonkwaiatisaks niahte kakonte asonkwatsennonniaten. * Oksa... Nonwa tehonwanentonnions ratironhiakehronon. † Okstentsi...

Onka onte ietiiatkathos Onka onte ietakere Katsenen tsi iotinonsote? Eksaa etho iakotonni.
Rawenniio ne karonhiake Rawenniio nonwentsiake Ne wahonni ratatkenhronni Sotsi tewanaiehne

Tokenske ii ne tionkwe, Tokenske ii onkwariwa, Ne nonwa roieshaonhatie Nok oni ne rawistos.

Tewakwekon hetsitwanenton, Onen kati tetewariwak Tewakwekon nonwentsiake; Onen kati twatonnharen Akwa hetsitewanonwenn Hetsitwanoronkwak oni

Kanekhere thoriwaieri, Kanekhere raksatiio Ahetsitewawenniioste Hetsitwanoronkwak oni :

Tsi nonwa wahennakerate Sonkwatsennonniatennire

Tewenron ki ronikonhriio Tewenron ki raksatiio Hetsitewariwaienthose Akwekon oni tsi tionnhe.

22

Akwwekon tetwariwak Nok twatonwesonnions Ionkwaiataterihon $\mathrm{Ne}$ twatatekenson Onen ronakeraton Hetsitwarharenionk we lesos wahalonni (3)

Wari twennakeraton Ionkwatewetonni Rawenniio tsi tionnhe Niio onkwe oni

$\mathrm{Ne}$ wahennakerate.

Wesonkwaskontakohe...

Tiotinakarontonha Konwanatsteristha Wasakotiwennahronke. Eronhiakehronon Wathonatonharenron Aseken asonthenne...

Tosa sewahteronsek Skennen swennontonnion. Aseken kariwiio Iakwariwenhawi Onen ronakeraton Ne hetsitwentenrane...

Entsiwaiatatsenri

Tsi iotinonsote

Nene katsenen okon

Ne ronahriskontie

Oniataraa sonha Ronwawenonniaton...

Nok tsi nahontsennonni

Ok sa wahonhtenti, Wahonwaiatisakha Ne ronakeraton Wahonwaiatatsenri Wahonwawenniioste... 
Akwekon wathontkenni Neronhiakehronon ;

Tehonwanoronkwanions,

Tsini raksatanoron

Ne tehonwanentontha... Tehonwanentonnions

Tsiaken twatatekenson, $\mathrm{Ne}$ ietinakeren :

Hetsitwawenniioste

Hetsitwasennaien

Onkwenrikonhrasaat

Hetsitewanonwehak.

23

Onkwe ratiatotenstha, Eronhiakehronon

Asonkwaiatotenste

Ne tionkwe ionkwentent

Raonha enhakweni

Enionteweienton

Kentiohk owanen nonkwe;

Iawet onwentsiase

Enwaton onkwe okon,

Eniewenniioke

Ka:onhiake aiente

Tsi neniontonnhokten

Tetsitwanonweraton $\mathrm{Ne}$ sonkwatonnienni, Onkwanikonhrakwekon Hetsiwanoronkwak ; Iahte kakonte, Jesos, Wakonwennarakwe, Takitenr karonhiake Iaskiatenhawite.

\section{4}

Twatontat kariwanoron Twakwekon twatsennonni Akwa ne Niio Roienha Onen ronakeraton.

Rotkon Roiatatokenti Aonekwensa Wari Raonniaton raieronke Nok oksa rotiataten

Rawen raronhiakehronon Iesos enhonwaiatseke Hetsiwasennanoronkwak Hetsitwasennanonwehak
Iesos tekwanoronkwanion, Iohehrakwat skwanonwes, Oneshon seskwaskontakwas, $\mathrm{Ne}$ ionk wariwanerahakskon

Wari tekwanoronkwanion, Sose seniniiakonne, Iahte senakwaienteri Iesos skwatewetonni.

Sose tekwanoronkwanion Okstentsi tsiatanoron, Iahte senakwaienteri Iesos takwehiaronni 
Tiotkon ietiiennitentas Iesos, Wari nok Sose, Othenon nionkwaiatawens Aiétiiataraseke.
'Twakwekon ietinakeren, Akwa twanikonhraasat, Nok onen entwatonnhokten Aetewakwekonhake.

Onkwe rotonhon Niio Roienha Tewatsennonni twatateken sonha Sonkwaskontakohe, sesonkwanerensionhe Taetsitewanonweraton.

Oksa tsitwaiatisakha Ietiiatonti katsenen ietiiatsteristha Oktiiotiatawensere tsitwakensere Ne nonwa sonkwatonnienni.

Konnonwese seksa onkwe satonhon Wakonwenniioste akwekon nakatonnhetston Onen nonwa enseskwaieritse Tsinikon takwarharatstennihne tsinahe

Tontahotiriwakwenhatie Ratironhiakehronon nonwa ronatsennonni, Ronton: Ahonwasennaien Niio

Tsini sonkwentenron sonkwawi nonwa ne Roienha

Tetewariwak, twatonnharen,

Tsi twatonraserons ne nonwa wahatonni ;

Roiewas, rawistos, tchasenthos ;

Tsinikon ionkwaientas hetsitwawenniiost.

Wari saiataterihon

Hetsateweton ne Sonkwawenniio, Takwaterennaiennihek

Senikwekonhatie Sose Roiatatokenti.

Rotonni Hiio Roienha

Tsinahe ne wahonnise

Akwa ioriwanehrakwat

Onkwe okon ne sakononks
$\{$ Ionwesen tatsitwariwakwase, $\{$ Sonkwennakeratse onwa.

$\{$ Ne rotitokensehakwe, $\{$ Ne ionkirharatstenniskwe.

$\{$ Tsi Iesos raksatanoron,

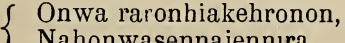


Wetreherke tewastoron

Tosa taionkwanonhianik

Tsi rentakere ne Iesos

Tokenske rotent ne Iesos

Tosa kati twatsarokwat

Toni saksatiio, Iesos.

Tiotkon oni ionkwarhare
$\{$ Naetsitewatkensera,

$\{$ Natsitwasennaiennira.

$\left\{\begin{array}{l}\text { Naetewatatkenhronni, } \\ \text { Tsi lesos ratatkenhronnis. }\end{array}\right.$

$\left\{\begin{array}{l}\text { Iotinonsote katsenen, } \\ \text { Ennekeri raonakta. }\end{array}\right.$

$\left\{\begin{array}{l}\text { Sase raiatakweniio, } \\ \text { Sase ne Sonkwawenniio. }\end{array}\right.$

$\{$ Tsi kento tiotkon ionkwentent,

$\{$ Ne Iesos tahatierente.

$\{$ Nok toni takwanoronkwa, $\{$ Tiotkon enkwanoronkwake.

$\{$ Askwentenre tsi ionkwentent, $\{$ Askion oni karonhiake.

\section{7}

Tsionkwe Niio hiatatienha Sesonkwaskontakwenhatie Nonwa sonkwennakerati

Tatsitewariwakwase : Gloria in excelsis Deo ! Ioiatatokenti kowa Waonkwatewetonhase, Tsi akwa kanonsaksenskwa :

Sonkwawire karonhiake $\mathrm{Ne}$ ionkwentent nonwentsiake; N Ronton ne ronatkon okon :

Wahakwa noneshonronon Iakotiohkowanen nonkwe. A kwa wennikonhriaks onwe :

Sesakotenrane nonkwe Aiakonaktaientake

Tsi iakotsennonni kowa :

Sonkwaswenskwe Rawenniio Shonikonhraseronniatha Roienha onkwe sahaton:
Irehre enkatketate 'Tsini iakoriwaneren

Tetewaiena ne tionkwe Akwekon tetewariwak Tsini wasonkwanonwene :

Tetewatont karonhiake Ne ratironhiahronon Aetsitewasennaien

Ne resaks nonkwanikonhra, Tenikwekon nonkwatonnhetst, Atsitewanonwehake

Eh ietewe tsi rotonni Eronhiakehronon okon Ne ronwawenniiostanions : $\mathrm{Ne}$ tsi ratatewentetha:
Ne nonwa tetewatoron I esos ionkwatewetonni Ionehrawat ionkwentenron. 
Areriia, areriia!

Eronhiakehronon ietinakeren

Tsini sonkwanonwes Iesos twatrori

Sonkwatsennonniatennire

Onkwe rotonhon tetewariwak :

Areriia, areriia !

Iotsennonni nonwa

Nonwentsiake Isi rotonni ne lesos

Sonkwawenniio.

Onen iontataskennha

Ronwasennaiens ne teieriwak :...

Eronhiahehronon ne iontsteniaron,

Niio ronwasennaiens,

Sonkwentenron

Tehonwanonweronnions

Nonwa rotonni ne teieriwak :...

Kenni wenniserate Rawenniio

Irehre: iahte kakonte ne Rienha

Sakotsennonniatennire

Nonwentsiakwekon kehrewatakwe :...

T'si sonkwariwaneraki Atan kenha

Sonkwaiatontion Niio

Onwa se shonikonhrenhon

Tsi sonkwanonwes sesonkwentenron :...

Tewatsteniaron kati, tewatateken Tetsitewanoronkwanion.

Ne rotonni

Aiakwawennakwekonhak

Tewatataskennha tetewariwak...

Iahte sewatiaktanion sewaronhiakehronon

Ken wasonte hetsisewakenseron

lesos onkwe wahaton watiswatatsterihate

Watsisewasennaienne $(2$;

Tesewariwakwenhatie tsini sewatsennonni

Karo kasene, Israerronon, Rotkon katsisewanonteratie Onen hetsisewennonksera

Raton tsi rohtaratié, onen watsitsionwentsiorenne

Akwa Rawenniio tsi tionnhe (2)

Onen satsisewaskontako nonwentsiakon tsi tiotekha. 
Kasewanenratironten niha aietsiennakeraton Otsistok tetsisewasterihatana.

Irehre: tho iakheiatenhawi tsi thononni Rawenniio Jatsiatakwennienhawit

Atsisewawenniiost nothenon sewanoronkwa.

Oksaok sewatkennisa onwentsiakwekon nonkwe Watsisewanoronkwanionna

$\mathrm{Ne}$ Wari hiatatienha atsisewawenniioste Naontesewennikonhrasahat.

Raonha Rawenniio tsi tsionnhe nok oni nensewenheie.

Niawen, Iesos, takwanonkwe onton Tsi onkwe wasaton, niawen lesos.

Iahte skwaswenhon iah se te iakwattokha

Iesos, aiesawennarakwake ne ken ionwentsiate ;

Onwentsiakon satsiatonti notkon sonkwaiesatha.

\section{0}

Niio hiatatienha Iesos

Sesonkwaskontakwenhatie

Sonkwennakerati

Tsionkwe kasene sewakwekon

Sonkwennakerati

Hetsitewawenniiost.

Oiatatokenti kowa

Waonk watewetonhase

Tsi kanonsaksenskwa

T'sionkwe, kasene sewakwekon 'Tsi ronakeraton. 'Tsionkwe...

Tsi kanonsaksenskwa : hetsi...

Sonkwawire karonhiake

Ne ionkwentent nonwentsiake Ronton ne ronatkon

Tsionkwe, kasene sewakwekon

Ronton ne ronatkon...

Sesakotenrane nonkwe Aiakonaktaientake

Tsi tiakotsennonni Tsionkwe kasene sewakwekon Ronwawenniiostha. T'sionkwe... Tsi tiakotsennonni...

Sonkwaswenskwe Rawenniio Shonikonhraseronniatha

'Tsi onkwe wahaton. 'Tsionkwe.

Irehre : enkatketate

Tsini iakoriwaneren

Ralatewentetha. Tsionkwe...

Tetewaiena ne tsionkwe

Akwekon tetewariwak

Telewatons karonhiake Ronatkonseriio okon

Hetsitwasennaien. 'T'sionkwe...

Ne resaks nonkwanikonhra Kenikwekon nonkwatonnhets, Hetsitewanonwehak. Tsionkwe..

Eh ietewe tsi rotonni : Eronhiakehronon okon 31

O ise swakwekon sewariwiioston Wasontate nonwa Niio Roienha Onkwe rotonhon Wari ioteweton 'Tesewariwak areriia. 
Karonhiakwekon eronhiakehronon

Nok nonwentsiakwekon ronatsennonni,

Onwentsiakonronon teiotonharenron

'Tsi ronakeraton Niio onkwe rotonhon.

Ii twakwekon ionkariwiioston

Wasontate nonwa Niio Roienha

Onkwe rotonhon Wari ioteweton

Tetewariwak areriía.

Tosa sewahteronsek skennen sewennonlonnion

Niio onkwe rotonhon, ronakeraton, Hetsisewentenrane, onen sewatsennonni, Iesos wahatonni, sonkwaskontakohe.

Swaiataterihon, ise ne tsionkwe, Si ronakeraton hetsiwarharenionkwe

Wasontate nonwa wasontanoron

Niio Roienha Wari roteweton.

Katsenen okon ronahriskontie, Oniataraa sonha ronwawenonniaton Ronwasennaiens ne Wari nok Sose.

32

Onkwe rotonhon Niio Roienha Wahonnise tioteronhiakwekon

$\mathrm{Ne}$ Wari wahoteweton

Tatsitewanoronkwanionne

Iesos Wari hiatatienha.

Kariwiio skionwentsiorenhon Iesos onkwe tsi satonhon Wahonnise iesaiatisaks Wahonnise tsiiesenonks.

Ionkwakenrenseronnihakwe Notkon nonwentsiakonronon Onen nonwa wasatoriane Onwentsiakon satsiatonti. Iahten tiiaionwe nonkwe Iesos tonsaharonhiakhasi. Etho sonkwaiatenhawi.

Niawen Iesos ne takwentenron Niawen ne takwanonwehon, Niawen tonsaseronhiakhasi Niawen etho skwentenronne.

Iesos aiesaienterhane, Iesos aiesennaien, Iesos aiesakonnienstake Iesos aiesennakeren.

Wari taiesanonweraton, Wari aiesanonwehak. Ise Iesos hetsateweton Iesos setwaiatat onton.

33

Takwahrori Wari Tsi naawenne sahatonni Tsini tsiatisakskwe Iesos tsiatatienha.
Onen iaenniseraieri Raiatat raksaa Wahatonni Iesos Iahte wakaltoken. 
Takwahrori, W ari T'sini kanonsotenne Tsi thotonni lesos Tsi iotierenne.

Akwa kanonsaksenskwa Iahten te iotekha, Ennekeri kentskaronkwen, Ethononni hanats.

Takwahrori, Wari, Onka ietsiatkensehakwe, Onka swanontenskwe Tsi sewatonkariakskwe.

Rononha kheiatkatho Kario iontsteristha Rctkon sakohrori Rawen: eh iaasene.
Takwahrori, Wari, Thenon iakolokaten Niate ionwentsiake 'Tsi Iesos ronakeraton

- Ne watenientenston Katsistokowanen ; Otsistok ne wakohrori Tsi thotonni Iesos

Takwahrori, Wari, Ohni iotierenne Asen nihati ne Ratikowanenskwe.

Wathonwanoronkwanion Ronwawenniiostha Owista, kenhie oni Akwekon nononkwa.

Wari, iesennitha

Nasakwaken I esos

Asakwawenniiost

Nonkwanikonhrake.

Wahonwateweton Niio Ro- Niio tsini honwaniha Onen kati nonwa [ienha Wahonwateweton Niio Sonkwentenrane ne Rawenniio Onkwe rotonhon, ionehrakwal Iesos enseskwaieritse. Taetsitwanonweraton Shetenrane naiakoronhiaken Ii tewakwekon.

[nonkwe. Twatsennonni nonwa Ken ionwentsiale Arekho ne si iawet Aseken Iesos sesonkwaskonta- Ne iontonhakwe Nonwentsiake.

[kwen Ne Niio enhonwateweton

Niio onkwe rotonhon.

Iesos onwentsiakon onen Ronnhoton, karonhiake Sonkwanaktaiennire.

Rotitiohkwakwekon Ne ronatk on karonhiake Tontahotiriwakwenhatie.

Rawenniio tsi tionnhe Ne Niio Roienha

Raronhiakehronon wahenron

Tsiaken, o Waria!

Roienha Niio

Onkwe rolonhon.

Ratironhiakehronon

Ok sa ok wahontrori;

Wari onen ioteweton

lesos twalateken

Niio onkwe rotonhon. 
Onen ietinonteratie Rotinontiio kowa

Aheren thonahtentionhe, lesos ronwaiatisaks ; Katsenen iotinonsote lahonwaiatatsenrí

Ne raksatanoron kowa

Ennekerike ranats.

Tokenske ne Rawenniio Tatsiswatontsothase, Akwa sakotohetstenni Rotinontiio okon ; Onkwanikonhra ne tsionkwe Senha rariwanonwes Natsitwawenniiostake Tsi sonkwentenronhatie.

lah se tehotakwatstenni Tsi thare nonwentsiake, Ne raotsokowatsera Nasakotenre nonkwe ; Iah tenhnon te hahawi Kento aiontsennonni : Ne sakotokatennire Karonhiake aiente.

Seníaken nisa tsionkwe Swanonsote swanonsote Tsiatisakwa Wari Iesos rotewe-

Tsiatontat teieriwakwa Ne konwaiats kariwiio ; Iah etho te swawennoten, Onen thatiriwakwa Ne ratironhiakehronon, 'Tehonwariwakwase Niio onkwe rotonhon.

Otsistok iakonatonni Tsi thonakeraton Iesos. Aetewanonteratie, Iesos tsitwatkensera, 'Twakwekon asen nihati
Tehonwanoronkwanionnon Iesos a kosatensne, Ratithawi kanoronson Nahınwawenniioste : Senha kati ne raonha Kanoron sesakowi Eh iahatiienterhane Karonhiake ieientha.

O wenniseriio kowa! Tsi ronwaienterhaon ; Rawenniio onwentsiake Tahototasionhatie ; Katsenenke thatasafa Asonkwaronhiakense ; Tekaiasontne enhasa Aiontsennonni nonkwe.

Tsi tesonkwanonks ne Iesos Natsitwaianenhawe Tosa tewateraiatak, Sonkwanatonnihatie; Iawet olsistok raonni 'Tsi ionkirihonnienni ; Enionkwaiatenhawite Katkeha karonhiake.

36

Ronwawennakeraton Ronwariwaienthose.

[ton. Ne se resaks ne twawenna Akwekon onkwaniknnhra, $\mathrm{Ne}$ tsi rariwanoronkwa Aetsitwawenniiost Tsini tewennontonnionkwa, Aetsitwawenniiost

Tsi tewatatiatha.

Tewahtenti twatarahtat, lesos tsitwaiatisakha, Twatenikonhraserenni Asonkwaiatonweskwen Ne nonwa tsi wahatonni : Sotsi sonkwentenrane lah se tionkwe te ken. 
Niawen kowa Sewenniio Skwentenrane, iakwaiesas, Onen seskwaskontakohe Teseronhiakasionhe :
Isehre : etho iaionwe Onkwe neniontontate Tsini sewennoten.

\section{7}

Sahatiken notistok rotiianer kowa Wahotitokense Niio onkwe rolonhon Ok sa ok wahonhtenti wahonwaiatisakha Iesos wahalonni.

Ok etho Sirisare notsistokowaton, Etho iahontaweiate ratiriwisakhe Ka nihonakeraton ronwanontiiostane...

Ne wahanosa Herot, wahoteronhienten, Wasakoriwanonton ratinikonhrason Ka nonwe enhatonni nene tsitwarharenion ?...

Ok sa wahonwarori, wahiron : Wetrerke Ronwanontiiostane enhennakerate, Ne ratonhakwe Wishe, ne rotokensehakwe...

Herot wasakorori, wahenron : Wetrerke, Etho ratonniane ne hetsiswaniente, Etho iaesewete, kento tsiswatohetste...

Ok sa ok wahonhtenti rotinontiioson Nok are sakonwaken ne oksîstok kowa, Iakonatonnihatie, aseken ne kahente...

Satkatane otsistok wahatinonsaken, Raksaa tsi renteron wahontiskwentaren, Wahonwawenniioste raotinihonhrakwekon...

Ethone wasakonon ratihawinontie Owista, kenhie, ononkwat, kanoronsonkowa Ronwatenientenstakon Niio nok onontiio...

Sasakonikonhrata raronhiakehronon Tosa ahontohetste tsi Herot thenteron Heren nahontohetste ronatasetonhatie...

Rotinontiio kowa ne ietinakeren, Hetsitwawenniiost onkwanikonhrakwekon Nok tsina twatsennonni twakwekon tetwariwak...

Rinonwes ne raksatiio, ne tsini haiatanoron Rinonwes. O ! ne raksatiio, sonkwanoronkwa. 
Ronwanatonkwa ne lesos; aseken sonkwanoronkwa Rinonwes; hetsilwanakeren, sonkwanoronkwa.

Ne tsini hatatkenronni, sase ne Sonkwawenniio, Rinonwes, hetsitwanakeren, sonkwanoronkwa.

Ne ralironhiakehron nonwa ahonwasennaien Rinonwes, ne ronikonhriio, sonkwanoronkwa.

Iah othenon tahonsennha, sase akwa raronhiakens, Rinonwes. O ! ronikonhriio, sonkwanoronkwa.

Iah othenon tahonoron, ne sesonkwaskontakohe Rinonwes. O! wasonkwentenre, sonkwanoronkwa.

Rinonwehak raksatiio, sotsi sonkwanoronkwakwe Rinonwes, enhinonwehake, sonkwanoronkwa.

Ne Iesos sonkwaiatison, raonha sakwaniahesen Rinonwes; ne riwennaierits, sonkwanoronkwa.

Ise swaronhiakehronon, tosa othenon swanosen, Rinonwes, ne riwenniiostha, sonkwanoronkwa.

lesos akwa kasenniio, akwa iosennatokenti Rinonwes; hetsitwanakeren, sonkwanoronkwa.

Tenhiriwakwase Iesos, ken wasonte nok ken wente, Rinonwes. O ! roliesen kowa, sonkwanoronkwa.

Ronnhek Iesos raksatiio! ionnhek oni ronistenha Khenonwes; ne ietinonwehak, ionkinoronkwa.

\section{9}

Ronnhek Iesos, taonken kasenniio Ronnhek Iesos ne Sonkwawenniio Akenikonhrakon wakatonnharen Kwawenniio wakerihowanate Ronnhek Iesos, ronnhek Iesos.

Ronnhek Iesos ! nonkwe okon sakononks Ne Niio nahonwanonwehake; Tenhnon oni ne sakohretsiarons Tiotkon ne iakoteweientontiesek. R...

Ronnhek Iesos! sakonikonhrahnirats Akwekon iakoriwanerakskon lienhre aonsakatatrewate Nok aonsonkeriwiiostonhake.

Ronnhek Iesos! akwekon iakoshare 
Tsini holanitenraskon kowa ;

Sakorharatstennis ne karonhiake

Iienhre: aonsakatatrewate.

Ronnhek Iesos! akwa riniahesenhak

Tokat enionkinikonhrotako

Notkonseraksen enkeiatekwate

Iah nonwenton oni tenionkisenni.

Ronnhek resos! watehinonweraton

Tsi tiotkon akwa nihakitenron ;

Tokat enwakenikonhranonwake

Enkakwisron enkerihowanate.

Ronnhek lesos! akwa wakatsennonni

Risennaiens okaristiiakon ;

Riiatkathos kanatarakon kenha

Nok oni rakenontens raieronke.

Ronnhek Iesos! tiotkon ronwasennaiens Akwekon ne ratironhiakehronon; Tiotkon enhonwanoronkwake oni Iesos akwa sakotsennonniatennis.

Ronnhek Iesos! nene Sonkwaw enniio, Tenhnon oni ne tewatateken; Ne karihonni akwa sonkwanonwes, $\mathrm{Ne}$ kati tiotkon hetsitwanoronkwak.

Ronnhek Iesos! sotsi ronikonhriio. Twanikonhrarak kati tewerhek Tosa natsitewanikonhraksate ;

Iahle ionttokhas ne ronwasentennis.

Ronnhek Iesos! ionnhek ne ronistenha Nok oni ionkwanistenha Wari!

Ionehrakwat tsi akwa ni kanonwes

Tsi roienha tehonwariwakwase.

Ronnhek Iesos! tiotkon nasakosenni Oktiwakwekon iakonkwetaksens

Nok nii tsiniiore enkonnheke, Iahle kakonte enkatroriseke: $\mathrm{R}$.

40

Twatonharen tetwariwak Sotsi awentanoron Rawenniio ne tsi tionnhe Onen ronakeraton.
Tsi roriwaneren Atan Ne sonkwakaronniaton Iesos ionkwaniseriio Sonkwatsennonnia tennis. 
Aionkwatetsirake Niahte kakont noneshon : Niio sonkwaskontakohe Tatsitwanoronkwanion.

Onen aionkwarewaton

Ne raonakwensera

$\mathrm{Ne}$ kati iotesenniaton

Tsi sotsi sonkwanonwes.

Ne tionkwawistotarhonne Otkon noneshonronon

'Teshowistotarisiaton

'Tsi ronwawenonniaton.

Ronikonhronni tehokwen $\mathrm{Ne}$ ionkisarontakwenne: Irehre : aiontsennonni Rotsennonniatakwenne.

Ratstarha ennekerike, Roiewas rawistose ;

Ii akwekon onkwariwa, Ii sonkwakonnienstakon.

Iesos tekonnoronkwanions Niawen skwaiatorenhon Nonwa onkwe wasatonni, Niawen taskwentenrane: Skwaskontakohe nonwa, Seiatekwatha notkon I onkinaskwaientakwe Seskwanerensionhe.

Iesos satanitenraskon, Tiotkon iesanonwehak, Tiotkon iesanoronkwak Tiotkon iesanentonsek, Satatiesatonhatie Nonwa onkwe sasaton ; Sotsi takwanoronkwa Iahte satatitenron.

Karonhiake tsi tsiteron, Tiotkon satsennonnihne, Nonwa seronhiakensere $\mathrm{Ne}$ kento onwentsiake. Ii tionkwe ionkwarihonni Satateronhiakentha $\mathrm{Ne}$ seriwaserakohe Tsi ionkwariwaneren.
Ronwanonwes, ronwanentons Ratironhiakehronon : Tetewatonts, tetwatkenni Ii senha tewakwisron.

Ionehrakwat, raksatiio, Raiatanoron kowa ; Sonkwanonwes, sonkwentenras, Hetsitwanonwehake.

Akwekon twatiskwentaren T'si ranats tsi rolonni ; Aetsitwawenniioste Onkwanikonhrakwekon.

Wari saiataterihon, Iesos Kristos hetsienha Akwa ne hetsateweton 'Tiotkon tsi hanakere.

Wari kanistenseriio Takwentenr skwaien okon Askwaterennaienhase Karonhiake aiakwe.

41

Ise sewaronhiakehronon Nonwa sewanehrako Tsini sonkwentenronhatie Iesos Sonkwawenniio Tsini ionkwaniseriio. Nonwa wenniserate, Sesonkwannhotonkwennire Twakwekon karonhiake.

Sotsi ne kariwiio Sonkwarihonniennire, Tsinenwe entionnheke Tsitewariwaierit Niahte kakont twarihonti Noriwakaion sonha : Onen tsitwariwanonwenn Iesos tsini hawennoten.

Wari okti ionehrakwat, Iesos hetsateweton Ne Rawenniio tsi tionnhe Niio nok onkwe oni. Taonken raksatiio N'hetsienha Iesos Kristos ! Tiotkon aiesasennaien Nonwentsiake karonhia ke. 
Tetewariwak tionkwe okon

Tsini sonkwanonwes Iesos

Tetwariwak tewakwekon

Tsini hotanitenraskon.

Niio ne onwentsiake thare Onkwe rotatonnihatie : Iahte sonkwatetsanitennis Tesonkwaswatetennire Sonkwaien okonha wahaton Atsitewanoronkwake.

Niare sonkwasetennihatie Tsini hoiatanehrakwat Ne ok akwa sonkwanatonnis Tsini hoiatanehrakwat

Tesonkwarhotonnis ne tionkwe Tsini ionkwentent akwekon.

Rowennarakwa ne Roniha Ne karonhiake thenteron Ne shonikonhraseronniatha Tekaiasontne renheions,

Ise swaronhiakehronon Swakwekon swanehrako Tsi watatkenhronnis Wari ionkwanistenha, Nonwa sewaterennaienhase, Iahten tsi kaieronni

Tsini kaiatanoron.

Onen nonwa twatateken Ionkwaiataterihon Iesos ne sonkwawenniio

Tontasonkwentenrane Shonikonhraseronniane Roniha Ne sonkwaswenskwe Nionkwariwanerahakskon.

Ioterientare Wari ne Tsinontaiawensere, Iesos ensonkwenheiase Tekaientannhareke Roienha wahotewentete Tsini akwa waskaneks Aiontsennonni nonkwe.
Ethoni kariwisahani

Naonsasonkwaskontako.

Rowennenhawe Rawenniio Tsini thotatierenhatie; Ok sa tehotatewentetha Tekaiasontne nok oni 'Tsinikon ne iehasentakwa Iawet raonha rahasens.

Tesonkwaiatatirontakwa Tsini thotatiataseton

Tsi rotenniote sonkwanontens $\mathrm{Ne}$ raowaratokenti

Ne akwa aetwatennate Naetewe karonhiake.

Aionkwatsennonniatskonhake Tsini sonkwentenron kowa, Hetsitewanoronkwak onwe, Hetsitewawenniiostak ; Twatontat aetwatsennonni, Tosa aetwatkaronni.

\section{3}

Tsinahe ieriwaneraks Kentiohkwakwekon nonkwe, Niio iahte hanonwese Thonwanonweronniontha Raonha kati Roienha Ne akwa satehniatoten Wathononweratonne.

Sahonwaiatinionte Ononsatokentonke Tsini ionkwariwaneren, Shoriwaseronniane

Nonwa sonkwatewentetenni

Raonha raonekwensa

Nok oni raieronke.

Etho Simeon rokstenha

Anen oni iweskwe Iesos ronwaiatorenne Ne ronwaiatisakskwe ; Tetsiaron wathiiatonti Tehonwanonweratonnions Sakotenranè nonkwe. 
Iesos akwa takwanoriwes, Iah sane te iakionkwe Ii oni enkwanonwene Niate wenniserake. Tiotkon eniakwatontate Tsinikon nenionkihrori $\mathrm{Ne}$ iesawennenhawe.

Tekwanonweronnions Iesos tsi onkwe satonhon Tekwanonweronnions Ise takwarihonnienni Iontatewennarakonhatie Ontatenonkwe nonwentsiake Tsini saieren.

Karonhiakwekon Saseronniennihakwe Onwentsiakwekon ; Sekon iahte senaiehne Asteson shewennarakonhaties Sanistenha nene Warı Tsine kawennoten.

Takwarharatstenni

Skennen kowa taiakionnheke Enhontsennonni

Ratinekenteron okonha

Seron : aionkiwennarakwake Iakinonkwe nok ionkiienha Onwentsiake.

Shenoronkwakwe Onwentsiake sekon si seskwe Akwekon nonkwe Senha tenhmon ratiksa okonha Ne karihonni satonhakwe Tosa tehatikanere Ne kariwaksen.

Ise Wari, Sose oni Iesos swakwekonhatie, Takwaterennaienhas

Tsi iakwes nonwentsiake: Katkeha eniakwenheie, Asakwaken Rawenniio 'Tsinenwe karonhiake.

\section{4}

Rononha kati

Raotiriwa akenhake

Ne rotikstenha

Eniontkaronni ratiksaa ; Okstentsi ioianerekwe Iahten tahonennakeraton $\mathrm{Ne}$ rotikstenha.

Kanoron kati Ionkitenronne ionkiienha Kariwanoron, Ise swaronhiakehrohon, Takwaienawasehek kati Tsinikon sonkwarihonnienni No Rawenniio.

Takwaianerenstak Nok takwanikonhrahniratsek Nonkwawatsira ; Takwawatsiranoronston Aionkiwennarakonhatie Othenon sonkwatenientense Ne Rawenniio.

lahten taonton lahten taskwawennarakwake Sewenniio ; Aseken takwatenientense Aiakiienawaseheke Aiakiiaterennaienhase $\mathrm{Ne}$ ionkiienha.

Sasehiarann, Sewenniio, Tsini salanitenraskon : Ii se ken onkwariwa Tsi seron : onkwe akaton.
Wari Saiatatokenti, Ise nonwa takwaienha, Askwaterennaienhase Nonwa nok iahte kakonte. 
Sose saiatatokenti

Ise sennita niskare

Kwennitentasehaties se

Askwaterennaienhase.

Tsionkwe Rawenniio

Hetsisewaien okonha,

Hetsiswawenniiost

Ase tsi tsionnhe;

Iosnore enwatohetste

Swateweienton tsi tsionnhe

Hetsiswaienterhane

Raonha hetsiswaiatisak.

Ionikonhraksat kowa

Tsi iakonnhe onwentsiake:

$\mathrm{Ni}$ kariwesha,

Ionkwetiio, ionkwetaksen

Iosnore ne watohetstha

Swarako kati ionwesen

Kowa ohnaken iahte kakont.
Iah ki te ionwesen,

Ahonwasennaienseke

Iesos ne Wari roienha

Niio ne Roniha, Rolkon

Oni Roiatatokenti. Amen.

46

Raton Rawenniio

Oksa takwatewentetas

Tosa sewatsterist

Iontonweskwatha.

Ok sa ok kati swatekwat

Swatennikonhrotakwatha

Hetsisewaienhaf Iesos

'Tsi rotehiaron nonwentsiake.

Tsini hoson Niio

Nene ionkwaretsiarontha

Asakwanonwehak

Sonkwaiatison ;

Onkweriasa akwa resaks

Ne nakotsennonniatake

Ok sa ahonhion ne Niio

Ahonwanoronkwake onwe.

\section{7}

Twateken twatkennisa

Tsini ionkwataskwat onwe ;

Rawenniio tsi rononsote

Karenniios tetewariwak.

Karo kihei

Tosa onsonkenikonrhen

Tsini hoianere ne Niio;

Aonhaa kariwanoron,

Ahinonwene ne tsinenwe

Aonhaa kariwanoron

Ahinoronkwake tsinenwe.

Aonton ken akesakseke

Onwentsiake kaienta on ?

Iahten; aseken raonhatsiwa

O Niio ken wente

Kasatsnent akeriasakon ;

Akwa onen wakonwenniioste

Tonsakateronweksi,

Ne wakiatahtonton,

Ne se ok onen skaskaneks

Nakonnoronkwake onwe.
Roianere rokwatse kowa.

Tsini seniseriio kowa, Sewenniio tsi iakionnhe, Ikehre ahinoronkwake Tsi nise waskenoronkwake.

Swakwekon sewariwiioston, Tesewaiena enskatne Skanikonhrat Rawenniioke Akenhake sewatsennonni.

Sewakwekon ase tsi tsionnhe, Tosa sasewanikonhren

Tsini tsisewentenron kowa

Satsiswaiatisa ne Niio.

\section{8}

Iah kati nonwenton

Tiia onsakonhiatonti, Ne ok nonwa tewakerenkenies

Tsini honikonhriio,

Siaton nakeriane

Tsinikon sonkwanonwes

A kwa enkatkawe onwe. 
Akwa iah kaneka N'atke takatsennonniate, Sonhatsiwa senikonhriiostha, Tiotkon iakotsennonni

Iesawenniiostha ;

Iah skariwat te iakonnis

Nonwentsiake iakotentas.
'Tokat kathe sonha Enskatatewennarisi, Ise tasenmonton tsi naskiere, Tosa tontakerhoton, Tosa enseskwatsten Tosa onsakatsennonni T'si ok onsakatatrewate.

Takwehiarak, Sewenniio, ionehrakwat tsini ionkwentent, tosa enseriwarako. Askwetokaten onkwanikonhrakon; ionkwarhare saianerenstakwa. Ionehrakwat, ionehrakwat tsini sonkwentenronhatie; tiolkon takwanikonhrahnirat, tosa takwatewentet tsinenwe.

O Iesos ! akwa kwanonwes,o lesos, iakwanikonhrakwekon. Iah othenon nonwentsiake, iah othenon te ionwesen, ise sonhaa kwasennaiens, ise saiatanehrakwat, ise ne onwentsiakwekon teiesariwakwase, tenkwariwakwaseheke.

Tetewasentotak ionkwariwiioston Iahte honwatkenses Iesos tsi renteron Ronwasentanionnis, ronwaiatanikonhrens, Arahonne nii, tosa tsitwaiatonti.

Ionehrakwat! katke ethoni tiawenhon? Tsini tiontieronnions nonkwe ionteroroks ; Tsini sakononks lesos ononsatokentonke, Serekhene nenne tsi enthonwahtentiase.

Tokenske, o Iesos, tsi sanikonhriio, Tsi iakwanakere satatenaktonni, Tiotkon takwatiennis, iah se te iesanonwes, Iah kati teskwenies nasheiatewentete.

Nahoten satkathos tsi satatiteron? Aoskon tokenske ne iontaweiatha Niahte iesaniente ok eken ionteroroks Ienaie, iontatswens, iakoskennhase oni.

Hetsitwasentennis tensonkwaiatoret, Iawet hetsitwannhas nasonkwahrewate, Iahta tewehiare raonhatsiwa roien Naetwatsennonni nok naetwatkaronni.

Ah! ne ionkiesatha ne khenoronkwakwe, Iah othenon etho teiononwaklekwe Tsi ionkiesatakwe tsinahe ne ionkswenskwe, Ethoni haierha Iesos tsi rolitare. 
Sehrewatak niha onen. Sewenniio, Eniesatsanike enionteweienton,

Ensehrewatakwe naonsaiontatrewate

Iahte kakont onwe aiesanoronkwake.

Tokat ne senonwes ne onkwanekwensa,

Okti tasennonton tsi nasatatieras;

Okti ionkwarhare tosa tesanonhianik

Aionkwaronhiaken naeseriientiio.

Teskanere Iesos ionkwatiskwentare

Tsini ionkwahterons n iskwatewentete;

Asatkatho nisa sanekwensanoron ;

Ne askwentenrale tsina teiakwasenthos.

lahte kakont onen ionkwaterientason

Akwa sonhatsiwa askwanoronkwake,

Aiakwahtentiate ne sariwatokenti

Nonkionkweta okon aiesanoronkwake.

Ronnhek sonkwawenniio lonnhek Iesos raosenna Atsitwanoronkwake $\mathrm{Ne}$ Niio Niio Rioienla Tsinenwe eniakionnlieke.

Sewenniio tsi iakionnhe Akwekon teskwanikonhratson 'Takwanonweratonheke Tsini iaweta takwentenron Sonhatsiwa takwaiatison Tokarat niskwenniserawi ; Seron aionteweienton Karonhiake aiontsennonni.

Arekho si iakwatlokha Takwaiatanonstatonhatie Skennen ti ionkwatatehiaron, Ise iawet takwehiaronnion. Ok sate skwaswatetenni Saianerenseratokénti Seron aiakotokaten Aiakoteweientontieseke.

Aouhaa takwentenron
Akwa lakkorinienston kowa, 'Takwanonten ne tsieronke Aionnhekon nonkwatonnhetston Seron aiakosnienontie Tosa aiakosenni notkon, skennen talaiontohetste 'Tsini kanoron onwentsiake.

Toni iaweta iotonhon Aiakwaiatenhon oneshon! Sekon takionnhetonhatie, 'Takwarhare ne karonhiake. 'Takwentenrhek, Sewenniio, Aionkwatsennonniatskonhake Tosa aiakwatiesate Sekon onkwawenk karonhiake.

Takwanikonhrahniratsek, Sonhaa akwanoronkwake

Aiakwakenronniheke Iontonwesk watha nonwentsiake 'Taionkwaswateke onwe Akwa aiakwakonnienstake Karonhiake aoriwa Aiakwatateronhiakente. 


\section{2}

Akwa tsini ionwesen onwe Sewenniio karonhiake, Asteson teiesakanere

Tiotkon iesawenniiostha

Ne ok katonrietha

Iakoniatorenue.

Tokenske tsini ionwentsiaksen Tsi tekatonnhakarias, Waskaneks onwe nakatonnhets Iaionwe tsinonni iokwats.

Ok oni naiawen n'akehre 'Tosa eh iekennontonnion. Kanon nakteson n'aketsenri Nostonha a katsennonni.
Ethone ok enkiewentane Ienkonrane karonhiake, lenwakenikonhraierine Iahte kakonte tsinenwe.

Katke, o Niio! ienkahewe Ne iatensk wannhohontiense?

Sekon katiken enionnise Enwakitenta onhatie?

Onen wakenikonhraientas Tsi isehre taiontentsa Tsinenwe kati enkwakwisron T'sinikon ne kariwiio.

\section{3}

Twatateken tetewariwak Rawenniı tetsitewariwakwas.

Ionnhek, ionnhek, ionnhek onwe Nonkwatonnhetston ne karonhiake

Onwentsiake oneshon oni Akwekon nonwa tsitewarihonti.

Twakonnienstak ne karonhiake Tionkirhare watenroseriio.

Akwa tiotkon ietewatkatho Tsi tenteron ne Sonkwaiatison.

Ionehrakwat tsini ionwesen $\mathrm{Ne}$ enskatne ientwatkennisa.

Ionkwarhare kati akwekon Eh neniawen n'enionkwariwiioston.

Tosa kati twariwanerak Ken tenhnon aetewenheie.

Iesos, Iesos, kwennitentase, Askion nisa aiakwatsennonni.

Wari, Wari kwennakeraton Takwaienawas etho naiawen.

\section{4}

Swanikonhrarak, sewariwiioston Tsini ionwesen ne karonhiake Tiotkon ronwakens ne Sonkwawenniio Tiotkon oni ronwawenniiostha. 
Akwa ne kaskaneks

Nakatsennonni ne karonhiake.

Karonhiake akwa ne ionehrakwat

Tsini akwa Niio ronwanonwes ;

Sakowis akoiatatokentison

$\mathrm{Ne}$ atorisenseratokenti.

Etho karonhiake ne kanaktiio, Tokenske tiotkon ionwesen kowa; Iah othenon teiakonikonhraksens Nok iah taonton aiontkaronni.

Akwa iakotent iiens nonwentsiake Tsi iah othenon teionnontonnion Tsini akwa ionwesen karonhiake Tsinenwe iahten kariwentane.

Tokat skennen enkeronhiakenseke Tsini theñnontors ne Rawenniio, Naonkiatawenseron nonwentsiake. Enwakerharekon karonhiake.

Kawenniio katkathos karonhiake, Wakehre : iokenrat nonwentsiake, $\mathrm{Ne}$ kati iah othenon te ionttokha Ienonwes iontonweskwatha sonha.

Onen karonhiake teiakotentson, Akwekon onen teiakotentson ; Kawenniio karonhiake iakotion Onen akwekon iakotion.

O Iesos! satanitenraskon kowa Karonhiake teskiatatironten, O Warı! kanistenseriio kowa, Wakenonktonse akonkenseke.

Sewenniio kwanonwes Ne askwentenrheke Aiawen tiotkon onen Akwanonweseke.

Iesos takwanoronkwa 'Takwanonwese Iesos kwawenniiostha Tsi iakionnhe.
Sewenniio, takwentenr Ne tsini ionkwentent; Tosa takwatewentet 'Tsini skwanonwehon.

Iesos seriwahtontha $\mathrm{Ne}$ kariwaneren, Sonhaa shetenranions Ne akwekon nonkwe 
Tiotkon ionkwaronhiaken Kento onwentsiake ; Ise, Niio, kwarhare Aiakwatsennonni.

Iesos kwasennaiens Tekwanonweratons Iesos sonhatsiwa Ne takwawenniio.
Tiotkon, Sewenniio, Takwentenronhatie, Ise sonhaa Niio Tiotkon kwaskaneks.

Ise, Sewenniio, Takwentenronhatie, Sonhatsiwa kwanonwes, sonha kwasennaiens.

Tsini seniseriio

Tontakwentenr Niio

Teionkweriasaweheston

Tsini ionkwaieren

Ne tontakwakanerak

Tsi iakwataraien ;

Takwaienawas, Iesos,

Tsi ionkwerientaksens.

S. Sesnonsake tsieratha

Tsi katsitsıararaks, Sesnonsake tsieratha Tsi kahionhatenion Shetenron notsitenha Akontitieseke, Nok kennaiakionkwe Iah teskwentenrane.

Aonkatetsirate Takesnonsawakon Ne wakiatakennhaton $\mathrm{Ne}$ ok skeniahesen Tsini sataniterron, Tsini konsentenni, Tosa takatewentet Ne skatatrewatha.

Takitenr, Sewenniio Ne katewentetha Tsini konnhoten Iesos 'Twakenikonrharha Nakeriwaierite, Takatenientense, Nok enkonnonwehake Akonnikonhriioste.
Sewenniio tsi konnhe

Sakatatrewate

Swakatatrewatakon

Tsi sanikonhriio

Niiaweta iotonhon

Takwahrewatakwen

Katiken ne shetenron

Nonwentsiakonronon.

$\mathrm{Ne}$ wakeriioskowa

Tsi wakatiesaton

'Tsinikon takitenron

Takonnhetonhatie

Ok eken konwennontion

Iahte skatsteristha

Kariwiio okonha

T'si skwatenientense.

Tewakatonharenrons

Tsi akwa ketsanis, Ti ken iosatste kowa Seronhiakentakwa, Niawen skennen ti konnhe Arekho skiatontion Niawen tsi sarharekwe Onsakatatrewat.

Takit@nr, Sewenniio, Tsini takenonwes

Tosa seriwarako

$\mathrm{Ne}$ tsini wakieren

Wakaterientasonhon

Tsiniiore konnhe

Enkonwennaierite

Takatenientense. 
Rawenniio sonkwarihotasienn:

Tsinikon ne kariwanoronson

Naieienterihake nonkwe okon

Karonhiake aieienterhane.

Ok si hanakere ne Rawenniio

'Tiotkon oni enhanakereke

Roronhison, raonwentsison oni

Roson oni tsini kanakere.

Sakoson Ronatkon ne karonhiake Nok sakoson nonkwe nonwentsiake Tetsiaron ne nahonwaienterhane Nok tsinenwe ahonwasennaien.

Raonikonhroskon ne Rawenniio Iah se othenon te haiatonte;

Tionkwe okonha tesonkwakahrenhawis Oktiwakwekon raiatanoron.

Saiatat ok Rawenniio tsi tionnhe, Iah tohka te hati Rawenniio

Nok tenhnon asen natehaiasere, Skanikonhra tsi asen nihati.

Niio Roniha ne sonkwaiatison, Niio Roienha sonkwaskontakwen, Nok Niio Rotkon Roiatatokenti Nene sonkwaiatatokentistha.

Ne ok thotati tsi sonkwaiatison, Naonsasonkwaskontako tenhnon Onkwe rotiatotenston nonwentsiake Roronhiakenhon nok rawenheion.

Thonatierenton nonkwe nonwentsiake Ronwawennontion ne Rawenniio, Ne sonkwaswatonne ne tionkwe okon Nakwekon aionkwatetsiraton.

Sesonkwentenron ne Niio Roienha, Onkwe rotatonni nonwentsiake ; Sesonkwanaktawi ne karonhiake Naonsatsitwa wennarak wake.

Tsiatak ni hoson noriwatokenti Iakoiatatokentistha nonkwe Eh iehoien ne iontsennonniatakwa Tsi ronwaiesaton, tsi ronwario. 
Rotiohkwison nene teieiasontha Ne ok enskat kentiohkwatokenti : lahte ieiatare iahte iakoien Naiontsennonni ne karonhiake.

Ienoronkwa tsi sakotenron Iesos Eniontsennonni iahte kakonte Iakonoronse ne kariwiioston Enionhriskon nonwentsiakonronon.

Katke onte tenkonkanerake, Sewenniio? ise konnonwes Katke iseke enkatorisen atorisenseratokenti ?

To onte kariwes enkeseke? onwentsiake kentonnis onwe. Sekon inonha ne karonhiake kiteron, wakatstaren onwe.

Kariwiio tewakswatetennis, wakenikonhrahnirats oni ; Oh sane sonhaa, Sewenniio, akonken, aetenikwekon.

Sewenniio, watkonnonweraton, onen kowanen waskitenre 'I'si wakatkawen ne kariwaksen, skatsteristha ne kariwiio.

Takwawis kanikonhraksensera, ne teskariwaserakwatha, Takwawis entkeriwaierite karonhiake enkatsennonni.

Akwa lesos takenoronkwa A kwa takenonwes Iesos, Tsi takeriwisahani

Aionnhekon akatonnhetston.

Onen kati konnoronkwa Wakonwenniioste kati Akatonnhetston ne oni kieronke Wakonwenniioste tsinenwe en[katonrieseke. Tokenske wahi takitenron Tsi iesaiatanentakton,
Ionehrakwat kati nii.

Iahte koniatsteriston, Iesos.

Akenikonhrakwekon kati Nonwa enkonnoronkwake; Wakerhare enskitenre Ne tsini takerharatstenni.

Oriwiio kasatslensera Sheiawi akatonnhetston Aonkenikonhrahniron A kesenni notkonseraksen.

60

Twakwekon ionkwariwiioston Sekon tewaksa okonha Tetewariwakwak

Tsini honikonhriio Iesos. Nok sonkwanatonnis T'sini hoianere.

Akwa ionkwatsennonnihak Ionkwatsennonnihak Akwa tehotatekwenni Rotketaton tsini Tsinenwe tsi sonkwanoronkwa. Ionkwariwaneren. 
Raowaronk sonkwanontens Sesonkwanaktawis

Tsi tiiakotsennonni.

Sesonkwarharatstanionnis Tsini hotsennonni

Ne sonkwarakwennis.
Ensakotonraseronse Tsinon tiakokwatse Ne ronwanoronkwa.

Shetsitewanonwehak kati, Taetewalskenni

Nahotsennonnihak.

\section{1}

Swatatiesatanions, swariwanerahakskon Sewennontonnion ken wenniseranorons Nonwentsiake tsionnhekwen nioserakeha Sonkwawi ne Niio sonkwatenientense.

Iah se tetsiakwawenniseraien noneshon Aseken iakotenniseratiesaton Sakawenniserawihne ne Rawenniio Tiakokaras onwe iakotetsiraton.

Onen wahonnise onwentsiake tsisewes Iahta tekontitas ne sewenniserat, Ionahtentionhatie akwekon tsi sewentas, Akwa ialı nonwenton taontakonkele.

Sewentorha kowa tsi sewariwiioston, lah othenon te ken sewariwaneraks, Iahte sewatsanis naesewatkaronni Oh kati naiawen naeswatsennonni?

Ne awennisera atanitenrasera, Nonwentsiake tionnhe, tosa tewatiesat, Iosnore enwawe ne kahrewatatsera Iahtiiaiehewe ensewententane.

Tewateweienton onwentsiake tsi tionnhhe Iahta sonkwenniserawi Rawenniio, Iah nohnaken taionkwateweientonke, Iahte kakent onwe noneshon wetewe.

\section{2}

[skwatha

Tsionkwe tosa swatatenikonhraten, swatewentet ne iontonweRaonha, akwa raonwatsiwa Rawenniio hetsisewanoronkwak Iahte ioskats ne atonwesentsera, sotsi seken iakonikonhrarios. Iah nonwenton skennen teionnontonnions kariwaksen iakoiotoron Ioskats tenlınon ne iakoteweienton, tiotkon nenne skennen ion-

Isi nison iakotsennonnihatie, iah othenon te iakoiatati. [nontonnion

Rawenniio teiontatemoronkwa ne ronwawennaieritonhaties. 
Kariwiio iakotienitonhatie katke sonha eniontsennonniate.

Iakotaskal onen waontonnhokten, iah othenon tetsieriwatsanis, Skennen kowa onen ne iakorhare ahonwaken roiatanehrakwat. Swatsennonni ne arekho othenon te iotaksen teswaiatoron, Isewehre are: akatonnhokten, tosa nisa iahta katsennonni.

63

Arekho te wakenikonhra Iah othenon te karonkha, Konnekennis, Iesos rakeni, Askenikonhrowanate.

\section{4}

Onen wahniakenne Sose nok oni ne Wari Wetreherke kanataien Wahotinatonti ; Sirisare wahonnelane Tsi thononsote Niio Wahonwaiataienthosere Iesos ne hiatatienha.

Etho Simeon rokstenha lesos wahoiatorenne, Wathoiatakwe, wahenron: Iahte skanoron tsi konnhe Iahte sketstanis ne kenheion, Onen Iesos wahiken: Aseken ne sakaonnheton Onwentsiakwekon nonkwe.
Sewenniio, akwa kaskaneks N'akonnikonhraierite : Wari saiatatokenti, Askon nakateweienton.

Anen oni akokstenha Jesos wahorihowanat. Wakenron : Onen ken ire Rawenniio tsi tionnhe. Wathiatonte onen Simeon Iesos ronwakonnienstha Wahniron: Tewatsennonnianion Rawenniio watsitwaken.

Ii rotiksten twakwekonhak, Iesos atsitwakonnienstake; Rahawe se ken onwentsia, Akwekon ne karonhia ; Raonha atsitwasennaien Ne ken twanakerenion, Raonha hetsitwasennaien $\mathrm{Ne}$ ken twanakerenion.

\section{5}

Swaronhiakehronon tetsisewariwakwas $\mathrm{Ne}$ lesos ronwario tsini sonkwanonwes Askwaienawase tsi ionkwariwiioston Aiawen asonkwentenre.

Akwa sewataskat ne sewatkonseriio Hetsiswanoronkwa ne sonkwawenniio Askion kati nise tsini tsisewanonwes Aiawen aetwakwekon.

Akwa iah othenon nii teionkwarihonte Eh iaiakwatati tsi renteron Niio : Ise kwaniahesen ne sewatkonseriio Takwentenr takwaienawas. 
Tewatiskwentaren, hetsitwawenniiostak Tiretha tsi nonwa rotasetonhatie Entsitwaienterhane nenionkwariwiioston, Ronatkon entewakwekon.

66

Sewenniio ken wenniseratenions Seron : taonsaieriwaserako $\mathrm{Ne}$ iakoriwanerahakskon nonkwe Tsi nonwa eniakawentontiete.

Takwanatonni tsi naiakwaiere Taonsaiakwariwaserako : Sasentontiete kowa nonwentsiake, Ethoni kariwes iahte sakon.

Saterientare wahi, Sewenniio Tsini ionkwariwanerahakskon, 'Takwentenr kati takwanikonrhata Askwanikonhraseronniheke.

Tikenha tsi naetewakseke, Senha oni ionkwaiotatiesek, Twaronhiakentak ne tewaieronke Ne se ok akwa ionkwakaronnis.

Tewaronhiakentak nonkwanikonhra, Tosa aetwennontonnionseke

$\mathrm{Ne}$ tsini iontonweskwatanions nonkwe Tsionkwarihonti ne kariwaksen.

Nonwa teionkwaiatoretonhatie Tsini ionkwariwanerahakskon, Aiokenton tsini tkariwaieri Noksa aonsetwatatrewate.

Hetsitewennitentas Rawenniio, 'Tetwatstarhak nonkwanikonhrakon, Aiawen tosa ahariwarako Tsini tsitewanikonhraksaton.

Tewatsiaken ietitenrhek nieiesas Nok tewerhek ; lesos wahitenre ; Ensesonkwaieritse Rawenniio, Iah nonwenton tasonkwatewentet.

Akwa sane iahte ionkwarihonte N'asonkwentenre ne Rawenniio, Ionkwarharek tsini honikonhriio, Tensonkwarhoton nentewenheie. 
Senha kati nonwa ionkwatsteniaron

Naetwaterennaienneseke,

Rariwanouwes ne Rawenniio

Jakokwisron ronwennitentase.

67

Sewenniio ken wenniseratenions

Seron taonsaieriwaserako

Tsi iakoriwanerahakskon nonkwe

Sewenniio, ken wenniseratenions.

Akwa sane iahte ionkwarihonte Asonkwentenre Rawenniio

Ionkwarhare, tsini honikonhriio, Ionkwarhare, naetewatsennonni.

68

Iakotaskat si eksaa ronwanonwes ne Niio Iahte iakothaharakwen tsi iakoriwiioston.

Sakononwes ne Rawenniio, tiotkon skennen ionn ontonnions Nahoten onsaionskaneke, Rawenniio se iontenro.

Oriwakon iakonkwetaksen tsiniiot iakotsennonni : Tiotkon iakonikonhrariotha iontonwesens onwentsiake.

Kaneka teiakorenkennis kento atonwesentsera Arekho okaseri nonwa entsiakonnhekon tsinenwe.

Okti iakononwaroriatha iakonikonhrotakwatha ; Iah se taiakotsennonniaten, ne ok tsiononwakte kowa.

Iesanoronkwa, Sewenniio iahte iakonikonhraksens lah se onka taiontatekwa tsini shenikonhriiostha.

Akwa iahte ionkikwatstennis ne ionkinikonhrotakwas Nok iahtaionkiataskatsten Niio hetsitewanoronkwa.

Tsi roiatanentakton lesos aetewatkatho ne tionkwe;

Ne se kakwenies aetewatkawe iontonweskwatha okonha.

Iakorhare aiontsennonni skennen tiiakoronhiaken, Nok oni ne iontonnhoktane ne iakonikonhriiostha.

Takwentenr, takion, Sewenniio, tosa iakwariwanerak, Sonhaa akwanoronkwake, nonwa nok oni tsinenwe.

Wari kanistenseranoron, tontakwannhe onwentsiake, lakwatekwasentakwa onwe tsi tsiteron karonhiake. 
O! swanatiio karonhiake sewanakere, ionwesen, ionehrakwat. Sewennakwekonhatie, sewanikonhrakwekonhatie tetsiswariwakwase asteson ne Niio. Saiatatokenti Niio, Sewenniio Niio Sabaoth. Wakontiskwentaren, ronwawenniiostha sakotsennonniatennis.

Sewenniio tsi konnhe Ionehrakwat wakitent Tsinahe tewakattoken Tiotkon konwennontonhatie lahte katsteristakwe Tsini skwatenientense.

Tsini wakeriwaien Ne wakiatorarakon Wakenikonhrariotakwe Tsi sotsi wakeriwaien Tiotkon akwa ketsanis, Tewakatonharenron.

Oktiwakiatisonkwa

Tsi akwa nikontsanis Satanitenraskon kowa Tosa takeriwarakwas Sanikonhriio kowa Tsiakente askrewate.

Niawen tsi sekon konnhe Arekho teskiatontion Kananon sane kiatakon
Kariwanerahaksera Ne wakiatakennhaton Natanitenrasera.

Wakatatiataksaton $\mathrm{Ne}$ tsini konsentenni li wakatatkaronnihon Watkaronniatserowanen ; Kariwaksenskwe okon Sotsi wakatsteriston.

Takitenr, Sewenniio, Tsitenr akatonnhetston Takitenr, takiatohare Iesos raonekwensake Takenikonhrotenstak Tsini sanikonhroten.

Satanitenraskon kowa Tosa seriwarako; Askenikonhranentakte Satanitenraserake ; Tsinenwe enkonnheke Enkonwennaierite.

I esos oriwiio skatatswense tsini kiatoten Tontakitenr kati, tosa tontakiatonti

Tewakenonhianiks, ehneken iakatkatho Iesos tontakitenr, satanitenraskon.

Eso takinonkon wahonnise takiatisakskwe lahte katontatskwe koniatekwenniskwe Sanikonhranonwaks, ne tsini kiatotenne.

Iahte kattokhakwe tsi kerhakwe enkatsennonni Eso kiatahtonskwe tsi konwennontieskwe, Skeriwaienteres, tsi wakatkaronni. 
Oia ok ethone aonkweron kateweienton, Ok eken isini, kataharakwaskwe, Ionehrakwat kati tsi sekon nonwa konnhe.

Iahta ketsaniskwe, sehrewatha iakonkwetaksens 'Tewakennhohontion, iawet ne noneshon;

Oh! wakitent kowa ne iahten skitenrane.

Tokenske iakotent sheiatontion noneshon wahi, Akwa iah nonwenton taieiewentane, Etho satekenat, kaneka shenakwase.

Wakenikonhrarios, ken wentatie nok asonthenne Tekaris nakeri tsini koniesaton, Nok wakiatisonkwa tsi skatatrewatanions.

Iahte kakont onwe otsirake kennakerathe, Takiatontisere ne koniatontieskwe.

O satiesen kowa! tosa takatewentet.

Wakatiskwentare sasitakta seskwaskontakwas, Ise takiatison, takatkarenrako

$\mathrm{Ne}$ aonkitenre sanekwensatokentî.

Skennhatense onwe, tosa kenha eh naonkieren? Ne nonkwe asteson tenkasentotake, Enwakiatateri naonsesanikonrhen.

Sewenniio onen iakahewe

Naskwentenre tsini tsiakwatatswens

Tosa enskwatewentete

Ne teskwanikonhratson kaiasake.

Satkatho tsini iateionenon

Onkwatonnhetst asitenre kati

Ionkwaterientason onwe

Aiakwateweienton, Sewennìio.

Tewakwekon ionkwatiskwentare, Ionkionsennha ne sataskwentenre

Takwentenr sanikonhriio

Naskwaiatokewate sanekwensa.

Watstarha akenikonhra lesos 2.

Watstarha akenikonhra tsini iesasentennitha (Iesos 6) 
Ionikonhraksat rakeni Tsini konsentennihatie Katehens tsi konwennaras Koniennitha, Sewenniio Takitenr takewennarak Wakenikonhranonwaktha Konniahesen akwa ise Etho keriwanentaktha Tiotkon enkonnoronkwake
Iesos tsi wakeriwaneren...
" rakeni tekasentotha...
“ satontat, skatatrewatha...
" askitenre takatontats...
“ tosa takeriwarakwas...
" tsini konwennontonhatie...
“ aseken takwenheiase...
“ tsi iesaiatanentakton...
“ tsinenwe ienkatonnhokten...

\section{4}

Rakiatisaks ne Rawenniio, rakenni; askeriason.

Sewenniio takesennion

$\{$ 'Tiotkon enkonnoronkwake $\{$ Iahte kakont enkonnonwehake

Akwekon waonkwaiatonti iontonweskwatha okonha Iah Niio teskwaiatonties $\{$ Ne kati ise skewenniiok $\{$ Ne kati ise ok koniaskaneks

Ionkwentent, ne iahte siatare, iah othenon te ionwesen

Kawenniio ok tsiatare $\{$ Tiotken ionkwarihonweskwani

Akwa ionlateronhiakentha, iienhre: aionkionnonwene Ne tenhnon ne Rawenniio $\left\{\begin{array}{l}\text { Kawenniio ok ronwaskaneks } \\ \text { Ok naheha wasakonnonwene }\end{array}\right.$ Tsiniiot iontatenoronkwa, iahten te tkariwaieri.

Are okti ionnowentha $\{$ Ne tsini iewennoten nonkwe $\{$ Ionton : kenonwes, iontatswens se ens

Tsini iontieranions nonkwe eso iakoiatahtontha;

Are iontontarhotonnis $\quad \begin{aligned} & \text { lienhre-ken: ionkinorinkwa, } \\ & \text { Akwa sane ens iontatkenronnis. }\end{aligned}$

Tsi ise iesaseronniennis, Niio iah etho te iawens.

Iah othenon te ietsanis $\left\{\begin{array}{l}\text { Iah se teskwanikonhratennis } \\ \text { Akwa skennen tsi iakonnhetie }\end{array}\right.$ Ise akwa sonhaa aonton askwanikonhraierite.

Watiesen askwanonwene $\left\{\begin{array}{l}\text { Tiiakenikonhrate nonkwe } \\ \text { Nok karonhiake aiontsennonnni. }\end{array}\right.$

Ne tsi iontieranions nonkwe iahte ienikonhrare.

Wakitent wakenonwehon $\left\{\begin{array}{l}\text { Tsini watonweskwatseroten } \\ \text { Ise, Niio, iahte konnonwehon }\end{array}\right.$ Sotsi wahonnise tsinahe koniateraiatakwenni.

Iahte wakerihontakwe $\quad\left\{\begin{array}{l}\text { Onen ki sakatatrewate, } \\ \text { Tiotkon enkonwennaieritseke. }\end{array}\right.$ 'Takitenr kati, Sewenniio, tiotkon takienawasehek.

Etho nakonnonwehake $\quad\left\{\begin{array}{l}\text { Ne tsi nise ni skwanonwehon } \\ \text { Tsini iesanonwes karonhiake. }\end{array}\right.$ 
Sewenniio tsinahe tsi konnhe Ne rakseronniennihatie Takiatanonstatonhatie

Aiawen akehiaraseke

Tsini iaweta skitenron.

Oriwakon akwekon nonkwe Aionkinikonhrotakohe ; Iah taonton ne akerako, Sonhaa konnonwehak.

Kaniseriio wakenisen Karonhiake thanakere

A katsennonni tsinenwe.

Ostonha wakathaharako

Nok sewakaharatonhatie:

Akeriane iawet rohtare

Riwennaienteritsihon

Tsinenwe etho nenwakieren Enhakateweientonke, Nok khti tsi enkatonnhokten Iahta taiakiatekhasi.

76

Atonwesentsera

Wakiatatonhon

Katstarha nonwa

Tsini wakieren

Etho satekenat

Iakotonwesen

Iakonikonhraksens

Ne tsiennhatens.

loriwanehrakwat

Tiakorenkennies

$\mathrm{Ne}$ iontonweskwatha,

Sase iokenrat

Kennikariwesha

Watohetstakwa :

Ionkwentenstetenni

Ionkwakaronnis.

Oh nahoten iontsta

Tsi iontonwesens?

Senha iosnoratie

Tsi iakonnheties ;

Onwar ionwesenne

Nok kennaheha,

Iontstarhakwa nonwa

Iahte tsionwesen.

Ne ki teskiatieren

Kahionhatenion

Iahta tekontitas

Kahnawaronnion

Etho akwa niiot

Nonkwennisera

Kontohetstakwanes

Iahta te tkonnes.

Iahte sattokhakwe

Satkatho nonwa

Tsinonni ieiatat

$\mathrm{Ne}$ shenonweskwe;

Tesationtanitha

Tsi teskanere :

$\mathrm{Ne}$ se sanonwehon

Otkenseroskon.

Iakotsanitenion

Ienakerekwe,

Iahte ietsaniskwe,

Kariwaneren,

Akwekon okenra

Onen iotonhon,

Sate wakenrotens

lakotentenion.

Iakoteweienton

Iakotsennonni

lakotonnhahere

$\mathrm{Ne}$ karonhiake;

lakotiesatanion

Kariwiioston

Iakotkaronnihon

Iahte kakonte.

Niio Sewenniio

Takitenr nisa

Tosa nonwentsiake

Akatonwesen

Akatatienhase

Ne karonhiake

Akatonwesonnion

Iahte kakonte. 
Sewenniio Takwentenr Tosa saiatakwa notkon Ne sheiatisonhon nonkwe Akwekon ne senonkwe.
$\mathrm{Ne}$ se oni askwentenrate $\mathrm{Ne}$ tsi tetsiakwaiatoretha Iakwaton iahte iakwaltokha 'T'sini ionkwariwanera hakskon.

Sewenniio tosa takwahrewat 'Tsi ionkwariwanerahakskon ; Sasanikonhrenhonhak niha Tsini ionkwariwaneren.

Ne se oni askwentenrate Iakwaronhiakens, iakwatorats, Iakwehre ne iataonsakene 'T'sini kwasentennis Niio.

Ne se oni askwentenrate $\mathrm{Ne}$ naiakwatiesate Ne se oni askwentenrate Ne naiakwentontietake [ken. Ise tesariwaserakwen Untaiakwentakon aiakwaronhia- Ieriwaneraktha nonkwe.

Sonhaa, Sewenniio, Sonhaa konniahesen, Ise tsienteritsihon Akwa tsini ionkwentent; Sasanikonhren kati T'sinikon konnikonhraksaton
Akwa ionehrakwat Tsi twanikonhraksaton Ionkwaniseriio lesos sonkwaskontakwen ; Sasanikonhren kati Tsini ionkwaieren [ton.

Sasehiarak tenhnon.takwaiati- Seriwanonwes tsionkwatatrewa[son.

Otkonseraksenskwa Ionkwaiatahtonton, Ionkwanikonhratenni, Ionkwakaronni onen; Satsteniaron kati, Iesos, Takwaiatanonstats Tosa ionkisenni nonatkonsera- Shonatatrewalon rotiatatokenti.
Wari senistenseriio, Sewaiatatokenti okon, 'Takwaterennaienhas Asonkwentenre Iesos Iaetewakwekonhake Tsi ronatsennonni

Tosa tsiron: Enhakitenre Niio. Ok sa nonwa tensatatiatoret. Wahonnise tsi tiennonks Rawenniio, tosa sateraiatak ensatsennonni, karonhiake iahte kariwakonte iakotaskat ronwawennarakwa.

Onen swakatatrewaton, Iesos rakeni, satanitenraskon. Takenikonhranentakte tsiatatake, tosa takatewentete, takwatontats, takitenr, wakeriwanerahakskon, Ne konnonwes, ne kontsanis. 
Swaskontako swatonnhetston Taiontonnhakariaseke! Watiesen naeswatsennonni ; Tosa noia swennontonnion

Tsi swatonnhisaks nonwentsiaOriwakon nonwentsiake Tewaser ne karonhiake

Iakohtonni karonhiake Akwekon iakotkaronniaton ; Ne naiakotsennonnihon Ok eken eniontetsirate.

Oh nahoten iotsennonniat Naiontonwesen nonwentsiake Nok ohnaken nonwentsiakon

Tsinikon ne ioskatstanion Iah cthenon te iorihonte; Ionnhe onwe nonkwatonnhetst Ne tewesak ne ioskats onwe

$\mathrm{Ne}$ ok tekeni te skaien Nonen enwatokten tsi tionnhe Ne karonhiake aiente Nok oneshon aieiatenne,

Taionkohte nakeriane Nakatsennonni karonhiake! Akkenron nonwentsiake Iesos ahii tisakseke!

\section{2}

Sotsi notiake wahonnise Tsi sewateraiatakwa

Iesos onen tetsisewennonks, Tosa tesewatahontakwek.

\section{Onen niha nonwa} Sas ewatatrewat

\section{Ne ken satekenat} Naesewatonwesenseke, Nonwa hetsisewaiatontion Ne sákotsennonniatennis

\section{Hetsisewentenron tsi} Ne ok katiken entsionnhekon Natsiswaníkonhraksate?

\section{Tiotkon ensewasereseke} Iakonikonhrotakwatha ; Katoken ensewaiatenne

\section{Ii nonwentsiake}

$\mathrm{Ne}$ ok tsioriwat Ionkwariwaien $\mathrm{Ne}$ kento tsi itewes Aetwesake Ne karonhiake.
Otsirake iahte waswas.

Sewentent tsini swaiatawens Ne sewatatiataksaton; Tsi teietsikanere Isionnhe Nok tokenske sewenheion.

Raonakwensera ne Niio Swatieniton nonwentsiake Akwa kati tesewalentson Tatsiswaiaterarake.

Aonha iotkaronniat kowa Aseken iahtaietsanike Nor.wa naiontetsirate

Rawenniio tatsisewennonks Tosa saswateraiatak Onen satsisewaiatisak, Satsisewatatrewaten.

83

\section{Raowenk Niio}

Onkwatonnhelston

Sotsi iokenrat

$\mathrm{Ne}$ tsini ionwentsioten, 
Onkwe iakotion

$\mathrm{Ne}$ iotsennonniat

Ronwaiatontion

Sakotsennonniatennis

Tewesak kati...

Ok saskennhase,

Tesek nonwentsia,

Sataterakwas

Owista, natakwennia ;

Ne nii iakwesaks...

$\mathrm{Ne}$ satonwesens

Iahte sattokha

Ne senoronkwa

Iontonwesk watha sonha -

Ne nii iakwesaks...

Ise senaie

Satatkonnienstha,

Ne senoronkwa

Okenra, oienkwara,

Ne niha sesak...
Hetsitwanonwenn

Ne Rawenniio

Kanikonhrata

Ne ioskats onwentsiake

T'waskanek kati...

Twatatkenronni

$\mathrm{Ne}$ ioianere

Eniakokwatse

Kento iontatkenronnis

Ne tewaskanek...

Twatennakerat

Sonkwawenniio ;

Rokwatse nonwa

Tsin: hotatkenronni

Twaskanek onwe...

Onkwe ienonwes

Aiontsennonni,

Kasewe kati

Sewati kariwaksen

Enswatsennonni...

84

Tiotkon ne tionkwe tewesaks Ise oni hetsiswarhare

Aetwatsennonni nahoten

Nok kati twaiatahtons

Ne swariwanerahakskonne

Nonwa swanikonhrariotha

Onwentsiake ionkwesakonhatie Tsini sewaiatoten ne kento;

Ionkwanikonratanionni

Tosa swanikonhriak,

Tsi iwatkathos othenon sonha; Tosa oni teswanonhianik;

lah se te kaien ionwesen.

Niahte haiatare ne Niio.

Oia wahi swarihonniennis

Kariwiioston sewahawe,

Akwekon kaswen ne kento

Aesewanikonhranentakte ;

Tosa kati sewaskanek

Okaseri enskarakewe

Ioiatoron ne sewatonnhetst

Onwentsiake iontsennonniatha Ensewariwiiostonhake,

Kenkaien sesakonnienstak

Ise tenhnon sewanikonhriak

ok onen tsini swaiatoten

Iah se tekonwatentoriatha

Niio raotanitenrasera ;

Oriwakon ehneken ok

Niate watseunonniatserakonte

Tsi renteron Rawenniio

Hetsisewanaktaseronniennis,

Kanaktiio ionehrakwat !

Raonha ensewakwekonhake

Kawenniio ensewakweni

Ensewati iontonweskwatha

Kennonweha enwatohetste

Nenswatateronhiakentake

Iahtakaieronnihake

A onsesewatatswenseke

Aetsitewanoronkwake

$\mathrm{Ne}$ asteson sonkwanoronkwa

Tetsitwariwaserako

Tsini sonkwaiatakennhatie ;

A etwatatkenronniheke,

Akwa aetwatatiesate ;

Enwaton ken atsitewaken

Nok onen tionkwetolenhake. 
Raw̉enniio sewatenro, saetesewatsennonni onwe Raiatakta sewenteron, akwa nonwa kwatsennonniase Iahte kakonte noneshon, eniakotentson nionkwetaksen Iahte kakont oni nise, enswatonnharen karonhiake

Kenni kariwesha kento swatateronhiakentakwe ; Nok ki nonwa iah othenon tetsisewanikonhraksatha $\mathrm{Ah}$ ! tokenske sewataskat, sewaiatatokenti nonwa, Ii tenhnon seken ionkwentent, kento iawet ionkwatiaktani.

Katke onte ienkahewe nene wenniseriio kowa, Etho nenionkwataskatston, tsi nise niiot sewataskat ; Ise kati kwaniahesen ne Rawenniio sewakwekon Askwaterennaienhase katke sonha tasetewatatken.

Tewatsiaken, twatateken, ne ok enskat nonwa tsi tionnhe, Tewatatiatakennhasek, kariwiioston tetewasniek Nok tosa kati nonwenton, tetwatatenikonhrotako, Akwa tenhnon tewakwisron aiakwariwaieritseke.

\section{6}

Iesos Rakeni, sanikonhriio kowa, Iahtaiairon tsini saiatanonwet

Iesos Rakeni, seniseriio, konwenniiostha nakenikonhra.

Saterientare, Sewenniio, wakitent A kwa tokenske iahte wakerihonte

Kariwaksenke iekiatakarenrekwen, Askiatakennha, tiotkon askienawase

Iesanonwestha akwa askiatanane Nok isinison akonnonwehonhatie

Takianerenstak, takiatatokentistak Tsini senonwes ethoni skiatotenstak

Akwa okstentsi kentonnis nonwentsiake, Ne se ok akwa kaskaneks karonhiake

Ise sonhaa okstentsi konnoronkwa, Ise sonhaa asteson koniatisaks.

Iaonwentsiathen akwa kahnekaskaneks Etho tsiniiot akwa koniataskaneks.

Aiawens kenha takeweiahontsontak, Nok karonhiake oksa ok iaketiete!

Kihei, rakeni, kihei, ok sa ok kihei Iesanonwestha oksa akiheiate. 
Ionkwataskat ken tewanakere

lonwentsianoron nenonkionwentsia

Kaien nonontatokenti Karfer,

Ok sateiot tsinonni honwario.

Konsennaiens Iesos nok konnentons

Konwenniiostha onwe tsi konnhe ;

Skatatswens tsi wakeriwaneren,

'Takitenr satanitenraskon.

Entakta waesario niesaswens Sason nokaristiatokenti ;

Niawen etho satatiteron,

Ksennaiens tsieronke, sanekwensa.

Shetenranionskwe Sitaeronon Ok ne rononha iesaswenhon

Ronnhere : asakwaiena, renhei,

Tekaientannhareke renheiat

O sitas! ohni sanikonhroten ?

Ise wahi hiaiatarakwen,

Ise hiarihonte nok hiannhaonhatie

Onwatsiok hianonten raierorıke!

Wakatiskwentare sasitakta

Aterhenrakon tsi saterennaiens

Ii akeriwa, Sewenniio,

'Tsini saiatawens takitenr.

Katkathos sanekwensa, ohneka

Tekiatieston tesathonkwaraton ;

Tier, Sak, San, tosa onen sasewentaf, Tetsiswakanerak renheionsere.

Onen thare Sitas ronkwetaksen Ratikwekonhatie sotar okonha, Iesos ronwaiatisakonhatie, Ne Sitas tehonoronkwanionne.

Iesaiena; Iesos, iesaneren Iesanaskwenhawison sotar, Niatekon iesakenrenseronnis, Iesakonrekhon ii akeriwa.

'Takwatkatho tsi kwaronhiakense O sewariwanerahakskon!

Sewatka niha tsini swaieren

Teiorihon ne kiatakwekon 
Tokenske aonha aoriwa Nonkwariwanerahaksera

'Tsi etho nihoiatawens Iesos

'Tsitewatatrewat twakwekon.

Tekoniatontsotanis, o Iesos !

'T'si iesasokwawesonnions

Sasitake tekenoronkwanions

Sanekwensa takiatoharet.

Kasewe swariwiioston okon, Thetsiswakanerak ne Iesos, Ohikta tehonwawehestanion Onekwensoskon rakonsakwekon.

Senha okeken konnonwestakwa Senha ok konsennaientakwa, Tsi teiesanonwarannhaston ; Ise sonhaa, takewenniio.

Skonsake iesennitskerosera Ise se tsiatanoron kowa! Ise sonhaa saiatanonwet! Ise Sewenniio tsi konnhe!

lesos wahaiena raoiasa 'Twatkathi, tsini ioiasakste ; Ronoronse, wahaiatienenne Ohahake tsi roiasakehte.

Ohni skwentenratha, Sewenniio, T'si saiasakehte Karferke! !i ionkwariwanerahakskon Onkwariwa tsi saronkiakens.

Tosa nise asakiasaketate, O Saiatatokenti kowa!

Ii aiakwakiasaketate;

li seken teionkwatentson.

Onen thare onontoharake, Etho enrenheie ne Iesos; li ensonkwenheiase ne tionkwe, Iah ken tatsitwanonwehake!

Onen enhonwaiatanentakte lesos tekaientannhareke Raosnonsa, raosita oni Sakowenniiosta ne sotar. 
Tewatkatho karonwarowanens

Ne tehonwasi:awehestakwa

Tekonwasnonsawehestakwa

Ii ethoni ionkwaieren.

Iesos onen waerontaketsko

Tsi iesaiatanentaktakon ;

Onen tsi heionsere iesarios

Sheiaterennaiennihaties.

Eh ikate kaiasakta ne Wari

Etho oni irate ne San

Sonkwanistenserawis ne Iesos

Akwa ronistenha ne Wari.

Raronhiakentha tsi roniatathens, Sahenron: Wakeniatathens!

Otsate ok tenhnon otsitsiatken

Tekaieston wahonwannhonto.

Skatatswens onen, o Sewenniio!

Onkerihonti kariwaksen;

Hetsitenron nene ranenskwaskwe

Takitenr oni nii, Iesos.

Takwentenr, Iesos, naiakwatstake

Onekwensatokenti kowa,

Ne teiotiakenhon sasnonsake,

Sasitake nok seriasake.

Iakwaskaneks, Sewenniio, Aionkwanikonhranentakte Saiasake tosa ne nonwenton Taonsaiakwariwanerake!

Sahonwaiatanentasi Iesos, Tekakanere ronistenha, Otstenrokon wahonwaiatata Ne sesakoskontakwen nonkwe.

Wakatiskwentare tsi tsiteron ; Onwentsiokon saheto tsiatat, Tiotkon sane Niio nisiatoten Tewakehtakon, konsennaiens.

Satatiatata nonkweriane, O satkaristiatokenti!

Takwatiatata oni nionkwentent

$\mathrm{Ne}$ seriasatokentike. 
Oriwakon tasatati tiatenro

Iah nonwenton takoniatontatse

Iah nonwenton tenskenikonhratako

Iah nonwenton Iesos rakatiennis

Naskwasenni naskwariwanerakten

Oriwakon tensewatkennisa

Iah nonwenton takwawennarakwake

Ise satkon takenikonhrotakwas.

Sasahtenti satennikonhriakt

Iah nonwenton tenkoniatsteristake.

Iah othenon te ketsanis kenheion

Kententsera, karonhiakensera

Naonhaa ketsanis niotaksen

$\mathrm{Ne}$ ketsanis, lesos rakatiennis

Ne ketsanis nakonikonhraksate Sewenniio takienawas niha

Ne ketsanis kariwaksenskwe okon

$\mathrm{Ne}$ ketsanis, Iesos rakatiennis.

89

Saonkinekwahestanion Setewennakerate,

Oksa ionkwanisentaon

$\mathrm{Ne}$ Niio karonhiake.

Akoren tenhnon

Tiakotati nethone

Twariwahnirat nonwa

$\mathrm{Ne}$ ionkiwennakarataton

Kariwiioston

Akwa kwawenniioste,

Akwekon iakwaienas

Tsinikon takwarihonniennis.

Okta tetewateronwek

Tsi rohtare ne Niio ;

Iah se te kanikonhrata

Raotiohkwatokenti.

O Sewenniio!

Akwa tewakehtakwen

Iahte katennowentha

Ise sewenniio tsi konnhe.
Tahonenne ronwatrori

Ne sesakoskontakwen

$\mathrm{Ne}$ rotitokensehakwe

Nahonwaienterhane.

Rokwatton kati

Ne kento nonwentsiake

Onka nonwa iekweni

Niahta honwawenniiostake !

Akwa rawennatokenti

Ionwesen tsi rohtare

Tsi tesakoswatetennis

Sakonikonhriiostha.

Iekwenies kati

Ronwanikonhraksatha ;

Ii se onkwariwa

Tsini hotatitenstetenni.

Saonkiiatewetonnion Ne ok ne ionkiiawi Sataionkwententaseke Nok naetewenheie. 
Tsi rawenheion

Iesos sonkwawenniio, Tewakwekon sonkwawi Aetionnheke karonhıke.

A kwekon tewanoronkwak Tsi ionkinekwaheston; Sasonkwanekoserawe, Sesonkwaiatokewen.

Oksa nethone Onkwawenk karonhiake Sontennhoton noneshon Nentilewariwaierite

Oksa kati, Sewenniio Akwa waskenonwene Tsi iahte sewakiatoron $\mathrm{Ne}$ iotakssn nahoten

Kanoron kati lahte iahonnisehon Onkwaterihonkohlen, Sewakatatiataksatanion

Akwa konwennaiesaton, Konuikonhraksatakon.

Tiotkon sane seskinonkskwe, Tekaontakwekonne.

Katstarha nonwa Naonsaskitenrheke, Naonkenaktotake.

Nakatsennonni karonhiake

Onen enkatewentete Iontonweskwatha sonha ; Rawenniio raowenna Enkianenhawe nonwa.

Iahte kakonte Entkeriwaierite Ne se ok iotsennonniat Iako!atennhese niotaksen.

Onen nonwa Sewenniio Enkatotarhoseke Tsi iesanikonhraksatha, Ensekswenseke notkon.

Tiotkon tsinenwe Enkonwenniiostake Nok enwakerhareke Nakonialkatho karonhiake.

90

Ionwesen ne iesanonwes Sew: nniio tsi konnhe Iah nonwenton te ieienteri Aiakonikonhraksen, [tsiake Iakotent ienonwes nonwenIah se ken te iontsennonni Sonhatsiwa, Niio, skweniese Askwanikonhriiostake.

Iah kaneka takatekwasen Niahtasiterontake;
Sonhaa nakenikonhra Ensaiat!sakseke.

Oktiwakwekon nonkwe okon Aionteriosere

Iah othenon taketsanike

Tsi nakonniahesenhake.

Etho natesatonwentsioni Sonha takwakanerak I ken nise seniseriio Asteson takwehiaras.

\section{1}

Tsiatontek tsionkwe wakewennentane lahta kakweni naiontsennonni Iontonweskwatha sonha nonwentsiake, Ok eken teiontonhakariatha. 
Riatatsenrion oneu ne rakenies Aiakotsennonnihake nonkwe ; Sakokaserokewas ne iontstarha Nok sakokwatștenni ne iakotent.

Ronwaiats Rawenniio tsi iakonnhe Raonha akwekon sonkionnheton Tokenske kati akwa sonkwaienha Sakononwes sakoien okonha.

Ionkwentent ionkwanikonhranentakon Tsini kanakere nonwentsiake Kense nenweha entewatonhokten, Ok eken entwaiataksenhake.

A etewatennikonhranentakte Tsi renteron ne Sonkwawenniio; Sonkwarharatstenni n'aiontsennonni, Iah oni tasonkwanikonhraten.

Eren sasewet, sewatenroseraksen Ne takwariwaneraktenni, Iahte kakonte watitewatekhasi, Iah onen taonsakwatsteriste.

Sewenniio, sonhaa nonwa skonnonwes, Skatatswens tsi iahte konnonweskwe; Sonhaa nonwa enkonnonwehake, Sonhaa enkoniatisakseke.

Wakerhare tsini satanitenron, Askitenre tsi nenkatonnhokten ; Sakatennikonhranentasi nonkwe. Sonhatsiwa enkonnoronkwake.

92

Kennikarihoten sonkwatenientense ne Rawenniio, " kariwanoronkowa tsini hawennoten.

Raonhaa ok ne Niio hetsewenniiostak, " tasennikonhrasaat sanikonhrakwekon hetsenonwehak.

Tosa okti kaiesha natsenaton ne Rawenniio, * nahiariwase othenon ensatati naseriwahni ate.

Akwa hetsennaientak ne Rawenniio awentatokentike, " tosa ethone othenon ensaioten

Shenoronkwak iesaienha, * nahonnise asonnheke.

Tosa sheriio, nonkwe * tosa oni aserheke : akherio.

Tosa seriwanerak kanakwa * tosa oni asennontonnionkwake naserheke: akeriwanerake.

Tosa ok asenensko, * tosa asatatewenniioste akoren akowerik. 
Tosa ok asheiennowenten, * tosa oni aesanowenhake. hake.

'Tosa ok asaskanekseke kanakwa * iah onakwatokenti taken-

'Tosa ok asaskanekseke akoren akowenk, " tosa aserheke : akatatewenniioste.

93

A siatarake ohaseratokentike Niio rawentawen * nok oni iawen : tatokentison.

Akwa senikonhrarak asaleweienton, ${ }^{*}$ tsini wenniseranorunson.

Entsiatisakha ratihenstatsi aonsaiesaronkwase * enskatha oni tsini onsakahewe.

Wenniseranoronson sahatonnhete lesos, * asatennikonhraseronni asialarane Okaristia.

Asentonliete kaieri riwasontashen iakawentontietha kowa * ne oni tsini wenniserake ionkiatenientense.

'Tosa aesewarake * ronwaiatamentakton.

Tosa oni ensaniake * wenniseratokenhaton iakohtiawenraton.

Ensatieseke ononsatokentike * tsini watenienten iakoties.

Kaneka iontatatekwaton kentiohkwakon ne iakoriwiioston; asetsaniseke oksa ok asateko nenseken.

Tokat oni aiesatekwate kentiohkwakon ne iakoriwiioston," tosa ensanoronseheke iosnore aonsaseriwaseronni.

\section{4}

Sewenniio tsi iakionnhe

Akwekon teskwanikonhratson

Takwanonweratonheke

'Tsini iaweta takwentenran.

Niawenkowa tisariwaierikowa

Niawen sekon iakionnhe,

Niawen arekho teionkwatetsiraton

Sekon takwentenrane.

Sonhalsiwa takwaiatison

Tokara niskwenniserawi ;

Seron : aionteweienton

Karonhiake aiontsennonni.

Arekho siiakwattokha

Takwaiatanonstatonhatie

Sùennentiionkwatehiaron,

Ise iawet takwehiaronnion.

Ok sateskwaswatetenni

Saianerenseratokenti

Seron : aiakotokaten

Aiakoteweientontieseke. 
Aonhaa takwentenron

A kwa takonnienston kowa

Takwanonten ne tsieronke

Aionnhekon onkwatonnhetston

Seron : aiakosnienontie

'Tosa aiakosenni notkon,

Skennen tataiontohetste

'T'sini kanoron onwentsiake

Toni iaweta iotonhon

Aionkwaiatenhon oneshon!

Sekon takionnhetonhatie

'Takwarhare ne karonhiake.

Takwentenrhek, Sewenniio

Aionkwatsennonniatskonnake ;

Tosa aiakwatiesate

Sekon onkwawenk karonhiake.

Takwanikonhrahniratsek

Sonhaa akwanoronkwake,

Aiakwakenronniheke

Iontonweskwatha nonwentsiake.

Taionkwaswateke

Akwa aiakwakonnienstake

Karonhiake aoriwa

Aiakwatateronhiakente.

Sewariwiioston

Nahoten isewehre

Tsi iahte sewanoronkwa

Tsini sonkwentenron

Karonhiake aiente

$\mathrm{Ne}$ lesos Sonkwawenniio?

Onen tesewatonharenron,

Sewaiatatsanitenions.

Rahawe nasakohrewate,

Sasewatatrewat kati.

Tesewennonks kariwiioston,

Tosa tewateraiatak

Kariwiiostonke ti tsionnhek

Kariwiiostonke swenhei.

Tionkwennonks ne kariwiio- Rawen : tesewatstekafa Tosa tewateraiatak: [ston, Tsi ienakerenion
Kariwiiostonke titionnhek Kariwiiostonke twenhei.

Onen wahonnise

'Tsi ken ionwentsiatekwe

Asatakon iakonnhekwe ;

lakorharenionkwe

Ne taiakoswateke:

Ne taiakoswateke ;

Ne tsini iakotentonne,

Ken wahoketote ne Iesos,

Tesakoswatetennire.

Irehre taiontkakwarisi

Watewateronhiak weksi.

\section{'Tekeni iawenre}


Nonwer:tsiakwekon nonkwe, Teietsiswatetennihek, Akwa sewakwisron sewatont Kaianerenseraksenskwa Oia onsaietsinikonron Nonkwe aieriwiioste

\section{Onkataonreni}

I esos ratik wekonne

Tsi ienakeronnion nonkwe; Wahonterientasa

Asakotirihonnien

Iesos ahonwaienterhann, Esotsi wahontsennonnianion, 'I'si waeriwiiostanion, Nok saiakorihontieseron $\mathrm{Ne}$ iakoiataksatakwe.

Akwa oriwakon Wahonwatiiesate, Watierite tsi rononnhe; Senha ok onhtenti $\mathrm{Ne}$ Iesos raoriwa, Sakoienahatie nonkwe ; Ok eken raotinekwensa lesos rosennowanatha [kon Ken ok nahe nonwentsiakwcWahonwaienterhaseron. ,

$\mathrm{Ne}$ iate ionenon 'T'si ionkwariwiioston

Raotinekwensa kenha.

Ronatewenteton

Iontonweskwatha sonha ;

lesos ronwanakerani

Tokati ni kanoron, tsionkwe,

$\mathrm{Ne}$ aesewatiesate!

Iawet aesewakenraton

$\mathrm{Ne}$ karonhiake aiente.

\section{Oh! tewanonwesek}

Naetwaiatarake

Ne rotiohkwison ne Iesos;

Sakowenniioston

Karonhiake aiente

Neniakoteweientonke;

Tiolkon kati twawennarakwak

Ne raotiohkwatokenti ;

Rannhaon se ne karonhiake

Aionkwaiatenhawite.

Sentiohkwatokenti, Takwanikonhrahnirats

Tsi iakwatonnhakarias. Wakatewentete

T'sinikon lakwaswase

Orien wakonnonteratie.

Enskialakennha nenkhieie, Nenkoniatontatiheke, Enkatsteriste kariwiio Enkswenseke kariwaksen.

16

$\mathrm{Ne}$ ok ieskenha satsisewatkatho Iesos hetsisewennenhawe. Israerronon wahonwawınnaswen, tahonwariosere karontake ronwaiatanentaktane tekaientannhareke.

lonehrakwat sentent, hiaiatontiesere netsienha, ken iotsanit sanikonhranonwakhe, ne tsi n'niawen: iakotent oni nentiaiheionsere sesaka onnhetskwe.

Sewatstaren ne nonwentsiakwekon, hetsisewaiatannhaten Ielesos, eniraterakwaton ne iorakwanentakon, eh tentiokarawe ionehrakwat, ne tsi neniawen enhonwario.

Tenkonsentho ne ken ionontenion, ensewanonlarisionko, tenkonsentho ionaterontonnianion, tewaterontiakhonnion enionhriskon ne rorontisahannhon, Niio Roienha.

Enwakwisron ne ken ionwentsiate tenwatonwentsiakarenron, enti ont onnekhon ne ronwatewenteton Niio Roienha, iakotieronnion skon ronwaronhiotakwen Rawenniio.

Sakotenre ne ronwaronhiotakwen, ne seken si thawehtakon; 
akonhaa enkeronhiaken nok enkiheie naonsakheskontako nonkwe ne kenoukwe, ne nonwentsiakon.

Saswatonnhet ne ken swaiataterihon, lesos nonwa wasonkwentenre tsi wahrere: aonsakheiatontako ne nonwentsiakon, aseken onen tahonwariosere ne Rawenniio.

Saswatonnhet ne ken swaiataterihon, Iesos nonkwe watsisewentenre; tasewahtenti sewaronhiakehronon, karo kasewe aetewakwekonhake nentsitowaiatata Iesos kenha.

Aionkwanikonhranonwaks Nonwa tsi ionkwatont $k$ ke Tsini honwaronhiakenton, Ne akwa sonkwaiatison.

Kanosasera rostonton, Ronwaiena, ronwaneren, Ronwanaskwaieutatieskive Sirisare iaharawe.

Asontakwekon Kaïfke Sotar ronwaiesatakwe Are wahonwakonreke ; Are wahonwarasentho.

Sotsi ionikonhrineken Tsi wahonwasokwaweson Akwa tishanekwensote Tsini honwaientanionskwe.

Onen tenhnon tsi roneren Senha ahonwaiesate; Ohikta rotiseronni

Tehonwanon warannhaston.
Onen iahten tsi shotonse Wahonwaiasaherhase ; Roiatoraraktonhatie Onen wahanontarane.

Akwa karonwarowanens Tehonwasnonsawestakon ; Tehonwasitawestakon Nok ronwaiatanentakton.

Akwa waharonhiakentsi Onen waerontaketsko, Aseken oronwaroskon Raiataniontakwenne.

Ok onen tsi karontote Ok iahatonriseraton, Ronwatentoriatonhatie Nok ronwasentennihatie.

Askwentenre, Sewenniio, Tosa aiakwariwaiesat Saronhiakensera okon Noneshon skwaskontakwaton.

98

Risentenni ne Rawenuiio Ionehrakwat ne tsi sekon konnhe; Kanonwe kenha iakatekwasen Iseke, wakerharekwen.

Skiatakennhas kwennakeraton, Takwaienha, satanitenraskon, Iah othenon te sanoronse Aseken Niio n'hetsienha.

Iotanitenra skarake Tsi kiteron naontasierate, Asatkatho akatonnhetston Asitenr aonsasetsient. 
Ionehrakwat wakatkaronnion

$\mathrm{Ne}$ tsi Niio riwennaiesaton

Niawen ne akiheiate

Ne tsini skatatrewatha.

A satekat akeriasakon

Iesanonwestha riakatsiroten

Tiotkon aiotonkotakonhak

$\mathrm{Ne}$ tsina konnonwehake.

Seniiatatake ne tsiatatienha, Etho askenikonhranentakte

Aiawen akeninonwehake

$\mathrm{Ne}$ tsini tsiatatenonwes.

Ronnhek Iesos, ionnhek oni Iesos roiasatokenti !

O ! aonsahinonwehak ;

Kaiasake sonkwenheiase

$\mathrm{Ne}$ tsini sonkwanonwehon

Ionnbek Iesos raoiasa.

Kaiasa tewasennaien Satiateraneken ne lesos ; loterontatehatonne

Wakarontanoronne kowa * Twakwekon tetewariwak Ionnhek Iesos raoiasa.

Kaiasa tewasennaien Waiunoron ioianiionte ; Ne saionkwatsiente onwe Tsi sonkwakaronni Atan.

Kaiasa tewasennaien Nene konwasennietha notkon Ne sesonkwaskontakwaton
Nok karonhiake ieshoteron.

Kaiasa tewasennaien Iesos raonekwensa kenha Akwa ioiatohareton Onkwe okon akotonnhetston.

Kaiasa tewasennaien Isi roiatanentakon Iesos; Sonkwatatis nok sonkwawis Naetwatsennonniate onwe.

Kaiasa tewasennaieu Raonha watsitwasennaien ; Kaiasake rawenheion Naonsasonkwaskontakwate.

Twanonwehak raoiasa Raonha entsitwanonwehak, Nok iawet entsitwasnienon Tsini sonkwatiasakehtati.

100

Raonekwensatokenti Nonkwe sakosk ontakwen Tewaiest nonkwakaseri Naetsitwentenrheke; Ii seken onkwariwa Ne tsini haiatawens Ionkwahretsiarontha kati Raoronhiakensera.
Atenhenrakon raonha Tehoterihotarhon ; Roterennaien, ratsanis Tsi ronwaiesatane ; Nok tenhnon ne kasatste Tsini sonkwanoronkwa ; Onen kati ontesenni Ne nonkwatsennonnialak. 
Sitas ne ronkwetaksenskwa

Onen wahoiatorenne;

Akwa wathonoronkwanion

Okti iehonowenton;

Ronwanakeranihatie

Niakoriwanerahakskon

Ehneken ieiatarase,

Nakon ronwatiesatha.

Iesos wahonwaialonti

Wahatatewentete,

Wahonwakenrenseronni

Iawet kariotaksenkwa ;

Wahonwakonsahaiakhon

Rakonsatokentike ;

Iah othenon takakweni

Tonsahatiatorete.

Ratsihenstatsikowahne

Iahonwanaskwenhawe ;

Oksa tasakoriwawas

Iesos wahotewentet

Akwekon oni ne Tier

Ne ronatenro kenha

Onton wahotonnhiiase

Ne wahonikonhrariot.

Onen nonwa Konskwiratne Iahonwaiatenhawe ;

Ne ronwennitentasere

Nasakotewentethas;

Barabbas ronkwetaksenskwa

Ne wahonwakonnienste

Etho sane ni twaierha

Ne tewatatkonnienstha.

Ronwaiatawitasionkwas, Akwekon ronwanerenks Skanoron ahrenheiate

Tsi ronwanonwarekhons;

Sewatka tsionkwetaksenskwa

Ise tesewatentston

Tsi nonwa nihoiatawens :

Ise kati sewenhei.

Iesos tekonnoronkwanions

Tesatonkwaraonhatie

Ohneka nok onekwensa

Entakta saesario.
Ohikta waeseronni

Wathonwanonwarannhast ;

Akwa ne raiatakwekon

Okti shanekwensote.

Sewatkatho sewanaie,

Teshariwaserakwas

Tsi sewatiataseronnis

Nok tsi sewatonwesens.

Onontoharake ware,

Rokehte tekaiasont ;

Ohahake ronoronse

$\mathrm{Ne}$ tsini iorontakste

Ah! oh kati na naiawen

Etho ioranentakon

Onkwariwaneraksera

$\mathrm{Ne}$ rateriwaketats.

Sahonwaiatanentakte Nok waerontaketsko;

Karonwarowanense ok

Raiataniiontakwen.

Tewatkatho tionkwe okon,

Ii ehni ionkwaieren ;

lah ken taetwatatitenr

Tsini ioriwatsanit?

Okti honwatentoriatha

Tsini honwenhratonnis;

Ok sa satiatanentasi,

$\mathrm{Ne}$ Niio akenhake.

Tosa kati Sewenniio,

Tontasatsnent, niiare

Etho akwaiatanentakt

Sataetewenheie.

Onen roweiennentaon

Wahatoriseraton,

Tsini hoteweiennison

Akwekon ronwannhatens.

Watwatonwentsiorenseron,

Satka nenhiariseron ;

Aonha ken nonkweriasa

Iah othenon taiawen.

101

Iesos tekonnoronkwanions

Tateiorihon tsieronke;

Tsini iesasokwaweson

Skanoron aesenheiaton. 
lesos tekonnoronkwanions Sakehte tekaiasonte ; Serenkhene iahasewe Onontoharake Karfer.

Iesos tekonnoronkwanions A kwa karonwarowanens Teiesasnonsawehestakon Nok tiesasitawehestakon.

Iesos tekonnoronkwanions Onen iahte tsi sahtonse Ohikta iakoseronni Teiesanonwarannhaston.

Iesos tekonnoronkwanions, Senheion tekaiasontne;
Tsini satanitenraskon

Oneshon skwaskontakwaton.

Aiawen akiheiate Ne tsina konnonwehake Tsi nise ni senheiaton Tsini skwakonnienstonhatie!

Akwa tekwanoronkwanions Ne tekaiasatókenti :

Shetenr iakoteweienton Nok niakoriwanerakskon.

A konwanenton tsinenwe $\mathrm{Ne}$ asenser،tokenti

Tsini sonkwen:enron Iesos

Tsi ronwaiatanentakton.
Tekwanoronkwanion Niio $\mathrm{Ne}$ sheiatsennonniatennis Akoiatatokentison, $\mathrm{Ne}$ sewakwekon karonhiake.

Askwentenre Iesos Kristos, Ionkwentent ne sheien okon Tsiakente seriwarako

Tsini kwanikonhraksaton.

Wari, Niio hetsienha Oneshon sonkwaskontakwen ; Tiotkon shetenranions nonkwe Iseke ionkwarharekon.

Ise swaronhiakehronon Ne Niio swakwekonhatie, Tiotkon askwaiatakennha; Tosa ionkwasenni notkon

Ise kwani okon kenha, Nok sewatokensehakwe, Tosa swaiatanikonrhen Tsi swenteron karonhiake.

Ise swaiatanoronson, Iesos Kristos swakwekonne, Kwennitha askwentenrheke Nonen tenskwaiatorete.
Ise Niio raoriwa Ne tsini tsi sewanonwes, Ietsiiesaton, ietsirio, Aiawen aetwahriskon!

Ise ne swariwanaton Ne nakwa kariwiioston, Askwaienawaseke Aiakwariwaierite.

Ise swaiatatokenti Iahte swanakwaienteri Karonhiake swatsennnonni Aiawen taetwatatken!

Ise, swaksa okon kenha, Hetsiswentenraton Niio Ne tsi ietsinekwaheston, 'Tiotkon askwehiaraseke.

Karonhiake swatsennonni, $\mathrm{Ne}$ niate swaiatotenson, Rwennitha, aeswarane Naetewakwekonhake.

Ahonwasennaien Niīo Roniha nok ne Roienha ; Satahonwatisennaien Rotkon Roiatatokenti. Etho... 
103

Sirisare waeriwihewe Wahiron: Iesos thare; Ok sa ok waeiakenne; Wahonwaiatisakha

* Niawen, ionton, wasakwaken Iesos Niio Roienha.

Akwa wehne iakaonni Tsini iakotsennonni Akaosa wahetskaronni Tsi rotohetstha Iesos

Ok etho tahose Iesos Tsi ronwaiatorenhon Iahte iontawennasetha Senha iontwennaientons.

Akwa ioriwanehrakwat Tsini honwakonnienstha Ieneratenhawinontie, lakoties ohahakeson.

Akwekon ieriwanontons Tsini sakotenrhakwe ; Otiake sesakaonnheton Otiake sesakotsienton.

Iakotsennonnihonhaties Sahonwatkatho I esos ; Raonha ronikonhraksens Tsini iontkaronniane :

Toha ronwatwentetane

* Iesos Niio Roienha.

Sahatkatho Sirisare,
Akwa ratstarha onwe;

Ralon : swentent ken swanakere, Iahte sewar!oronkwa

Nonwa hetsiswentenrane...

Swakwekon sewanehrakwas Tsini iakonkwetaksens Senha sane twaserohens Ii ionkwariwiioston Tiotkon tsitwanakwatennis...

Kennaheha, twatateken, Tsiontennikonhkarhathos, Iahten teshonwanonwese, Akwa ronwatwenteton Ieriwisaks nahrenheie...

Ionkwaterientare nonwa Tsini haiatanoron : Ronwaiatanekherenskwe Ne ronwaiesatakwe Iah ostonha te iienrhakwe...

A onsetwatatrewate, Eso tsi tionkwetaksens; 'Tiotkon hetsitwawennonties Rawenniio tsi tionnhe; Ne konwaiats wahonwario...

Tontakwentenr Sewenniio Tosa tontakwahrewat Akwekon eniakwatkawe Kwanikonhraksatakwa, Sonha enkwanonweseke Jesos Niio Hiaienha.

\section{4}

Sirisare sewaienha, tosa ii akeriwake sewatstaren, ne tenhnon sewatstarakwak ne ise sewariwake. nok oni ne ietsiien okonha akoriwake. Tionkesnonsaweheston, teionkwasitaweheston, nok iakosetanion nakstien okon tsini ionkiieren.

\section{5}

Takitenr, o Iesos, takitenr

Tsiui sanikonhriio kowa;

Tosa satkatho wakeriwaneren, Iahte kakonte ne katstarakwa. 
Oriwakon tsinahe konnhe, Iawet takenentsawakontie ; Senha ok wakkwenion tsi koniatontion Ii akwa wakatiesaton.

Ok eken tewakenonhianiks, Sekon akonniahesenhake;

Tsini iaweta konnikonhraksaton, Sekon ken enwaton waskitenre.

Iah nonwenton takiewentane, Tiotkon tewakserentonnions ;

Akwa tiotkon ne tikennonton nionkwa Tsini wakitent tsini wakieren.

Iesos tisa wenninekenhon Nenshetenre iontatkenronnis Teskanerak kati tsiniskatatswens Iawet teiorihon nakweriasa.

Sewenniio tkariwaieri Satrewatha kariwaneren; Wakonion n'askrewate nonwentsiake, Nok tosa takrewat nonwentsiakon.

Wakatewentet ne kieronke, Sennonton tsini satatieras Niate karonhiakenserake isatst Takitenr tenhnon nenkatonnhokten.

Iesos onen sewakerhare, Akwa ne wakaterientasa Niatekon enkatatrewatakwake Naonsakonikonhraseronniate.

Saronhiakenseratokenti Nok oni saiasatokenti Iahte kakout tenkkanerake, tiotkon Etho enkatekwasentakwake

O Wari kanistenseriio, Hiakweniatserawi n'hetsienha Nashetenre naonsaiontatrewat, Takitenr aonsakatatrewat.

Taetewatatenonwehak ii tewakwekon, roriwison ne Iesos. Raonha raoriwa kento tsi ionkwalkennison Hetsitewanonwehak Rawenniio tsi tionnhe. Hetsitewatontats tsinikon roriwison. Ro- 
riwison ne lesos nii tionkwe taetewatatenonwehake tsiniiot ne raonha tsini sonkwanoronkwa. Ronronkha ne rotiriwiioston noh oni ratinoronkwa tsini horiwison ne Niio.

107

Okni kwaieren, kwaien okonha. nah nahoten kwanikonhraksaton! takwahrori. Ne ken karihonni takwaiasonnienni tsi kwaiatinekenhon n'Egiptke * Saiatatokenti Niio. Saiatatokenti sesatste. Saiatatokenti sonnhe onwe, takwentenr.

Nahoten niah tekwanikonhraieriton? Kheriio n'Egiptronon naiawens aetsionnheke, nok nise takwatewenteton naionksokwaweson.

Ohni kwaieren, iotenataratkon karnakon, nok nise takwakonrekhons nok takwasokwawesons.

Ohni kwaieren, otstenrokon tionekinekenhon ne kwaniatanawentakwen, nok nise otsate tenhnon otsitsiatken takwaniatanawentakwen.

Ohni kwaieren kheriio ne Kanaanronon ronwatinontiiokwe naetsionnheke. nok nise kahnia takwaientanion.

Ohni kwaieren kwasennowanaton nok kwaiatanoronston. nok nise kaiasake takwaiatanentakton.

108

Tsini ionkwaiatawenskwe Akwa ionkw sntentonne, Iahte ionkwarihontakwe Aionkisarontakwen, Ne tsini hoiatawenskwe Iesos saharonhiaken ; Onen iahten tetsionkwentent, Onen se shotonnheton.

Tsinahe thoriwaneren Ne sonkwaniha Alan, Ne ionkwaiatoronhatie Wetewennakerate; Tsinahe shotatonnheton $\mathrm{Ne}$ Sonkwaniha Iesos Ne sonkwaianerenstakwa 'Tsi ionkinekwahestha.

Enskat iehoriwane! en Ne sonkwaniha Atan Akwekon sonkwakaronni Akwa iahte kakonte; Tsinahe shotatonnheton Ne Sonkwaniha Iesos Tiotkon aontonseke Aetewatsennonni.
Arekho tsi rawenheion. Iesos tekaiasontne lotehatonne nelhone.

Ne tekaiasontne ;

Tsinahe shotatonnheton Oia tsini tsiawenhon, Akwa iorontanoronhon Nene tekaiasontne

Ionkwanaskwaientatiene Notkon oneshonronon Ne ki ionkwannhesehakwe Aionkwatsennonnihon; Tsinahe shotatonnheton $\mathrm{Ne}$ Sonkwaniha Iesos, Tiotkon sonkwaienawase Aetwatsennonni.

Iahte honwaienterihne O.ı wentsiake sireskwe, Iontennowentakwe nonkwe Ne tsini haierhakwe; Tsinahe shotatonnheton Ne sonkwaniha Iesos, Akwa tiotkon senha nonkwe Ronwaienterihatie. 
Niawen kati, Sewenniio. Tsi nonwa kwaienteri, Tosa iakwariwaiesat Tsini skwentenronhatie. Akwa tewatataskennha Ne tewatatekenson ; $\Lambda \mathrm{kw}$ ekon tetewariwak Iesos shotalonnheton.
Tosa othenon ne nonwa Teionkwanikonrharen, Ne ok tewennontonnionhek $\mathrm{Ne}$ tsi Iesos shotonnheton, Saheto ion! waronhiaken Ne kento nonwentsiake, Ionkwarharek karonhiake Ienionkiiatinionte.

\section{9}

Tekwanonweraton Iesos ken tsiteron, okaristiakon sataseton Onen satatonnheton, tesariwaserakwen ionkwariwanerahakskon. Iesos konnonwes tsi satatonnheton; karonhiake onen tsisenonton, sannhotoukwen onen karonhiake, takwannhotonkwenni karonhiake. Tetwariwak, twatonharen. Areriia, areriia, aiesanenton, aiesasennaien. Areriia, areriia.

110

Twatsennonni twatateken Twakwekon twatonharonnion; Onen Iesos shotonnheton Roronhiakenserentaon.

Tosa aetwatsaniseke Tsi kenheions twaieronke Aseken ensewatonnhete Iahte kakont entsionnheke.

Ne tenhnon tewatsanisek Naetwariwanerake, Aseken kariwaneren Iakoiatentha noneshon.
Sewonniio askwentenre, Nonen eniakwatonnhokten $\mathrm{Ne}$ naelwakwekonhake Iahte kakont karonhiake.

Ahonwasennaien Niio Ne Roniha nok Roienha, Rotkon Roiatalokenti Tiotkon ahonwasennaien.

Tsiniiohtonne tsinahe Nonwa nok iahte kakonte Tiotkon eh naiawenseke Tahta hariwentane. A men.

\section{1}

Atsitwasennaien tatsitwanenweraton Iesos shotonnheten, roiatanehrakwat -Ne onkwe rotonhon Wari kaiatakon, Akwekon roteweiennison nonwentsiake Akwekon thennontons shotatenerension Tsi ronerenne ne kenheion.

Onen thoiakenhon tsi raiatatakwe Satewatarenhon ne asenhaton nonta Tehoteroronte; iotsennonniat kowa Karonhiake nok nonwentsiake.

Satsennonni, Wari saiatatokenti, Iesos otstenrokon ronwaiatatenhenne 
Onen shotonnheton sonkwatokatennitha

Tsi iah othencn te honoron

Tsiariskonhonne, Wari, sasonkwenheiase, Aesanonwehon sataesenenheion Onen kati nonwa sataetsiatsennonni Iaskwatatiase tsi renteron.

112

Ne nonwa wenniseriio lesos shotatonnheton Rotatewennaieriton tsinikon thotatihne Onen enwakiheion nonwentsiake Asen nenwata enskatatonnhete

Rasennion kenheionsera sakoteronkwen sotar Otstenrokon thoiakenhon akwa rotakweniase Tehoteroronte tsi tehoswathe lahte skaieronni tsi asonthenne

Tsini haiatoten Iesos, iah taonton naiotken Raieronke notstenrokon Niio ni haiatoten Sate se hniiatoten ne Roniha Sate hninakere onwe tsinahe.

Sitaeronon okonha tsisakorihonnienni Nahontsennomni tsinenwe iahte hotinonwehon, Ok eken ronwaswenhon, ronwanosas, Nok wahatiriwisa nahonwario.

O Iesos takwawenniio sonha ok sanonwehon Naiesaiatanentakte tekaientannhareke Ne senheiaton nasheskontakwate Nok ashetenrate iahte kakonte.

Twakwekon nonwakenwente akwa tewatonnharen Nok oni tewatsennonni tsi Iesos shotonnheton, Ronwaken norhonkehne skennen sronnhe Ronwaken nokarasneha sronnhiio.

Onen ne Ratikwekonne tewawennakwekonhak 'Tatsitewanonweraton Iesos tsi sonkwentenron Sonwahahonniennì ne karonhiake Katke sonha ientewakwekonhake.

113

Twatonnharen ne ionkkwariwiioston Tsi nonwa naonkwaiatawen Sonkwatsennonniatenni ne Iesos Kristos Ne tsini sakononwehon nonkwe 
Ken wenniseratenion shotonnheton Akwa ionkwariwatokense

Ii onkwariwa tsi ronwaronhiakenton Ii onkwariwa tsi shotonnheton.

Tetewariwak kati tewakwekon Tsini sonkwentenron ne Iesos

Akwa tewakwisron tetewariwakwak Tewenron: tokenske shotonnheton.

jriwakon tsini hotiieren Ne ronnehron ne Sitaeronon ;

Asakwario, akwa iatasakwakonten Sakosennion ne tsi shotonnheton.

Onen tesonkwawistotarisien Ne iahte tsitewanaskwa tesken Aseken onen sonkwaienha iotonhon Ne Iesos Kristos tsi shotonnheton.

Tewatsiaken kati ne twatatekenson Ne tewatenientenstakwake Tsini ionkwatsennonni tsisonkwentenron $\mathrm{Ne}$ lesos Kristos tsi shotonnheton.

Iesos wahatesenni Rotanitenraskon Ronikonhriio kowa Iesos wahatesenni Onwa atsitsion nonkweriasa Saeto sane tionkwetaksenskwa Irere : taiakwatatenonweseke Twatatitenrhek, tewatontatsek Akwekon tsitewatatrewat.

Tetsitewariwakwas

Tsi rasennion notkon

Nok tehowistotarhon

Tetsitewariwakwas, Hietsîtewasennanoronkwak Ne Rawenniio karonhiake; Tekaiasontne tesonkwatson ne tionkwe Kaiasanoron tewanoronkwak Tewakwisrontie nonwentsiaks.

Tsini ionkwaiatawens

Kento tsi itewes Tewanikonhraksatha, 
T'sini ionkwaiatawens

lawet ne kaiasa sonkwawis

Tsi kati niiot ne rotketaton

Ii ionkwariwa raoiasatokenti

Tewatketatsek, tosa twatiesat,

Kaiasatokenti enwaton.

Wari Iesos n'hetsienha

Akwa sententonne

Sahonwaiesate,

Wari lesos n'hetsienha

Iawet tieseriasawestakon.

Senisienseriio satakatstaton

Tsini skwanonwes kaiasakta sistakwe

Takwentenrkati, takwaienawas

Tiotkon aionkwatakatstaton.

115

Shotonnheton lesos, akwa shotonnheton: * twatonnharen, niawen twatsennonnianion.

Twakwekon entsitewatonnhete ionkwariwiioston ; * Iesos tentsitewatatken, ensetsitewatierenhake.

Etho nentionkwaienseke tsini thoien Iesos; * onen iahten tsi twaiatisahaseke.

Akwekon entionkwetiiohake karonhiake, * iah othenon taionkwaiataksate.

Iahta tentsieronwekon teieronwekonne, * iotohelston entewatkonseriio.

Hero! ionkwaiatateri ; Iesos shotonnheton, * rotenientenstakon tsinentwaiatotenhake.

116

Kanatiio! o jonatar:ehrakwat!

Tsi tsiteron, iakotonnhahere,

Raiatakta Rawenniio tsi tionnhe

Asteson iaetwakanerak.

Ne ok ieienteri iakotsennonni

Ieiotohetston tsini ionwesen,

lahte tkaien naiakonikonkraksen

Iahten oni naiakotentane.

lahte kakonte iawet iakorarhon

lahta taonsaiakowerakwe

Iahte tkaien naiakonikonhraksen

Iahten oni naiakotentane. 
Teioswathe kowa tsinon tieteron, Akwekon teiakoteronronte, Iah oni taonsaiakokarawe Iah oni tentsiaiheionsere.

Iahtaonsaiontkatho ne iotaksen, Iah oni tiakonikonhrotakwas;

Iah othenon tetsiakokwenies notkon, Iakohton tsini iakotsennonni.

Sewenniio, sonha tsiatiio kowa, Sonhaa sheiatiiostha nonkwe;

Ne waesarane karonhiake

Sonhatsiwa enkoniaskaneke.

Sonkerihonti ne kariwanıren, Akwekon oni ne ienekirha Sakseronni tsinikon wakenenskwen Kaskaneks se karonhiake iake.

Tikate enkatateronhiakerite Tiotkon enwakentontietonhake; Enkhetenranionheke ne iakotent, Tiotkon enkaterennaienneseke.

\section{7}

Areriia. Ken wenniserison Rawenniio, tewatsennonni ken wente, nok tewatonnharonnion. - Ronwasennaien Rawenniio, aseken roianere, aseken iahte kakont rotanitenraskon kowa.

Rawenheion ne karihonni ionkwariwanerahakskon. Nok shotonnheton, naonsasonkwaskontako ne tionkwe.

Hetsitwasennaien Iesos Sonkwawenniio Nonwa karonhiake Tsi iesawenonton Roiatanehrakwat Roiatanonwet onwe Hetsitewasennaien Tatsitwanonweraton.

Onontaharake Etho wathatano Ronwanonteratie
Ronistenha ne Wari Nok ratikwekonne

Tehonwanonweratons

T'si karonhiake ne shretane.

Ii oni ken wente Twawennakwekonhak Ise Wari oni Saiatatokenti Atsitewanenton Tatsitwariwakwase

Twakwekon kati ne tewenron.

Swanuhohatokenti ne sewannhotonkwe Karonhiake nouwa tenswatennohonti 
Ionsataweiat ne Sonkwawenniio

Tsinon noneshon sesonkwaskontakwen.

Wahariwakweni, wahariwasenni

Ne kariwaneren nok ne kenheion

Ne nonwa shaniente ne rannhotonkohe

Ne karonhiake iensonkwaiatiniont.

Tehonwakanere ratikwehonhatie Ronwaiatannhatens teiontekhasionhe

Wahontieren ok wathateweiarikte

Tsiniiot tsitenha ne karonhiake.

Hetsitwasennonnias swaronhiakehronon

$\mathrm{K}$ aronhiake tisewanakere hetsitwanontonnion

Swaiatatokenti ne ietsisewaiatenhawiton

Ise tsionkwe okon skennon swennontonnion.

Ok eken tiotkon ne sewakwekonhatie

Etho se renteron okaristiiakon

Etho asteson sonkwentenranions

Hetsitwanoronkwak kati nonwentsiake

Nok entsitewaken karonhiake.

Satkon Niio saiatatakenti

Tasatsnente onkwanikonhrakon

Askwentenre aiakwateweienton, , Sasatekwat notkonseraksenskwa.

Karo, Satkon satanitenraskon Karo nisa, askwanikonhrata ; Takwarihonnien tsineniakwaierhake Karonhiake aiakwatsennonni.

Satkon saiatatokenti Ise takwaiatison

Kwennita ken iakwataraien

Takwatiennire skwaiatawen

Onkwanikonhrake kasennakerate, Etho tenskwaswatetennire, Tiotkon aionkwanikonhratoken Othenon sonha naesonweskwen.

Kasatiennire onkweriasakon Satekal etho satsiratokenti Asakwanonwene Niio

A onhaa aiakwaswen iolaksen sonha 
Onkwennasa oni kwawenniiostha

Ise ne takwawennison,

Kariwiio aiakwahtarakwak

Iah nonwenton taiakwalwennaksat.

Satewenniiost onkwaiatakwekon; Eren sawit ne kariwaksenskwe okon, loianerenstakwa takwaiesten, Asteson naskwaiatonweskwen.

Takwentenr nisa takwanikonrhala Asakwaienterhane ne ronwaniha

Raonha oni ne ronwaienha

Ise oni tehiatatenonwestakwa.

129

Satkon, takwatekwaten

Satsiratokenti

Onkwanikonhrakon

Nok askwaienawase

Tiotkon naionkwariwiiostonhake.

Akwa ne ionkwarhare

Ne saianerenstakwa,

Nene teionkwatonwentsioni.

Akwa icnkwaterientare

Iah othenon te sanoronse

Tsini sonhatsiwa naskwentenrheke.

Tiotkon takwentenranions

Tsiatak niioriwake

Akwa ne kariwanoronson.

Takwanikonhrowanat

Raiatatake Niio

Nok oni tsini haiatoten.

'Takwatokaten karonhiake, Akoiatatokentison

Tiotkon sakotsennonniatennis.

Tiotkon takwaienawas Natsitwanonwehak Niio

Onkwanikonhrakwekon.

Takwanikonhrahnirat

Tosa othenon hetsi-

Tewasennisek ne Niio. 
Takwanikonhrahnirat

Naiakwateweienton

Nok naiakwatsteniaron.

Tosa aiakwatehensek

Tsi ionkwariwiioston

Nonkwehne nonkwatike

Nok takwanikonhrata

Aiakwatsaniseke

Kariwaneren oriwiio

Aseken kariwaneren

Telwatekhasiatha ne Rawenniio

Noneshon iakoiatentakwa

Takwanikonhrahnirat.

Tsi ionkwariwiioston

Tosa aiakwatsaniseke.

Aiawens tsi neniakwatonnhokten

Naskwentenre ne karonhiake

Tiotkon naetewakwekonhake.

123

Satkon saiatatokenti takwatekaten, Nonkwanikonhrakon niakionkwe.

Iahten terıskwas watetennire

Ne ok neniakwaiatahton

Sonhaskwanikonhrowanatha

Ise sanikonhroskon

Takwatienhas nisa.

Oneshonronon, onkwe okon oni Asteson tehotiienawakon

Naionkwataskatsten ne kariwaksen

Askwenterre katakwannhe nisa.

Takwarihonnien naiakwattokhake Aionkwateweientontieseke;

Onwentsiake ase tsi iakionnhe

Nok neniakwatiatisa.

124

Satkon Saiatatokenti, ise takwaiatison:

Satekat satsiratokenti, ne takwaswateten

Nask waiatakennha aiawen aiakwaienterhane

Tsini iothahinon karonhiake. 
Iah othenon te sanoronse Sewenn io tsi iakionnhe, Teskanerak, kwennitentase, tsini ionkwentent

Ne sheien okonha, takwentenr nisa, takwaiatakennha, Notkouseraksen aionkwateriiohake.

Satkon shetenranions onwe tsi iakoriwiioston, Tsini satanitenraskon, tosa takwaiatonti,

Takwaiatahnirat tenhmon, aiawen aiakwatsteristak Tsini sariwison naiontsterist nonkwe.

Ise Sewennio tsi iakionnhe, airsasennaienseke I esaniha nok Iesaienha, ise oni Satkon saiatatokenti Aiawen aiakwatsennonni karonhiake tsineniakwenheie Etho naiawen, ethenaiawen.

\section{5}

Otsiratokenti

Tsi takwanonwes

Iesos Sewenniio

Nonwentsiakwekon

Ne takwanonwestha

Naskwatekaten

Naskwanonwehake

Nonkweriasakon.

Iakotonnhahere

Ne karonhiake ;

lakotsennonniaton

Tsi iesatkathos;

Tesaswathe kowa,

Tsiiatiio kowa,

Saiatanehrakwat,

Saiatanonwet.
Ioriwanehrakwat

Raiatanoron

Rakiatanoronkwa Sase kiataksen. Aonsahinonwenn Iate haiati

Ahikonnienstake Iahte kakonte.

Toni honwanonwes

Ne karonhiake!

Toni iekenhronnis

Ken ionwentsiate!

Oh! tewaskiıneksek

Natsitewaken

Naetwatsennonni

Iahte kakonte.

Sewenniio, akwa kwawenniiostha, ken tsiteron okaristiiakson Ii onkwariwa tsi sanekwensarihon, ne tsi ionkwariwanerahakAkwa wahi ionkwariwanehrakwas tsini takwanonwes_skon. Ne tionkwe; akwa kati onkwanikonhrakwekon Iakwaskaneks akwanonwehak oni iahte kakonte.

lonehrakwat, ionwesen kowa, ionehrakwat, ionwesen Sewenniio, tsinonni tesiteron. Iaonweskwani, nok iotonnharen nakenikonhra tsi tesiteron Sewenniio. Kieronke nok nakeriasa, ionatsennonni teiesakanere, iesawenniiostha, sonhatsiwa aiesaskaneke. 
Takwehiarak, Sewenniio, ionehrakwat tsini ionkwentent, tosa enseriwarako. Askwatokaten onkwanikonhrakson; ionkwarhare saianerenstakwa. Ionehrakwat, tsini sonkwentenronhatie; tiotkon takwanikonhrahnirat, tosa takwatewentet tsinenwe. O Jesos! akwa konnonwes. O Jesos! iakwanikonhrakwekon. Iah othenon nonwentsiake, iah othenon te ionwesen, ise sonhaa kwasennaiens, ise saiatanehrakwat, ise ne onwentsiakwekon teiesariwakwase tenkwariwakwaseheke.

Ioriwanehrakwat, raiatanoron kowa, tahatsnente tsi iehasentakwa. Rotaseton okaristiakon, kanatarok kenha rotasetonhatie, sakotsientanire onkwatonnhetston okon. Ah! tokenske rakenonwes, akeriwa tsi kento rotatiteron. Risennaiens kati, riwenniiostha oni, losa oni tetiatekhasi ahatekate nakeriane, iahte kakont ahinonwehake. Nene Jesos Raweriniio, raonha Sonkwai atison.

\section{0}

Hetsitwawenniiost Niio Roienha tsi iehasentakwa, karonhiake nontare, ii sonkwaniente. Irehre: aiakwakwekon, tionkwe twanehrako, ne tsi sotsi sonkwanonwes. Patiens okaristiakon, sonkwarhare tsi renteron, karonhiake sonkwawire. Sonkwaienawasere ionkwentent nonwentsiake.

\section{1}

Twatsennonni nonwa twakwekon, thare ne Niio Ro:enha, ratatewentetasere kento tsi iehasentakwa, thanekwensarisere raonekwensatokenti. Karo kati, Sewenniio, karo kase, sonhatsiwa enkwanonwehake. Ne ok enskat nonwa karonhiake, tiakwaskaneks etho ionwesentserakont, takwentenr neniakwenheie, enkwaienterhane.

Ken ire ne Rawenniio Ken tsi iehasentakwa; Oriwiio tsi katontats, Iah sane te hiatkathos, Onkweriane rotatonni, Akwa rotatastoton : Onwa sonkwanontens

Ne okaristiiakon.

132

Tasatsnent Iesos Tontakatiennira nisa

Tasatsnent Iesos

'Tontakenikonhrahnirat.
O Iesos seskwaskontakwen, Tekaientannhareke Etho sanekwensarihon, Ne skwaiatohareton : Sekon tsi ielasentakwa Saien ne sanekwensa ; Siataseronniatonhatie Onkwatonrihetston okon.

Karo kase koniatisaks, Satanitenron

Ise akwa koniatisaks Naskatienhase. O lesos 
Satanitenraskon

Tsitenr nakatonnhets

Satanitenraskon

Tenikwekonhatiesek

Takwaskontakwen

Tsi iesaiatanentakton.

Takwaskontakwen,

Senheion tekaiasontne.

Sewariwiioston okon

Hetsiswahonkarawis Iesos

Sewariwiioston okon

Kasewe rotenniote.

Aonha entewatsennonni, Entewake raieronke ;

Aonha entewatsennonni

Naetionnhek onhake.

Kanon senha tieweiente

Iontathonkarawis nonkwe

Ne raowaratokenti

Ranontens nonkwatonnhets.

Tokenke tsi ionkwataskat Raonha Sonkwawenniio

A kwa ratatewontetha

Naetewatsennonni.

Ne se watenientenstakon Iahta tahononhianike
Ronatsennonni

Ne ratironhtakehron on ;

Tsini shetenron

tah ken nii caskitenre

Katewentetho

Natonwesentseraksenskwa ;

Katewentetha

Notkon aoriwa okon.

\section{4}

Ne se watenientenstakon

Nensonkion karonhiake.

Karo kasewe, wahenron, Ne tsini sonkwanoronkwa, Karokasewe, wahenron, Sewatkens tsini ionwesen.

Ii onkwariwa senheion Nok sekon tisatoktani ; Ii oni onkwariwa

Kento saiataseton.

Ionkwanikonhrahniratstha Sawaratokentı kowa; Ionkwanikonhrahniratstha Naionkwasenni notkon.

Askitenre, Sewenniio Akeriwanoronkwak Tsi takwatiennis niakionkwe, Tosa akatiesate

135

Itewe, twat ateken, tsi Iesos tesonkwahonkarawis, Itewe, twatateken, rotenniote ne raieronke.

li sonkwennis, karo kasewe, ne sewententenion onwentsiake, Akwekon kaseronni, hasewe enkwanontenseke.

Inon tionkwatiaktani tsi akwa tewanakere onwe, Niatekon kanakere naionkwakaronniate onwe ;

Ne ok enskat akwa ionkwaien ne Iesos raowaratokenti, Ionkwanikonhrahnirats tosa aionkwasenni notkon.

Kariwanoron kowa tsi Iesos sakononwese nonkwe, Ne iakotent okonha ne sakoteranentaktennis, Irehre : aionwe tsinonni henteron karonhiake :

Ne rotenientenstakon, tsi sonkwatiennis tsi renteron. 
Itewe twatateken, tsi Iesos tesonkwahonkarawis, Itewe Iwatateken, tsi sonkwennonks ne sonkwanonwes; Tosa aetewakonnienste, ne iokenrat sonha nonwentsiake, Tiotkon tewannhatensek tsi tionkwanakte karonhiake.

136

Tsi rohasen $\left\{\begin{array}{c}\text { Oh ! nahoten katkathos, Iesos Sewenniio tsi kon- } \\ \text { nhe? }\end{array}\right.$ Etho tsiteron, satatwentetonhatie tsi rohasen.

Konsennaiens $\left\{\begin{array}{l}\text { A kwa konwenniiostha Okaristiiakon tsiteron, } \\ \text { Akwa konnonwes, akenikonhrakwekon konsen- } \\ \text { naiens. }\end{array}\right.$ Ionehrakwat $\left\{\begin{array}{l}\text { Takwanontens tsieronhe, ne ionnhekon onkwa- } \\ \text { nikonhra, } \\ \text { Nok tsiatatake skwanikonhranentaktha, ionehra- } \\ \text { kwat. }\end{array}\right.$ Sewenniio $\left\{\begin{array}{l}\text { Akenikonhra kenha akatonnhetston nok kieronke. } \\ \text { Iahte kakonte, iahta kariwentane, Sewenniio. }\end{array}\right.$ Ionehrakwat $\left\{\begin{array}{c}\text { lesos, satatkenhronni ii iakionkwe takwanoronkwa } \\ \text { Takwentenrane, ii kwanikonhraksaton, ionehra- } \\ \text { kwat. }\end{array}\right.$ Sewenniio $\{$ Sonha ne tsiatanoron, ise akwa takwanonwese,

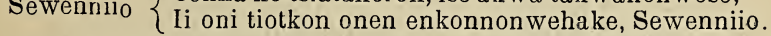

Akeriane $\left\{\begin{array}{l}\text { lesos tasatiennire, tosa nonwenton aiotaksen } \\ \text { Aiawen nonwa tiotkon aesonweskwen akeriane, }\end{array}\right.$ lahtekakont $\left\{\begin{array}{l}\text { Iesos, takatsteristha, onen nonwa enkoniatontatse, } \\ \text { Aontiesenhake a konken karonhiake iahte kakont. }\end{array}\right.$

\section{7}

Tetewariwak $\left\{\begin{array}{l}\text { Tsini sonkwanoronkwa Iesos } \\ \text { Tsinikon sonkwentenranion. }\end{array}\right.$

Onwar ok onkweriane, akwa rotiataten

Tewatsennonni kati tetsitewariwakwas.

Iesakowanen $\left\{\begin{array}{l}\text { Akwekon ne iesakowanens } \\ \text { Sonhaa kwawennijostha; }\end{array}\right.$

Saiataseton sane waskwatenientense

Nok teskwaswatetennis nakonienterene.

Teseniteron $\left\{\begin{array}{l}\text { N'akatonnhetston iahte kakont } \\ \text { Sonhatsiwa saniahesen; }\end{array}\right.$

Ionakwen niha notkon, wakskwen iahte kakont,

Iah se taonkteronne, Iesos rakatienni.

Raonhatsiwa $\left\{\begin{array}{l}\text { Hetsitwanoronkwak ne lesos; } \\ \text { Ne sonkwatsennonniatennis; }\end{array}\right.$ 
Ne watenroseriio onkwatatekenha,

Akwekon sonkwaisnha hetsition nonkweri.

Tsini skwanonwes $\left\{\begin{array}{l}\text { Nahoten enkatonraseron? } \\ \text { Akwa wakonwenniioste }\end{array}\right.$

Enskat wakienterhaon, iakotonnhahere

Ne sakonhienterhane Okaristiiakon.

O sewenniio! $\left\{\begin{array}{l}\text { Iah othenon te sanoronse } \\ \text { Tsiatanonstat nakatonnhetst, }\end{array}\right.$

Tosa ioiataksatak, iotaksen nahoten.

Aonkeriwiioston, nok akatsennonni.

Iesos akatonnhetston

Saiataskatstenni ;

Sonha saiataskaneks

Sonha sanoronkwa.

Ne akonnhekon lesos

Nakonnoronkwake

T'si askwatienniheke

Okaristiiakon.

Seron aonkientake Tsi onkwe satonhon, Naketsientaniheke Akotonnhets nonkwe.

Satatewentetakwa

Tsi iehasentakwa

Nask wariwaseronnien

Na!akwatsennonni.
Tsi kanataraieronni

lieks ne tsieronke.

Tsi katsitsiaieronni

Iwat sanekwensa.

Tokenske nakatonnhets

Iawet watonkariaks, Tsini waskaneks onwe

Naseiatienhase.

Wakenontonks, o Iesos ! N'askataweiaten, Taetsiateraneken Oksa nakatonnhetst.

Onen wakoniatkatho I esos Sewenniio Tonsaskweriasateni Ise aionnhekon.

Karo kase, karo Iesos rakeni, sanikonhriio kowa. Iah othenon te wakonweskwanis, iah othenon onwentsiake. Karo ise sheionnheton ise seskontakwas nonkwe. Sotsi konnonwes, rakeniseriio, karo kase, karo, Iesos, ise seskontakwas nonkwe. Wahonnise tsi wakeronhiakens, koniatisaks. Takitenr, konnonwes, koniatisaks.

Ken kaien taiehawi raotinatarok Ratironhiakehronon ionkinontenre.

Tesakorhotonnis kaniseriio Watenroseriio Sonkwawenniio. 
Ne sakonoronkwa, raiatanoron Kaowenk karonhia ne sonkwaiennis.

Ne nokaristiia tekanerenston Aiakaonnhete ne karonhiake.

Iakonikontriio, iakoiat:saks, Iesos raweriasa iakawehtennis.

Iesos sonkwaienha hetsitwarori Nasonkwentenrheke tsini ionkwentent.

Hetsitwawenniiost tsi iakionnhenion; Iononwakte okon ensonkwatsiente.

Kento hetsitwasennaien, akwa raonha ne Sonkwawenniio, Rotaseton sane nok iawet ronwakens nene iontontats.

Tatsitewatontsolase, tewatstarha tsi ionkwariwaneren, Nentewatatkenronniheke, entwatkatho ne rotiesen.

Takwentenr nisa, o Iesos! takwalkarenrako tsini ionkwentent Twatkatho ostonha oni, tosa tetewatekhasi.

Ronwasennaien asteson Niio Roienha sesonkwaskontakwen, Raonha ronwaiatisaksek nonkwanikonhra okonha.

142

Twakwekon tewatskwentaren 'Tokenske ioriwanehrakwat Nok tatsiwariwakwase Iesos ken okaristiiakon Iahte kakonte renteron.

Saheto ioteriwaseton Raieronke raotonnhets, Tiotkon kanatarakon kenha Sase akwa tionkwehtakon.

0 ! tosa teionkwanonhianik Natsitwawenniiostake, Atsitwawennaieritseke Iahte kakont tsinenwe.

O Iesos! akwa ionehrakwat T'sini satanitenraskor: Tokenske ii onkwariwa Tiotkon kento sataseton.

Thawenninekens rohasen Tiotkon rowennarakwa Iesos Tahatsnente karonhiake.

Ne kati ionkwariwiioston, Tatsitewanonweraton

Tsini honikonhriio Iesos

Tsi oni nihotontatskon.

Akwa kwennitentase, Iesos, Askwentenre ne ionkwentent, Askwanikonhrahniratsek Akwanoronkwake onwe.

Wakwasennaien onwentsiake Ontaiakwanikonhrasal Etho akwasennaiensek Katke sonha karonhiake. 
Ken renteron onwe ne Iesos.

Hetsitewasennaien.

Roiatasetakwenhaties Sonkwawenniio Iah taonton ahonwaken Onen thare Iesos ne roiatanehrakwat Sewatiskwentaren, tsionkwe okonha, Hetsisewawenniiost

Hetsisewanenton tsinihonikonhriio Nok tsini hoiatanonwet

Hetsitewanonwenn ne sotsi

Sonkwanonwehon :

Hetsitewanenton

Ne sonkwentenranions

Tetsitewanonweraton.

Twanikonkrarak Niio ken renteron Ken rotaseton okaristiiakon . Twatiskwentaren nok twakwekon Twateweienton, hetsitwasennaien.

Taonken, Iesos, takwentenranions, Takwatsteristha, ne oriwakwekon Seriwahtontha kariwaksen, Asteson kwawis, seianerenstakwa.

Akwa tokenske, Iesos takwaienha, Takwaiatison nok seskwaskontakwen, Takwatsientanis nise tsieronke, Ne skionnlietstakon, tsiniskwakonnienstha

Akwekon oni niaiheionsere Sheiatakennhas tsi sheien okonha Sheiatanonstats nonwentsiakon Nokkaronhiahe sheiatenhawitha.

Katewentetha atonwesentsera, Katatensera, akwekon kasenna ; Konnonwes, Iosos, konnoronkwa Nok tsiatatake katiatanentakta.

Ise, o Wari, nonwa teskwakanere Ionkwatkennison, I esos tholaseton, Aesarane nasonkwentenre Tsini hanonwes etho naiakwaiere. 
Swatkonseriios, swatsennonni onwe

Swatennitenron, askwaienawase

Nasakwanenton ne lesos Krislos,

Onkwatonnhetston setsi rakonnienstha.

Ronwatinenton Roniha, Roienha, Nok oni Rotkon Roiatatokenti ;

Asen nihati, enskat ok Niio,

Oktiwakwekon ronwatisennaien. Amen.

145

Hetsilewanehrakwas Niio Roienha

'Tsini sonkwanonwes, tsini sonkwentenron.

Rotasetonhatie okaristiiakon

Akwa, akwa hetsitewasennaien.

Tsinenwe ontionnheke, Iesos, enkwanoronkwake Enkwawennarakwak, enkwawenniiostake.

Takwanikonhriiostak, satanitenraskon ;

Tiotkon, tiotkon akwanonwehake.

146

Iesos tekwanonweraton

Tokenske ken sataseton

Ionehrakwat takwanonwes,

Asteson skwentenranions.

Iesos sonkwaiatanonstaton

Naionkwaronhiakenhon

Iesos wasonkwahahisas,

Karonhiake eniakwe

Iesos sonkwentiohkwarakwen,

Raonha rotatiesaton,

Raonha shoseronniaton

Tsi ionkwariwaneren.

lesos onen kwennitentase,

Takwanikonhrahnirat

Aiesasennaientatiesek, Iahtakariwentane.

O Iesos konsennaiens, Okaristiiakon

Tsisehton sataseton

Sotsi tsiatanoron

O saiatanehrakwat!
Karonhiake katkeha

'Isi tsiteron onwe

Iahten tsi sataseton

Katke enkwatkatho

Katke sonha. 
Ratironhiakehronon

Tatehontatote

Ronatiskwentaronnion

Tsi iehasentakwa

Karonhiake nontare Tehoteroronte ;

Akwekon sonkwaniente Ratasetonhatie-

Ioriwatsanit! Iesos Tesonkwatkenseres ;
Akwa tsi ionkwataskat

T'si sonkwatiennire

- Aiawen onkweriasa

Akwa tahaiatak, Iahawite onwe

Tsinonni henteron.

Ise oni, o Wari, Seriasa askwani Nasakwanonwehake A kwa iahte kakont.
Iesos Sewenniio, Tekonnonweratons Akwa sataseton Okaristiiakon.

Niawen, Seweniio, Satanitenraskon ; Akwa ionehrakwat Takwentenronhaties

Tokenske Rakeni

Wakeriwaneren ;

T'si seniseriio

O Iesos, takitenr.
Takiatokef niha Sakatatrewate; Akwa iah nonwenton Eh taonsakiere.

Wakerihonte ken Naskataweiaten? Enskat tasatati Nok enskiewentane.

Koniaskaneks Iesos, Koniatisaks oni Wakonion nakeri Askataweiaten.

Iesos rakeni, akwa koniaskaneks Tosa sentoren naskataweiaten

Iesos rakeni, akwakonnonwes Tsiena nakweri, etho tsiterontak.

Seniseriio, takwanonwes onwe, Takwentenr askion nakwanonwehake.

Takwenheiase tsini skwanonwehon Aiawens oni nii akwenheiase.

Skatatrewatha tsini wakieranion, Takitenr, Iesos, aonsaskiatokef.

Takitenr kati, nakoniatorenne A ontasatsnent nakeriasakon. 
Sotsi wakataskat

Iesos Kristos rakatiennis

Sotsi wakataskat

Iahte wakerihonte.

Niawen, Sewenniio,

Tsini skenoronkwa ;

Akwa akeriane

Satatenonsotani

Oh nakiere nonwa

Takonnonweraton?

swaronhiakehronon

Takwaienawas niha.

Rakeni, konsennaiens, Takatiennis onwe
Tekonnonweratons

Akenikonhrakwekon.

Iesos konnekennis, Tosa takiatonti, Tiotkon tsiterontak Satatenonsotani.

Wakonwenniioste Tsi konnhe, rakeni, Tosa konnhek nii, Ise sonnhek kiaiakon

Ise, Wari, askon Ahinonweseke $\mathrm{Ne}$ Iesos hetsienha Kento nok karonhiake.

\section{1}

Riiatisaks akenikonhrakwekon Tsi renreron ne Sonkwawenniio; Riiatkathas tsinonni hotaseton, Onwentsiake iawet roronhionnis.

Akwa iotsennonniat

Ise Iesos sonhaa konsennaiens.

Tsini iotsennonniat

Iotonkote nakeriasakwekon

Tsi konnonwes, Iesos Niio,

Tsi konnonwes.

Rakatiennis ne nahaiatiioste

Akatonnhets nahnikwekonhake

Wahiiena ne riiatanoronkwa

Ok tsinenwe iahte hiiatonti.

Takionnhetha. ise saiatanonwet; Oriwiio tsi tekonkanere;

Ne wakroris tsi wakeriwí́oston,

Ne skonnhekon, lesos tsi koniataks.

Oh nahoten tenkonnonweratonte Sewenniio tsini ski tenranion Tesek onen tsi wakonion nakweri Tesek oni n'akkaseri okon. 
T'siniiore enwakerhenseronne Nonwentsiake tsi takwatennieton Wakniraton, enkonwennarakwake, Karo kihei, tosa skonwennonti.

Sewenniio takonwennentasere 'Tiokon nonwa akonnoronkwake, Tosa skonnhek nensewakenikonrhen Nakewenna tsi konrharatstenni.

Twatiaken tewataweiat ne tsi iehasentakwa Ne thenteron Iesos onkwe tesakorhare Akwa tsi ronikonhriio sonkwaiatisaks onwe Twatatkenronnihek kati tsini sonkwakonnienston.

Ne enionkwannhotonkwase isi renteron ne Iesos Entsitwariwaientere tsi ionkwariwaneren Aseken rariwanonwes niakaweriasanonwaks Nok akonikonhrakwekon onen tsi ronwanonwes.

Takwatontats, Sewenniio, akwa tsi kwanekennis, Takwarisien nonkweriane tsini kaien niotaksen : $\mathrm{Ne}$ tenhnon takwariwataas ne kariwiio okon $\mathrm{Ne}$ ok onsaiakwatsterist iesasennaientakwa.

Ise sewaiatatokenti karonhiake sewenteron Ise ohenton, o Wari, akwa kwennitentase, Takwawennakaratatsek ne tsi sakwasennaiens Nok tsi sakwawenniiostane tsi swataskatstenni.

Niio Sewenniio, sonhaa skowanen $\left\{\begin{array}{l}\text { akwekon teiesanonweratons } \\ \text { ii oni tekwanonweratons. }\end{array}\right.$ Tsini satsteristha nonwentsiakwekon $\left\{\begin{array}{l}\text { ionkwanikonhrasennies } \\ \text { sakowanatanions. }\end{array}\right.$ Tsini ioianere ne ken karonhiake $\left\{\begin{array}{c}\text { tewatkathos iotsistokwaron- } \\ \text { nion } \\ \text { tsi nateionkwaswatetenni. }\end{array}\right.$ Ne sasennaientak tsini skwentenranions $\left\{\begin{array}{c}\text { iakionkwe nonwen- } \\ \text { tsiake } \\ \text { sateskwaiatison. }\end{array}\right.$ Ne ken karonhiate ne ken ionwentsiate $\left\{\begin{array}{l}\text { akwekon tsini kaniatare- } \\ \text { nion } \\ \text { Niio hetsitwasennaien. }\end{array}\right.$ 
Ise oni nene swatiesen okonha $\{$ tetsiswanonweraton

Ise oni nene swatiesen okonha $\{$ tetsisewariwakwas.

Ne ken iorakote ne ionkwatonniennis $\left\{\begin{array}{l}\text { tsini iakionnhekwen non- } \\ \text { wentsiake } \\ \text { ise, Niio, takwasahani. }\end{array}\right.$

Tiotkon kati onen enkwanatonseke $\left\{\begin{array}{l}\text { tsini tkarakwınekens } \\ \text { ise, Niio, takwasahani. }\end{array}\right.$

Kenkwitehne kati akennhake oni

f entiekehne asonthenne oni $\{$ atsitwasennanoronkwake.

Swaronniakehronon ne tesewatkenni $\left\{\begin{array}{l}\text { tetsisewariwakwasehatie } \\ \text { letsisewarensaronniontie. }\end{array}\right.$

lakwaskaneks oni nasakwasennaien $\left\{\begin{array}{l}\text { nonwentsiake tsi iakwes } \\ \text { nonwa nok tsinenwe. }\end{array}\right.$

Toni iotsennonniat!

Wahakatienhase lesos,

Toni iotsennonniat!

'Tsi iakenikwekon.

Akeriasakon renteron

'Tirehta ratasetha, Iesos iakenikwekon

Toni iotsennonnial!

$\mathrm{Ka}$ onte nontawe Iesos wahakiatisake?

$\mathrm{Ka}$ onte nont we

Tsi nahakkonnienste?

Iah se te wakerihonte

'T'sini wakeriwaien.

Ka onte nontawe

Tahakitenrane?

Senha ken sewaien, Sewaiatatok ntison, Senha ken sewaien Naeswatsennonni?
Kawenniio twakehtakon

Raonha Rawenniio

Kaien nakatsennonni

Tsini swatsennonni.

Iotek nakeriasa

Tsinahinonwehak Iesos ;

Iotek nakeriasa

Ne ronwanonwestha

Tosa riiatanikonrken

Tsinenwe enkonnheke,

Akeriane renteron,

Iotek nakeriasa

Asewenniioke,

o Iesos! nakenikonkra, Asewenniioke

Akwekon tsi konnhe.

Skatatswens tsini wakieren, Akwa skatatrewatha, Iesos, a kenikonhra Asewenniioke.

155

Iesos ken tsiteron Ionehrakwat satanitenraskon Takwaskontakwaton Nok satatewentetonne Sewenniio, askwentenre Tosa takwariwatake Askwaienawasehek Nakwanikonhraierite
Aiesasennaien

Nonwa ise okaristiiakon

Akwanonwehake

Tsini iesanonwehonhatie

Aseken tisariwaieri

Nok taiakwaiatanonstaton

Ii oni iakwehre

Asakion onwatonnhetston. 


\section{6}

Ratasetha Sonkwawenniio Kento tsi iehasentakwa. 0 ! hetsitewawenniiostak Tsiniiot ne karonhiake.

lesos ise saiatanonwet onwe, Kento satatkenronnion : Askitenre sanonsatokentike Takonriwakwaseheke.

Ne kaskaneks akienterhane Tsiatakta iakotsennonni Sonhaa koniatisakseke Akentonni onwentsiake.

Onwentsiake sewanakere Kasene tewakwekonhak
Twasennaien tsi rakowanen, Tsi oni ronikonhriio.

Sonhaa, Iesos, takonnhetha Sonha kati konnoronkwa

Kento tsi ikes nonwentsiake, Nok oni ne karonhiake.

O Wari! Iesos senikwekon, Hiawirawi onkwe okon ; Ise kati kwanistenha, Sannhatsera onkwe okon.

'T'si ises ionkwaiatentonne, Askwanentsawakonhake, Tosa aiakwathaharako, Akwarane karonhiake.

\section{7}

Sewenniio, takwatsennonniatennis

Tawataskatstennis

Tsi takwatiennis nonwentsiake

Takwanoronkwa ionehrakwat

Tiotkon satatewentetonhaties

Onkwatonnhetston

Senontens ne sawaratokenti.

Ionkwatsennonni, Sewenniio

Onkweriane tekwanonweratons

Nok iakwaskaneks

Iahte kakont akwanoronkwake

Ise satekat nonkweriane,

Tiesanonwestha nakatsiroten

Aiotekhake

Iawet tstnakwaiatisakseke.

- Tetewariwak tewakwekon Iesos hetsitewasennaienhek, $\mathrm{Ne}$ onkwarenna

Tsinenwe tsini sonkwanoronkwa.

\section{8}

Okaristiianoron

Twakwekon ne tionnhekon ;

Akwa teionkwehtakon

Niio raiatakwekon.
Oriwiio thotati

Iesos Sonkwawenniio, Rawen takwanakeren, Ietsitenr ne iakotent. 
Rowi ratsihenstatsi

A hatkaristiionni, Raonha raowenna

Ne roieratonhatie.

Ken kanataranoron Niio ne raowaronk; Nok ne notsitsia kenha Niio raonekwensa.

Sewenniio, tisehton, Ken nonwa takwentenron, Karonhiake tsiteron Nok okaristiiakon.

O ktiwakwekon sane Kenteron sanikonhra; Iahnonwent on kaneka Taiesaiataioha.

O wenniseranoron! Ne Niio sonkwentenron. Akwa tewatsennonni Tsi Niio sonkwatiennis.
Nok tehnon tewatsiaken, Tosa hetsitwatiesat, Tosa tsitwanakeren Sitas ne ronkwetaksen.

Akwa rotkaronnihon, Niio rotewenteton Ne nonwa rotkontakon Roronhiaken noneshon

Iesos senikwekon onwe, Satkon Saiatatokenti, Takwentenr ise askion N'onikonhratokenti.

O Niio! sonhatsiwa Tsiatakweniio kowa; Sonha oni tsiatanoron Nok satanitenraskon.

Sonha kati kwaniahesen, Takwariwaseronnien Tsineniakwatonnhokten, Ok aiakwatsennonni.

Ionehrakwat, o Sonkwawenniio!

Ionehrakwat satanitenraskon

Ionwesen akwanonwehake

Senha kati nise takwanonwes.

1 Tiotkon kati enkwanonwehake

Aseken takwentenranion;

Tiotkon kati enkwanonwaheke

Tsinenwe eniakionnheke.

Senha aionwesenhake

Sonha akwaskaneke,

Aiakwalsennonni akwanonwehake, Aseken eh niskwarharatstenni,

Sonhaa takwanikonhriiostha.

Seshetsientane nonkwatonnhetston

Akwa, Jesos, takwatontats

Tsini satanitenraskon,

Aiakwatsennonni, akwanonwehake

Sonhaa akwaskanekseke.

Swakwekon swaronhiakehronon, tsionkwe okon oni, Twakwekon tsitwanonweraton Niio ne Roienha

Onwatsi warawe ken iehasentakwa. 
Akwa ioriwanehrakwaton tsi naawen nonwa; Sonterisi kanatarok nene otsitsia oni; Iesos iehenteron ne raiatakwekon.

Iesos ise takwanonwese ioriwakenniaton, Asteson tontakwatkensere kento iakwanakere ; Ionkwentent iakionkwe tontakweutenrane.

Onen ki tekwanonweraton, Iesos ken tsiteron, Iakwatontats Isini saieren, isen: iahte kakonte Enwakataseton okaristiiakon.

Sonhaa ki oni kwaniahesen, Iesos takwaienha, Iah othenon te sanoronse, ioianere sonha. Tiotkon shetenratha iakotent ohonha.

Iesos tokenske kwanonwese, Wari takwaienha Tiotkon asonkwaianerenste Iesos tsiatatienha Kento rotaseton okaristiiakon

Ise sasenseratokenti, saiatat ne Niio lesaniha nok lesaienha, ise one Satkon Saiatatokenti, tekwanonweratons.

Taswatotek swariwiioston Swanehrako ne tsi rohasen Iesos tahatsnente ne karonhiake Okaristiiakon tihenteron.

Sewanehrako ne karonhiake, Iawet ne tetewakanere, Oriwiio ratironhiakehronon Tiotkon ratikwekonhaties Iesos

Tokenske ronatkonseriios Ronatiskwentare tsi Iesos Renteron nonwa tsi iehasentakwa, Ionehrakwat tsini honwanentons.

Ise, Wari, kwennakeraton, Nonwa akwa ionkwatsennonni Tsi hetsitwatkathos netsienha Iesos

Tsi renteron tsi iehasentakwa.

Ne kati kwanistenseriio, Atsennitentase netsienha Asonkwentenre ne tsini ionkwenten Tsi itewes kento nonwentsiake. 
Ise sewaronhiakehronon

Tetsisewakanere ne Iesos,

Askwentenre nok hetsisewahrori

Onen tsi sakweriasawis

O Iesos! watkwatontsothase

Akwa tionkwanikonhrasahaton,

Nokoni akw. watkwanonweraton,

Askwentenre askion karonhiake.

162

Aonton ken, okaristianoron, Naiesaken ne tiakawehtakon

Nok iahtaiesanonweseke?

Ise, Iesos, ne okaristiia

Takenontens ne sawaratokenti

Nakonnhekon notsiratokenti.

Oh! iawekas nakwa nakatonnhetston

Ioien onwe naesanonwene

Iotentonne tsinahe n'akatonnhets

Iah se akwa te sanoronkwakwe,

Onen nonwa akwa wateswateten

Naesaken okaristiiakon.

Iahte skaien tsi ionkwene ionwesen,

Ne tsionwesen tsi iehasentakwa ;

Watohetstha nonkwe iontonweskwatha

Iesanonwes, ionwesen tsinenwe.

Twakehtakon Iesos tsi sataseton,

Ne sateiot takonkanerake,

Teioswathe onen nakenikonhra

Saiatisaks naesenikwekon ;

Karonhiake enskiatatokentiste

Nonwentsiake onkwe satatonni

Enskonnhekon tsini sanikonhroten

Enw்akati nonkwe iontonwesens.

Ionehrakwat, Iesos, ne senheiaton $\mathrm{Ne}$ iakionkwe tsini skwanoronkwa, Ionehrakwat nonkwe teieronwekon, Iahte iekens tsini skwentenranion. Oh naiawen, Jesos, nakkweni tiotkon Nonkwe okon aiesanonwene.

Nonwentsiake tsinonni sataseton, Naiesaken oni karonhiake?

Iah othenon te iakotsennonniatskon Niahte ionton : Iesos rinoronkwa 
Ne kaskaneks naiesanoronkwake

Tsi tsiteron okaristiiakon ;

Oh nahoten iakoien nonkwe okon

Iatakene tsi saiatanonwol!

Iah nonwenton taonsakoniatonti

'Tiotkon nonwa enkonnoronkwake.

To niiore Iesos niienkahewe

Nenkatkatho ne wenniseriio,

Nenwakati ne ken ionkionwentsiate,

Nenkonrane onen karonhiake?

Etho akwa, Iesos, iesanoronkwa,

Iah nonwenton taiesaiatonti ;

Askitenre, o lesos! teseskiatak

Karonhiake askwatsennonniaten.

163

Akwa wakeronhiaken

Nakenikonhrakon,

Ne waheronhiakentha

Tsi Iesos rinonwes.

A keronhiakehronon

lesos hetsroriana

Tsi akwa ni kaskaneks

Ahiienterhane.

Karo nienekanonwaks

Ne iakoniatathens,

Senha riiatanonwaks

Iesos ne rinonwes.

Roiatanonwet onwe

Nene riiatisaks :

Asteson ne tikehre

$\mathrm{O}$ ! ahiiatkatho.
Tiotkon nakenikonhra Iaonsennha onwe Tsi iah tiiaontkatho Raiatiio kowa.

Akwa raonhatsiwa Ne riiatarakwas:

Aiawen nakatonnhets

Tahiaterahneken.

Ahatekate onwe

Nakenikonhrakon :

Tiotkon ahinonwesek

Tsiniiore konnhe.

Katke enkoniatkatho, Iesos, karonhiake

Katke eh ienskiteron

Tsi ionwesen kowa.

Nonwa ken wente, twatateken okon, Rawenniioke $\mathrm{Ne}$ ionkinonkon ionkwaiatateri : twatsennonni kati, Tatsitewanonweraton Iesos sonkwentenron nonwa kenwente.

Ioriwatsanit wakeriwaneren, ioriwatsanit Tsini konwennontion. Ohni sanikcnhroten tsi takwataweiatenni? Iesos, enkwaiesate, skennen tasatati, enskiewentane

Akwatokenske akenikonhrakwekon wakerientaksens [ton. Tsini konwennontion : akwa skatatrewatha tsi konnikonhraksaWakaterientason, Iesos, iah nonwenton takonwennontı. 
Iesos tokenske tsi tewakehtakon ken sataseton Okaristiiakon. Etho sataseton, akwa tsiatakwekon, Tsini tsiatanoron, konsennaiens Iesos, tekonnonweratons.

Tiotkon tsinahe takwaiatononstals, takwanonwese A kwa ionehrakwat, satanitenraskon, sekon takwentenron, Takwawis ne tsieronke, tsini siatanoron, akwa konnonwes.

Ionwesen akwa tsi takwatiennire; tekonsteriatha Enskwanontenseke; konhiennitentase, Iesos karo kase, Sotsi ákwa kaskaneks atsiterontako akweriane.

165

Ionwesen tatsitwariwakwase Nok oni naetsitwasennaien Renteron Iesis okaristiiakon Ionehrakwat akwa ionehrakwat Wasonkwatsennonniat Nok wasonkwatienhase.

Akwekon kati tetwariwak Ne tsi Iesos sonkwentenronhatie.

Entakta tsi ronwariosere Iesos Okaristia ne roriwison;

Oriwiio sonkwariwisahani

Aseken sotsi sonkwanoronkwa ;

Hetsitwanenton kati

Hetsitwanonwenn oni.

Tionkwehtakon ken okaristia Tsi renteron Iesos Niio Onkwe, Raieronke raonek wensa oni

Raotonnhets ionatasetakon

Ne kanatarok kenha

Otsitsia kenha oni

Karonhiake ieshenteron ne Iesos

Tsi raweientehtakon Roniha

Ionehrakwat oni nonwentsiake

Tihenteron okaristiiakon

Ratironhiakehronon

Tiotkon ronwasennaiens.

Akwekon Iesos sakohonkarawis

Rotenniote okaristiiakon

Sakoien okonha ne sakononteris

Raieronke nok raonekwensa;

Akwa ne ion hi akwat,

$\mathrm{O}$ ! rotanitenraskon. 
Okaristiiakon sonkwaniahesen

Iesos ne ionkwanikonhranonwaks

Ionkwanonwaktani sesonkwatsientha

Nok oni tesonkwasnie ionkwentent, Sonkwanikonhrahnirats

$\mathrm{Ne}$ ionkionnhatsioha.

\section{6}

Iesos kwawenniiostha akwakwekon Okaristiiakon tsi sataseton

Ise skwaiatison skwatsteristonhatie

Ise Sewenniio akwekon tsi iakionnhe.

Tsi senheion ne takwaskontakwaton Nok okaristia takionnhetstakon;

Akwa ionehrakwat Iesos takwanonwes

Satatewenteton, ii aiakionnheke.

Wari kwennakeraton nok skwaienha

Wari tsiati Niio ronwanonwestha

Saiatatokenti takwaterennaienhas

Iesos ne hetsienha asakwanonwehak

Ise sewatkon sewaronhiakehronon

Sewaiatare lesos tsi rotaseton, Sewatanitenraskon skwaiatanonstate, Tosa raieronke aiakwatiesate,

Iesos tehwanonwerators, satatewenteton iakionkwe onkwariwake. Ise takwannhotonkwennis karonhiake: tiotkon ioiote notkonseraksen aionkwanikonhrotako. Tiotkon notkon ionkwanikonhrotakwas; askwentenre, Iesos, askwaiatakennha. Etho naiawen.

168

Konsennaiens Iesos ontatenikonhrasahate Niio okaristiiakon sataseton

Ise Sewenniio nakatonnhetston

Kennikonhroktakwa tsini tsiatanoron.

Iakotaskat wahonnise ronwaken lesos

Onwentsiake sireskwe ronwawennahronken

Iah nonwa te ikens tsi iehasentakwa

Nok tewakehtakon kento renteron.

Sahrenheie Iesos tekaiasontne

Iahte hototasion Nito tsini haiatoten 
Nok ne kento tsi iehasentakwa

Rotaseton onkwe tsini haiatoten.

O Iesos! saiatanéhrakwal kowa, Askiatohare sanekwensalokentike;

$\mathrm{Ne}$ skatstarat oi iokweniatseraien

Aonsaiakoskontako akwekon nonkwe.

Satatiteron okaristiiakon, seron :

Aionnhekwen nakotonnhetston nonkwe;

Aiakehiarakwake tsi kheiehase

Akheiatsennonniaten nenkheiatienhase.

O Iesos! sataseton okaristiiakon,

Konnekennis a kenikonhrakwekon

Askatokaten saiatanehrakwatsera

Karonhiake tsinonni ionwesen kowa.

169

O Iesos, tekonnoronkwanions

Toni iotsennonniat o Iesos!

Senontens nonkwatonnhetston; Tsi takwawis ne tsieronke,

Tosa kati aiontiesate

'Tsini seialanoronstha.

Heren sesheiatenhawitha Niakoriwanerahakskon, Nok ne tesheiatatirontha Ne tiakoriwaieriton. lah na etho te shetenrase Ne ratironhiakehronon.
Askitenrheke, Sewenniio, Tosa raetiatekhasi, Tiotkon aonkenaktotake Iataktane tsi tsiteron.

\section{0}

Iesos raweriasatokenti, Tetewariwakwas nonwa, Etho se ok non tkaiakens Naiontsennonni nonkwe okon.

Ise Iesos, shenikonhrahnirats nonkwe, Ise takwaialahnirats iakionkwe, Iseke iakorharekwen nonkwe okon. Satatewenteton tsuli takwanonwes.

Ise shenikonhrahnirats Nonkwe onwentsiake iiens, Ise sheiataskatstennis Eronhiakehronon okon.

O Iesos takwaskontakwen, Takwanekwensarise, Askwanakton, Sewenniio, Seriasatokentike. 
Raweriasanonwet kowa,

Iotekha tsi takwanonwes, Iesos,

Sonha saianere, sonha tsiatanoron,

Saiatatokenti, satanitenraskon-

Asewenniioke nonkweriasa,

Wakion nonkweriasa okon. Amen.

171

Ise Wari Watere, Taonken sataskit! Iesos hetsenoronkwa Hianoronkwa oni.

Raweriasatokenti

Ne Sonkwawenniio

Tsi iotanitenraskon

'Tetewanonweraton.

Onen sonterakewe Tsi sariwaneren, Ne raonha hiarori Iesos n'hetsenonwes.
Tetsiatennosen kenha Rasar shotonnheton; Tsiatatekenha Marta, Tsiatsennonni niha.

Kaiasakta Iesos Tesatontsotakwe : San nok oni ne Wari Sewakwekonhatie

Satstarha tsi raiatat Ne tsiatatenonwes: Nonen sholatonnheton Satsennonni kowa.

Tsiatahonsatat, tsionkwe okon, Tsi netsisewasnorakwa Iesos, Raton: ionehrakwat tsi kwanonwes

Nok iahte sewatsennonniatskon. Tiotkon sane kwentenranions, Katke onte enseskwaieritse, Enskwanikonhraierite?

Sewenniio, etho naiawen, Tsini waskaneks seriasatokenti ; Tokenske eso ionkwariwaneren, Nok onen nonwa Kwanikonhraseronniane.

Sewenniio, takwasennion, Ise satiatakweniiost nonkweriasa ; Iahte kakonte iakwahnirats, sonhaa enkwasennaien, Enkwarıoronkwake.

Tiotkon sonkwaiatisaks Iesos Nok tesonkwaswatetennis; Onka aiekweni iahtahononwesere Ne rotanitenraskon kowa? 
Seriasatokentike, Sewenniio, Akwekon ionkwatekwasentakwa,

Ise sonhaa takwawis skennen,

Sonhaa takionweskwatennis.

Iakotaskat, Sewenniio,

Ne konwaniahesen seriasatokenti,

Tsi ok nahoten naiakoiatawen,

Tiotkon skennen kowa

N'akonikonhrakon.

Tekweriasawehestanion,

Ionkwariwanerahakskon ;

Ne tenhnon ionkwarharekwen

Tsi satanitenraskon kowa,

Kwanekennis, sasanikonrhen

Tsi kwanikonhraksatanion,

Karo akwekon naiakwenheie,

Tosa aiakwatanikonrhen.

Ionkwanikonhrakontakwen

Tsi neniakwakweni

Enkwanikonhraseronni,

Sewenniio, tosa seriwarako

Tsi iakonkwetaksens

Iesasentanionnis.

Iesos raweriasatokenti, Tetewariwakwas nonwa, Etho se ok non tkaiakens

Naiontsennonni nonkwe okon,

Raweriasatokentike Akwekon tewatekwasen.

Tewatotarho tsi ionkwehne Iah nonwenton skennen te ken ; Iesos hetsitwaiatisak, Roien naetwatsennonni.

Tihaweton tsi ronwahason Ne tekaientannhareke; Iokaronte raiatake

- Iaontasetakwake nonkwe.

Ionkwasere notkonseraksen Naionkwanikonhrotako,
Ka naetwatekwaseke

Tosa aionkwanonterane?

Oh! hetsitewanakton Iesos A hatien nonkweriasakon, Saonkweriasat aonton Tsi entsisewanoronkwake.

$\mathrm{Ne}$ hetsitewanoronkwatak Tewakweni nonkionkweta Ietinikonhrenhawitak Nahonwanoronkwake oni.

Aweriasalokenti kowa, Iotekha tsi takwanonwes; Tontakwaiatatironten Ethonon aiakwatorisen.

Iesos seriasatokenti, Tionkwaiatak nonwentsiake. 
T'sitenr nisa, tsileur Sewenniio, Ne sentiohkwa Kentiohkwatokenti, Eso nonwa iotkenrenseronni, Ionkwarhare enseiatakennha.

Kwennilentase takwentenrat Tsini ionkwanoronkwa seriasakenti.

Sewenniio hetsiatanonstatsek Ne Tier raonaktake irate, Sakwaniha eso roronhiaken, $\mathrm{K}$ wanekennis atsitsennonniaten.

Shetenr nisa, shetenr, Sewenniio, Iakonkwetaksens iesasentennis, lakotent iahte iesaienteri, Shetenr aonsaiontatrewate.

Sewenniio, skwanistenserawi Iah nonwenton te ioiatoronlon, Wari ne ionkwaterennaiennis Ienionkwatatiase tsi tsiteron.

.175

Kweh! sanonsanoron, Sanonsatokenti, T'si thotinonsote Iesos, Wari, Sose.

Etho thawenonton Raronhiakehronon, Wahrenhase Wari : Tekonnoronkwanion.

Taonken tsiatiio, Niio senikwekon, Ise ne hetsienha Onwa watonstre.

Tontakenron Wari : Rakiatawenk Niio, Raonha thennonton, Eh naiawen niha.
Ethone ne Niio Onkwe rotatonni Kaiatakon Wari Ioiatatokenti.

Tewanonsanonwenn $\mathrm{Ne}$ kanonsanoron, Kanonsiio kowa, Iononsanehrakwat.

Iah nonwa te skaien Sitaerononke; Inon niehotiha N'eronhiakehronon. Iesos, konsennaiens Niio ne Hianiha Ne Wari saienha Nok Sose hiehiaron.

Ise Wari, tsiatanoron, Iesennakeraton karonhiake, Sonhatsiwa kwaniahesen, Oneshonronon ionkwanosas, Ioiole naionkwakaronni, Watoratis nonkwe okon ; Shetenr niakoriwanerahakskon. Tosa, Wari, takwatewentet. 
Askwentenre, tosa othenon Aioiatoron nonk watonnhetston Nok takwaiatakennhasek. lakwaskaneks iakwakwekon ; Taontaskwanerakeionkwenten Iah othen on te sanoron Nasonkwentenre Rawenniio.

Senistenseriio, askiteron

Katke sonha karonhiake tsiatakta.

Wakatsennonni tsi takenoronkwa, Wakonweskwani ne akennontonnion, Ne teskiatieren katsitsianoronson Oserakhonson wakonnhetonhatie.

Katke ienkahewe wenniseranoron Entenikwekonhake karonhiake?

Tsiniiore ienkonrane, o Wari, Sasenna wakenikonhrahnirats.

Wakonweskwani akonnaton Wari Tohka konnaton tsini wenniseres Nok kattokas, o satanitenraskon! Sheiatakennhas ne iesaniahesen.

178

Tekonnoronkwanions, Wari, Ise tesentenhawitha,

Iah okti te iakostontstha, Ne iesennitentase ;

Asatontate tsi ionterennaiens

Sheien okonha nonwentsiake iiens.

Sonha hiakonnienstha ne Niio, Takwaiatanonstat iontkaronniatha

Niatekon tsini iawenserons Aiontkaronni nonwentsiake, Nehtiioke, kaniatarake, Iah nonwenton skennen te ken, Ionkwahteron naionkwasenni notkon Nok aionkwaiatente nonwentsiakon.

Tsi iiens nonkwe nonwentsiake Ne sakat taiontstekafa Kaniatarake kowa, Tiotknn takaweralase. Iotteron taiakolieronnionse Aieskone nok naiaiheie. 
Iakotaskat iakotehiaron

Konwanoronkwa ne Wari,

Iakotaskat iakorennhaon

Ionton: ise, TVari, takienha,

Iah nonwenton taiakotewentete

Tiotkon oni eniakoiatakennha.

Aontaskwannhe, o Wari, Tsi neniakwatonnhoktane, Askwaienawakonhake, Askwaterenuaienhase

Ethone tsi netsienha karonhiakeson

Tentre tensonkwaiatoretana.

Niio hetsicnha, senistenseriio Sheiatakennhas iaiheionsere,

Takionweskwatennis,

Konniahesen, konnoronkwa,

Tiotkon enkonnoronkwake.

Sheia wis niakonikonhraksens Skennen aonsaionnontonnion, Nok iahte sheiatewentetha Niakoriwanerahakskon.

Shetenre ne teionsenthoserons Iakotaskat iakorenrihaon Nok ne teiontonnhakarias

Ionton: Ise, TVari, takienha.
Wakenistensen ne Wari, Ionehrakwat wakenonwes Tiotkon wakitenranions, Wakenistensen ne Wari, Ionwesen akhenaton, Wakonweskwanitha.

To onte, Wari, riakotaskat Iesawi nakonikonhra?

$\mathrm{Ka}$ onte naietsenri nonwentsiake Aiawens nenkatonnloktane Netho naion wesenhake?

[waks Tosa taonkenikonhrarha notkon Tsinitsion sewanikonhranon- Nok akonrane karonhiake.

\section{1}

Wakenaktaien karonhiake, rakerharatstenni Rawenniio, Iakiatatekenha ne Iesos nok istenha ne Wari. 
Tokenske onte ken, Wari ne sanistenlıa? Katiken karonhiake tisennakeraton? Onka iesarihonnienni netho naesewennoten, Atsenatonkwake Iesos tsiatatekenha?

Kaiatonseranoron ne ionkwarihonniennis, Kahawe Rawenniio raowennatokenti, Waton : tsi ensaterennaien, ensiron: Takwaienha Karonhiake tesiteron, aiesawenniiostake.

Ionkwatenro Iesos, ii onkwariwake Tsinonni kanonsaksen ronakeraton, Tehokwen nonkwaronhiakensera okon, $\Lambda$ kwa sesonkwaierenhon tsini sonkwanoronkwa.

Ranonweskwe lesos, onwentsiake sireskwe, Asakonaton nonkwe rontateken okonha ; - Ne ionkwatokatakwa iah ok sewatieren te ken Sonkwaronhiakense nok sonkwenheiase.

Katke ienkahewe wenniseranoron kowa Enkatewentete ne ken ionwentsiaksen? Enkatsennonni karonhiake Raiatakta lesos, tiotkon tenkeriwakwe.

182

Takwaien okonka, Wari saiatatokenti, Onen iakwahnirats enkwanoronkwake, Tsinenwe onwentsiake eniakweseke, Enkwakonnienstake, enkwasennaienheke tiotkon.

Ne ionkwahniraton, takwaien okonha, Wakion nonkweriasa, ise sawenk onwe, A kwa ronatonte ratironhiakehronon, Wakiatonti oneshonoron enkenoronkwake ne Wari.

Tsinikon enionkwanikonhrotako notkon, Nok enionkwannhane niahte ioianere, Oksa enskatekwate, enkiron : eren saset, Iah ise te sawenk nakweri, ne Wari aowenk.

Okti oriwakon aionkwanakwase notkon, Ne tiotkon ioiote aionkwakaronni :

Iahtaontkweni ne Wari teiotson, Teioniarorarakon nohniaraksenskwa.

Tiotkon kati tewaniahesenhak ne Wari, Iahta iontkaronni ne konwanoronkwa, Eniakoienawase akonwasenni notkon, Aonha tiotkon iotkwenionhaties Niio ronistenha. 
183

Ka nitewes ionkwatonhahere Ne Wari akoien okonha, Onkwahenton ioken ken wente? Tekonwarensaronnions.

Ka nitewes tsi karenniioson

Iakotontenion ne kento?

Kanistenseriio ionkwaienha, Tewentanoronst ken wente, Ition nonkweriasa ne Wari Aositakta tsi kaiatare.

Asiena, o Wari, tsi kwawis Nonk wanikonhra nok tsi iakion-

Ionkwarhare enseskwaieritse rnle, Iah nonwenton taontohetste. Katke sonha karonhiake.

Ise sewaronhiakehronon, Ietsinontiio ne Wari, Aiakwawennakwekonhake 'Tsi teniakwariwakwase Taetewaieste nonkwarenna Tsi nahote̊n teieriwakwa

Aionkwanikonhrahnironhake Tsi tewasennaiens ne Wari ; Tosa taonsaionkwateriwatenion Tsi nonwa nionkwanikonhrotens ;

Akwekon wahtons ken nonwenNok oni ne ioianereson ; [tsiake, Nok ne tokenske iesanonwes

Ise, Wari, satanitenraskon, Tesnekanerak sheien okon, Tiotkon sheiatanonstatsek Ne iontkaronniatha okonha, Asheion ne tiakoriwaieris Aiakoiatatokentiste, Nok niakoriwanerahakskon Sheion aonsaiontatrewate.

184

Ronatsennonni ratironhiakehronon Sontaweiate Wari karonhiake, Tsi raweientehtakon ne roienha Ionaktiio konwennakeraton.

Iaetewatenniete netho nonkwanikonhra Tsi ieteron ne Wari aetewakwekonhak. Iahte kaien natsennonniat kento nonwentsiake, Tewaskaneksek kati aetewarane.

Tetiokaras onwe nonwentsiake, Tiotkon iakonikonhraksenserons, Aiawen katke sonha iakonrane Tsi tsiteron, Wari, karonhiake.

Tiotkon iokwisron notkonseraksen Aionkwakwa, Wari, tsi kwanonwes, Nok iah nonwenton taiakwatontate, Tiotkon senha enkwanoronkwake.

To neniontsennonni sheien okonha, Wari, nonen ensewakwekonhake!

Tiotkon teniesariwakwaseke, Iah nonwenton taesewatekhasi. 
185

Ise sewaien okonha, konwennakeraton karonhiake, T'aesewariwakwase kanistenseratokenti ;

Aesewatnanelake nonwa ne kawennanoron ;

Ionnhek, ionnhek ne Wari, ionkwanitensen karonhiake, Ionehrakwat tsi ionkwanonwes, aetion nonkwanikonhra, Karenniioson tetwariwak, tiotkon enkwanonwene, 'Tiolkon enkwasennaien, tiotkon tenkwariwakwase.

Onkwanikonhrakwekon nonwa iakwahnirats : Wari, enkwanoronkwake tsinenwe eniakionnheke, Nok neniakwatonnhokten teniakwariwakwase Ionnhek, ne Wari ne ionkwanoronkwa ; tiotkon.

\section{6}

Sonhaa, Wari, iakwakweken kwaniahesen, Takwaien okon, takwaterennaienhas tiotkon.

Iah nonwenton te ioiatoronhon kariwanerahakserakaion, Iah nonwenton te ioiataksaton, ok si ranoronkwa ne Niio.

Iahte ionakwaienterhaon, kaiatiio naotonnhetst Kaiatakon rotiataten ne akwekon roteweiennison.

Iah othenon te ionoronse aionkwaia takennha, Kaiati ioianerenstakwa, iotanitenraskon kowa.

Aonhaa ionkionweskwatennis nene kanistenseriio, Ieiotohetslon tsini wattokha, akwekon ioriwaieriton.

A onhaa tewentenhawitha, teionkwaswatetennis, Kanousanoron, ne karonhia iakonnhotonkwennis nonkwe.

Tsiakotsientha teiakokonhentonnions iahte iakowentetha Iakonikonhraksenserons, tiotkon iakonikonhrahnirats.

Wari, konwennakeraton ratironhiakeron on Nok oni iontekwasentakwa niakoriwanerahakskon.

187

Iseke, Wari, nonkwe okon iakorharekwen. Konsennaiens entiekehne, asonthenne.

Iah othenon te saiatoronhon ne iotaksen, Hetsienha Niio, sheien okonha nonkwe okon.

Iah othenon te sanoron Rawenniioke, Iesanontiio nonwentsiake nok karonhiake.

Tokat ahakon ne Rawenniio aonkwatsehak, A katsennonni akonnonsonnien, kanonsiio.

Kenonwes, Wari, akonsennaien, akonneken Takonriwakwase tsini saiatanehrakwat. 
Iah nonwenton te saiatoron Ne kariwaneren, Wari aontaskwatkatho, Shetenr ne sheien okonha.

Ise shennhotonk wennis Nonkwe kanatatokenti, Ise sheiawis aiontsennonni Tsinonni iakonnhe onwe.

Askion tosa aiakwaienterhane Ioteriwatehat,
'Tosa nonwenton aioiatoron

Nonkweriasa ne iotaksen.

Eso sakwanikonhraksaton

Ne sonkwaiatison, Ise satsenikonhraseronni Tosa asonkwahrewate.

Ise senistenseriio kowa, Ne kwennakeraton, Sheiatanonstat iesanonwes. Ne iontkaronniatha okon.

Iotsennonni Wari, akwa tewatonnharonnion, Konwennakeraton ratironhiakehronon, Konwaiatanehrakwas, konwasennaiens.

Sataetewatsennonni karonhiake tsi ieteron. Etho ens naiawen.

190

Ikehre akesennaien Karonhiake konwakowanen; Aiakawennakwekonhak Akwekon eronhiakehronon.

Satontat kati, Waria, Sonlraa kento satenata, Satontat kati, Waria, Iesanentons sheien okon.

Ise hiawi Rawenniio Ne kennonni iakwanakere ; Shetenr kati, shekonnienstak Kanesatakeronon okon.

Tokenske akwa ionkwentent Asteson kwanataksatenni; Tsionkwanikonhrenhonhaties Ne tsini saiatanoron kowa.

Akwa nonwa iakwaskaneks Naonsaiakwatatrewate, Aonsakanatiioste Akwekon akwanoronkwake.

Kwanekennis kati onen Ne askwawennakaratate,
Asonkwentenre netsienha Asonkwatonnhakanonniste.

Aiawen iahte kakonte Tosa onsakwanikonhraten Aiakwariwaierite

Tsi ionkwannhas kariwiioston.

Aionsaionkwarihonti Tsini ionkwakenrenseronnis Ne kariwakatensera Akwekon oni natatswenhon.

Ii kati aiakwariwaierile Ne teskariwaserakwen, 'Tosa aiakwatsanisek Naeronhiaken nonwentsiake.

Ethoni skwarihonniennis, Tsinahe onwentsiake ises ;

Tsiaken sanistenseriio, Sonha akwanakeranihek.

Shetenr onkwatatekenson Nakte sonha tienakereniors ; Saonkwanikonhrat kenhak Tsi akwasennaienheke onwe. 
191

Ionkwanistensen ne karonhiake, Entiekehne oni, Akwa tiotkon tionkwakahrenha- Asonthenne oni. Ne tsini ionkwarıoronkwa [wis; Ionkwariwiioston okon, Asteson tionkwennonks 'Tsinonni kenteron.

Iakohtien tsennare nok tsiatare Ienoronkwa oni tsi iehawe

Akwa iahtaiontkaronni ;

Tsinikon iontkaronniatha

Iwehre: aiontsennonni nonkwe Asteson ionkwaterennaiennis Naetewateweienton

Tsinenwe entewenheie

Natsitewatkatho

Iesos karonhiake.

Iakoiatakennhas

Sasennatokenti.

Tsiaken, senistenseriio kowa, Takwentenr nonwentsiake ion-

Skanoron aionkwasenni [kwentent

Wari,hetsienha Sonkwawenniio Ne kariwaksenskwe okon ; Hiawennarakwakwe nonwen- Takwaiatakennha Senha onte karonhiake [tsiake; Taiakwatohetste. Nonwa iahte hiawennonties, 'T'sini hianoronkwa, Saiatatokenti.

Iah nonwenton te saiatoron[hon,

Askwentenrheke ne kwaniahe-

Oktiwakwekon sheiatanonstats Nehtiioke nok kaniatarake, Karhakon nok kanatakon, Tsinikon iesaniahesen.

Askwaiatanoustatseke [sen ;

Ne ieriwanerahaktha ;

Wari, Wari, Wari,

Wari, askwentenre.

192

O Wari! kwennakeraton, Sonhatsiwa tsiatanoron, Hiaiatanoronston Niio Roienha hetsatewelon.
'T'sini ionkwakaronni Ef Ise tesariwaserakwa : Karonhia takwannhotonkwenni, Kannhotonkwe tsinahe.

Onwentsiakwekon ne tsionkwe Paonha Niio hetsienha Swatsennonni, ionkwentenron, Ne sakoiatison nonkwe, Iotontaton, joteweton Iesos sesonkwaskontakwen.

Wari, saiataterihon, Aseken Iesos hetsienha Tsiatakon onkwe rolonhon, Sonkwatsennonniatenniton.

Ise iesaiatarakwen, Entsateweton ne Iesos; Tsini sariwaieriton $\mathrm{Ne}$ iesaiatarakwaton.

Nok oriwakwekon roson Ne tetewakaneronnion.

Wari, ise takwaienha, Wari, iesennakeraton Tosa askwaiatatkawe Tsinenwe eniakionnheke.

Aiesasennaien lesos, Nok ne Wari hiatatienha; Ahonwasennaien Niio Rotkon Roiatatokenti. 
193

Ise Waria konniahesen, Tsiatanoron, saiatatokenti, Sasitakta katiskwentarha, Wakitent, wakeriwaneren.

Risentenni ne Rawenniio, [nhe, Aiawen ne akiheiate Ionehrahwat ne tsi sekon kon- Ne tsini skatatrewatha. Ka nonwe kenha iakatekwasen!

Iseke waherharekwen.

A satekat akeriasakon Iesanonwestlia nakatsiroten, Skiatakennhas kwennakeraton Tiotkon aiotonkotakonhak, Takwaienha satanitenraskon, Ne tsina kounonwehake. Iah othen on tesanoronse ; Aseken Niio netsienha.

Ionatanitenra skarake T'si kiteron naontasierate
Seniatatake ne tsiatatienlıa, Etho askenikonhranentakte Aiawen akeninonwehake Ne tsini tsiatatenonwes.

\section{4}

O Wari! senistenseriio, Konnoronkwa konnonwes.

Asteson iesanentonhaties Teieiasontha okon.
Wakonion akeriasakwekon, Tsiena, akatsennonni.

Kaskaneks a kwa ionehrakwat Nakonwennarakwake.

Takienha, konniahesenhaties Hetsienha ahinonweseke Takitenr, takienawas. N'ahiken karonhiake.

\section{5}

Wari, tekonnoronkwanion, Tsiati ioianerenstakwa, Rawenniio senikwekon, Onen satsennonni kowa.

Takitenr, Sewenniio, tontakiatoret, Onka onte nonkwe wahakiatorenne? Sotsi wakahterons tsinahitstanike; 'Takitenr, rakeni, takiatanonstate.

O Wari! tosa sahteron, Niio rakennhaonhatie;

satontat kariwanoron

$\mathrm{Ne}$ tsini hoierenhatie.

Io! onen takrori tsi nakarihoten Ne saiatenhawi waskenonsorenne, $\Delta$ katontat onen : helsewennahnolon Niio Rawenniio twakwekon tsi tionnhe. 
Akwa rakennhaonhatie.

Rawenniio ne tsi tionnhe

Irehre; hetsienha aonton

Riienha onkwe enhaton.

Oh kati neniawen, seronhiakehronon,

Enhiateweton nonkwe ratonsere?

Rirharatstenni Niio rakiatawen

Iahtakienterhane kanakwa nahoten.

Iah othenon tahonoron

Niio ne Sonkwawenniio:

Roiatatokenti Rotkon

Enthatsnente ne tsiatakon.

Ii ne ken kiteron riseronniennis

Niio Rawenniio netsewennenlawis ;

Tsini sewennoten etho ens naiawen,

Sotsi wakitent ne Niio rakiatawen.

Ethone Niio Roienha

Onkwe rotonhon.

196

Wennitanoron kowa

Wari awennita ;

$\mathrm{Ne}$ Wari twanistenha

Tetewariwakwas.

Tewanonsaseronni

Katsitsianoronson;

Tsi teionkwariwakwen

Tewaienha Wari.

Onwa kati iahte kakonte

Kwaiatarakwas istenha

Akenkake tsinenwe.

Tsiniiot ne karakwa

Teioswathe kowa,

Senha ne twanistenha

Teioteroronte,

Nonwa kwennakeraton

Wenniseranoron

Nok iakwariwahnirats
'Ts'ise takwaienha.

O Wari tsiatanoron

Ise haro kase,

Asientho kariwiios,

Askwaiatakennha,

Tentkatsitsianekare

Naonkwanikonhrakon,

Nentwariwaierite,

Etho ens naiawen.

Watonwesentseraksen

Takwaiatanonsta.t, Nok askwanatonhase

Tsini saianere, Aosennatokenti Tewarihowanat, Nentwariwaierite Entewatsennonni.
Wari saiatatokenti, Onen iakwahnirats

Nakwanoronkwake tsinenwe, Aiawen aontaskwatkatho,
Askwanoronkwake oni ; Askion tsini sanikonhroten, Naiakwakwenieseke Nakwanakeren nonwentsiake. 
Kwawis, o Wari, ase tsi iakionnhe, $\mathrm{K}$ wawis oni neniakwatiatisa ;

Tiotkon serhek : nene kheien okonha Iakwehre nii : ionkwaienha.

Nene konrharatstennis nonwa, Tiotkon ken enkehiarake, Ne ki onen keriwahnirats ; Tiotkon enskienhahake Tsinenwe enkonnheke.

Iakotaskat ne iakowennahniron, Iahte kakonte iesanoronkwa ; Ensheiateraswiiostenniheke Nok ensheion karonhiake.

Iesennakeraton ne karonbiake, Iakwakwekon tekwanonweratons ; lesanehrakwas eronbiakehronon, Iesanentons tsini tsiatanoron.

Ii oni takwanoronkwa niakionkwe, Asteson takwaterennaiennis ; 'Teseniriwaienawakon Iesos Naskwentenre aiakwatsennonni.

Akwa iah othenon te sanoronse Shenikonhrahnirats ne iakotent, Shetenranions ne teiakokonhentons, Karonhiake teshennhotonkwennis.

Ionkikaronni ne iakisot kenha, Tsionkiiatekwaton karonhiake ; Iakwatsanise naiakwatkaronni, Askwentenre, askwaiatakennha.

Tontakwatkatho, satanitenraskon, Askwatokaten tsini skwanonwes ; Satontatsek tsi iakwaterennaiens, Sonhatsiwa akwa kwaniahesen.

Ise Wari, sanikonhriio kowa, Ise takwalewetonni lesos, Ise skweni nenionkwatonnhoktaon Karonhiake akwaiatorenne. 
199

Rawenniio hetsiswaien okon

Hetsitwanistenserawi ;

Naesewaiatakennhaseke,

Sewasennowanat ne-Wari,

Sewatkatho nikenliohkwa nonkwe,

Akwa niate ionkw totenson,

Tsi kenteron iontekwasentakwa,

Ne tsini iotanitenriiskon.

Rawenniio sonkwaien okonha, Sonkwanistenserawi tionkwe

$\mathrm{Ne}$ naionkwaiatakennhaseke

Tewasennowanat ne Wari

Tiotkon iawet ionkwahretsiaronnions

Naontetwariwaierite;

Ionkwasnienons tsi tewaronhiakens,

Iawet ionkwanentsawakontie,

Eren sioiatenhawitha notkon

Tsi teionkwakwatasehonnes ;

Oriwakon iokwisron noneshon, Aonhaa watkwenies ne Wari.

Kawenniio ionkwanikonhraksens, Iah skennen te tewennontonnion, Senha ionkwateranentaktennis

Tsiniiot nə ionikonhraksens, Oksa aetewennitentase

Tionkwanikonhrasahatonhak, Iokontatie entwaiewentane, Entsionkwanikonhriiostake.

Niatekon tsini iawenserons Naiontkaronni nonwentsiake, Nehtiioke nok kaniatarake, Iah se nonwenton skennen te ken. Kawenniio akonwehiarane, Tokenske wakonwaniahese, Ok sa ok iotonte tsi ionkwentent, Ok sa ok eh nitiotieraton.

lakotaskat si iakotehiaron Si konwanoronkwa ne Wari ; Iakotaska ne iakorennhaon, Iontons : ise Wari takienha Ne kanonwes nakonwaniahese, Ne se ok teiotonwentsioni Aionkwalatakennhaseke, Tosa aetewatkaronni. 
Ise sewariwanerahakskon, Hetsisewatsanis Rawenniio;

Tsi kenteron aeswatewkasen, Entsisewariwaseronnien.

Ise sewariwiioston okon,

Hetsisewanonwes Rawenniio,

Eh oni nise sewatekwasen,

Ensewaiatanonstatseke.

Sonhaa konniahesen, Wari takwaienha ; Takwaiatanonstatsek Onwentsiake iakwes ; Nok nenwakitentane, Nentkiheionsere. Takwaterennaienhas Aiakwatsennonni.

Onka akheniahese? Sonha tsianoron, Sanikonhriio kowa, Satanitenraskon ; Ise hetsienha Jesos Niio Rawenniio; Ise hetsennitentas Nasonkweutenranion

Akwekon iontekwasens Tsinon tesiteron, Tsini iakohteronse Naiontkaronnianion, Salontat kati, Wari, Eh akatekwasen, Ise iaskwatatiase Tsi renteron lesos,

Ise Niio hetsienha, Hianoronkwa kowa, Rotatenonsotani Ne sanikonhrakon, 'Tsiati ne ioianere Iahte saiataksenkon.

Ionkwarhare kati
Ritsanis Rawenniio, Wakeriwaneren ; Eso tewakalentson Nahakrewatake ; 'Tosa takatewentet Nentkatonnhoktane ; Shetserientaseronni Onsahakitenre.

Wakatennikonhrisa Nenkateweienton, Akwa tsini senonwes Etho nenkierhake ; Enkhennhaseke oni Ne kheiatsteristha Aiesanoronkwake Sataionkwaieren.

Tsini skatatrewatha, Ne tekasentotha ; Aonsakiewentote Nakenikonhrakon : Aiawen iahte kakont Akonwenniioste, Akonsennaienheke Oni karonhiake! 
Sanikonhriio kowa, Sanikonhrowanen, Takwanikonhriiostak lakwanikonhraksen, Ise katsitsianoron Karonhiake tiotonni.

Sanikonhranehrakwat Tesentenhawitha ; Ise tsiatiio kowa. Nienakere okon ; Taiesariwakwase Ne ronatkonseriio

Iahte saswatanitskon Iesennitentase ;

Ise kanonsanoron Iontekwasentakwa ; Senistenseranoron, Takwatsennonniatennis
Aiesaiatahnoton, Aiesennakeren, Akwa oriwakwekon, Sariwaieriton; Skwenies ne karonhiake Nonkwe shennhotonkwennis

Iesaniahesen kowa Tsiontonnhakanonnis ; Ise seshetsientanions Teiakokonhentons : Takwaiatanonstatsek Iontkaronniatha okon.

Akwekon iesatsanis Nonwentsiakonronon ; Iesakonnienstha kowa Eronhiakehronon. Takwaiatatironten Tsinonni satsennonni.

202

Takwaien okon Wari saiatatokenti

Takwanoronkwa ne iakionkwe,

Takwanoronkwatha naiakwateweienton,

Tosa aiakwatkaronni, nok isinison

Aionkwatienitonhatie nasonkwentenre Rawenniio.

Satontat, Wari, nakwa akwanoronkwake Ne iakwaskaneks iakwakwekon

Ne nonwa iakwehre tsi teionkwariwakwen

Taontaskwakanerake nok naskwentenre,

Askwaterennaienhase tsini ionkwentent nonwentsiake

Ionkwententaon sasonkwakaronni Atan

Tsi ionkwaraon kariwaksen,

Ionḳwaiataksenhon, iawet tionkwaiatenhon

Tsi tsiteron karonhiake; oh ! askwentenre

Nise iahte saiatoron aonlaskwennonk karonhiake.

Hiawenniioston Rawenniio nonkwe okon ;

Ronikonkronni, hiawirawi ;

Etho kati niiot tsi tekwakaneronnions

Tsiniiot ne iontatienha ; takwentenr kati,

Takwaien okon iakionkwe, seriwaierit, sawiraien.

Tionkwe okonha, tewasennowanat Wari ; Tewasennaien, twaniahesen ;

Iah se te ionkwentent, nentewanoronkwake

$\mathrm{Ne}$ entsionkwaiatatako ionkwanaskwaien

Notkon nonwentsiakonronon; enionkwenteron karonhiake. 
Ise, o kwennakeraton, Nonwentsiake iakwanakere, Sasitakta kento taiakwe ; Kwawire nonkwanikonhra, lahte kakont eniakwehiaraseke Tsini jaweta takwentenranion.

Iah se takwaiatanikonrhen, lah nonwentou, iah nonwenton.

Tetiokaras nonwentsiake, Watatoratis notkon nonkwe, Tiotkon iokwisron naionkwakwa, O Wari, Isi kwanoronkwa. Nok iah nonwenton taiakwatontate, Tiotkon tenhnon enkwanoronkwake.

Hetsienha sonkwaskontakwen, Sate skwaienha niakionkwe ;

Tiotkon teskwaiatatirontha

Tsinon teioswathe kowa, Tsinikon sawiraien nonwentsiake, Sawiraientak ne karonbiake

$\mathrm{O}$ wenniseriio kowa Neniontkatho sheien okonha Skonsake teioteroronte Ok ne tenshotiswateten ! To kati nenionwesenhake Teniesakanerake tsinenwe!

Ionwensen konwaienha Aonton Wari, K asene twatateken Tewennitentas.

Tetewariwakwas?

Tetewatkenni ; Ronatkonseriios Ietinakeren

Tsinikon nawentawen Tewehiarasek

Naetewasennaien

Tsi tewenteron

Tetewatont akwekon, Tetewariason,
Tewatekwasentawak Tsi tekenteron.

Ise ne takwanonwes, Aonhaa ionwesen Ne taetewahtaren Onkweriasakon.

Sonhaa tsiatiio Ne karonhiake; Aonhaa ionwesen Iesanoronkwa.

1: Ken kaiatonserake Iawet kwatkathos Nok ionkwatsennonnianion 'Tsi iakwenteron. 
Iahte kakonte kati, Wakion, o. Wari, Onkwawenna, onkweri, Naskwentenrheke.

Onwari jonkwaienha, Kanistenseriio, Ne iakwaniahesen, Ionkwaiatakennhas.

Onwari ionkwaienha, $\mathrm{Ne}$ ionkwatsteristha, Teionkwaswatetennis Kariwiiostonke.

Onwari ionkwaienha, Iakonikonhriio, Ionkwatontatihatie

T'sini ionkwanonwes.

Onwari ionkwaienha, Akwehon ionkawis, Teiekaneratsihon

Tsini ionkwaiewas.
Hetsiennitak netsienha, Asonkwatkatho, Onikonhratokenti Onen asonkion.

205

Onwari ionkwaienha, Ne iakwanonwehak, Tiotkon iakwenterontak A kaweriasakon.

Onwari ionkwaienha, Ne Kanesatake, Ionkwaiataterihon

Tsi ionkwanistensen.

Wari ise takwaienha, Wari iesennakeraton, T'siaken tontakwaiatat Tsinenwe niakwa tonnhokten.

Ahonwasennaien Niio Ne Roniha nok Roienha, liotkon Roiatatokenti

'Tiotkon ahonwasennaien.

\section{6}

Takitenr, Wari takwaienha, Tsini iahte sonkwatstenni lahte kakonte konnatennis Tsi wakitentahonhaties,

Oriwakon sate iakonkwe Sate iontateniahesen, Teiontatenikonhrateni Iontatenikonhraksatha Ne ok skennen onkennontonnion Tsini ienonwes nonwentsiake Ne ok iahtakatkaronni, Ise enskenonnatieseke, Seriane enkalekwasen. Iahta iakoialakennha, Isinon iaetewatkatho

Tsi kaien naiontsennonni.

[tsiake,

Toni ionkwentent nonwenNe ok kaien naiontstaren Tsi iakatehiarontie nonkwe Iakoronhiakenhonhatie, Iakesaks natonwesentsera, letsenries ionikonhraksat, Okaserokon tsi iakonnhe Nok oni tsi iaiheions.

$\mathrm{Ka}$ onte non nakatekwasen Tsina tekatonnhakarias? Ise, o Wari, konniahesen, Ise skwenies naskitenre Ise wakonhion nakweriasa Ethok non ni kanaktio 'Takenatonhas karonhiake Tosa akatatkaronni. 
'Tetewaiena swakwekon Sewaronhiahehronon, Taetewarensaronnion Wari tewennakeraton.

O Waria, tasatontek Tsi nonwa kwasennaiens, Kwanoronkwa, kwaniahesen, Ise kwennakeraton.

Tsini ioiatanehrakwat Iakatohetstenni onwe Onwentsiakehronon okon Eronhiakehronon oni

Tsini ioiatatokenti Shonikonhraseronni
Jiswenniio tsi iakonnhe lionwanakonni ne nonkwe.

Aonha kanonsanoron Ne ral.ıweiatennine Ahaia tanoronstake, Ahaialatokentiste.

Ia! te kanakwaienteri, - Iotennitenraskon kowa ; Konwennitentase nonkwe Naiakoiatanonstate.

Ise nene sewentenron Ise naskwaroriheke Tsi ki niioiatanonwet Kanistenseriio kowa.

\section{8}

Ikehre : Wari akhesennaien

Ne kento konwennakeraton,

Tsiniiot ratironhiakehronon

Akkweni takeriwakwase.

O Wari! tokat enkonnakeren

Tokenske enkatsennonniate ;

Sonha kati tsiniiore skonnhe

Iosatste enkonnoronkwake.

Swakwekon sewaronhiakehronon,

Wari tetewanonweraton ;

Tsi nonwa nikkwenies kesennaiens

Nok oni tekeriwakwases

Ne kati, Wari iahte kakonte

Tsinenwe enkonnonwehake:

Tiotkon enkonwennaieritseke,

Katkeha akonhienterhane.

Tokat enkehre : akatsennonni

Tsini iotsennonri ne Wari,

Iahte kakonte akhenakeren

Akwekon nakenikonhrakon.

Tokenske sotsi wakenonwehon

Ne iontonweskwatha :

Onen kati sakatatrewate,

Sonkwati ne iontkaronniatha. 
O Wari, ise sanikonhriio, Takitenr takewennarakwak ; Satkatho tsini wakitent kowa, Tsinenwe enkonniahesenhak.

Wari, satanitenraskon kowa, Niawen tsi takatsteristha.

Takaterennaiennihek kati, Nakkweni akateweienton.

209

Sewatka onen ne tsionkwe Teswataterensaron ;

$\mathrm{Ne}$ tesewarensaronnion

Ne ioiatanehrakwat.

Tewatenro nonwentsiake Wari tewanistensen, Ne teionkwahtarakonhak Naetewasennaien.

Iakwennakeraton Wari Twasennowanat onwe Iahte iotatsennaksaton Aosennatokenti.

Akonwanenton ne Wari Skonwatekwatha notkon, Konwatsanis nonwentsiakon Tsini kasennanoron.

\section{Kwasennaiens, o Wari,} Ne nonwa ken wente; Sanikonhriio kowa, Satanitenraskon.

Satenata ne kento, Sanonsatokenti, Takwaiatanonstatsek

Takwaien okonha.

Tsini tsiatiio, Wari, Iahte saiatoron

Ne nonwar si siatat Saiatatokenti.

Ise skwa natiiostha Ken iakwanakere;
Rannhaon ne Rawenniio Taioniarorarake Ne ionkwanikonhrotakwas, 'Tosa aionkwasenni.

Iokweniatseraien Vari Naionkwaiena wase T'sini iontatienawase Ne iakowiraienton

Si renheionsere Iesos Rawirawi ne Wari Nonkwe okon nonwentsiake Tosa aiakotenton

Ise kati takwaienha, Wari, Iesos netsienha, Iakwatatekenha Iesos $\mathrm{Ne}$ niakwateweienton.

\section{0}

Takwatsennonniatennis Takwaiatakennhas.

Tawaiatanoronstha, Takwaien okonha, Takwaterennaienhas Tosa akwasenten.

Ise hetsienha Iesos Sesonkwaskontakwen, Rawenniio tsi tionnhe Niio onkwe oni.

Kanistenseranoron, Kanistenseriio, Takwateremnaienhas Karonhiake iakwe. 


\section{1}

Niio hetsienha,kanistenseriio, Ne Sonkwawenniio lesanoronkwa eronhiakehronon Tsi sonkwanistenserawis

Takwaiatanonstatsek

Tosa aionkwasenni

Kariwaksen, sotsi kanakere.

Hiaiatarakwen

$\mathrm{Ne}$ Sonkwawenniio

Naskwatsteriste

Ionkwentent okonha ;

Askwentenrheke kati,

Akwa tsi kwaniahesen

Katakwannhe tsini

Ionwentsiaksen.

Oneshonronon

Tiotkon ionkwanosas

Iokwisron kowa

Naionkwakaronni,

Iwehre : akonkweta

Enwaton nonkwe okon

Enwatkweni

Niahtaskwentenrane

Sonkwanoronkwa
Niakionkwe ;

'Takwarihonnien kati

Nasakwanoronkwake, Takion nisa tsini

Tsenoronkwa.

Takwentenranion

Tsi ionkwatehiaron,

Askwentenranion

Neniakwatiatisa ;

Senha skwatsteriste

Eniakwatonnhoktane

Karonhiake akwaienterhane.

Aiawen kati, Takwaien okonha, Aiakwakweni

Aionkwarihonti

Ne iotaksenskwe okon

Kwanikonhraksatakwa

Iahte kakont

Askwaiatonweskwen.

Kwasennaiens, Wari, kwennakeraton

Tsiatanoron, saiatanehrakwat,

Akwekon nakoiatatokentison

Karonhiake sheiatohetstenni.

Onkweriane tekwanonweratonnions ;

Ise akwa kanistenseriio,

Sonhatsiwa iesaniahesen nonkwe,

Karonhiake askwannhotonkwase.

Kariwanerenke tionkwaiakenhon, Sekon nonwa tiotkon ionkwasennies ;

Ise kati sonhaa kwaniahesen,

Askwentenre aionkwataskatston.

Askwatontatse satanitenraskon, Askwatkatho ne tsini ionkwentent, Senha aionkwanikonhraientane 'T'si senonwes ne sheien okonha 
T'si tsiteron iakwatekwasentakwa, Tosa kati takwakenronnihek ; Sonkwawi niakionkwe ne Rawenniio Takwaienha akenhak tsinenwe.

Ise iah othenon te sanoronse Ne tsi hiawenniioston netsienha ; Tsiaken askwaterennaienniheke Karonhiake asakwaienterenn.

Tsiatanoron, Wari, Niio netsieuha Onontiio okon jesanentons, Konhiatarakwas nistenha aonton Iahte kakont enkonnoronkwake.

Enkakwisron aiesasennaienhek Aiesanoronkwake oni nonkwe ; $\mathrm{Ne}$ ok enskat skennen kennontonnion Niah kaneka teniesawennonti.

Akenistenseratokenti kowa, Teskanere wakonwennentase, Katke sonha nenkonwennontieseke Askitenre karo akiheie.

Sewenniio tekwanoronkwanions Niawen tsi takwanistenserawi, Seron aonkateweientonhase Kheien okon iakoriwiioston.

Twatateken, akwa twaniahesenhak Ne Niı sonkwanistenserawi : Enionkwaienawase nonwentsiake Aionkwariwaieritonhatie.

Ionkwentenron tsi ionkwatewetonni Niio Roienha sonkwaskontakwen, Enionkwentenre tsi nentewenheie ; Enionkwanaktonnien karonhiake.

Takwentenr, o Wari, hetsienha sesonkwaskontakwen, Satontat tsi kwennitentase, satontat iakwatekwasentakwa Seriasatokentike

Karo nontasatierat, eh ionkwatiskwentare Tsi kwennitentase, takwaterennaienhas. 
Ionikonhriakt, o Wari! naontaskatkatho, Wakeriwaneren, nok sane satontatskon.

Iahten sane nonwenton niahta shetenrane $\mathrm{Ne}$ iesaniahesen, tosa takatewentet.

Hetsenoronkwa Iesos nok hiawennarakwa, Atsennitentase nasonkwawennarakwe.

Takwarihonniennihek tsinaiakwaiere N'aiakwasennaien ne seriasatokenti

Eh tahakatekwate Iesos tsi tsiteron, Raton: was, istenha, ensariwaseronnien.

Eniesawennarakwe, ensewennarakwe ; Ise kati, Wari, tekwawennakanere.

215

Konhiennitentase Askwehiaraseke,

Niio hetsienha,

Takwaterennaienhas, Nonwa skennen tikes Nok nenkatonnhokten, Niio hetsienha.

lahte sanoronse Othenon watseneken Niio hetsienha, Aseken roriwison N'enhiawennarakwak Ne tsi iakonníenstha, Niio hetsienha.
Takwateweionni Tiotkon si hanakere. Niio Roienha, Iah sane nonwenton Te saienterhaon Kanakwa nahoten, Niio hetsienha

Kwariwaienthose T'sini iakionnhoten Niio hetsienha; Tosa kati nonwenton Askwaiatatkawe Tsinenwe iakionnhe, Niio hetsienha

Kweh ! sewawatsiranoron Iesos, Wari, Sose.

Kwanentons, kwasennaiens, kwanonwes...

Wakwawenniioste tsi konnhe...

Wakion tsi nenkatonnhoktane...

Tiotkon enkwehiaraseke...

Nenkeronhiaken enkiron...

T'sionk we ietsisennanoronkwak...

Ietsisennaniahesenhak...

Rononnhek nonkweriane...

217

O Wari, o Wari saiatatokenti !

Senistenseriio, tsiatanoron kowa

Tekonnonweratons keriasakwekon ;

Askitenre, ista, tosa takiatonti. 
Nahoten kati saskaneks ? kien. Nahoten tesatonwentsioni ?

Ne ok nii kesaks nakhetenre nonkwe.

A hinonwehake lesos, ne kaskaneks :

Takewennarakwak, askon, konnekennis

Nahinoronkwake nene tsiatatienha ;

Iahte shewennonties niesennitentase.

Okstentsi keriwanonwes, kien, Tsi nahoten nonwa seniente.

Etho ki niawenn, Iesos hetsenonwenn.

Ista, sekon skawennat aonsakatati :

Oh kati nenkiere nahinonwehake?

0 ista ! askeni ise seriasa

N'akkweni netsienha ahinoronkwake?

Tokenske iahte sarihonte, Sonhaa, akwa iahtaskweni

N'asateweienton natsenonwehake.

Ne se aoriwa wakoniatorenne Askon kati, ista, n'akkweni ii oni, Sase konkwetaksen nahinoronkwake Ne rakewnniio Iesos tsiatatienha

Tsiatarak, kien, akotiohkwake Ne Iesus raweriasake

Etho iontkennisas naionterennaien.

Io, ista, eh niawenn tsi skerionnienni N'akataweiate Iesos raweriane Etho niha nikier, etho kiterontak Etho nonwe konnhek, etho oni kihei.

\section{8}

I

Twasennaien Wari

Niio ronistenha

Nonwa watonsere

Tewanonweraton. Ave Maria.

Twasennaien Wari

Saontaweiate

N'onaraseneha...
Twasennaien Wari Ionkwatewetonni Sesonkwaskontakwen...

Twasennaien Wari

Roiatiniionte

Ne raksaa Iesos...

Twasennaien Wari Sahoiatatsenri

$\mathrm{Ne}$ roiatontionne... 
II

Konsennaiens Iesos

Tsi saterennaiens

Atenhrakon Orif

Senonwes nakiron: $A$. $M$.

Konsennaiens, Iesos, lesaiesatanions

Iesasokwawesons...

Konsennaiens Iesos

Ne iohiktatsanit

Tiesanonwarannhori...

Konsennaiens Iesos

Tsi saiasakehte

Onontoharake...

Konsennaiens Iesos

Saiatanentakon

Tekaiasonteke...
III

Shotonnheton Iesos

Hetsitwawenniiost

Iotsennonni Wari

Tetewariwakwas; $A$. $\boldsymbol{M}$. Nonen sonsarete

Iesos karonhiake

Iahoken ne Wari

Tetewariwakwas...

Karo kase Satkon

Saiatatokenti

Tontakatiennire

Wari takienawas...

Kwanentons, o Wari !

Kwaiatanehrakwas:

Senheion, sesonnhe

Shionnhelon netsienha...

Nonwa karonhiake

Satsennonni kowa

Shetenr sheien okon

Ken iakwanakere...
Onen watennitoktane

Wari awennitatokenti.

Nok iahta kheiatewentete

Akenistenseratokenti.

Onen ictokte wennitiio, Wari awennita, wennitanoron, Tiotkon enkonnonweseke, Wari Isen : kien enkoniatakennha, Tiotkon.

Ionehrakwat takitenranion

Tsinahe koniennitentase;

Arens wakenikonhraksenskwe, Teskwaikaton ne karonhiake, Nok takenikonhrahniraton. [taren,

Iawetowanen wakatiskwen- Aiawens, akonken karonhiake.

Wari, akta tsinon tsiatare, Nok takewennakarataton

Tsinonni thenteron netsienha.

Teskwariwaserakwenni Tsinikon konniahesaon; Iahta koniatewentete.

Ethone tsi koniennitentase, Konnoronkwa nonwa nonwen[tsiake, 220

Ise sewaronhiakehronon, Akwa sewatkonseriios, Niio satesonkwawenniio Tiotkon sewakwekonhaties.
Tiohton nisewaiatotenson, Tiohton nisewentiohkwake, Iataiairon nonwentsiake Toni tsiion karonhiake. 
Akwa ehneken ratiiatare

Wishe, Kafrie, Rafae,

Neh akwa ratisennowanens,

Akwa ratiiatanoron.

Tsini sonkwanonwes ne Niio, Tewaiatatson sonkwawi

Saiatat raronhiakehronon

Nasonkwatsteristonhatie.
Ietinonwenn, ietikonnienstak Neronhiakelıronon okorı, Ietinenton, ietinakeren

Tsini honwanonwes Niio.

Ise sasenseratokenti, Iesaniha, Iesaienha Nok Satkon Saiatokenti, Kwanentons, kwawenniostha.

221

Sasakoiatisa Niio Ratironhiakehronon, Oksaok wahotokense

Ne tsini honwanonwes, Oksa wahrere : ionksennaien Iahte kakonte ratiiatanoron Iahte kakonte ne karonhiake.

Onen kati Rawenniio.

Sakoiatanoronston

Tiotkon tsi nonwe renteron,

Tehonwatiskwentarhon

Ronatsennonni ronwanenton : Ne kento nonwentsiake ;

Twakwekouhak hetsitwasen- Nok ne ratironhiakehronon

[naien Sakotisennies tsini ionkinonwes,

Tewakwekonhak tetewariwak. Sakotisennies notkonseraksen.

Tiotkon kontinikonbrare

Notkon noneshonronon

Naionkinikonhrotako

Ratironhiakehronon;

Akwa ionkiiatanonstals

Nene iontkaronniatha ;

Ionkihretsiarons, ionkinonwes ;

Tewatsteniaron ietiwennara-

[kwak,

Tewatsteniaron ietinonwehak.

Sakorihonte ne Niio

Naionkiiaststeriste,

Nok naionkiienawase

Ne kento nonwentsiake,

Ne kati ietiiennitak

Ratironliakehronon

Okstentsi ronteweientons

Tsi ionkiiatsteristha,

Twatsiaken kati, tewakwekon, Ii onkwariwa tsi ionkwentent,

Twateweienton tewatatekenson 'Tewatontatsek tsini hatiierha

Twateweienton ietinentonsek. Tewatontatsek tewakwekonhak.

$2 ? 2$

Sose tekonnonweratons, ise Iesos ronistenha, teseniterontakwe. Ionkwariwanerahakskon, jse satanitenraskon, takwaterennaien-

[has.

Saiatatokenti Sose,shetenr niakononwaktani,sheiaterennaienhas. Ise Iesos hetsehiaron iakoriwiioston okon, iesenniahesenhaties. Hetseriwawase niha ne nonwa sonizwatsteristha, Ariwawakon-

[kowa.

Shetenr kentiohkwatokenti nene rotiohkwison lesos, sheiateren[naienhas.

Tsi nentiakwenheionsere, kwennitentase iakwenhei wenheion[tatokenti.

Sose saiatatokenti, kwanekennis, karonhiake iaetewakwekon[hake. 
223

Sose tekwanonweronnions, Akwekon kwatsennonniase, Tsini hianonwes ne Niio.

Sose, ise lesos hetsehiaron, Hetseneken asonkwentenre, Ionkwariwaneren.
Sose Wari teseniterontakwe 'Tetsiaron nisa takwaterennaienTsinentiakwenheionsere. [has

Sose, akwa saiatanehrakwat, Aiawen taelewatatken Katkeha karonhiake.

\section{4}

Sose roiatatokenti Rawenniio roiatarakwen, raonha lesos rawehiaron, raonha nonwentsiake tsini hoteweientontieskwe, Iesos nok Wari tsi sakotsteristakwe.

Iesos Wari sose Kawatsiranoron, Akwa ionehrakwat Kwawatsiranonwes Tsi tekwakanere.

Onka aiekweni Aieriwaiena?

Wari ioteweton, Iah sane nonwenton Tionakwaienterhaon.

Onwentsiake ranats Ne Roienha Iesos ; Oniatara sonha Ronwarvenonniaton Ne Niio hiatatienha.

Onkwe ratatonnis, Nonkwe sakaonnhets, Ronwaiatenhawis Nok ronwastarontha Ne sakoiatison.

Eronhiakehronon Wahotinehrako ; Onwentsiakeronon Wahontsennonniate Sesakoskontakohe.

Wahotisterihen Kario rontsteristha Nok ne rotiianer Wahonwatkensera, Wahonwawenniiost.
Niio hiatatienha Satehniiatoten, Wahowiron Sose, Wahawatsironni Owatsiratokenti.

Skanikonhrat ronnis Raotinikonhra, Iesos, Wari, rose, Sonkwawatsirawis Naionkiatakennha.

Ok sa ok thotasawe Ne raiatakwsniio, Sonkwanekwensaris, Sonkwaronhiakense, Ii ne iakionkwe.

Ronwennakeraton Eronhiakehronon, Onwentsiake thare Nasonkwaribonnien Ne ionkwentent okon.

Ok si senakere Nok satehiarontie Tsini skwasahani, Satatenientense, Saieritonhatie.

Seksatatokenti Sheiatohetstenni Rotiksten okonha, $\mathrm{Ne}$ iesanehrakwas T'sini sehtaranis 
Tsi satehiarontie Teshewennakanere Wari, Sose oni; Ne thonennontontie Iahta sewennonti.

Tesewatatenikonhraieritonhatie ; Enskat seriwaswens, Naiontatewenniiok Tsi iakotehiaron.

Onkwatatken onton Ne sewawatsira ; Etho aiontkatho Kaneka ionskaneks Naiontatkwatako
Iesos, Wari, Sose, Askwanikonhrata Etho nataiakwatatenoronkwake Kento nonwentsiake.

Iesos, seskwaieren Tsi salehiarontie ; Teskwanatonnire Tsini ieiothahinon $\mathrm{Ne}$ tsini tisenon

Sewakwekonhatie $\mathrm{Ne}$ Wari nok Sose Takwaiatarako Aonsakwaieren Nonwa nok tsinenwe. Etho naiawen.

Iesos, Wari, Sose, Soasen, Anen sewawatsiralokenti, takwentenr ne ken iakwataraien ; etho natakwawatsiratotenst tsini sewawatsirotenne sekon onwentsiake siseweskwe. Akwanakeren tsinenwe eniakionnheke, nok onen eniakwatonnhokten, aonsakwaieren ne karonhiake, iaionkwakwatse. Etho naiawen.

\section{7}

Twatateken, tetsitewariwakwas

Ne nonwa nonwentsiakwekon nonkwe

Iakorakwen ahonwennakeraton,

San Watis raotiohkwa aonton.

Tetewaiena kati

Aetewakwekonhake

Tewatonhek : akwa iakotaskat

Iakotion iakononwarahtontha.

Wahonnise onwentsiake sireskwe,

Rawennake nok raweiennake

Satesakorihonnienniskwe nonkwe

Naiontkawe ne iontkaronniatha.

Iahte hoienterhaon ne kakwiio

Iahten oni ne kahnekakonson;

Ohnekanos ok rahnekiratakwe,

Ne rawenne raronhiakehronon.

Tsi onen wakoserareste nonkwe T'sinahontatenonwaroriate, Wasakotenre tsi wasakotkatho Ne niahta onsaiehnekirhake. 
Wahaterio notkonseraksenskwa Ne iakononwarahtontha nonkwe ; Eronhiakehronon wathatiiena Iahte kakonte wakonwasenni.

Ehni honnhaon Iesos ne Sán Watis, Eso sakotenrhakwe wahonnise; Sekon nonwa aonsasonkwentenre Naonsetewatonkwetiioste

Karonhiake kati nonwa nontawe Naonsetewatatrewatanion : Tosa aonsetewahnekirhake Iakononwarahtontha nahoten.

Ne iakokwas nohneka tsi ionttokha ; Iakokwas tsi iakoriwiioston, lakokwas tsi iakoien nonwentsiake, lakokwas oni ne karonhiake

Ieiotohetston iakotenstetennis Kario iakoiatotenstha nonkwe Netho nieiatotenne tsinahe Tetiehere nonwa nonwentsiakon

O San Watis! ise kwennakeraton, Salontek tsi wakwawennentase Iah onen taonsaiakwahnekihra, Ise enkwanakeraniheke.

O lesos! iesanonten Otsate, otsitsiatken, Takwentenrhek ne aiakwakweni Onkwe naiakwaiatotenhake.

Sewatka nohneka

Naeswasenni notkon

Nok taesewatentsa

Naeswatsennonni.

Karonhiake akwa tewesaksek.

Iahte tsienekirha

Tiotkon iakotsennonni,

Akowenk karonhiake

Nenionteweienton.

- Onen nonwa, twatateken, Irehre Rawenniio,

Tsi sakononwes nonkwe:

Tosa tsienekirhak
Akwa kanonsiio

Iahte ienekirha ;

Tsiniiot teioswathe

Tsinonni ieteron.

O.ase tsi tsionnhe ! Sewanikonhraientann Tsi ionowen nohneka Nok iakokaronnis.

Iahte ieienteri Aiakononwarahton, Skennon eniontehiaron Eniakonnhiioke. 
K anon tienakere Ne iahte ienekirha Kennakeraseiot, Iawet karonhiake.

Tewatka nohneka Tetwatatenoronkwak Na etewaienterhann Tsi tiakotsennonni. 229

Teiakok watasehonnes Notkon ase iakonnhe, Ne iahte iakokwatstenni Tsi iakoristaiennis : Iakonnhas taienonniakwe, Waton iahte iotaksen, Ne iakononwaroriatha Nok iakoiataksatha.

Kaswens ne kariwiioston Taienonniakwe nonkwe; Ne kaswatha tsi iakokwas Ne ioianerenstakwa : Akwa iakowennaksatha, Ia koweiennaksatha, Iakonnhas kanaiesera, Kanakwaiesatsera.

Iahte tsiakoriwiioston Ne teienonniakwanions ; Iahte shonwanonwes Iesos, Otkon ok skonwanonwes, Katsiraksen iontekhatha Akwa ieiatakwekon. $\mathrm{Ne}$ tenskiatsirateniate Tsi iotekha noneshon.
Wari twanistenha, Ise kwaniahesen, Takwaterennaienhas Naiakwattokhake.

\section{Saiatatokenti}

San Watis iesaiats,

Tsini sarihoten

Nen'aiakwaiena.

A kwekons teienonniakwa Iakotenstetanionnis, 'Tiotkon oni iakonnhastha Aiehnekakastake. Iakoterakareraston, Niio ronwanakonnis ; Iah se tehaonweskwani Taiakohenretanion.

Teienonniakwa iokwenion Raiatanoron kowa San Watis ronwaniariakon Ne taionkwaswateten : Sekon iawet etho niiot Tsiniiot iakoniariaks, Iahte tsiakonontsistonte Tsini iahte tsionttokha.

O Iesos! kwennakeraton, Iahte saienterhaon [kwe $\mathrm{Ne}$ iontonweskwatha nonNe naiakwariwaswen, Takwentenr aiakwakweni Aiakwatatienhase, Aiakwatonwesenseke Tsinenwe karonhiake.

Onen nonwa swalorisen, Karonhiake swatsennonni Ise, Iesos swakwekonne, Sekon nonwa sewakwekon.

Ise, Tier, iesakowanen Ne Iesos ratikwekonne ; Raonha hiaiatarakwen, Akwa hiaiatanoronston.
Satehorihonte nonwa Sanaktake rentskwahere, Rom kanatatokentike Sakwaniseratokenti.

Saiatatokenti kowa, Teskwakanerak, takwentenr, Tsi nonwa nionkwaiatawens, Ionkwariwiioston okon. 
Ioronhiakens ne sentiohkwa, Aseken ronwakenronnis, Ronwakenrenseronnianions Nene ratsteristonhatie.

Iesaiatanentaktonne Naonnise kaiasake; lawet ehni honwaierha Ne n'Ariwawakon kowa.

Antre saiatatokenti, Akwa tekwanonweratons, Ne Iesos senikwekonne Nok tsiatatekenha ne Tier

Sasatkatho ne kaiasa Niesaiatanentaktane Wasenhase, oh! wasiron, Kaiasiio, konnoronkwa.

Ise kaiasatokenti Hienheiase rakiatison, Ise shakeskontakwaton Konhienheias oni nii.
Ise satesenentawen, Tier, Kor seniiatatokenti, Seninikonhrakwekonhak, Askwaterennaienhase.

Ronwanenton karonhiake, Ronwanenton nonwentsiake Nasen natehaiasere, Ne Rawenniio tsi tionnhe.

\section{1}

Wahonnise si konnonwes, Konhiaskaneks, iseke kihei ; Aiakiariskon ne Iesos N'ahirane karonkiake.

li oni, twatateken Aetewariskon Iesos, Satsitewaronhiakense Nene sonkwaronhiakense.

Ronwasennaien Roniha, Nok ne Roienha, nok oni Piotkon Roiatatokenti Nonwa nok oni tsinenwe.

Wari seniienha Soasen nok Anen; Seniiatanoron, tekwanoronkwanion.

Hetsitsiatereha ne Sonkwawenniio; Sesonkwaskontakwen ne Wari roienha.

Hetsisenirori netsitsiatereha Tsi akwa kaskaneks nahinonwehake.

Sahaiatat Niio Roniha, Roienha, Satehonnas Rotkon Roiatatokenti.

233

Ne nonwa, twatatekenson, Atsitwasennaien ne roiatato-

Kento si kanataien [kenton

Raonha sonkwatsterist, Sonkwaiatanonstatha, Ronwasennaien Saksarie Tetewariwak nonwa.
Saksarie tsinahe sironnhekwe Rawenniio raoriwa Tiotkon ratsteristakwe Nok kariwiio sonha ; Enskat ok ratsaniskwe Ne kariwaksenskwa. Ronwasennaien Saksarie Tetewariwak nonwa. 
Taetsitwanonweraton

Rawenniio tsi tionnhe

Akwa ne sonkwentenron,

Ensonkwatsteristake

$\mathrm{Ne}$ roiatatokenton

Akwa sonkwanoronkwa. Ronwasennaien Saksarie 'Tetewariwak nonwa.

Kwasennaien, Saksarie, Ne nonwa ken wente ;

Sanikonkriio kowa, Satanitenraskon.

Satenata ne kento, Sanonsatokenti.

Takwaiatanonstatsek, Takwaien okonha. Saksarie, Saksarie, Askwaterennaienniheke.

Ok sa tisatasawe, Ase Isi sonnehekwe, Shetenranionskwe nonkwe, Ne ok saiotekwe.

Ise skwanatiiostha Ken iakwanakere ; Takwatsennonniatennis,
A kwa kati tetewatkenni Tsiswanoronkwanion Ne roiatatnkenti Saksarie twatatekenson ; Atsitwanakeren Tsini hoteweienton. Saksarie tsitwasennaien Aetewawennakwekon.

\section{4}

Takwaiatakennhas.

Takwaiatanoronstak, Takwaien okonha ; Takwaterennaienhas . Aiakwatsennonni.

Hetsennitentas Iesos Sesonkwaskontakwen, Rawenniio tsi tionnhe, Niio onkwe oni.

'T'si iseskwe tsinahe Tsi kanataienton, Shenatonniskwe nonkwe Karonhiake ientha.

Ii nonwa takwatkatho Ken nonni ionkwentent; Takwaterennaienhas Taionkwaswatheke.

\section{$23 う$}

Raswe Saksarie saiatatokenti, 'Tekwakanere takwaienha, Takwatsterist ne sheien okonha, Hiawennarakwa ne Rawenniio, Atsenatonhas twaterennaientakwa T'sina teionkwatonwentsioni.

Wahonnise siseskwe nonwentsiake Satewenteton tsi sonnhe, Owistanoron okon oni, lahte saiatakste tsieronke, 'Taseswateten niahte iakoriwiioston Asennholonkwas karonhiake 
Tsi ieseskwe tsi kanataienton Iesetakwe tsiniiot notsitenha ;

Iahte setsaniskwe kaienkkwire Nok oni iahte setsaniskwe

T'si karontote aiesanerenke

Nok oni aiesaiatoten.

Onen oieri niioserake

'Tsinahe saronhiaken tsieronke, Senheion tsina tesawisenheion ; Kawenoke iesaiatontion, Karonhiake iawenonton satonnhets Kaiatiio tsiniiot karakwa.

Hetsennitentas ne Sonkwawenniio, Takwentenr, takwateremnaienhas

* Aiawens aonsahonikonrhen Tsini ionkwariwaneren, Nasonkwentenre, nasonkwanikonhrata Ne ioianere tsinaetwaiere.

\section{6}

Onen nonwa rawentawen Nariwawakon Nikora ; Tsini ieskahes sahrenheie Roiatatokenti kowa.

Ne ronwaiatanehrakwas, Ronwanonwes, ronwanentons, Ronwaniahesenhaties Niakoriwiioston okon.

loriwanehrakwatenions 'Tsini hoieren Nikora Iakononwaktanionnis Sesakotsientanionskwe.
Ne ronwawennakeraton Nene teiontstekafanions, Ratiksa okonha oni Nene ronteriwaienstha.

Tetsitewatennakerat Tsini thoriwaierikwe, Tsini honikonhriiohne, 'Tsini horiwiiostonne.

Saiatatokenti kowa Askwatatiase nonwa, Ii oni katke sonha Sataetewatsennonni.

Ahonwasennaien Niio Asen natehaiasere : Roniha, Roienlia, Rotkon Oni Roiatatokenti.

\section{7}

Akwa ioriwatsanit Tsini hotiiatawenskwe Ne thonatierenton Rotiriwiioston : Iahte honwatikwatstenni, Ronwatiiesatakwe, Iesos sakowennahniratskwe
Niate kanatake Ne ronwatiswenskwe Ronwatikwanionskwe Ne raonatakwennia : Ronwatinerenkhon Iahonwatiiatonti Ne tsi tiakaonwentsiokwaton. 
Ok etho iaotken

$\mathrm{Ne}$ ratiieronke ;

Atonkariakon wahotirio

Nok oni nothorasera ;

Iahte tewenniseraien

Iahtahonwatiiesatake

Okwire ronwatiientanions.

Iohnekatarihen

Iahonwatiskowe

Wahonwanasitiake

Nok wahonwanatsio,

Otsirake oni

Iahonwatiiatonti,

Iahten kati tahontsente.

Iahte sakotirakwa

Ronwatihrewatha;

Iahte hatitsaniskwe

Ronwatironhiakentha ;

Ne ratinoronkwa ;

T'si sakoienha Iesos ;

Iah kati tahonwawennonti.
Katiken aonton

Naieriwahnoton

$\mathrm{Ne}$ enrate Iesos,

Ensesakoieritse,

Iakonikonhkatste,

Iahte tsiakorihontion

Rawenniio raoriwa.

lah kariwa te honnonni

Ne raonatakwennia ;

Ronton : katiken tionnhiio

Tsi tionnhe nonwentsiake?

Ne tenhnon iakonnhiio

Iesos karonhiake

Nonen sakotsennonniatenni

Iotohetston iononwaktekwe

'Tsi iontateriioskwe

Iahtaontohetste,

Iesos karonhiake

Ensakotsennonniaten.

Etho ieniakokwatse

Nonen enwaton ne ionskanek-

\section{8}

Tewatsennonni kenwente Nonwa seken rawentawen Marten roiatatokenti ;

Twatsennonni twatonnharen.

Ise nonwa ne sentawen, Akwa tekwanonweratons; Askwaterennaienhase

Sekon nonwentsiake iakwes.

Ise satsennonni nonwa, li tenhnon ionkwententsion : Takwentenr, takwaienawas Sataetewatsennonni.
Iakwentonnis onwentsiake, Iakwaskaneks karonhiake; Iakwakwekon aiakwenhei

Tsi nise ni senheiaton.

Ne satesonkwaiatison $\mathrm{K}$ wanekennis hetseneken Eh nasonkwentenre nii Tsi ki nise nihientenron.

O sasenseratokenti! Iesaniha, Iesaienha, Satkon saiatatokenti, Tiotkon aiesanentonsek.

\section{9}

Tetewariwakwas Firamenn, tewakwekon tetewariwak Tsini iakoiataterihon, iakonikonhrahnironne, Tsi werhakwe tosa nonwenton akienterhane kanakwa ; Tiotkon tsinenwe enkonnheke enhiwenniioste ne Niio.

Asen iawenre niioserake si kanakere Firamenn, Rom konwaiats tsi kanataien iakonwaiatenhawite; 
Rasenna Tiokresien etho raiatakweniio kenne; Rariwaswens kariwiioston, rerhakwe : akeriwahtont.

Tiokresien wasakawenhase ne Firamenn : ionkeniniak, Takenron : tosa ionkeniniak ; rakewenniio se Iesos, Iah othenon kakatensera tatewakatonwentsioni, Karonhiake tenhnon ahakon ne rakewenniio lesos.

Saerihokten ne Firamenn, Tiokresien wahonakwen. Wasakonnholon ne Firamenn, watesakowistotarho ; Wahrere ne ronontiio: katkeha enwatontate, Tsi konwannhotonkwe Firamenn, taiakotkense ne Wari.

Nok kaieri niwasontashen konwannhotonkwe Firamenn, Iahte iakaweron : katontat tsini iontateskanekskwe. Onen wakonwasokwaweson, akwa ki iahte watontals. Iakotatewennaieriton tsini howenniioston Iesos.

Wahonakwen ne ronontiio, ethone wahatennhane Iakoristonitietha, ethone wakonwentiake Firamenn, A wenke iakonwaiatonti nok konwaiatanonstaton ; Sakomnhaon ne Rawenniio ne ratironhiakehronon.

Tiokresien ne ronwanakonni, senha onen ronakwenhon, Wahenron ; ahenna isewatst. Onen wakonwaiahakhon; Riononha tonsahotikwate, tkonktons raotienkwire. Sekon are sahonatako neiatahotikontake.

Awa ionehrakwat Tiokresien tokenske tsi ronakwenhon, Onen wahenron : Sewaniariak. Onen wakonwaniariake. O saiatatokenti kowa Firamenn! askwentenrheke, Askwaterennaiennilieke nasonkwentenre ne Iesos.

Tewanenton ne Kateri, Ioiatatokenti kowa, Iahte kanakwaienteri, lesos ok rowenniioston.
Iah onka te sakotenras; Ne kati wahatennhane Nakonwaiena Kateri, Sekon sane si kaksaa.

Maksimen, ronkwetaksenskwa, Iah othenon te hotsanis Ethone ronontiiokwe ; Raswens ne kariwiioston, Irehre : Akeriwahtont.

Nene kaksatokenti, Wakenron : Takeronhiakent, Takerio, ne kaskaneks.

Sakoronhiakentanionskwe Niakoriwiioston okon ; Nene sakoriohtakwe Trsi sakoweiennaswenskwo
Sakenheie ne Kateri, Ne ratironkiakehronon Wakonwaiatanoronste, Rawenniio sakonnhaon. 
Onontoharake Sina, Akwa onontatokenti, Etho wakonwaiatata

$\mathrm{Ne}$ ratironhiakehronon

Seksatokenti kowa, Ise iesennakeraton
Nene konteriwaienstha Askwaterennaienhase

Niio, ise Sewenniio, Iesaniha, Iesaienha, Satkon Saiatatokenti, Tiotkon aiesasennaien.
Aietikonnienstake Ratitsihenstatsi okon : Ratiiatanoron kowa Rawenniioke nonkwati.

Tiotkon ietiwennarakwak Ronwawennenhawe Iesos: Iontatkaronnianions

Ne ronwatiwennonties.

Onen nonwa twatateken, Aonsatewatatrewat ; Ionkwarihonti niotaksen, Kariwiio twatsteristak.
Ehni wakenikonhroten, Takiena;vas, Sewenniio, Tiotkon akateweienton, Tiotkon akonnoronkwake.

Ahonwasennaien Iesos, Ne ratsihenstatsi kowa, Okaristiiakon tiotkon

Tahonwanonweratonseke

Sateietisennaiensek N'hiatatienha Niio, Rotkon Oni Roiatatokenti, Enskat ok nashen nilıati.
Satkon saiatatokenti, Sheiatienbas sheien okon Ne nonwa rotiniakhe; Asheiatatokentiste.
Wari saiatotokenti, Asheiaterennaienhase Aontahiniriwaierit N'oriwatokenti kowa
Ise lesos Sewenniio, Shetenr ne ken rotiniakon, Tahiatatenoronkwake, Kanoronkwatseriiohak.

Iakotaskat nonwentsiake Niakoniaks Rawenniioke : Teniontentsa karorhiake Nok oni nontatien okon.

Tewateranekenseratokenti roson ne Iesos
Ne tsiatakhaton watontha

Oriwatokenti kowa.

Kanoron naiontiesate; Okstentsi ioriwatsanit, Aienrheke iontatrona: Karo kihei, tosa nikier.

Sewaiatatokentison, Swanakere karonhiake, Ietsiiaterennaiennihek Iontatrona nonwentsiake. 
Tsi nonwa nenwakiote Wakonwenniioste, Wakonhion, Sewenniio, Asianerenste niha.

Takenikonhrahnirat, Katiken enkkweni

Nii akonhatsiwa

Akateweienton.

Sewenniio tsi konnhe, Takiatatokentist, Takwanikonhrahnirat, Iesos, Wari, Sose, Sewaronhiakehronon, 'Takwaiatanonstat, Takwaterennaienhas Nakateweienton.

Tsi nonwa ni wakiole, Ise Sewenniio, Ise ki koniotense, Ise sawenk onwe. $\mathrm{Ne}$ kati konnekennis Takiatanonstatsek Akeriwaierite Takatenientense.
Sewenniio tsi konnhe, Niawen tsi wakiote, Saheto, Sewenniio, Tenwakwisenheion, Sekon tsi enwakiote; Aseken Hetsienha, Onwentsiake sireskwe, 'Tiotkon roiotenhon.

Tsinise naskennhaon, Onen, Sewenniio, -Wakeweiennentaon ;

Wakonwenniioste Tsi wakeriwannhikon Sakatatrewate : Tosa seriwarako, Satanitenraskon.

Ise takatsteristha, Saronhiakehronon, Tekonnonweratontha Tsini skitenranion .Konrharatstenni nonwa, 'T'si enkatsteniaron, Takatentsa niiaake Tsinonni tsiteron.

Shetenr, Sewenniio, iakwatatenonkwe Tsi tetieseronniatha ieronhiakens nonwa : Ieronhiakentakwa eso tsi ronwaskaneks Taiesakanerak tsı ionwesen kowa.

Sariwison wahi aiontatienawase Ontateniha, ontatien okonha ;

Ne kati wahonni koniennitentase Sasheiatiniont ionkiiateweton.

Sariwison oni aiontatitenrheke Rontatekenson onwentsiake iiens. Tosa takwawennonti, sasheiatontako Iakiienterihne, ionkwatenro kenha.

Hetsennhaon, Sewenniio, raronhiakehronon Tahawistotarisi tsi ronwasaronte ; Asehiarann niha sanekwensa kenha Ne tionekwahonne tsi tekaiasonte. 
246

Ionehrakwat sonkwanoronkwa ne Rawenniio tsi tionnhe; Tiotkon sonkwatsteristouhatie, sonkwaienawasehatie; Iah se othenon te tiotokte tsini sonkwentenronhatie.

Ne rinonwes $\left\{\begin{array}{l}\text { ne Rawenniio tsi tionnhenion } \\ \text { tiolkon enhinonwehake. }\end{array}\right.$

Taonken roiatanehrakwat! taonken thoriwaieri ! Tiotkon tsi hanakere onwe, tiotkon enhanakereke, Iah othenon te honoronse, iah othenon tahokweni.

Oktiwakwekon ranakere, akwekon tihawenniio, Oriwakwekon raienteri tsini iawenhon tsinahe, Tsini ieniawensere oni, akwa raienteritsihon.

Akwekon sonkwawis nilewaks nok ne tewatseronniatha : Sonkwaiatanonstatonhaties, tiotkon sonkwaiatakennhas, Nok oni sonkwarharatstenni atorisenserakonte.

Iakotaskat ionteweientons, akwa wahi iotsennonniat, Tiotkon akwa ionwesen kowa, tiotkon skennen ionnontonnion, Nok tsiakotsennonniatonhaties tsi ronwanonwes ne Niio.

Ise Sewenniio, takitenr, takiatik iesanonwestha ; Nonwentsiake nok karonhiake tiotkon enkonsennaientak; Akwa niahte karennakonte tiotkon tenwakeriwakwen.

\section{IESOS RAORIWA TAKWENTENR.}

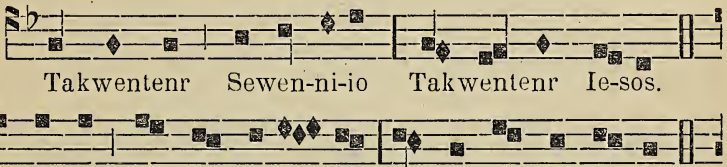

Takwaton-tats Se- wenni - io, Ta-kwaton-tats Ie - sos.

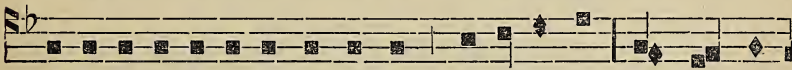

Ni-io ie-sa-ni-ha,ka-ronhia-ke te-si-te-ron, Takwentenr

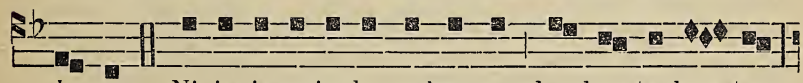

Ie-sos. Ni-io ie-sa-ienha, onkwe se- she-skon-ta-kwa-ton,

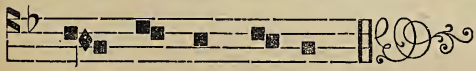

'Ta-kwentenr le- sos. 
Niio, Satkon Saia | tatokenti,

Asenseratokenti, | enskat ok Niio,

Iesos, Niio a | kwa hiaienha,

lesos, Niio tsiatat enha sate | seniiatoten,

Iesos, Saienha ne Wari iahte kana | kwaienteri,

Iesos, tiotkon si senakere, tiotkon oni en / senakereke,

Iesos, iesennakeraton | karonhiake,

Iesos, iesennakeraton tia | koriwaieris,

Iesos, akwa iesaia | tanehrakwas,

Iesos, ise Niio ne | sesatste kowa,

Iesos, ise Sewenniio tsini iawense | re tsinenwe,

Iesos, iah olhenon / te sanoronse,

Iesos, sanikonh / riio kowa,

Iesos, ise satontatskon | ne nonwentsiake,

Iesos, sennitentonne | nonwentsiake,

Iesos, iesawennaierits non | wa karonhiake,

Iesos, seriwanoronkwa kowa iahte iena / kwaienteri,

lesos, akwa hetsewennaieriton | Niio hianiha,

Iesos, sonhaa tsini saronhiakenhon onkwe / akoriwa,

Iesos, sonhaa tsini she / noronkwa nonkwe,

Iesos, sonhaa tsini senonwes ne skennen / akenlake,

Iesos, ise takwaiatison nok / seskwaskontakwen,

Iesos, sonhaa sariwaieriton ne kariwiio / se okonha,

Iesos, sonhaa takwawenni | io niakionkwe,

lesos, seriasakon ion | tek wasentakwa,

Iesos, ise sheien okonha | ne iakotent,

Iesos, ise akotsokowatsera nia | koriwiioston,

Iesos, kwahniha se | niseriio,

Iesos, sonhaa te / saswathe kowa,

Iesos, ise ne raottokhats ra ne hianiha | karonhiake,

Iesos, iahtiiaiehewe tsi | ni saianere,

Iesıs, aiakionnhe | kon tsi sonnhe,

Iesos, ise akotonwesentsera ne | ronhiakehronon,

Iesos, ise sherihonnienni ne se / wakwekonne,

Iesos, ise tesheswatetenni ne rotiiaton noiatonse / ratokentison,

Iesos, ise shesastenserawi ne kariwiıston ron / watiriohton,

Iesos, ise shenikonhrahnirats ne | iakoronhiaken,

Iesos, ise sheiawis niahte iena | kwaienteris,

Iesos, ise shenikonbriiostha ne ie | sawennenhawe,

Iesos, ise shenatsennonniatennis akoiatatoken | ti okonha,

Iesos ise seriwahtontha | ne kariwaneren, Takwaiatokef Iesos.

Iesos ise seriwahtontha ne / kariwaneren, Takwatontats Iesos.

Iesos ise seriwahtontha | ne kariwaneren, Takwentenr Iesos.

Takwa | tontats Iesos, Takwawennarak Iesos. 


\section{RATIKWEKONNE RAONENTAWEN SONHA.}

Iontaw :
T 2

Ioneh- rakwat, Sewenni-io, tsi-ni she-iatsennon-

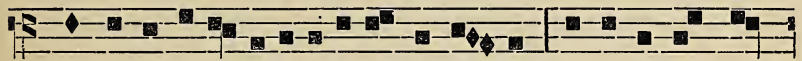

nia-ten-ni ro-ti - iatatokenti ken wen-te karonhiake she-

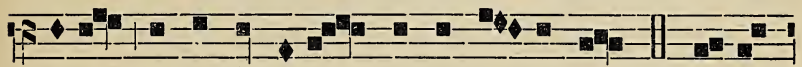

teron, iah-te ka-konte wahontsen-non-ni. Se-wen-

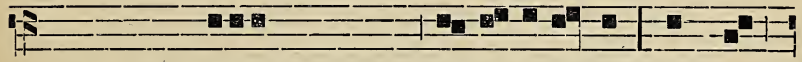

niio, ise shetenranions ia - ko-ri-wi-io-ston; ta - kien-

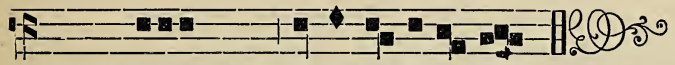

teritsilion akwekon tsi-ni ka-tie-ranions. Ahonwa...

Takw : (sk. 24). Rawenniio sewalenio (sk. \う3).

T 8.

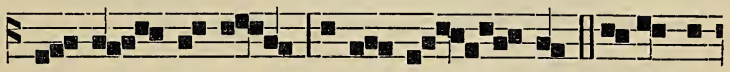
A - re - ri - ia,
a-re-ri - - ia. Io- nehra-

5-二-

kwat, Sewen - ni-io, shesenno-wanaton ne she-nonwes,

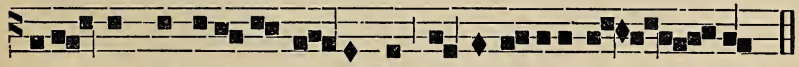
ionehrakwat sariwahni - raton tsi rona-terihon - - te. a.

Sewariwiioslon, naholen (sk. 161). Solsi wakalaskal (sk. 187). 
IOKARENRE.

T 8.

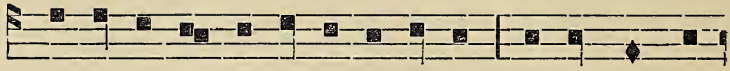

Teswa- ta- te - nonwehak ne tsionkwe, tsi- ni kwa- no-

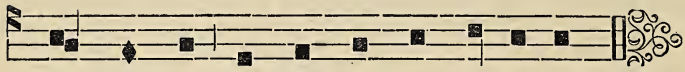

ron-kwakwe, ra-wen ne lia-wen-ni-io. Salkon Niio. 8. e.

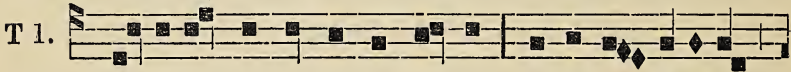

E - so en-kwanonwe-ne o- nen entsiswa - ie-ri-te,

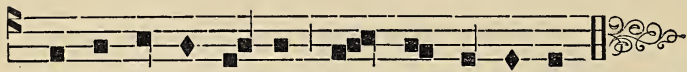

tsi-ni io-ri-wa-ke kwa-te-nienten-se. Rolaskal 5-6. $i$.

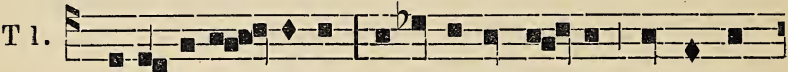

Rona-taskat onwe ro-ti-nikonhri - io; a-se-ken

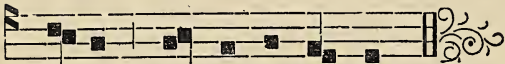

$\mathrm{Ni}$ - io en-honwatka- tho. Sewenniio le ok. 6. $u$.

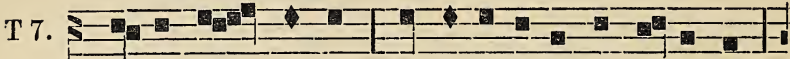

T'o-sa swar-ha-rek tsi ia-tewatsothos, a - rekho

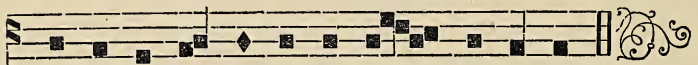

tsi se-wa- ta - te-ni-konhra - se-ron- ni.

Sewenniio ialute. 7. e.

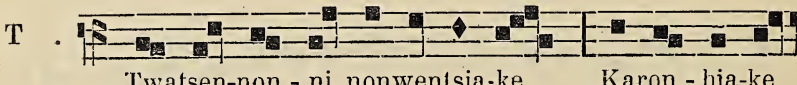

Twatsen-non - ni nonwentsia-ke, Karon - hia-ke
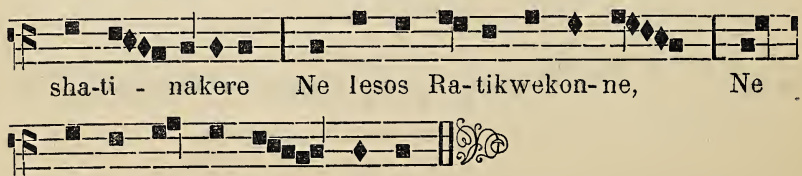

te-hon-wa-ri-wa - kwase. 
Ne teskwaswatetenni Ne teskwaiatoretane ; Akwekon kwennitentase, Swatontat takwaienawas.

Karonhiake sewannhotons, Nok oni sewanhhhotonkwas, Aonsaskwaiatokewe

Tsini ionkwariwaneren.

Ise tsietsitsientanionskwe Niakononwaktanihakwe,
Ne aonsesewatsiente

Nonwa ne nonkwatonnhetston.

Aiawen, nonen ensrawe Tesonkwaiatoretane, Tosa ahariwarako

Tsi sakwanikonhraksaton.

Ahonwasennaien Niio Ne Roniha nok Roienha, Rotkon oni Roiatatokenti, Nonwa nok oni tsinenwe. E. N.

v. Tehonarenihon kariwiioston nonwentsiakwekon.

R. Tsiniiore iotonwentsiokte ronwatiwennahrouken.

1on Ros.

T 1.

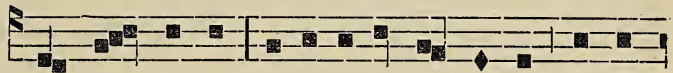

Nonkwe sonha en-ietsinaskwen-ha-we, nok en-

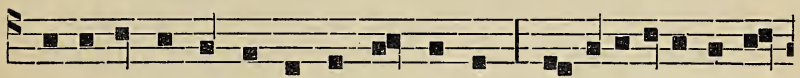

ie-tsi-sokwaweson tsi tiontken-ni-sas ten-ietsitaste tsi ra-

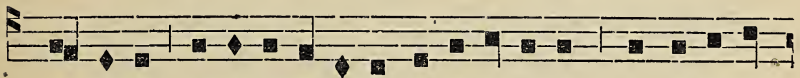

ti - teron ro-tinon-ti-io-son ra-ti-ko-rason ii a-ke-ri-

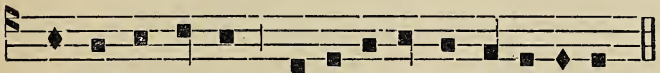

wa-ke, ne senha ta-on-re-ni ka-ri-wiioston. $5 e$.

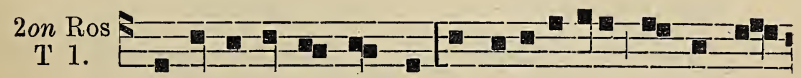

Sewa-ia-tahni - ronhak,nok sewateriio notkon-se-

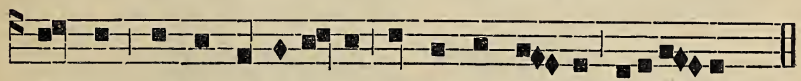

raksen; nok iahte kakonte enswatsennon- ni, areri - ia. 5-6o. 


\section{SH : RATIKWEKONNE NOK KARIWIIOSTON RONWATIRIOHTON RAONENTAWEN.}

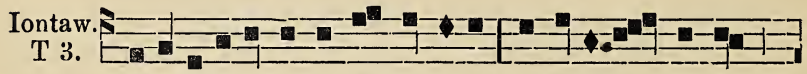

Roti-ia-tatoken-ti, Se-wenniio, en-icsasen - na-ien,

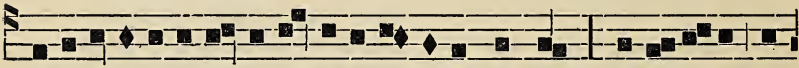

enhati-rihowana- te tsi-ni sa-ia-tanehrakwat, a-re-ri-ia, a-

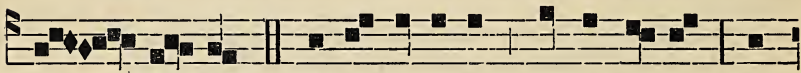

re - ri - ia. Enkonsennaien, Sewenni-io, nok

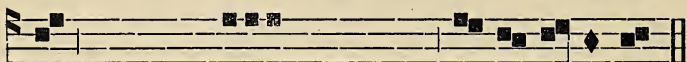

enkonnienste sasennatokenti iah-te kakonte. Ahon...

Takw: (sk. 24). Areriia (sk. 237). Akwa lsini (sk. 137).

Hetsitewanenton

Ne tesonkwaswatetennis ;

Hetsitewanenton

Sonkwatsennonniatennis. Sonkwanonkwatserawis.

Kaniseriio kowa Sonkwanonwes kowa;

Iahte sonkwaswatha

Tsini ionkwentent kowa.

Hetsisewasennaien, Swaronhiakehronon ;

Sewenron niiawen

Tsini honikonhriio.

Are wetwaiatahton,

Watsitwaiatonti ;

Oksa sesonkwennonks,

Sesonkwahaharatha.

Ionkwasaronte notkon

Sasonkwanerensi ;

Ok eken raonha

Sonkwanikonhrahnirats.
Ratsientha sonkwatonnhets, Ratetsientseriio;

Kanonkwatseriio

Asteson sonkwentenre

Tsini ionkwaiewas ;

Akwa onkweriane

Rotatenonsotani.

Akwekon tesonkwasnie

Tsini hoianere ;

Sonkwaseronniennis

Tsini honikonhriio.

Tiotkon tewatekwasen

Ne raweriasakon;

Hetsitwawenniiost

Tsini sonkwanoronkwa.

Raonhake asteson Ionkwarharekonhak ; I ken sonkionnhetha Sonkwakwatstenni oni.

Teswalotek (sk. 192), 


\section{IOKARENRE.}

T 3.

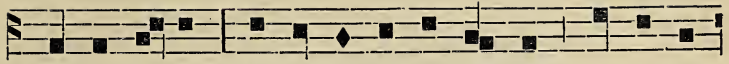

Sewen-ni- io, she-iatsennon-nia-ten- nis ne ie-sa-

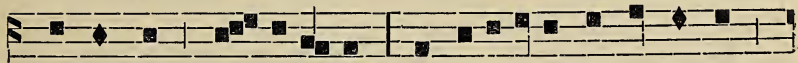

noronkwa, a-re-ri-ia; se-sheie-ri-ti ka-ron-hia-ke

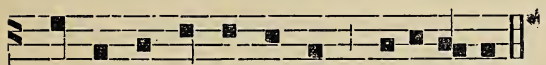

ne ic-sa-wenna-rakwa, a-re-ri-ia. Salkon Niio. 8 a.

T 7.

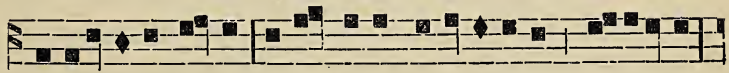

Rotiia-tato-ken-ti karonhiake shatinakere, a-re-riia,

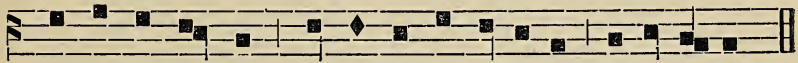

nok iahte ka-kont e-tho enhon-to-risen, a-re-ri-ia.

Rolaskal. $7 i$.

T 2 .

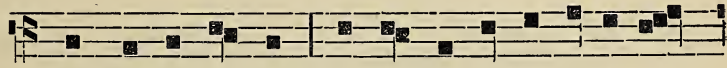

Rion-wasen- na-iens ne Ra-wen-ni-io aste-son

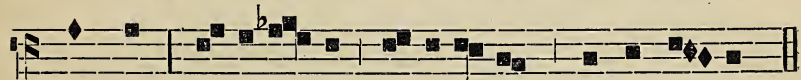

iontons: a-re-ri - ia, a-re-ri - ia, a-re-ri - ia.

Sewenniio to ok. 2 e.

$\mathrm{T} 2$.

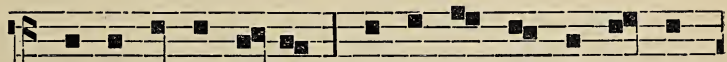

Ten-ia-ko-ia-ta-the tsi-ni-iot ne ka-ra-kwa,

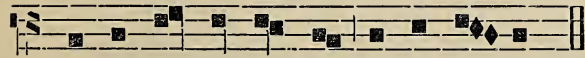

en-honwa-ken Ni - io, a - re - ri - ia. Sewenniio ialıle 2 a.

Ralikw : Twalsemnomi (sk. 238). K. R: Ne ioriwalsanil (sk. ?44). 
v. Sewaiatatokenti Rawenniioke, teswatonnharonnion, areriia.

R. Hetsisewaiatarakwen ne Niio asewakwekonhakwe, areriia.

Ion Ros.

T 2.

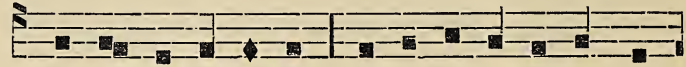

Te-ios - wa-the k'-wa ten-ia-koswa-the-ke ka-

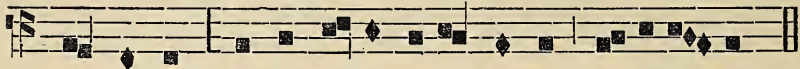

ron-hia-ke iah-te ka-kon-te tsi-nenwe, a-re-ri - ia.4.e.

v. Wenheiontanoron Rawenniioke nonkwati, areriia.

R. Tsi ronenheion rotiatatokentison, areriia.

2on Ros.

$\mathrm{T} 2$.

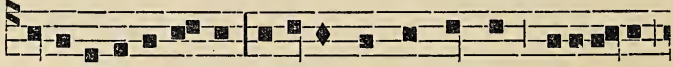

Sewaia-tatoken-ti, tesewatonnharonnion, areri-ia ;

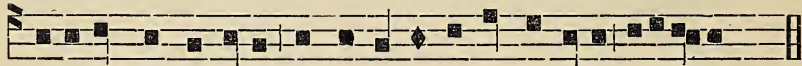

ensewakweko hake iah-te kakonte liawenniio, areri- ia. 8. $a$.

\section{KARIWIIOSTON RONWARIOHTON RAWENTAWEN.}

Iontaw.

T 7 .

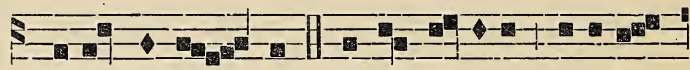

Enhatsennon - ni Rawen-ni-ioke roia-la-

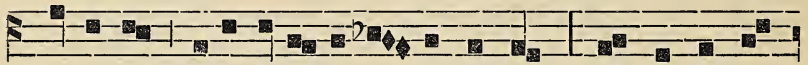

token-ti kari-wiioston ron-wariohton; nok en-ho-sen-

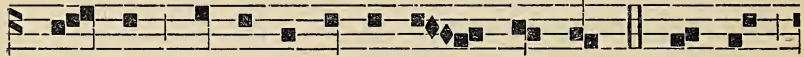

na - ien iah-te ka-kon-te tsi - nen - we. Ta- kwa-

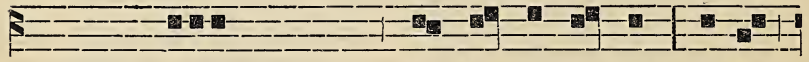

tontats, Sewenniio, tsi ka-te-ren-na-iens ; tsiata-

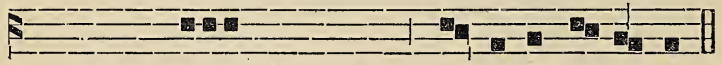

kennha nakwatonnhetsion tosa a- konwa-ka-ron-ni. Ahon...

Takw: (sk. 63). Kanaliio (sk. 173). 


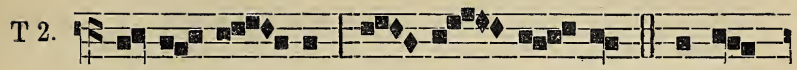
A - re - ri - ia, a _- re - ri - ia. Nete-

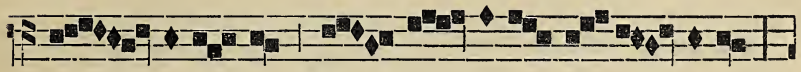
ionk - kane - re iah - te j-iens a - sa - takon;

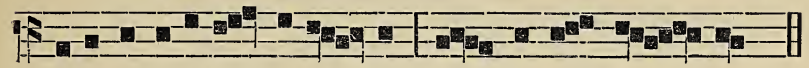
ok eken teniako- swate - ke iah - te ka - kon - te. a. Katke onle (sk. 14l). Tasatsnent (sk. 179).

\section{IOKARENRE.}

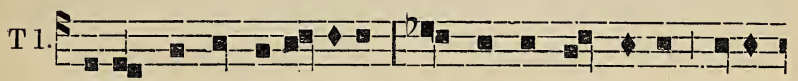

Ne enionksen-no-wanate onkweh-ne nonkwa ti, i- i

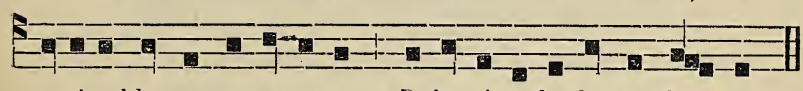

o-ni enkhe-sen-nowana-te Rake-ni-ne-ha ka-ron-hia-ke.

Satkon Niio 1 .

$\mathrm{T} 3$.

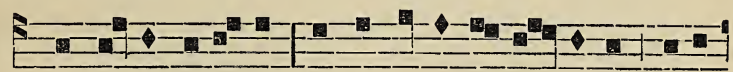

Ne te-ionkkane-re ian a-sï-ta-kon te iiens; ok e-

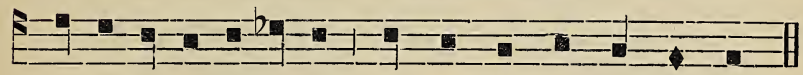

ken ten-iakoswa-the-ke, ra-wen ne Ra-wen-ni-io.

Rotaskal. 3 e.
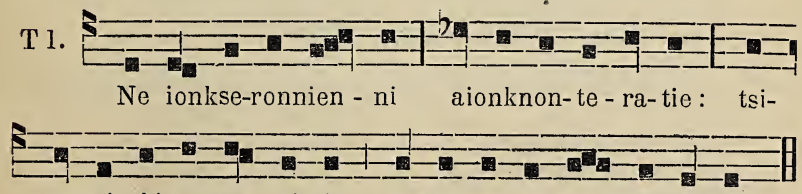

nonni tki-te-ron ni-i,- e- tho en-ie-te - ron-ta-ke.

Sewenniio to ok. 6 a. 
$\mathrm{T} 1$

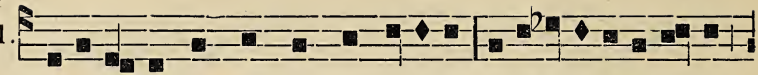

Kaneka enion-kse-ron-nien-niheke ensakoia-tanoronste

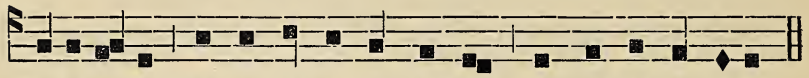

Rakeni-ha ka-ronhia-ke then-te-ron, rawen Rawen-ni-io. Tewakehtakon Rolkon. $6 u$.

$\mathrm{T} 2$.

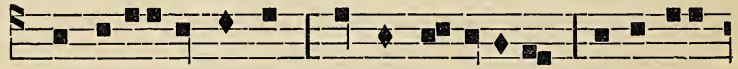

$\mathrm{Ne}$ io-ri - watsa- nit ronwa-ti-ie-saton Ne tho-na-

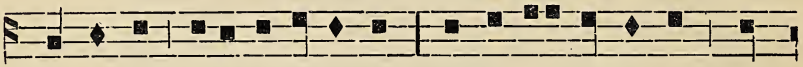

tierenton ro-ti-ri-wi-ioston, Ne ka-ri-wi-ioston tsi

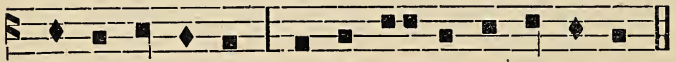

roniwa-ti-riohton, Ne Ie-sos ronwenhe-ia-se.

Niate kanatake

Ne ronwatiswenskwe

Ronwatikwanionskwe

Raonatakwennia

Ronwatinerenkhon

Wahonwatiaionti

'T'si tiakaonwentsiokwaton

Ok etho iaotken

Ne ratiieronke

$\mathrm{Ne}$ atonkariakon

Nok nothorasera,

Ronwatiiesaton

Nok ronwatiriohton,

Kahniien ronwatiientanion.

Iohnekatarihen

Iahonwatiskowe

Wahonwanasitiak

Nok wahonwanatsio,

Otsirake oni

Iahonwatireke ;

Iahten kati tahontsente.
Iahte hontsteristha

Ronwatihrewatha, Iahte hatitsanis Karonhiakensera, Ne ratinoronkwa Sakoienha Iesos, Iahten tahonwawennonti.

Katiken aonton Naieriwahnoton $\mathrm{Ne}$ enrate lesos Ensakoieritse, Iakonikonkatste Iahte tsiakonoron Rawenniio raoriwa.

Iahte hatinnhatens. Raonatakwennia, Katiken iakonnhes Nonkwe nouwentsiake. Ronton ne iakonnhes: Iesos karonhiake T'si sakotsennonniatenni. 
Aietinentonnion, Nonwa nok tsinenwe, Ne nasen nihati Raonhake Niio:
Roniha, Roienha, Piatikwekonhatie

Rolkon Roiatatokenti. Etho naiawen.

v. Akwa hetsiatanoronston, Sewenniio.

i. Ne ise sariwake rotewenteton tsi ronnhe.

lon Ros.

T 8.

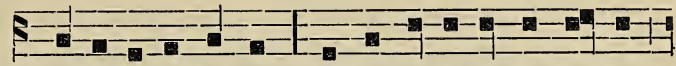

Ro-ia-ta-to-ken-ti iah-ie ho-rihontion Ie-sos

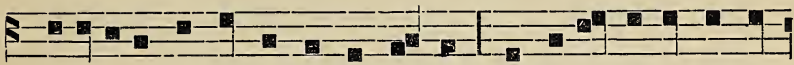

ra-oriwa; ne rostonton ronwa-rio, iah o-ni te sa-ko-tsa-

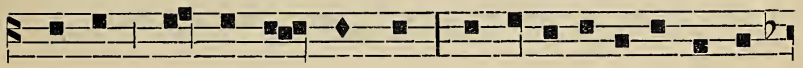

ni-kon ia - konkwe - taksens, teshia-tieren ne io-nonsah-

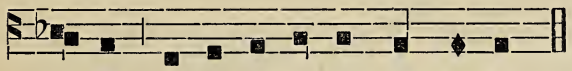

ni- ron otsten-ra-ke kentskwahe-re. 8. $e$.

v. Teshiatieren thoriwaieri tkerhite ioianiionte.

R. T'sini hoieren nonwentsiake haronhiake enwatehiaron.

2 on Ros.

$\mathrm{T} 1$.

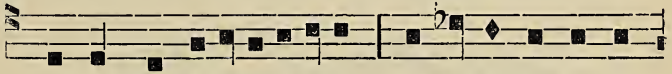

Ne iienhre: a-iakene-seke, ion-ta-tewen-tet na-

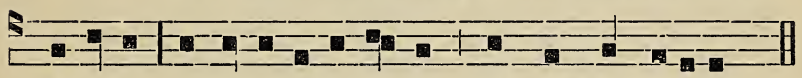

ka-onha, iontkrtat nako-ia - sa, nok ionknonte-ratie. 1. $u$.

\section{KARIWIIOSTON RONWATIRIOHTON IRAONENTAWEN, SONHA.}

Iontaw.

$\mathrm{T} 1$.

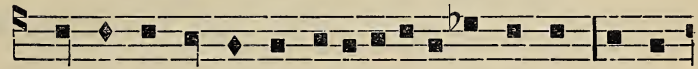

Tsi-ni honttokhakwe ro-tiia-ta-token-ti-son, enion-

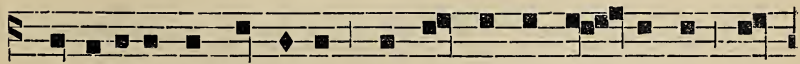

tro- ri-seke nonkwe okon, nok kentiohkwato - ken-ti ten- 


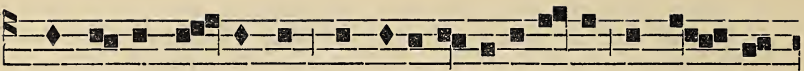

hon-wa-ti- ren - se-ron ; nok o-ni ra-o-ti-senna iah-te ka-

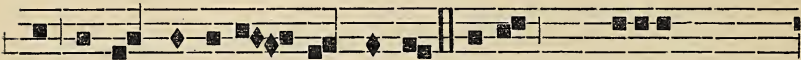

kont enkon-wa-ti-na - conkwake. Sewatonnharen swaiatato-

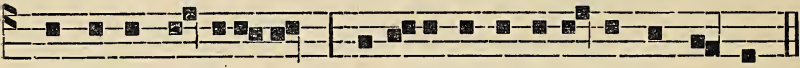

kenti Rawen-niio-ke ; tetsi-sewanonwera-ton tsi-nen-we.

Ahon... Takw. (sk. 68). Katke onte (sk. 141).

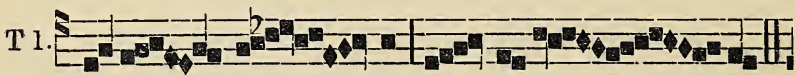
A-re - - ri - - ia, a - re-ri - - ia.

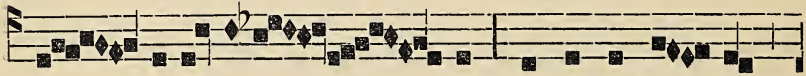

Tia - ko-ri-waie _ _ ri aiontsen-non - ni

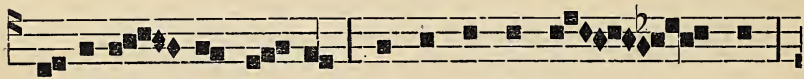

ra-ia-ta - kta $\mathrm{Ni}$ - io, ta-ion-tonnharon - - nion

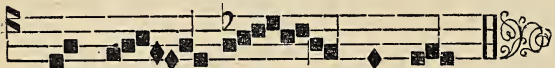

iah-te ka - kon-te. $a$.

Tsinahe 7 niwasonlashen, ken kaien taieriwakwe :

T 8.

Ne ia-koron-hiaken-hon tsi-ie-ien-thoskwe a-ion-

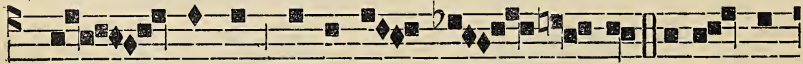

tsen - non-ni nenieien - tho - ko. Sako-wi

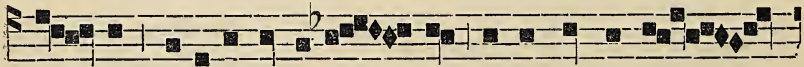

Ie - sos a-onsahonwaie - ren a-ionhriskon ra - 


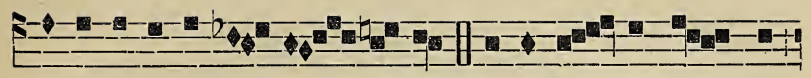

on-ha tsi roronhia - ken - hon. Ienka-he - we ten - hnon

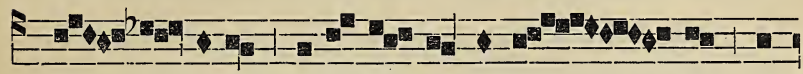

ra - on-ha ensa-ko- tsennonnia - ten en-

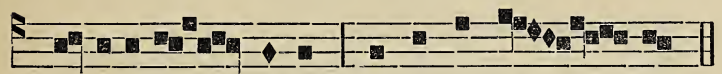

se-sako-ie - ri-tse iahte ka-kon - - te.

Ionelirakwal sonkw. (sk. 235). Akwa Iesos (sk. I41).

IOKARENRE.

Tierennakelskwallıa (sk. 243).

T 8. 年国-

Kariwiioston ronenhe - iaton toni ioron-hiaken-se-ratsa-

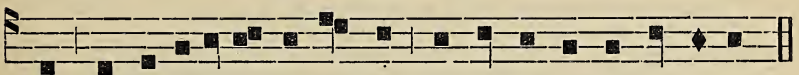

nit akwa ro-tironhiaken-hon ta- hontentsa ka-ron-hiake.

$\mathrm{T} 1$.

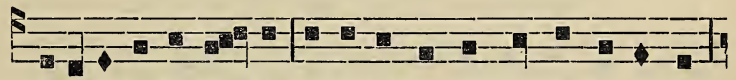

Rati-ieronke ken-ha o-kenrokon skennen wa-to-risens,

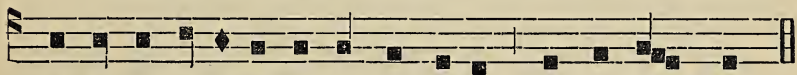

nok tenhonwati-ren-sa-ronnion-he-ke iah-te ka - kont.

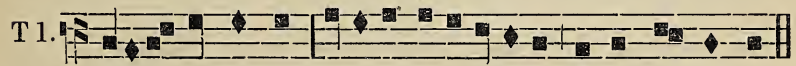

Ise ie-tsiriohton ne Niio ra-o-riwake, hetsiswa-nenton.

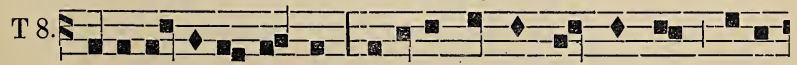

Roti-ia - ta-to-ken- ti Ro-ti-ri- wahni-hra-tstakwen ra-o- 


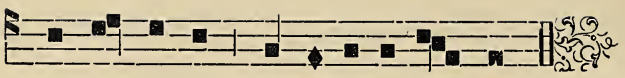

ti-ne-kwensa ne ka-ri- wi-io - ston.

Ne ioriwalsanil (sk. ?44).

v. Teswatonnharonnion nok sewatsennonnihak, swaiatatokenti.

R. Hetsisewaiatanoronston ne Rawenniio karonhiake.

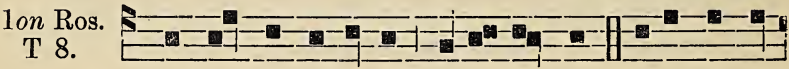

Nonwa ka-ronhiake ra-o-nawenk, rotikenhron-

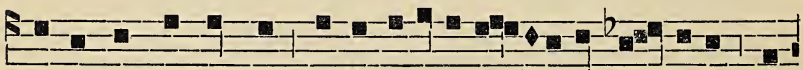

nihon ionwentsiaksen, onen sesa-ko-ie - riti Rawen - niio te-

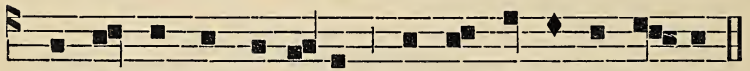

honwa-nekwen-sa-ri - se ne son-kwaronhia-ken-se. 8. $a$.

v. Eniontorisenseke iahte kakonte karonhiake rotiiatatokenti.

i. Aiawens aionkwanaktaientake tsi ratiteron.

$2 o n$ Ros.

T 6 .

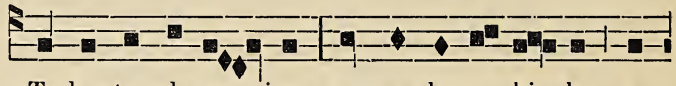

Te-hontonnharon-nions nonwa karon-hia-ke ne

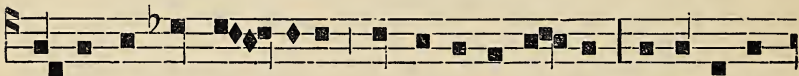

lesos sonkwawen - niio ronwaianen-ha - wi ; tsi-ni io-sa-

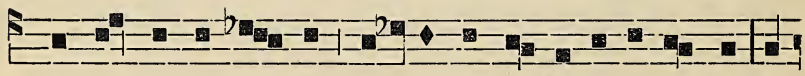

tste ron-wanonwe - hon, rona-tewen-te- ton tsi rononnhe ; en-

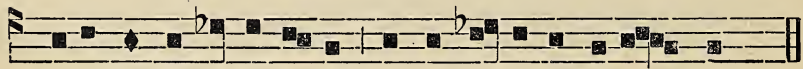

hatikwekon-hake le-sos ka-ron-hiake iahte ka-kont. 6. a. 


\section{ROTIIATATOKENTI RATIRIWAWAKON RAONENTAWEN SONHA.}
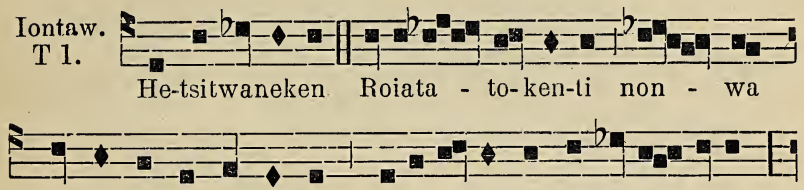

ken wente rawen-lawen, roia-ta-rakwen ne Rawen-niio;

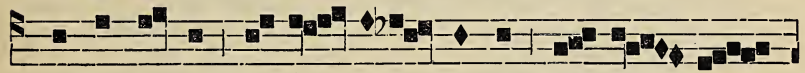

karon-hia-ke ro-ia - tano-ronston iah-te ka-

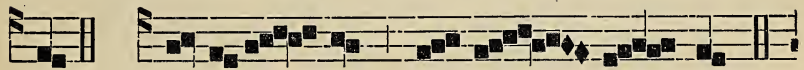

kont. (s/) A-re-ri - ia a - re - - ri - ia.

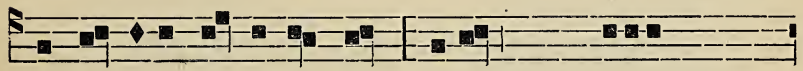

sewen-niio, sa-se-hia-rann tsi-nikon thoriwaieriton

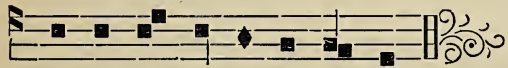

onwentsia - ke tsi i - re-skwe. Ahon...

Takw. (sk. 24) Tewalsennonni (sk. 23l).

T 8.

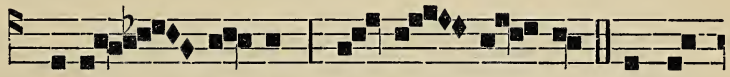

A-re - - ri - ia, a. re - ri - ia. Powen-

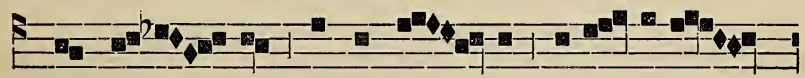

nen-ha-wa - kwe liawenni - io sa-ko-rihon-

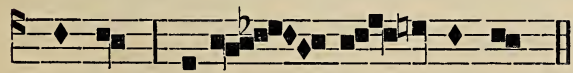

nienni, kari - $-w^{-}$io-ston. $a$

Ronnhek Sonkw. (sk. 136). Tewakwekon (sk. 141). 
IOKARENRE.

T 7.

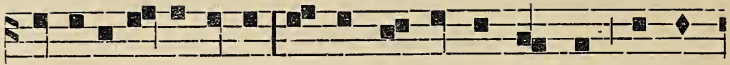

Ne roia-ta- token-ti ne nonwa rawen-ta- wen, tsi ron-
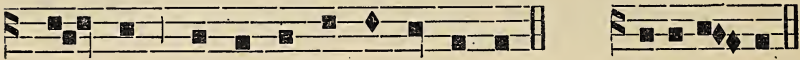

nhe-kwe $\mathrm{Ni}$-io rowennen-ha-wakwe.

(sh.) A-re-ri - ia.

Salkon Niio. 5 e.

$7 \mathrm{~T}$

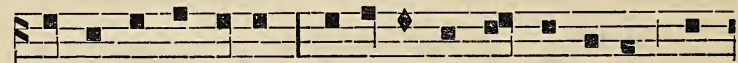

Ro-ta-taskennhaon u-ko-ri-wa-wa-konha-ke ne
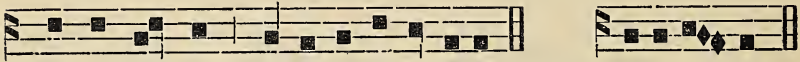

Rawen-ni - io ra-o-ia-nerense-ra.

(sh.) A-re-ri - ia.

Rolaskal. $7 i$.

T 3.

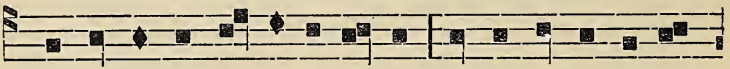

Ne ro-stonton ro-kowa-na-ton Rawenni-io a-ka-
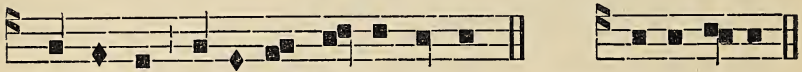

tste-ri-ste ra-o-tioh-kwa-to-ken-ti.

(sh.) A-re-ri-ia.

Sewenniio lo ok. $3 e$.

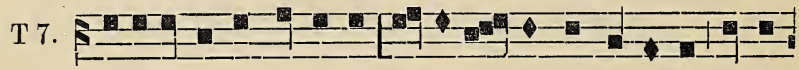

Sewa-riwawakon okon, ta- tsise - wanon-we-raton ne Ra-
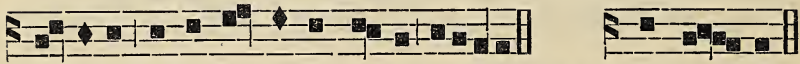

wenniio, ta-tsiswa-ri-wakwa-se, areriia. [tokat: wakwa - se.

Tewakehtakon asen 5-6. e.

$\mathrm{T} 5$.
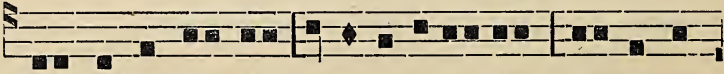

I Kentiohkwakwe-kon ia-ko-ri-wi-io-ston Ron-wasen- 


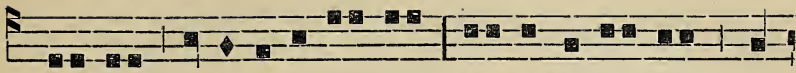

na-iens ro-ia-ta-to-ken-ti, Ne ka-ron-hia-ke e-

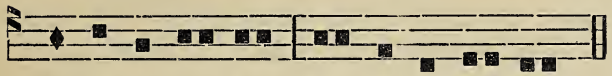

tho ra-we-non-ton Non-wa ken wen-te.

Raonhatsiwa

Tsi roteweienton

Sekon si ronnhe

Rosennaientakwe

Ne Rawenniio

Tiotkon ratsanisk we

Kariwaneren.

Akwa raswenskwe

Ieriwaneraktha,

Ratrewatakwe

Nahowennarakwe,

$\mathrm{Ne}$ ok iekenha

Ne sakotenrhakwe

Iakoieshaon.

Ahonwaneken Iakonorwaktani

Sakotsientakwe,

Iahte honoronse
Ne iahte tsionsons, lesos ahoneken

Naonsaionton.

Twateweienton

Roiatatokenti

Nentsitwaneken

Nonwa rawentawen,

Tokenske nii

Ensonkwentenrane

Nentewenheie.

Tentesonkwannhe

Nentwenheionsere

Nenionkwatsterist

Nonwentsiakonronon,

Tsi tiakolkwatse

Ensonkwentenronna

Ne karonhiake.

Etho naiawen.

T 6.

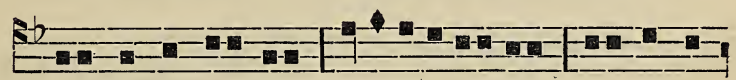

II Kentiohkwakwe-kon iakoriwi-io-ston Ron-wasen-

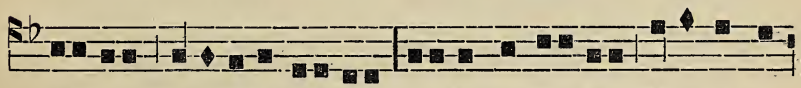

na-iens ro-ia-tato-ken-ti, Ne ka-ronhia-ke Etho rawe-

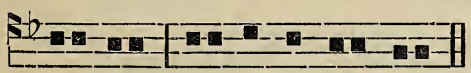

non-ton Nonwa ken wen - te.

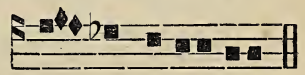

$\mathrm{E}$ - tho na-ia-wen.

T 1.

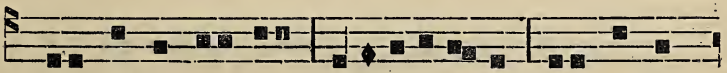

III Kentiohkwakwekon iako-riwi-ioston Ronwa-sen- 


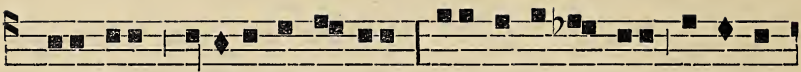

na-iens roia-ta-token-ti, Ne karonhia-ke e-tho ra-

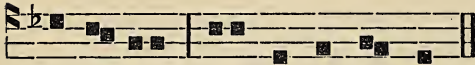

wenon-ton Non-wa ken wen-te.

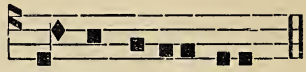

E - tho na-ia- wen. $\mathrm{T} 6$.

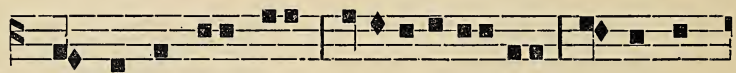

IV Kentiohkwakwe-kon ia-koriwi-io-ston Ronwasen-

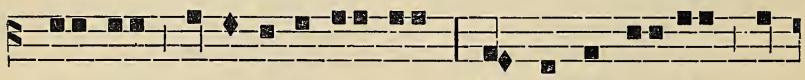

na-icns ro-ia-ta-to-kell-ti, Ne ka-ron-hia-ke e-

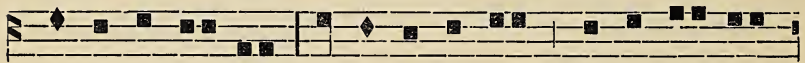

tho ra-we-non-ton Nonwal kén wente, nonwa ken wen-

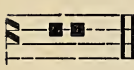

le.

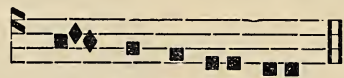

$$
\text { E - tho na-ia - wen. }
$$

T 6.

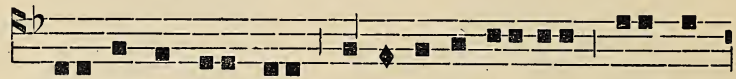

V Kentiohkwakwe-kon ia-ko-ri-wi-io-ston Ron-wa-

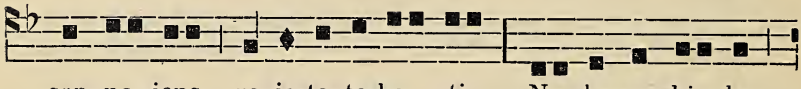

sen-na-iens ro-ia-ta-to-ken - ti, Ne ka-ron-hia-ke

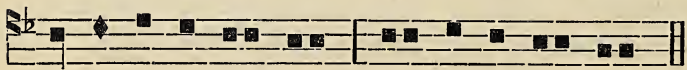

e-tho ra-we-non - ton Nonwa ken wen-te.

T 6.5

VI Kentiohkwakwe-kon iako-riwi-io-ston Ron-wasen-

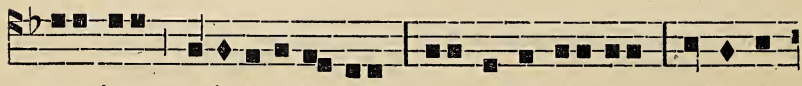

na-iens ro-ia-ta-token-ti, Ne karonhia-ke e-tho ra- 


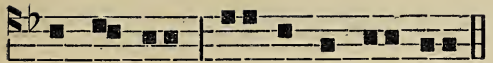

we-non-ton Nonwa ken wen-te.

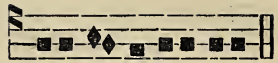

E- tho na-ia-wen.

v. Rononwehon ne Rawenniio nok roiatanoronston, $(s / l)$ are.

R. Ronaktawi kanaktanoron karonhiake $(s h)$ ares iia.

1 in Ros.

T 1.

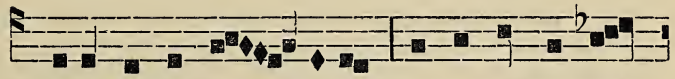

I-se ne Ra-wen - ni-io hetsewen-nen-ha-

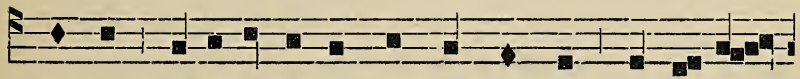

wakwe, i-se she-rî-hon-nien-ni nonkwe ta-kwate-
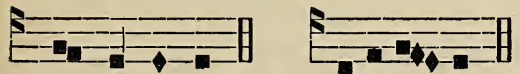

ren - naien-has. $\quad(s / h)$ A-re-ri - ia. $1 e$.

v. Ne roiatatokenti sakohaharaton nonkwe Rawenniioke $(s / \iota)$ ar.

R. Onen kati nonwa rokwatse kowa karonhiake (sh) ar.

\section{$20 n$ Ros.}

T 1 .

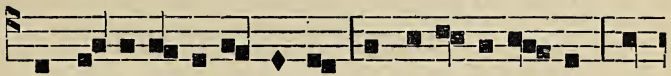

Rawen-ni-io rononwehon nok roko-wana - ton ro-

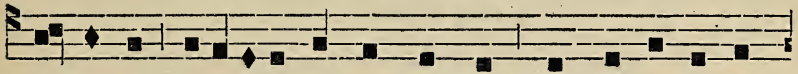

ri-honten a-sako-ri-honnien nonkwe nok ka-ron-hia-ke

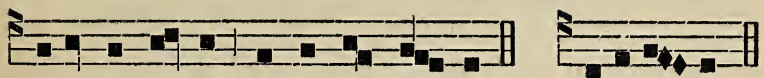

ahatsen-non-ni iah-te ka-kon-te. (s/l) A-re-ri - ia. 10.

Ariwawakon kowahn kenha Ros.

T1.

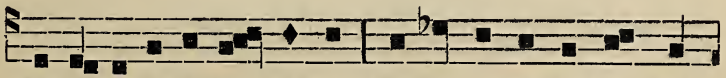

Ne a - ri-wawakon ko-wa kenhne, onwentsiake tsi- 


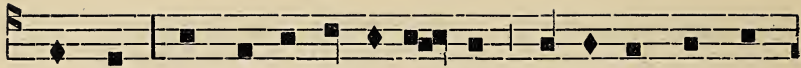

reskwe, ken wente ka-ronhia-ke ie-lıa-we-non-ton
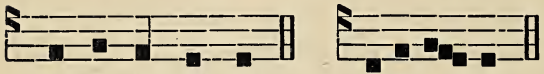

a-hatsennon-ni. (sh) a-re-ri - ia.

Rti. kariwiioslon sakoriholasienni nonkwe Ros.

T 1.

I-se tesheswa - teten-ni rao-tiohkwatoken-ti le-sos,

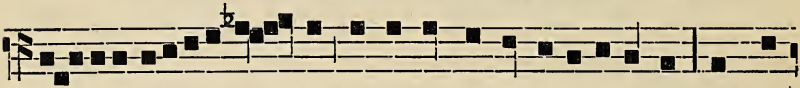

N. saiatatoken - ti, tsiatakennhaon kariwi-ioston, hetsen-
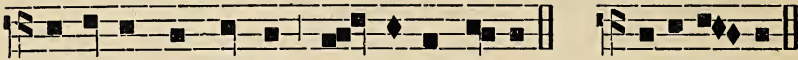

nit asonkwenten-re $\mathrm{Ro}$-ienha $\mathrm{Ni}$-io. (s/h) Are-ri - ia.

\section{RRTt. RATITSIHENSTATSI NOK IAHTE HATITSIHENSTATSI.}

Iontaw.
T 1.

Kari-wi-io ten-ie-ia- to-re-te na-koia-tato-ken-ti

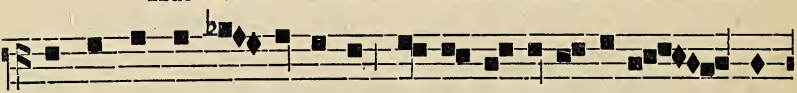
nok en-tiewenni - nekenne a-kwa io-te-ri-wakwa - ri-

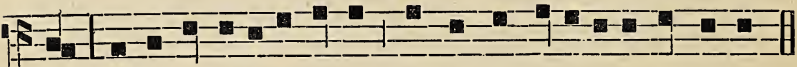

sion, ie-ha-we nakaweriane ne $\mathrm{Ni}$ - io ra-o-ianeren-se-ra.

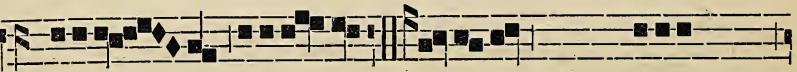

(sh)Areri - ia, areri-ia. Tkariwaieri nahonwasennaien 


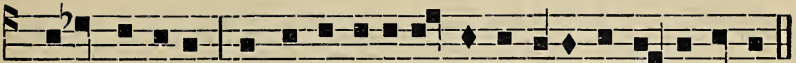

ne Rawenniio, nok takonwariwakwase ra-o-sen-na-tokenti.

Ahon... Takw. (sk. 68). Kanaliio (sk. I13).

T5.

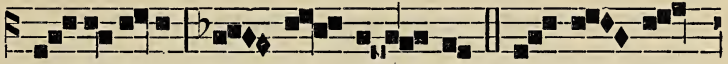

A - re-ri-ia, a - re - ri - ia. Ro - ta - skat

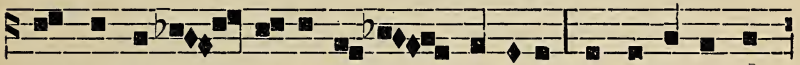

ronkwe ne ro - tsanis ne $\mathrm{Ra}$ - wenniio; akwa raskaneks

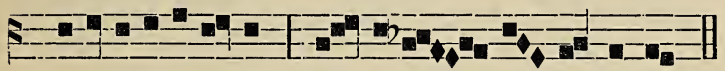

a-nariwaie-ri - te ra-o-ia - ne - ren-se-ra. $a$.

Swanikonhrarak (sk. 137). O lesos konsennaiens (sk. 185).

IOKARENRE.

Tierennakelskwallia (sk. 249).

T 1 .

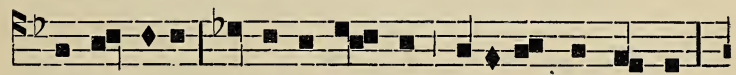

Sewen-ni-io tsi-ni takwa- wi io-ia-ne-rensta- kwa,

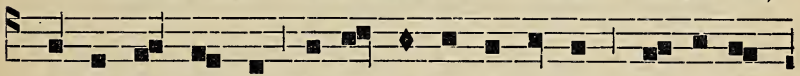

wa-ke-non-sta-ton te-ioh-nanet ok e-ken tsi-ni wa-

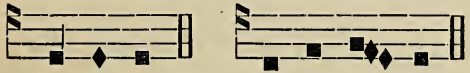

ke-hiaron. (s/l) A - re-ri - ia.

$\mathrm{T} 1$.

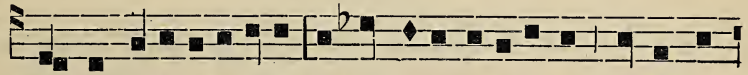

Tsiaken, tisa-riwa-ieri, tıotkon sari-waie-ri-ton: e-tho nen-
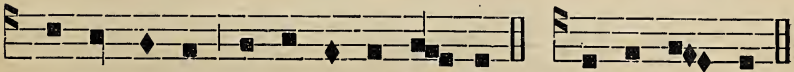

satsennon-ni tsi waka-tsen-non-ni. $(s / h)$ a - re-ri - ia. 
T 3

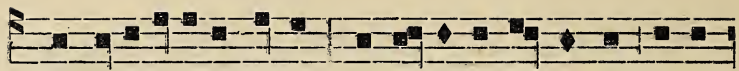

Thoriwaie-ri rattokha ne ro-ia-ta-to-ken-ti, tsi ron-
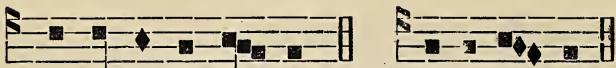

nhe ro-te- we-ien - ton. (sh) A-re-ri - ia.

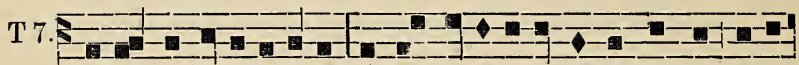

Rotaskat thoriwajeri, ne Rawenniïo tsi nenhononkse, tsini-

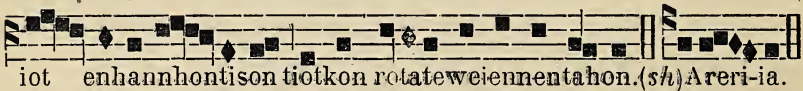

Kenliohkwakwekon (sk. 250).

v. Rononwehon ne Rawenniio nok roiatanoronston, $(s / t$.$) are.$

R. Ronaktawi kanaktonoron karonhiake, (sh.) areriia.

I Ros.

$\mathrm{T} 1$.
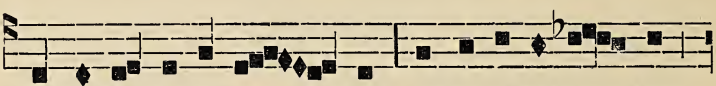

Teshiatie- ren ne ron - kwe akwa rattokha - kwe
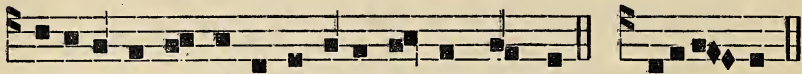

ne rotenonson-ni otstenrake wahentskwaren. (sh.) areri - ia.

v. Rawenniio wahohaharate tsinonni iohahiio, (sh.) areriia.

R. Tsiniiore karonhiake iahatsennonni, (sh.) areriia.

II Ros.

$\mathrm{T} 1$.

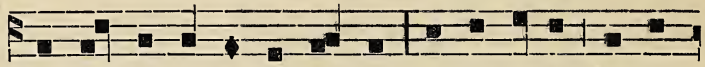

Ronkwe rawen- tawen non-wa nonwentsiake roswen-

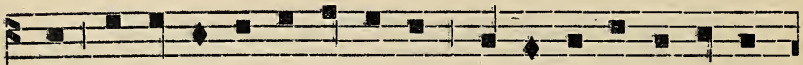

hon, ro-tie-ni-ton ka-ri-wi-io, rokwatse nonwa ka-ron-
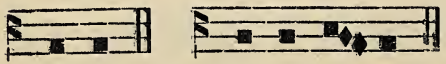

hia-ke. (sh.) A - re-ri - ja. 


\section{IOTIIATATOKENTI IAHTE IOTINAKWA- IENTERHAON.}

Iontaw.
T 5. Sewenni-io, io-nehrakwat tsini sheten - ron io-

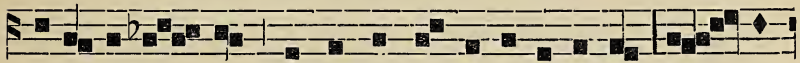

iata - to-ken - ti nonwa ken wente karonhiake she - te-

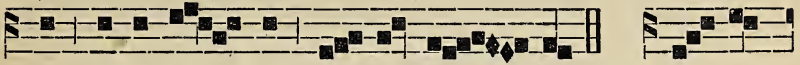

ron, ialite ka - kont on-tsen-non - ni. (sh.) A - re-

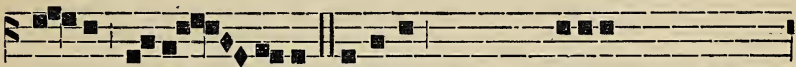

ri - ia a-re - ri-ia. Iakotaskat iaht , iakoiatorohon ne kari-

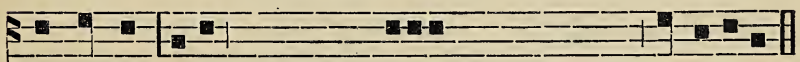

wa-neren, ne iakotahitakhe liawenniio raoiane-ren-se-rake.

Ahon... Takw. (sk. 63). Kulke onle (sk. 141).

T 8.

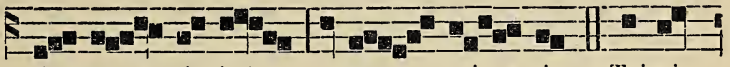

A - re - ri-ia, are - ri - ia. Tsi-ni

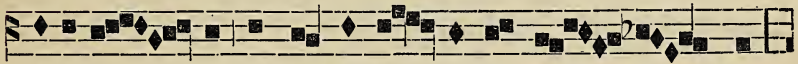

kaia-ti - - io nok io-kwatse sa-tonnhe - - tston,

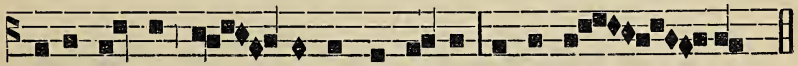
ionehrakwat tsi - ni hianonwehon Rawenni - - io. $a$.

Swanikonhrarak (sk. 13i). Telewariwak tsini (sk. 181.)

IOKARENRE.

TI.

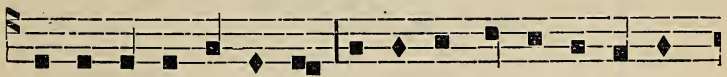

Ne io -ia-ta-to-ken-ti wat-tokhakwe iahte io-na-
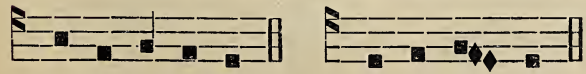

kwaienter-ha-on. (sh.) A-re-ri - ia. Salkon Niio. I $i$. 
T 1

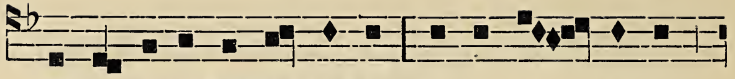

Wa-ta-te-ni-konhra - rakwe to-sa 0 - thenon

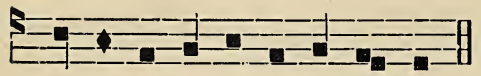

a. - io-ia-to-ron a-o-tounhets.

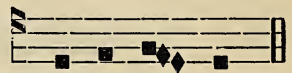

(s/l.) A-re-ri - ia.

Tewakelılakon onkwe. 5 a.

T 3 .

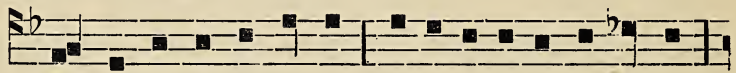

Iah - te io- ien-terha- on io-te- Ii-wate-ha-tanions,

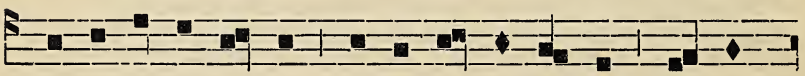

en-ka- ia-tarha - ke ne kentiohkwa-no- ron ron-wa-
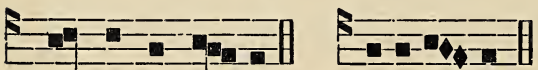

kens Rawenni - io. (sh.) A-re-ri - ia. Sewenniio to ok. 3 a.

T 3.

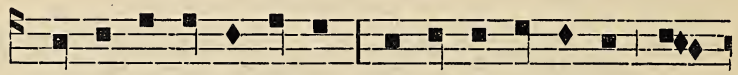

Akwa tsi-ni tsia-ti-io: o-nen hianonwehon Ra-
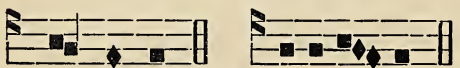

wen-ni - io. (sh.) A-re-ri - ia. Tewakehlakon Rolkon 5 o.

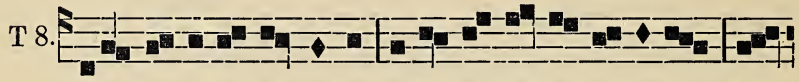

I-se Iesos, ie- sa- ienha Ialste ka-nakwaien-te-ri, ka-

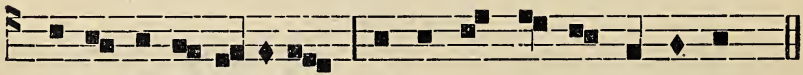

ronhia-ke she-ie - ri-ti, T'si ie-sa - nonwe - hon on-we.

Ise sonhaa tsiatiio, Saiatanehrakwat kowa, Ionehrakwat tsi senonwes Ne naiesanoronkwake.
Ne teiesennitiohkwaton Niahte ienakwaienteri, Tiotkon iesanonteratie Tiesariwakwasehatie. 
Askwentenre, Sewenniio, Aiawen tosa nonwenton Aionkwariwaientake Ioteriwatehat sonha.
Ahonwasennaien Niio Roniha nok ne Roienha, Rotkon Roiatatokenti. Satahonwatisennaien. E. N.

T 5 .

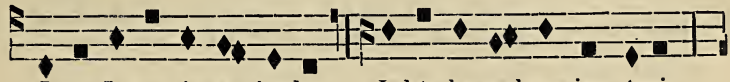

I- se Iesos, ie-sa - ienha Iahte ka-nakwa-ien- teri,

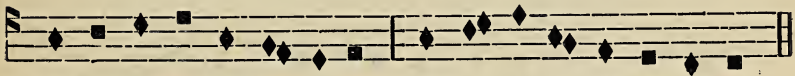

Karonhia-ke she-ie - ri-ti, Tsi ie-sa-nonwehon onwe.

v. 'Tsini kaiatiiohne satonnhetston, (sh.) areriia.

к. Raonha hianouwehon ne Rawenniio, (s/l.) areriia.

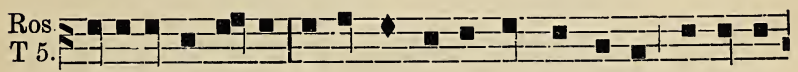

Raonha ne Ie-sos hetsenonwehon nonwentsiake, a - siena
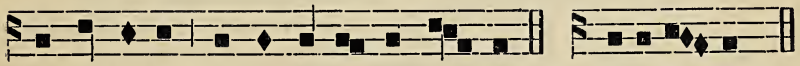

ka-ronhiake hia-nakta-se- ronnien - ni. (s/l.) Are-ri - ia. 7 e.

\section{IOTIIATATOKENTI IOTINIAKONHONNE.}

Ionlaw : Sewenniio (sk. 257). Tuk. (sk.63). Areriia (sk 257). Akwa tsini (sk. 137). Swantkonlırarak (sk. 137). Twanikonlırarak (sk. 181.

\section{IOKARENRE.}

Tierennakelskwalha (sk. 257).

$\mathrm{T} 3$.

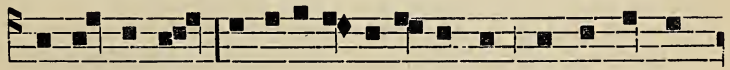

Ranonwehon Niio io-ia-ta-to-ken-ti nonwa awen-

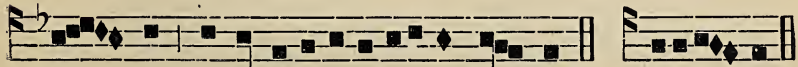

ta - wen, ne tsi kaia-ti-io a-otonnhetston. (s/l.) Areri - ia. 
$\mathrm{T} 4$

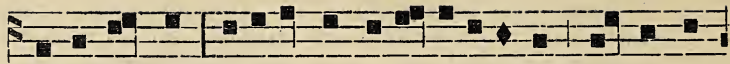

Ionehrakwat ioriowanens sari-waie-ri-ton, a- kwa ia-
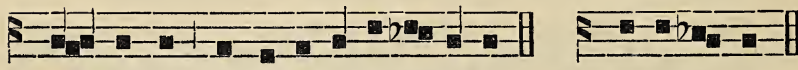

kwaskaneks akwa na-ke-ra-ni- he-ke.

(sh.) A-re-ri - ia.

$\mathrm{T} 8$.

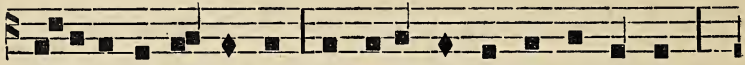

E-so saronhiakenhon nok saio-tenhon nonwentsiake;
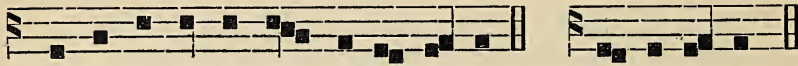

onen ie - io-he a - sa-to - ri-sen. (sh.) A - re - ri - ia.

T 3.

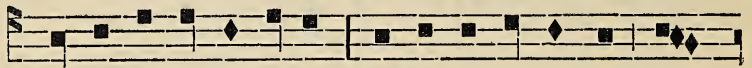

Akwa tsi-ni tsia-ti-io, o-nen hianonwehon Ra-
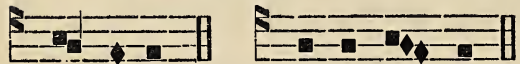

wen- ni- io. (s/..) A-re- ri - ia.

T' 8 .

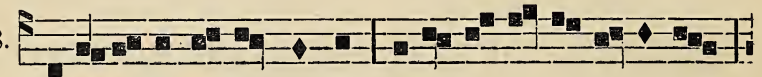

Tae- te-wa-ren-sa-ronnion Io-ia- ta-to-ken- ti nonwa

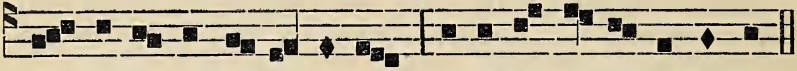

A - wenta-wen ka-ron-hiake le-ia-we-nonton kcn wente.

Raonha Rawenniio

Rononwehon nonwentsiake, Ionwesen, iontonweskwatha, Akwekon iokenhronnihon.

Ioronhiakenton ieronke, Tetsioriwaserakwaton, Ionontenskwe naotonnhetst Tsini waterennaienskwe.
Iesos, shesatstenserawi

Akoiatatokentison,

Siena nonwa awentawen Tsi ionkwaterennaiennis.

Ahonwasennaien Niio Roniha nok ne Roienha, Rotkon Roiatatokenti, Satahonwatisennaien. E. N. 
v. Raonha hetsenikonhraieriton ne Rawenniio, (s/.) areriia.

R. Onwa kati iahte kakonte hiatsennonniatenni Kristos $(s h)$ a.

\section{Ros.}

T 8.

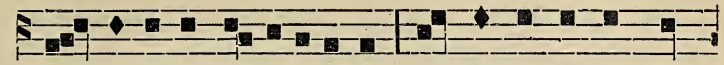

Tes- kiatieren ka-ri-wiioston ronkwe rahninons kat-

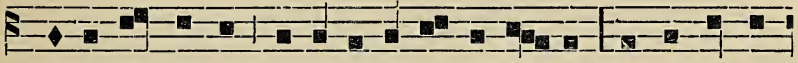

staro-kwa-norons, inon ra-we-sa-konha - tie, enskat waha-

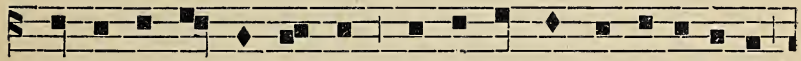

tsenri nakwa ka-no-ron, wa-ha-tenhni-non tsi-ni hoien

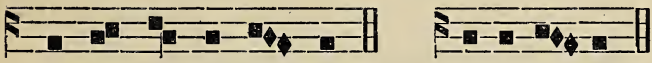

nok wa- ha - hni-non - te. (s/.) A-re-ri - ia.

v. Takwaterennaienhas, N... saiatatokenti, (sh.) areriia.

R. Aiawen laiakwatentsa tsini sonkwarharatstenni Kristos, (sh.) areriia.

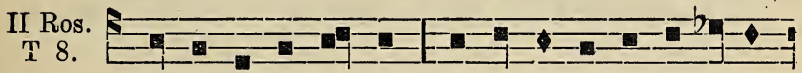

Io- ta-ni-ten-ra-skon iahta te-io-nonhia-ni-he-

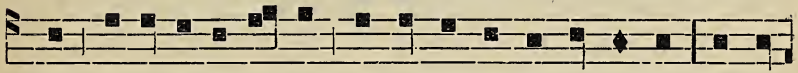

kon aion-ta-ti-ten-re ia-koten-tenion o-konha, nok o-
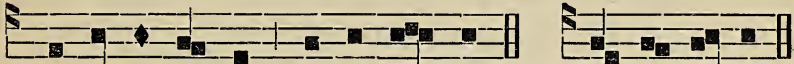

ni kanonweskwe a - io - io - ten. (s/l.) A - re - ri - ia.

\section{ONONSATOKENTI AWENTAWEN.}

Iontaw.

T 8.

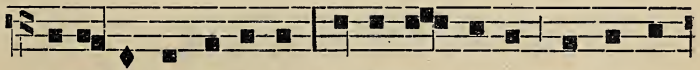

Te-io-te-nonhia-ni-ton tsi tewen-teron : ken ronon- 


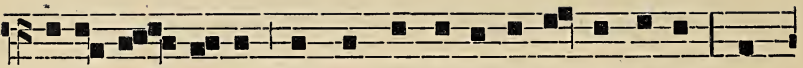

so-te ne $\mathrm{Ni}$-io nok kannhokaron-te ka-ronhiake, nok

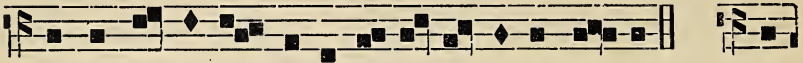

a-kon-wa- na-lon-kwake Isi ro - naktaien $\mathrm{Ni}$ - io. (s/.) A-

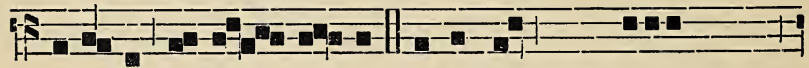

re-ri-ia, a - re - ri - ia. Ionehrakwat, Sewenniio, ionwe-

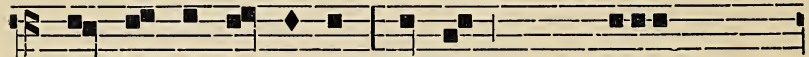

sen tsi te-si - te-ron; ia-onweskwani nok iotonnharen

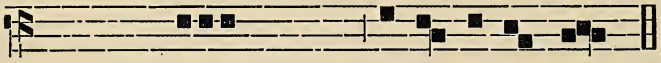

nakenikonhra tsi ie- ha-sen-ta - kwa. ahonwa.

Takw. (sk. 53). Katke onle (sk. 183).

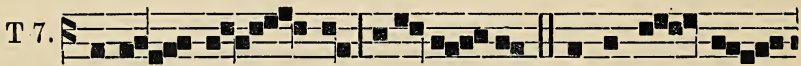

a-re - ri - ia, are-ri - ia. Twatiskwen-!a-

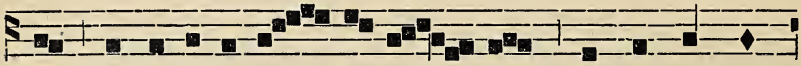

ren tsi ren-teron $\mathrm{Ra}$ - wenni - io, he - tsi- twa-sen-

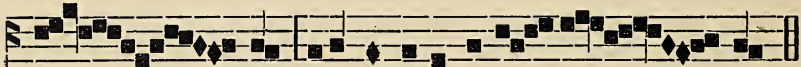

na - - ien raonon-satokenti - ke.

$a$.

Sewenniio tsi (sk. 53). Twakwekon lwatis. (sk. 183).

\section{IOKARENRE.}

T 7.

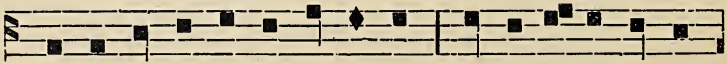

A- ka-non-sa-lo-ken-ton-ha-ke, Sewenni-io, tsinen-

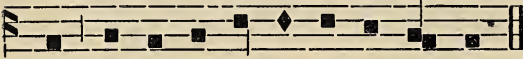

we ne sa-ta-te-non-sa-ra-kwenni. Salkon Niio. 7 o. 


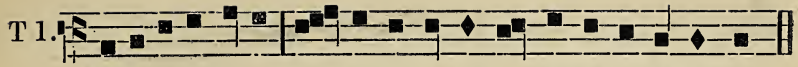

Niio ra-ononsa ion - terenn-aien-takwa kanonsa-takwen.

T'ewakehtakon asen. I $u$.

T 1.

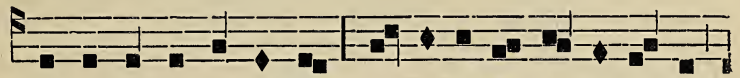

Akwa io-non-sahni-ron Rawenni-io ra-o-non-sa :

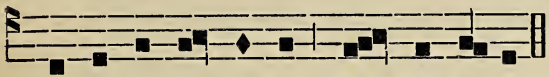

a-kwa o-tsten- ra-ke kentskwahe- re. Sewenniio to ok. 6 a.

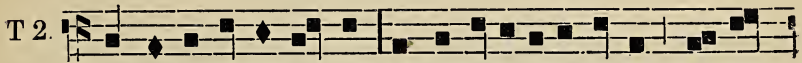

Akwa kanonsano-ron Rawenniio ra-onon-sa, ne te-

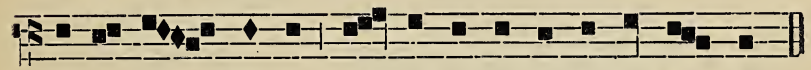

wa-te - nien - tenslon tsi - ni ionwe-sen ka-ron-hia - ke.

Sewenniio iahte. 2 a.

T 6.

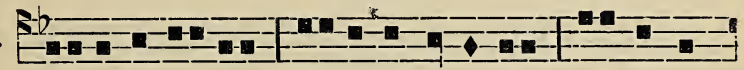

Twanikonhra-rak Ni-io ken renteron Ken ro-ta-

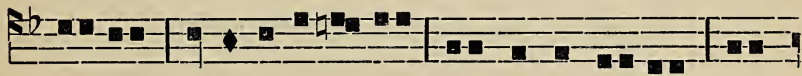
se-ton o-ka-ri-sti-ia - kon Twa-tiskwenta-ren nok

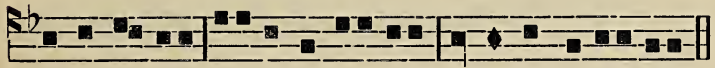

tewakwekon, Twate-we-ien-ton, hetsitwasennaiens. (sk. 134.)

v. Sewenniio, akwa kwawenniiostha.

R. Ken tsiteron okaristiiakon sanonsatokenti.

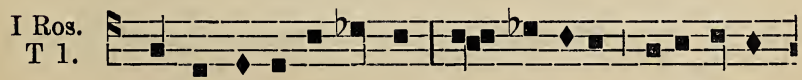

Ranon-sa-token-ti-ston $\mathrm{Ra}$ - wenniio ra-o-nonsa- 


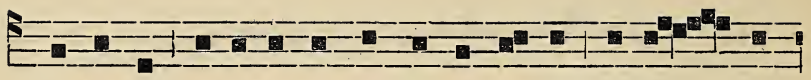

to-ken-ti ; a- seken sonkwarha- ratsten-ni, ell-sa - ko-

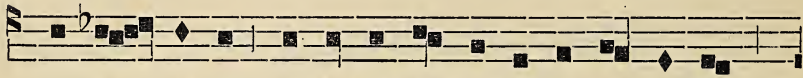

wenna - rakwe. e- tho ronwen-ni-ten-ta-se ra-wen:

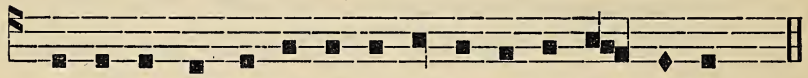

o- thenon nenskwaneken-ni - he-ke, e- tho nen - iawen. 10.

II Ros.

T 6.

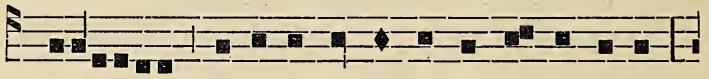

$\mathrm{Te} \quad$ - - io- tenonhia- ni-ton tsi te-wen- te-ron!

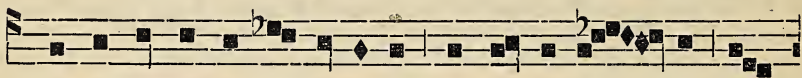

akwa tokenske ro-nonso-te ne Ra-wenni - io, nok

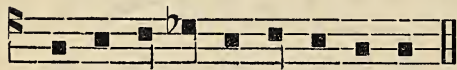

kannhoka-ror-te karonhia-ke. 60 .

WARI IOIATATOKENTI AWENTAWEN SONHA. I.

Iontaw.
T 2.

Wa - ri tekwanonweronnnions iahte sa-nakwaien-

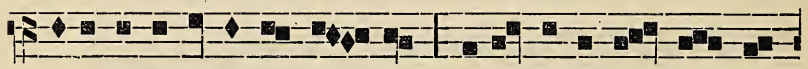

te-ri hetsa-te-we-ton le - sos, raon-ha ka-ron - hia nok

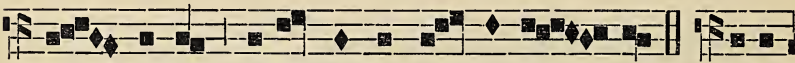

on - wentsia io-nehrakwat Ra-wenni - io. (s/.) A-re-

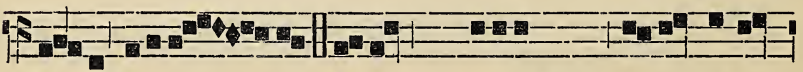

ri - ia, areri - ia. Akwa kariwiio tsini wake-nikonhro- 
但ten,ririwaienthose ne Rawenniio akwekon tsini ka-tie-ranions. Ahon... Takw. Wariaorenna (sk 29).

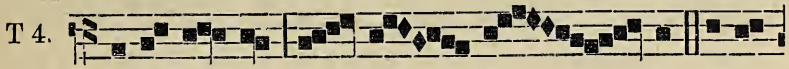
A-re-ri - ia, a - re - ri - - ia. Sate-

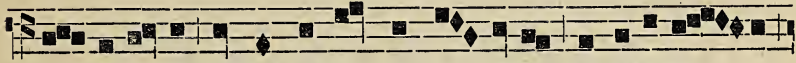

we-ton, Wari,nok iah nonwenton te sanakwa ienterha - on

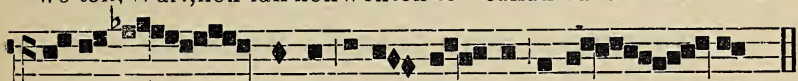

$\mathrm{Ni}$-io he - - tsienha takwa-te - rennaien - has. $a$.

lse Warine

Tsialanoron Wari

Onwari ionkwaienha

Takwaien okonha

Koniennitentase

Kwasennaiens Wari

Ikelıre : aklıesennaien sk. 208 Takilemr Wari

219 Sewalka onen ne

¿I5 Iesennakeraton

203 Rawenniio helsis...

$2: 0$ Ionwesen konwa.

218 Niio lielsienlla senis.

:06 Tekonnoronkwanions sk. 215

217

210

211

214

202

201

IOKARENPE.

T 3.

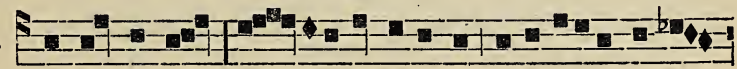

Sonha- tsi-wa Wa - ria tsiatanoron tsiati ioianerensta-

s
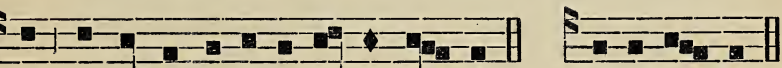

kwa iah enskatha te sa- ri-wannhi-kon. (sh.) A-re-ri - ia.

Satkon Niio, $3 \mathrm{e}$.

T 4.

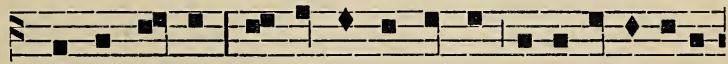

Karonhia-ke sa-tsennon-ni nonwa to-sa askwaiata-
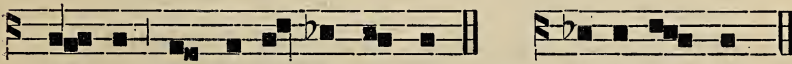

tka- we on-wentsia-ke ia- kwes.

(shi.) A-re-ri - ia.

Tewakehtakwen onkwe. 4. e. 
T 8.

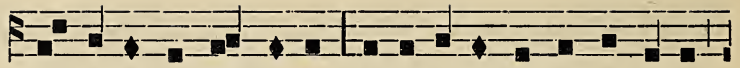

'Tsiariskonhon netsienha Iesos sasonkwaronhiakense
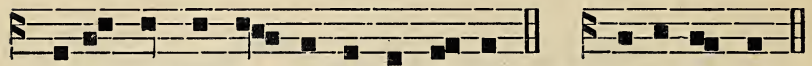

sa-te-tsiatsennon - ni ka-ronhia-ke.

$(s / \iota)$ A-re-ri - ia.

Sewenniio lo ok. 8. e.

$\mathrm{T} 4$.

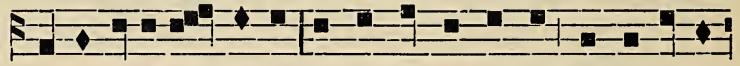

Wari sanikon - hri-io takionweskwatennis hetsenni-ten-
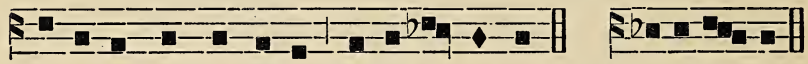

tas tasonkwannhe lesos Ni-io hetsienha. (sh.) A-ré-ri - ia.

Tewakehlakwen Rolkon. 5 e.

T 1.

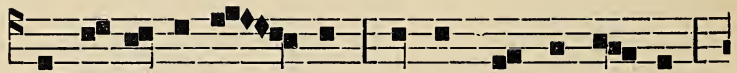

I 'Te-kwano-ronkwa - nions Ne kwenna- ke-ra - ton,

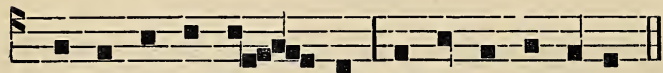

Ie-sos ne hetsien - ha $\mathrm{Ni}-$ io onkwe 0 -ni.

'Tokenske tsiatakon

$\mathrm{Ne}$ onkwe rotonhon

'Tsi hiariwawire

Raronhiakehronon.

Onka aiekweni Aiontéweienton, Aiesasennaien, Saiatatokenti.

Tsini tsiatanoron, Sheiatohetstenni

Eronhiakehronon

Nok akwekon nonkwe.

Sonkwatsennonniaton Ionkwariwiioston
Hiaiatanoronston

Ne Soukwawenniio.

Asakwisron, Wari, Askwaiatakennha Tosa ionkwasenni Ne noneshonhronon.

Askwanikonhrata T'si naiakwaiere Asakwanakeren lesos ne hetsienha.

Ahonwasennaien Roniha, Roienha Niio oni Rotkon Roiatatokenti. E. N. 


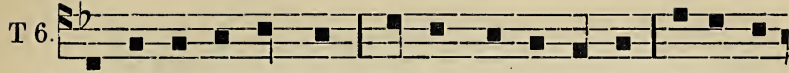

II Tekwanoronkwanions Ne kwenna-ke-ra-ton, Iesos ne

$-6$

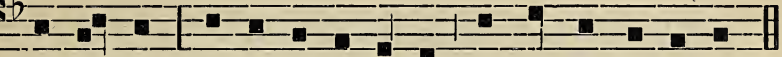

hetsien-ha $\mathrm{Ni}$-io onkwe o-ni, $\mathrm{Ni}$ - io onkwe 0 -ni.

T 6.

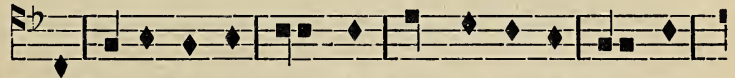

III Te-kwanoronkwanions $\mathrm{Ne}$ kwenna-ke- ra-ton Ie-

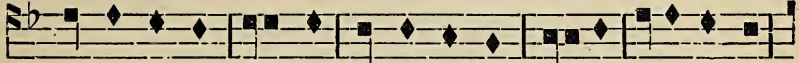

sos ne hetsienha $\mathrm{Ni}$ - io onkwe o - ni, I esos ne hetsien-

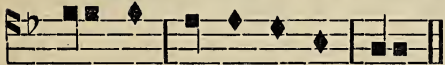

ha $\mathrm{Ni}$ - io onkwe o - ni.

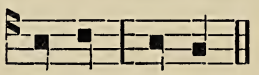

Amen, amen.

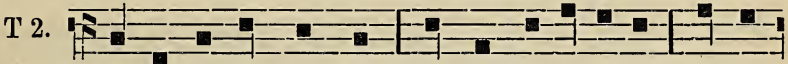

IV Tekwa-noronkwanions Ne kwennake-raton, le-sos

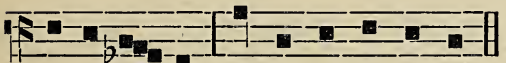

ne hetsienha Ni-io onkwe 0 - ni.

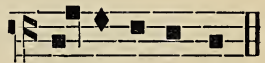

E- tho na-iawen.

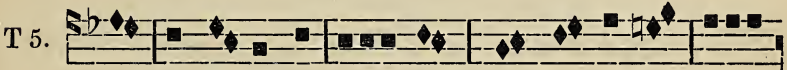

$\mathrm{V}$ Te-kwanoronkwanions Ne kwen-na-ke-ra - ton,

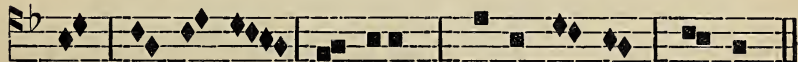

Ie - sos ne he - tsienha Ni-io on-kwe 0 - ni.

T5.

VI Tekwa-no-ron-kwanions Ne kwenna-ke - ra-ton,

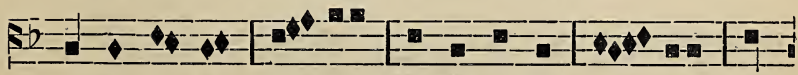

Ie-sos ne he - tsien-ha $\mathrm{Ni}$-io onkwe o - ni,. Ie- 


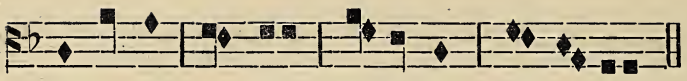

sos ne he-tsienha Ni-io on-kwe o- ni.

v. Takwaterennaienhas saiatatokenti Niio hetsienha, (sh.) ar.

R. Aiawen taiakwatentsa tsini sonkwarharatstenni Kristos, (sh.) areriia.

I Ros. Saialatokenti (sk 82). 4 e.

II Ros.

T 8.

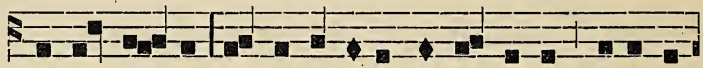

Iotsen-non-ni, eniontonheke nonkwe okon; a-seken

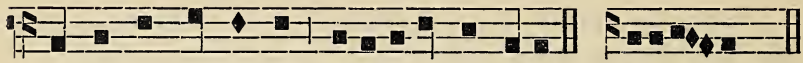

io-tatken-ron-ni-hon ra iatarakwen Niio. (s/ı.) A reri-ia. $6 u$.

II.

Iontaw.

T 2.

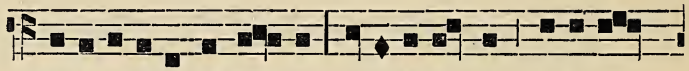

Sa-iatatoken-ti Wa-ri, iesa-ia-ti-saks ia-kori-

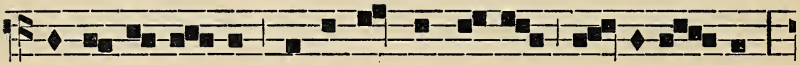

wi-io-ston o-kon ionte-kwasen-ta-kwa se-riasa - kon,

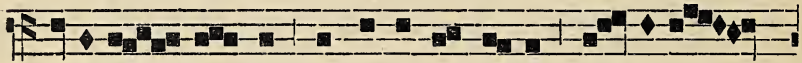

ie-sania - he-sen, ie-sen-ni-ten-ta-se a - sheten -

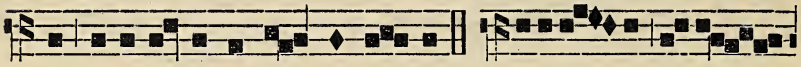

re ashe-ia- le-renna - ien-ha-se. (s/ı.) Areri - ia a-re-

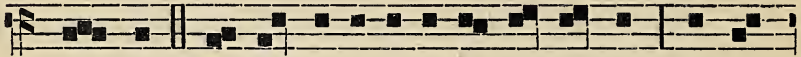

ri - ia. Wa-ri, tsiati io- ia-ne-ren-sta-kwa, se-ni-

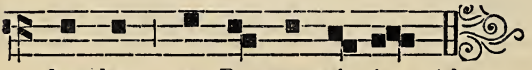

kwekon ne Ra-wen-ni - io. Ahon. 
T 8

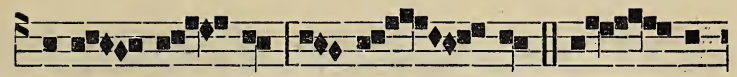

A-re - ri - ia, a - re - ri-ia. Sa - ta-

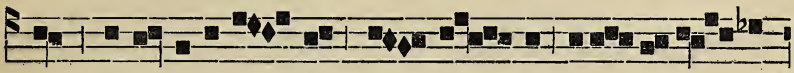

skat, sa-ia-ta-token - ti Wa - ri - a, a-ie-sa-nen -

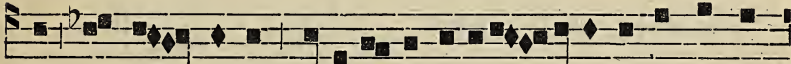

ton nonkwe o-kon; aseken takwatewe . ton-ni te-hoswa-

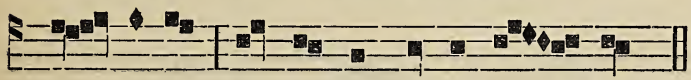

the ko-wa Kri-stos Sonkwawenni - io. $a$.

Ise, Wari, tsiatanoron, Iesennakeraton karonhiake Sonhatsiwa kwaniahesen, Shetenr niakoriwanerahakskon. Nok takwaialakenphasek.

Oneshonronon ionkwanosas Ioiote naionkwakaronni, Watoratis nonkwe okon; Tosa, Wari, takwatewentet.
Askwentenre, tosa othenon Aioiatoron nonkwatonnhetston ; Askwatkatho tsi ionkwentent Iakwaskaneks iakwakwekon Taontaskwanerake,ionkwentent, Iah othenon te sanoron Nasonkwentenre Rawenniio.

\section{IAKONIAKHE AKOHASERA}

Satkon (sk. 233).

Iontaw.

T 1.

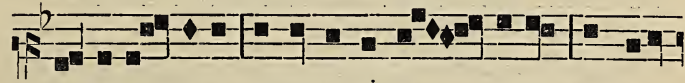

$\mathrm{Ni}$ - io I - sraer ta-tsi-se $\frac{\text { ni }}{\text { wa }}$ rah - neken nok a-e

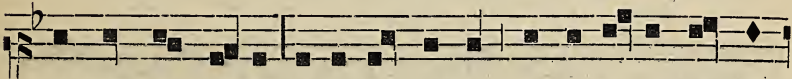

swakwekon - ha-ke ne sa-ko - tenron tehni-ha-se tsionkwe-

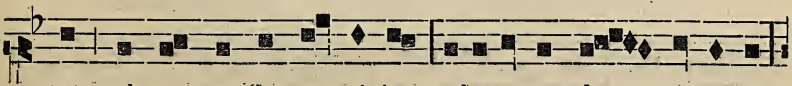

tat, nok nonwa, Se-wen-ni-io, a-kananon she - ienawäs 
2

a-ie-sa-sen - naien - seke.

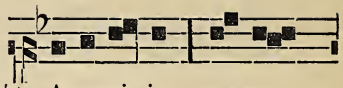

(s/i) A-re-ri-ia, a-re -

-2
10

ri - - ia. Iakotaskat akwekon ronwatsanis ne Ra-wen-

7-

ni - io; ne iakotahitakhe raoia-nerense-ra-ke. Ahon.

Takw. (sk 68).

T 1.

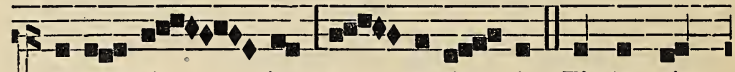

A-re - ri - - ia, a - re-ri - ia. Thaten-niet

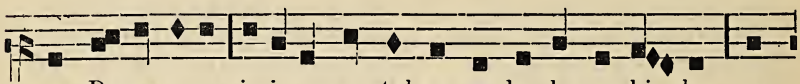

ne Rawgrnno io-iane-renstakwa sonha karon-hia-ke na-

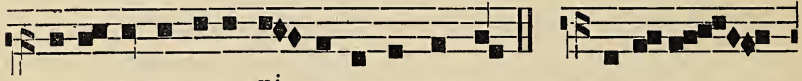

onta-we he-tsise $\underset{\text { wa }}{\mathrm{ni}}$ ia-tanon-statsek. $a .(s h)$ Are- ri-

3.

ia. Atsi-se ni- wen- $^{\text {nen- re Rawenniio ro-ta - nitenron ne ro- }}$

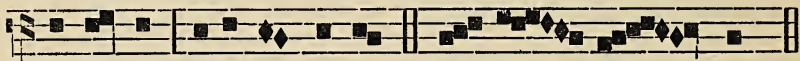

ronhi-son raonwen-tsison. A - re - ri - - ia.

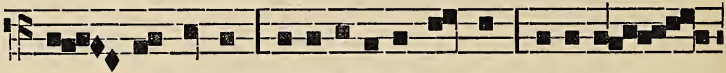

Ken neniawen eniako-ianerensten onkaki

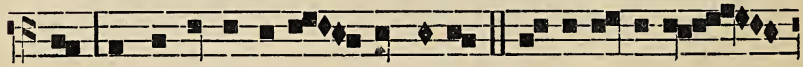

ok ronwatsanis ne Ra - wenniio, A-hiaia-neren -

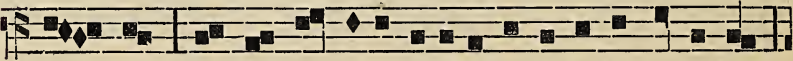

- - sten ne Ra-wenniio ontahatennie-te ka-ronhiake 


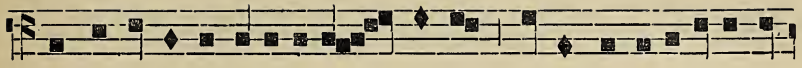

nok a-satkatho io-ianere sonha niate wenniserake tsi-

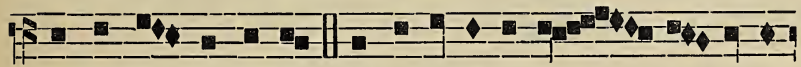

nenwe en - sonnheke. Nok a-satkatho sa - - ko - ti-ien-

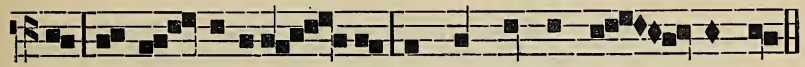

ha ne she-ien okun - ha skennen kenhak sa - nonskon.

Ise lesos Sewenniio (sk. 233).

T 8.

Rawen-ni-io ensa-ko- ten-ra-nion tiako-riwaie-ri ne-

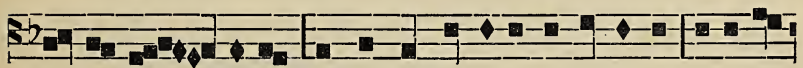

ne ronwa - tsanis teniontatken ontatien okonha sakona-
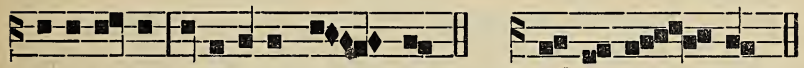

tere o-kon ensakaon sken - nell. (s/l) A-re-ri - ia.

\section{TSI KAHNIOTE OKARISTIA}

Iontaw.
T 2.

Sonkwa-nontens ne Rawen-niio o- riwi-io ne raie-

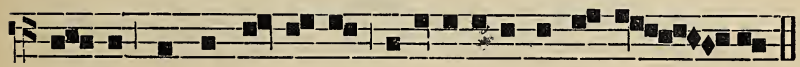

ron - ke; sonkwatsteronwi nae-tewake twatsennon - ni.

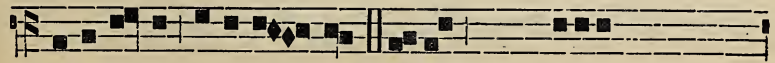

(s/l) A-re-ri-ia, a-re-ri - ia. Tetewariwak hetsitwasennaien 
F-

Niio karon-hia-ke. A-se-ken a-ste-son se-sonkwen-

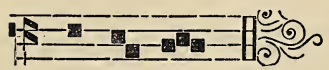

tenra-nions, Ahon. Takw. (sk. 49).

T 7.

A-re - ri - ia, a - re - ri - ia. $\Lambda$-ke-

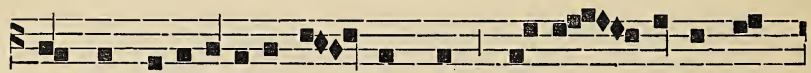

waronk o-ri-wiio ia-kon - nhekwen, nok a - ke-nekwen-

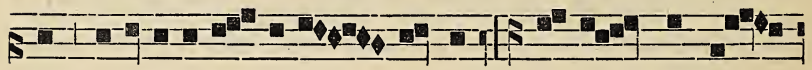

sa o-riwiio ieh-neki - rha-tha; ne i - raks na-

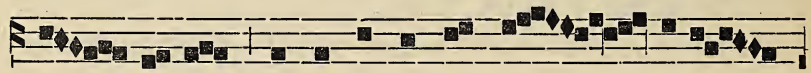

ke - wa-ronk nok rahne-kir-ha na - - kene.

5-2

- - kwensa nakon-ha - ke raia - tare, nok i - i kiata-

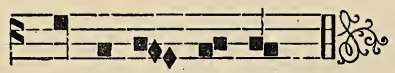

re ra-on - ha-ke. $a$.

Tsi enhonekarhase: Aetewawennakwekonhak. Oh nahoten (sk. 97).

T 7. S-

A-kwa tsinikon ensewa - natarhakse-ke ne ka-

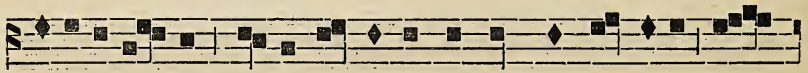

natarano- ron, se- we-ia - ra-sek ne Sonkwawen-niio son- 


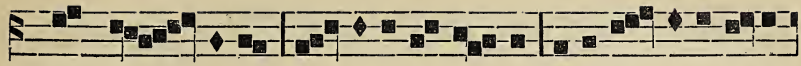

kwenhe - ia-se. Tsi-niiakon ka - ti enieri - waiesa-te

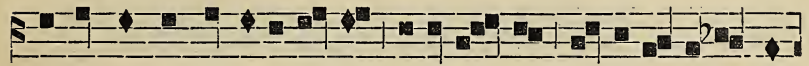

ne kakwanoron oka-ri - stia, ra-ieron-ke Iesos ra-0-nekwen-

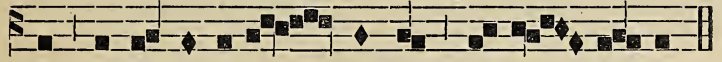

sa enhon-waie-sa - tanion a-re - ri - ia,

\section{TSI RATHONNIANE}

Iontaw.
T 7.

Tsionkwe o-kon, o-nen to-ha en - ra-we ne Ra-

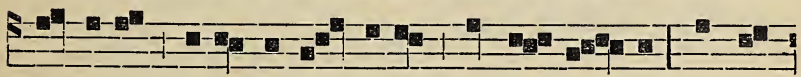

wenniio nasa-kos-kon-ta-ko nonkwe o - kon, nok o-

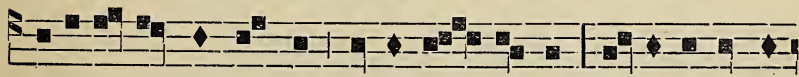

ni ense-watsen-non-ni seweria - sa-kon tsi onen en-tsi-

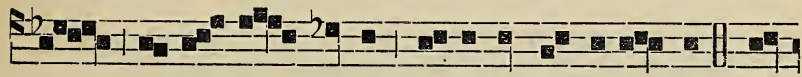

se - wa-wenna - hronke tsi-ni hawen-na-no-ron. Se-

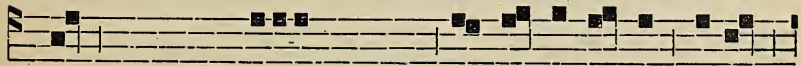

wenniio sheianerenstenni ne sheien 0 - kon; eren

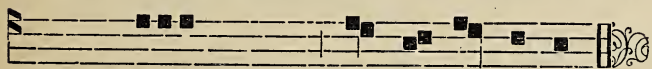

sheiatenhawitenni ne iont-ka-ron-niatha. Ahon...

Takw. (sk. 39). Karo kase Iesos (sk. 109).

T 8.

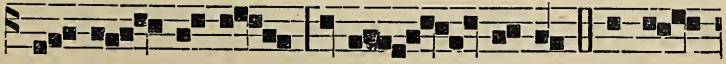
A - re - ri-ia,
$\mathrm{a}-\mathrm{re}$
ri - ia.
Takwa- 


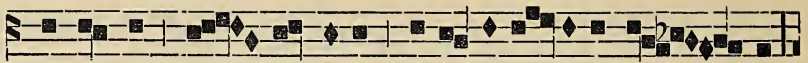

na-tonhas, Se - wenniio tși-ni sa-ta - nitenra - skon,

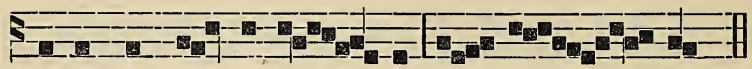

a-sonkwenna - ke-ra - - tse ne - tsien - - ha. $a$.

Iesos rakeni sanikonh... (sk. 153). Okarisliianoron (sk. 190).

IOKARENRE.

T 8.

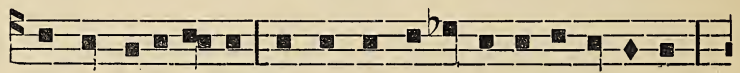

Tsi nenhatota- si nonwentsiake sesakoskonta-ko-he

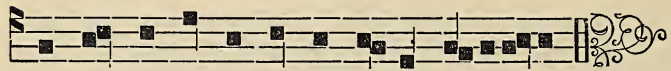

e-niontsen-nonni nonkwe o- kon, a-reri-ia. Salkon Niio. 8 a.

T 8.

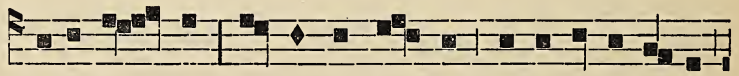

I-se tsion-kwe nonwentsia-kwe-kon, sewarharek ka- ti

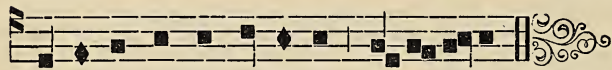

entsi-s -wenten-re Ni-io, a-re-ri-ia. Niio Sewenniio. 2 a.

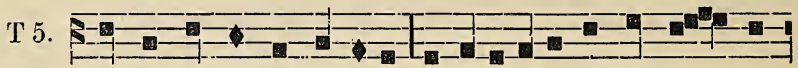

Onen tentre Rawenniio enthoia-tanehrakwaton - ha-

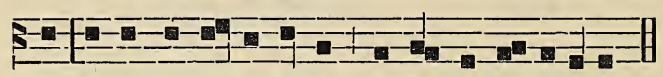

tie tenioswate-ke kowa netho-ne a-re-ri-ia.

Tewakehtakon akwa. 5 a.

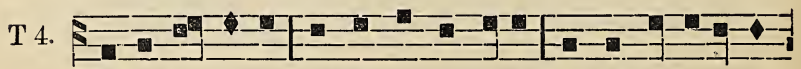

Ni-io Ro-ienha sonkwatonniennire, sonkwariwaseron-

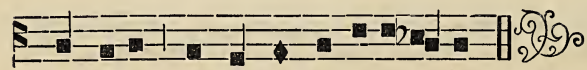

niennire onwentsia-ke a-re-li - ia. Sewenniio ise. 4 e. 
T 4

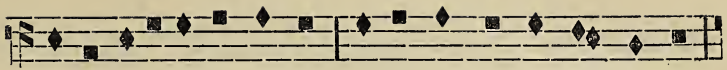

Ni-io sata-niten-raskon io-nehrakwat ta-kwanonwes

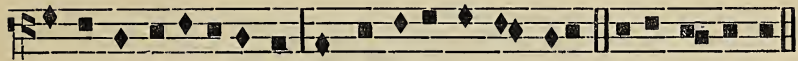

Aionkwatetsiratonke Skwariwaseronniennire. Etho na-iawen.

Wahonnise tsi kwarhare, Tsi kwaskaneks, kwaiatisaks, Aiawen aiosnorenne Ne raontaskwentenrane.

Ne kwennitha, Sewenniio, Ontaskwennakeratire Aseiatekwate notkon Sonhaa asewenniioke.

Ontasatsnent karonhiake Ontaskwarihonniennira
Tsi akwa naiakwaiere Ontakariwaierike.

Onen twatonnhakanonni Aionkwarihonti onwe Ne kariwaksenskwe okon Ronwanikouhraksatakwa.

Ne tenhnon aetwatsterist $\mathrm{Ne}$ kariwiio nahoten Ne akwa`rariwanonwes Ronwanikonhraieritstha.

v. Tesannhokonti karonhiake.

R. Aontahatsnente sesakoskontakohe.

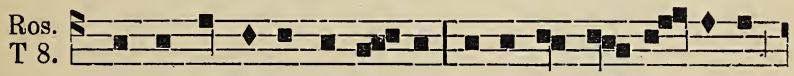

'To-sa sahte-ron, Wari - a, hiani-konnra - non-wehon

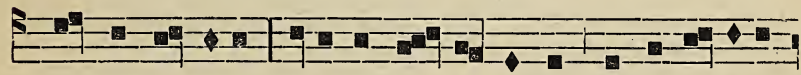

ne Ra-wen-ni-io, o-ia nentsia- to - ten-ne, nok en-sa-wi-ra-

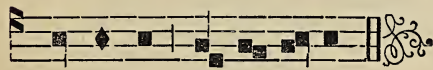

ien-ta-ne, a - re-ri-ia. $8 e$.

3 DEC. SAKSARIE RTI. 1on WENT. KAHNAKWAKE.

Iontaw.

T 5 .

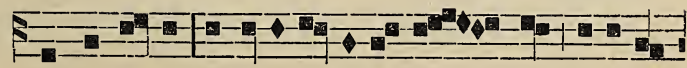

Sewenni- io wakeri-howa-na-ta - - nion sa-iane- 


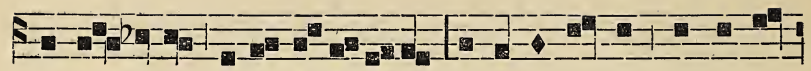

rense - ra ro-ti-nonti-io-son; wakenonwehon akwa jo-

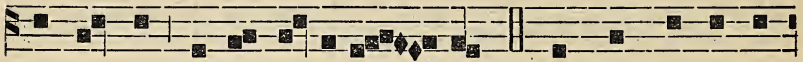

ne-hrakwat sawen-na-token - ti. Swentiohkwakwekon

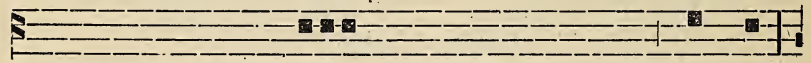

ne tsionkwe tatsiswanonweraton ne Raw nniio tsi Isionnhe

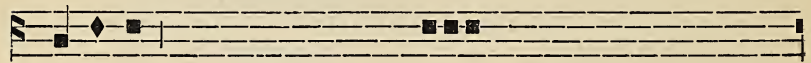

a-se-ken asteson sonkwentenranions, nok oni enwation

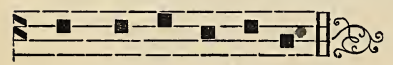

sonkwarha-ratsten-ni. Ahon. Tukw. kowa (sk. 42). Are. (sk 255).

Hetsitwasennaien nonwa Hetsitewennakeraton Ne kento raotenata, Saksarie Roiatatokenti.

Sekon si hanekenteron Wathoswateten ne Niio, Wahonikonhraientane, Ok sa ok wahariwiioste.

Raonha rakwenion Ennias Wahotokaten oriwakon Nonwentsiake iontsennonni, Nok noneshon iontkaronnis.

Ethone wahatewenlete Ne iontonweskwatha nonkwe ; Wahatateriwisahas Niio raoriwa okon.

Iah sane te horiwaien; Ok eken kati rokwisron
Wahatateronhiakente

Ne raieronke nonkwati.

Tikate nasontakwekon Roterennaientatieskwe; Nok ne isini wenniseres, Onkwe sakoseronniennis.

Rokwenion kentiohkowanen Tsiakotatrewaton onwe, Iahten tetsiakotsteriston, Ne iakarıwaientakwe.

Iah katiken iah taonton Naonsetewatatrewate; Tewalsiaken tewatontatsek Nensonkwahretsiaron.

Hetsitwawenniiot Niio, Nok oni Roienha Iesos. Nok twanikonhrakwekonhak Saksaria hotsitwasennaien. E.N.

Kenlıolikwakwekon (sk. 250). Ionehrakwal (sk. 178). 
T 3.

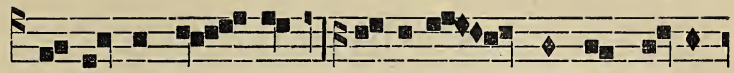

Ro-ta-skat ron - kwe ro- ta-ta - skennhen ro-io-

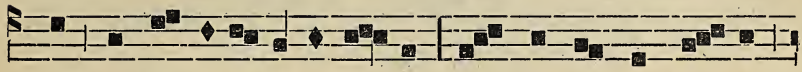

te, tiotkon enha-ie-wa-ta - ke. To - kenske kwahro-ris,

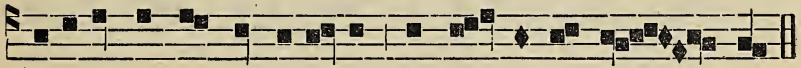

enhotakwennia-ien-ni-heke ronnhas tsi-ni ho - - ien.

\section{IOKARENRE.}

Sewenniio (sk. 257). Tsi nakonwehiarane. Ion lok. ken kaien taieriwakwe.

T 1.

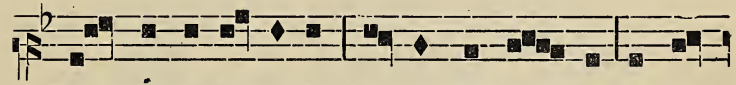

Tsionkwe se-wat-katho he - lsiswarha - rek iah o-

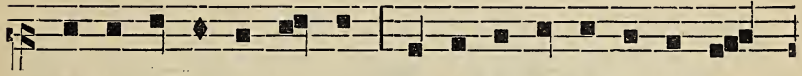

thenon te ho-no-ron-se se-sa-koskonta-ko-he non-

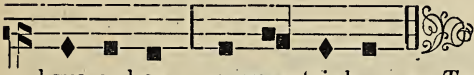

kwe o- kon nonwen-tsiake. v. Teshannhohonli.

II Iok : v. Takwaterennaienhas Saksarie saiatatokenti.

R. Aiawen taiakwatentsa tsini sonkwarharatstenni Kristos.

Ros: Saksarie (sk. 83) । a.

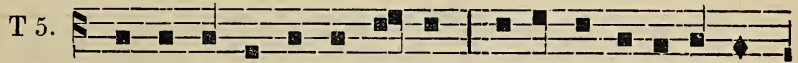

Ra-ton-hakwe ne San Watis: hetsiswa-ha-hase-ron-

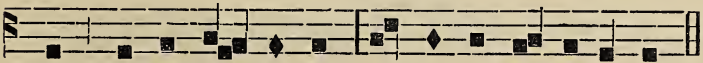

nien $\mathrm{Ni}$-io Ro-ienha en - tsiswenna-ke-ra-tse. 


\section{TSI RATHONNIANE}

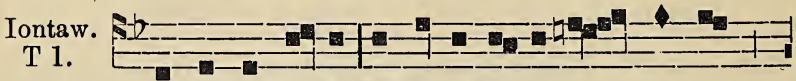

Teshannhohonti Karonhiake; onen skennen

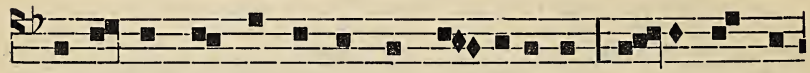

on-ta- hatsnent ne nensonkwenna - ke-ratse ; i - se tsionkwe

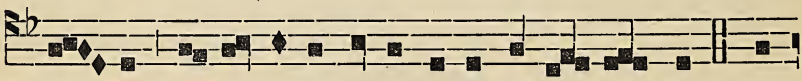

o - kon, se- warharek entsiswa-nakta-se - ron - nien. Se-

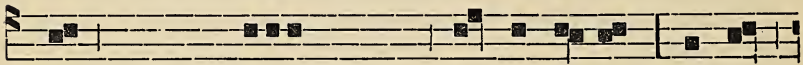

wenniio, saiatanehrakwat ne ka-ron-hia-ke; sonha-

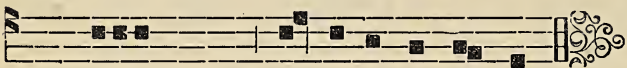

tsiwa sason tsi - ni-kon iakwatka- thos. Ahon...

Takw : (sk. 39). lesos oriwiio (sk. 146).

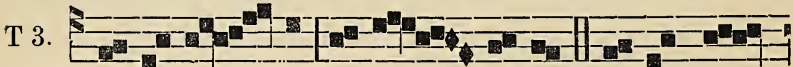

A-re-ri - ia, a-re - - i - ia. Ie-sos, kwar-

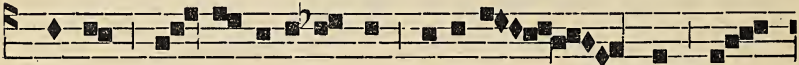

ha-re, to - sa a-sani-sko, se-riwah - - tont ka-

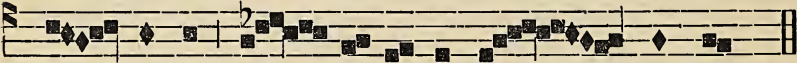

ri - waksen; she - ne-ren-si sen - - tiohkwa. $a$.

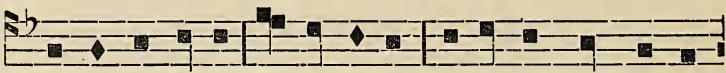

Tesannho-honti, ka-ronhiake: on-ta-hatsnent ro-ta-

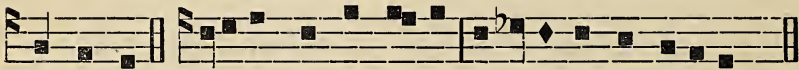

ni-tenron. 2. Ise ne tsionkwe okon hetsisewaskontakohe. Tes. 


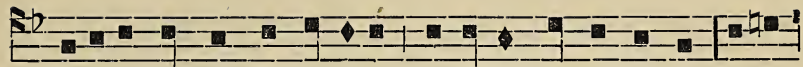

sasa-nikonhren, Se-wenniio, tsi-nıkon kwasenten-ni, tatsia-

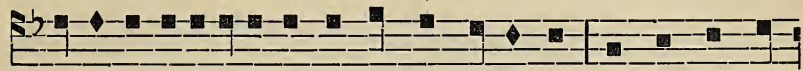

tasnorat aon-tare nensonkionwentsiorenne sonkwatsennon-

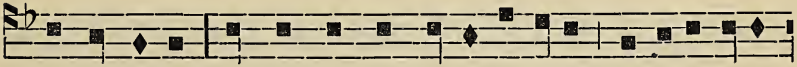

niaten-ni-re, tiotkon ionkwarha-re tsi-nahe tionki-riwane-
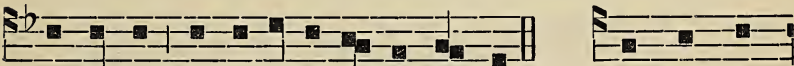

rakten-ni ne tho-nalieren-ton nonkwe. Tes. Ion-kwa-na-

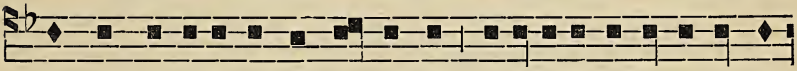

skwaien tsinahe notkon-se- 1 aksen ; raonha enhakweni netsien-

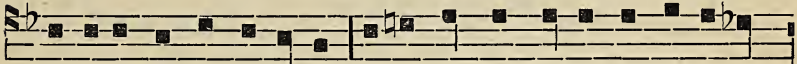

ha ense-sonkwaneren-si ; io-nehrakwat tsi-na ionkwaia- ta-

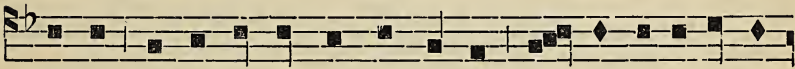

wenhon niahta sonkwatonnien-ni-ron ne-tsienha ne na-son-

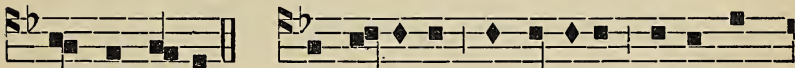

kwaskonta-ko. Tes. Teskwanerak, Sewen-niio, tsi-ni ion-

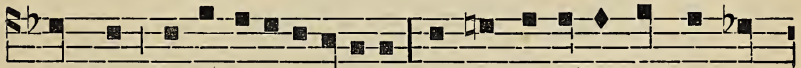

kwentent ok sa kati a-ontare son-kwa-ri-wase-ron-nien-ni-

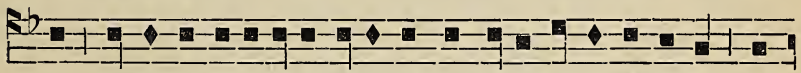

re; ok sa ok a-ontare rariwah-ton-tane ka-ri-wa-neren, ne
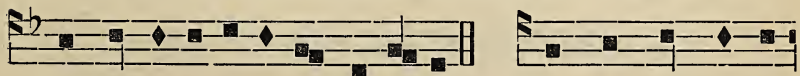

ionkwa-ka-rewa-ta-nion tsi-na-he. Tes. Skennen swennonton- 


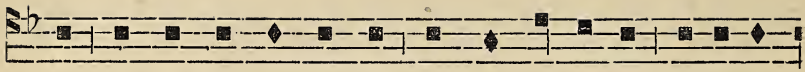

nion,skennen swennontonnion nonweutsiakwekon ; onwatsi

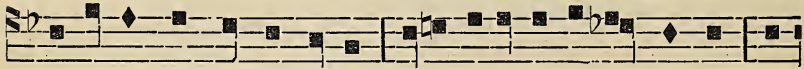

ok onen swatsennonniane ; tosa te-sewatonha-ren-ron : wa-

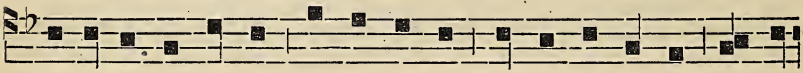

katen-ni-ten-raskon; ii kwaien-ha nonwentsiakwekon; ii ten-

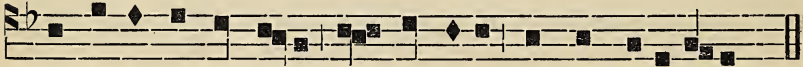

hnon Niio kwawenni-io, kwentenrane, wakwaskontako-he.T'es.

Twanikonhrarak (sk. 184).

Ros.
T 8.

I - se ken ta-: e-ti.n ;, o- ia ken ensakwarhareke? Hetsi-

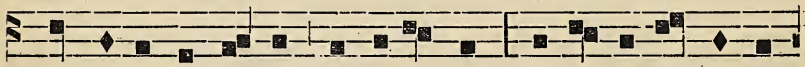

swahro-ri San Watis: Ken ni-iawens : te-ie-ronwe-kon-ne

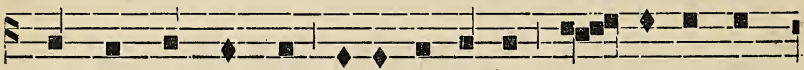

tsiekens, tsiontonnhetst ia-ka-wenhe-ion, ia - kotent tion-

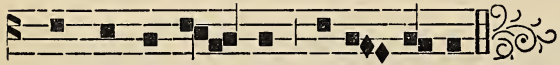

tatswa-te-ten - nis, a-re-ri-ia. 8. $a$.

8 DEC. WARI SONTIATATA. I WENT.

Iontaw.

$\mathrm{T} 1$.

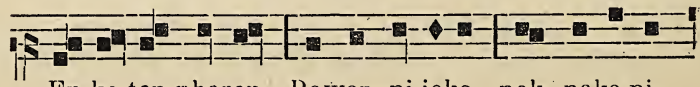

En-ka-ton-nharen Rawen-ni-ioke nok nake-ni- 


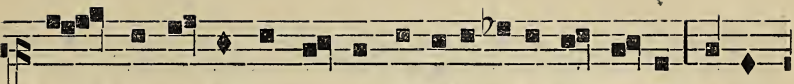

kon - hra en-watsennon- ni Ni-ioke ra-kewen-ni - io ; a-se-

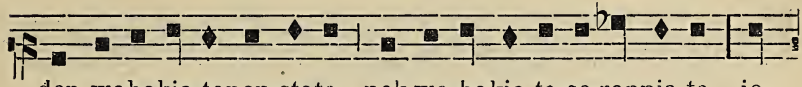

den wahakia-tanon-state nok wa-hakia-ta-se-ronnia-te io-
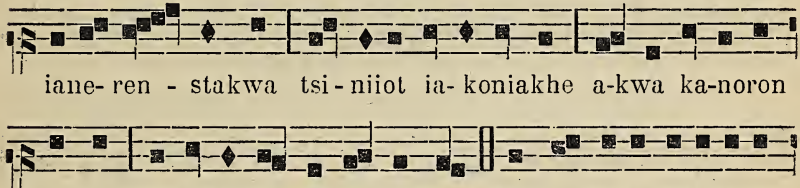

son-lua ia-ko-tiata - se-ron-nia-ton. Sewerl-ni-io,enke-riho-

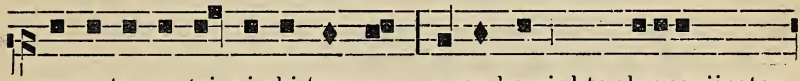

wana-te ne tsi-ni ski-ten-ron; a-se-ken iahte sheweniioston

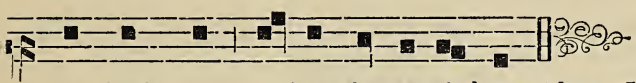

ne ionkswens na-ion-tka-ronni-hon. Ahon Takw. (sk. 49).

T 4. $\overline{1}$ 二-

A-re - ri - ia, A - re - ri - ia. Akwa tsiati-io, Wa-

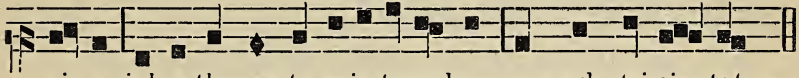

ri-a, iah 0 -thenon te saia-toronhon onwarh tsi sia-tat. $a$.

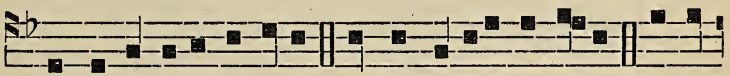

Akwa tsiatiio Wari-a. 'Akwa tsiatiio Wari- a. Iaho-

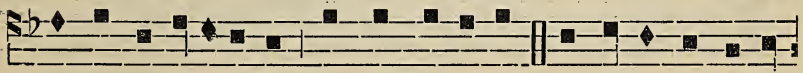

thenon te saia-to-ron onwarh tsi siatat. Iah o-thenon te sa-

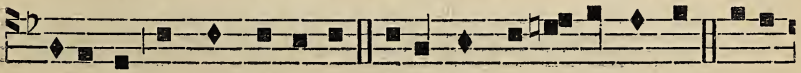

jatoron onwarh tsi siatat. I-se skwana-ti - iostennis, I- se 


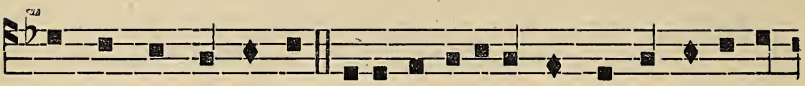

skwatsennonniatennis. I-se sheia-tano-ronstha sheien okon-

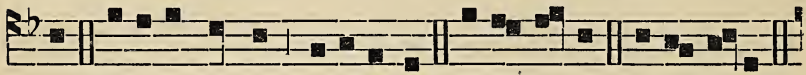

hon. Sheiatakennhas ne ie-ie-sas. O Wari - a ! O Wari- a!

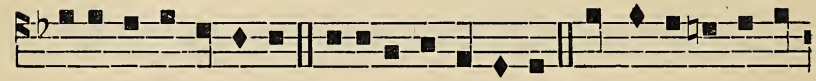

I-se sattokha kowa. Sata-nitenron kowa. Takwaterennaien-

52

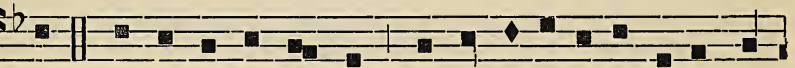

has. Takwahretsıaronkwas Rawenni-io Iesos asonkwen-

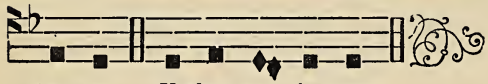

ten-re. E-tho na-ia-wen.

Tekonnoronkwanions Wari (sk. 201). Iesos akalon. (sk. 182).

IOKARENRE.

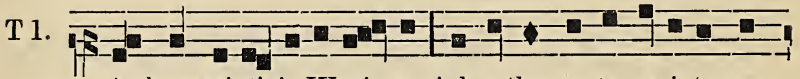

A- kwa tsiati-io Wari-a, iah o-thenon te sa-iatoron-

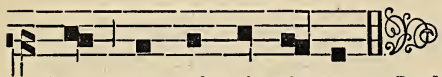

bon onwarh tsi sia-tat. Salkon Niio. 6 .

$\mathrm{T} 5$.

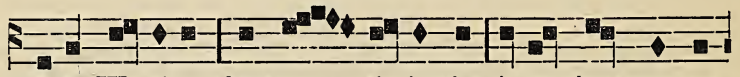

I- se Wa-ri-a, skwana - ti - iostha, i-se skwatsennon-

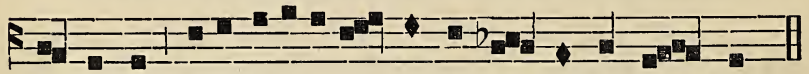

niaten-nis, i-se sheiatano - ronstha ne sheien o - kon.

Tewakehlakon onkwe. 5 P.

T 8.

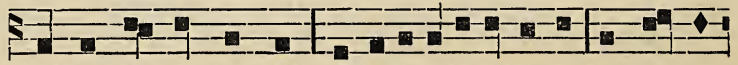

Sonhaa hiakonnieustha, Wari saiata-tokenti, Ra-wen-ni- 


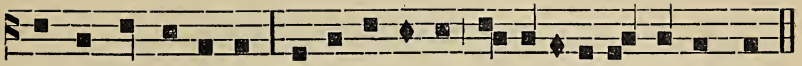

io sonkwaia-ti-son, iah onka etho te sakoia-la-no-ronston.

Sewenniio lo ok. 8 a.

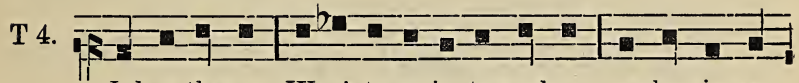

Iah o-thenon, Wari, te sa-ia-to-ronhon; a-skwaie-na-

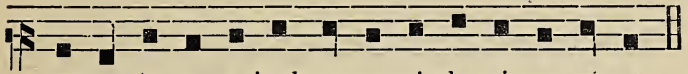

wa-se to-sa a-ionkwa-sen-ni ka-ri-wa-né-ren.

Tewakelitakon Rotkon. $4 \mathrm{e}$.

v. Onwa ken wente iotiataten Wari ioiatatokenti.

R. lahte ioiatoronhon kariwanerahakserakaion.

I Ros. Saiatalokenli (sk. 82). 1 a.

II Ros.

T 1 .

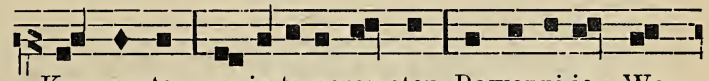

Ken wente ro-ia-ta-noron-ston Rawenni-io Wa-

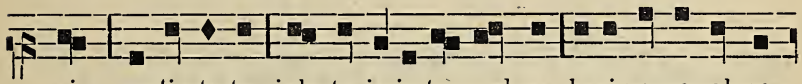

ri sontia-ta-ta, iah-te io-ia-to-ronhon kari-wane-rak-se-

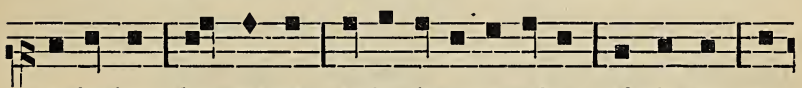

ra-ka-ion; ken wente te-io-nia-ro-ra-rakon ohnia-re ne

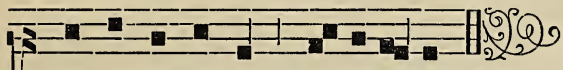

notkon-se-raksen, a-re-ri-ia. $6 i$.

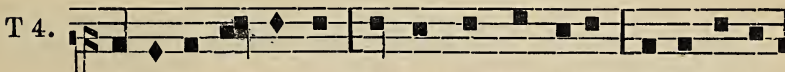

Niio hetsa-tenniet nensonkwaskon-tako. akwa enha-

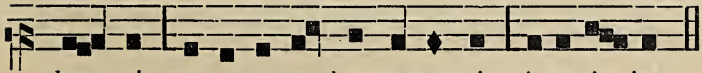

kwe-ni a- on-sa-son-kwa-neren-si. A-re-ri - ia. 
III. TSI RATHONNIANE.

Ionlaw : (sk. 273). Karonhiake (sk. 109). Satsleniaron (sk. 110;. Trualiaken (sk. 188).

Ros. karo ne, 17 Dec.

$\mathrm{T} 8$.

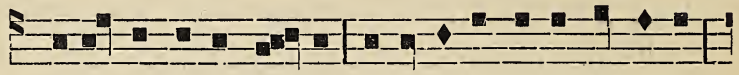

Sa-taskat kowa, Wa-ri, sa-tonla-ton tsi-ni hiahro-ri

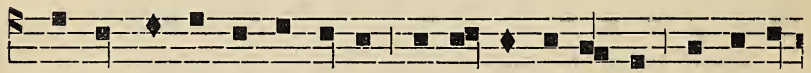

ne thonnhaon Rawen- ni-io; ne to- kenske e - tho nensa-ia-

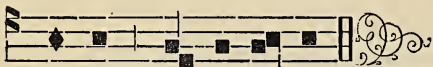

tawen, a-re-ri-ia. 8 a.

Onen ne 17 Dec.

T 2.

O Sewen - ni-io te-ioswa-the ni-sia-tolen ka-sat-

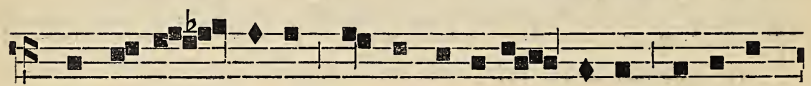

snent karon - hiake tesheswil-te-ten - nihek te-tia-ko-

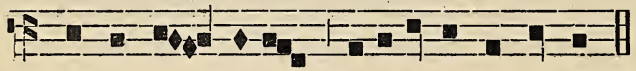

ni-kon-hra - karas a-sa-takon ia-konnhe. $8 e$.

IV. TSI RATHONNIANE.

Iontaw : (sk. 278). Iesos karo (sk. 110). Tesannhohonti (sk. 278). Kento (sk. 183).

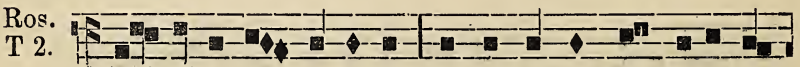

O iesenna - ke-raton akwekon nonkwe, iesarha- 


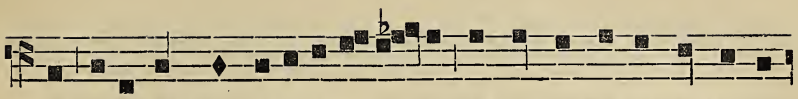

re a-ontaskwennakera-ti - ra ionkwanaskwaıen notkonse-

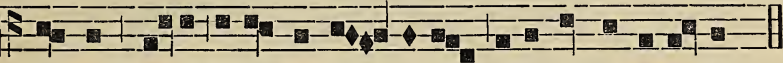
raksen, i-se ne ta-kwaia - ti-son a-onsaskwanerensi. $2 a$.

21 DEc. ATONWA RTI 2on Went. (sk. 238).

Ros.

T 8.

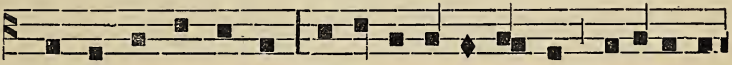

Ne tsi waskatka-tho, a-tonwa, saton-ta-ton; iakotaskat

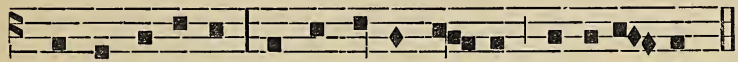

nenionton-ta-te niahte ia-kotka - ton, a-re-ri - ia. $8 e$.

TSI NAHATONNI. I WENT.

ASONTHEN.

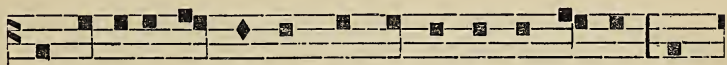

Rawenniio tsi tionnhesonkwawi ne Ro-ien-ha rashotonnheton, a- re-ri - ia

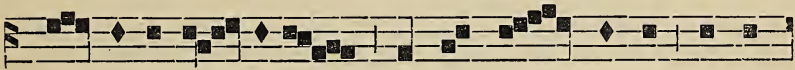
nen - sa-ke ka - ratie nonwentsia - kwekon ka-ron-

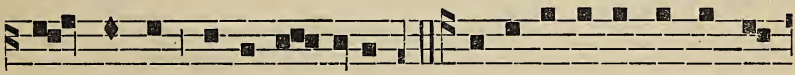

hia-kwekon ratste-ri-sta ne. Ka-se-ne to-sa swenni-ten-

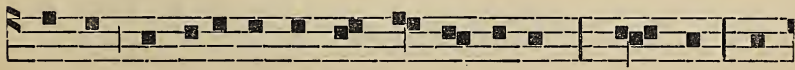

tonhak, ken i-re se-sonkwaskon [ta-ko-he (s/t) ta-kwen]; a- 


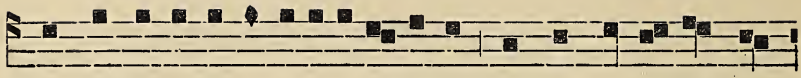

kwekon te-tsi-te-wa-te-ratana niha tenionkwari - wakwen-

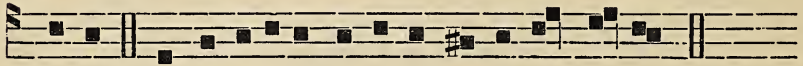

hatie. Ka-ren-niioson te-tsi-te- wa-ri-wa- kwas. Rawen...

A - re-ri - ia, a - re-ri - ia, a-re - ri-ia.

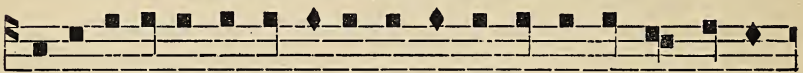

Raon-hatsi-wa liawenni-io nonwentsiakwekon ratsia-nen-ha-

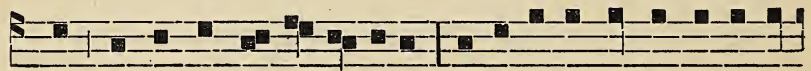

we akwekon te-ha-ka-nere ka-niata-rakwekon io-tahon-

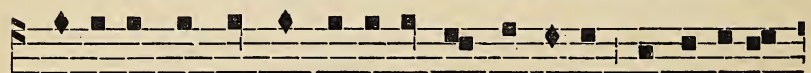

sa-te tsi nennhannhanne e-tho nen-wa-tiere, nok o- ni ra-
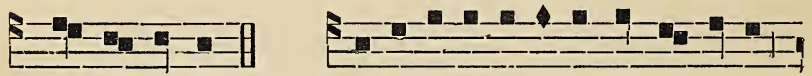

on-wen-tsison. Ka: A. Kase-ne te-tsi-te-wa-nonwe-raton

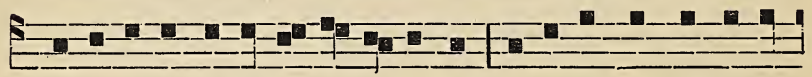

tewastoron te-tsi-te-wa-ka-nerak ne sesonkwaskon-ta-kotwatonnharen Sonkwawenni- io non-

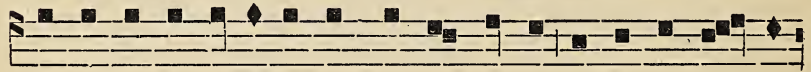

he nonwa wa-sonta-te sonkwenten - ra-ne, te-wa-ta- hon - sawa wenni-se - ra-te se sonkwaskon-takwen twakwekon te-wen-

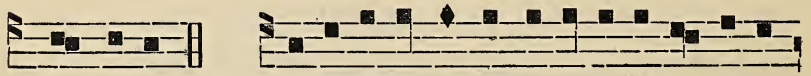

tat ka-ti. Ka: A. A horwasonna-ien Ro-niha nok lioienha ron.

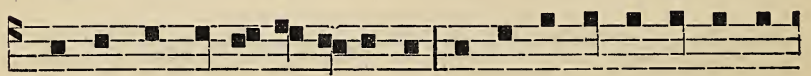

nok Rotkon Ro-ia-ta-to-ken-ti. Tsi-ni iohton-ne tsi-na-he 


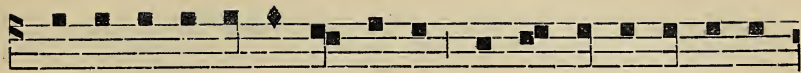

e-tho naioh-ton-ha- ke nonwa, tiotkon o-ni tsi-nenwe

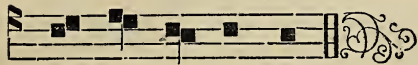

E - tho na - ia- wen.

Iontaw :
$\mathrm{T} 2$

Te - kwanon-weronnions Ie-sos tsi ta-kwaton-

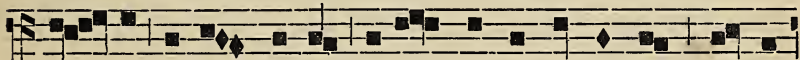

nien - ni takwa-niente a-ia-wens ka-ronhia-ke askwa-

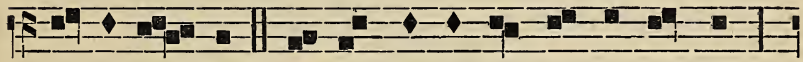

ia-ten-ha - we. Se-wentiohkwa-kwe-kon ne tsionkwe,

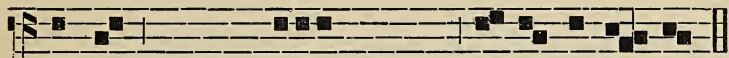

se- watsteniaron Rawenniio rao-ri- wa o- kon-ha. Ahon.

Takw. kowa (sk. 42).

T 2.

A-re-ri-ia, a-re - ri - ia. Tc-

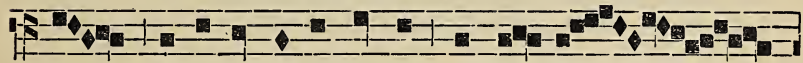

sa

te-se-waton-ha-renron wakwa-hro

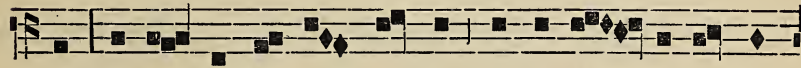

ri ko-wa-nen io - tsen-non-nial Ie-sos $\mathrm{Ni}$ - io Ro-ien-

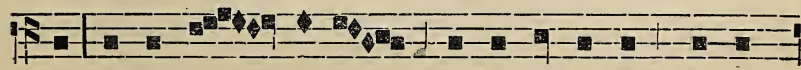

ha, onkwe ro - ton-hon. Ka-ro ki-se-ne nonwen- 


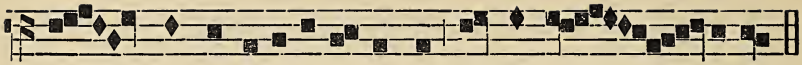

tsia - kwekon hetsilewanenton ne Rawen - - ni-io. $a$.

T 6

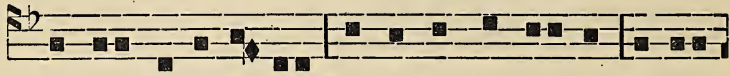

Swentiohkwa-token- ti nonwa swatsennon-ni $\mathrm{Ne} \mathrm{Ni}$ -

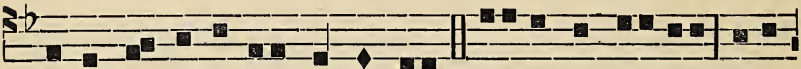

io Ro-ien-ha nonkwe ro-ton-hon. ${ }^{*} \mathrm{Ne} \mathrm{ka-ron-hia-ke} \mathrm{etho}$

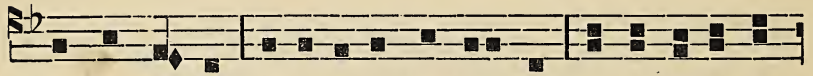

thawennonton. * Ii o-ni te-twatken-ni tsi te-ion-te-ri-

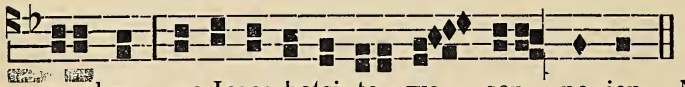

wa-kwa ne Iesos hetsi- te - wa - sen - na-ien. Ne.

Ne wasontanoron, eronhierakehronon

Akwa teiontkennionkwas ne rontonnions :

* Ronwasennaien, tehotiriwakwen. Ii.

Sonkwaskontakohe, tsini kaiatonkwe,

Ne notkonseraksen shatekwatane,

* Kariwaneren shariwahtontane. Ii.

Ahonwasennaien Roniha, Roienha,

Nonwa wasontate onkwe rotonhon,

* Nok oni Rotkon Roiatatokenti. Ii.

0 ise swakwekon (sk. 124). Sennakeraton (sk. 118). Wahatonni (sk. 11\%). Tsionkwe Niio (sk. 122). Rotonni. Niio (sk. 121).

ENTIEKEHNE.

Iontaw.

$\mathrm{T} 7$.

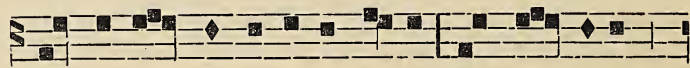

Soukwaton- nienni ne ra-ksaa Ra-wen-ni-io

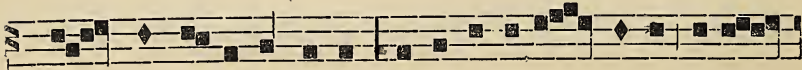

son - kwawi ne Ro-ienha Shariwa-seron - niane no' wen- 


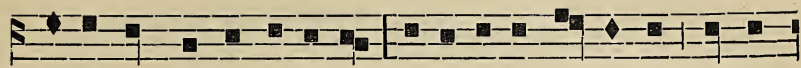

tsiake skennen ta-kenha-ke, en-hasenna-no-ron-ke onwen-

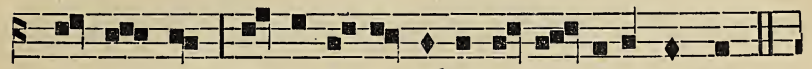

tsia-kwe-kon nok ne ka-ronliake ro-ia - ta-nehrakwat.

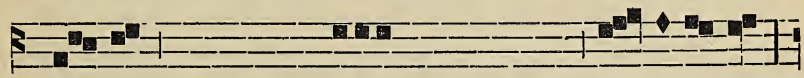

'Ta-tsitewariwakwase Niio Roienha ka-ren-ni-io-son;

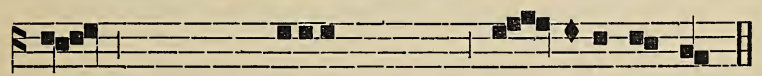

a - seken ioriwanehıakwatenions tsini ha-ie-ranions. Ahon.

Takw. sk. 49). Areriia (sk. 287).

T 5.

Onen wathonno-hon-ti ne $\mathrm{Ni}$ - io ka-ron-hia-ke, ORo-natsen-non-ni ko-wa ra-ti-ron-hia-kehro-non A-

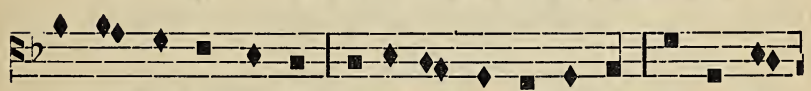

nen ton-ta-hatsnen-le ne he-tsi-twarha-re-kwe Onwentsiae-twa-kwekon-ha-ke kario ronıste-ri-stakwe Na-tsi-twa-

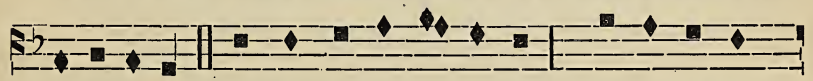

ke wa-rawe. Teskwaskwate-ten - ni-tha $\mathrm{Ni}-\mathrm{io}$ takwaia-torenne. I - se sko-wanen-ko-wa A-kwa we-sa-

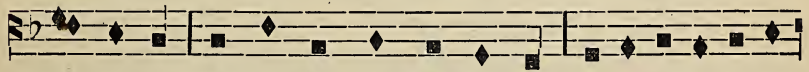

wen-ni-io Tiotkon si se-na-ke-re sa-leswaia-ta-nota-tkenron Io-ne-lirakwat skwanonwes iahte ionkwarihon-

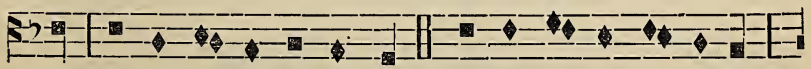

ron Nasen-se - ra- to- ken - ti. te Ionkwari- wa-nerakskon
Ii ton-sa-ionkwa-ri-waKaron-hia-ke tsi-te-ron. 


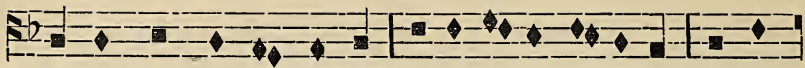

se- rakwen nonwentsia-ke, I-se wa-sa-tke-ta-te, akwa

Iahte tswens nonwentsiake, Kario io-ti-nonso-te, e-tho

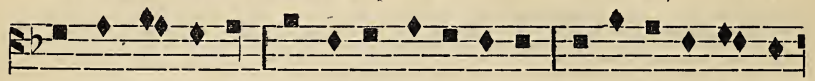

ne wa-sie-ri-te li aionkwa-ie-ri-ton. Eh na-sa-tiato-ten-

or-kwe wa-saton, Sahtontha ne ie-na-ie. Wasa-ta-ti-ten-ste-

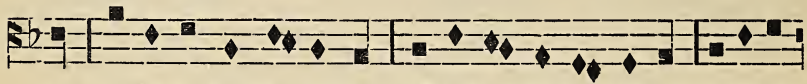

te tsi-ni ionkwaia-totens Wasohre tsi na-sie-re a-onsa-

ten akwa. wa sa-ta-tie-sat. Ka-ri-wa-ne-ren te-se - seriwa-

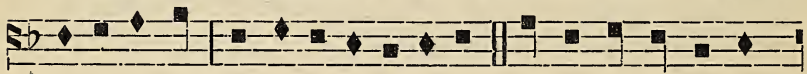

ionkieronhak Ne ni-i sekhe-ieren. Ni-io ne Ie-sa-ni-

se-ra-ko-he, Iah sa-ne te tsiente-ri. Salkon ronwanonwe-

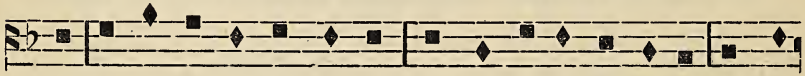

ha, takwawis ne Hetsienha, Sonkwa-iane-rensta-ne ; takwastha sason ne ra-ie-ron-ke, Teskwaswateten-ni-lak ne sa-

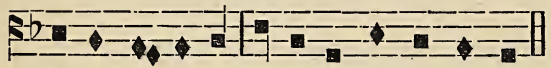

nikon-hra-ta-as, Iesos sakwaien-terhann.

tsi-ra- to-ken-ti. Iesos sakwanonwehak. Etho naiawen

Akwekon lelwariwak (sk. 119): Rotonni (sk. i2l).

\section{IOKA RENRE.}

T 1.

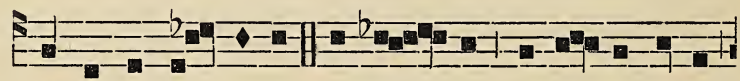

Ne Ni-io Ro - ienha tsi-na - he iah-te ka-konte

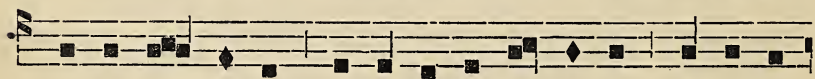

ra-ia-ta - takwe ra-on-hakon ne $\mathrm{Ni}$-io ra-on-ha

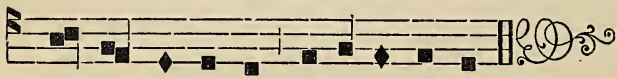

o - ni $\mathrm{Ni}$ - io ni-ha-ia-to-tenhne. Salkon Niio. 5-6 i. 


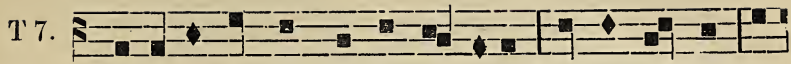

Sa-koten-ron nonkwe Rawen-ni-io ; thoten-nie-ton ne

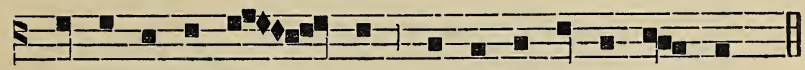

se-sa-koskon-ia - - kwen to-sa a-iontka-ron - ni.

Tewakehlakon onkwe. $7 \mathrm{e}$.
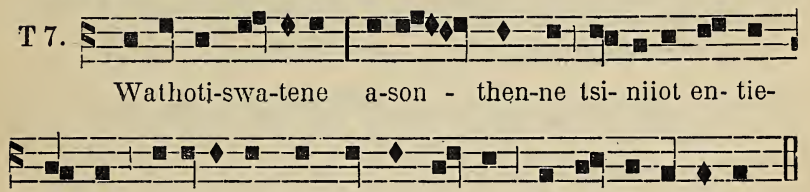

kehne sakonoronkwa Rawen-ni - io tho-ti - ri-wa-ie-ri.

Sewenniio to ok. 5 i.

$\mathrm{T} 4$.

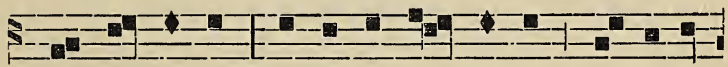

Ra-wen-ni-io ro-ta-ni-ten - raskon iah- ta-on-

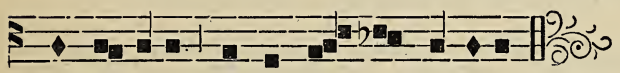

tontsa-hate tsi-ni ho - ni-konhriio. Sewenniio, iahte, 4 e.

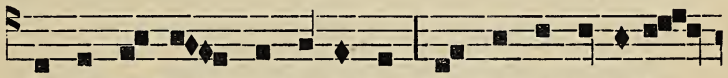

Ie- sos te- kwa - nonwe-ratons, Non-wa wasennake-

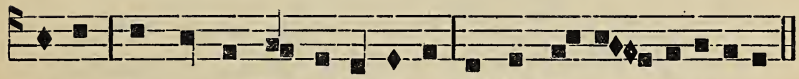

ra-te Tiotkon tsi Hia-ni-ha Ni-io, Nok tiotkon si senake-re.

Saksatiio, tsiatanoron, lonehrakwat waskwentenre, lonkwariwanerahakskon, Skwariwaserakwenhatie.

Ne skwariwaserakwatha Ne tsini saiatawenhon, Saronhiaken, sawistose, Nok ieiesas sariskontie.
Akwekon satrewatakwa Oukwe okon akoriwa, Kasenna, kakatensera Nok iontonweskwatha okon.

Wari tekwanonweratons, Akwa saiataterihon :

Ne Niio hetsateweton, Iahte senakwaienteri. 
Ahonwanenton Roniha, Nok ne Wari Hiatatienha,
Satèhonwatinentonnion

Rotkon Roiata'okenti.

v. Niio Roienha onkwe rotonhon, areriia.

R. Nok wasonkwennakeratse, areriia.

Ros.

T 1.

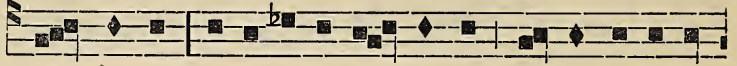

Kun wente Ie-sos ro-na-ke - raton, ro - tonni ne se-

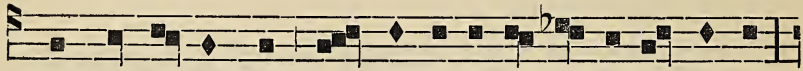

sonkwaskon-takwen; ken wente te-hon-wa-ri-wa-kwa-se

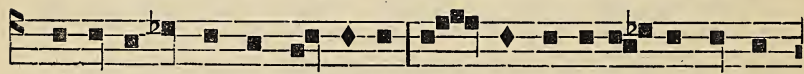

ra-tironhia-kehro- non okon; ken wente ro-na-tonnharon-

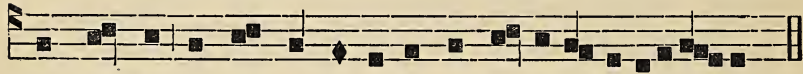

nions, rontons : Ronwasennaien ka-ron-hiake $\mathrm{Ni}-\mathrm{io}$, areri - ia. 1 a.

\section{DEC. ETIENN RTI. 2on WENT}

Ionlaw: (sk. 242). Takw.(sk. 24). Areriia (sk. 243): Asonthenne (sk. 113). Walıatonni (sk. 112). Swaronliakehronon (sk. 113).

Iokarisnre.-Ne Niio (sk. 290). Ne ioriwalsanil (sk. 24ł).

v. Akwa hetsiatanoronston, Sewenniio.

ห. Ne ise sariwake rotewenteton tsi ronnhe.

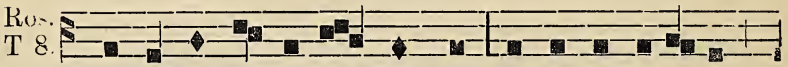

Walıonwa-ia-ta-ten Etienn tia-ko-ri- waie-ri

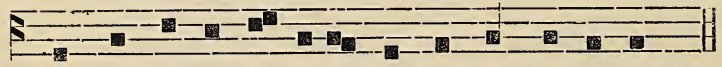

nonkwe sonhi nok e-so wa-hon-witstar-ha-se. 8 e. 
27 DEC. SAN RTI. 2on WENT.

lonlaw: (sk. 23i). Takw. (sk. 24). Aleriia (sk. 237). Twalsteniaron (sk. 118). Onkwe rolontion (sk. 117). Swalonlek (sk. 117).

IOKarenre. - Ne Niio (sk. 290). Twalsennonni (sk. 238). v. Tehonarenihon.

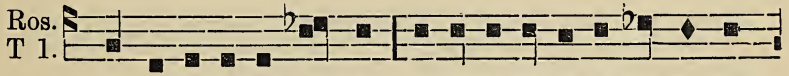

San Roia-ta-to- ken - ti ra-onha tsi-ni ho-nonwehon

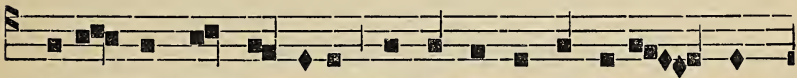

Iesos Sonkwawen-niio, ro-taskat ro-ri-hota - sien-

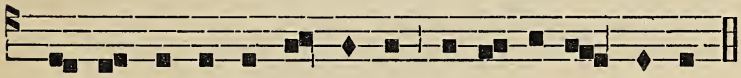

ni ne ka-ri-wa-no-ron-son.tsi-ni ia-wen-se-re. 1 o.

\section{DEC. EKSA OKON KENHA. 2on WENT.}

lonlaw : (sk. 245). Takw. (sk. 24). Areriia (sk.246). Onkwe rotonhion (sk. 117). Twalonnharen (sk. 130). Akwekon tetwariwak isk. 119).

IOKarenRe-Ne Niio (sk. 240). Ne ioriwalsanil (sk. 119).

v. Herot raserohen wasakowentho eso eksa okon kenha.

i. Tsi tkanataien Wetreher, Tafit raotenata.

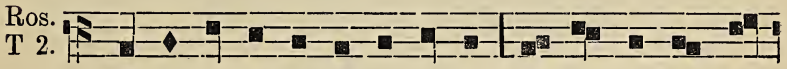

Kentionko-wanen neksa okon le-sos ronwen- he-

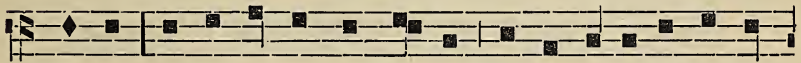

ia - se, ne ron-kwetaksen He- rot ro-tennhaon a-hon-wa-

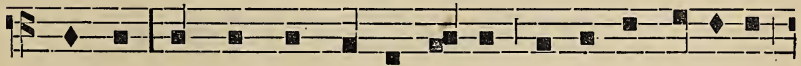

wen-tho. Ronwa-kens ka-ron-hia-ke sa-kawen-he-ia-se 


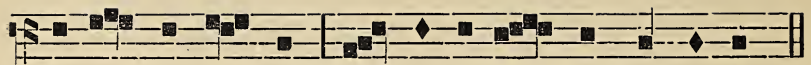

a-ste - son ron tons Kwa-sen-na-iens Se-wen-ni-io. $2 a$.

Tsi ionsakahewe 8 nonta-lsini honentawen ken kaien iokarenre aionste.

Etienn (sk. 243). San (sk. 238). Eksa okon kenha (sk. 247).

Ros. Tenhnon ne sakul lsini haonentawen.

\section{A WENTATOKENTIKE}
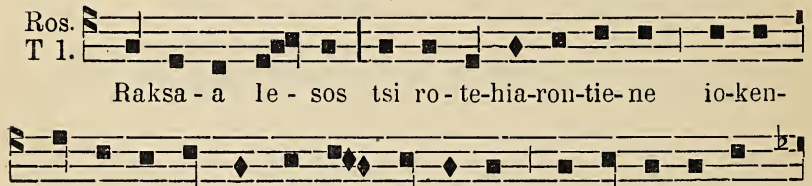

ta-on- ha-tieskwe tsi-ni hattokha nok tsi-ni tho-ri-

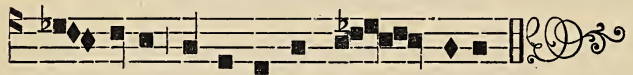

wa - ieri Ni-io-ke nok on - kwene. $6 a$. v. Niio Roienina.

TSINA TEION'TATENORONKWANION $20 n$ WENT

Karo kase. lontaw: (sk. 288). Takw. (sk. 53). Areriia (sk. 287). Tawatonlal (sk. 120). Onkwe rotonhon (sk. 117).

IOKARENRE.

T 6 .

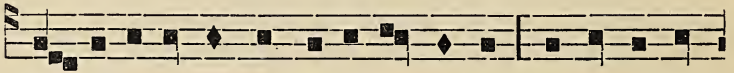

Io - ri- wanelırakwat tsi-ni ia- wenhon; a-kwekon ro-

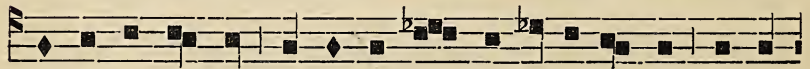

te-we-ien-ni- son onkwe ro- ton-hon, ro-te-we-ton ne Wa- 


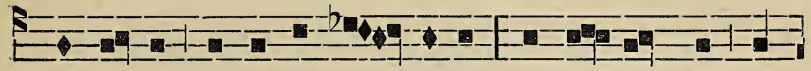

ri iah-te ka-nakwa-ien - te-ri, nok te - ho-kwen non-

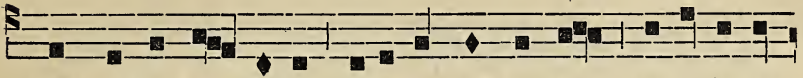

kwa-ronhia-ken - se-ra ta-e-te-walkwe-ni ra-o-ia-ta-
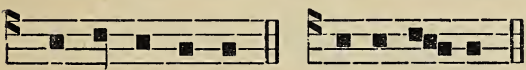

nehrakwa-tse-ra. (s/) A-re-ri - ia. Salkon Niio. $6 u$.

T 3.

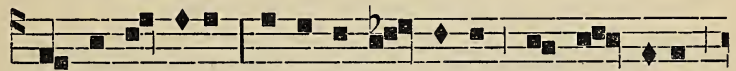

Ne wason-tate tsi senna-ke - raton, Se-wen - ni-io

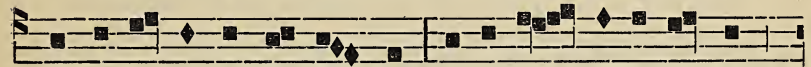

tsi-nonni kanon-saksen - skwa, ra-ti-ron - hiakehro-non

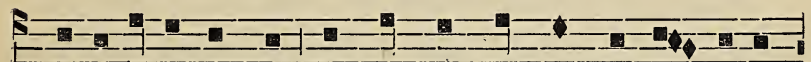

te-ho-ti-ri-wakwen skennen ken-lıak nonkwe ia - ko-ni-

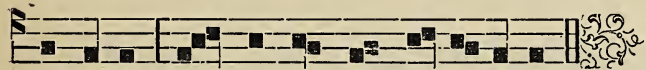

konhri-io. Te - kwanon-we-ra-tons lesos.

Tewakehtakon onkwe. $3 i$.

$\mathrm{T} 4$.

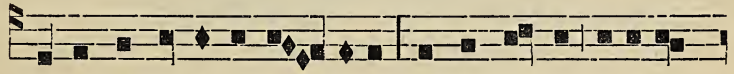

Onka iah-la-ia-ko-ne-hrako ion-ki-hro-ri ro-ti-to-

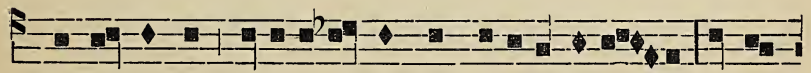

kense - hakwe : roia-tane- hrakwat roieshaonha - tie : hetsi-

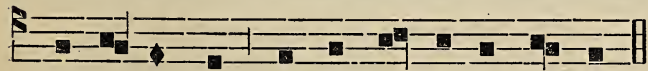

twano-ronkwak, son-kwa-non-wehon tionkwe.

Servenniio lo ok. 5 o. 
T 1.

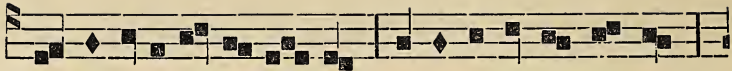

Wa-ri, saia-ta-te- ri - hon hetsa-te-we-ton Ie-sos,

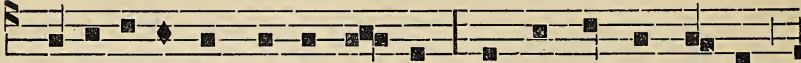

hiaia-ta-no-ron-ston ne $\mathrm{Ni}$ - io, son- ha hia-konnienston
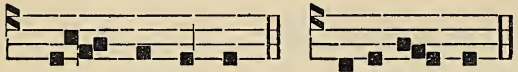

non - wentsiake. $(s / l)$ A-reri - ia. Twakelilakon Rolkon. $1 \mathrm{i}$. lesos tekwanoronweratons (sk. 291). v. Niio.

Ros.
T' 1.

$\mathrm{O}$ - - nen onkwe ro-tonhon $\mathrm{Ni}$ - io lio-ien-ha

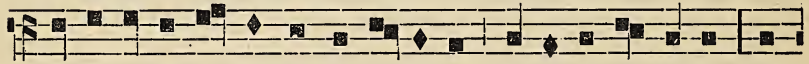

sa-tehni-ia-to - ton $\mathrm{Ni}$ - io Roien-ha ok si ha-na-ke-re; on-

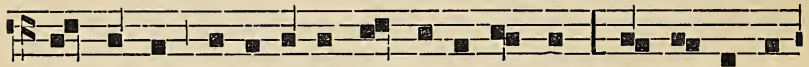

kwe tsi-ni ha-ia-to-ten, iah-te ho-ni-sen; $\mathrm{Ni}$ - io tsi-ni

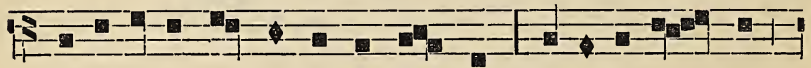

ha-ia-toten, iah - te ho-ni-sten- sen. Te-tewa-ri - wak
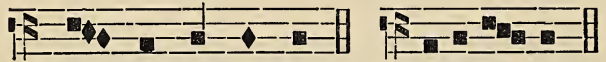

twa - ton- nbaronnion. (s/l) A-re-ri - ia.

6 JAN. IESOS WA'THONWANORONKWANION. 1 ON WENT.

Iontaw.

T 2.

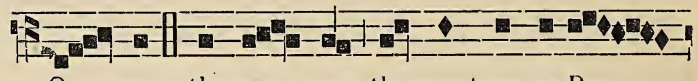

O - nen thawe - non thennontons ne Ra-

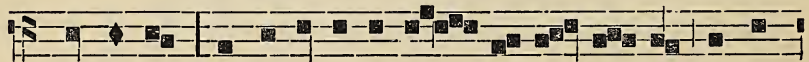

wenni-io, nok ra-hawe tsi ro - te-ri - hon-te kakwe- 


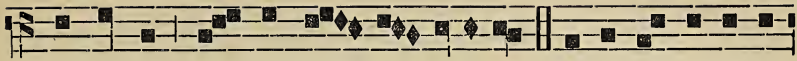

nia-tse-ra, nok ka-wen - ni - io-se-ra Ni-io hetson ra-ia-

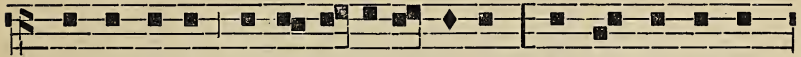

takwe-ni-io sa-ia-to-re-ta-tse-ra, nok ne Ro-ien-ha

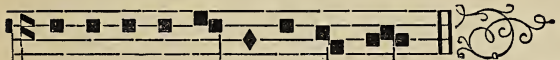

a- on- ta-ha-ri-wa-ie-ri - te. Ahon. Takw. kowa (sk. 42).

$\mathrm{T} 2$.

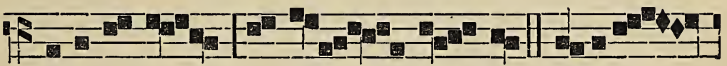

A-re-ri - ia,

a - re - - ri - ia. Waakwa-

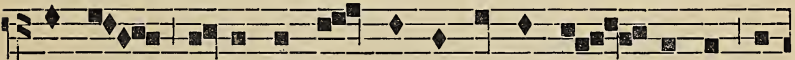

tka- tho o- tsistok ko - wa tsi tka-ra-kwi - nekens nok

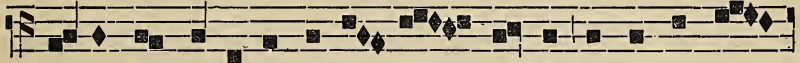

ta-ia-kwahtenti iakwaba - wi - nontie sakwawenni-

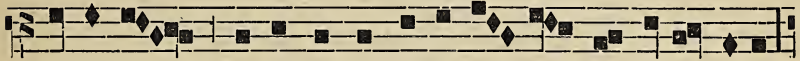

io-stane ka-no-ron sonha te-sa - kwa-nonwe-ra- tonne

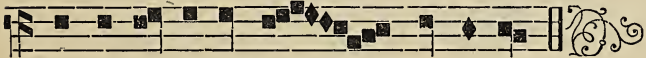

ne wa-ra-we ne Ra - - wenni-io. $a$.

T 1.

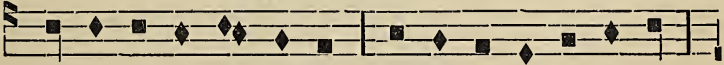

Tsiastoron,tsionkwe o-kon, Shetsiswa-ni konhra- ieTo saswa-ten - nowentak! O-tsistok ne ioh-ta- re,

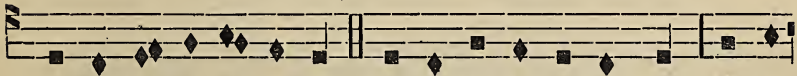

wentho-se-re ne Ie-sos. Se-wa-ti tsi ren-te-ron $\mathrm{Ne}$ se-

Waton: ne Rawenni -io. A-kwa en-haonweskwen Skennen 


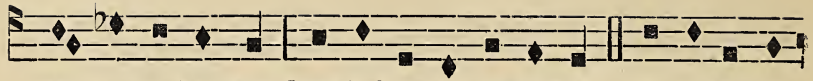

we - ria-sa-kwekon, Swerhek, a - hinon-wehak. O-wista ron-

ni - se a-e - se - wa-ta-te-wen-te-ta-ke. Ne wa-te-nien-

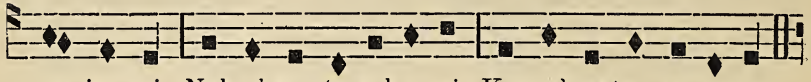

wa-jen-ni Nok olıne-ta nok c-ni Karonkwatse-ra-noron. ten-sta-kon $\mathrm{Ni}$ - io ni ha-ia-to-ten, Onkwe nok o-non-ti-io.

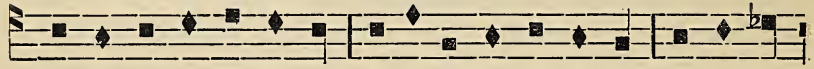

Waha-ti-ken no-tsistok Ne ro-ti-ia-ner kowa, Ok-sa wa-

Wahonwa-ia-ti-sak-ha $\mathrm{Ni}$-io raksa io-tonhon, Ronwawen-

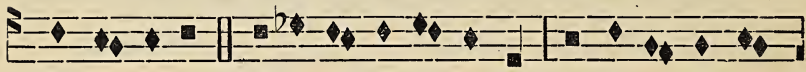

kon-wa-se-re. Ie-ti-na-ke-ra-ni-hek A-he-ren tho-nah-

ni-io-stane. Oksa ronwa-ton-ta-ti Iah-te ho-ti-ni-

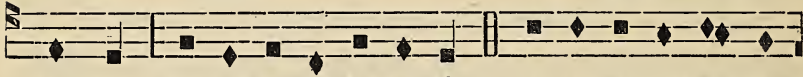

tention, Ie-sos ronwa-ia-ti-saks. Ii o-ni sonkwa-non-

sko-hon, $\mathrm{Ne}$ tsi- ni honwaskaneks.

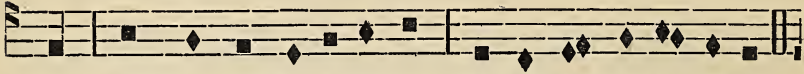

kse, Tiotkon sonkwa-ia-ti-saks; He-tsi-twa-nonte-ra-tie.

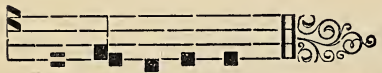

E - tho na-ia-wen. Salialiken (sk. 128). Onen ietinon. (sk. 127).

\section{IOKARENRE.}

$\mathrm{T} 2$

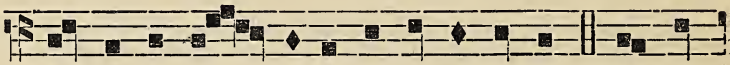

Ok si ka-na - ke-re Ni-io lio-ien-ha. Son-kwa-

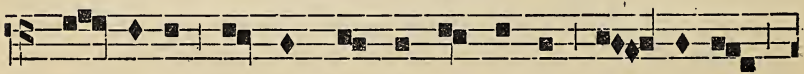

wen - ni-io se-son-kwas-kon-ta-ko-he ken wente 


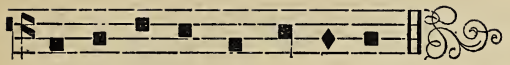

ro-to-ta-sien nonwentsiake. Salkon Niio. 5-6.

T 1.

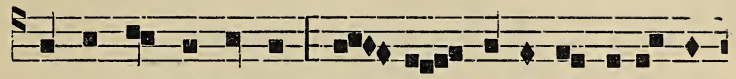
I-no' tho-nahtention nas-hen ni-ha-ti ra-ti-sen-

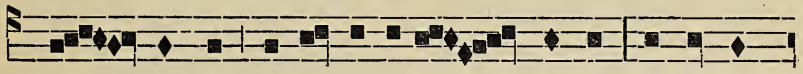

no - wanens a-honwa-ia-ti - - sakha, o-tsistok

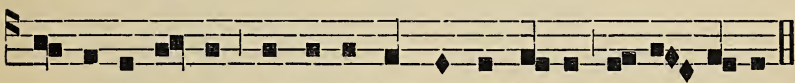
ro - ti-na-ton-ni tsi-nonni then-te-ron Ie-sos a-re - ri - ia.

Tewakehtakwen asen. 10.

$\mathrm{T} 3$.

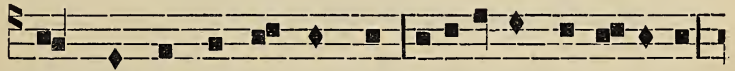

Swentiohkwakwekon tsionkwe, tatsi-sewanonwe-ra-ton

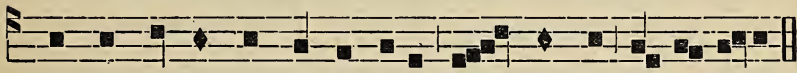

nonwentsiakwekon Rawen-niio tsi tsionnhe a-re-ri-ia. Sewenniio to ok. 2 e.

T 7.

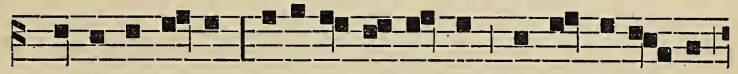

Asen ni-ha-ti ro-ti-ia-ner ko-wa ka-tsisto ko-wa

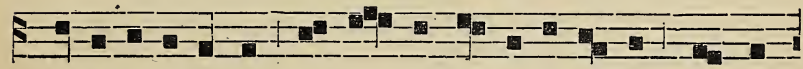

ne ro-ti-na-ton-ni tsi-non-ni tho-nake-ra-ton ron-wa-

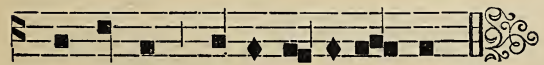

non-ti-io ro-ti-non-ti-io - son. Sewenniio iallle. $7 i$.

$\mathrm{T} 3$.

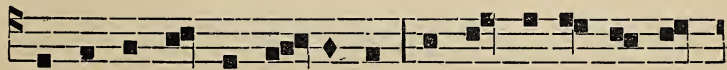

Ie-sos tekwa-non-we- ratons, Nonwa we-sa-ien-ter- 


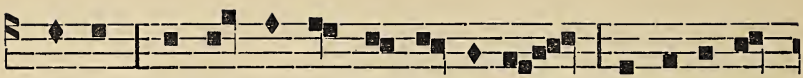

ha-ne, Na-he-ren tha-ti-na-ke-re

Ne ra-ti-sen-

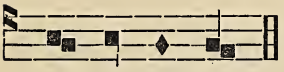

no wanenskwe.

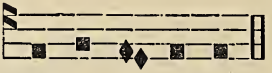

E-tho na-iawen.
Ne iahte hokwenion Herot Tesakonikonhrakenni Wahotokaten notsistok Tsini haweriasaksenskwe.

Niawen, Iesos Sewenniio, Tsi seron : ionkenekwahest: Ne sane kanoronstakon Naionkenekwahestakwe.
Niawen takwatokatenni $\mathrm{Ne}$ tsi iahte sanoronse; Ohnekanos watesteni Akwa otsitsia iotonhon.

Aiesasennaien, Iesos, Nonwa satatiatotasion: Sate ietsisennaienhek $\mathrm{Ne}$ asenseratokenti.

v. Ratisennowanens tenhonwanonweratonna.

R. Tenhonwawenniiostana kanoron sonha.

T1.

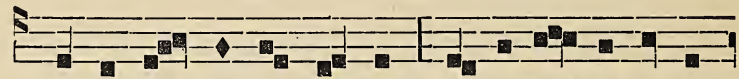

Onwa ken wen-te nakwah io-nehra-kwat tsi-ni

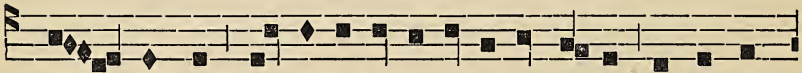

ia - wenhon: a - sen ni-ha-ti ro- ti-non-ti - io ronwa-ien-

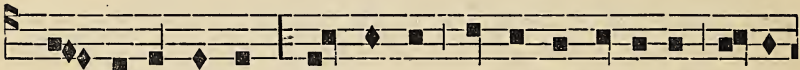

ter-ha-on Ie-sos; ken wente, wathahne-ka-te-ni le-sos

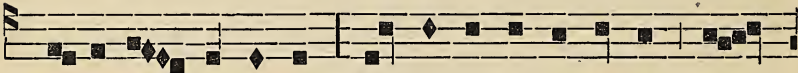

o-tsi-tsia io-tonhon; ken wente, kahion-hakta Sor-

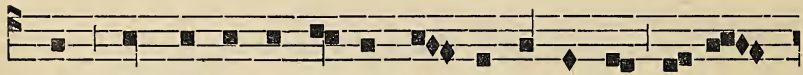

ten, San Watis wa-ho-ne- kwa-he-ste le- sos se-son-

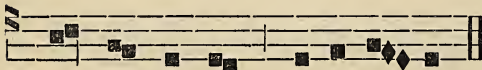

kwaskon- ta-kwen, a-re-ri - ia. $5 i$. 


\section{$-301-$}

\section{NIIOHNE.}

lontaw. (sk. 296). Takw. (sk. 68). Onka onte. sk. 119). Rinonwes (sk. 128).

IOKARENRE ( $s k$. 298).

v. Sonhaa, Sewenniio, konwenniiostha.

r. Katewentetha noneshonronon aoriwa okon.

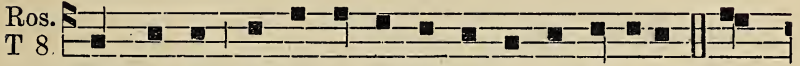

Konhienha, oh-nio-tieren e-tho-ni skenierase? ii

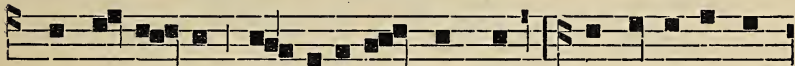

nok hia-ni - ha ke - ni-ia-ti - sakskwe, Oh niotieren ta-

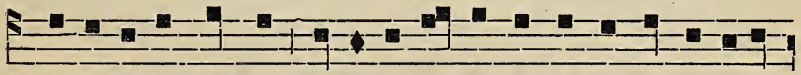

ke-ni-ia-ti-sakskwe ? io- te-ri- wi-son na-katste - ri-ste ra- o-

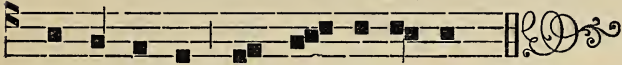

ri- wa sonha ne Ra-ke-ni-ha. 5. o.

IESOS RAOSENNATOKENTI. 2on WENT.

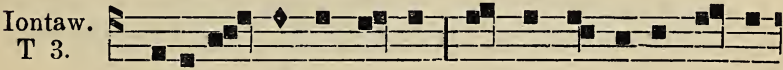

Rao-sen-nakon le-sos a-kwekon taion-iontso-

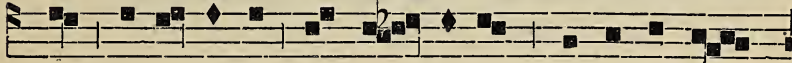

ten, karon- hiake on- wen - tsiake nok onweritsia-

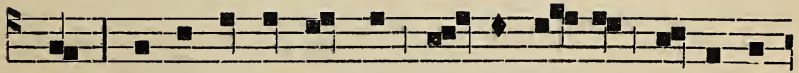

kon ; nok a-kwekon nonkwe a - ion-tro-ri tsi Sonkwa-

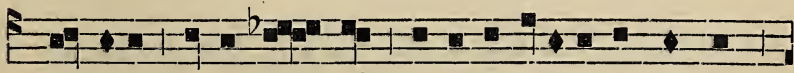

wen-niio lesos Kris-tos sa-te-ho- ti-ia-ta-ne-hrakwat 


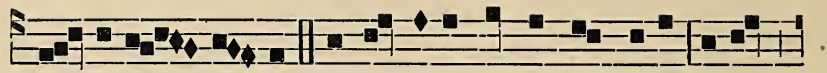

$\mathrm{Ni}$ - io Ro - ni - ha. Sewenni-io takwawen-ni-io, io-ne-

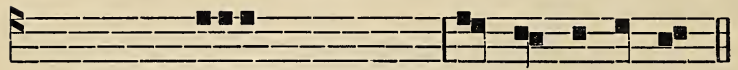

hrakwat tsini tsennanoron non-wentsiakwekon. Ahon. Takw. (sk. 53). Ronnhek (sk. 129).

T 8.

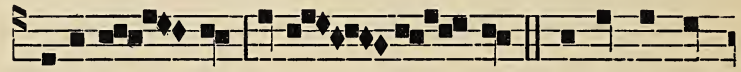
A-reri - ia. a-re - ri - ia. Raon-ha Ra-

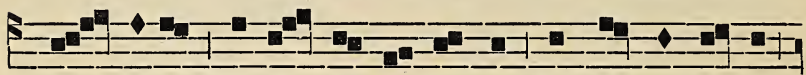

wen - ni-io ro-sen - na - ne-hrakwat, nok nonkwe o - kon

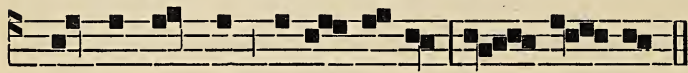

ie - konnienstak ra-o - sen-na - to - ken - ti. $a$.

Ronnhek Sonkwawennio (sk. 136). Tsionkwe Niio (sk. 122).

\section{IOKARENRE.}

T 1.

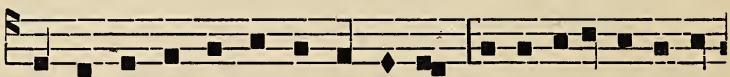

Nenhonwasen-na-nia- he-sen-hake I esos ra- osen-na-to-
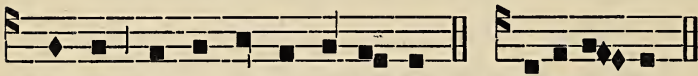

ken-ti ka-ronhia-ke enien-te. (s/l) A-re-ri - ia.

Satkon Niio. 2. e.

T 7.

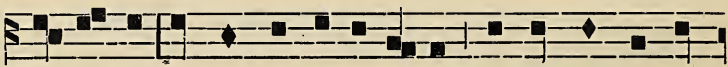

E- tho-ne Sonkwawenni-io Ie- sos rasennion ken-he-

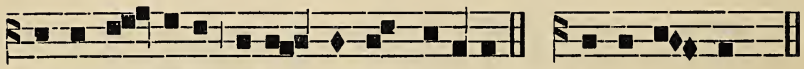

ion sahren-heie teka - ientannhareke. (s/l) A-re-ri - ia. 
Rolaskal. 7. a.

T 3.

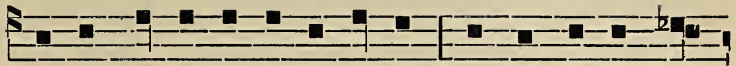

Sa-nekwen-sa-to-ken- ti, le-sos, takwaskon- ta - kwa-

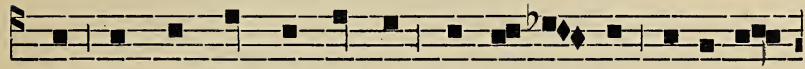

ton takwentenr ia-kionkwe ka-ron-hia-ke askwaia-

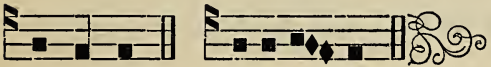

tinion- te. (sh) A-re-ri - ia. Sewenniio to ok. 3. $a$.

T 8 .

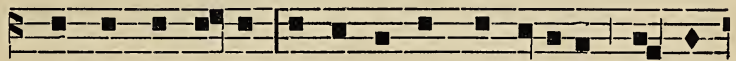

Askwenten-ra - te, Iesos, tsi takwen-he-iase, to - sa

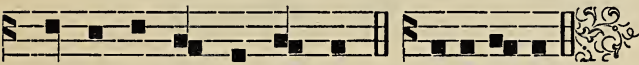

a-ionkwasen - ni no-tkon. (sh) A-reri - ia. Sewen. iahte. 5-6e .

Iesos tekwanonweralons (sk. 299).

v. Takonwanonweraton Iesos raosennatokenti, $(s h)$ areriia.

R. Nonwa nok oni tsinenwe, $(s h)$ areriia.

T 1.

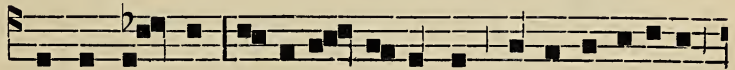

Entse-sen - non ra-0-sen-na Ie-sos a-seken raonha

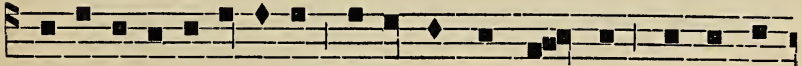

en-se-sa-ko-ia- to-kewe ra-o-tiohkwakwe- kon a-ko-ri-

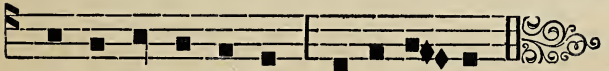

wa-ne- ra-hakse- ra, a-re-ri - ia. 5-6. $a$.

$\Gamma 1$.

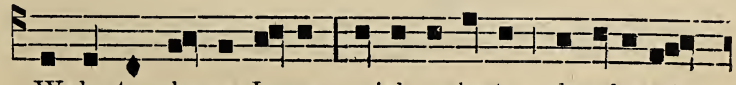

Waha-tennha-ne Ie-sos a-ieh-ne-ka-ta oh-neka tsi 


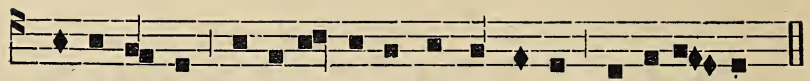

katse-to- ton nok o-tsi-lsia io-tonhon-hatie. A-reri - ia.

v. Sonhaa.

18 JAN. \& 22 FEV. TIER RTi. (sk. 127.)

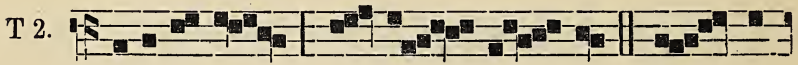

A-re-ri-ia, a - re - ri - ia. Tier i-

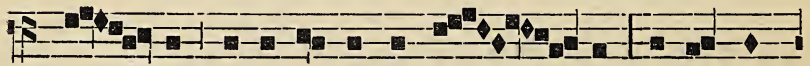

se - ke tsi-ni-iot otstenra - $\quad$ ke en-ki- tioh-

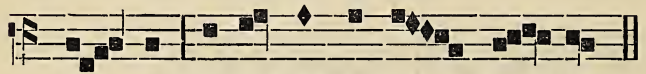

kon - ni na-ki-tiohkwa- to - ken - ti. $a$.

v. Ise 'Tier wakonnatonkwe.

R. Nok iseke tsiniiot otstenrake enkitiohkonni nakitiohkwatokenti.

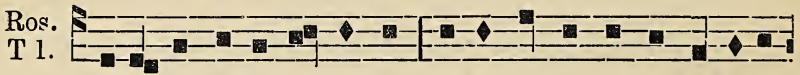

I-se Tier ie-sa-ko-wanen Ra-li-kwekonne nok niakori-

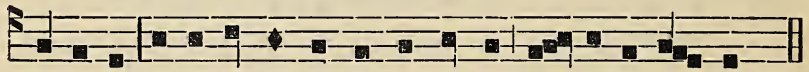

wiioston; lesos hiawi iennhotonkwatha ne karonhia-ke. 1.e.

Alonwehiarane ne Kor Rli.

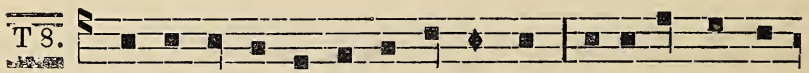

Kor, Ie-sos hetsewen-nenha-wakwe she-rihonnien-ni

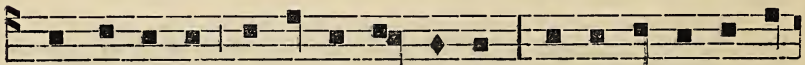

nonkwe okon ne ka-ri-wi-io-ston hetsen-ni-tẹntas Ni- 


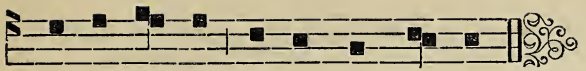

io Ro-ien- ha a-sonkwen-ten - re. 8. $e$.

v. Hiaiatarakwen Rawenniio, Kor saiatatokenti.

1. Aserihowanate kariwiioston nonwentsiakwekon.

25 JA.. \& 30 JU. KOR. RT. (sk. 237). Ros. Kor (sk. 304).

Ise Tier... v. Ise Tier. (sk. 304).

\section{TSI NAONTATHASERON, $20 n$ WENT.}

T 8.

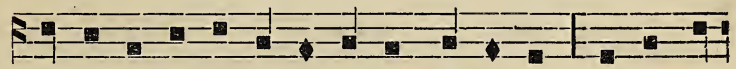

Te-sa-koswa-te-ten-ni- re nonkwe o-kon a-iontsen-

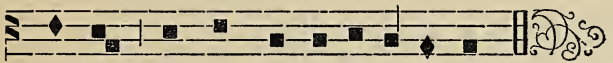

non-ni sentioh-kwa Isra-er-ronon. Tes... 8. $a$. Tes...

A seken intkathon ne kkahrake * ne sesakoskontakohe. T'es...

$\mathrm{Ne} h$ 'tsennhaon * tsi sakorihonniennire akw.kon nonkwe. Tes.

Alion... Tes... Tsini... Tes...

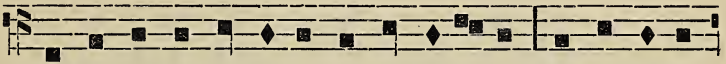

Sa-tontek, Sewen-ni-io, takwa-iena-was, nok takwaia-

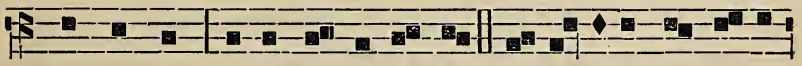

tanonstats tsini tsen-na-no-ron. Sewen-niio kwenni-ten-

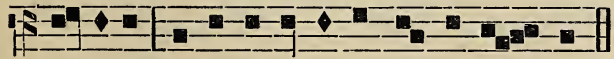

ta- se-re, satontat tsinikon en-kwane - ken. Ahon. Salontek. Tekwanoronkwanions (sk. 266). 
Iontaw.
T 1.

O-nen teskwawi, $\mathrm{Ni}$ - io, sa - tanitenra-se-

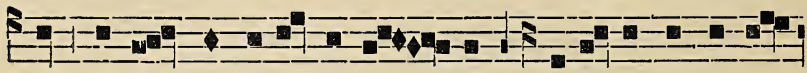

ra tsi sa - non-sato- ken-ti - ke, ne tsi-ni tsenna-no-

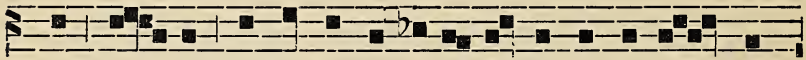

ron, $\mathrm{Ni}$ - io; ne wahon-ni iesa- sen-naiens onwen - tsia-

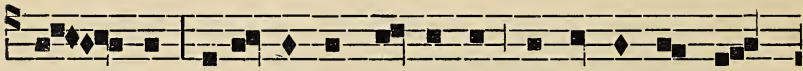

kwe - kon ione-hrakwat ka-nanon tsi nenseskwa-ie-

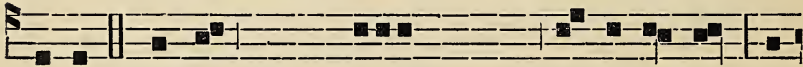

ritse. Sewenniio sonha skowanen aiesa-sen-na-ien, ka-

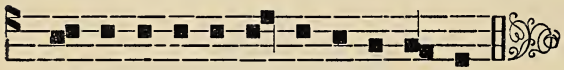

ron-hiake a.ie-sa- wenni-iosta - ke. Ahon...

Tukw. (sk. 58). Onen wahniakenne (sk. 143). Areriia (sk. 269). Tokul.

T 8 .

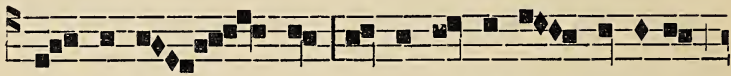

$$
\text { O - nen sken - - nen te seskiatak Se - wenni-io, }
$$

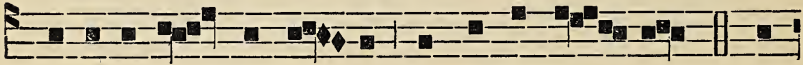

e-tho na-ia - wen tsi - ni skerha-ratsten - ni.

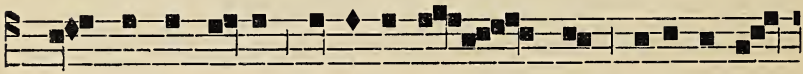

se - ken kkara-ke ii ri-iatka - - thon sesakoskon-

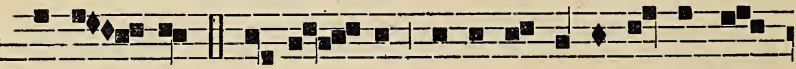

ta-ko - he. Ra-on - ha tensa-kos-wa-te-ten nonkwe 


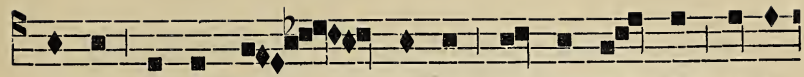

o-kon, a-iontsen - - non-ni ne sentioh-kwa Isra-

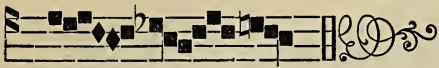

er.

Ise swaronhiakehronon (sk. 132). Kasene, kasene (sk. 116).

IOKARENRE Tierennak (sk. 294).

10 Ioriwanehrakwal (sk. 294). 20Wari, saiataterihon (sk. 296).

T 3.

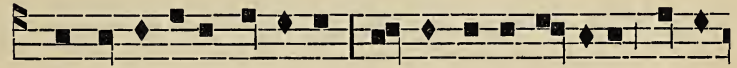

Watho-iatakwe Si-meon rasnon-sake raksa-a, waho-

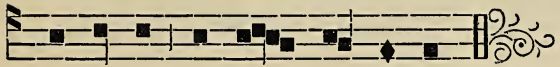

sen-naien ne $\mathrm{Ra}$ - wen-ni- io.

$4^{\circ}$ Tesakoswa... (sk. 305). Tekwanoronkwanions (sk. 214).

v. Takivaterennaienhas saiatatokenti Niio hetsienha.

R. Aiawen taiakwatentsa tsini sonkwarharatstenni Kristos.

Ros.

T 8 .

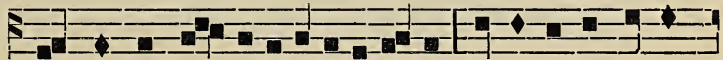

Ken wente Wa-ri ioia-ta-token-ti waho-ia-ten-ha-wi-

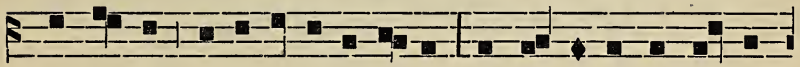

te Ie-sos o-nonsa-token-ti-ke nok Si-meon roksten-se-

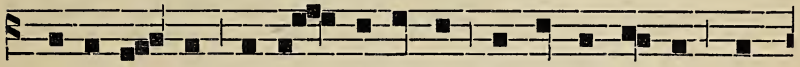

ra-to-ken - ti watho - ia-takwe wahenron:O-nen, Se-

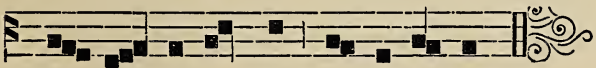

wen- ni - io skennen a - ki-he-ie. 8. e. 
A WENTOKON TSINIIORE 7 NIWASONTASHEN.

KAHASERES. Takw. $\{k .34)$.

\section{I}

Iontaw.

$\mathrm{T} 4$.

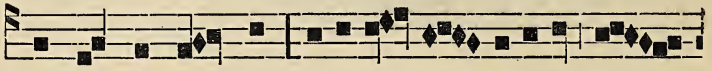

On-wentsiakwe - kon a-ie-sa - wen - ni-ioste Ni -

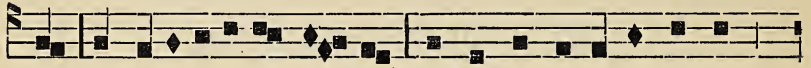

io, nok taie-sa-riwakwa - se nok ta-kon-wari-wakwa-se

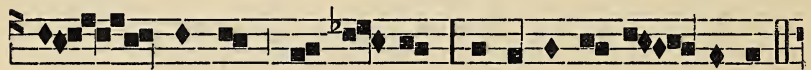

sa - - sen-na - to - ken- ti, sonha-tsiwa sko - wanen.

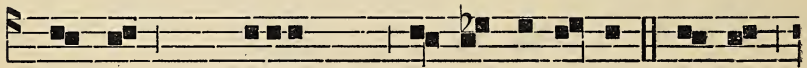

Tsionkwe okon sewatonn - ha-ren $\mathrm{Ni}$-io-ke, he-tsi-

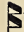

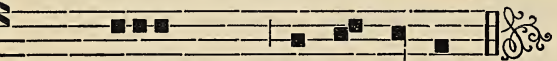

sewasennaien naesewatsen-non-ni. Ahon...

T 4.

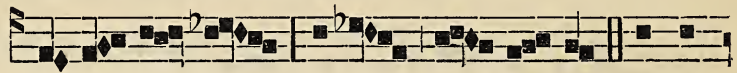

$$
\text { A - re - ri - ia, a-re - - ri - ia. He-tsi- }
$$

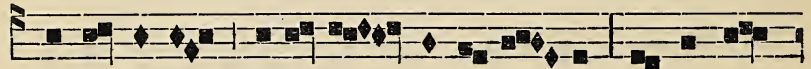

swasen-na-ien te-tsiswa - nonwe-ra - ton ne Rawen-

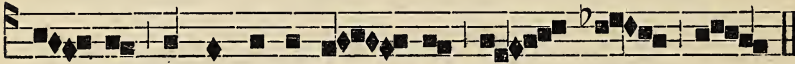

ni - io, swaronhiakehro - non o - kon. $a$.

T'sionkwe (sk. 134). Twrtaleken (sk. 137). Nonwa ken wenle (sk. 194).

II.

Iontaw.

T 4 .

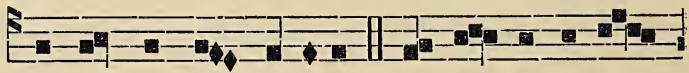

Sa- ton- tat, Se-wenni-io, tsi kon-hienni-ten- 


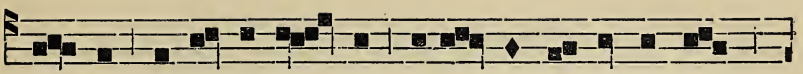

ta - se, askia-ta-ken - nha, to-sa ta-kwa-tewen-tet,

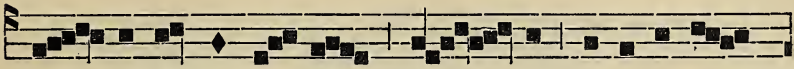

to - sa tak-kenron-ni, Ni - io ta-kiata-non-

8二 -17

sta - tis. Ra-wennio tehakswatetennis nok rakia-ta-non-

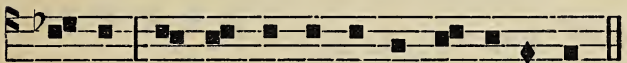

sta-tis; on-ka ka-ti enkhe-tsa-ni-se-ke. Ahon...

T 6. Areri - - ia, a-re - ri - - ia. Se-

今-ar

wen - ni-io sheionwe - skwaten - nis ne ie - sa-

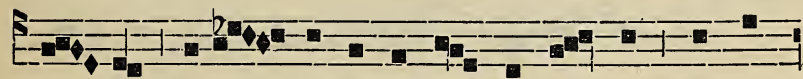

non-wes, i-se - ke tenionton - nha-ron-nion iah-te

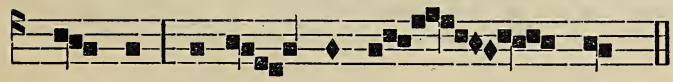

ka-kont en-ion - tsen-non - - ni. $a$.

Iesos wahalsenni (sk. 172). llewe (sk. 180).

III

Iontaw :
T 3

O- ri-wakwekon tsini skwaie-rase, Sewen - ni-io,

E-

akwa te - kwaia-to-re-ton tsi-ni sa-ie-ren a - seken 


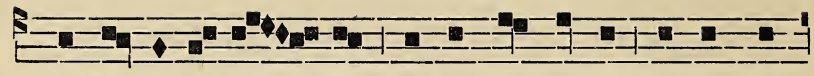

ionkwa-ri-wa-ne - ren nokkwawennontion tsi-ni skwa-

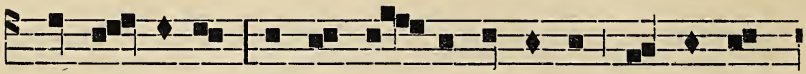

te-nien-ten-se, tenhnon sa - sen-no-wa-nat sa- sen-na-

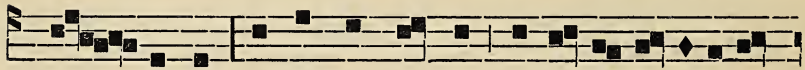

to - ken-ti, nok askwenten - re tsi a-kwa iahti-ia-ie-

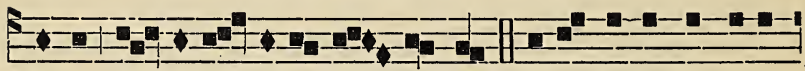

hewe tsi - ni sa - ta-ni-ten - ra-skon. Iako-taskat iahteia-

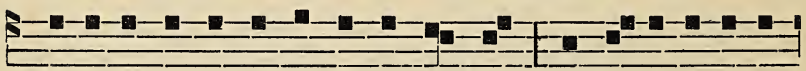

ko-ia-to-ronhon ne ka-ri-wa-ne-ren, ne ia-ko-ta-hi-tak-

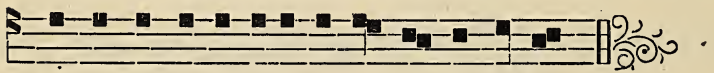

he Rawen-ni-io ra-o-ia-ne-ren-se- ra-ke. Ahon...

T 1.

A - re - ri - ia, a-re - - ri - ia. Sewenni-io,

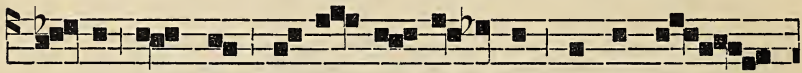

i - se sa - wenk na-ke - ni - kon - hra; nok tsi kon-

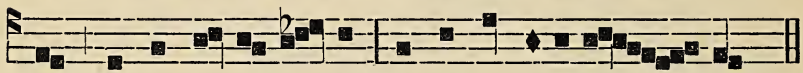

nhe wakonwenni - io - ste, enkonwennarakwa - ke. $a$.

Takwehiarak (sk. 179). Sewenniio kwanonwes (sk. 138). Okarislianoron (sk. 190).

IOKARENRE AWENTOKON. ROS.

T1.

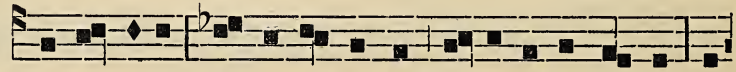

III Se-wenniio, ok tensennon-ton a-onsasketsien-te; nok 


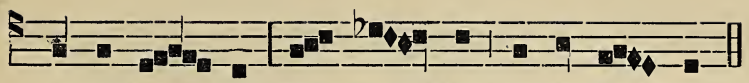

rawen Ie - sos: Tken-non - tons, sa-siewen - tann i. $u$.

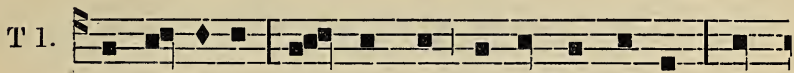

IV Se-wen-ni-io, ta - kwentenr wa-tiakwenhe-ie, en-

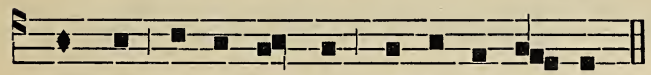

skweni ok sa skennen ti ska-nia-ta - re. 1. $u$.

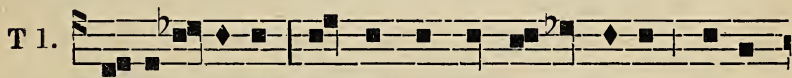

V Sa-sewa-rorok ka-nenhaksenskwa o-konha ne io-

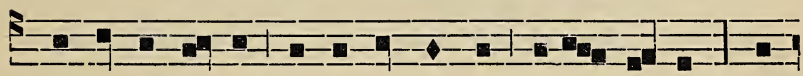

ton-nihon, nia-re swento-kon-nianion a-ia - kontho, ka-

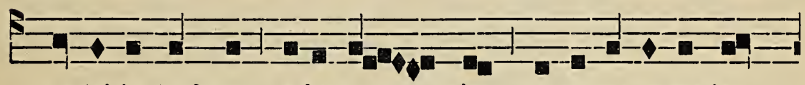

na-tsi-io tenhnon a-ke-non - skon er-se-wa-te-we-ien-

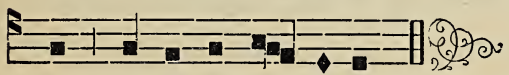

ton, rawen liawen-ni-io. 1. $u$.

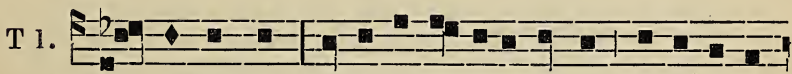

V1 Io - nekrakwat tsi-ni-iot ka-ri-wi-ioston tiako-nikon

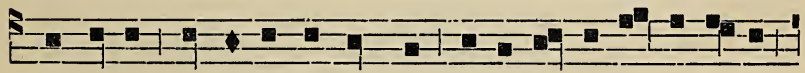

hratenies; ne ia-kowis non-kwe ta-on-sa-hon-tieren-ha-ke

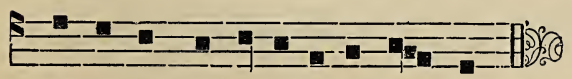

Ie-sos ronnhe nako-nikon-hra-kon. I. 0 . 


\section{TSIATAK NIWASONTASHEN.}

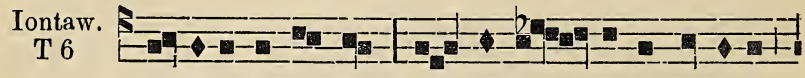
Sa-ta-niten-ra-skon, a - skiten - re, Sewenniio,

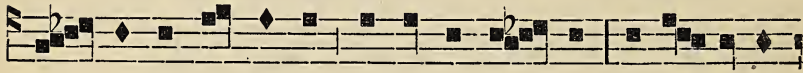

a - se-ken to - kenske iah-te kat-tok - ha wa-ke-ri-wa-

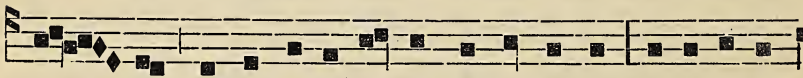
ne - ren; akwa se-wa-ka-ton-nha-kanon-ni sa-ka-ta-tre-

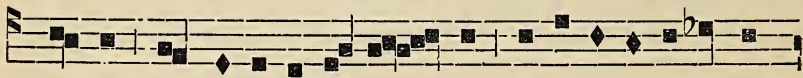

wa-te, a-kwa sa-sa-ni- kon - rhen nake-ri-wane-rakse-

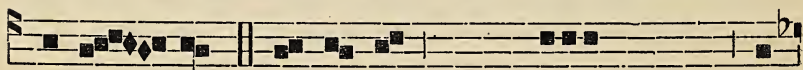
ra 0 - kon. Se-wemniio katennikonhrenhawitha tsinon-

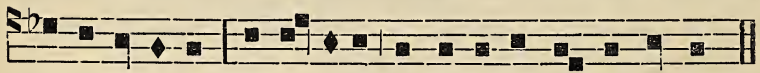
ni te-si-teron; Sewenni-io, son-hatsiwa konnia-hesen. Ahon. Takw. (sk. 34).

T 8. Sewenni - io, taki-tenr, so - tsi wa - kitent,

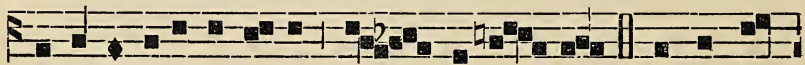
iahti-ia-iehewe tsi-ni kon - wennon - tion. Konienni-

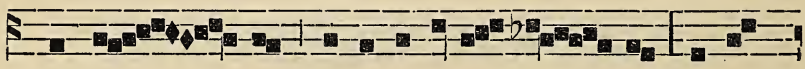
ten-ta - se, to-sa se-riwa - ra - ko tsi ia- 


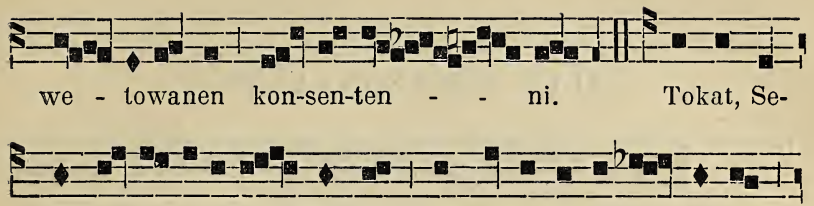

wenni-io, a-saka - henhion nonkwariwa-ne-ra - ksera,

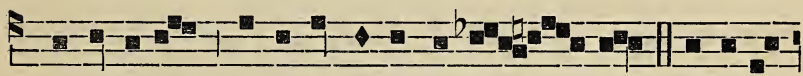

onka ka-ti iah-ta-iakoh-te-ron-se - - ke. Iah ki non-

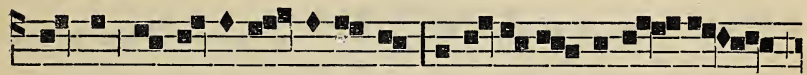

wenton ta - ie-ri-wa- saha - te tsi sa-ta-ni - tenra - skon,

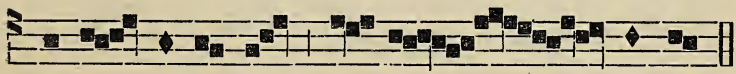

wake - rha-re-kwen en - ski - _ - ten-re.

Twatateken (sk. 225). Nonwa ken wenle (sk. 194).

IOKARENRE AWENTOKON (sk. 75).

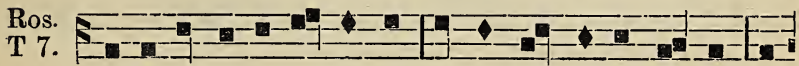

Wasakohro-ri ro-kwatse ne kannha-tse ra son-ha: Oh-

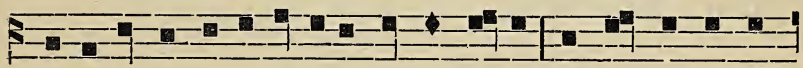

niotieren iali-te sewa-iote ken wentatie? Nok wa-honwenha-

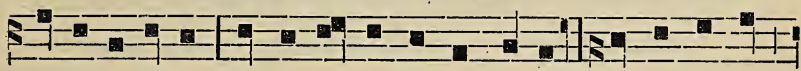

se ta-ho-niron: Iah on-ka te ion-kinnhaon Eh wa-se-we

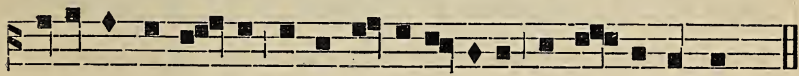

ne akonwentsiake; tsi-ni tka-ri-wa-ie-ri enkwa-ka-riakse. $7 a$. 


\section{IAIAK NIWASONTASHEN.}

Iontaw.

Aski-ten-re ni - sa, Sewenni-io, a- seken kon-

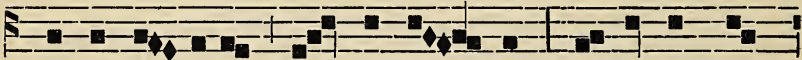

hienni-ten - ta-se ken wen-ta - tie, a - se-ken, Se-

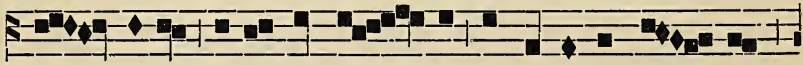

wen - ni-io, sa-ni-kon-hri - io nok o- ni sakwa - tse

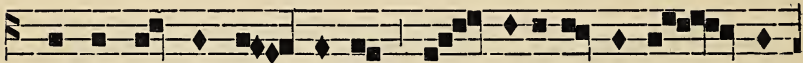

nashe-ten- ra-nion - he-ke ne na-ie-sa-nia-he - sen-

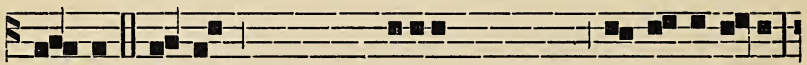

ha-ke. Sewenniio, takewennarakwak tsi konhienniten-ta-se;

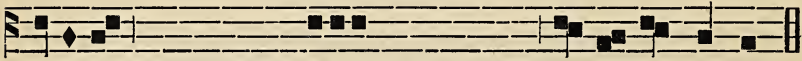

a-seken ionehrakwat tsi wakiewas io-ia-ne-ren-stakwa.

Ahon... Takw. (sk. 34).

T 8.

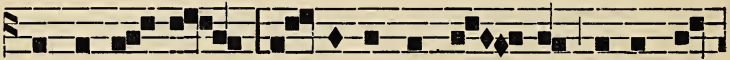

Sewenni - io, . lo - sa takwahre - wat tsi kwani-

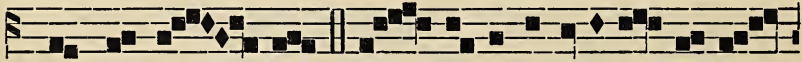

kon- hraksa - - ton. Sen - ha askwaiatoha - re, se-

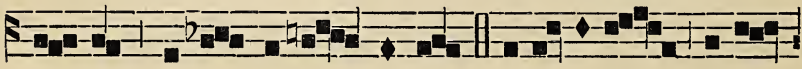

ri - si tsi-ni ionkwa - ie-ren. O-ia ni-ha askwe- 


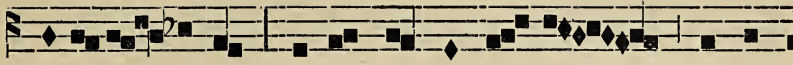

riaso-ten - ste tenkwanon-we-ra - ton iahte

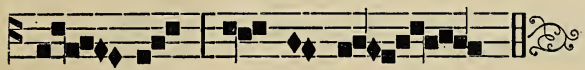

ka - kont tsi - nen-we.

Iesos wahalesenni (sk. 172). Ilewe twalaleken (sk. 180).

IOKARENRE A WENTOKON (sk: 75).

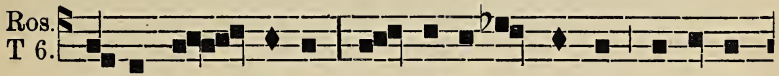

I - se kwen - tenron, kwa-ri-ho-ta-sienni ka-noron

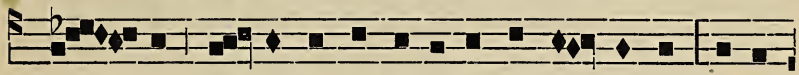

son - ha a - koren twake-ri-wa-kenwa - tanion, rawen

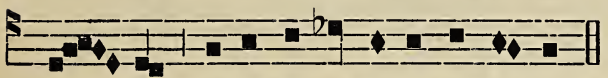

Ie - sos, sa-kawen-ni Ra-ti-kwekon-ne. 6. $a$.

\section{WISK NIWASONTASHEN}

Ionlaw. (sk. 308). Sewenniio (sk. 312)

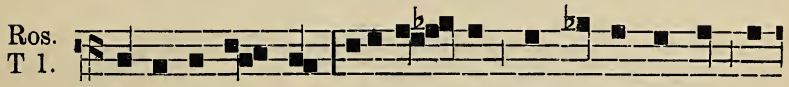

E-tho-ne Ie - sos iahonon-ke te-haron-wekon,wa-

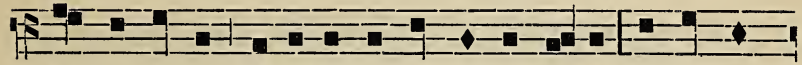

ha-wen-ha-se : Nahoten teskwatonwentsioni ? Sewen- ni-

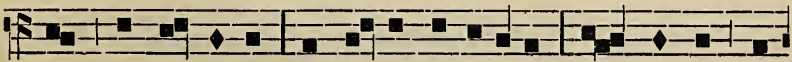

io, akken -se-ke. Nok Ie-sos wahoro-ri : I - skensek, wa- 


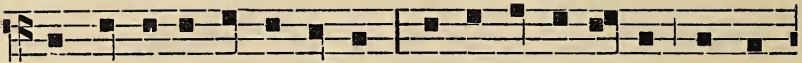

satsien-te tsi ti-seh-takon. Ok sa ok sa-ha-ken, nok wa-

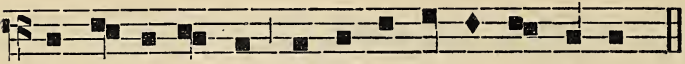

ho-se-re le-sos, wa-thononwe-ra-ton Ie-sos. I. $e$.

\section{TSI NAONTATAKENROSERAWE.}

T7.

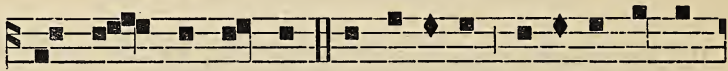

Ta- kwa- wen-na-rak, Sewenni-io, a-seken iah-ti-

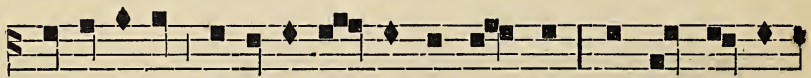

ia-ie- he-we tsi-ni sa-ta - ni-ten-ra-skon, nok tsi - ni sa-

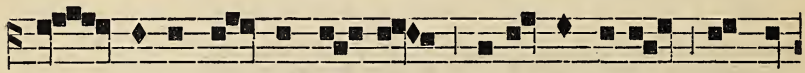

ta - ni-ten-ra - skon ko - wa, a-sa- ton- ta-te, askwen-

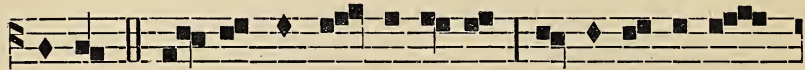

ten-re. Ta-ki-tenr, Se- wenni-io; a - seken akwa

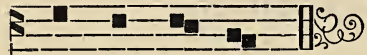

kon-nia - he - sen. Ahon. Takwa.

T 1

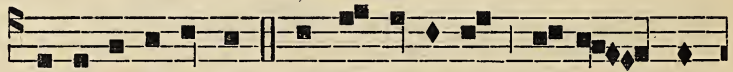

Tsi-tewata-trewat onkwe - riasakon o-ken - ro-

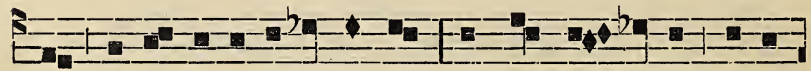

kon, te-wa-ta-teronhiakentak, ionkwenton - tietak te-te-

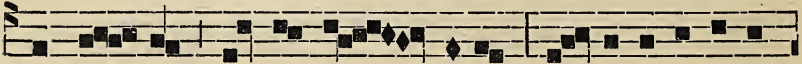

wa-sen - tho Ra-wen-ni - - io-ke; a - seken ia-ti-ia- 


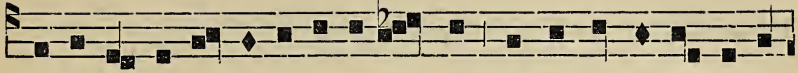

ie-he-we tsi-ni ho-ta-nitenra-skon iah-te ka-konte a-on-

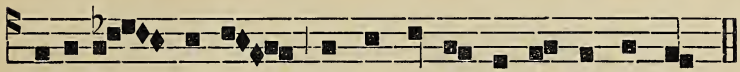

sahoni - konrhen onkwa-ri-wa-ne-ra-hakse-ra.

$\mathrm{T} 2$

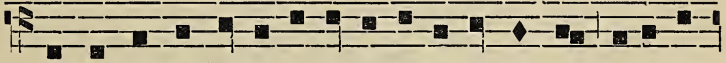

Te-watonnha-ta-ko a-on-sa-io-ia - neren- ne a-on-son-

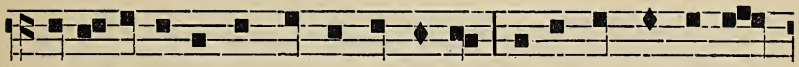

tera-kewe tsi ionkwa-ri-wa-neren; to-sa ionkwa-nisko

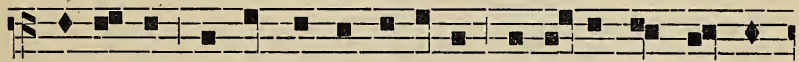

tsi 0 -nen nentsitwen-heion-se-re na-e-te-wen-non-ton-

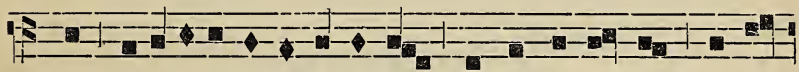

nion a-onse-tewa-ta-trewate, nok iah ta-on-ton a-on-

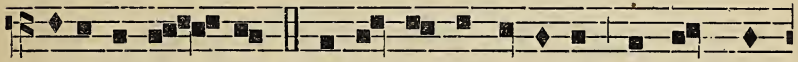

se-tewakwe - ni. * Sa-ton-tat, Lewenni-io, nok ta-kwen-

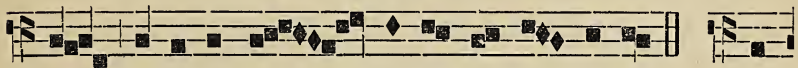

tenr, a-seken to - - kenske kwa-sen-ten- ni.

'Ta-

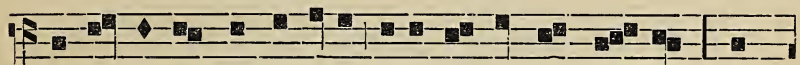

kwaie-nawas, Sewenniio, i-se takwaskon-ta-kwen, nok

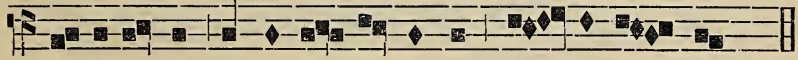

tsi-ni a-kwa nitsenna - nehrakwat, ton - takwan - nhe. Sat.

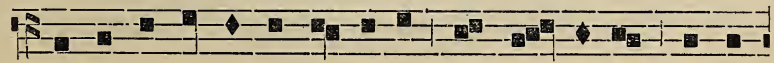

A-hon-wa-sen-naien lio-ni-ha, nok Po - ien-ha, nok Ro- 


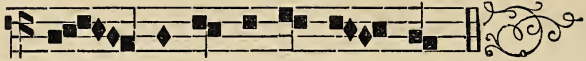 \\ tkon Ro-ia - ta- to-ken - ti. Sat...}

Iontaw.

$\mathrm{T} 1$.

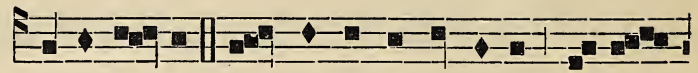

Ashe-ten- re a - kwekon, Sewenni-io, nok iah-

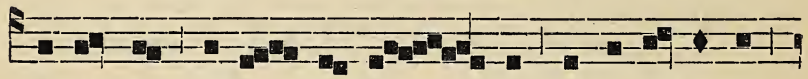

te itswens tsi-ni - kon sa - - son, iah-te sa-tka-thos

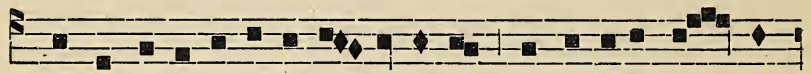

onkwe a-ko-ri-wane-ra - hakse-ra, kawen-niio tsion - ta-

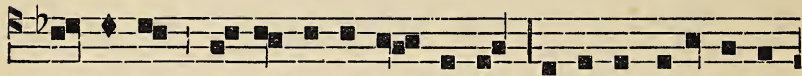

tre-watha; ok e-ken enshe-ten-re, a-seken i-se $\mathrm{Ni-io}$

年-

Sewen - niio tsi ia - kion - nhe. Taki-tenr, Ni-io,

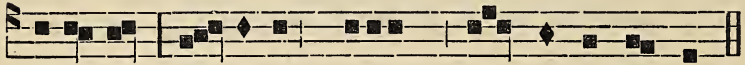

ta-ki-tenr ; a - seken iesaniahesen na- kwatonnhetston. Ahon.

Takw. (sk. 40).

$\mathrm{T} 2$.

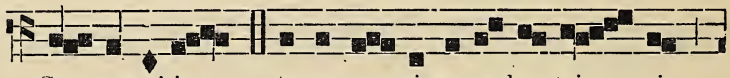

Se-wenni-io, to-sa se-ri-wara-ko tsi - ni

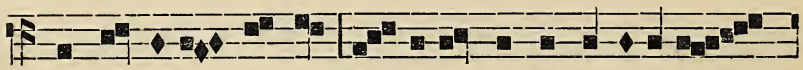

ionkwa-ri-wa - ne- ren, to - sa askwahre-wa-take tsi-

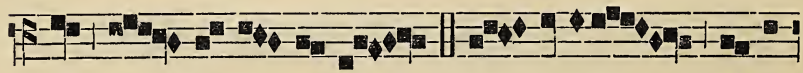

ni ia - kwatie - ra-nions. Se - wenniio to-sa 


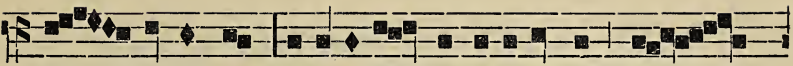

sa - se-hiarann o-riwaka - ion kariwaksen o-

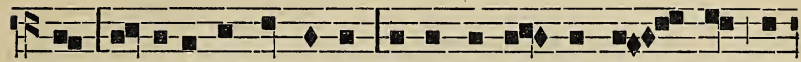

kon, ok-sa a-ionkwa-rane ne shetenra-nions non - kwe, ne

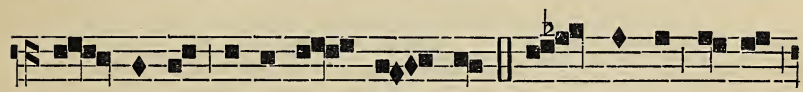

wa - hon-ni sotsi ion - kwen-tent. Ta - kwannhe,Ni- io,

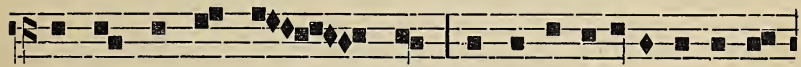

ne takwaskon-ta - - kwen, nok a- on-saie-sasennanen-

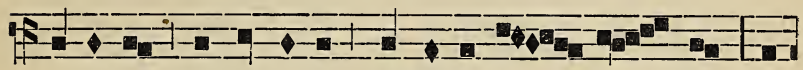

tonseke, Sewen-ni-jo, takwaia-ta - non - stat, nok

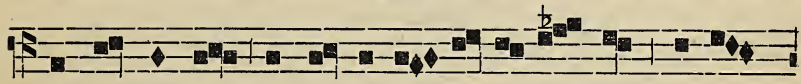

askwenten-re ionkwa-ri-wa-ne-ra-hakskon, ne a-

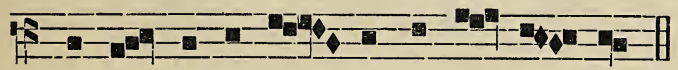

skwenten-ra-te tsı - ni tsenna - no - ron.

Akwekon iokenral (sk. 19). Telewasenlolak (sk. 135). Onen (sk. 150).

\section{IAKAWENTONTIETHA KOWA.}

Iontaw.

T 8.

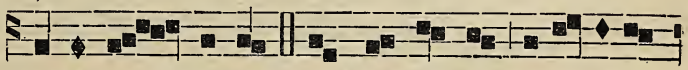

Satsia-ta - trewat. ne wahon-ni ka-ri-wane-

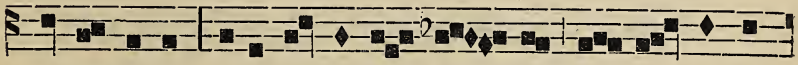

ra-hakse-ra a-on-son-te-no - ha - re e - tho-ne wa. 


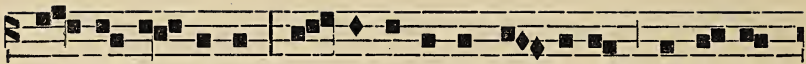

to - ken en - rawe ra - on-ha ne Rawen-ni-io en-sa-ko-

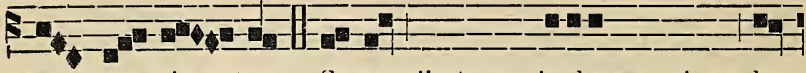

tsen-non-nia - ten. Sewenniio tosa aionkwasenni ne ka-

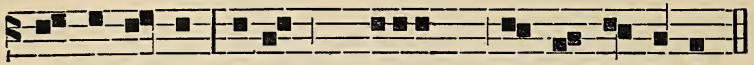

ri-wa-ne-ren; i- si takwawiten kari- wa-ksen okon. Ahon.

Takw (sk. 39).

T 2.

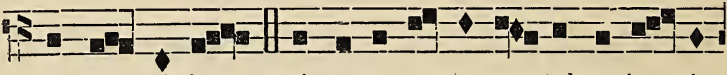

Sewen - ni- io tsia-ta-no-ron ko-wa takwa-ia - ti-

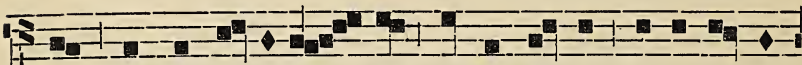

son, takwenni-se-ra - wis nonwentsiake akwa-sen-na-

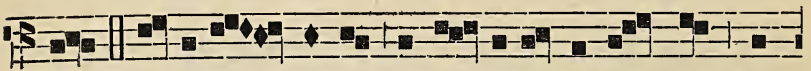

ien. To-ni ia - we-ta tsi-na-hetsi iakion- nhe kwa-

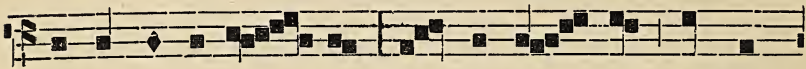

ni-konkraksa-ta - nion kwa-wen-non - tion ionkwa-

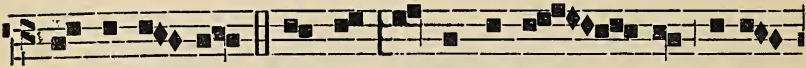

ta-trewa-te. Niawen sekon ia-kion - nhe, a-rek-

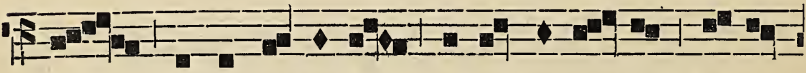

ho teskwa-ia-tontion neionkwa-na-non ka-ri-

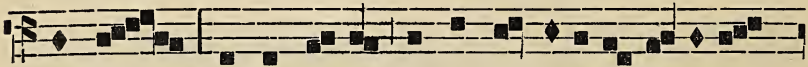

waksen ionkwa-ia- ta - kennha-ton sa-ta-ni-ten-ra-se- 


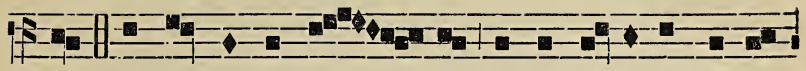

ra. 'Ton-takwentenr ni - - sa, tsitenı o-ni nonkwaton-

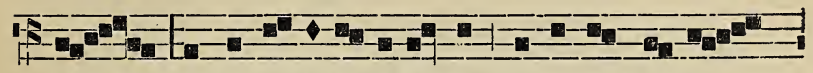

nhetst, askwa-ia-to-ha-re Ie-sos ra-o-ne-kwensa-

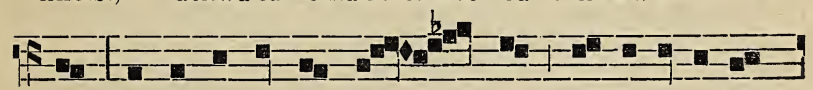

ke takwa-ni-konhro - ten - - stak tsi -ni sa-ni-kon-

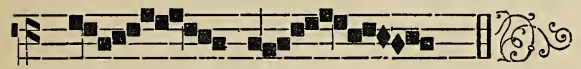

hro - - ten.

Sewenriio ken w. (sk. 144). O lesos (sk. 185). Onen (sk. 150).

IOKARENRE AWENTOKON (sk. 75).

T 2.

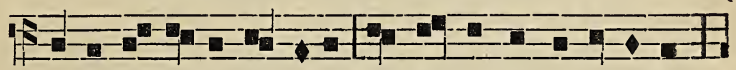

A-serhe-ke Sewen-niio, Nonwa tsi wenta-noronson,

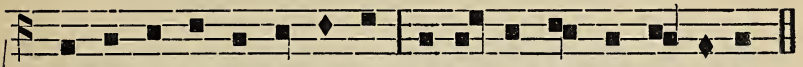

Akwa aion-kwa-ton-he-ke O-thenon ionkwenni-ta-ke.

Askwanikonhrahnirato

Tsi ionkwentontietha kowa,

Tsi neniakwakwenieseke

Aiakwariwaierite.

Onen tewaronhiakentak

$\mathrm{Ne}$ tewaieronke kenha

Tosa aontsteristake

Tsini watonweskwatakwa

Akwekon nonkwanikonhra, Asiteure, Sewenniio,

Ne aiawentontietake

Kariwaksenskwe okonha.

Ionehrakwat takwentenras

Ne tsi seron: nonwentsiake.
Tenshatiriwaserako

Tsi ionkwariwaneren.

Aionkwaronhiakenhonhake Akwa tetieseronniatha Ne teseriwateniatha

Karonhiakensera sonha.

Akwa onkwariwa kowa

Tsini ionkwariwaneren,

Satanitenraskon kowa,

Tosa takwariwarakwas.

Ahonwanenton tsinenwe

Ne nasenseratokenti

Ne ionkwariwisahani

Tsi nonwa ne wentotenson: E. N. 
v. Shetenr, Sewenniio, shetenr ne sheien okon.

R. Tosa iahte kakonte askwanakwase.

Ros.
$\mathrm{T}$ 8.

Sonkwenni- serawis ne Rawen - niio ne ken wenni-

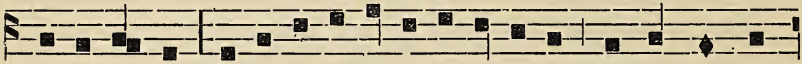

se-ra-tenions, ta- on-se-te-wa-ri-wa-se-ra-ko tsi ionkwa-ri-

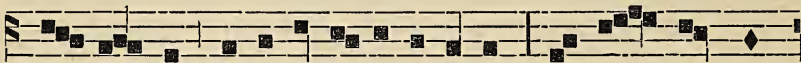

wa - ne - ren; te-wa-ri-wa-ie- ril ka-ti tsi ia - ka-wen-

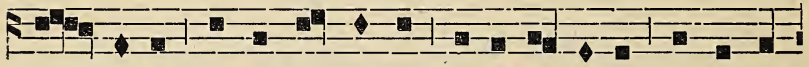

ton - tietha, twaronhia-kentak te-wa-ie-ron-ke, nok tewat-

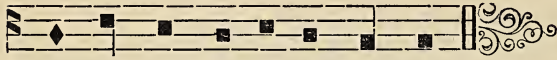

starhak nonkwa-ni-kon- hra-kon. 8. $a$.

\section{IAKAWENTONTIETHA KOWA.}

Iontaw.
$\mathrm{T}$ 4. Se- wen- ni-io, a-onsa- sehia-rane she- tenra-nions

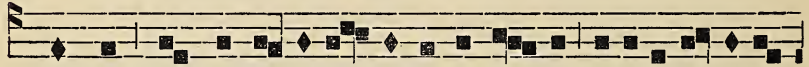

nonkwe, nok iahti - ia-ie - hewe tsi-na - he sata-niten-rasen

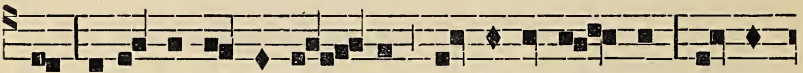

ra, tosa na-ion-ki-sen - ni ion-ki-ka-ron - nis; e-ren

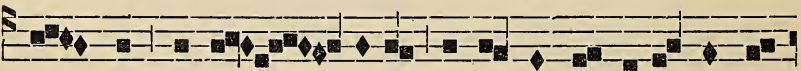

sha - wit, Sewen - ni-io ne tiotkon ion-ki-ni-konhro-

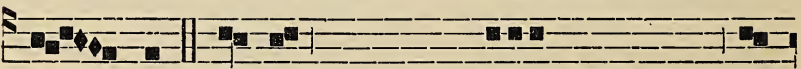

ta - kwas. Se-wenniio, katennikonhrenhawitha tsinon- 
820-10 -

ni te-si-te-ron; Se-wenniio, sonhatsiwa konnia-hesen. Ahon • Takw. (sk. 39).

T 2.

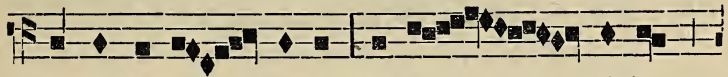
Hetsi-twasen - naien liawen - - ni-io,

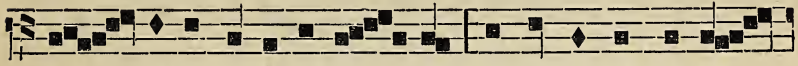

a - seken ro- ta-ni-ten - ron, nok iah-te kakont tsi-

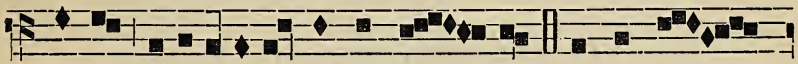

nenwe enlıota-niten-raskon-ha - ke. Rawen-ni - -

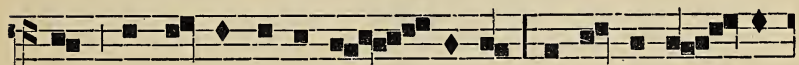

io, iah o-thenon te sa - - noron iah-ti - ia-ka . - he-

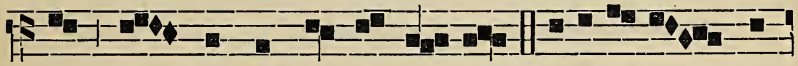

we on - kwennon-ton-niontse - ra. Iako-ta-skat iont-

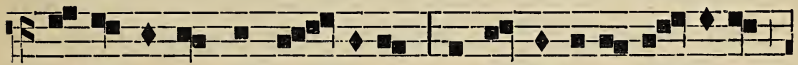

tokha ronwawen-na - ierits tiotkon ia-ko-ta-hi-takhe

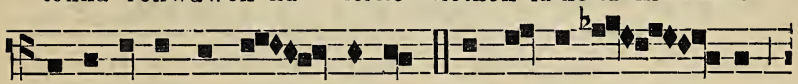

ra- 0 -ia-ne-ren-se - ra-ke. Askwe-hia-ra - - ne,

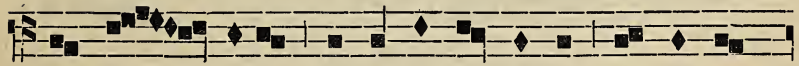

Se - wen - ni-io she-ia - ta-nonstalsek she-ien o-

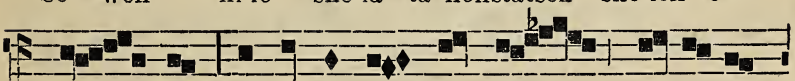

kon - ha, she-ia-ne ren - sta- kwak ne ka-ri-

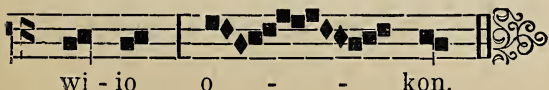

wi - io $0 \quad-\quad$ kon. 
Sewenniio tsinale (sk. 149). Akwa tsini (sk. 137). Ken ire (sk. 179).

\section{IOKARENRE (sk. 321).}

Ros.

T1.

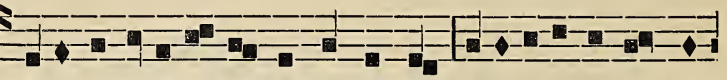

Tosa swatrori naho- ten swatkathon, ioriwanehrakwat ko-

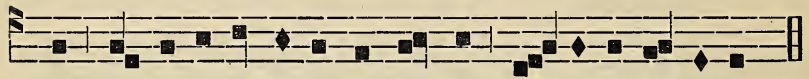

wa, tsi ok onen ensho-ton-nhe-ton ne Ni-io Ro-ienha. $1 u$.

\section{IAKAWENTONTIETHA KOWA.}

lontaw. (sk. 318). Takw. (sk. 39).

T 8.

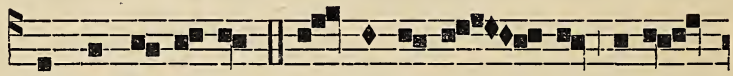

Wakwa-nia-he-se, la - kwa-ia- ti - son, ne tsi-

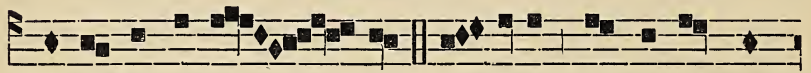

te-ron ka-ronhia - - ke. I - seke ka- ten-ni - kon-

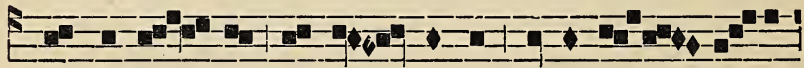

hrenha-wi - tha, kon-ne - kennis takswate - ten-ni

50 -

he - - ke. Tetioka - ras nonkwanikonhra-

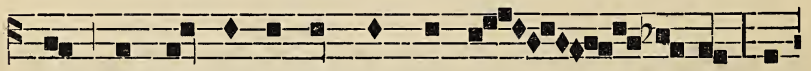

kon ionkwentent niahta skwentenra - - ne, iah-

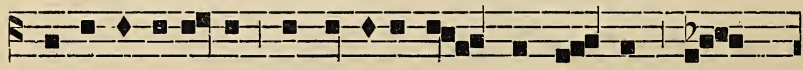

ta-iakwato-ri-sen, Sewenniio, tsi kwenni - tha ; to - 


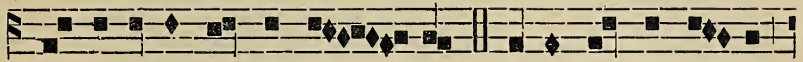

sa ka-ti askwa-wennon - ti. Katakwentenr ni - sa,

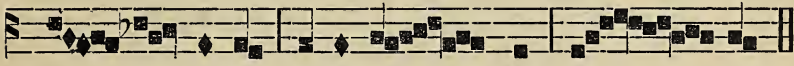

Se - wen-ni-io, ka-takwen - tenr ni - - sa.

Sewenniio lsi (sk. 141). Alonwesentsera (sk. 149). Ratasetha IOKARENRE (sk. 32i).

Ros. T 8.

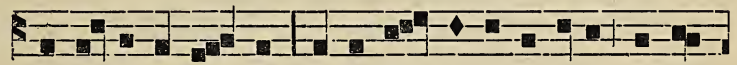

Tonta - ti skaia - tat ne ionkwe,watiohenre-te, waken-

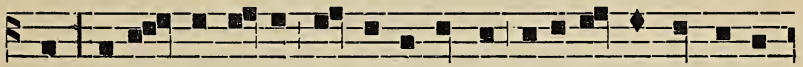

ron: Io-ta - skat kowa ne sa-nisten-ha iesa-te- we-ton, ie-sa-

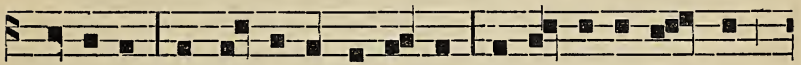

tstaronte. Nok Ie-sos ton-tahenron : Senha ia-ko-ta - skat

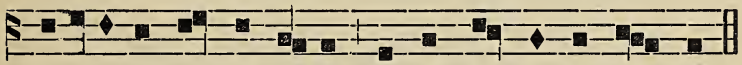

sako-rihonnien-nis $\mathrm{Ni-}$ io nok ron-wa-wen-na-ra-kwa. 8. $a$.

\section{IAKAWENTONTIETHA KOWA.}

Iontaw. (sk. 319). Tukw. (sk. 39). Sewenniio (sk. 320). Tsiatonlek (sk. 158). Swalaliesalanions (sk. 14?). Tetewa. (sk. 181).

IOKARENRE (sk. 32l).

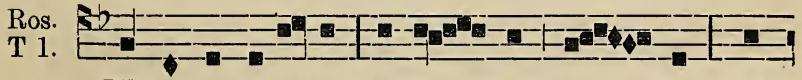

Waha-non-ta-ra-ne e- tho - ne le - sos, nok

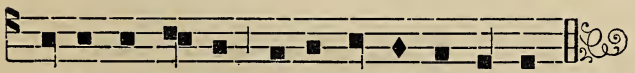

e-tho wa-hontien o-ni ra-ti-kwekon-ne. 1. $u$. 
19 MAR. SOSE RTI 1on WENT.

Iontaw.
T 5.

Tesonkwannhe nok sonkwa-ia-ta-kennhas Ra-

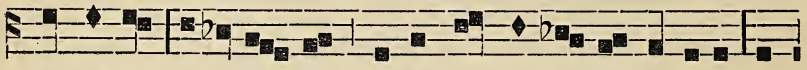

wenni-io ra-onha-ke enwatsennon-ni onkweriasa nok

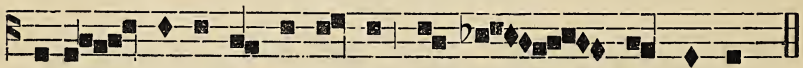

rao - senna-to-ken-ti-ke ionkwa - - rha - rekwen.

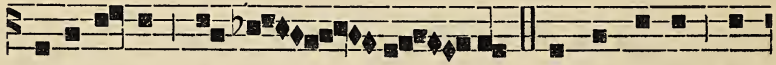
(sh) Are-ri - ia a-re - - ri - ia. Sewen-ni-io, he-

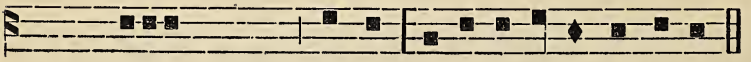

tsatsteristha Israer, satontek, i- se he-tsi-terons So-se. Ahon.

Takw. (sk. 58). Sose saialatokenli (sk. 224).

T 8. 5 Rotaskat ronkwe ro-tsa - nis Rawen - -

5-2

- ni-io, raskaneks a-hariwaie-ri - te ra-o-ia - ne-ren-

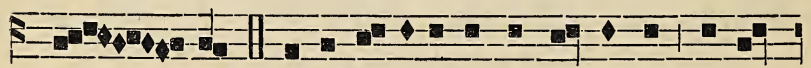

se - - ra. Thori-wa-ie-ri rokwatse onwe ra-ia-

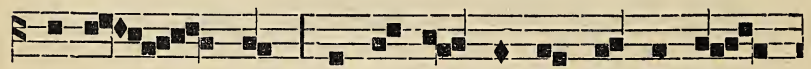

ta-no - - ron; tenhon-wa - ren-sa - ron-nionhe-

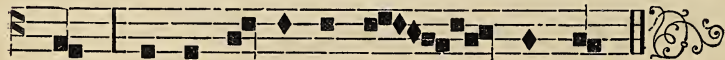

ke iah-te ka-kon-te tsi - nen-we. 
T $5.5-2=-10$ A-re-ri - ia, a-re - $\quad$ - ri - $\quad$ - ia.

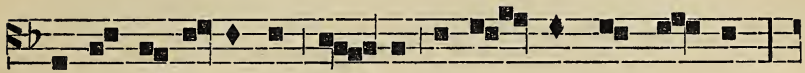

Tekwanonwe-ratons So - se hiano - ron-kwa $\mathrm{Ni}$ - io,

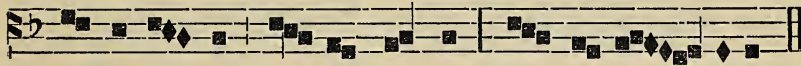

kwa-niahe - sen, ton - taskwannhe, kwen-na-ke - raton. $a$.

Sose lekwanonweralons (sk. 223). Ionwesen (sk. 195).

IOKARENRE.

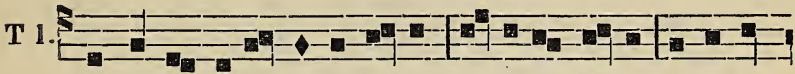

Jakob wa-hote-weton So-se ro-nekwe Wari a-on-ha
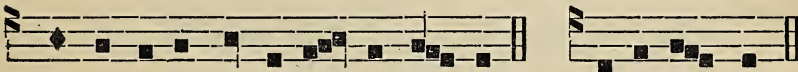

ionkwa-te-we-ton-ni Ie - sos Kris-tos. (sh) A-re-ri - ia.

Tierennakelswallıa (sk. 299).

T2.

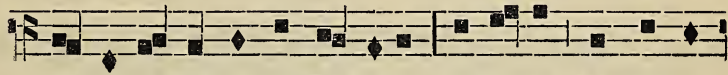

Ra-ronhia-kehro-non Ka-bri-ie thoto-ri Ra-wenni-

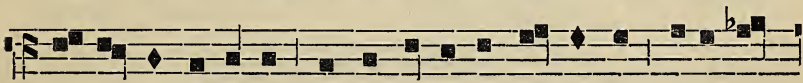

io $\mathrm{e}$ - tho ia-ha-re tsi tkana-ta-ien $\mathrm{Na}$-sareth a-hahro-

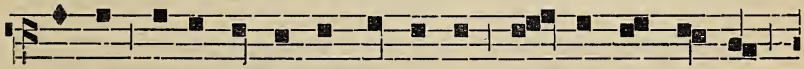

ria-na iah-te ka-nakwa-ien-te-ri ro - ne-kwe So-se
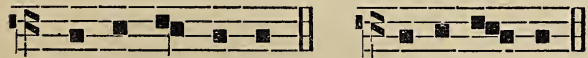

(te-hasnie - hakwe.) (sh.) A-re-ri - ia. 


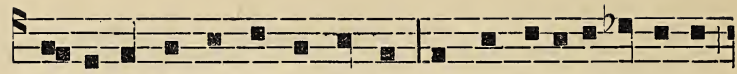

Ne ra-ti-nonnhakwe ka-tsenen wahon-wati-ia- tatsenri

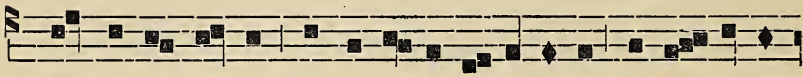

Wa-ri nok So-se nok raksa-a ra-iationni en-ne-ke-ri-
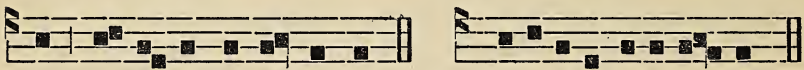

ke (tsi kon-tekwaien-takwa.) (s/l.) A-re-ri-ia, a-re-ri - ia.

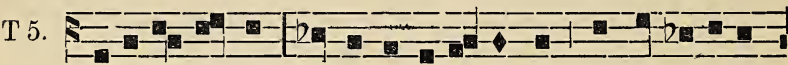

Rotasa-wa-tie a-sen ni-jo-se-rashen enthro-se-raie-

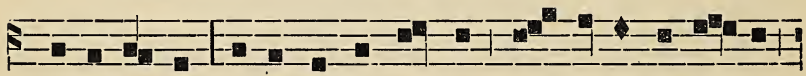

ri-te le-sos i-ienrhakwe nonkwe: Ro-ien- ha ken So-se
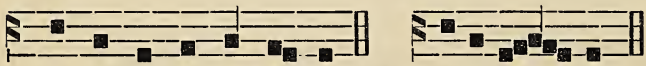

(ne ok ne ra-we-hia-ron.) (sh.) A-re-ri - ia.

$\mathrm{T} 2$.

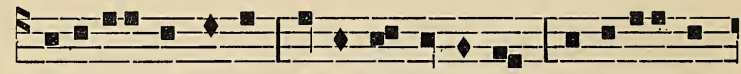

Iesa-nentons Sose eron-hia-kehronon, Ne ie-sa-nen-

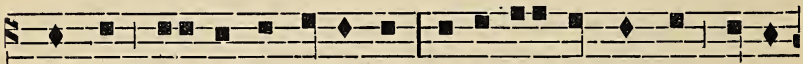

tonkwa tsi-ni tsia-tano-ron; Ii o-ni iakionkwe kwaia-

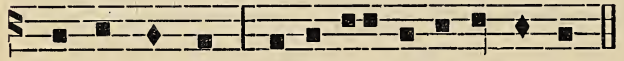

ta-nehrakwas, Tsini sa-ia-ta-to-ken-ti.

Hiaiatanoronston

Ne Sonkwawenniio,

Akwa hiarihonte

Sheiatsteristakwe,

Sheiatakennhaskwe

Iesos, Wari oni,

Hetsehiaron hiaiatison.
Sahennakerate Sonkwaskontakohe Onontiio Herol Tsi rorioserene, Nok wathetsiatakwe, Ok sa weswateko Egiptke weswatekwasen. 
Sotsi iotsennonniat

Tsini hetsitenron

Ne raksaa lesos;

Aseken tokenske

Ahonariskonhon

Eksa okon kenha,

Ethone sahonwatirio.

Askwentenre, Sose, Askwaienawase, Aionkwakwisrontie Kariwiiostonke
Nok neniakwenheie

Askwaiatorenne

Tsi tsiteron karonhiake.

Aietinentonnion, Nonwa nok tsinenwe, Ne nashen nihati Raonhake Niio: Roniha, Roienha, Ratikwekonhatie Rotkon Roiatalokenti. Etho naiawen.

25 MAR. SAHAHRORIANA. 2on WENT.

Iontaw. (sk. 268). Takw. (sk. 29). Areriia, (sk. 269).

$\mathrm{T} 2$.

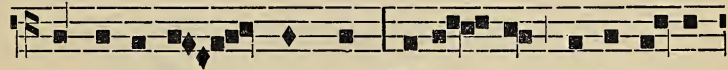

Tekon-noron - kwanions, Wari - a, tsiati io - ia-

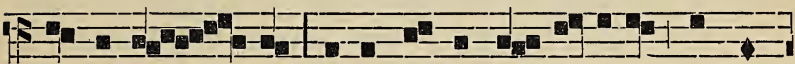

ne-rensta - kwa, se-nikwe-kon Pia-wenni-io, son-ha

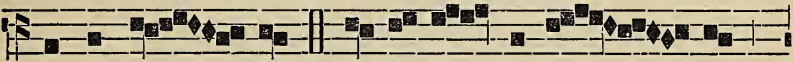

hiakonnien - stha. Sa-tonta - ton tsi - - ni

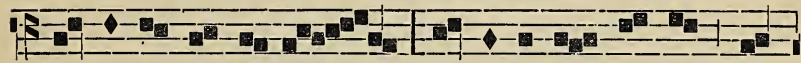

hiahro-ri Ka-bri - ie

ra-1'onhiake- hro- non thonn-

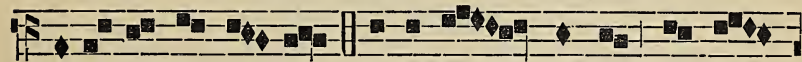

haon Rawen-ni - io. Ni-io he - tsienha ta - kwa-

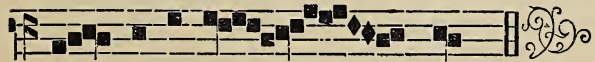

te - ren-naien - - has.

Wari lekonno. (sk. 208). Kakwirie (sk. 111). Sewar. (sk. 180). 


\section{IOKARENRE.}

Tierennakelskwallıa (sk 28\%).

T 8.

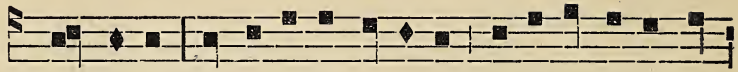

Thonnhaon Rawenni-io Kabri-ie a-hahro-riana Wa-
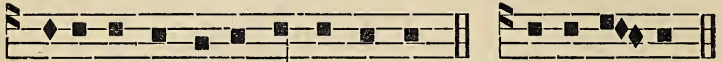

ri-a iah-te ka-nakwaien-te - ri. (s/.) A-re-ri - ia.

$\mathrm{T} 1$.

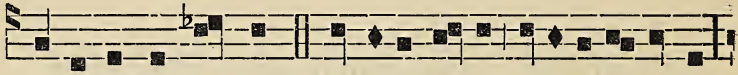

Tekon-noronkwanions, Wari-a, tsiati io-ianerenstakwa
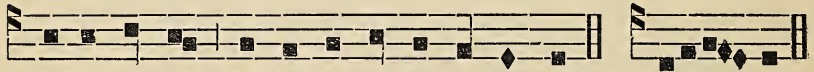

se-ni-kwekon Ni-io, sonha hiakonnienstha. $(s h)$ Areri - ia.

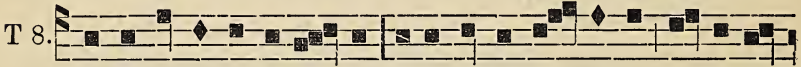

Tosa sahteron Wari- a, hianikonhranonwehon ne Rawen-

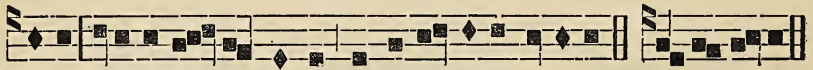
niio, oia nensia-to- tenne, nok ensa-wiraientane. (s/ $/$ ) A-re-ri- ia.

T 8.

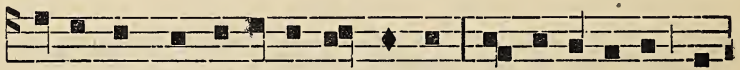

Ra-owenk Rawen-ni-io tsi konnhe; e - tho na-iawen tsi-
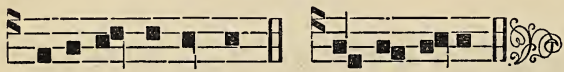

ni honi-konhroten. (sh.) A-re-ri-ia. Tekwanoronkw. (sk. 264).

v. Wari tekwanoronkwanions tsiati ioianerenstakwa, $(s h)$ are.

r. Rawenniio senikwekon $(s / \iota$.) areriia.

T 8.

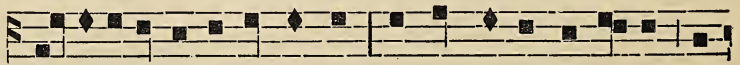

Ka-briie raronhiakehronon wahrenha-se Wari-a, wa- 


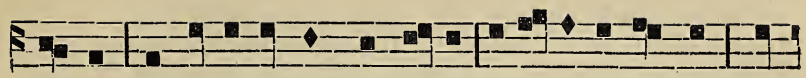

henron: Tekonnoronkwanions tsiati io-ia-ne-renstakwa Ra-
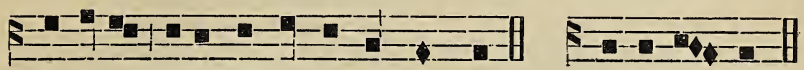

wenniio senikwekon hiakonnienstha. (sh.) A-re-ri - ia.

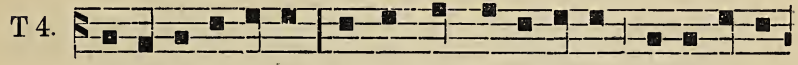

Ro-tokense kowa wasonkwen-nake-ratse ta-sakotken-

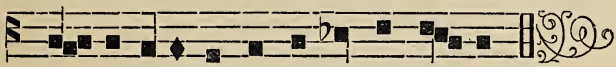

se - ra ra-otiohkwa ne Rawenni - io. v. Shelenr (sk 322).

\section{IESOS RAORONHIAKENSERA.}

lonlaw. (sk. 322). Iahle tsieriwakwa Ahonwa... Takitenr (sk. 167). Aionkwa. (sk. 163). lesos tekon (sk. 16j).

IOKARENRE AWENTOKON (sk. 75).

T 2.

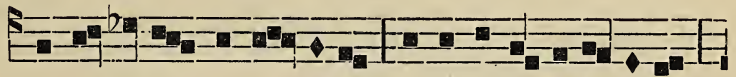

Ie-sos ra-o - ien-tannha-re, A-steson tetwaka-nerak.

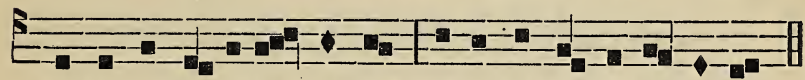

Tiotkon twata-1e-hia - rakwak Tsi-ni sonkwaronhiaken-se.

Okstentsi roronhiakenton Onkwariwaneraksera, Skanoron nahawenheion 'Isina thaterientakari

Ronwakonsaien ne lesos Nok ronwanitskeroserhon, Etho se ken ni twaierha Tsi ietikenhronnis nonkwe.
Nẻ ronwakenrenseronni, Nok iotehat takionhionnı, Nok wahonwatewentete, Ronkwetaksen shonwentenron.

Wahonwasere ne Iesos, Akwa wahonwaiesate, Raonha ronwahrewaton, li tenhnon teionkwatentson. 
Iesos okstentsi ohikta Tenonwanonwarannhaston, Teshoriwaserakwaton Tsini ionkwariwannhikon.

Akwa wathawisenheie Iesos tsi rorontakehte, Entsitewaienawaste Onkwaiasa entwakehte.

Wahonwaiatanentakte Ne tekaientannhareke,
Etho wasonkwenherase Ii ionkwariwanerakskon.

Akwa tekwanonweratons Iesos raoientannhare, Ise, ne hiaianerenston, Sonhaake ionkwarharekwen.

Sewenniio, kwatonronni Tsini satanitenraskon. Onkwe sesheiatontakwen, Sheiatsterist iahte kakont. E. N.

v. Kwasennaiens, Iesos, nok kwanentons.

R. Aseken saiasatokenti takwaskontakwaton.

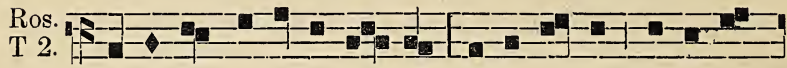

Abraham netsiswani - ha roska-ne-kon na-haken

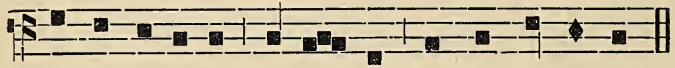

nakwenni-se-ra ; rotka- thon, rotsen-non-ni-hon. 2. a.

\section{TSI NAONTATENENTON.}

T 7.

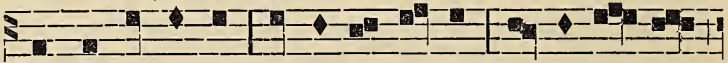

Ron-wa-sen-naien Ro-ienha Ta-fit; ronwanen - ton

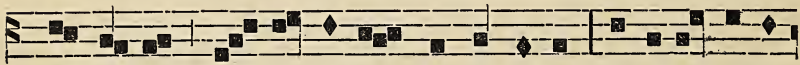

ne tha-re ra-sen-na-kon Rawen-ni-io; I-sra-er iesen-

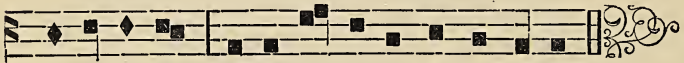

na-ke-ra-ton; ie-sa-sennaien ka-ron-hiake.

Sewenniio ken wenniseratenions (sk. 145).

$\mathrm{T} 2$

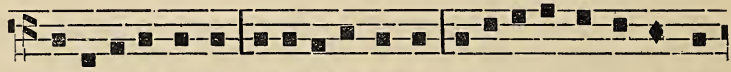

Saiata-token-ti, saia-tatoken-ti, saiata-token-ti Sewen- 


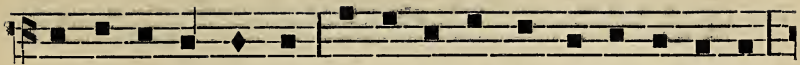

ni- io tsi ia-kionnhe. Karonhiakwekon, onwentsiakwekon

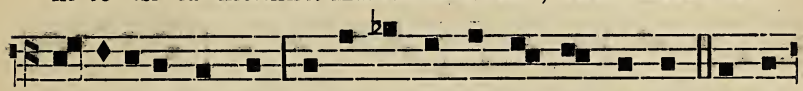

sa-ia-tanehrakwat; ie-sa-sen-na-ien ka-ron-hia-ke. Ronwa-

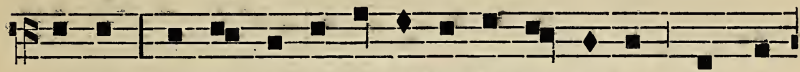

nenton ne tha-re ra-senna-kon Rawen-ni-io; ron-wa-

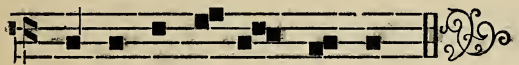

sen-na-ien ka-ron-hia - ke.

Tsi ensakonenton : (eniontnanela).

'I' 1

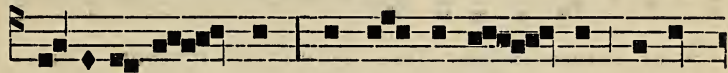

Si- ta-e-ro - non wa-ha - ti-nen - ta, oksa

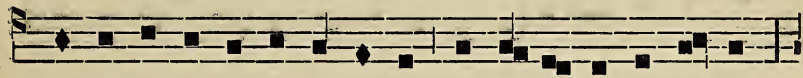

wahon-ne-te tsi tha-re Ie-sos, nok o-ni wahon-ni-ron

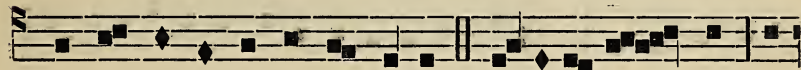

Ronwa- sen-na-ien ka-ron- hiake. Si-lae - ro - non wa-

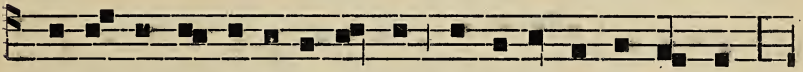

ha-titska-ron o-ha-ha-ke-son nok o-ni wahon-ni-ron :

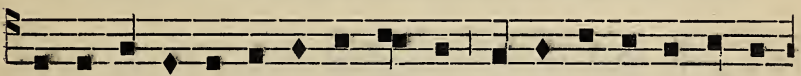

Ronwasen-na-ien Ro-ıen-ha Ta - tit, ronwanen-ton ne tha-re

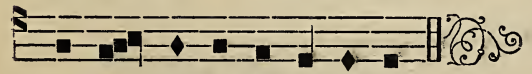

ra- sen - nakon Ra-wenni- io.

v. ... in pace. R. In nomine Christi. Amen. Sirisare (sk. 162). 
$\mathrm{T} 1$

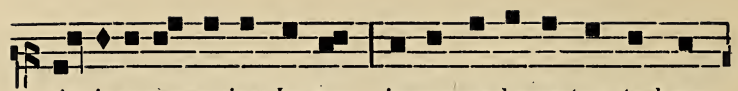

A - iesa-sen-naien Ie- sos, ie-sen-na-ke-ra-ton ta-kwa-

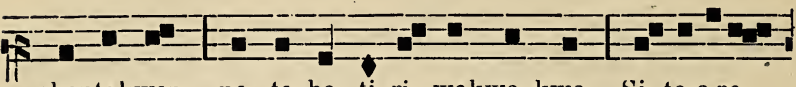

skontakwen, ne te-ha-ti-ri-wakwa-kwe Si-ta-e-ro-

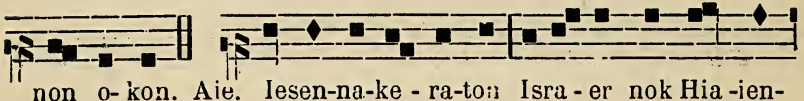

non o-kon. Aie. Iesen-na-ke - ra-toii Isra - er nok Hia-ien-

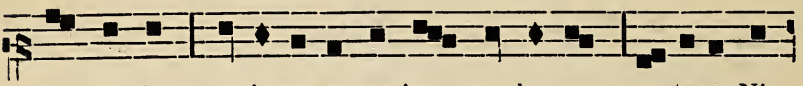

ha $\mathrm{Ni}$-io, a-ie-sa-sen-na-ien son-ha-a, on-tase $\mathrm{Ni}$ -

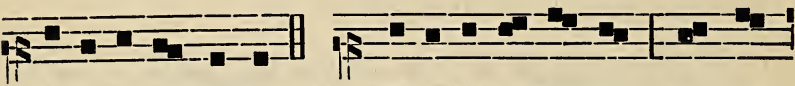

io ra-o-sen-na-kon. Aie. E-ronhiake- hro-non, ka - ron-

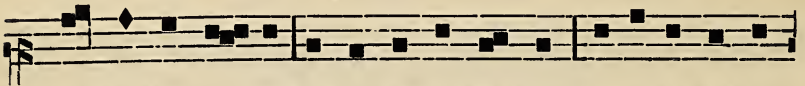

hia-kwekon 0 - ni nok nonwentsiakwekon i-se she-iatsen-

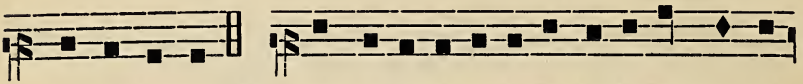

nonnia-tennis. Aie. Nonwa ie-ti-na-keren te-te-watken-ni

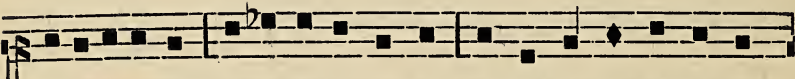

Si-ta-e-ronon, te-wa-teren-na-ien nok tetwa-riwak ka-ren-

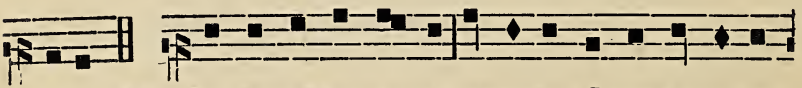

ni-io. Aie. Ne nonwa ken wente tewenron: Ronwasennaien

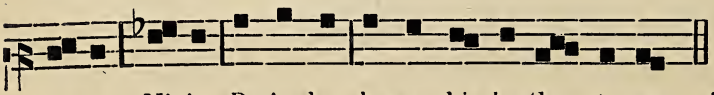

Ie-sos, Ni-io Ro-ienha, ka-ron-hia-ke then-te-ron. Aie. 
T 2. 可Ken i - re ne Ra - wenni- io ro-se-nontie kana-ta-to-

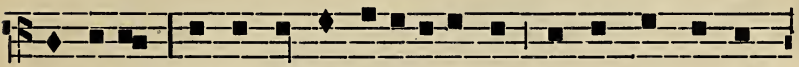

ken-ti-ke wahon-ni-ron Sita- e-ro-non : Sonkwatsennonnia-

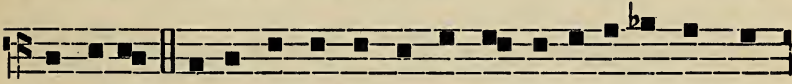

ten-ni-re. * Ra-tikwi-renha - winon-tie, te-ho-ti - ri- wakwen-

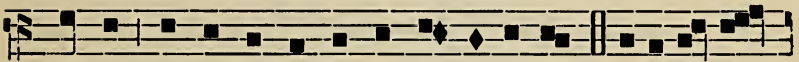

ha-tie: A-hon-wa-sen-naien ka-ronhiake. Sa-e-ri - wa-

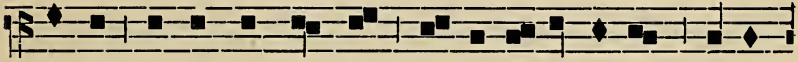

ron-ke kentiohkwakwe-kon tsi ken i-re Ie-sos Si -ri-

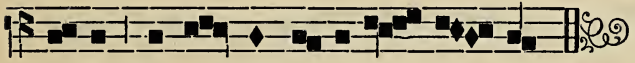

sa-re tonta - honwa-te-ra - ta - na. Rati.

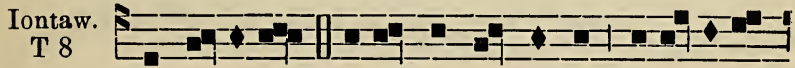

Sewen-ni-io, to-sa takken-ron-ni, to-sa takia-

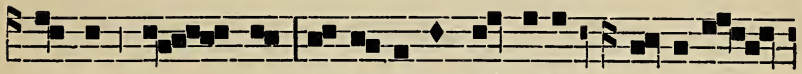

ton-ti non - wa; a-ske-ni-konhra-rake a-ontas-

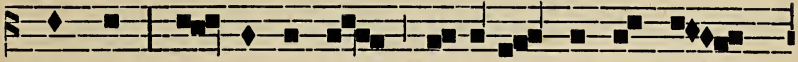

kwannhe, to - sa nonkwe ie-sero - hens ne ionks-

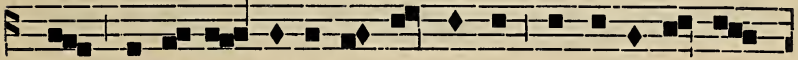

wens ionta-te - wenniiost tsi konnhe, askia-ta-non-sta-

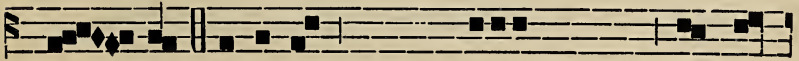

tse - ke. Ni-io Sewenniio, ohniotieren iawet ta - kwa- 


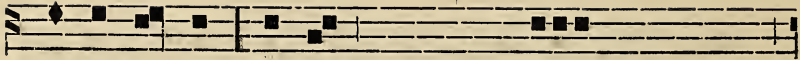

te wen-te-ton, kat-ketats akwekon nonkwe akoriwa-

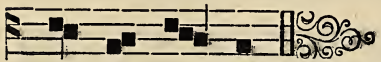

ne-ra-kse - ra. Sew. Takw. (sk. 39).

T 2.

$\mathrm{Ni} \mathrm{-} \mathrm{-} \mathrm{io} \mathrm{Sewen-ni-io,} \mathrm{ohni-o} \mathrm{-} \mathrm{tie-ren}$

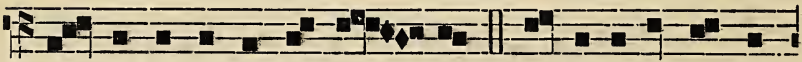
ia - wet takwa-te-wen-te - ton. A - ko-ri-wa-ne - ra-

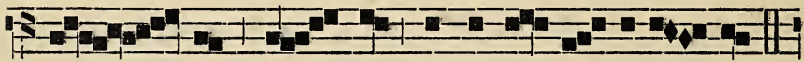

kse - - ra non - kwe ie-sannhas askre-wa - te.

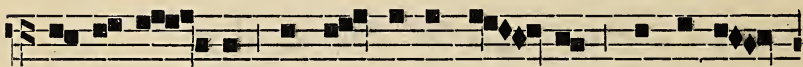

Se-wenni - io, konien - ni-ten-ta - se iah-te ski-

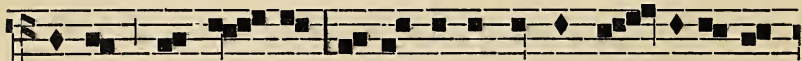

tenron, eh ka - ti ua-on-kia-tawen tsi nen - senonwe-

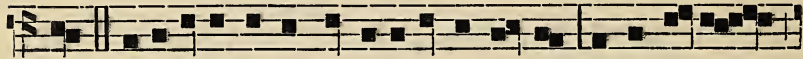

ne. Iaki-ni okon kenha ie-saniahesa- on, Sewenni-io,

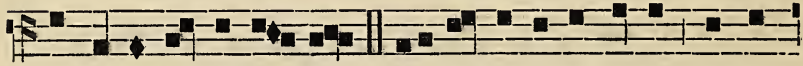

nok sheia-ta-nonsta - ti. Ii ia-wet o-tsi-nonwa iah on-

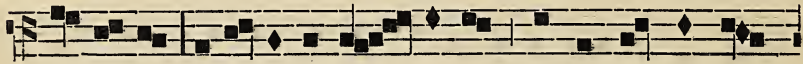

kwe tesken; iokenra nie-ia - to-tens ionken-ren-se-ron-

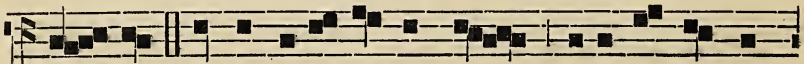

ni - hon, Ne iontohetsta-nionskwe okli ion-kie - ron- 


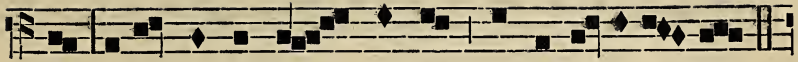

skwe te-ion-non-ka-ren - ronskwe nok ionkie-sa-ta - kwe.

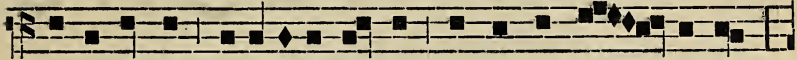

Io-nehrakwat ro-tise-ra-hreston tsi ionkkenron - ni-hon.

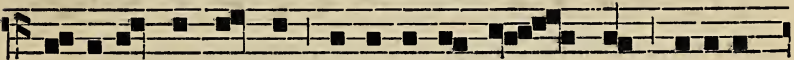

te-ho-ti -khasionkwen ne katse-ron-nia - tha, ie-iohe

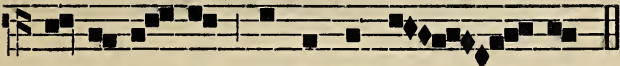

te-ho-ti - ien na-kwatia-ta - - - wit.

Salialkatho (sk. 167). Tetewasenlotak (sk. 135).

IOKARENRE. (sk. 331).

Ros. $\mathrm{S}$ 8.

Sakohro-ri Ie-sos ra-tikwekonne 0 -kon: Onen te-te-

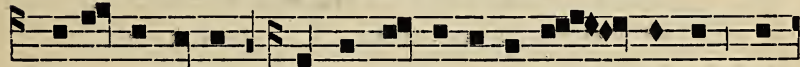

watekha-sionhe; nonen en - sewa-ka-ton - nheton en-

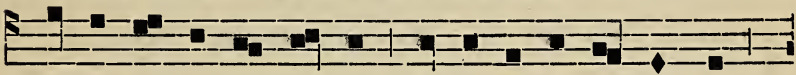

kwahente Ka-ri - re - ne, e-tho tente-wa-ta-tken

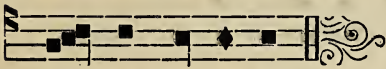

en - swatsennonni. 8. $a$.

\section{OKARISTIAKOWAHNE}

Iontaw.

$\mathrm{T} 4$.

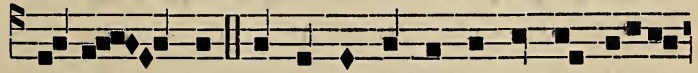

li tenh - non en-te-watsennonnia-ta-kwe rao-ia- 


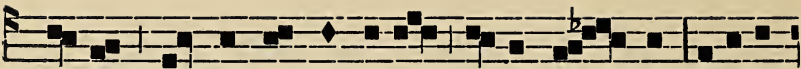

sa-kon ne Sonkwawenni-io Ie-sos Kri - stos, raonha

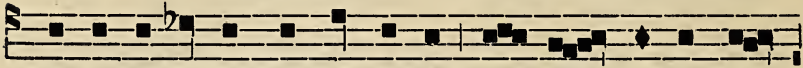

sonkwaskontakwen sonkionnheton, nok se - sonkion-nhe-

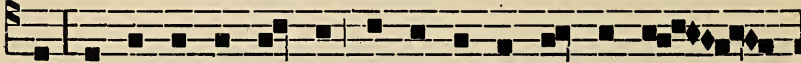

ton se-sonkwania-kenton, nok ensonkwatsen-non-nia-

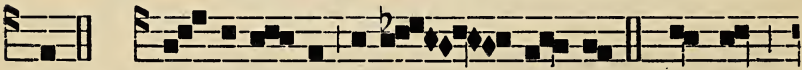

ten. (sh.) A - re-ri - ia, a-re - ri - ia. $\mathrm{Ni}$ - io

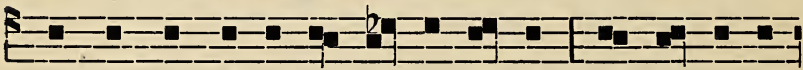

ta-kwen-tenr nok ta-kwa - ia-ta- non-stat, ta-kwaswa-te-

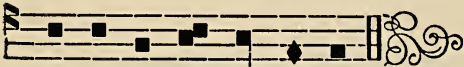

ten nok askwentenrhe- ke Ii. Takw.(sk. 53). Sew. onen (sk. 147).

T 6.5.

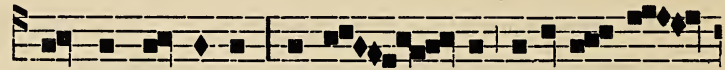

Kristos ne $\mathrm{Ni}$-io Roien - - ha sakowen-na-

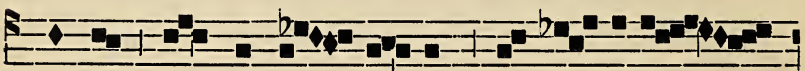

lakwen non-kwe ron - wa - rio, ron-wa - ia-ta-

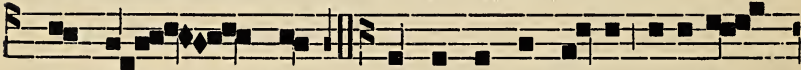

nen-ta - - kton. Ne wahon-ni Ni-io ro-iata-

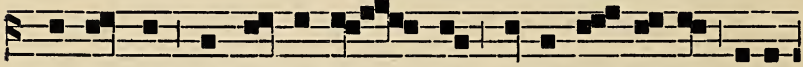

no-ron-ston, nok ro-senna - wi ka-senna-no-ron neio-

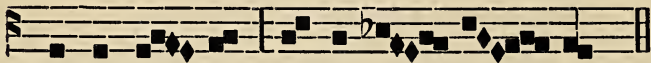

to-hetsten - nis no-sen-na o - kon.

Rataselha (sk. 190). Okaristiianoron (sk. 190). Telewalonls. 


\section{RONWAIATẢNENTAKTONNE}

Niio Sewenniīo (sk. 336). Raonekwensalokenli (sk. 164).

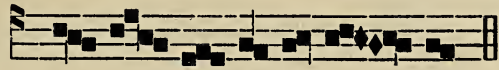

...pependil. Ve-ni - te a-do-re - mus.

Tsi tenienoronkwanion kaiasa ken kaien taieriwakwe :

T 6.

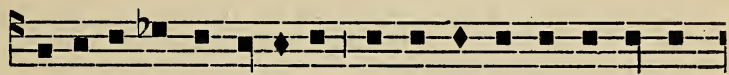

Onen tahon-wa-rio-se-re sonkwani-konhra-ta - askwe

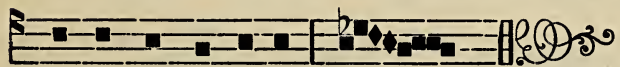

ne Sonkwawen-ni-io.

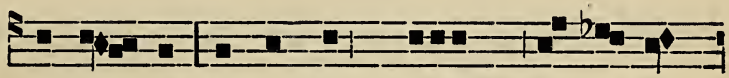

O IE - sos. Ionkwentenl sonkwanikon/trahni-rals-

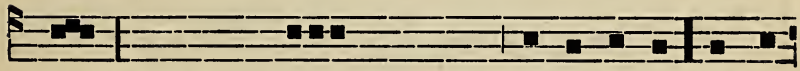

kwe; * onen tahonwariosere sonkwaiatanonslalakwe : ionkwen-

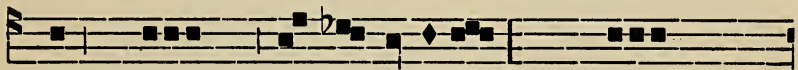

tent oni nentite - wenhe - ionse-re; * onen sonkwenheion-

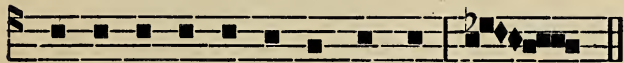

se-re ne se-sa-ka-onnlelskwe.

O IEsos. Sewenlent ne sewaiesas ietsikenrenseronni; ' onen hetsisewaiatontiesere netsisewenlenrhakwe :-Onen nonwa onwentsiakwekon Iesos ronwaiatannhalens; onen tahonwarioser'e.

Satanitennaskon. Akwa tokenske wahonwakenrenseronni Iesos; * Onwentsiake sekon tsi ireskwe :-Oll se nonte nahotkenseroten wahokenrenseronnis:" Iesos wahorio rotanileniaskon. 
Satanitendaskon. Raonliatsiwa tsini hoialanelurakwat ; onen tahonwariosere iah othenon te honoronsellakwe :-Naholen wahostonte sesakotsientanionskwe; * nahoten wahostonte ne sesakaonnhetskwe.

Takwatontats. Akwa tokenske, akwa tokenske; ; lesos wahorio kariwaneren :-lesos wahorio iontalkcnionnis ; * Iesos wahorio ne kanenskwen.

TaKWatontats. lesos wahorio ne kanaiesera; " Iesos wahorio iakotewennotatskon:-Onen kati aetewaswen ne kariwaneren ; " ne se wahorio netsitewaniahesenne.

Takwentenr. Onen serinikonhriak kariwaneren ; * iah nonwenton taonsaiesatontalse:-Aiawen iahta ionkwariwaneren ; iahta horonhiakenhon lesos.

'TAKWENTENR. Aiawen iahtaionkwariwaneren; * iahtahawenlieion lesos : - Aiawen iahta ionkwariwaneren ; " karonhiake akwekon aionkwawenniioke.

TakWEntenr. T'sionkwe, lsionkwe hetsisewaiatisak; * Rawenniio tsi lionnhe.

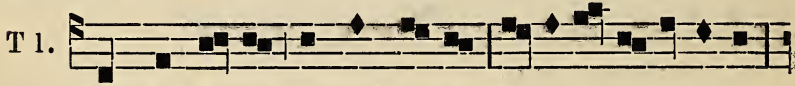

Ronnhek Ie-sos, ionnhek o - ni Ra-o-ia-sa- token-ti,

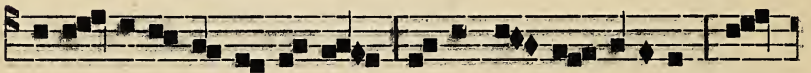

Atsi - te-wanonwe-ha- ke Ie- sos sonkwenhe-iase. *'Twa-

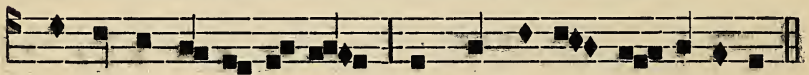

kwekon te- te-wa-ri- wak: Ionnhek Ie-sos ra - o- ia-sa.

Kaiasa tewasennaien

Wahiio ioianiionte

Ne saionkwatsiente onwe Sonkwakaronni Atan. Twa...

Kaiasa tewasennaien, Konwasennietha notkon, Wahatesenni ne lesos, Ethone sahonwario. Twa...

Kaiasa tewasennaien, Iesos raonekwensa

A kwa joiatohareton Onkwe akotonnhetston. Twa...

Kaiasa tewasennaien,

Etho ioranentakon

Nonkwe iakoriwaneren

Iesos roriwahtonton. Twa...

Kaiasa tewasennaien,

Tewanonwehak oni,

Tatsitewanonweraton

Iesos sonkwanonwehon. Twa.

Ahonwasennaien Nilo

Roniha nok Roienha, Rotkon Roiatatokenti, Asenseratokenti

Ahonwawenniiostake Nouwa nok iahte kakont. E. N.

ısi nen/lalkarislienliawe: Iesos raoientannhare (sk. 331). 


\section{WENTAKTA}
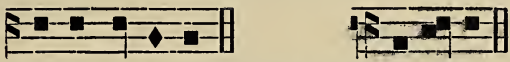

Lumen Christi. De-o gra-ti-as. ...genua. Leva-te.

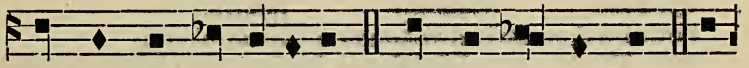

'Takwentenr, Sewenni-io. 2. Kristos, ta-kwentenr. 2.Ta-

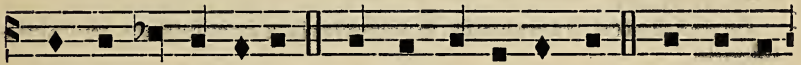

kwentenr,Sewenni-io. 2. Kristos takwatontats. 2. Kristos, ta-

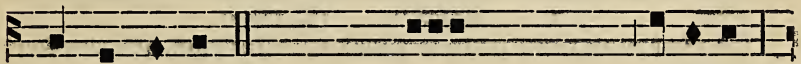

kwawenna-rak. 2. Niio iesaniha, karonhiake te-si-te-ron,

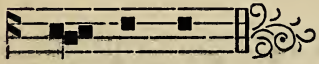

Ta - kwentenr.

Niio iesaienha, nonkwe sesheiatontakwen,

Niio Satkon Saiatatokenti,

Asenseratokenti enskat ok Niio,

Wari saiatatokenti, iahte saiatoronhon sasatiatata.

Wari saiatatokenti, Niio hetsienha,

Wari saiatatokenti iesakowanen iahte ienakwaienteri,

Wishe S $t i,-$ Kabrie S $t i,-$-Rafae $\mathbf{S} l i$,

Ise sewaronhiakehronon sewaiatatokenti,

Ise sewatkonseriio sewaiatatokenti,

San Watis $\mathbf{S} t i$,-Sose $\mathrm{S} t i$,

Ise, kwani okon kenha sewaiatatokentí,

Ise, sewaienterihne tsinontaiawensere,

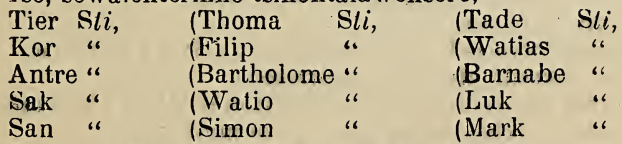

Ise, sewaiatatokenti, I - sos sewakwekonns,

Ise, sewaiatatokenti, sewaiatonserisahannhon noiatonseratokentison, 
Ise, sewaiatatokenti, Iesos hetsisewanonteratieskwe, (Ise, sewaksa okon kenha sewaiatatokenti, Etienn $S t i,-$ Roren $\mathbf{S} l i$,-Vensan $\mathbf{S} t i$,

(Fabien, Sebastien oni seniiati,

(San, Kor oni "

(Kosme, Tamien oni “ “

(Gerve, Prote oni

Sewakwekon sewaiatatokenti kariwiioston ietsiriohton.

Silfestr Sli, Ambroes Sli, (Ierom Sli, (Nikora S $l i$,

Krekwer " lOkustin " (Marten "

Sewakwekon seriwawakonne sewaiatatokenti,

Sewakwekon sewaiatatokenti, kariwiioston takwarihotasienni,

Antwenn S $t i,-$ Benoe $\mathbf{S} l i$, - Bernar $\mathbf{S} t i$,-Dominik $\mathbf{S} t i$,

Franswe $\mathrm{S} l i$,

Sewakwekon Rawenniio hetsisewariwawasehakwe sewaiatatokenti,

Sewakwekon sewaiatatokenti karhakon tisewanakerekwe.

Wari Watere $\mathbf{S} l i$, (Lusi $\mathbf{S} l i, \quad$ Sisir $\mathbf{S} l i$, Anastasi S $t i$, Akal " Anies " |Kateri “

Ise sewaiatatokenti iahte sewanakwaienterhaon, nok sewaterehonskon,

Sewakwekon sewaiatatokenti karonhiake tisewanakere,

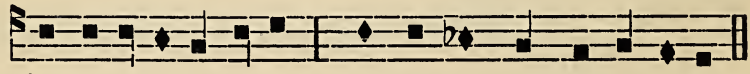

Takwaie-nawas nisa, $\left\{\begin{array}{r}\text { Takwentenr Sewen-ni-io. } \\ \text { Takwaton - tats Sewen-ni-io. }\end{array}\right.$

Tosa aionkwarane nonwentsiake ne iont | karonniatha, Tontakwannhe Sewenniio.

'Tosa aionkwariwaientane ne ka / riwaneren,

Tosa aiakwaienterhane ne sa | nakwensera,

Tosa oktontaiawentsi aiakwa | tonnhoktane,

(Tosa taionkwanenhraienniheke notkon no / neshonronon,

(Tosa aiakwatstake kanakwensera,atatswenhon nok naweria | taksensera,

(Tosa aionkwennontonnion ne kanakwa $i$ iesatsera,

(Tosa aiakwatkaronni naionkwa | teseronti,

(Tosa taiaon | wentsisonkwe,

(Tosa akannratarinesek, aiontonkariake, nok a | ionteriio,

(Tosa iahte kakonte aionkwa / tetsiraton,

(Ne tsini iahte saswenhon onkwe asatatonni Wari | kaiatakon,

Ne tsini seron: akheionwentsio | renne nonkwe,

Ne tsini sennakeraton | nonwentsiake,

Ne tsini iesanekwaheston nok oni tsini / sentontieton,

Ne tsini iesaronhiakenton resaiatanentaktononi te / kaiasontne, 
Ne tsini skwenheiase nok otstenrokon | satiataten,

Ne tsini tsi | satonnheton,

Ne tsini ietsisenonton | karonhiake,

Ne tsini teskwatennietenne Rotkon Roia | tatokenti,

Ne tsinatenteskwaia | toretane,

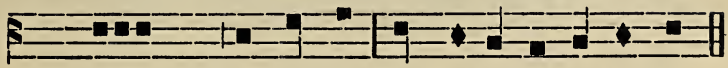

Ionkwariwanera-hakskon, Kwenni-ta ta-kwatontats.

Aiawen askwentenrheke tsini ionkwa / tsiioha,

Aiawen tosa askwari / wakwase.

Aiawen askwanikonhrataaseke naonsaiakwatatrewa|te onwe,

Aiawen tatsnieke tiotkon naontehiaronseke ne sentiohkwa | tokenti,

$\Lambda$ iawen asheiatahtonte ne ieriwahtontha ne kari | wiioston,

Aiawen skennen takenhake ne rotinon | tiioson,

Aiawen niakoriwiioston skanikonhrat a | kenhake,

Aiawen akwekon aionkwanikonhrahnironhake saianeren | serake,

Aiawen askwanikonhranentakte ne ka ronhiake,

Aiawen nonkwatonnhetston, ne sate iakionkwe asheskontako tsi tiotekha ia / hte waswas,

Aiawen askwatonnienniheke ne iakionnhekon non/wentsiake,

Aiawen asatontatseke tsi iakwate ! rennaiens,

Sonha Niio | Hiaienha,

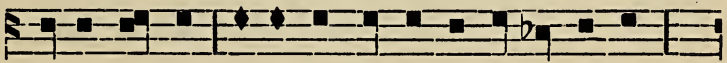

Iesos Kristos se-ri-wahton-tha ka- ri- wa-ne-ren,

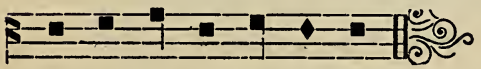

Ta-kwa-ia - to - kef le - sos.

$\{$ Ta-kwa-ton-tats Ie - sos.

Ta - - kwentenr Ie- sos.

Kristos takwatontats. 2. Kristos takwawennarak. 2. Takwelltenr Sewenniio. 2. Kristos takwentenr. Takw. (sk. 24).

Tsi ienenrinekens, ken niiol taieriwakwe :

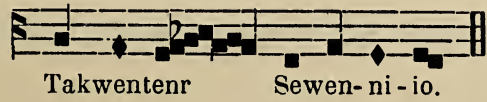


T 8.

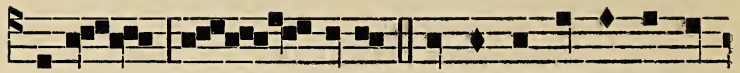

A-re - - - ri-ia. Hetsi-twa-sen-na-ien ne

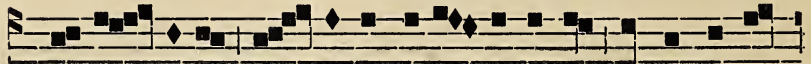

Rawen-ni-io, a - se-ken rota - ni-tenron nok iah-te ka-

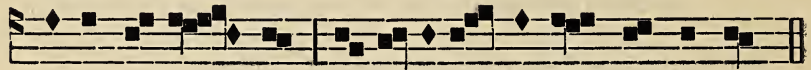

konte tsi-nen - we, en - ho-ta-ni - tenra - skon-ha-ke.

Iahta leieriwakwa: Iesos Kristos.

T6.

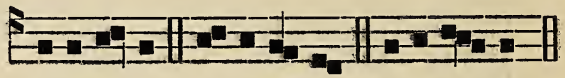

Tsi roialaraon. A-re-ri-ia, a-re-ri-ia, : a-re-ri - ia.

Swentioh... (sk. 17). a.

Ros.

T 8.

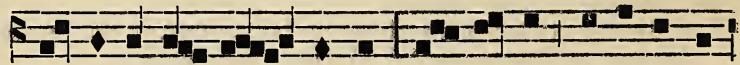

Orhon-ketsi en - takta, a - rekho te-tiorakwi-

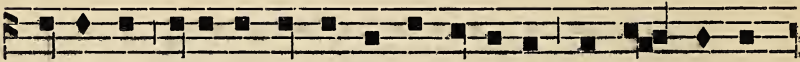

nekenhon ieiawe-nonton Wari Wate-re, nok ska - ia-tat

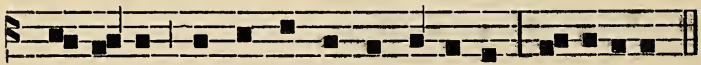

Wa-ri-a a-iont-ka-tho tsi ra-iatat a-re-ri-ia. 8. $a$.

\section{TSI NONSAHATONNHETE}

Rawenniio tsi lionnte (sk. 285).

Iontaw.

$\mathrm{T} 4$.

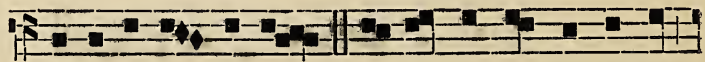

Onen shoton-nheton

Ie- sos sonkwaskontakwen 


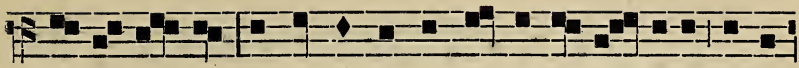

a-re-ri - ia, sho-nikonhra-se-ron-ni ne Ro-niha tsi sa-

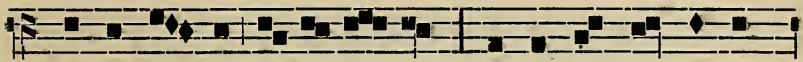

kwasenten - ni, a - re-ri - ia, sasonkwe-hia-ra-ne

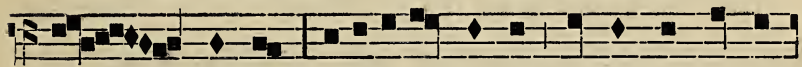

ne tionkwe ro-ia-ta-nehrakwat, ra-sennion kenhe-

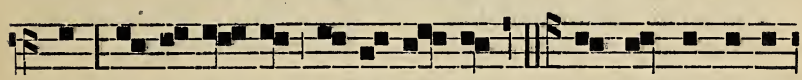

ion, a-re-ri-ia, a-re-ri - ia. Se-wen-ni-io ta-

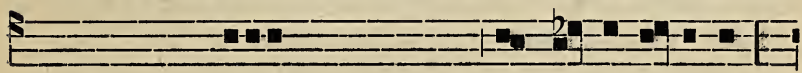

kwatenientense tsienteri tsini tiawens na-kweriasakon,

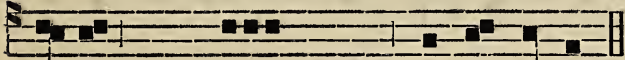

takienteritsihon nok onen seskwatonnhetse. Ahon.

Takw. (sk. 42).

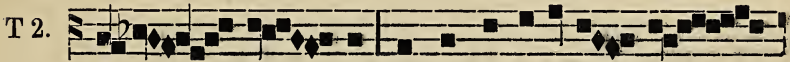

Ken wen - te rawen-ni-se-rison $\mathrm{Ra-}$

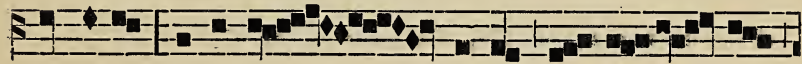

wenni-io tewatsen - - nonni nok e - tho - ne

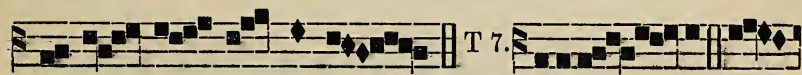

te- wa-ton - nha-ronnion. Areri - ia, a-

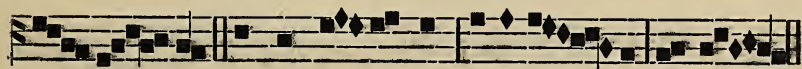

re - ri - ia. Sonkwaskontakwen onen Ie - sos Kristos. 


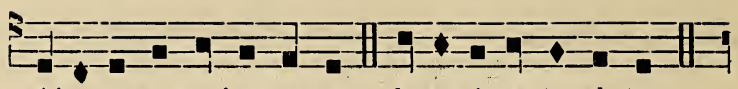

Alıonwasen-na-ien nonwa Iesos shola-lonnheton.

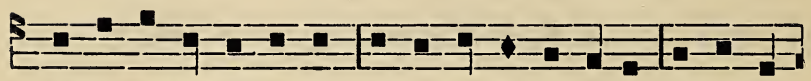

Shoni-konhra-se-ron-ni Ni- io akwa Ro-ni-ha Tsi ronwa-

To-sa se-watsa- ni-sek Tsi ia - i-heions nonkwe; Onen kon-

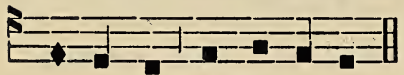

senten-ni skenuen on-ton.

wasen-nion ne kenhe-ion.

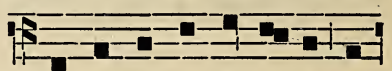

Takwahro-ri Wari - ia, na-

Nok ra-ti-ken- nia - te ra-

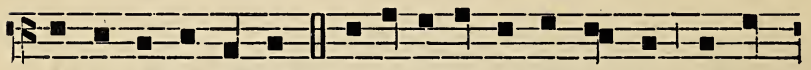

hoten wasat-katho? T'si ra-ia-ta-takwe le-sos ka-keti- rouhia-kehronon. Waho-niron : iahte shenteron, o- nen

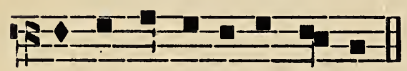

ron 0 - nia-ta-ra- a son-ha. seken sho-tatonnhe-ton.

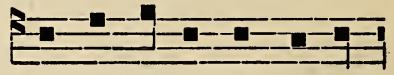

Tionkwehtakon sho-tonnhe-

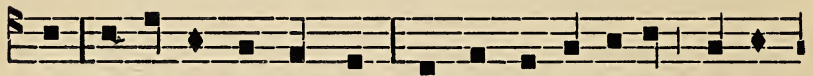

ton; iahte ka-kont ronnhe, Askwentenre Iesos ne na-

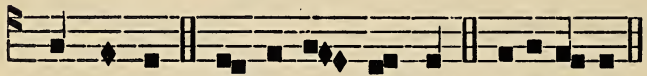

skionnheke. E-thona - ia-wen. A-re-ri-ia.

Alsilwasennaien (sk. 170). Oh naholen (sk. 97).

IOKARENRE.

T 8.

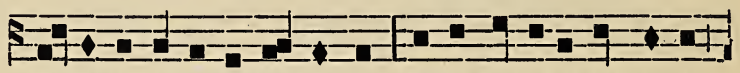

Sa-iatat ra-ronhiakehronon karon-hiake tahatsnente,

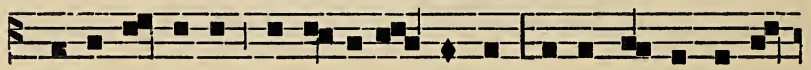

eh iatha-ta-ne tsi ra-ia-tat Ie-sos wahatstenra-karha- 


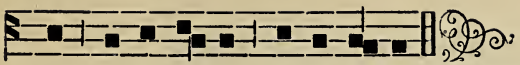

tho, a-re-ri-ia, a-re-ri-ia. Salkon Niio. 5-6. $i$.

T 7.

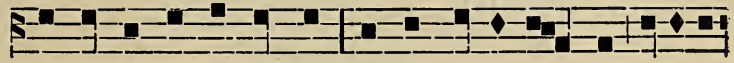

Wa tia-onwentsi-sonkwe akwa io-nehra-kwat aseken

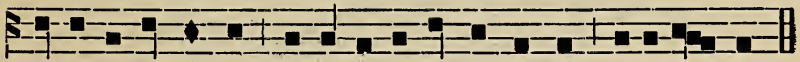

raronhiakehronon karonhiake tahatsnen-te, a-re-ri - ia.

Rolaskal onwe. 7. a.

T 7.

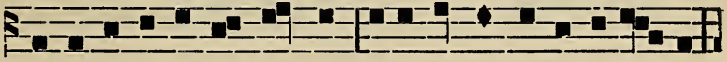

Tsina ho-nate-ron-hien-ten ratinonnhakwe tsi thaia-tat,

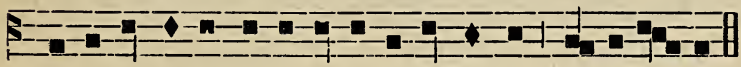

eh na-a-wenne tsi-ni-iol a -honen-heion, a - re-ri - ia.

Sewenniio lo ok. 5. $i$.

T 8.

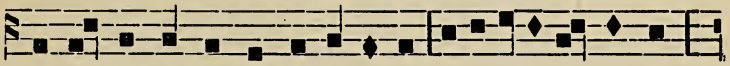

Ra-ronhia-kehronon sa-kohro-ri io-ti- riwi-ioston-ne :

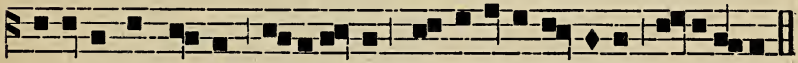

tosa sewah-teron, i-se lesos he-tsi-sewaia-tisaks, a-re-ri-ia.

Sewenniio iahte. 8. e. Ken wente (sk. 345).

Ros.

T 3 .

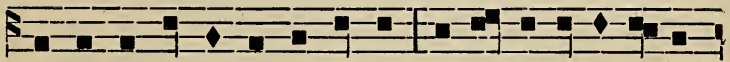

Wakontkatho iotsten-ro-wanen, wakontieren ok akte

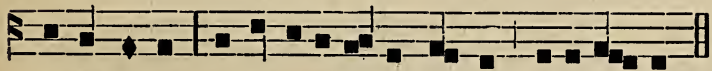

skaha-witon tsi thaiata-takwe Ie- sos, a-re-ri - ia, I. $a$. 


\section{NONTA TSINAHE THOTONNHETON.}

Ionlaw : Onen (sk. 344.) Takw. (sk. 68). Areriia (sk. 345). Twatonnharen (sk. 171). Riiatisaks (sk. 187).

1OKARENRE.

$\mathrm{T} 7$.

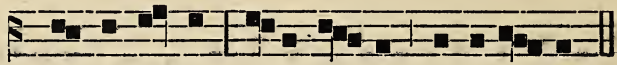

$\Lambda$ - re-ri-ia, a-re-ri - ia, a-reri - ia.

Salkon Niio.-Rolaskat.-Sewenniio lo ok.-Sewen. iahle. 7. e.

T 8.

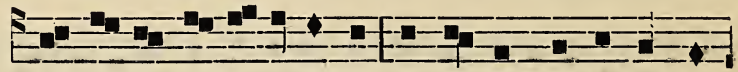

Twatsiaken twata- tekenson, Twakwekon twatonnharon-

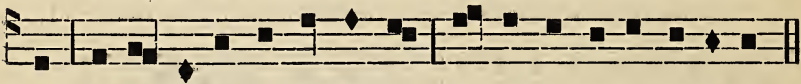

nion: O-nen Ie-sos shotonnheton, Ro-ronhia-kense-renta- on.

Ronwaiatat otstenrokon, Ionenhiakste kanontekon, Skennen kowa thoiakenhon, Twakwekon twatonnharonnion.

Rotatewennaieriton Rawen : enskatonnhete; Ne kati ionkwarharekon Ii entsitwatonnhete.

Ne niate swaiatotenson, Iesos hetsiswanentonnion; Sotsi se ken sonkwanonwes, Akwa rotanitenraskon.

Ronwaswenskwe sakosennion Nok noneshonronon okon, Akwekon oni kenheion; Iah othenon tahonoron.

Ne akwa tionkwehtakonhak Rohnhe Iesos, akwa ronnhe,
Iahte kakont enhronnheke. Tosa tewatennowentak.

Sewentent, Sitaeronon, Hetsiswaiatanentakton Ne nakwa sonkwaiatison, Twakwekon sonkwentenranion.

Akwa sakotsientanionskwe Ne iakononwaktanionni, Nok oni sakaonnhetskwe Onen tsi iakawenheion.

Twakwekon twatsteniaronNe tsi ionkwariwiioston, [nion Iesos sonkwahretsiaronnion A etewateweienton.

Ahonwasennaien Niio Ne Roniha, nok Roienha, Rotkon Roiatatokenti, Iahte kakonte tsinenwe.

v. Onen Iesos shotonnheton, areriia.

R. Onen ionkwaiatateri entsitewatonnhete, areriia. 


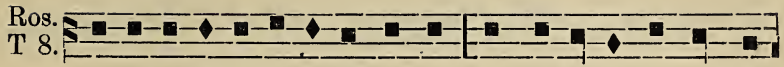

lonsa-ka-hewe sa-tekon nonta, kannhokaronton kannho-

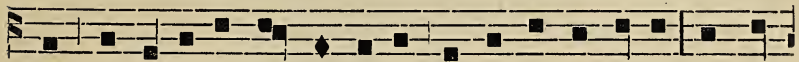

ton, wa-thata-ne Ra-wen-ni-io, wa-sa-kawen-ha-se : wakion

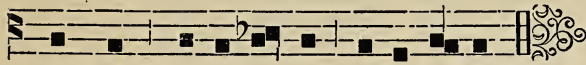

skennen 'a-re-ri-ia, a-re-ri-ia. 8. e.

AWENTOKON TSINIIORE KARONHIAKE ENSRETE.

Iontaw.

T 8.

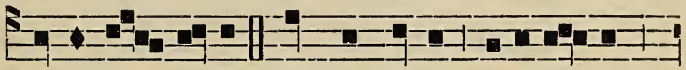

Sewatsen - nonni tsionkwe okon, a-re-ri - ia ;

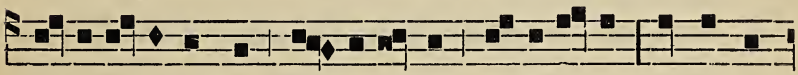

te-se-wa-ri-wakwas ra - o-son-na, a - re-ri-ia, thetsi-se-

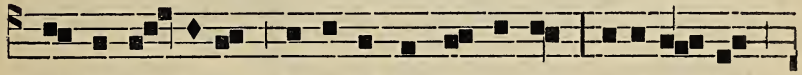

wanonwe-ra-ton Iesos tsi sho-tonnh

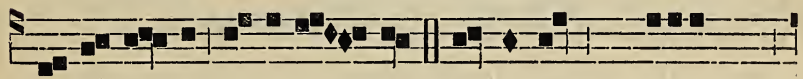

a - re-ri - ia, a - re-ri - ia. Sewatonnhare sewaiatatoken-

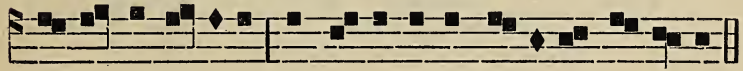

ti Rawen-ni-io-ke; thetsi-sewanonwe-raton tsinenwe. Ahon.

Takw. (sk. 72),

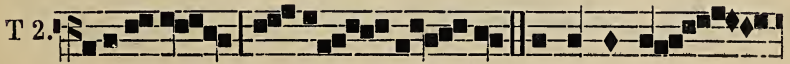
Areri- ia,
a - re - ri - ia. Twatonnharon- 


\section{H-T- nion, lesos shotonn - heton ensesonkionnhe - te. $a$. \\ Helsitwasen... \\ Onen tsionwesen. Iesos wahate... Twatsennonni. lesos tekwanon... Onen nonwa.

$\begin{array}{cl}\text { sk. } 174 & \text { Ne nonwa. } \\ 112 & \text { Tsini ionkwa... } \\ 172 & \text { Tekwanonwe.. } \\ 170 & \text { Shotonnheton. } \\ 196 & \text { Iesos, lesos. } \\ 101 & \text { Twatonnhaien. }\end{array}$ \\ sk. 171 IOKARENRE (sk. 348).

\section{TSI IENENRINEKENS.}

Salonlek (sk. 305). Rotiialatokenti raotiriwa. (sk. 341).

\section{Iontaw.}

T 4.

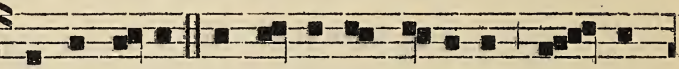

Rawen-ni-io ka-ron-hiake thenteron ra - ke-

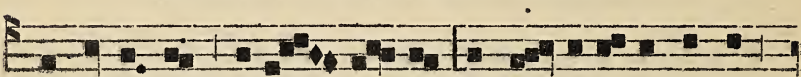

wenna-rakwen, a-re - ri - ia, nok tsi ri-ri-wa-ien-nis

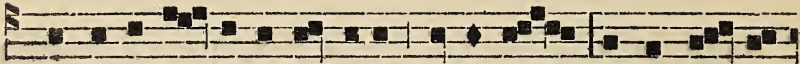

nakwate-ren - naien-takse-ra, ne a-kwa rakwa-ta - hon-

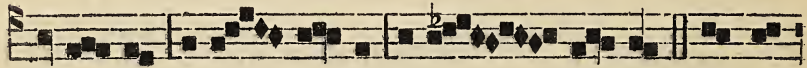

sata - ti, a-re - ri - ia, a-re - - ri - ia. Se-wen-

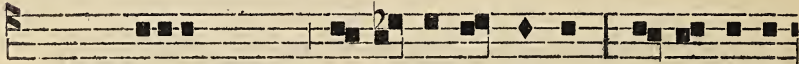

niio takenikonhranirats en-kon-no-ronkwake, ta-kia-tahnt-

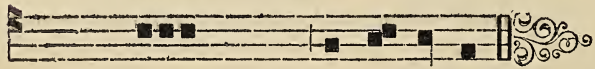

rats, nok iseke katekwasen-takwa. Ahon... Takw. (sk. 41).

T 8.

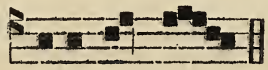

A-re - ri - ia. Helsilwasennaien (sk. 344). 
Niio Sewennio. Ionehrakwat. Shatonnhelon. sk. 188 Ken ire.

235 Jesos wahale...

173 Twataleken. sk. 179

172

225

OWATSIRATOKLNTI IOn WENT.

Iontaw.

T 1.

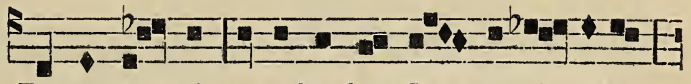

Twatsennon-ni te-wakwekon Ra - wenni - io-ke,

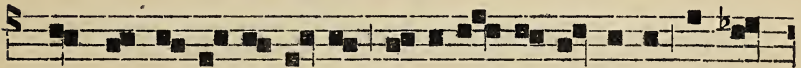

ionkwen-ta-to-ken--ta-se O-wa-tsi-ra-to-kenti ia-wen-

B-口EE-

tawen ro-natsen-non - ni ra-ti-ronhia - kehro-non;

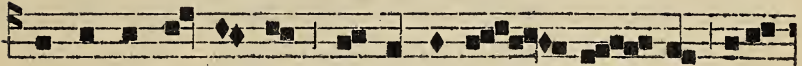

nok ronwa-sen-na-iens ne lio-ienha $\mathrm{Ni}$ - io, a-re-

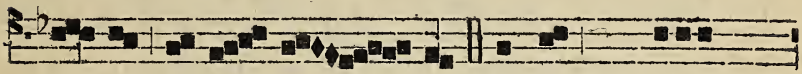

ri - ia, a-re - ri - - ia. Onkwe okon enhonwaia-

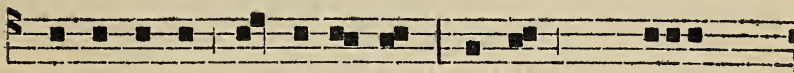

ti-sa-ke ne Ra-wenni - io, nok enhonwasennaien niate

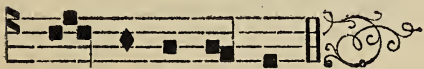

ka - wa-tsi-ra - ke. Ahon... Jakw. (sk, 42).

T 1.

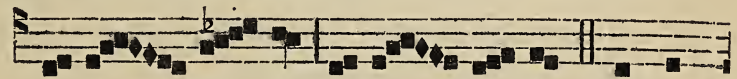

A-re - ri - ia, a-re - ri - ia. Ra-wen-

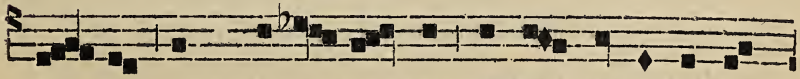

ni - io, a-kwa io-ne - hra-kwat sa-ko - nonwehon non- 


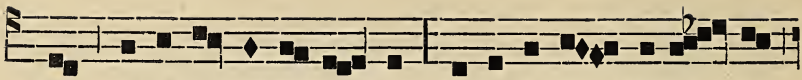

kwe sa-ko-wi-ne Po-ien - ha to-sa a-ion - tkaron - ni

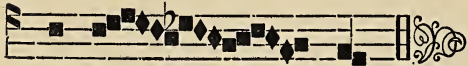

no-ne - - shon.

lesos Wari (sk. 224). Ise Wari (sk. 269). Oh nahoten (sk. 97).

IOKARENRE.

loriwanehrakwat (sk. 294). Warı saialalerihon (sk. 296).

T 1 .

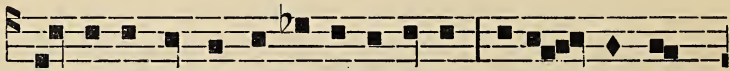

So-se rat-tokha nok thori-wa-ie-ri ro-ri - hon-ten

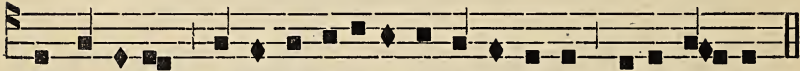

Rawenniìo tahasnie raowatsi-ra-to-kenti, a-re-ri - ia.

T 8.

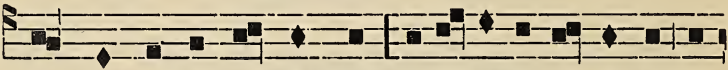

Swentiohkwakwekon tsionkwe, ta-ie- tsinonwe- ra-ton ra-

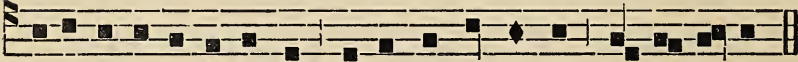

o-tiwatsi-ra-token-ti Iesos, Wari, So-se, a-re-ri-ia.

Tekwanoronkwanions (sk. 266). v. Niio Roienha (sk. 292). Ros. Anen (sk. 296).

T 3.

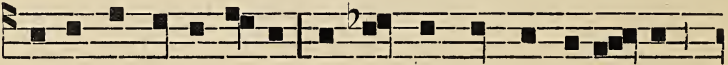

Ii kenonnhatse-ri-io khe-nontens kheien okon-ha,

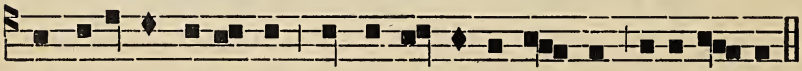

kheia-tewen-tetennis kheien 0 -kon tsi konnhe, a-re-ri - ia.

v. Onen lesos (sk. 348). 


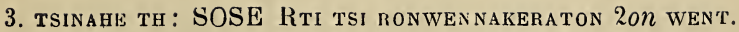
Ne sakarennal. Sose raw : (sk. 326). Ros. Thoriwaieri (sk.82).6. u. T 8.

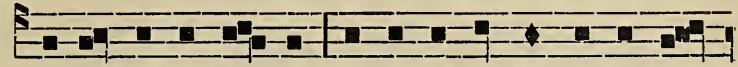

Tokenske kwahro- rıs : enswa-ni- konhraksen nok ten-

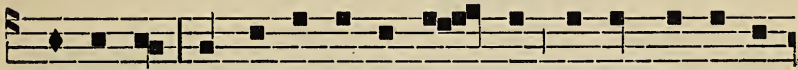

swasentho ; nonkwe enion-ton-we - sen, nok tenhnon iaten-

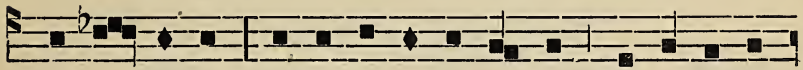

se- wa - ta-ton tsi se-wa-ni-konhraksens, ten-se- waton-

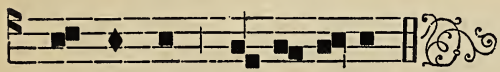

nha-ronnion, a - re-ri-ia. v. Onen Iesos (sk. 348).

3 MAI. KAIASATOKENTI. 2on WENT.

Iontaw : li lenlınon (sk. 337). Takw. (sk. 24). Kristos (sk. 338). T 8.

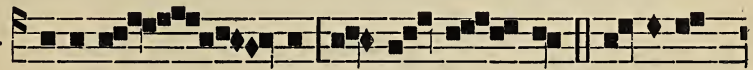
A-re.ri - - ia, a - re - ri - ia. Ka-ia-sa

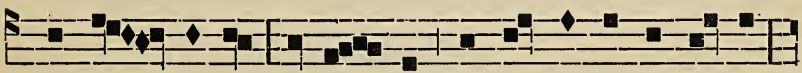

twasen - na-ien ne le - sos se- sonkwaskontakwa-ton

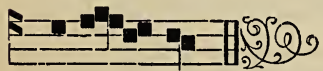

no-ne - shon. a. Ronnliek lesos (sk. 129). Ken renl. (sk. 184).

\section{IOKARENRE}

Nenilonwasenna. (sk. 302). Iesos raoientann... (sk. 331).

v. Eniontkatho kaiasatokonti karonhiake, $(s / \iota$.) areriia.

R. Tsi entesakoiatoretane ne Rawenniio nonkwe okon, $(s /$. $)$ are. 
Ros. T 1 .

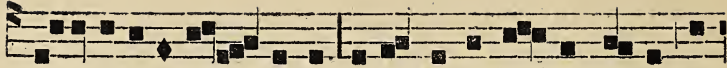

Kaiasa tewasen - naien, sathia-terahne- ken le-sos te-

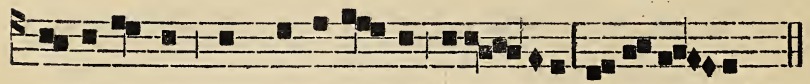

te-wa-riwak. Ionuhek ne Ie-sos rao - iasa a-re-ri-ia. 5-6 a

4on Sh. Ros.

T 2.

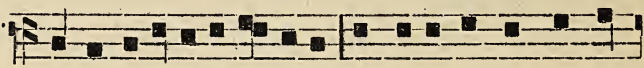

Onen rakenineha sake, nok kanikonhraksen-se-

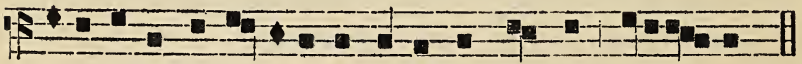

ra-io-te-ten sewe-riasakon tsi-ni kwa-hro -ri. a-reri -ia. 2. $a$.

5on Sh. Ros.
T 8.

Se-wa-nek nok en-tie-tsi-ion nakwa a-e-

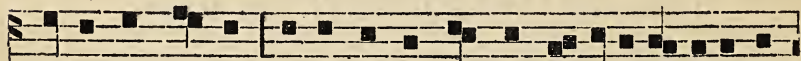

se-watsen-non-ni hetsi-swano-ronkwa Ra-keniha, a-seken

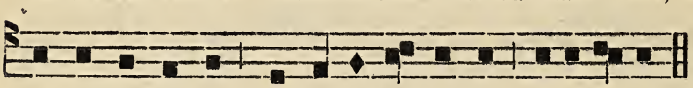

takwanonwehon nok ti-se-weh-takon, a-re-ri-ia. 8. $a$.

KARONHIAKE SARETE 1 IO WENT.

Iontaw.

T 7.

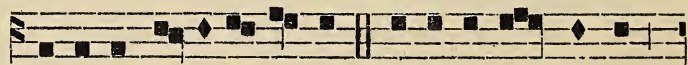

Nahoten, Ka-ri-re-ro-non, sewanehra - kwatha

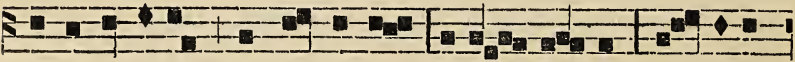

teswakanere ka-ron-hiake? a-re - ri - ia; tsi-ni-iot 


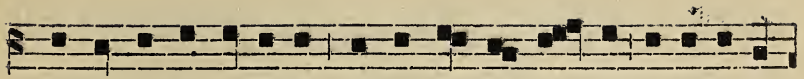

swatkathos karonhiake shawe-non-ton-ha - tie, ok he sekon

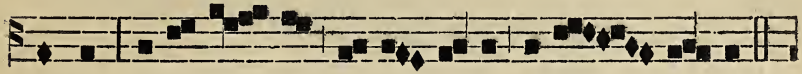

tentre, a-re-ri - ia, a-re-ri-ia, a-re - ri-ia.8. $a$.

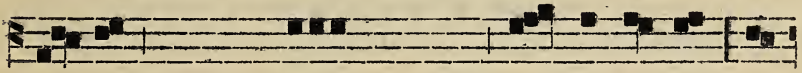

Se- wentiohkwakwekon tsionkwe, sewa - tonnha-ren, a-

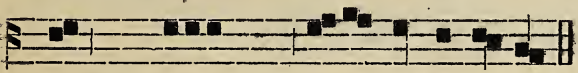

kwa tatsisewariwakwase ne Rawenni-io. Ahon. Takw. (sk.49).

T 8.

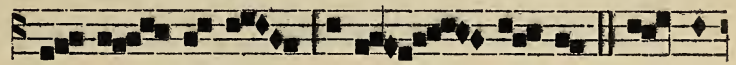
A - re - ri-ia,
a-re - - ri - ia.
O - nen

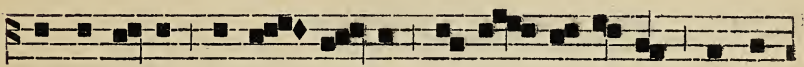

Rawenni-io karon - hia-ke ie-sha-wenonton wa-sa-

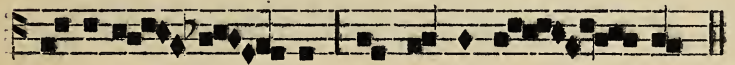

ko-ia-ten - - ha ien-nhotonnion - kwe. $\iota$.

Swannhohatokenti (sk. 174). lonehrakwal (sk. 178).

\section{IOKARENRE}

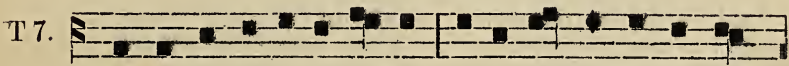

Na-ho-ten, Ka-ri-re-ro-non, ie-se - watkathos ka-ron-

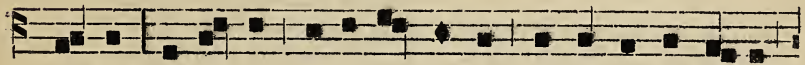

hia-ke? ne le-sos iesha-we-nonton nonwa ka-ronhia-ke

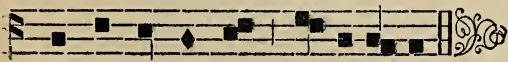

ok l.e na ten-tre, a-re-ri-ia. Satkon Niio. 7. o. 
T 8.

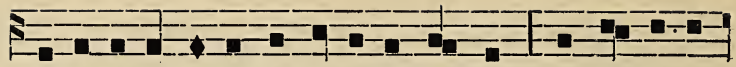

Eh ia-hatahton- te tsi ioronhio-ronnion, nok ia-tha-ra-

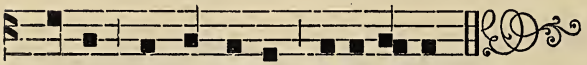

ta-te ka-ronhia-ke, a-re-ri-ia. Rotaskal onwe. 2. $e$.

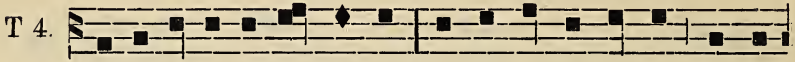

Watesa-ko-ia-son-tha-se ne Ra-tikwekon-ne nok wa-

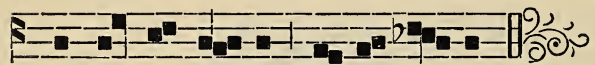

thontekha-sion-ko, a -re-ri - ia. Sewenniio to ok. 4. é.

T 8 .

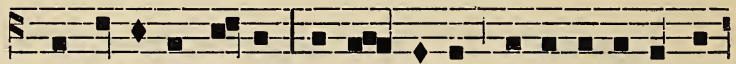

Eh iahatien Ie-sos ka-ron- hia-ke tsi ra-weienteh-ta-

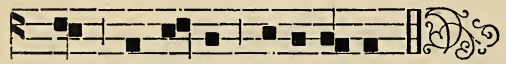

kon Ro-ni-ha, a-re-ri-ia. Sewenniio iahte. 5-6. $e$.

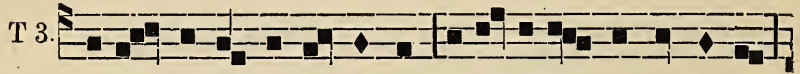

Ie-sos, seskwa-ia-ton-takwen,Tsi iotekha nonwentsiakon,

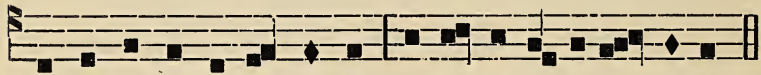

Karonhia-ke tsi-se-nonton, e-tho akwa-ia-ti - sakha.

Ne tsi satanitenraskon,

Seron: ii akeronhiaken

Nonkwariwaneraksera

Naonsaskwaiatokewe.

Iesakwatton nonwentsiakon Senonksonne ne sentiohkwa Katkaron tsi iesarhare

Karonhiake asheiathe.

Sekon aesennhaseke $\mathrm{Ne}$ satanitenrasera
Karonhiake askwenteron

Tsina tenseskwaiatakwe.

Sonhaa akwanoronkwake

Tsinenwe eniakionnheke

Nakwanoronkwake oni

$\mathrm{Ne}$ karonhiake tsinenwe.

Atsitwasennaien Niio

Ne Roniha nok Roienha

Nok ne sahotinikonhrat

Rotkon Roiatatokenti. E. N. 
v. Niio karonhiake ieshawenonton rotonnhahere, areriia.

R. Nok Rawenniio tehonwariwakwase, areriia.

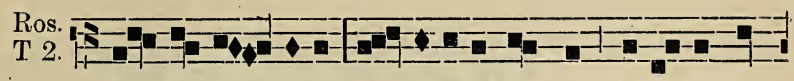

o Se-wen - ni-io, sa - iata-nehrakwat, i- se ne non-

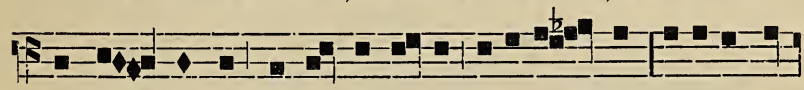

wa ken wente ne ka-ronhia-ke ie-senon-ton, tosa takwa-

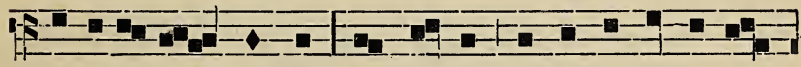

ia-1onti ia - kionkwe, kwenni- tha, askwa-nakta-se-ron-

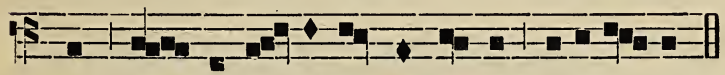

nien, e - tho a - iakwatsennonni, a- re-ri - ia. 6. u.

'NIIOHNE ONEN KARONHIAKE SHAWENONTON.

lontaw: Nahoten (sk. 354). Takw. (sk. 68). Areriia (sk. 355). Akwa lesos (sk. 141). O skwanatiio (sk. 146).

IOKARENBI: (sh. 355).

v. Niio Roienha iahatien karonhiake, areriia.

1. Tsi raweientehtakon ne Roniha, areriia.

T 8.

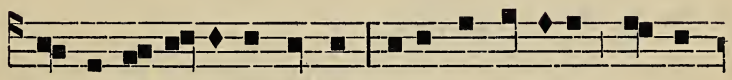

Kwahrori tsi neniawen-ne naeswa-hiarake no-nen

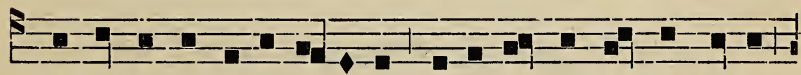

tsi ienwaten-ni-se-ri- he-we tsi o-hen-ton ne kwahrori,

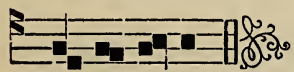

a-re-ri-ia. 8. a. O Sewenniio. v. Niio karonhiake. 


\section{WENTAKTA R. RTI RAWENNISERA}

Roliialatokentison raoliriwa (sk. 34l). Areriia (sk. 344). Satkon Sli. (sk. 175). I. K. seriwaht. (sk, ?8). Olsiralokenli (sk. 178).

ROTKON RTI RAWENNISERA 1on WENT.

\section{- Iontaw.}

T 8.

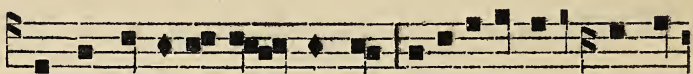

Rotkon Roia-ta-to - ken-ti raiata-noron nonwen-

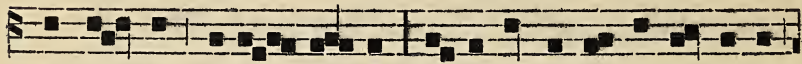

tsiakwekon, a-re - ri - ia, iah o-thenon te ho-nekherens

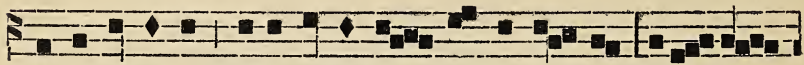

tsi-ni io-tieren, o-riwakwekon ratste-ri-stha, are - ri-

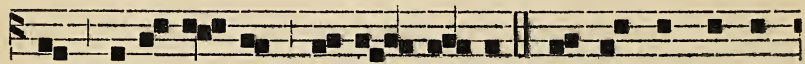

ia, a-1e-ri-ia, a-re - ri - ia. Ni-io satkon nas-

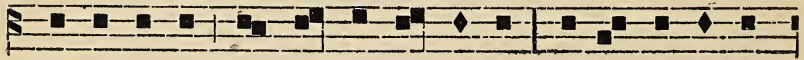

kwa-te-katen non-kwa-ni-konh-rakon a- io - tekha-ke

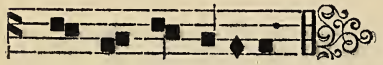

nakwa-nonwehake. Ahon. Takw. kowa (sk. 42).

T 2.

A-re - ri-ia. Karo ka-se, satkon sa-ia-ta-

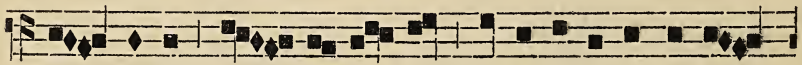

to - kenti, she - ia-tien-has nakawe-riane sheien 


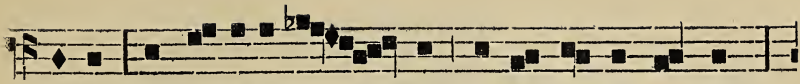

okon, nok she-iate-ka - ten nako-ni-konhra-kon

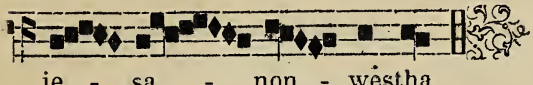

ie - sa - non - westha.

T 1.

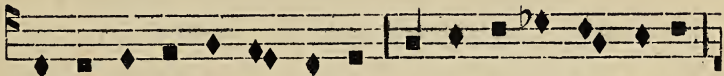

Ka-ro ka-se, Satkon on-we, Ontaskwaron-hia-ti-re, Ionkwentent, takwaien- ha, Ka-ro sa-tie-sen kowa.

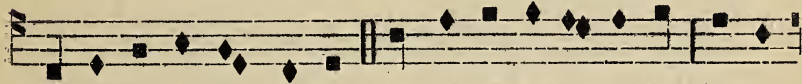

Ne sheia-ne- ren-stakwa. Ne ta-kwa-ia-ta-kennhas, SwaniA- on-taskwaswa-te-ten. Teskwato-ri-sèn-tho-se, Teskwa-

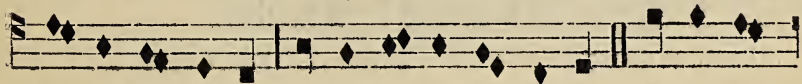

konhrahni-ratstha Swani-konhri-io-stakwa. O le-sana-ton-ni-ha-tie Takwakonnienstonha-tie. Iah-te ion-

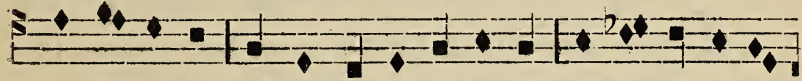

swathe kowa, Takwentenr a-wen-tehak Onkwa-nikonhrakwari-hon-te, Iah-te swaie-na-wa-se, Iahta ionkwaia-

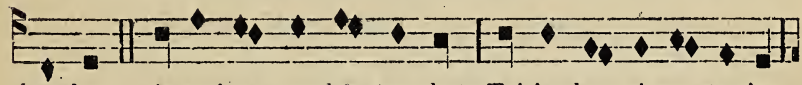

kwekon. Asalsient nonkwatonnhets Tsi io-ka-ri-wa-lanion nerek. Akwa-ni - konhranetskat, Askwa-ni-konhrahni-rat

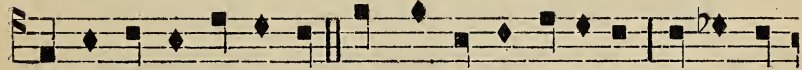

Nok ashe - ia - nerenstak. A - sheion sheien o-kon, Akwa iaAskwana-ton-ni-ha-tie. Takwentenr,Sewenni-io, Askwani-

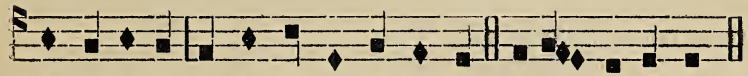

korharenion A- to - ri- sen-se-rakont. konhraierit Karon-hia-ke a-iakwe. Etho na-iawen. Karo kase (sk. 359). Salkon saialalokenli (sk. 175). 


\section{IOKARENRE}

T 3.

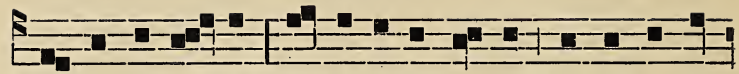

Wahontkenni - sa né Ra-tikwe-konne kanonskono-

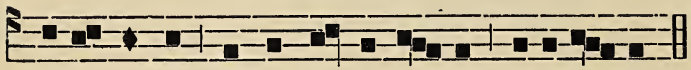

ie-ri nonta, wahon-te- ren-na- ien, a-re-ri - ia.

Salkon Niio. 5-6. a.

T 8 .

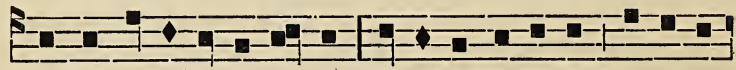

Rotkon Ro-ia-ta-to-ken-ti wasa-ko-tienhase, wa-sa-ko-

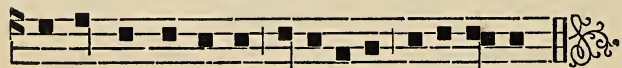

nikonhrahnira-te, a-reri-ia, a-re-ri-ia. Rolaskal. 8. a.

T 1.

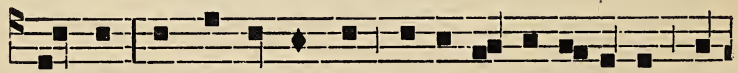

Tsionkwe nonwentsiakwekon ti-se-wa-na-ke-renion, ta-

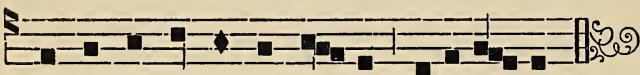

tsiswanonwe- ratons $\mathrm{Ni}$ - io, a-re-ri - ia. Sewen, lo ok. 6 a.

T 8.

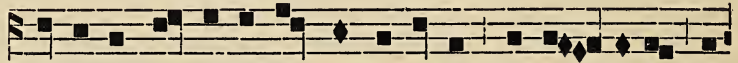

Ratikwekon-ne wahonhronke tsi-ni. ie-wen-notens nia-

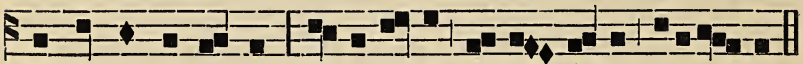

te ionkwe-to-tenson, a-re-ri-ia, a-re - ri-ia, are-ri - ia.

Tewakelilahon Rotkon. 5-6. $i$.

T 8.

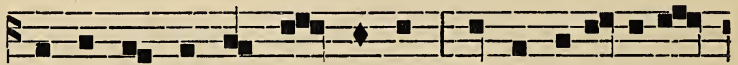

Ka-ro ka-se, Satkon onwe, a-kwa sa-ia-ta-to- 


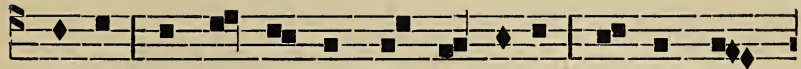

ken- ti, Kwenni - tha ionkwentent okon Takwen-tenr

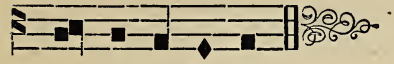

askwa- tien-ha-se.

Ontaskwennakeratira, 'Takwaswatetenniheke, Askwanikonhratokensten Tsini sarihonwe kwani.

Asatien ne onkweriane, Asatekate satsire, Aiontonkotakonhake

Ne tsina kwanonwehake.

Sewenniio, onkwennason Aiawen askwawenonni,
Asteson tsini senonwes Aionkwatharakonhake.

Heren askwite niolaksen; Tiotkon skennen akenhake. Taiakwatatenonwehake, Ise askwakonnienslake.

Atsitwanonwehak Niio Roniha nok ne Roienha Nok tehiatatenonwestha Rotkon Roiatatokenti. E. N.

v. Rotkon Roiatatokenti, areriia.

R. Entsitewarihonnien oriwakwekon, areriia.

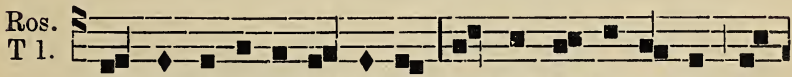

Ken wente io-ni-tiohkonni Kentiohkwato-ken-ti, a-

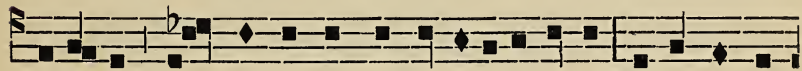

re-ri-ia, Ken wente Rotkon Roia-ta-token-ti Ra-tikwekon-

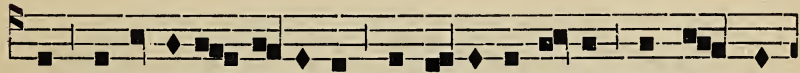

ne wate-sakos-wa-te-ten, wasa-ko-tennie-te nonwen- tsia-

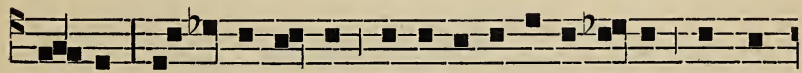

kwekon ka-ri-wi-io-ston ta-ha-ti-riwa-re-nia-te nonkwe

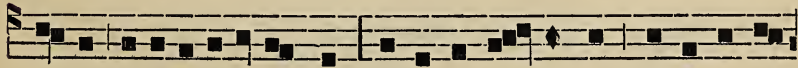

o-kon a-sa-koti-rihonnien : entia-ka-weh-takon nok enionte- 


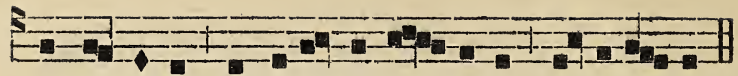

nekwa-heste, karonhia-ke en - ien-te, a-re-ri-ia. 1. $a$.

ASENSERATOKEN'TI. 2on WENT.

Iontaw. T 8 .

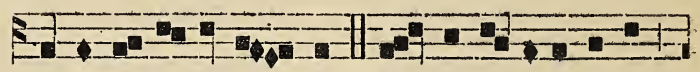

A-tsi-te-wasen-na = ien A - sent-se-ra-token-ti

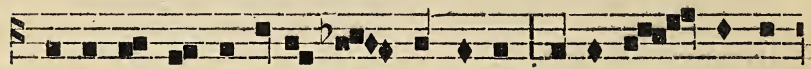
shaia-tat ok ne Niio Ra-wen-ni-io, he-tsi-te - wanen-

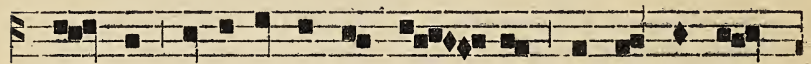

ton - nion, a-seken sonkwenten - ron ne nonwentsia-

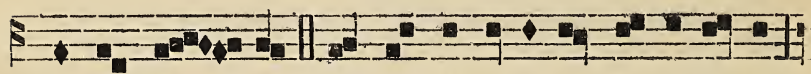

kwekon tsion - kwe. Ni - io Sewenni- io tsi ia-kionnhe,

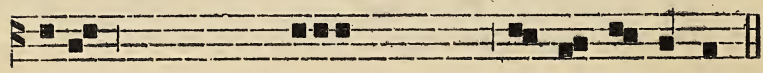

akwa ionehrakwal tsini tsennanoron nonwentsiakwekon.

Ahon... Takw. (sk. 53).

T 8. S-

A-re-ri-ia, are - ri-ia. Sonha tsiata-

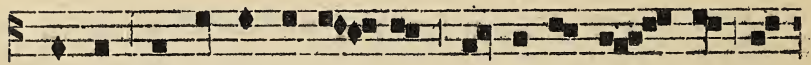

noron, $\mathrm{Ni}$ - io Sewenni - io ta-kwaia - ti - son, ie-

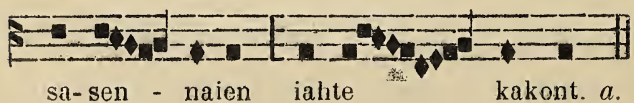




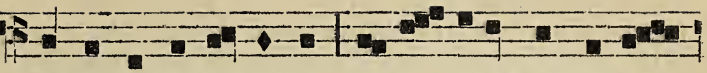

Teani-ronhia-keh-ronon tehni - riwakwakwe hia-

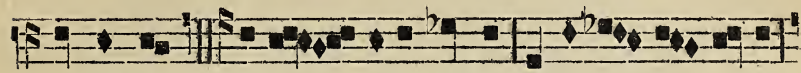

tonhakwe. * Saia - ta-to-ken-ti, Sa-ia-ta - to - ken-ti,

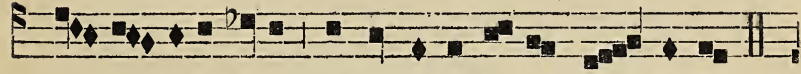

Sa - ia - ta-tokenti, Se-wenni-io Ni-io Sa - ba-oth.

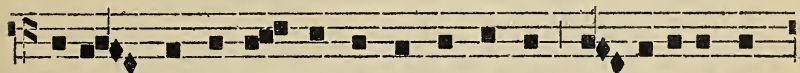

+ Iaon - wentsiana-non onwentsiakwekon sa - ia-tanehra-

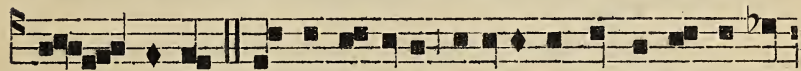

kwa - tsera. A-sen ni-ha-ti raonhakon Ni-io ka-ronhia-

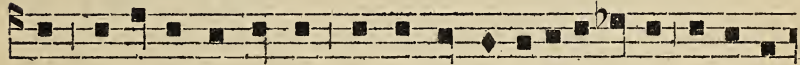

ke Po-ni-ha, Ro-ienha nok Rotkon Roiata-to-kenti, ashen ni-
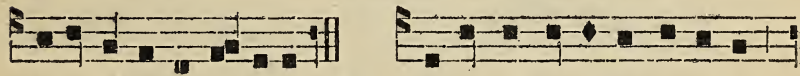

hati, shaia-tat ok Ni-io. * Saia... Ahonwa-sennaien Ro-ni-ha

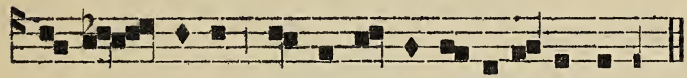

nok Ro - ienha, nok Rotkon Ro-ia-ta-to-ken-ti. † Iaon...

T 1.

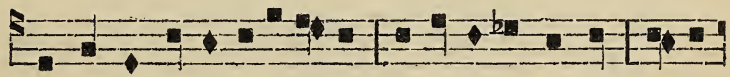

I - se Sashense-rato-kenti, Sa-ia-la-nehrakwat, ashen

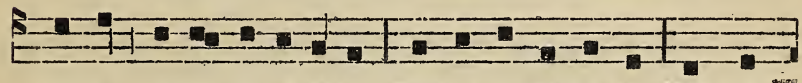

nitsion, Sesia-lat ok Niio Tiotkon se- na-ke- re, Nok iah-

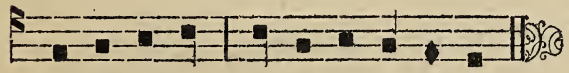

te ka-kon-te en-se-na-ke-re-ke. 
Ise, Sashenseratokenti, Tiesariwakwase Orhonkehne nok ieka. rahane Iakoriwiioston Ne nonwentsiakwekon Ne tienakerenion.

Ise, Sashenseratokenti, Tiesatontsothase, Iesennitha, iesawenniiostha, T'sini tsiatanoron, Ne iakorharenion, Ne nashetenranion.

Ise, Sashenseratokenti, Eronhiakehronon Iesanentons tiotkon ne rontonnions: Saiatatokenti, Saiatatokenti, Saiatatokenti.

Ise, Sashenseratokenti, Sesiatat ok Niio, Iesaniha nok Iesaienha, Ise oni Satkon Saiatatokenti, Tekwanonweratons.

Saionkinekwahetstanion (sk. 157).

\section{IOKARENRE}

Tierenakelskwalha (sk. 302).

$\mathrm{T} 1$.

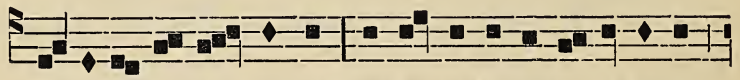

Ta-ie-sa-non-we - ra-ton, sonha Ashense-ra - iokenti,

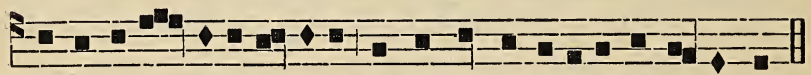

ok si sena - kere tsi-nahe,nok nonwa,nok oni iahte kakon-te.

$\mathrm{T} 2$

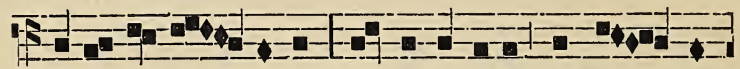

Ahonwa-sen - naien Ni-io Roniha, nok Ro - ien-

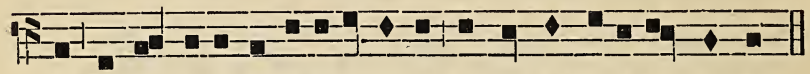

ha Rotkon oni Ro-ia-ta-token-ti, nonwa nok oni tsi-nen-we.

T 3.

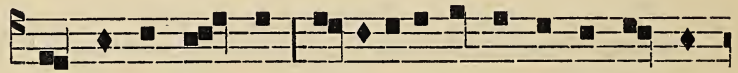

A- kwekon non-kwe ta-ie-iena tahonwa-ri-wa-kwa-

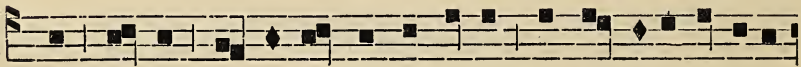

se Ni-io Ro-ni-ha, nok Roienha, Rotkon o-ni Ro-ia-ta-

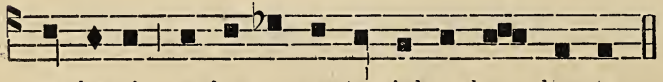

to-kenti a-honwa-nenton iahte ka - kon-te. 
T 5 .

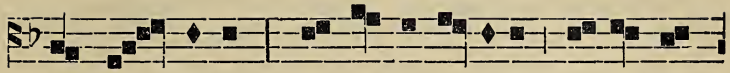

Ne a - kwekon ro- te-we-ienni-son, a-kwekon

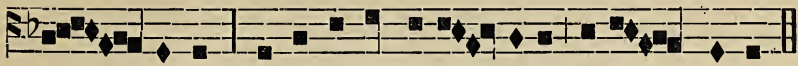

then - nontons ahonwawenni-io - stake ia-hte kakont.

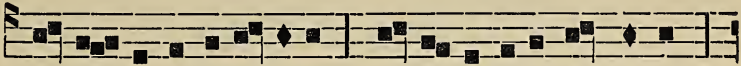

, O Sashense-ra-to-kenti, * I - se te-ioswathe kowa,

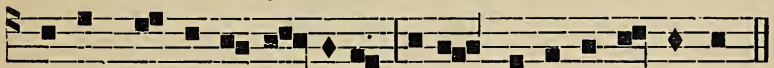

Takwaswa-te-ten -ni-lıe-ke, Akwa akwa-noron-kwake.

D Askwentenre oni nonwa Akwawenniiostake onwe Tsinenwe eniakionnheke Ne takwenniserawihne. $\cdots \rightarrow-\cdots+$

F Sewenniio tsi iakionnhe, Ise ne takwaiatison, Ise takwanikonhratha, Akwa tekwanonweratons.
Iesos seskwaskontakwen, Seriwahtont ne iotaksen, Shesenui notkonseraksens, 'Tosa aionkwakaronni

Satkon Saiatatokenti, Satekat ne ionkweriane Sanonwetseratokenti Ise takwarihonnienni. E. N.

v. Aietisennaien Roniha nok Roienha, nok Rotkon Roiatatokenti.

R. Aietiriwakwase tsinenwe.

Ros. $\mathrm{T}: 4$,

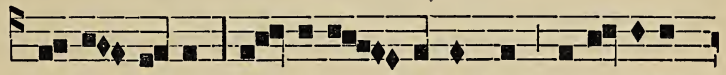

Se- wen-ni-io, sa - ia-ta - nehrakwat, le-sa- niha

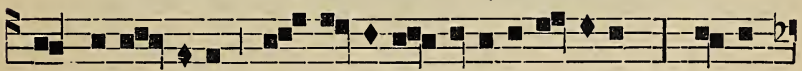

nok le-sa-ienha, Sa-tkon o-ni Saia-tato-kenti, tsiata-

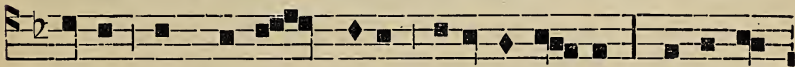

noron nonwen-tsia-kwekon, takwaia-ti - son ; onkwani-

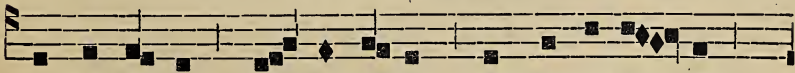

konhrakwekon, kwa-sen-naiens, kwawenni-io - stha, 


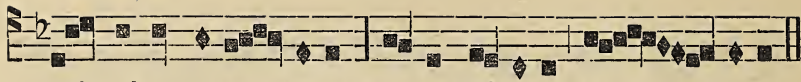
nok tekwanonwe -ratons nonwa nok oni tsi - - nenwe.

T 8.

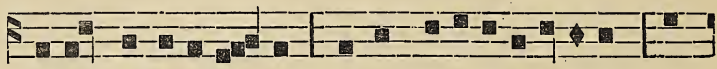

Tosa tesheia-to - ret, iahta ta-iesa-ia-tore-te; ok

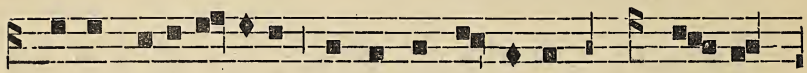

he nentsiesa-ie-ra-se tsi nenshe-ie - ra-se nonkwe

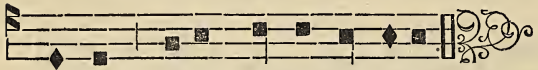

sonha ; rawen ne Rawen-niio. v. Sonhaa. (sk. 301).

NIIO RAWENTAWEN. 1on WENT.

Iontaw.
$\mathrm{T}$ 2.

sonkwanontens ne Ra-wenni-io, ori-wiio ne

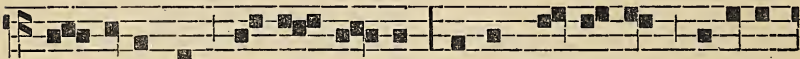

ra-ie-ronke a-re - ri - ia, sonkwatsteronwi nae-te-

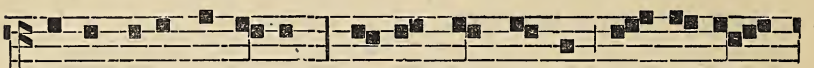

wake, tewatsennonni tsi sonkwentenron, a - re - ri -

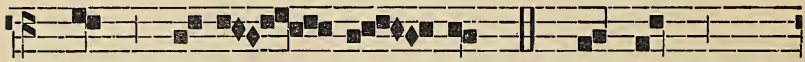
ia, a-re - - ri - ia: Te - tewariwak,

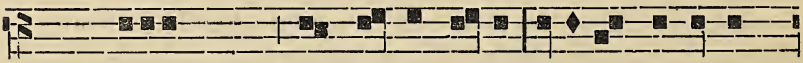

hetsitwasennaien Niio ka-ronhia-ke; A-seken as-teson

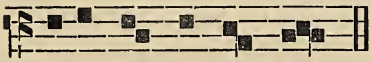

sesonkwenten-ra - nions. Ahon...

Tahw. (sk. 42.) Areriia. (sk. 272). 


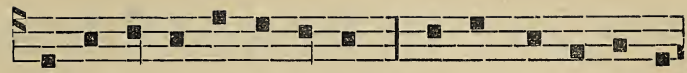

A - e- twawennakwekonhak Ta-tsi -te -wanonwe- ra-ton

O- nen te- te - wa-riIe- sos ka-na- ta-ra-

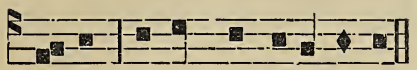

wakwak.Niate karenni-ioson. no-ron. Ne son-kwatenniotase.

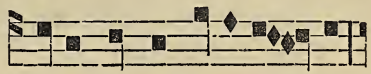

Akwa Iesos raieron - ke

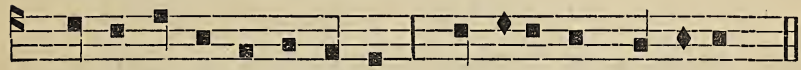

Ro-ri-wi-son a-ie-kse-ke Ra-o-ne-kwensa o-ni

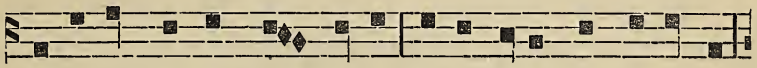

Neakwa ionkwatsen - nonni Rawenniio sonkwatien-ni. Ta-ie-ha - wi ka - ron - hiake Ne ka-nata - ra -kon onwe

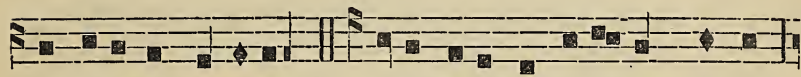

Ne kati se twaiatat, Katsitsianoron 0-ni.
Onen tewatonnharonnion nonwa.

Tsi tionn-he Rawen - ni - io

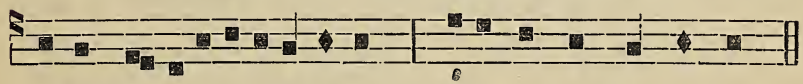

Tsini wenniseranoron kowa.

Niio ne sonkwennonkon Iawet kento ron - wario Nok ronwa-ne kwensa ris

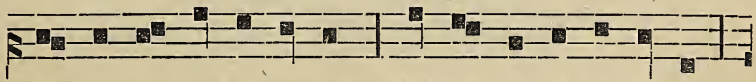

Ii ionkwa-no-ha-re-tha, Nok ii ion-ki-ien-nio-tha

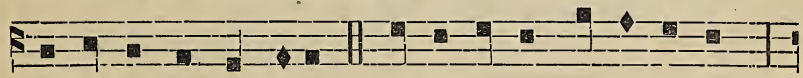

Iakwatonnhetstha oni. Akwa twatonnhaka -non-ni,

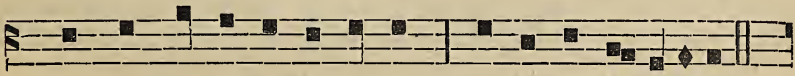

Twaten - ni-konhrase - ronni Tsi twaia- ta - rasere. 


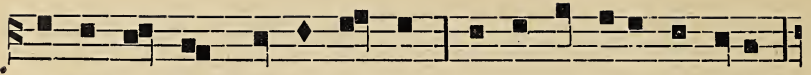

Tewatsia - ken, twari- wa-tka Ionkwanikonhrotakwatha

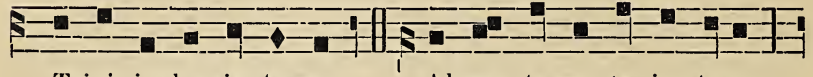

Tsini- ionkwaia-to-ron. Ak-wa te-wa- te-rien-la-sa,

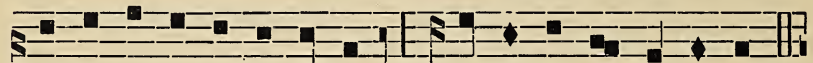

akwa te-wa-ta-tkahen-hion Onen sonkwa-tienni - re.

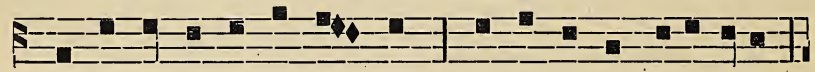

I-ieks tia-ko-ri-wa - ie - ri, Ionkwetaksens iieks 0-ni :

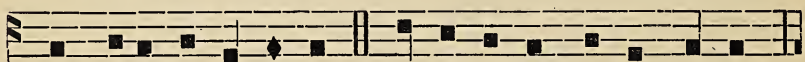

Iah sate iaka-onnhetsl. Akwa ne ion-tsennon-nıatha

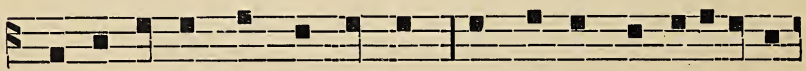

Tsi-ni-a-kon, ne iont-10-kha. Ne tenhnon ia -i-he-ia-tha
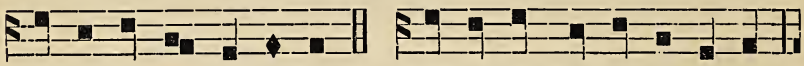

Ia-ko - ri-wa - ie - sa-ton.

Ken ka-ien ne ia-konnhekon,

Ken ka -na - ta ra-kon kenha

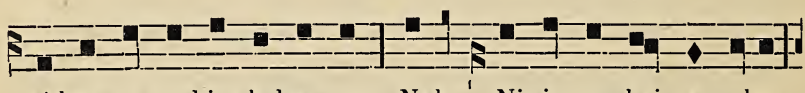

Akwa e-ronhia-kehro-non $\mathrm{Nok}^{\prime} \mathrm{Ni}-\mathrm{io}$ sa-koi - en okon

Ie-sos rawen-ni-io-se- ra, $\mathrm{Ra}$ - ie-ronke, raon'kwensa

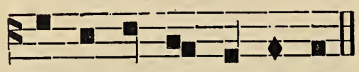

'Tosa ie- ri - wa - ie-sat. Tokenske sonkwanontens.

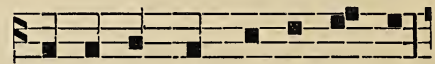

I - se ne skwenie-sakwe -kon, Eh-ni skwa-ni -konhroten - stak,

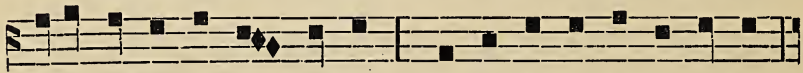

Iesos sa -ta - ni- ten - ra-skon A-ia-wen a-kwanon -we-hak
Sahtont ka-ri- waksen o-kon Tiotkon akwawennarakwak 


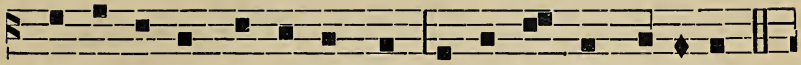

Ne ionkwari - wa- ne-rakskon Ionkwari - wa - ienta-kwe.

Nok a-e -twakwe-kon-ha - ke 'T'sia-takta ka - ronhiake.

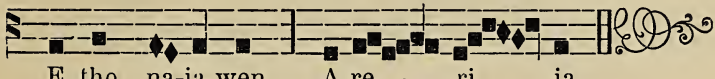

E- tho na-ia-wen. A-re - ri - ia.

Oh nahoten (sk. 97 ) Ionwesen. (sk. 272.)

IOKARENRE.

T 1.

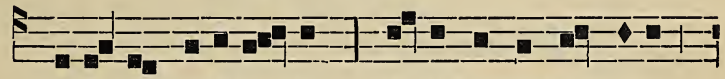

Saha-sa nokari-stia Ie-sos Sonkwawen-niio

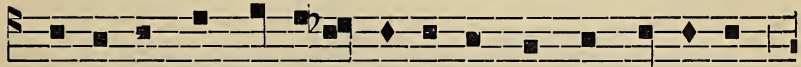

ro.ha -sen nok sa - hori-hon-ten ron-wa -wen- nenha-we

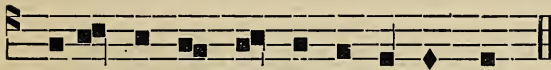

a-ha - ti - ha- sen - se - ke tsi- nen-we. Salkon Niio 5-6. $i$.

T 2

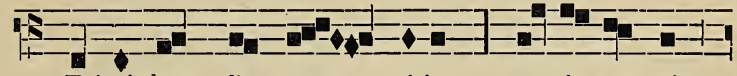

Tsi-ni-kon la-wen - ni-io ro-tie-ra - nion

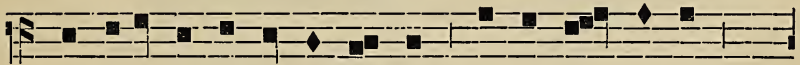

ne io-ri- wa-ne-hrakwate-nions, io-na-te - ro-ron

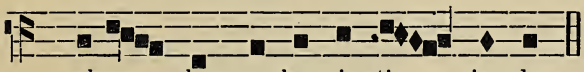

akwe - kon o-ka-ri-sti - ia-kon. Rolaskat. 2. e.

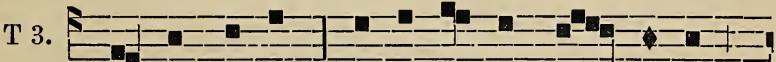

Ra - wen Ie -sos : Te-sewak, nok i - se ·wak :

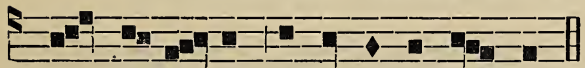

ii kieron-ke ka-na-ta-rok ken - ha.

Sewenniio to ok. 3. a. 
T 5.

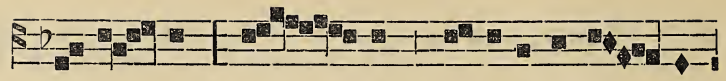

Ionwe - sen tio - - tkon ta-tsitwanonwe - ra-

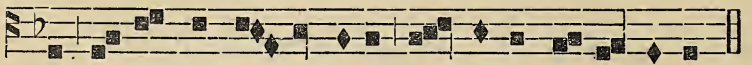

ton tsi sonkwenten-ron Iesos sonkwawi kakwa-noron.

Sewenniio iahle. 5.-6. a.

T 3.

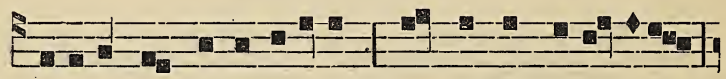

Tetewatonts swariwiioston. Twakwekon tetwariwak

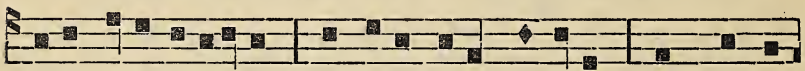

Niio o-karisti-iakon Hetsi-te-wanen-tonnion, Sonkwanon-

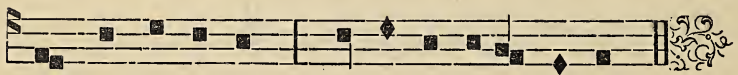

wes, sonkwanontens Ne ka-na-ta-ra-no-ron.

Entakta tsi rawenheion

Watrakwe kanatarok,

Wahaterennaienhase

Raieronke iotonhon.

Rawen: Etho nenswaiere

Naetwakwekonhake

Sonkwanontens raieronke Irehre : naiekseke. Ionnhekon nakotonnhetston
$\mathrm{Ne}$ iakoteweienton

Nok ne rotenientenstakon

Karonhiake eniente.

Tosa onka iakonorons

Tsi akwa naieiere,

$\mathrm{Ne}$ tsi rotenniote kowa

Ahonwaienterhane ;

Twatatitenr, tetwatkenni

Hetsilewanonwehak.

Twawenniiost (sk. 105).

v. Shenataranonten karonhiake tiawenonton, areriia,

r. Akwekon kahawe tsini iontsennonniatha, areriia.

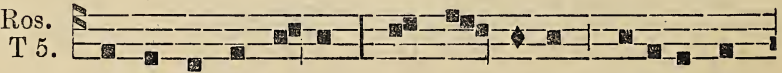
Rc-ten-nio-te Ie-sos son-kwanontens raie-ronke 


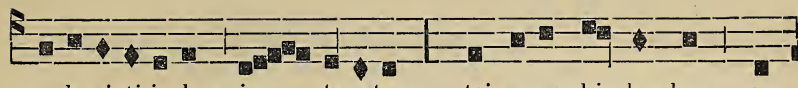

o-karisti-ia-kon io - taseton; tsi ro-ronhia-kenhon se-

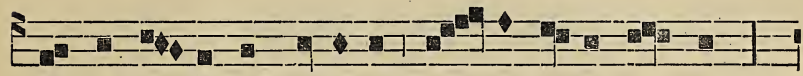

son-kwehia- ra-kwenni-ha-tie son - kwani-konhra - tas

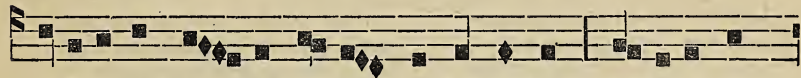

ioia-ne-rensta - kwa on-kwa - ni-konhrakon, e-thone son-

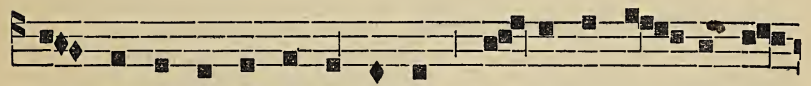

kwarha-ratsten-ni ka-ronhia-ke, e - tho ien-ion-kwakwa-

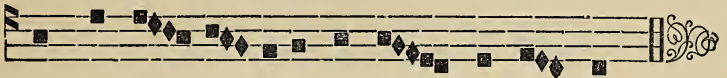

tse a -re - - - - ri - - ia.

nente - wa - te-we-ien-ton tsi tionnhe. 5. $i$.

\section{NIIOHNE TSI NAONTENERATONNIATE.}

lonlaw : Sorkwanontens. (sk. 366). Takw : (sk. 49). Areriia (sk. 272). Aonton ken (sk. 193). Rîiatisaks (sk. 187).

TSI IEIAKENSERE.

$\mathrm{T} 4$.

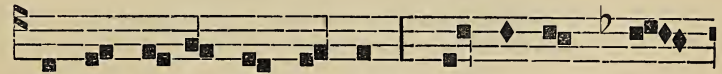

Hetsi - te - wa - wen-ni - iost ne ak- wa Son-

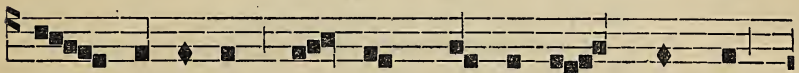

kwa - wenni -io, a - kwa ro-ta-ni - ten-ron,

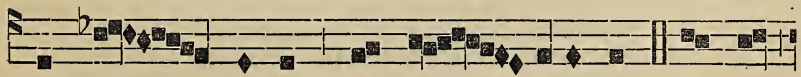

son - kwa - nontens ne ra - - ieronke. To-ken- 


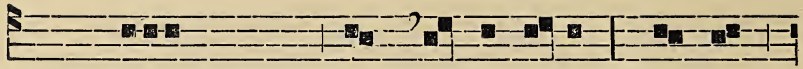

ske renteron Iesos o - ka - ri-sti - ia-kon, li on-

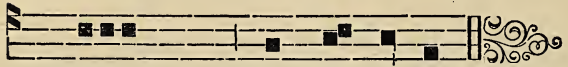

kwariwa tsi ren-te-ron-Iakon Ahon. Telewalonls. (sk.370)

lesos tekwanonweratons Tokenske ken sataseton Ionehrakwat takwanonwes Asteson takwenteuranions
Iesos sonkwaiatanonstaton Naionkwaronhiakenhon Onen wasonkwahahisas Ne karonhiake iaiakwe

Iesosonkwentiohkwarakwen lesos ne kwennitentase Raonha rolatiesaton Raonha shoseromnialon Tsini ionkwariwaneren Takwanikonhrahniratsek Ne nakwasennai!'nseke Tsinenwe eniakionnheke.

O Okarisliianoron (sk. 90).

Raotin itarok eronhiakehronon Nonwa iakonnhekon iakoriwiioston ; Tsini sonkwanonwes ne Roienha Niio Sonkwaienni raieronke.

Kanataranoron Iesos raieronke Kanekwensanoron ne raonekwensa Akwa se ken Niio akwa raieronke Nok akwa raonekwensa.

Iesos konsenuaiens okaristiiakon Ioriwanehrakwat etho sataseton Senha ionehrakwat iieks ne tsieronke Akwekon oni ieiesas.

Ieriwaiesatha ne okaristiia Iakotentascre tsi neniontonnhokten Nok ionteweientons waeiatarane Ne karonhiake eniente.

Askitenre, Iesos, ne akhenakeren Eronhiakehronon tsini iesanonwes, Nok nenkatonnhokten etho enkerane Tsi iakotsennonni kowa. 
Hetsitwasennaien ne Roienha Niio Sonkwatenniotase okaristiiakon Aietisennaien Roniha nok Rotkon Satehatiiatanoron. E. N.

Tsi ionlorisenlakwa tenieriwakwa Twawenniiost. (sk. 105). lesos tekwanonweralons (sk. 196.) lonehrakwat. (sk. 178.) Twanikonhrarak, (sk. 99.) Niio raorenna (sk. 99).

\section{IOKARENRE.}

Sahasa (sk. 369.) v. Sonhaa Sewenniio (sk. 301.)

Ros.

$\mathrm{T} 1$.

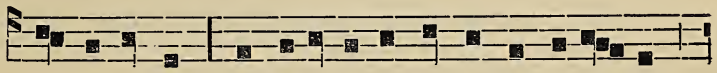

Sa-sa-sto- ron tsi teka-na-to-ken-se-ron iaha - se,

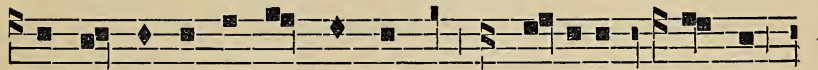

ia - kotent, te - ie- ron -we- kon, iontsino - ka- tha

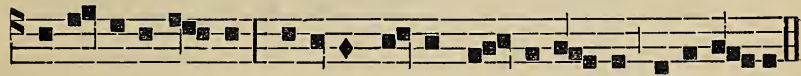

ashe -ia-ti-nionte akenonsi - ne a - kenonskon a-re-ri - ia.

Rolenniole (sk. 370.) v. Slienalaranonten.

\section{IESOS RAWERIASATOKENTI 1on WENT.}
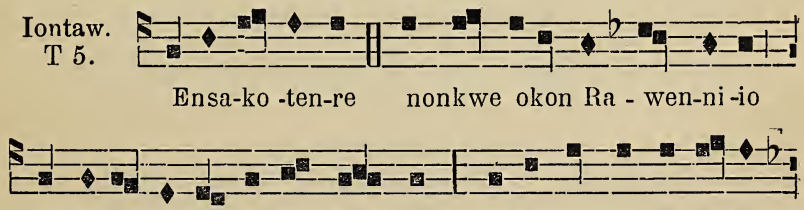

ne rota - ni -ten -raskon ko - wa sa -kononwes sa-ko-na-

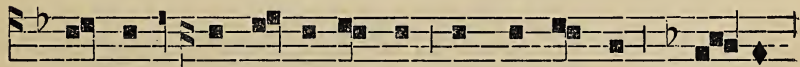

ktawis ra-we- riasa-kon iah nonwenton ta - sa- 


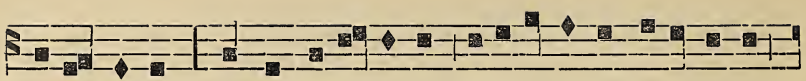

koia -ton-ti, akwa iako-taskat raonhake ia-korharekwen

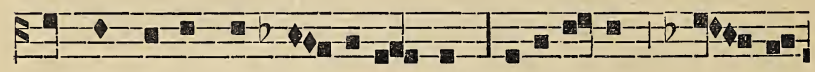

ronwaskaneks ron-wa - ia-ti - saks, a -re-ri-ia, a - re-

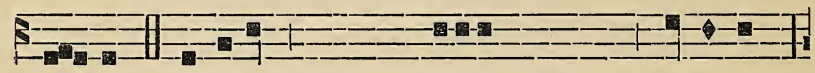

ri - ia. Te-tewariwak Rawenniio raotaniten - ra-se-ra,

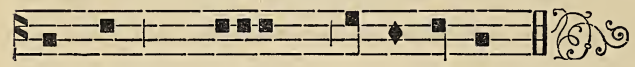

nonwa nok tsinenwe tewa-ri-ho -wa-nat. Ahon. Tukw : isk. 58.)

i' 1. EA-re-ri - ia, a-re-ri - ia. As-kwa-nake-

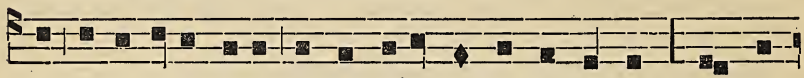

ren wakenikonhriio, waken-nitenton nakweria-ne, nok en-

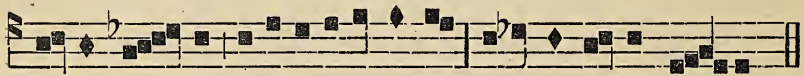

sewat-sen - ri en-iaonrisenta-ne sewatonnhetston o - kon. $a$.

Tsitenr nisa (sk. 200). lesos raweriasalokcnli. (sk. 97).

IOKARENRE.

Tierennaketskwallia (sk. 369).

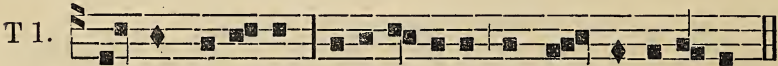

Askwa-nake-ren wakeria-si-io nok wa-ken-nitenton. 
$\mathrm{T} 2$.

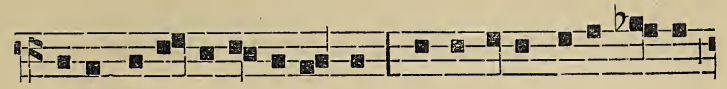

Iesos rawe-riasa -token-ti, sonhatsiwa sa-ri-hon-te

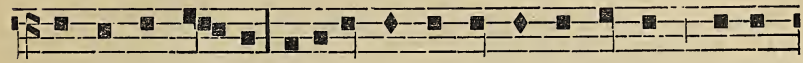

ne Rawenni - io, ahiatkatho, a - hiano-ronkwake, askwa-

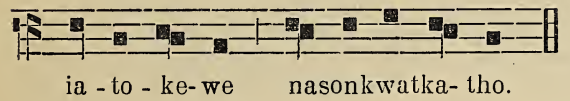

T 8.

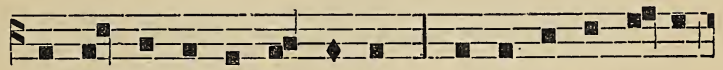

Kani - konhraksense - ra-kon se- ria-sa- to -kentı

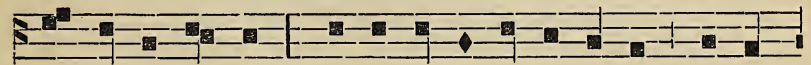

ia -wet io-sko - hon, ne wahon-ni ka-ronhia-ke iot-sen-

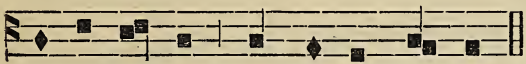

nonni nonwa nok iahte ka-kont

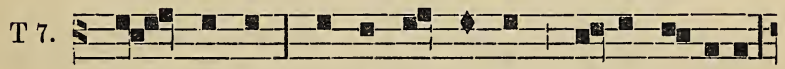

Ta -kwen-tenr takwa- na-klo-tas, Ie-sos, se -riane ;

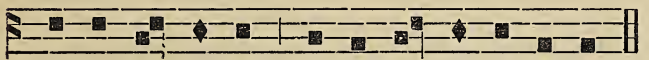

sa-on-kwe-ria-sat nonkweria-sa a-on-ton.

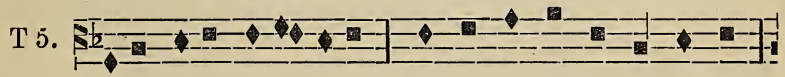

Iesos ne takwaia - lison Nok o-ni-takwaskontakwen,

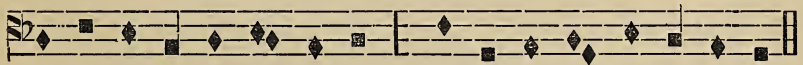

Ok si tsial a - tienha Ni-io, lia-on-lia se se-ni-ia-tat. 
Ne tsini takwanonwehon, Karonhiake tisenonton Tesariwaserakwaton

Tsi sonkwakaronni Atan.

Iesos ne takwanerension Tsi ionkwanaskwaientakwe Ionkwaswens notkonseraksen, Tiotkon askwaiatakennha.

Tosa askwanaktannhese Ne seriasatokentike,
Etho se ietsientha nonkwe Akwa aiontsennonnianion.

Asikwe tsi iesahason Ne tekaientannhareke, Sheiawi aiontekwashen Nonkwe okon seriasakon.

Ronwasennaien Roniha, Iesos oni ne Roienha, Rotkon Roiatatokenti Ne tehiatalenonwestha. E. N.

v. Teiotennhohontion Iesos raweriasa tekaiasontne,

R. Etho aietsiente nonkwe ioianerenstakwa okon.

Ros.

T 1.

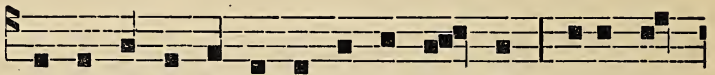

Tsi Ie-sos ro-ia -tenton ia-hon-ne - we, sahontto-

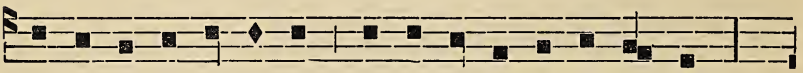

ke o-nen ra- wenheion, iahta thonwa-si- na- ri - ton,

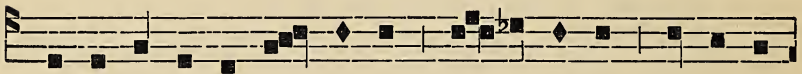

sa-ia -tat tenhnon ne so -tar a - sikwe ra-na-ha-

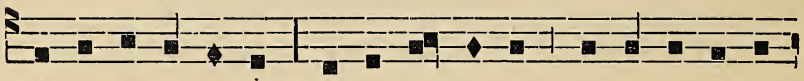

ta-ke wa-ho-ha-se ta-ka-ia-ken-ne o-nekwensa nok

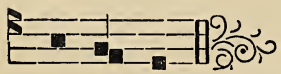

oh- ne- ka. 
10 JUN. RESIS RTI 1on WENT.

AKWESASNE.

Ionlaw : Kariwiio. (sk.254.) Takw : Kowa.(sk. 43.)Areriia (sk.255)

Hetsitwasennaien nonwa

Hetsitewennakeraton

Ne kento raotenata

Resis roiatatokenti

Sekon sihraa, Rawenniio Okstentsi wathoswateten Wahonikonhraientane Oksaok kariwiioston.

Serenkhene tsi hatatis Ok nahonwawennaronke Iesos, Wari sakonatons Ne ratennikonhroriatha.

Ethone wahatewentet Ne iontonweskwatha nonkwe Wahatateriwisahas Niio raoriwa okon.

Iah sane te horiwaion Ok eken kati rakwisrons

Ne nonwa twatatekenson Atsitewasennaien Ne Roiatatokenti Kento tsi tkanataien Raonha sonkwatsterist Sonkwaiatanonstatha Ronwasennallin ne Resis Tetewariwak nonwa.

Resis tsinahe ronnhekwe, Ravenniio raoriwa, Tiotkon ratsteristakwe $\mathrm{Ne}$ kariwiio sonha Enskat ok ratsaniskwe $\mathrm{Ne}$ kariwaksenskwa.

Tasalsnent lesos (sk. 679).
Wahatateronhiakente

Ne raieronke nonkwati.

Tikate asontakwekon Raterennaientatiese, Nok ne tsini wenniseres, Nonkwe sakoseronniennis.

Rokwenion kentiohkowanen Tsiakotatrewaton onwe Iahten te tsiakotsteriston Ne iakoriwaientakwe.

Ii katiken iahtaonton Aonsetwatatrewate, Twatsiaken tewatontatsek, Sekon se sonkwahretsiarons.

Hetsitwawenniiost Niio Nok oni Roienha Iesos Nok twanikonhrakwekonhak Resis hetsitwasennaien. E. N.

Tatsitwanonweraton Rawenniio tsi tionnhe Akwa ne sonkwentenron Ensonkwatsteristake ; Ne Roiatatokenti

Akwa sonkwanoronkwa.

Akwa kati telwatkenni Tsitwanoronkwanion Ne Roiatatokenti Resis, twatatekenson, Atsitewanakeren Tsini hoteweienton, Resis hetsitwasennaien Aetewawennakwekon.

IoKarenre.-Sewenniio (sk. 255). Tierennakelshwatha (sk. 347). Ros: Resis (sk. 83). I a. 
24 JUN. SAN WATIS SAHENNAKERATE. 1on WENT.

Iontaw. T 1.

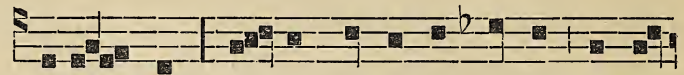
A-re - ko , on - kwe tsi wa-ka- ton-hon raksen-

s-

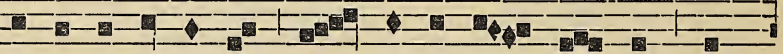
na-wi Ra-wenni-io tsi - ni waksen - no - ten, nok

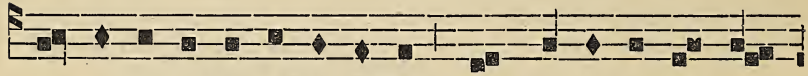
ra-kwa-wi iahta ketsa - ni-ke a - on -ta - ke- wen-ni-

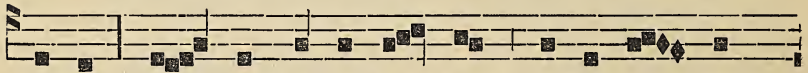
neken, a - kwa io-ne - hra - kwat ra-kia-ta - kenn-

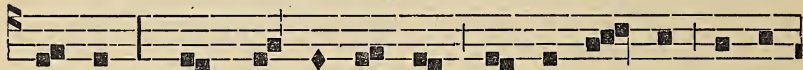
ha - on, nok nia - ke- wen-na te -skia-tie - ren ka-ien-

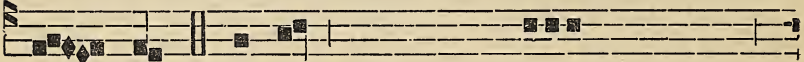
kwi - re Tkari - waieri nahonwasennaien ne

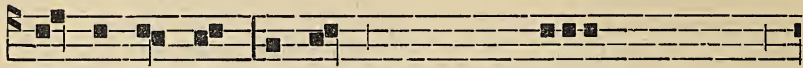

Ra- wen-ni - io, nok takonwariwakwase sasennatokenti son-

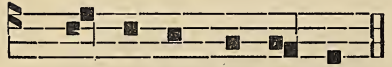
hal -si-wa skowa - nen. Ahon. Takw. (sk. 58).

$\mathrm{T} 2 . \overline{\mathrm{T}} \mathrm{9}$ 二- 二 A - re -ri -ia, a - re - ri - ia.

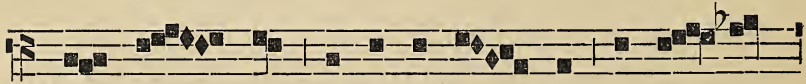
Se - ksa - a ro-wennenha - we Rawen - 


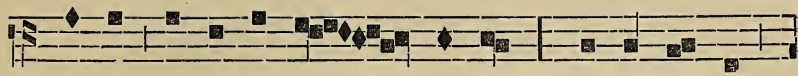

ni -io, en-ie-sa-na - - tonkwe, entshente se

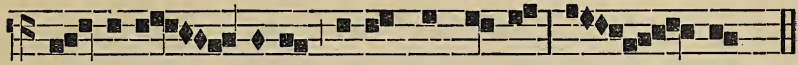

Sonkwawen - ni-io natse - ha -ha-se-ron - - nien. $a$.

Twalaleken. (sk. 225). Ken renteron onwe (sk. 184.)

IOKARENRE.

Tierennakelskwatha (sk. 302).

T. 3 .

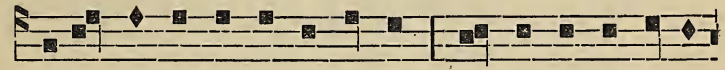

A-ri - sa-wet Sa-ka -ri ro -ne wa- o-wi- ra-ienta-

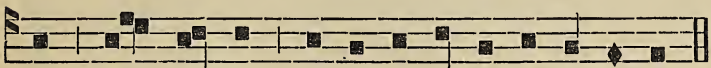

ne San Wa-tis ro-ha-ha-se-ronnienni Ni- io.

T. 8.

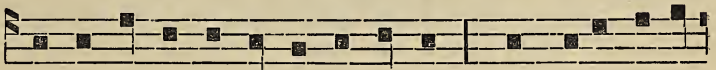

Iahta hahneki- rha-ke notsi-tsia, nok o- ni iako-

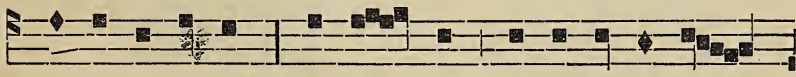

nonwa-rahtontha, nok e - so ten-iontonnharon-

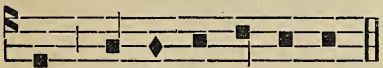

nion nenhenna-ke - ra -te.

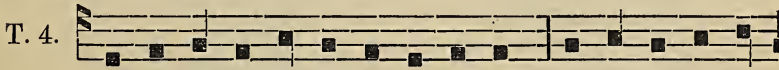

Ronwariwa-nontonskwe ro- ni- ha na-ho-ten en-hon-

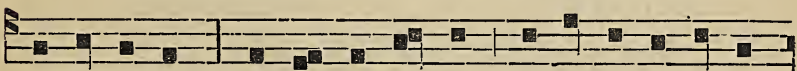

wanatonkwe, nok wa-ha - ja - ton : san Watis enhonwa-

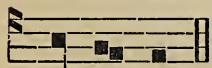

ia-tse - ke. 


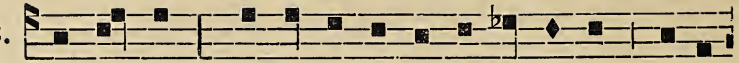

sek·sa - a Ra-wenni- io rowennen-ha-we enie-

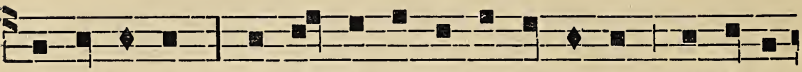

sa-nu-tonkwe ; en-tshente se Sonkwawenni-io natshaha-

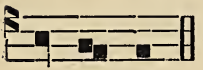

se-ronnien.
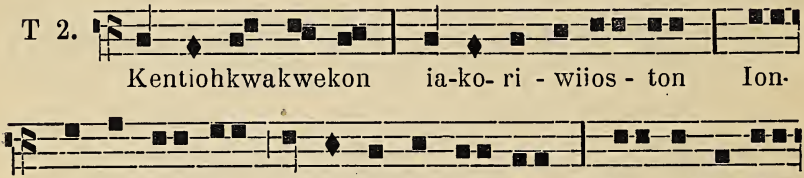

tsennon-nia- tha tsi ro-na-ke-ra - tou Ne-ne San Wa-

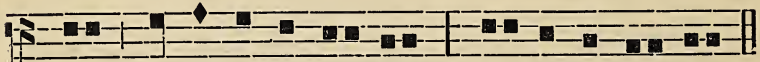

tis ro-ia-ta-ne-hra-kwat, Ra-ia-ta-no-ron.

Ok si hotenron

Areko rotonni

$\mathrm{Ne}$ lesos Kristos

Né Sonkwawenniio

Roriwahtonton

'Tsi ioiatoronne

Raotonnhetston.

Watıoswathene

Raonikonhrakon

Wahatonnharen

Sahoienterene

Niio Roienha

Kaiatakon Wari

Raiatatakwe.

Wahosennaien

Raonikonhrakon

Wahonentonnion

Ne raksaa Iesos

Nok iahte kakont

Wahowennioste

Wahononwene.
Sahatehiaron

Wathaiatorete

$\mathrm{Ne}$ tsi onkwene

Ieriwatsenrietha

Kariwaneren

Ne wahatsanike

Wahatotarho.

Wahatekwasen

Iah onka te tientha

Ne tsi raweron:

Etho skennen kowa

Ahisennaien

Ahinouwehake

Ne Rawenniio.

Watroseriake

Eso nioserake

Karhakon sonha

Ne raonhatsiwa

Ronikonhro ni

Waharonhiakente

Ne raieronke. 
Nok Rawenniio

Wahonikonhrata

Ienakerenion

Asakotokaten,

Onkwe rotonhon,

Sakoskontakohe

Akwekon nonkwe.

Akwa kwennitha, Saiatatokenti, Aesarane

Asonkwentenr Iesos
Tsi satsennonni

Etho iaiakwawe

Neniakwenheie.

\section{Aietinenton}

Roniha, Roienha

Nok oni Rotkon

Roiatatokenti,

Tetewaiena

Eronhiakehronon

Ietisennaien.

v. Thonnhaon ne Rawenniio San Watis

R. Ahohahaseronnien ensakoskontako.

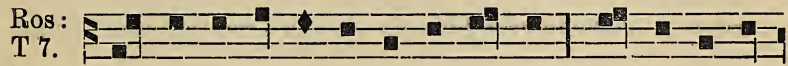

Ne sonkwennake- ra -ti raksa - a, sen-ha ra-ia-

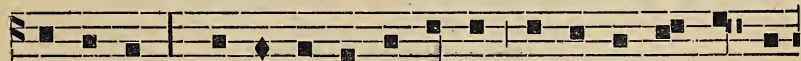

ta-no-ron tsi -ni- iot ro- to -kense ; se- sonkwaskontakwen

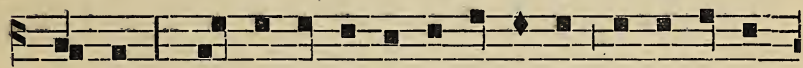

ra-wen : Tsi-nia-kon ia-ko-na -ke-ra- ton ia-htenhonwa-

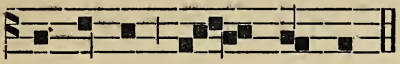

to- hetsten San Wa-tis.

29 JUN. TIER, KOR ONI. Ion WENT.

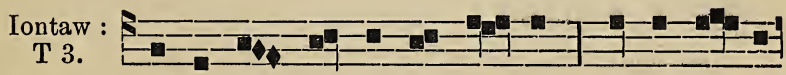

Onen skien-te - res ori - wi - io tsi ronnha-on

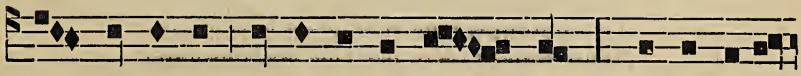

Ra - wenni-io Raron-hiakehro - - non a-hotsiakwa- 


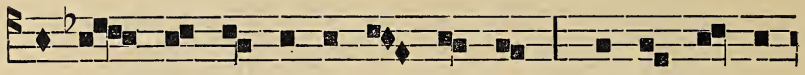

ri - si He-rot tsi ra-kennho-ton, nok tsi ionkwa-

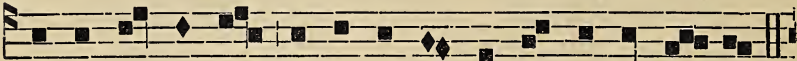

rhare ak-wekon tsi.ni kentiohkwasi - ta - e - ro - non.

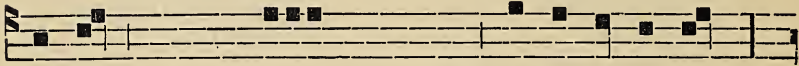

Se-wenniio takwatenientense nok takiente-ri-tsi- hon,

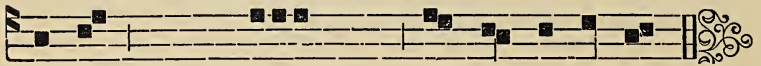

nok se - riwaienteritsihon tsi - ni ka-tie-ranions. Ahon.

Takw : kowa. (sk. 161). Rawenniio sewalenro. (sk. 153). Areriia (sk. 304). Sewariwiioslon. (sk. 161). Onen nonwa (sk 227).

IOKARENRE. (sk. 237).

T 2 .

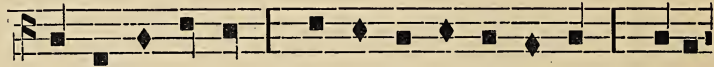

Tsialakweni- jo, Tier sa-ia- ta- to- ken-ti,

I-se

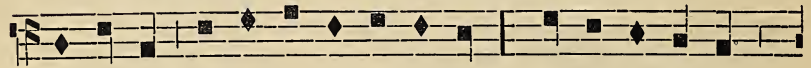

skowanen ne ka-ri- wi- iostonke, Hiari-honta-kwe

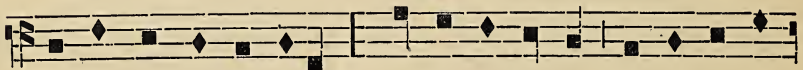

Ie-sos Sonkwawenniio

Nasatste -ris- te ra -o-tiohkwa-

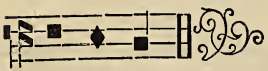

to- kenti.

Hiaiatarakwen ise sonbaa hiawi Iennhotonkwatha thenteron karonhiake Wahienhase : Ko, ise Tier wakonnhane A sheiatiniont neniesawennarakwe. 
Tiotkon tsinenwe entenikwekonhake Ise satatis, skewennenhawe kowa, Katke nonwenton tesaterientawenrie Nasherihonnien nakitiohkwatokenti.

$\mathrm{Ne}$ iakotiten raohonwakon Noe Akaonhaa iahte iakoskonhonne, Ne teskiatieren ne Tier raohonweia Etho satita, karonhiake iensete.

Satehnikwenies Ariwawakon kowa Ne saoriwat tsini hoterihonte Hetsitwanonwenn, hetsitwawennaierit Ronwakowanen teieiasontha okon.

Tewatateken tewawennakwekonhak Tewanentonsek Asenseratokenti

Niio Roniha, Niio oni Roienha Nok oni Niio Rotkonseratokenti. E. N.

Ros:

T 1.

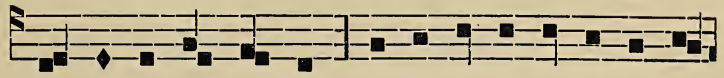

Ken wente $\mathrm{Si}$ - mon Tier ia- ha- rathen te-ka-ia-son-

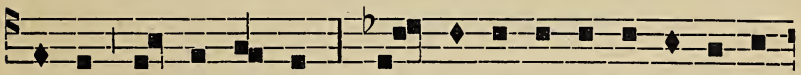

te-ke a - re-ri - ia. Ken wente ne ra-ha-we kannho-

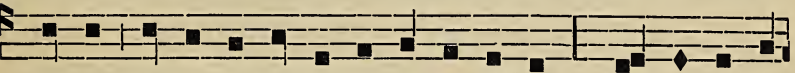

tonkwa ka- ronhia-ke ia- ho- ra-ne le-sos. Ken wente Kor

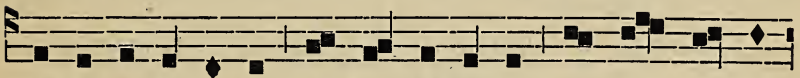

te -haswa-te - tenni nonwentsiakwekon ron-wa - nia - ria-

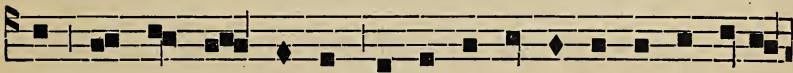

kon ra - o - ne-kwensa ro -ri -walıni-ratstakwen ka-riwi-

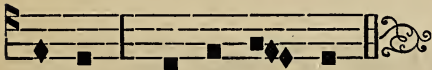

ioston, a-re-ri - ia. 5. $i$.

Ne 6on July. Ros : Tier Rli (sk. 83.) 7. a. 
2 JUL. WARI IONATARENAWIHNE

ARISAWET. 2on WENT.

Ionlaw: Wari. (sk. 264). Areriia (sk. 269). Tsialanoron (sk. 219). Tonwesen. (sh. 214).

IESOS RAONEKWENSATOKENTI. 2On WENT.

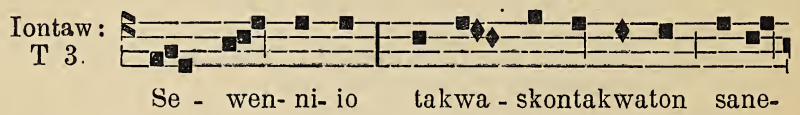

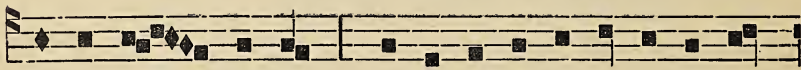

kwensato - kenti, takwakonnienston tsi takwenten-

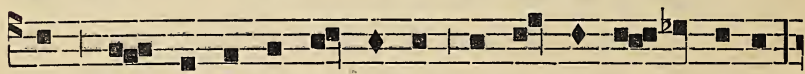

ron ion - kwa-ri- wi-ios - ta- on takwa-ia-ta - rakwen

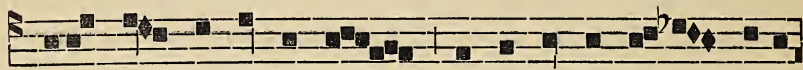

ae - twa - kwekonha-ke tsi-nonni te - si - teron

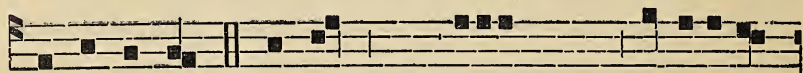

ka- ronhiake Tetewariwak Rawenniio raotanitenra-se-

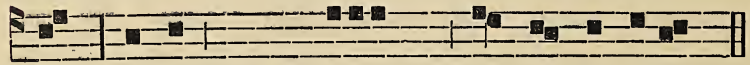

ra, nonwa nok oni tsi nenwe tewa- ri - ho-wanat. Ahonn.

Takw : (sk. 53). Sewenniio onen. (sk. 147).

T 5

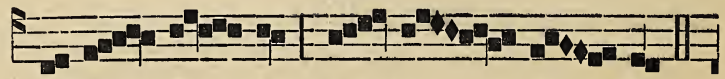
A - re - ri - ia, a - re - ri - ia. 


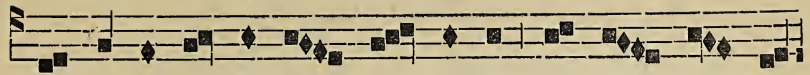

Ie - sos ra-o - ne-kwen - sa - noron io - ia - to - ha-

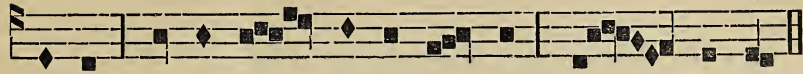

re-ton na-kotonn - hetston non - kwe o - konba. $a$.

Raonekwensa... (sk. 164.) Twakwekon. (sk. 183).

\section{IOKARENRE.}

Nenlonwa... (sk. 802). Telewalonts (sk. 370).

v. Takwentenr nisa, takwaien okonha,

n. Ne seskwaskontawaton sanekwensatokenti.

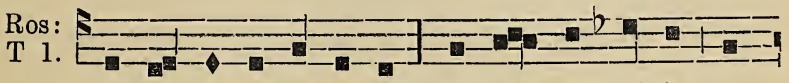

Tatsi- twa-nonwe-ra -ton Sonkwawen-ni- io ra-

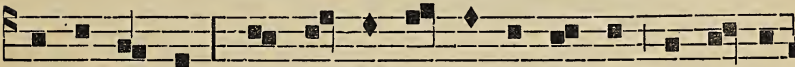

o-nekwensa ne se-sonkwaskontakwa-ton ro-ta- te-

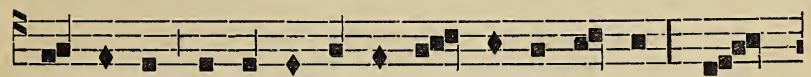

wente-ton sonkwawis ra- 0 - ne-kwensa- no - ron 0 -

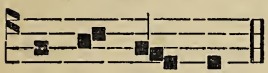

ka-ri - stia - kon. 1. $e$.

JUL. 26, ANEN IOTI. 1on WENT

lontaw: Tawalsennonni (sk. 387). Takw : (sk. 42). lesos Wari Sose (sk. 225). Wari seniien/ıa (sk. 228). lokarenre (sk. 2j9)

AUG. 1. TIER RTI SAHONWANERENKON ORONKWASA.

lonlaw: Onen (sk. 381). Takw : (sk. 24). Areriia (sk. 304). Rawenniio sewalenro (sk. 153). Sewarwiioslon (sk. 111.) 
IOKARENRE. (sk. 237).

v. Ise Tier wakonnatonkwe

R. Nok iseke tsiniiot otstenrake enkitiohkonni nakitiohkwato kenti.

Ros: Ise Tier (sk. 304). Kor lesos (sk. 304)

AUG. 6. IESOS SAHATIATATENI.

lontaw : Raosennakon (sk, 30l). Takw: (sk. 68). O swanaliio (sk. 147). Areriia (sk. 302). Ronnhek Sonk... (sk. 136). Toni iotsen... (sk. 189),

\section{IOKARENRE.}

Nenhonwa... (sk. 302). Iesos seskwaiatonlakwen (sk. 356).

v. Iesos takwanatonni tsini saiatanehrakwat,

ir. Satatiatolasi onontoharake.

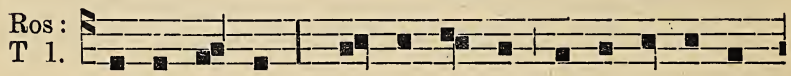

Ie-sos Kristos sa- hni- ia - tat ne Ro-ni-ha, sa-

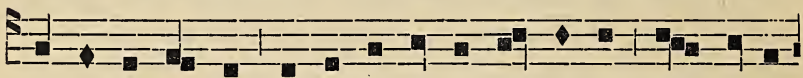

te- ho -naswa -the asisen ni -ha -ti ken wente ra - tikwe.

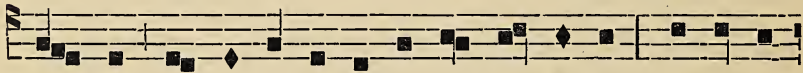

kon - ne ro - natkathon o-nonto - ha - ra -ke satha - tia-

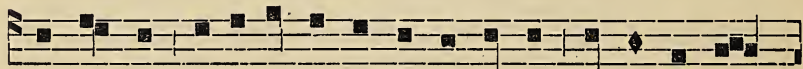

ta -te - ni ra-o- ia-ta-ne-hra-kwatsera wathote-ron-

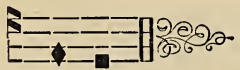

ronten. 1. e. 
AUG. 10. ROREN RTi 2on went. (sk. 242).

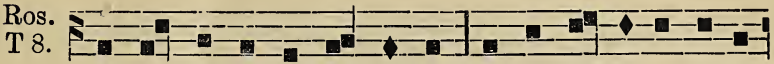

Roren ro-ia-ta - to - kenti wa-ha- wenhase ne ro-

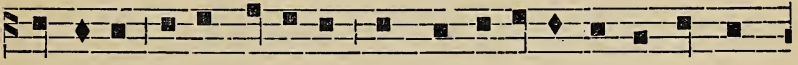

nonti -io rotennha-on-ne skennenha nahonwaskonten wa-

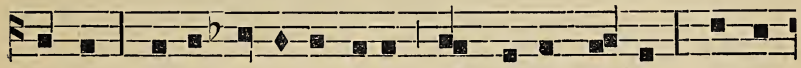

henron : Skiata- ka-ra-ti io-ri, skwetar nok i-sek: kheia-

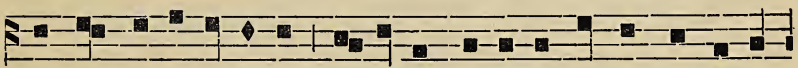

khonni ia-ko-tentenion aotso-ko -watsera kentiohkwatoken-

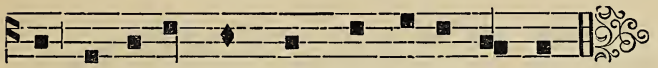

ti ne en-ion-kwakwatsten ka-ronhia-ke. 8. $a$.

AUG. 15. SATKONWASKWENHAWE WARI. 1 WENT.

Iontaw. $-b$

T 8 .

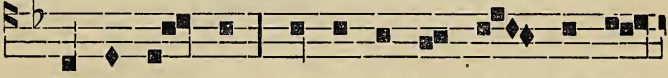

Twatsennon - ni te-wakwe-kon Ra - wenni-

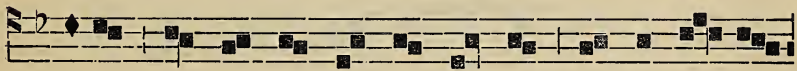

io-ke ion -kwenta - to - ken -ta - se $\begin{cases}\text { Wa- ri } & \text { ka- ron- } \\ \mathrm{A}-\mathrm{n} \text {-n io - ia- }\end{cases}$

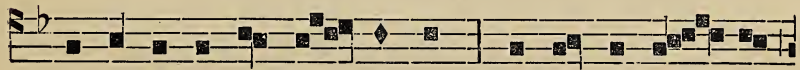

$\left.\begin{array}{l}\text { hia - ke tsi ia - we - nonton } \\ \text { ta -to-ken- ti ia - wen - ta-wen }\end{array}\right\}$ ronat - sen-non - ni 


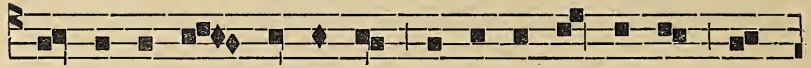

ra - ti - ron-hia - keh-ro- non nok ronwa-sen -na -iens ne

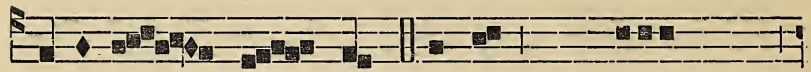

Roienha Ni - io. Akwa tkariwaieri tsini wake-

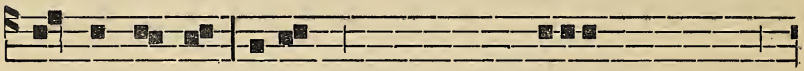

ni -konhroten ririwaienthose ne Rawenniio akwekon tsini

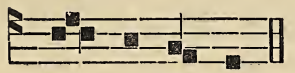

ka - tie -ra - nions. Ahon,

Takw : kowa. (sk. 42). Ronatsennonni (sk. 204).

$\mathrm{T} 5$.

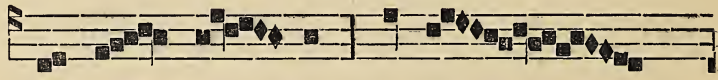

$A-r e-r i$ - ia, A-re

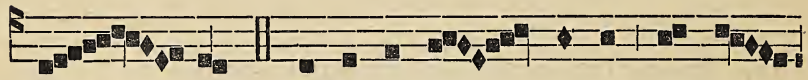

ri - - ia. Ta-konwaskwen - ha-we Wa-ri -

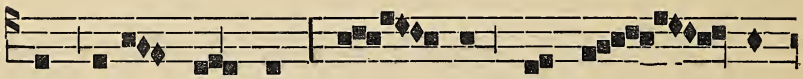

a karon - hia - ke, te - - hon-tonn-ha - - ron-

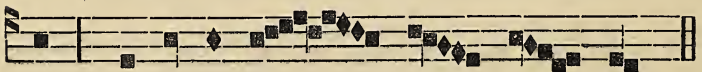

nions ra-ti-ronhia - - keh - ro - non. $a$.

Jolsennonni. (sk. 205). Oh nahoten. (sk. 97).

IOKARENRE.

Tierennakelskwatha (sk. 290). 
T 7.

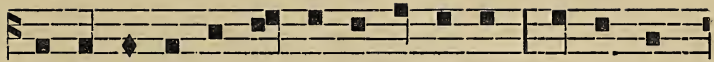

Tsi-na-ta -konwaskwenhawe Wari - a wathontonn-

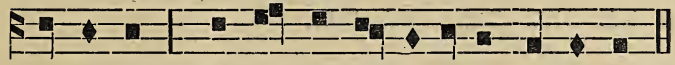

ha-ronnion ra- ti - ron-hia- kehronon o-konha.

T 8.

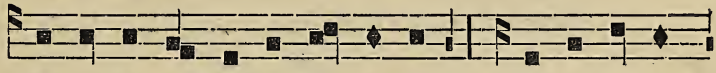

Ka-ron-hiake Wari ie - te-ron hiate-ia - te-

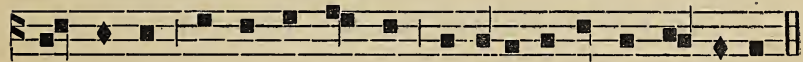

ra - hneken le-sos ro-ien - ha iotsennonni iahte ka-konte.

T7.

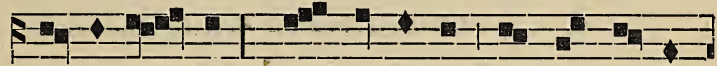

Sonha Wa - ri, hia-konnienstha ne Rawen-ni-

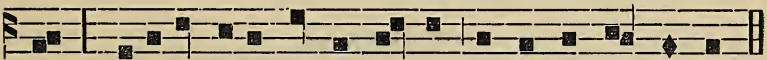

io, aseken takwate- we-ton-ni ne se - sonkionnheton.

$\mathrm{T} 1$.

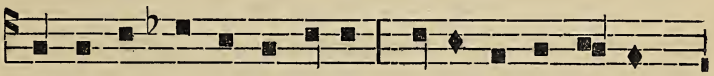

Wari, ie - senna- ke- ra-ton ra-ti - ronhia-ke- hro-

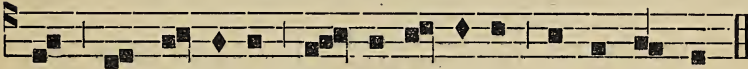

non nok a-kwe-kon no- neshonronon ie -sa - tsa-nis.

v. Iotsennonni Wari, akwa tewatonnharonnion,

$\mathrm{R}$, Konwennakeraton ratironhiakehronon.

Ros.

T 8 .

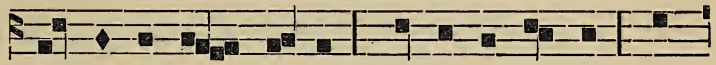

Ken wente Wa - ri - a ie -ia-wenon-ton ka-

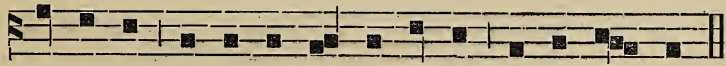

ronhia-ke, ne roien- ha hnikwekon iahte ka - kont. 


\section{AWERIASATOKENTI WARI}

Iontaw : (sk. 268). Takw. (sk. 29). Weriasanoron (sk. 200). Takwentenr (sk. 219). Kasene (sk. 116).

Ros.

T 5 .

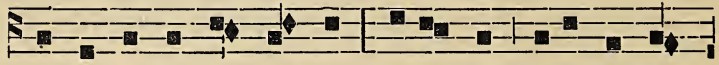

Ionwesen na-kwe-ria-ne, a-se - ken io-ri- wa-ne-

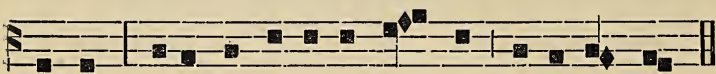

hrakwat tsini ha-kie-ra-se nia- hte ho-no-ron-se.

SEPT. 8. SONNAKERATE WARI. 2on WENT.

lonlaw : Wari (sk. 264). Takw : (sk. 58). Tekonnoronkw... (sk. 201). Telewaiena (sk. 216)

\section{IOKARENRE.}

T 8.

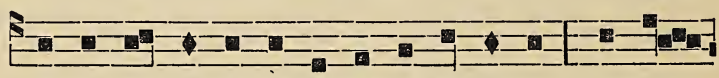

Onwa ken wente ionkwenta-to -kenta -se Wari -

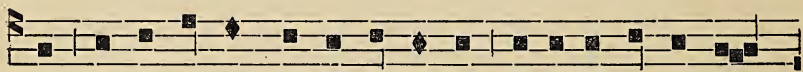

a a-wentawen- $\left\{\begin{array}{l}\text { io -na-ke-ra-ton } \\ \text { konwaniakte-ni }\end{array}\right\}$ a- ko-sot o-kon ken-

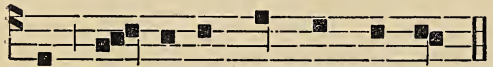

ha A - braham tenhnon Ta.fit. Salkon Niio. 8. e.

T 6.

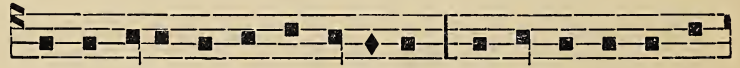

O-nonti- io na -kawatsi- ro-ten Wari-a te-ka-wa-

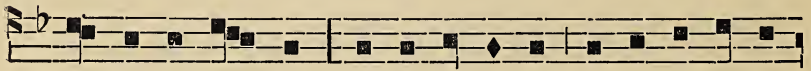

tsi - ra - ren-sa - ron, te-wenni- ten-tas a-ion-kwaia- ta- 


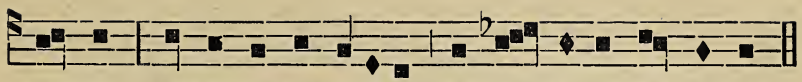

kennha nok ia-ionkwata-tiase tsi ren-te-ron roi-en-ha.

Tewakeh!akon onkwe. 6. a.

T 8.

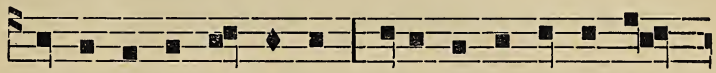

Onkwanikonhra -kwekon Iesos tatsi - twari - wa-

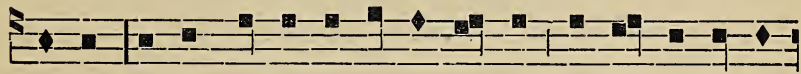

kwase nonwa ken wente wenta - no -ron tsi $\left\{\begin{array}{l}\text { io }- \text { na-"ke- ra- } \\ \text { io }- \text { nia - }\end{array}\right.$

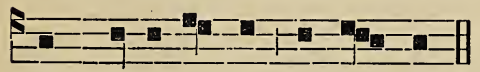

$\left.\begin{array}{l}\text { ton. } \\ \text { kon. }\end{array}\right\}$ roni-sten -ha Wari - a. Sewenniio to ok. 2. e.

T 7.

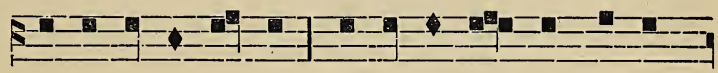

'Te-watonnha -ron-nion ne ken wente tsi $\left\{\begin{array}{l}\text { io na- } \\ \text { konwa- }\end{array}\right.$

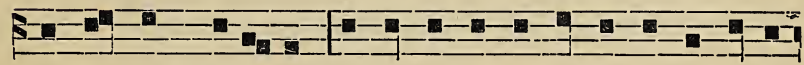

$\left.\begin{array}{l}\text { ke -ra - ton } \\ \text { niakten -ni }\end{array}\right\}$ Wari -a, a - iawens ai-on-kwate-ren -naienha-

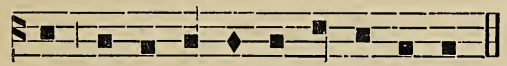

se a-sonkwentenre Ie-sos Kristos. Tewakelılakon Rolkon. 7.i.

v. Onwa ken wente ionakeraton (konwaniaktenni) Wari ioiatatokenti,

R. Ne ionkwatewetonni tehoswathe kowa.

Ros.

T 1.

Senna-ke-rat-se - ra- to- ken- ti

Tsi te-tsia-te -ra- ne- ken So-se

\} Wari he-tsien- 


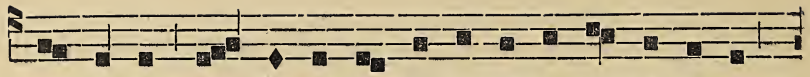
ha $\mathrm{Ni}$ - io tio - ha-wi- ton na -kotsennonnia - tat-se- ra

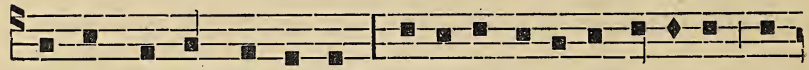

nonkwe nonwentsiakwekon, a- se-ken takwatewe-lonni te-

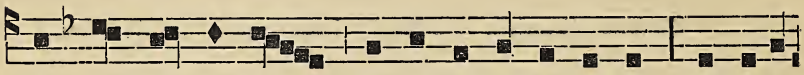
ho-swathe ko-wa Je-sos Sonkwawenni-io a-sata-

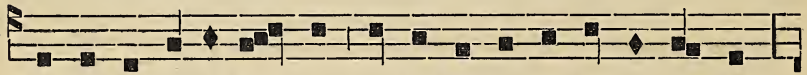

kon teson-kwaia-ta - kwen sonkwana-tonni te -ios- wa -the

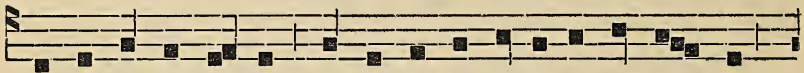

ra-sennion kenke-ion nok sonkwahahawi karonhia - ke

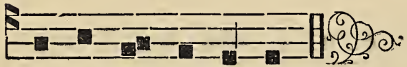

ai- a - kwatsennonni. 6. o.

WARI TSI IONIKONHRANONWAKON.

Iontaw.
T 1

Ka-ia - sa-kıa konti-ken-nia-ta- kwe Ie - sos

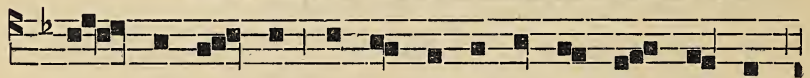

ro - ni-sten - ha nok o-na-ra-se - ha Wa - ri - a

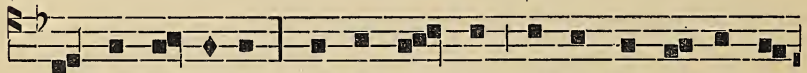

ro - ne Kle-o-fas nok Sa -lo - me nok tenhnon W'ari Wa-

E-

te- re. Tsi-sathonwisen ken irate hetsienha enkenhake 


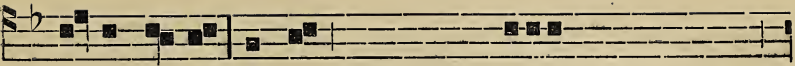

rawen Ie-sos. Wahawenhase hnikwekonne : Ken ikate sani-

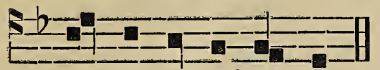

sten-ha en -kenha - ke. Ahonwa... Takw : $s k .29 . \mid$

T 4 .

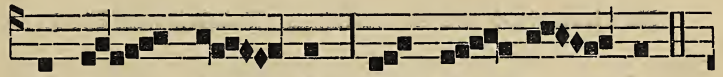
A-re - ri - ia, a-re - ri - ia.

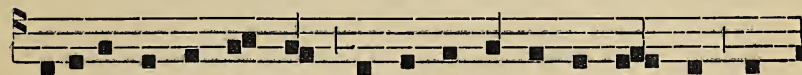

O i -se se-wakwe-kon ne sewa-to-hetstonha - tie, te-

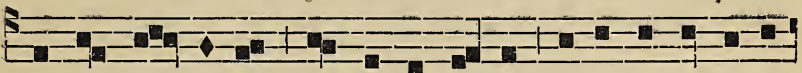
se-wa - ia - to- ret, nok se- watkatho ka-si- ken ka-nikon-

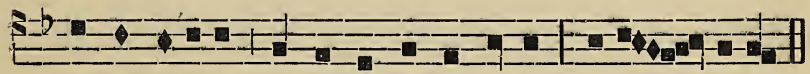

hraksenseraien tsi-ni- iot a -ke-ni-kon-hraksen - se- ra.

T 2.

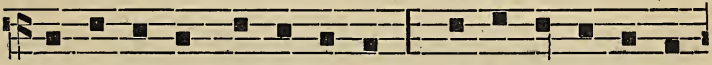

Ie - ka-ta -kwe ka -ron-takta A - on-ton ken nai-on-tro-ri

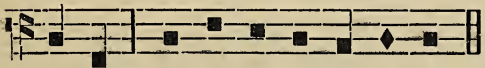

hakwe Ra-ia-ta-ni-iont Ie -sos. nonwaks Tsi-ni -iot wakenhe - ie.
Wari sot-si watstarTsi-ni io-ni-konhra-

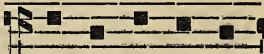

$\mathrm{Ne}$ io -nikonhraOn ka iahten ta-

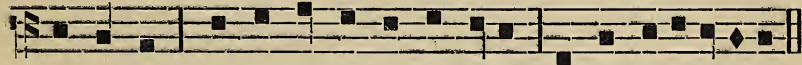

rio-ta-kwe $\mathrm{Ne}$ ok enskat ne roien-ha O-nen renheionsere iontstaren $\mathrm{Na}$-konwatkatho ne Wari Tsini ioronhiakenne

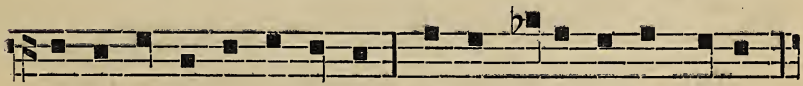

Onka iahta konwenten-re Tsi-ni Ionikonhraksenskwe 


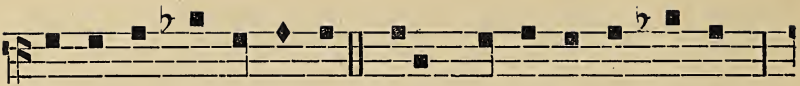

ro-ien-ha sahonwa-rio. I-i se-ken onkwa-ri - wa,

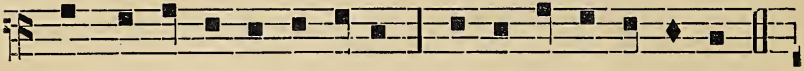

$\mathrm{Ne}$ ionkwari -wa-ne -rakskon, Hetsitwaron-hia-kenton.

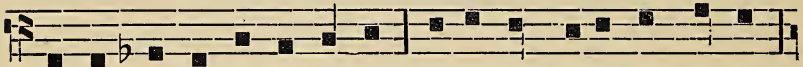

Wari akwa takwanonwes Takwentenr a-e -twa-hriskon

Siaton nonkwanikonhrakon Tsiui honwaronhia-ken-ton

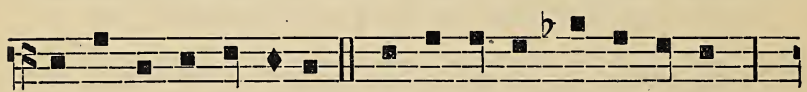

Sakat a-e-twatsta-ren. Hetsenni- tak ne hetsienha Tiotkon ai -a-kwe-hiarak. Tiotkon a-tsi-twenten-rhe- ke

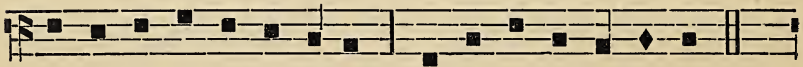

A- on-ta -ken-ni-konhrasat

A - on- sa-hi - nonwehak

Tsi nenwe ne entsionnheke

Tsi-ni honwa-ie -sa-ton.

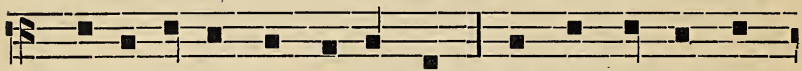

Ra-ia - ta-kta ne hetsien-ha Wa-ri te-twa-te-

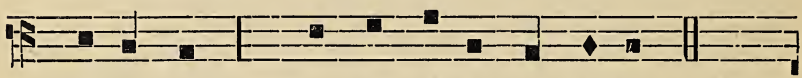

ra-ne-ken Sa - kat te-wa-ron-hiaken.

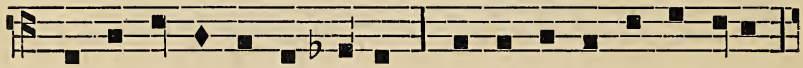

To-sa a-e-twa-tie - sa-te Ne ra - o-nekwensa kenha

Nok tsi -ni sonkwanon-wehon Hetsre-hiaron asonkwentenr

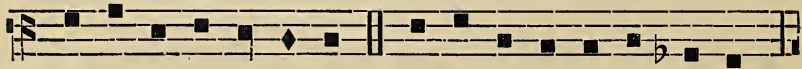

Tsini ia-kionkwetaksen. Netenhnon tewatste - ris-tak

Tsinenwatonwentsioklen. 'To-sa ka-ti a-ka-ie-sha 


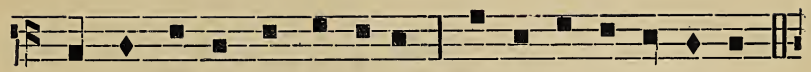

Ne ok a-e-twa-ska-ne-ke A- tsi-te-wa-senna-ien.

Tsi ron-wa-ia - ta-nen-takton Tsi ro-nekwensa -ri-hon.

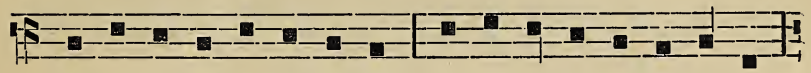

Nonen en-iakwatonnhokten Wari hetsennit netsienha

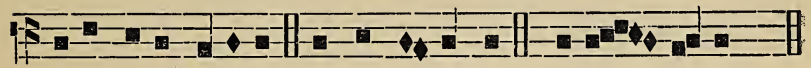

karonhiake iaiakwe. Etho na-ia-wen. A-re - ri-ia.

Ise Niio helsienha (sk. 212). Iesos lekonno... (sk. 165).

IOKARENRE.

Tierennakelskwallıa (sk. 265).

T 3.

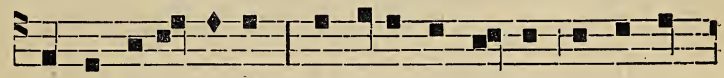

Ka-ni ieha-wenon Wari-a netsien-ha, ra - on-ha

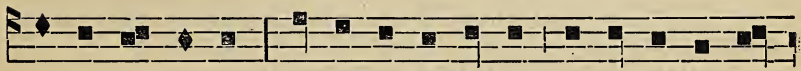

hetse - no - ronkwa, hetse -nonte -ra - lie sa-ha-nonta- ra-

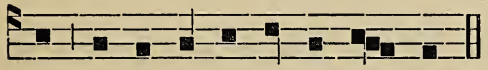

ne ta-sonkwanekwensa-ri - se.

T 1.

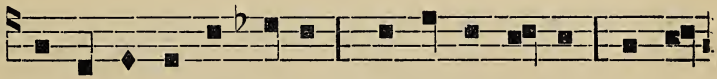

Onka iahta iontsta-ren na-konwa-tka-tho tsi- ni.

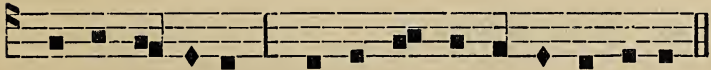

io- ron-hia-kenne sahon-wa - rio ro-ien-ha Wari. 
T 6.

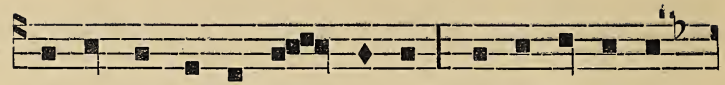

Sia-ton nonkwani- kon - hra-kon tsi- ni honwaron-

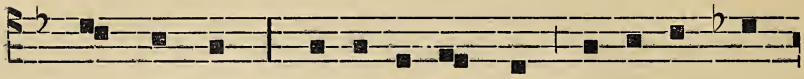

hia - ken-ton Ie-sos ne-tsien -ha tiotkon a - ia-

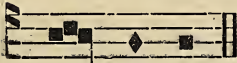

kwe- hia-rak.

T 2.

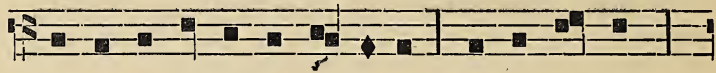

Ka thonte tsi-ni tsia- tl-iohne, Ie-sos, nonwa

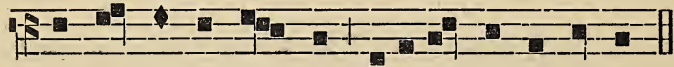

iahte ska- ie- ron- ni i- i kwaia -ta -ksa-ton.

v. Takwaterennaienhas Wari iesennakeraton kariwiioston ronwatiriohton

R. Etho istakwe raoiasakta netsienha Iesos.

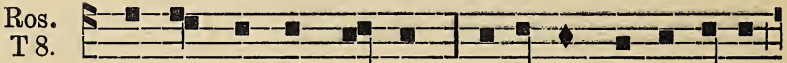

Watstarhakwe Wa-ri, tsini - iot wa-kenhe-ie

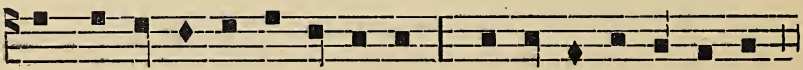

tsi ie-ka-ta-kwe karon- taḱta ; ronwannhatens ro-ien- ha

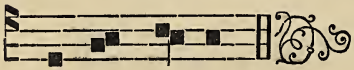

tsi tren-he-ions. 
SEPT. 29. WISHE RoN KOWA. 2on WENT.

Iontaw.

T. 3.

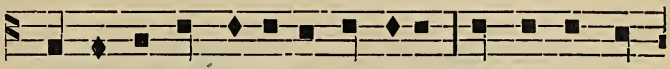

Hetsit-wa-senna-ien Ra-wenniio, swakwekon swa-

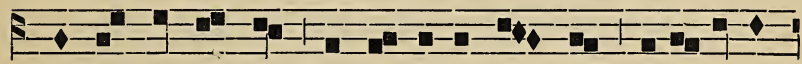

ronhia - ke- hro-non. se -wa -te-ri - hon - te hetsi -se-wa-

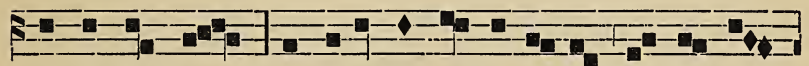

wenna-ra - kwa natsiswawenna-ie -ri - te tsi - ni - kon
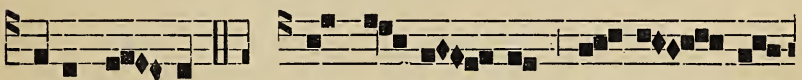

enthata - ii. (s/.) A - re - ri - ia, a - re - ri-

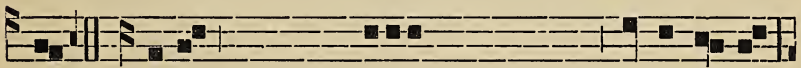

ia. Tewatonnharen tetsitewatonraserons ne Rawenni-io,

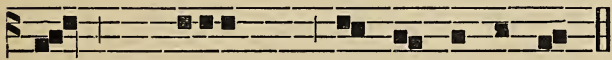

a - seken asteson seson - kwentenranions.

Ahon... Takw : (sk-49). Swaronliakelironon (sk. 143).

T 8

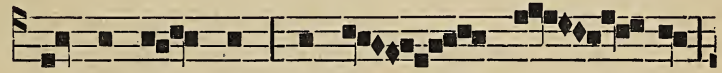

A - re-ri - ia. a-re - - ri - - ia.

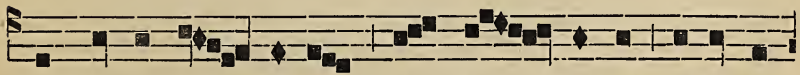

Swa-ronhia-ke - hronon, swa - Isen - nonni askwaia- 


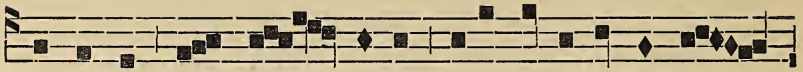

takennha non-wen - tsiake, tosa asonkwahrewa -

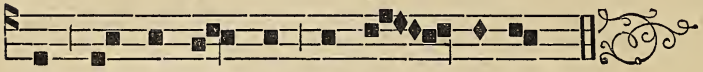

te ne tenson - wa - ia-to - re-te- $a$.

Akwa wakeronhiaken (sk. 194). Telewariwak (sh. 181).

IOKARENRE.

Tierennakelskwalha (sk. 295).

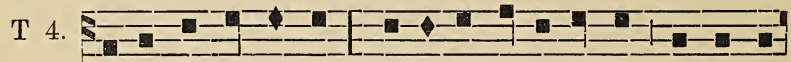

Rati-ia-ta-no-rons ra-ti-ronhiakehronon ronwaien-

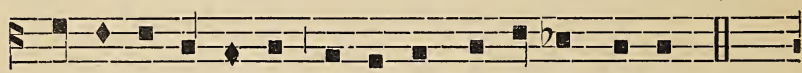

nis ne Ra-wenni- io tsi ia-kwa-te-ren - nai-ens.

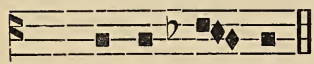

(sk.) A-re - ri - ia.

T 7.

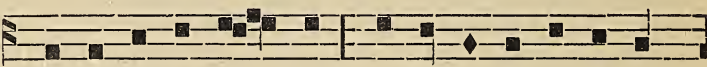

Ne ronwako -wa - nen ra-ti - ron-hiake- hronon

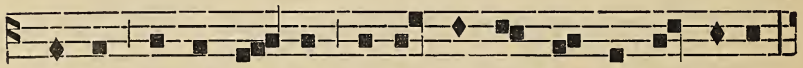

Wishe wa-ha-ri - ste notkon-se-ra-ksen, nok wahenron:

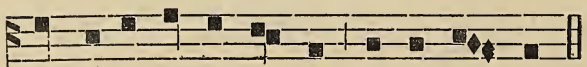

Ronwasenna-ien $\mathrm{Ni}$ - io, a - re -ri - ia. 
T 8.

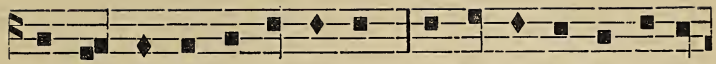

Se -ron -hiake -hronon Wishe, wakonri - honten teshe-

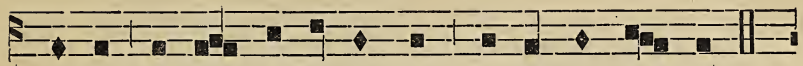

ia- tak nonkwe a-ko-lonnhetst neniontonnhok - ten.

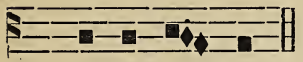

(sh.) A-re-ri - ia.

$\mathrm{T} 8$.

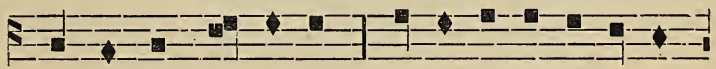

Swaron-hia - ke - hronon, tat-si - se -wanon-wera-
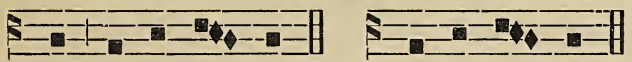

ton Ra-wenni - io. (sh.) A-re-ri - ia.

T. 2.

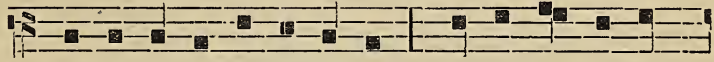

Sa- ia -ta- to - kenti $\mathrm{W}$ ishe, Sa-tkonse-ri - io

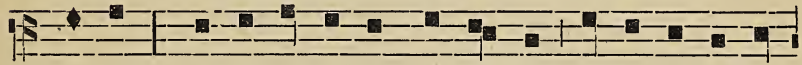

ko- wa, I- se shesen-nion ro-nat-kon, sotsi ra-ti-na-

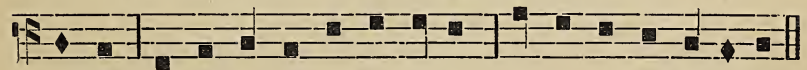

iehne ; I - se ie-sen-na-ke-ra-ton Ra-ti - ronhiakehronon.

Kwennitha, satanitenron, Takwannhe ne sentiohkwa, Tosa ionkwasenni notkon Ionkwateriiohatie, Ionkwanosas ionkwaswense, Ionkwaiesatonhatie.
Ise seronhiakehronon, Hiaiatarakwen Niio.

Ise takwatsteristonhatie, Takiatakennhenhatie, Tosa aseriwarako Ne tsini konwennontion. 
Aiawen askwatekwaten Otkon oneshonronon, Askenikonhrataseke Tsini hanonwes Niio Nok askienawaseheke Akeriwaierite.

Swaronhiakehronon okon, Niio swakwekonhatie, Askwaterennaienhase
Akwa aeswarane, Onen eniakwatonnhokten Aetwakwekonhake.

Skanikonhrat aetionni Sewaronhiakehronon, Hetsitwasennaien Niio Roniha nok Roienha, Rotkon Roiatatokenti, Iahta kariwentane. E. N.

v. Sakorihonten ne Rawenniio ratironhiakehronon. (sh.) Ar.

$\mathrm{R}$ Aionkinonnhatieseke nok aionkiatakennhaseke. (s/l.) Ar.

Ros.

$\mathrm{T} 1$.

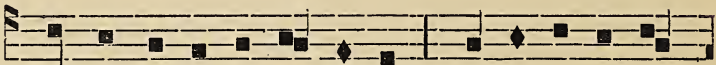

Wishe ie-senna- ke - ra -ton

ra - ti - ronhia -ke-

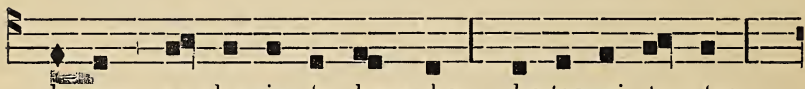

hronon, a - skwaia - ta - kenn -ha, he-tsenni - ten- tas

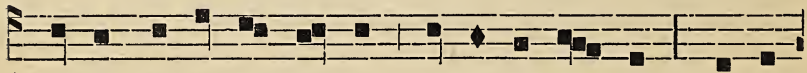

tiotkon a- son-kwenten-re Ro-ien-ha $\mathrm{Ni}$ - io, a-re-

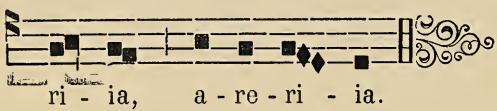

oct. 1. KARENSATOKENTI. 2on WENT. (sk. 264).

Takwaien (sk. 203). Tsiatanoron (sk. 219). Niio hetsienha (sk. 218).

OCT. 2. WARI AONISTENSERA. sk. 268).

Kwasennaiens (sk. 218.) lonkwanis... (sk. 207). Sewenniio (sk. 160). 
IOKARENRE (sk. 330).

v. Sonha tsiakothonwisen okon tsini kiakonnienstha ne Niio

R. Iesos oni hetsienha raonhaa tsini honwasennaiens.

OCT. 3. WARI IAHTE KANAKWAIENTERI. (sk. 280).

v. Tewatonnharen tsi tewasennaiens Wari iahte ionakwaienterhaon

R. Aionkwaterennaienniheke Rawenniioke Iesos Kristos.
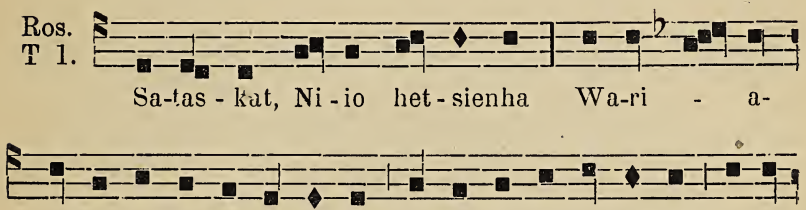

iahte se-na-kwaiente-ri ro-ta-te-nonso-ta-ni Potkon

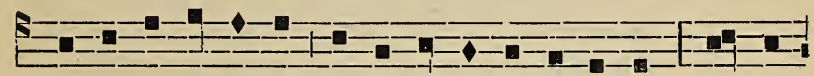

Ro-ia - ta- to- ken-ti ka-nonsa-no-ron se- ria-ne, son- ha,

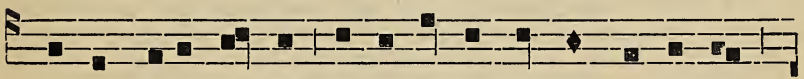

a - kwa io-ne -hra-kwat hianonwehon Sonkwawen-ni- io
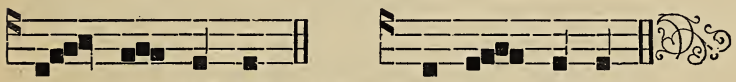

Ie - sos Kristos. (21 Nov.) A - re - ri-ia.

OCT. 24. RAFAE RARONHIAKEHRONON. (sk 397).

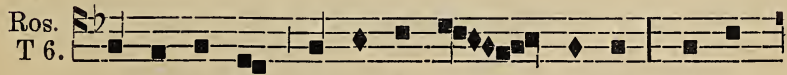

Ii ne Rafae ke-ron-hiake - - hro-non ra-ia- 


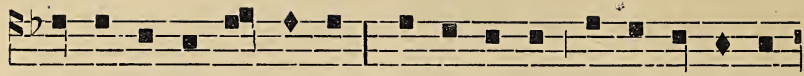

ta-kta Ni-io tki-te-ron; i - se tenhnon ta-tsiswa-non-we-

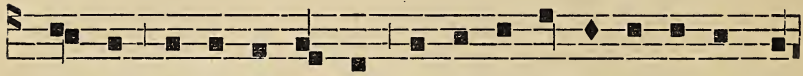

ra-ton swari-ho-wa-nat tsi-ni io-ri-wanehrakwat tsiE- =-

ni ho -ie - ren.

No. 1. ASONTHENNE WAHONTERENNAIEN. 1on. WENT.

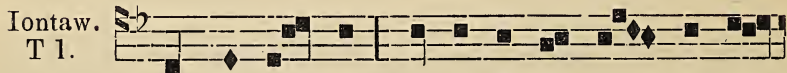
Twatsennon-ni te - wa-kwekon Ra - wenni-

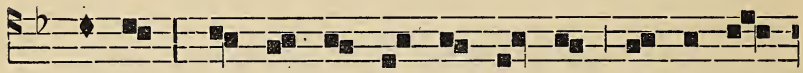
io - ke, ion-kwenta - to - ken - ta - se Ro -ti - ia-

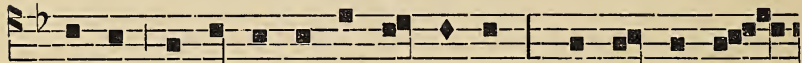

ta - to - kenti- son ra - o - nen-ta - wen ro-na-tsen-non -

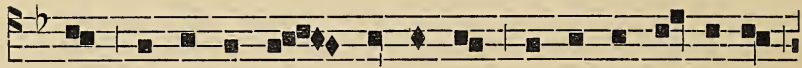

ni ra-ti - ron-hia - kehro-non nok ronwasen-naiens

S-2

ne Roien -ha $\mathrm{Ni}$ - io. Se-wa-tonnharen sewaia- 


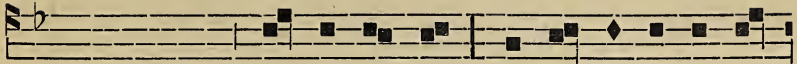

tatokenti Ra - wen-ni - io - ke tet -si - se - wanon-we-

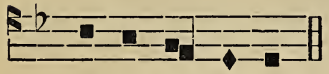

ra-ton tsi - nenwe.

Ahon... Tahw: (sk 49.) Rawenniio sewatenro (sk. 153.)

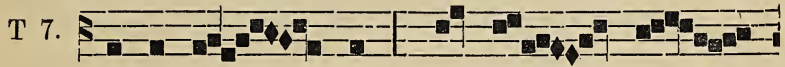

$$
\text { A-re-ri - - ia, a - re - - ri - }
$$

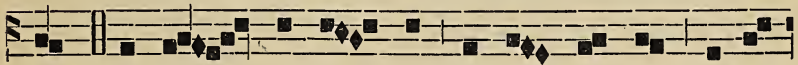

ia. Ka-ro ta -se - wet tsi tki - te - ron, swani-

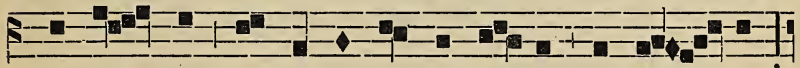

konhra - ksens, nok se -wa-wi - sen-heions nok i - - i

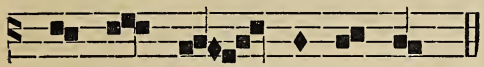

en - kwa - ie - - nawa - se. $a$.

Tekwanoronkwanion. (sk. 166). Ionehrakwat. (sk. 178).

IOKARENRE.

Tierennakelskwalha (sk. 369).

T 1

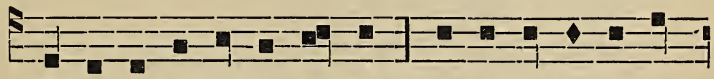

Wakheken io-tiohkwatsa- nit nia-te ionkweto-ten-

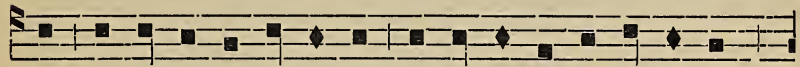

son, niate ie-wenno-tenson, watie- ta-ne tsi thenteron

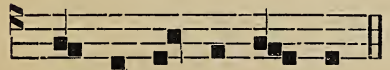

$\mathrm{Ni}$ - io ka - ronhia - ke. 
T 8.

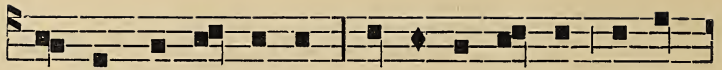

Nia-wen, Se-wen-ni - io ta-kwaia -ti - son tak-wa-

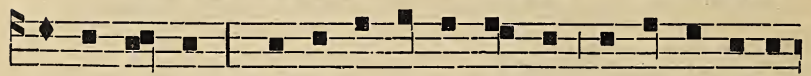

skonta-kwa-ton sa -ne-kwensato-ken - ti niate iakwawa-

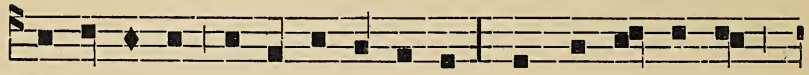

tsi-raien-tons, nia-te ionkweto- tens nok io- ne-hra-kwat

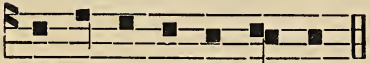

ta-kwasenno- wa-na - ton.

T 8.

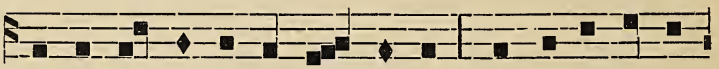

Hetsi -swa -nenton Ra-wen - ni -io, swaia- ta - to- ken-

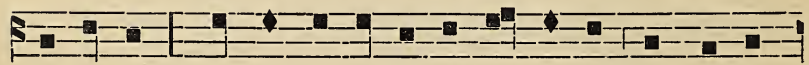

ti 0 - kon, swakwekon teswa-tonnha-ronnion nok ta-tsi- .

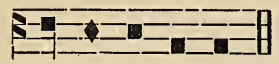

swari - wakwase.

T 8.

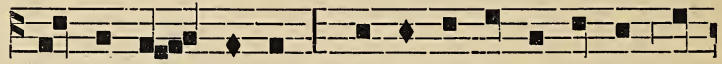

Ken-tiohkwa-no-ron ro-ti-ia-ta-to-kenti ron-

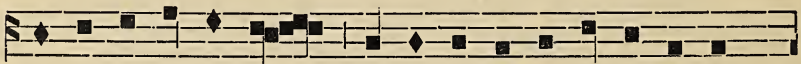

wakens Rawenni- io

ronwasen-nai-ens ka-ronhiake,

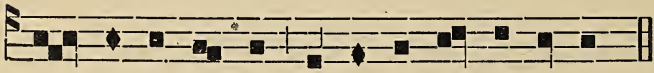

ra - on -ha $\mathrm{Ni}$-io sa-ko - ia - ta - no-ronston. 
T. 8.

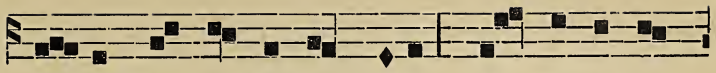

Te- kwa -no - ron-kwanions Niio, Ne she-ia-tsen-

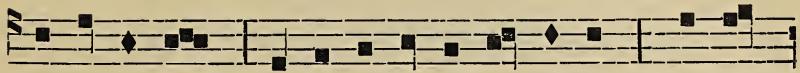

nonniaten-nis

$$
\text { A -ko -ia - ta -to - ken-ti - son, }
$$

Ne swa-

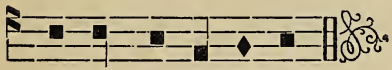

kwekon ka-ron-hiake.

Wari, Niio ne hetsienha, Akwa satanitenraskon, Ise swaronhiakehronon, Askwaterennaienhase.

Ise, kwani okon kenha, Nok sewatokensehakwe, Ise Iesos swakwekonne, T'osa skwaia tanikonrhen.

Ise, Niio raoriwa Ietsiiesaton, ietsirio, Ise, swarihowanaton $\mathrm{Ne}$ tsi ionkwariwiioston
Ise, swaiatatokenti Iahte swanakwaienterhaon, Ise, sewaksa okon kenha, Hetsisewentenron Niio.

Ise, ne sewatsennonni, $\mathrm{Ne}$ niate swaiatotenson, Twakwekon tetewaiena Aetwawennakwekonhak.

Atsitwasennaien Niin Roniha nok ne Roienha Ne satietisennaien Rotkon Roiatatokenti. E. N.

v. Teswatonuharonnion nok sewatsennonnihak, sewaiatatokenti, R. Hetsisewaialanoronston ne Rawenniio karonhiake.

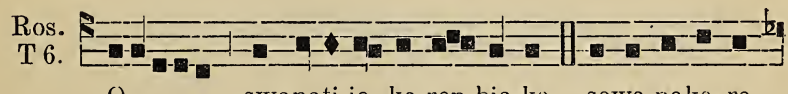

o swanati-io ka-ron-hia-ke sewa-nake-re

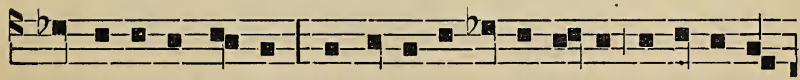

swa-ia-ta-to-ken-ti, iah o-thenon te tiotokte tsi-nii ionwe-

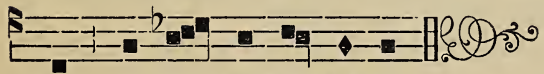

sen se- wa-kwekon Ni- io.

Nov. 21 (sk. 264). Ros. (sk. 40l). 
KaHASEIES AWENTOKON TSINIIORE ENHATONNIANE. Takw: (sk. 34$)$.

Iontaw :

T 6 .

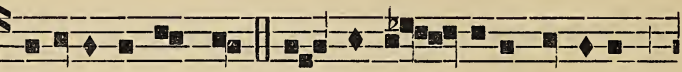

Sa-tanitenra-skon a - ski-ten - re, Sewenniio,

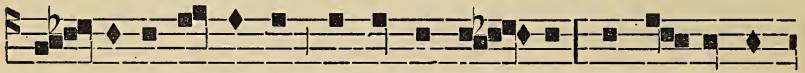

a - seken to-kenske iahte kat-tok - ha wa-ke - ri-wa-

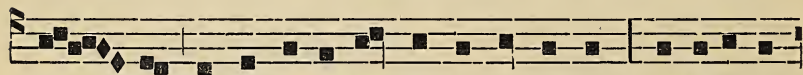

ne - ren akwa sewa-ka-tonnha-kanon-ni sa-kata-tre-

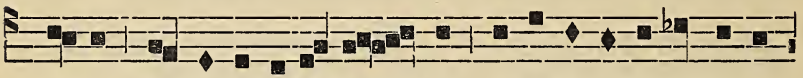

wa-te, a-kwa sa-sa-ni-kon - rhen na-keri- wane-rakse-ra

E-

0 - kon. Se-wen-ni-io, katennikonhrenhawitha tsinon-

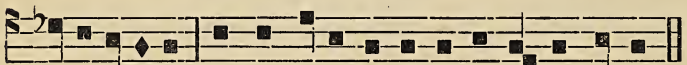

ni te-siteron. Sewen-ni-io sonhatsiwa konniahesen. Ahon...

T 8.

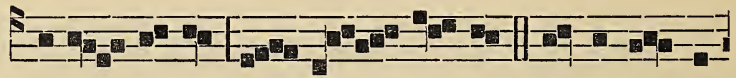

$$
\Lambda \text {-re - ri-ia, a - re - ri - ia. Ra-wenni - io }
$$

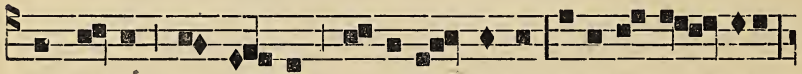

rattokha, ra-satste, ro-ni-konhri-io, ensakohre - wate

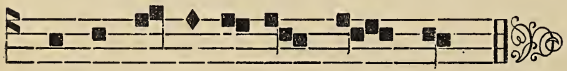

niahte tsia-ko-ta- tre - wa - ton. $a$.

Tsionkwe (sk. 134). Tsini seniseriio (sk. 139). Tsi rohasen (sk. 181). 
lonlaw: Onen teskwawi (sk. 306).

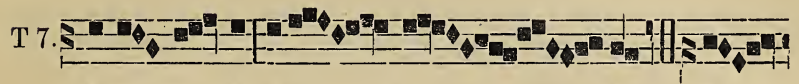
A-re - ri - ia, are - - ri - _ ia. Son-

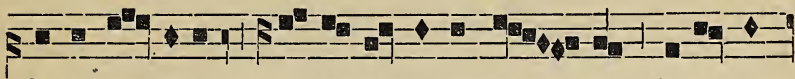

ha Sewen-niio tsiata-no-ron,skowa - nen, ie-sa-sen-

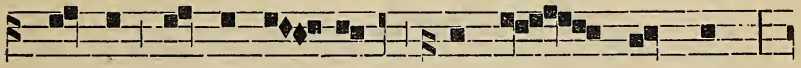

na - ien ka - rouhia - ke nok ie - - wen - ni-

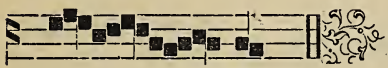

io - - stak, $a$.

lesos rakeni (sk. 153). Solsi oliake (sk. 151). Tesos kwaw. (sk ${ }^{*}$ 196).

Iontaw :
T 1.

Sonha te-sennontons, Sewen-ni-io, akwekon

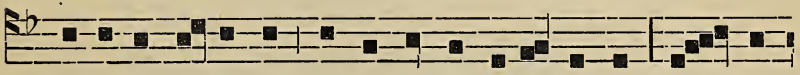

ie-sawen-na- rakwa, nok iah onka ta-ie- kweni a - on-

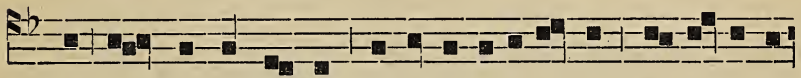

ta - ie - sennhonta-se; a- seken i-se sa-son ka-ronhia on-

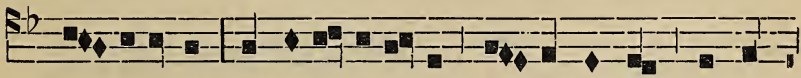

wentsia o- ni nok tsini iotie-ren ka-ronhia-ke son-ha- 


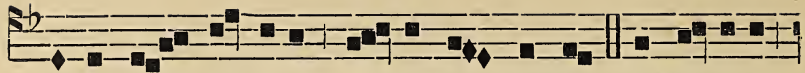

tsiwa, Se - wen-ni-io o - ri- wa - kwekon. Se-wenni-io

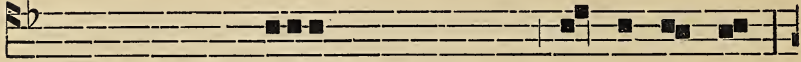

tsi konnhe, iahtiiaiehewe tsini saia - ta - nehra-kwat

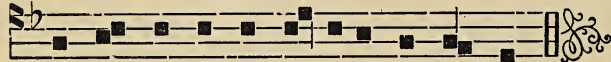

ta-ki - tenr tsi- ni sa- tani- ten-ra-skon. Ahon...

T 7. S二-1 A -re-ri - ia, a-re - ri - ia. Iah onka sa-

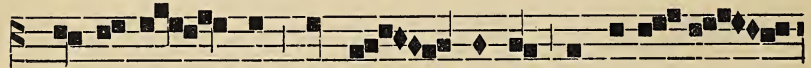

tehnia-to - ten Rawen - ni-io ronwanon-ti - -

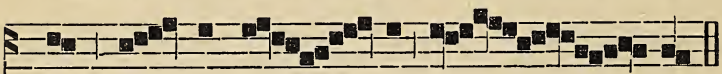

io ro - $\mathrm{ti}-\mathrm{non}-\mathrm{i}-\mathrm{io}-\quad-\quad$ son, $a$.

lonehrakwal (sk. 191). Tiolkon (sk. 152). O lesos (sk. 185).

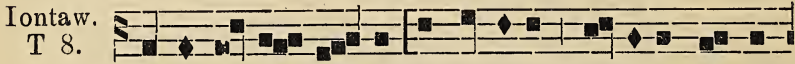
Aski-ten-re ni-sa, Sewenniio, a-seken kon-ien5一ni-ten - ta-se ken wenta - tie; a-seken,Se-wen - ni-io,

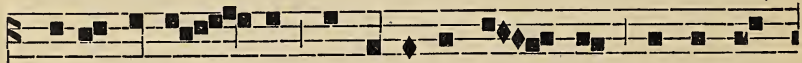
sa-ni-konhri - io, nok o-ni sa-kwa - tse nashe-tenS- ra-nion-heke ne na-ie-sa - niahe - senha - ke 


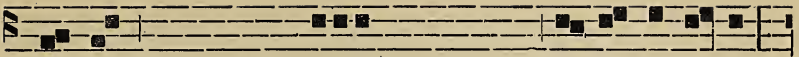

Se- wennilo, takewennarakwak tsi kon-ienni - tenta-se,

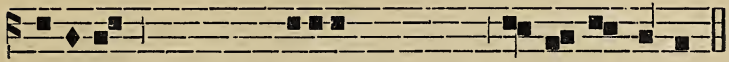

a-seken ionehrakwat tsi wakiewas iola-ne-renstakwa. Ahon.

T 1.2. A-re - ri-ia, a-re - ri - ia. te-tsi-swa-ri-

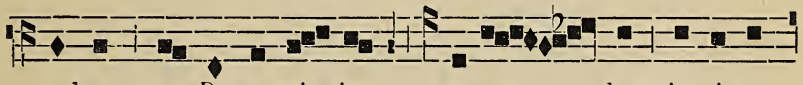

wakwas ne Rawenni - io, a-se - ken io-ri-wa-

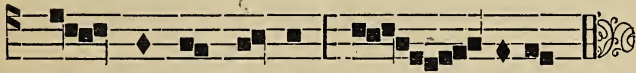

neh - rakwat tsi-ni ho - ie-ren. $a$.

Swalatiesalanions (sk. 142). lesos oriwiio (sk. 146). loriwanelır... (sk. 179).

ROS. OKONHA AKENNHAKE.

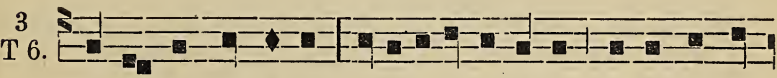
Onka tsiothonwisen o-ie-ri io-wistaien, tokat enskat

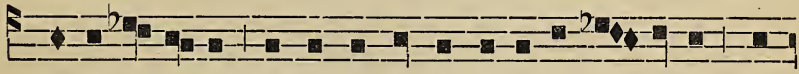

a- io-wiston-ti, iah ken ok sa ok ta-ka-ha-se - ro-ten na-

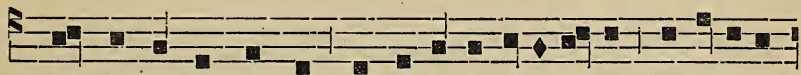

ion - he-we ka-nonskon na-we-sa-ke a-o-wi-sta tsi-niio-re

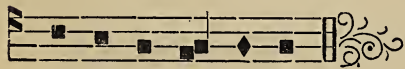

ia- on- sa- ka-tsen-ri. $6, a$. 
T 1 . 5-

Sewenni - io asontakwekon wakwataha- ro-we, iah-

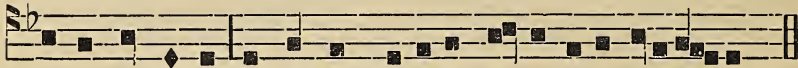

te ionkwanawi ; ne tenhnon tsi takennhaon enwakaharonti.l.u.

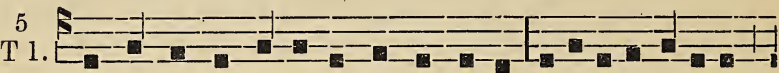

Hetse-wen-ni- iosta-ne Rawen-ni-io ononsatokenti-ke

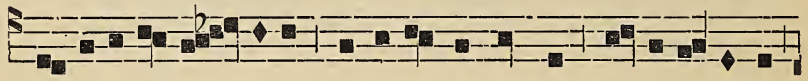

nok iaonse- hia - rane tsi ro-nikonhraksens tsiata-te-kenha

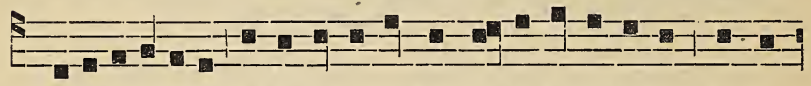

i- se sariwake, ok he ta-satsiakwari - si tsi nahoten he- tse-

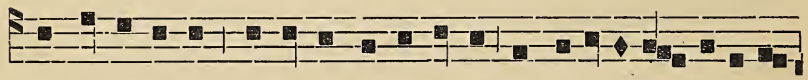

wenni-iostane; nia-re h€-tsia-tisakha nok saseni-ri - wase-ron-

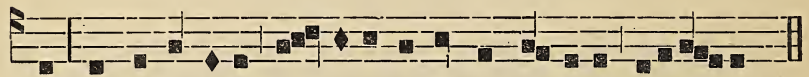
ni ; nok tsi tentese e - thone entsewen-ni - ioste, areri - ia. I.u.

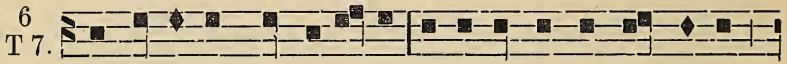

Khetenre ionknontera-tie onen iakwakwekon-ha-tie

5-解-

ashen nonta iah-te shotiien na-honne-ke; tokat iahtakhe-

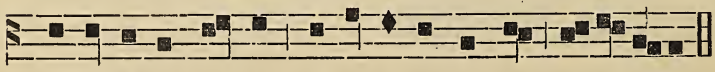

na-ta-ranon-ten-re, en-iako-no-ron-se, a-re-ri- ia. 7. $e$. 


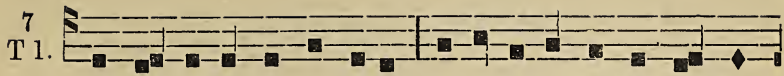
Iah ta - onton karon-ti-io a-on-hiani- ionten wa- hia-

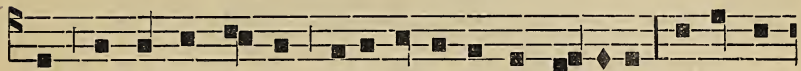

ksen,nok karon-taksen a-onhia-ni-ionten wa-hiios : tsi-ni ka-

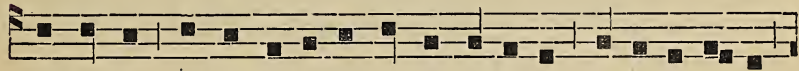
ron-ta-ke iahte wahiani-iontha wahi-ios, en-ie-rontiake,

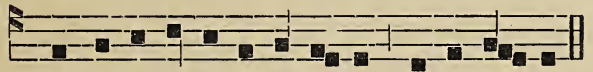

nok otsi-rake +n-iako-ti, a-re-ri-ia. 1. $i$.

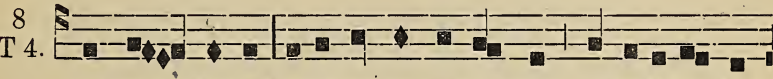

- Oh nen - kiere? a-seken ne ra-kennhas sa-hakeri- hon-

E二

tako tsi kkowanen-ne: iah ta-onsonton a - kientho; kate-

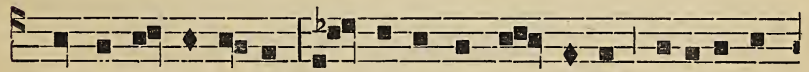
hens ta-ka-tsa-ri - te; ka - token tsi nen- kiere aontiesen-

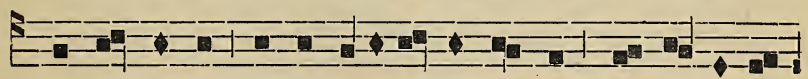

ha-ke nonen enwa-kate-ri - hon-takwen, a - ion-ke-na-

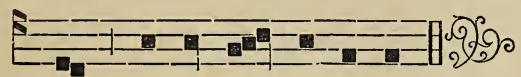

kton tsi ia-ko-non-so-ton. 4.e.

T 8.

Ken ka-ien ka-ia-ton : a-ke-nonsa ion-te - rennaiens

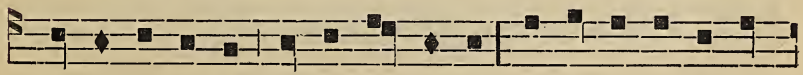

ka-non-so-takwén akwekon nonkwe; nok ni-se ion - tekwa- 


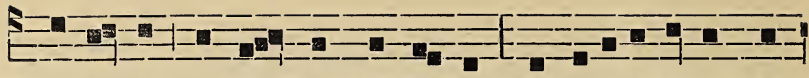

sen-ta-kwa ie-nenskwas tsionniaton. Nok sakota-tiskwe ken

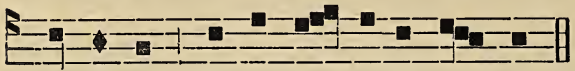

wenta-tie o-non-sa - token-ti - ke. 8. $a$.

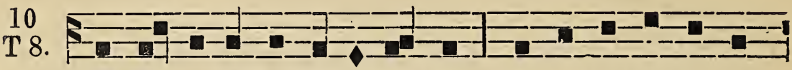

Shotia- to-kewenha-tie ronkwe ro- tatkenhronni-hon

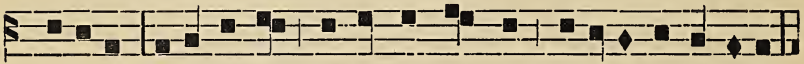

raonha, a-seken ne ion-tatkonnienstha 'nsakokenron Niio,

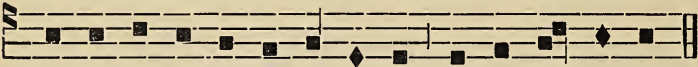

nok en-sa- kosen- no-wa-na- te ion-tatkenhron-nis. 8.e.

T 5.

I -ia-nere tsini ho-ie-ren teionhon-takwekon niahte

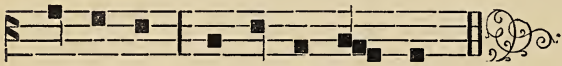

ion-ta-tis se-sa-kotsin-ton. 5, $a$.

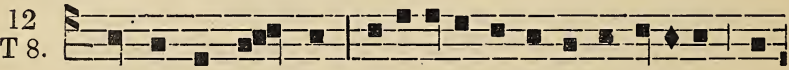

Sa-ia-lat ron-kwe, Si-ri-sire tahahtenti, iahare ne

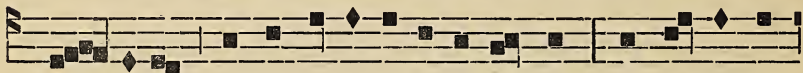

Se - ri-ko, wathonte-ra-ne ra-ti-nenskwas, ne a-kwekon

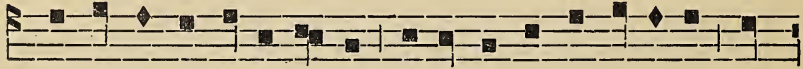

wahonwakwa tsini ho- ien, wahonwakenren- seronni, wa-

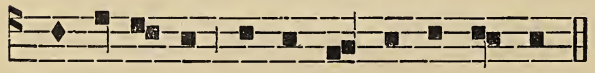

honwa-rio si se-renkhe - ne ti sronnhe. 8. $a$. 
T3 2.

Sa - ia-tat tsi-ni ha-ti sahalto - ike tsi o-nen sa-

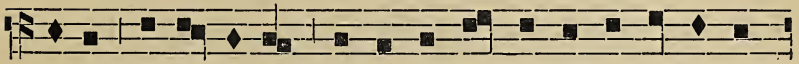

haton, sa-ha-ke-te, nok watho-non-we-ra-ton ne Rawen-

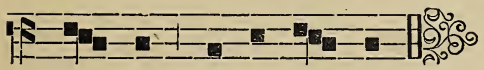

ni - io, a-re-ri - ia. 2. $a$.

14

T 1.

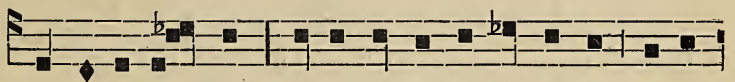

Ne tio-tieren - ton se-wesaksek ka-ron-hiake a-e-

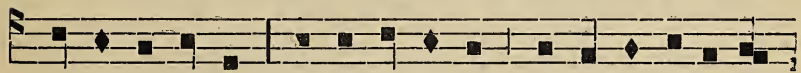

se-watsennon-ni, nok $\mathrm{Ni}$-io sen-ha en - se-tsi-se-wa-ie-

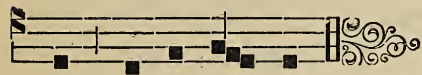

sten, a-re-ri - ia. 1. $e$.

15 T 4.

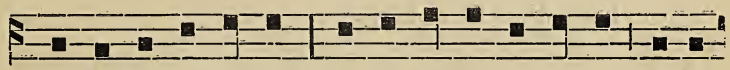

Ro-to-ken-se kowa wasonkwennake-ra-tse ta-sa-

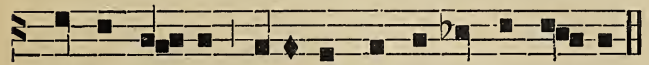

kotken-se - ra ra-otiohkwa ne Rawen-ni - io. 4. $e$.

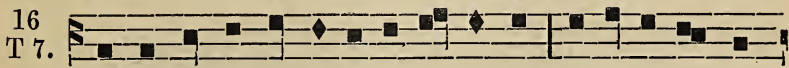

Ka ki ok nonwe en-tie-sa-hon-ka-ron, sa-ta-te- na- kta-

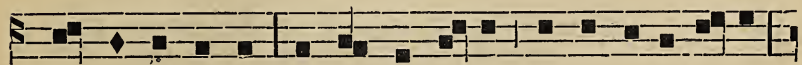

ra-kwas ohna-ken nok en-hienha-se ne hiahonka-ra-wi : 


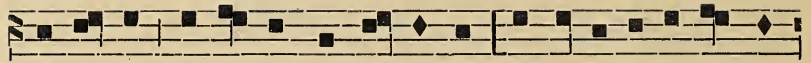

Tiaten-ro, o- henton ie-sa - salien, nok ten-iesa-ren-sa- ron-

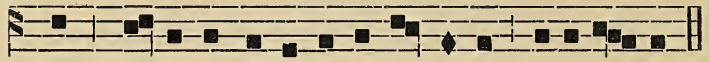

nion no. seron-ni sewa-tekwa-he-re, a-re-ri-ia. 7. $a$.

17 4.

Oh-ni ha-ia- toten ne Kristos ? Onka ron-wa-ien-ha ?

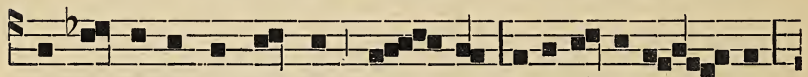

ta-hon-niron a-kwe-kon: Ta - tit. Tahata-ti le - sos.

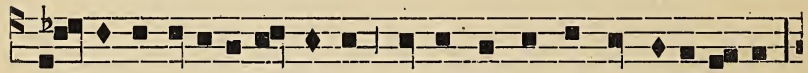

Na-hoten a-0-ri-wa Tafit ro-na-tonkwa Rawenniio, raton :

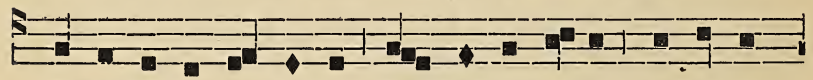

Rawenni-io ra-wen-ni Son-kwawenni-io: satien tsi

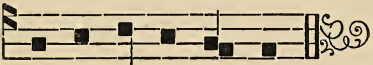

kewe- ien-tehta - kon. 4. $e$.

18.

Watrakwe ka - ti ro-ia-ta-ken-he-ion ra-o-nakta

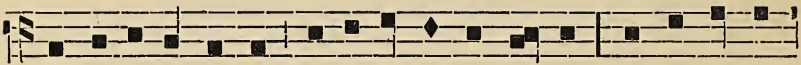

tsi ra-ia-tionnikwe, wahosen-na-ien $\mathrm{Ni}$ - io, nok a-kwekon

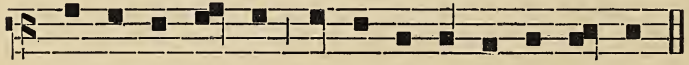

ne wahontka- tho wahonwa-sen-na-ien $\mathrm{Ni}-$ io. 4. e.

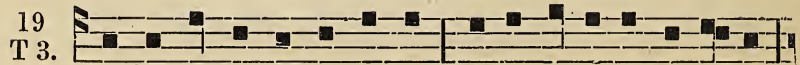

Wahatka-tho ronon- ti - io onka ia-ko-lekwahe- re 


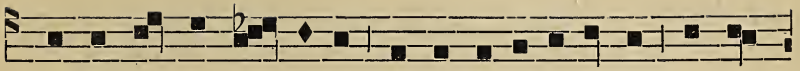

nok waho- ken sha - ia tat iah-te ho-tia-tawi-ton ion-ten-

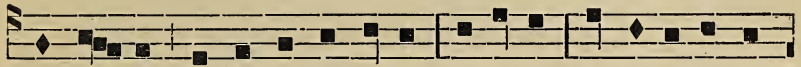

niota- kwa, nok wahawenha-se: Tiatenro, oh niotieren sa-

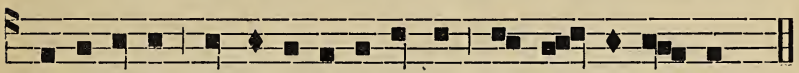

ta-we-iaton iah-te satia-ta-wi-ton ion-ten-niota-kwa. 3.e.

20

T 3.

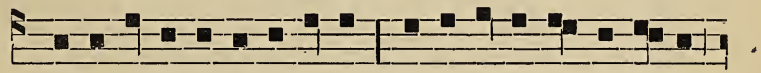

Wahaien-te-re-ne Ro-niha tsi e-tho si io- wi-sta-he

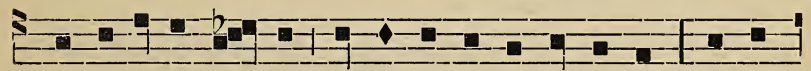

ni thothrori Ie - sos: Hetsienha sho-ie-wenta-on. Nok wa-

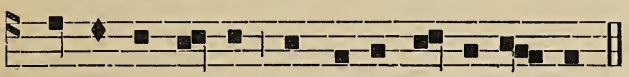

ha-ri- wl - io-ste nok ra-onkwe-ta o-kon. 3. $e$.

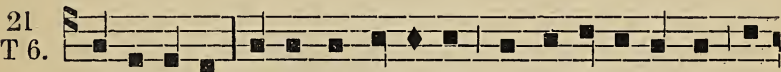

Iekonhiawi akwekon tsinikon konka-ro- ta- nihne, a-

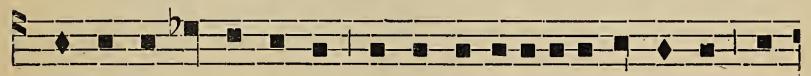

seken ta-ke-ne-ken-ni, iah ken te io-te-riwison-honne a-

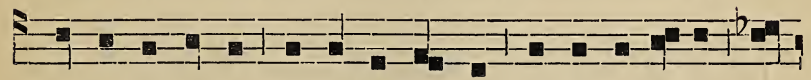

on- satsi-tenron ne- ne tsiaten-ro hetska-ro-ta-ni tsi

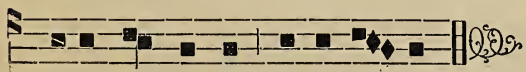

ni-i kon-ten-ron? a-re- ri - ia. 6. a, 
22

T 1.

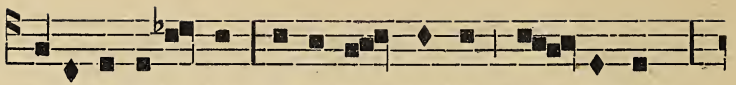

Aon-sa-ietsi-ion ne sa-te-tsionkwe a - kowenk

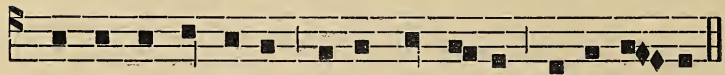

nok satsi-tsion Niio ra-owenk $\mathrm{Ni}-\mathrm{io}, \mathrm{a}-\mathrm{re}-\mathrm{ri}$ - ia. 1. $i$.

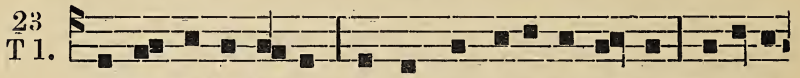

Wahalkatho Ie- sos nok wahrenhase, wahenron : Takenia-

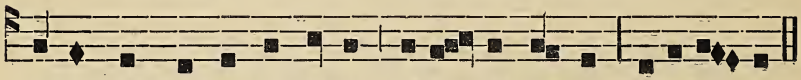

hesen, kien, tsi ti-seh-takon ne se satsien-te, a-reri - ia.

Eniontsle oni lsini iolatenron karo ne 7 niwasontashen.

Akta tsi Rathonniane.

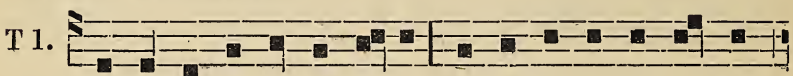

Tokenske on-we kwahrori, ia- hta-on-lo-kten nonkwe

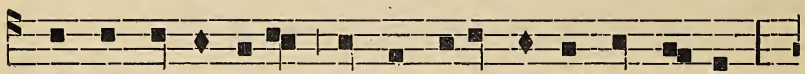

nonwentsiake iiens tsi ok etho nen-ia-wen-se - ron

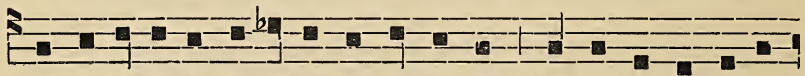

Enwa-tohetste karonhia nok nonwentsia,iah nonwenton nake-

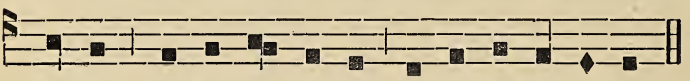

wenna ta-kon-to - hetste, rawen Rawenni - io. 1. o.

TSINI KARENNOTEN TEIERIWAKWATHA AHONWASENNAIEN TSI IONTAWEIATAKWA.

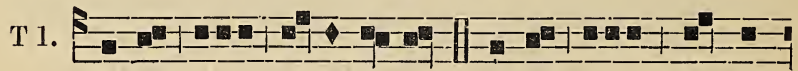
Ahon... ta-to-ken-li. T'si-ni... ha - ke

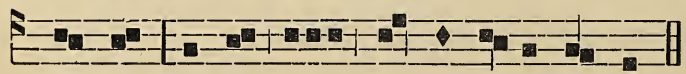

non-wa, tiotkon... we e- tho na-ia - wen. 


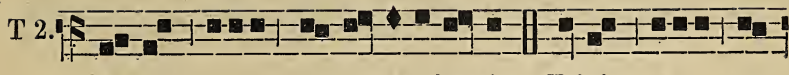
A- hon...
ro - ia-tato-kenti.
Tsini....
ton-

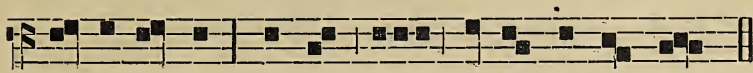

ha-ke non-wa, tiotkon...

e- tho na-ia - wen.
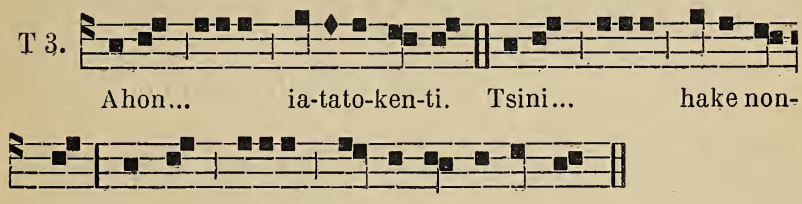

wa, tiotkon... we e-tho na-ia-wen.

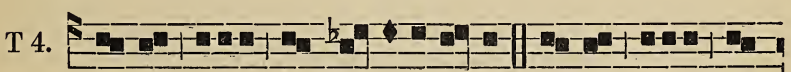

A- hon... ro- ia-tatoken-ti. Tsi-ni... ton-

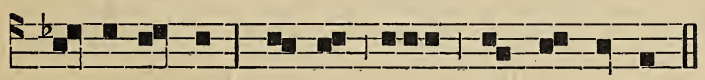

ha-ke nonwa, tiot-kon... tho na-ia-wen.

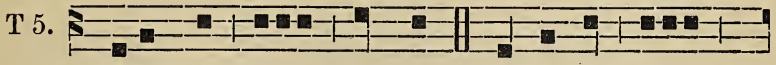
Ahon-wa...... ken-ti. T'sini ioh.....

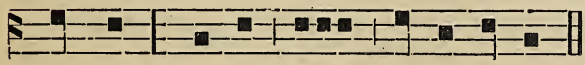

non-wa, tiotkun..... tho na-ia -wen.

$\mathrm{T} 6.5$ 二
A - hon - wa..... ro - iatato-kenti. Tsini.....

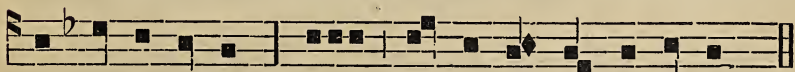

ton- ha-ke nonwa, tiotkon... nenwe e - tho - na -ia- wen. 
T 7. 5-
A - hon...... ia - ta-to - ken- tl. Tsi.ni......

5-

ha - ke non-wa, tiot-kon...... we et-ho naia - wen.

T 8. A-hon...... ro- ia- ta-to-ken-ti, Tsini......

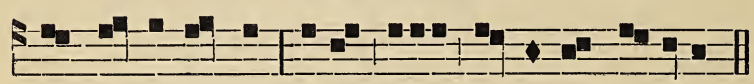

ton -ha- ke nonwa, tiotkon...... we et-ho na-ia-wen.

TIERENNAKETSKWATHA OKON IOKARENRE.

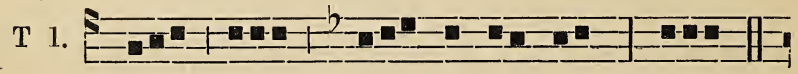

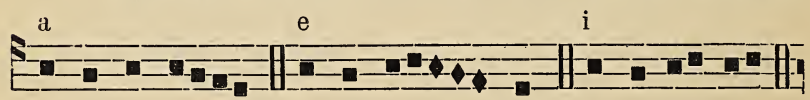

年二-

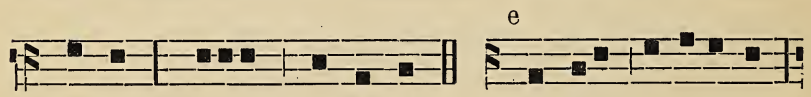

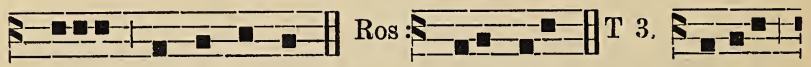

a

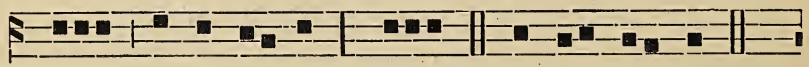

S-1 


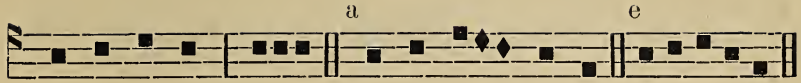

a

T 5.

s

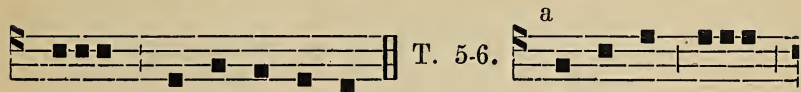

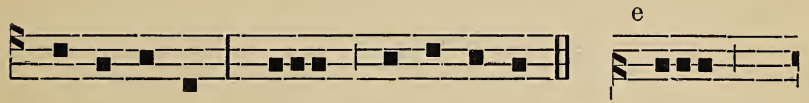

F“

i

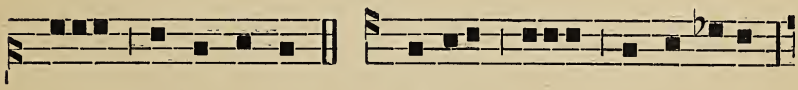

0

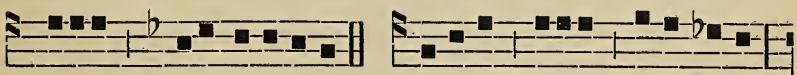

a

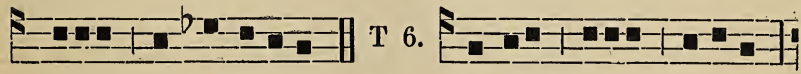

e

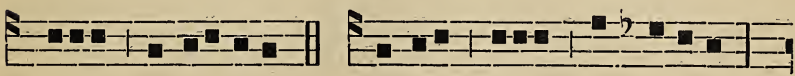

i

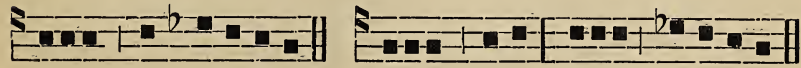

S 


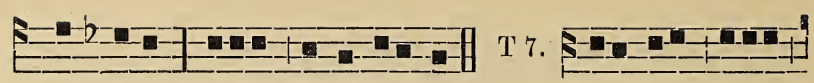

a e i

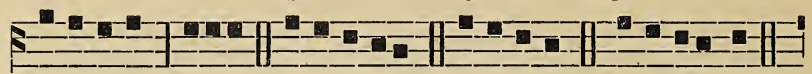
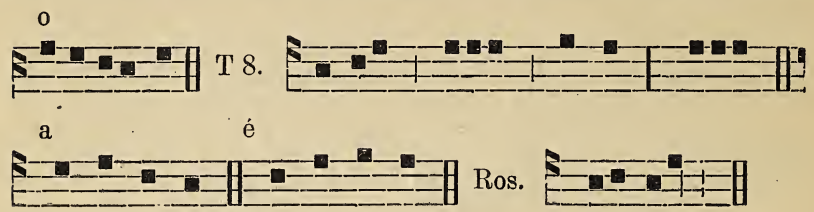

ONEN 


\section{IERENNISAKSTHA.}

Ratikwekonne.............. 237

Sh : Ratik. nok K. Ronw ... 240

K. Ronwariohton........... 242

K. Ronwatiriohton........... 245

R. Ratiriwawakon ........... 249

R. Ratitsihenstatsi nok .... 254

Ioti. iahteiotinakwaierterhaon ......................

Ioti. iotiniakonhonne........

Ononsatokenti ................

Wari ioiatatokenti I .........

Iakoniakhe .....................

Isi kahniote okaristia ......

I Tsi rathonniane.............

Saksarie Rti.. ...... .........

II Tsi rathonniane...........

Wari sontiatata...............

III Tsi rathonniane. ..........

IV “ " " ......

Atonwa Rti ....................

Tsi nahatonni asontben....

“ " entiekehne..

Etienn Rli .....................

San Rti ......................

Eksa okon kenha .............

$\Lambda$ wentatokentike ............

'Tsina teiontatenoron.........

Iesos wathon wanoron........

Niiohne .........................

Iesos raosennatokenti ......

Iier Rti .......................

Kor Rti .......................

T'si naontathaseron..........

Awent. tsiniiore 7 niw .......

7 Niwasontashen............

6 " 6 ............

5 “ $\quad$ "............. 315

Tsi naontatakenroserawe.. 316

1 Iakawentontietha kowa.. 319

II

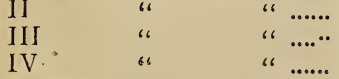

Sose Rli .........................
Sahahroriana .............. 329

Iesos raoronhiakensera..... 331

Isi naontatenenton........... 33?

Okaristiakowahne........... 337

Ronwaiatanertaktonne.... 339

Wentakta.................... 341

Tsi nonsahatonnhete......... 344

8 nonta..................... 348

Awentokon tsıniiore ........ 349

Tsi ienenrimekens ........... 350

Owatsiratokenti .............. 351

Kaiasatokenti................. 353

Karonhiake sarete.......... 354

Niiohne ....................... 357

Rotkon Rolatatokenti....... 358

Asenseratokenti ............. 36?

Nio rawentawen ........... 366

Tsi naonteneratonniat..... 371

lesos raweriasatokenti...... 373

Resis Rti.................... 377

San Watis ..................... 378

Tier Koroni...................... 381

Vari ionatarenaw. Aris... 384

Iesos raonekwensatokenti.. 384

Anen .......................... 385

Tier Rti. sahonwanerenkon 385

I sos sahatiatateni........... 386

Roren Rti .................. 387

Satkonwaskwenhawe...... 387

Aweriasatokenti Wari..... 390

Sonnakerate Wari ........... 390

Wari tsi ionikonhranon..... 392

Wishe ......................... 397

Karensatokenti ............... 400

Wari aonistensera........... 400

Wari iahte kanakwaienteri 401

Wari tiakonnhe iakori ..... 268

Rafae......................... 401

A sonthenne wahonteren.... 402

Sakonwaiatenhawe Wuri.. 405

Kahaseres awentokon...... 406

Rosennaiens awentokon.... 410

Ahonwasennaien ............ 416

Tierennaketskwatha okon. 418 



\title{
IONTERENNAIENTAKWA
}

\author{
NIATE WENNISERAKE AIERIHOKTEN ORHONKEHNE
}

OKARASNEHA ONI.

\section{H \\ RASENNAKON RONIHA, NOK ROIENHA, NOK ROTKON ROIATATOKENTI. ETHO NAIAWEN.}

IESOS ROTERENNAIENTAKSERISON.

Takwaienha ne karonhiake tesiteron, aiesasennaien, aiesawenniiostake, aiesawennarakwake nonwentsiake tsiniiot ne karonhiake tiesawennarakwa.-Takwanont ne ken wente iakionnhekon niate wenniserake, sasanikonrhen nionkwariwaneren, tsiniiot nii tsionkwanikonrhens othenon ionkinikonhraksaton nonkwe ; tosa aionkwasenni ne kariwaneren, akwekon eren sawit niotaksens, Etho naiawen.

RARONHIAKEHRONON RAOWENNA.

Wari, tekonnoronkwanions, ise tsiati ioianerenstakwa, Rawenniio senikwekon, sonhaa tsini hiakonnienstha ne Niio, Iesos oni hetsienha raonhaa tsini honwasennaiens.-Wari saiatatokenti, Niio hetsienha, takwaterennaienhas ionkwatiwanerahakskon nonwa nok oni tsi nentiakwenheionsere. Etho naiawen.

\section{RATIKWEKONNE RAOTIWENNA.}

Tewakehtakwen Niio Roniha iah othenon tehonoronse, roteweiennonni karonhia onwentsia oni.-Tewakehtakwen Iesos Kristos raonhatsiwa Niio Roienha Sonkwawenniio onkwe rotonhon, ne roiatison Rotkon Roiatatokenti, Wari roteweton iahte kanakwaienteri. Ethone si hoterihontakwe Konskwirat ronwaiatskwe, roronhiakenhon ne Iesos ronwaiatanentakton tekaientannhareke, rawenheion ronwaiataten, onwentsiakon iehokton, nok asen watontha niwenniserake shotonnheton. Karonhiake ieshawenonton, etho kati nonwa ieshenteron tsi raweientehtakon ne Roniha iahothenon tehonoronse. tentesakoiatoretane neniakonnheke nok oni niakowentaon. - Tewakehtakon oni ne Rotkon Roiatatokenti, kentiohkwaien otiohkwatokenti, onwentsiakwekon sakentiohkwat, iken teiontatseronniennis akoiatatokentison, sewaterakewas iakoriwaneren, entsiontonnhete niakowentaon, iahte kakonte ientsiakonnheke. Etho naiawen, 
Niio, ise Sewenniio tsi iakionnhe, iah othenon te sanoronse, satontek; ise oni Wari saiatatokenti, iahte senakwaienteri; Wishe satkon seronhiakehronon ; San Watis saiatatokenti; Tier Kor oni leses hetsiseniwennenhawakwe ; sewakwekon ne sewaiatatokenti karonhiake tisewanakere; ise oni hetseriwawase ne Sonkwawenniio Iesos, sewakwekon tsiatontek: onen wakeriwaienterene tsini wakeriwaneren, kennontonniontserake, kewennake keweiennake oni, ii akeriwa, ii akeriwa, ii akeriwa kowa. -Onen kati, Wari saiatatokenti iahte senakwaienteri, Wishe Satkon seronhiakehronon, San Watis saialatokenti, Tier Kor oni, Iesos hetsiseniwennenhawakwe, sewakwekon ne sewaiatatokenti karonhiake tisewanakere, ise oni hetseriwawase ne Sonkwawenniio Iesos, sewakwekon kwennitentase, askwaterennaienhase, aonsonterakewe tsi wakeriwaneren. Etho naiawen.

\section{OIERI NIHAWENNAKE NE NIIO.}

Saiatat ok Niio hetsewenniiostak, raonhatsiwa Rawenniio tsi sonnhe.-Tosa hetsennaiesat Rawenniio raosenna.-Satsterist niawentatokenton Niio rawennisera.

Shekonnienstak iesaienha aionnise asonnheke.-Tosa sheiaset nonkwe.-Tosa satenakwaiesat.-Tosa senensko. - Tosa sheiennowenten.-Tosa saskaneksek kanakwaiesatsera.-Tosa saskaneksek akoren akowenk, naserheke : akenensko.

\section{TSIATAK NIKAWENNAKE NE KENTIOHKWATOKENTI.}

Tsiatarak ohaseratokentonke, Niio rawenniserake, nok oni tsi iawentatokentaserons.-Satsterist iawentatokenton okonha.Aonsaiesaronkwase arahonne enskat tsini ioseres.- Ahiatienhase Iesos arahonne tsi nonsahatonnhete.--Satsterist iakawentontietha, kaieri niiokwenhraron konwaiats, entakta ne wentanoronson, nok oni ne kaieri niwasontashen karoha tsi nonsahatonnhete.-Tosa sewarak ronwaiatanentaktonne wenniserate.Aontaseriwaierite othenon tentsenonweronniontake ne R. nensatonnisahase.

\section{IAKORIWIIOSTAKON.}

Sewenniio, tewakehtakon akwa oriwiio tsinikon ionkwarihonniennis ne Kentiohkwatokenti, aseken ise tisawenninekenhon, iah taonton asennowente.

IAKORHAREKON.

Sowenniio, akwa wakerhare nenskienawase tsi nakiere ne karonhiake akatsennonni ; ne enskitenrate tsini sonkwatsennonniatenni Iesos Sonkwawenniio, aseken etho niskwarharatstenni. 
RONWANONWESTHA NIIO.

Sewenniio, sonhatsiwa konnonwes akenikonhrakwekon, aseken iahtiiaiehewe tsini saianere, nok ise sariwake khenonwes akwekon nonkwe tsi nii nikatatenonwes.

\section{TSIONTONNHAKANONNIA THA.}

Sewenniio, akwa sakatatrewate, skeriwaswens tsi wakeriwaneren iken ise seriwaswens tsi ieriwaneraks. Ne ki skatatrewatakwa tsini konnenwes, sonhaa tsini satanitenraskon. Takitenr, sasanikonrhen; ne askitenrate tsini sonkwenheiase Iesos Sonkwawenniio. Akwa tewakennikonhrasaalon, askienawase tosa nonwenton taonsakiere tsinenwe enkonnheke nok taonsakeriwaserako. Etho naiawen.

\section{OHASERATOKENTI.}

Ne iekwenies, ioianere aieiatarake ohaseratokentonke, aseken aonhaa waterennaientakseranoron ohaseratokenti. Tsi iehasens ronwariwaiennis ne Rawenniio Iesos raieronke nok raonekwensa. Iawet sonkwaiakhonnihaties ne lesos tsinikon sonkwatsennonniatenni sasonkwaronhiakense, sasonkwenheiase oni. Ioiauerenserison ne Kentiohkwatokenti waton : T'siatarak ohaseratokentonke Niio rawenniserake nok oni tsi iawentatokentaserons. Ioriwatsanit kati ieriwaneraks ne iakohaseronties nethone.

Ioianere aierensokten tsini kahaseres nok riiare 1on Aonsahonwatatrewaten ne Rawenniio tsinikon ronwanihonhraksaton, 2on ahonwasennaien, ahonwawenniioste oni tsi iakonnhe, 3on Ahonwaneken tsinikon teiakotonwentsioni, $40 n$ tahonwanonweraton, nok 5 on aionterientasa.

\section{SEWA'TATERONKWENNI.}

Ioianere aonsaiontateronkwase sewennitatson, tokat kaieri niioriwake tsioseratson, 1 on kenkwitehne tsi iakawentontietha kowa. 2on akennhake Rotkon Roiatatokenti tsi rawentawen, 3on kanennakehne, asonthenne sahonterennaien, nok $40 n$ koserake sahatonni. Akwa ieriwaneraks, iawet iakorihonties tsi iakoriwiioston ne iahte tsiontateronkwennis arahonne enskat tsini ioseres, aseken waton ne Kentiohkwatokenti : aonsaiesaronkwase aralionne enskal lsini ioseres.

Tsini ioriwake tsiontateronkwennire, 1. aiontiatotarako tsi ionkwehne nok aiairon :

Sewenniio tsi konnhe, ise saterientare tsini wakeriwaneren aseken iah othenon te sanekherens, takitenr kati aonsakehiarane 
tsinikon konnikonhraksaton, aontiesenhake taonsakeriwaserako tsi sekon skennen ikes nonwentsiake tosa askrewate nonwentsiakon tsi nenkatonnhokten.

Onen tenhnon nonwa akwa skennenha aierihokten oieri nihawennake ne Niio, tsiatak nikawennake rie Kentiohkwatokenti nok tsiatak nikontiriwanerahakserakweniics, sewalierens oni aiontorisen aontonseke aonsaiontatehiarakwen tsini ionnontonnionkwa, tsini iontatiatha nok tsini iontieranions, kanonskon, atsle, ononsatokentike nok oni ka ok nonwe itiens.

2. Ahonwaneken ne Rawenniio asakonikonhrataa aieriwaswen tsi iakoriwaneren : aierensokten, noh niare taieiatorete : 1. aonhaa tsini ioriwatsanit ne kariwaneren aseken ioiataksatha nonkwatonnhetston; 2. tsina teiotenenhianiton ensakohrewate ne Rawenniio nonwentsiakon enskat ok oni aiakoriwanerenhake ne ioriwatsanit; 3 . tsini iakokaronnis ne kariwaneren, iken tsaeriwanerake iahte tsiakonaktaien ne karonhiake; 4. aonhaa ne kariwaneren roiatanentakton Iesos tekaiasonteke, ne rawenheiaton sasonkwenheiase ; 5 . tsini kariwaneren ronwanikonhraksatha ne Rawenniio raonbatsiwa tsini haiatanoron, tsini honîkonhriio, tsini hotanitenraskon akwekons oni iahtiiaiehewe tsini hoianere.

3. Aionterientasa iah nonwenton taonsaieriwanerake nohnaken, nok ne senha aiontewentete ne iakonikonhrotakwatha okonha aienrheke, enhiriwaienhase ne ratsihenstatsi tsinaonkiatawen, tsinenhakierase, nok skennenha akonikonhrakwekon oni aierihokten tsiontonnhakanonniatha.

4. Tsinonni ieteron aierihokten tsiontateronkwennitha, nok ethone taiontontsolen tsi renteron ne sesakoronkwennis ne ok iekenha aiairon : Niio, enkonrihoklase tsini wakeriwaien niolaksen, nok oni nise, rakeni.

Ethone aiontonteke tsi nahoten ensakohrori ne sesakoronkwennis aienrheke : akwekon enkeriwaierite tsi nahotén enhakwatenientense. Nok akwa akonikonhrakwekon aierihokten tsiontonnhakanonniatha.

5. Nonen tsiontateronkwenni, tosa ok sa ok aionhlenti; ioteriwison tenhnon tahonwanonweraton ne Rawenniio, nok ok sa ok aieriwaierite tsi nahoten sakotenientense sewatateronkwennike. Kariwakson aienisko, aseken oriwatokenti kowa iotonhon ne teskariwaserakwen.

\section{OKARISTIA.}

Iahtiiaiehewe tsini sonkwanoriwehon ne Iesos Sonkwawenniio rotatiteron okaristiakon, raweron: tiotkon aiakwakwekonhake nonwentsîake niakoriwiioston, aontonseke aiakwakwekonhake iahte kakonte ne karonhiake. Iotkate tsi iekwenies aieiatarane. Arahonne aiontsteriste eh naieiere tsi nonsahatonnhete, etho 
nionk wannhaon ne'Kentiohkwatokenti iawen :Ahiatienhase Iesos arahonne tsi nonsahatonnhete. Tiotkon aiehiarake tsini waton ne kaiatonsera ionteriwaienstakwa: Ne ronwanaktannhese ne Sonkwawenniio n'akaweriane katkeha oni ne raonha ensesakonaktannhese ne karonhiake.

Tsi ieiatarasere 1. Aiontaizkawehtakon tsi renteron okaristiakon : aierihokten ne iakori wiiostakon 2. Ahonwaskanekseke : aierihokten ne iakorharekon, 3 ahonwanonwehake : aierihokten ne ronwanonwestha Niio. 4. Aonsahonwatatrewaten, aierihokten tsiontonnhakanonniatha ; 5. Akta tsi ieiatarasere aiontatkenhronniheke nakaonha, aierihokten tsiontateronkwennitha nok: Sewenniio. iahle wakerihonle asalaweiale ne kialakon, enskal kali aonlasatali nok enskaiewenlane nakalonnlıelslon. 3 .

Nonen iakoiataraon aierensoktsn nok niare 1. Tahonwanonwaraton ne Iesos, 2. ahonwanonwehake akonikonhrakwekon, 3. ahonwaneken nahoten teiakotonwentsioni. 4. Ahonwawenniioste eiatakwekon 5. aionterientasa tosa nonwenton ahonwanikonhraksate.

Aionttaerennaienhase ontalien okonha, iontatekenha, iontenro nok oni ne iakowentaon.

Aiakehiarake tsi iahte iakoterihonte aieiatarane ne iahte ieweiente ne kariwakweniioson kariwiiostonke. Ioteriwison kati ne iakıwiraien aiontaterihonnien nontatien okonha ne ionterennaientakwa ionteriwaienstakwa oni, nok aionattennhane aieiatarake tsi sakorihonniennis.

\section{IONTATKENTSIOKHAS.}

Ioteriwison akwekon niakoriwiwiston onen si iakoiataraon aiontsteriste nasakokentsiokhawe, aseken niahtiiekaieri tsi iakoriwiioston narekho teiakotkentsiokha, raonha Ariwawakon enkoriwiioston nensakokentsiokhawe.

Nonen e̊nhariowanate tsi enrawe nariwawakon nensakokentsiokhawe, akweken n'arekho teiakokentsiokha aiontatsennaren ok sa ok, aieiatarake tsi sakorihonniennis akwekon oni aieriwaierite tsi nahoten ensakonatonhase.

\section{TEWATERANEKENSERATOKENTI.}

Oriwatokenti kowa ne Tewateranekenseratokenti. Ne kati iakoniakhe aienrheke : tosa akatiesate, ahonwaîatisakhe ne sesakoronkwennis, aiontsteriste aieiatarane; nok tosa othenon naiontsteriste nohneka nok iontonwesentseraks ‘nskwe okon kaswens ne kariwiioston, 


\section{IONTATIATOKHAS TEIAKOKONHENTONS.}

Tsi iakononwaktani, ahonwanonksa ne sesakoronkwennis, tosa aienisko tsiniiore iahte tsionttokha. Ahonwawenniioste ne Rawenniio tsini iakoiatawens, aiontsteriste aieiatarane nok asakoterennaienhase iontatiatokhas teiakokonhentons, nok oni ne tewateriwaserakwatha kowa. Tokat arekho te iakoiatonseronni onka eniakoiena nakowenk sonha, ioteriwison eh naieiere sekon si iakonikonhraieri.

\section{TEKAIASONTE AOHAA.}

Akwa tekonnoronkwanions $\mathrm{Ne}$ tekaiasatokenti.

Shetenr niakoteweienton Nok niakoriwanerakskon. (2).

TSI IEHASENTAKWA.

O Iesos seskwaskontakwen, ikehre nonwa akonnonteratie onontohorake, iawet akatkatho saronhiakenseratokenti ; askitenre, askenikonhrenhawite nakatatkenronniheke, naonkennitenton, aonsakatatswenseke nok akonnoronkwake oni tsini skwentenranion nethone; ikehre: taonsakeriwaserako tsini wakeriwaneren, takonnonweratonseke tsini skwanonwehon niakionkwe; ikehre oni : taonsakeriwaserakwase ne iakotiaktani tsi tetieseronniatha, nok oni ne sekon teiontcnnhakarias nonwentsiake akwatatekenson.

Ise nonwa, saiatatokenti Wari, sakwenion hetsenonteratie ne ne Sonkwawenniio si hoiasakehte onontoharakeson ; ethone takwaienha iotonhon kaiasakta ; askon netho naonkenikonhrotenhake tsini sanikonhrotenne nethone, iaontane nakeriane nakeriwanoronke onwe tsi sonkwaronhiakense nok tsi sonkwenheiase. Etho naiawen.

Hetsitewanonteratiesek Isos tsi ronontaraonhatie.

Akwa okti sanekwensote Tsi nonwa (2) roiasakehte.

Siaton nonkwanikonhrakon, Tsini honwaronhiakenton Tiotkon aiakwehiarah.

Tokenske onwe, Sewenniio, Akonhaa ne konronhiakenton; Sekon kati iahte skrewatha, Ne tsini (:) sanikonhriio.

Skaialontseratson aiairon:

v. K wasennaiens, lesos, nok kwanentons.

т. Aseken saiasatokenti onkwe sesheskontakwatori. 
1.-IESOS RONWATEWENTETON N'AHLENHEIE.

O lesos Sewenniio, satontaton tsi teiestiatoreton nok iesatewenteton ; askitenre akwekon akatontate tsi ok nenskeriwisabase tsi naionkiere nonkwe tosa aonkenakwen ise tenhnon akonwenniioste, akonnikonhraierite oni tosa aonsakeriwanerake.

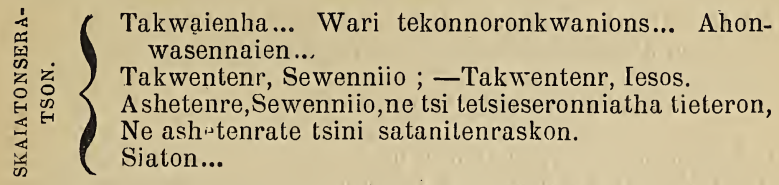

Iorontakste sarontakehte, Iawet onkwariwaneraksera

Ioranentakon ne tsiatake; Ne tsini (2) shenonwes nonkwe.

\section{II.-I IESOS ROIASAKehte.}

O Iesos Sewenniio, watesekwe nonkwariwanerahaksera tsi wasatketate ne tekaiasonte, askitenre tosa aonsakeriwanerake ne ioriwatsanit tsinenwe sekon enkeseke nonwentsiake ; okeken akatstarhakwake tiotkon tsini konwennaiesaton, nok taonsakeriwaserakwaseke.

Ioriwatsanit! Rawenniio, Raiatienens tsini iorontakste :

Onkwe etho nihonwaieren Raonha (2) sakoiatison.

\section{III.-IEsos wahaiatienenne.}

O Iesos Sewenniio, iawet ii onkwariwanerahaksera sawisenne tsi wasiatienenne: askitenre akesatsteke kariwiiostonke, tosa akennikonhroktake tsini skwatenientense nonwentsiake, akwekon tenhnon aonkeriwaieritonhatie.

Kanon wase, ise Waria ? Kanon wase ! Hetsenonteraties, Onwa tsi ok onontoharake Hetsienha (?) ensonkwenheias.

\section{IV.-IEsos Wathiaterane Ne ronistenha.}

O lesos Sewenniio, iahothenon te seninakwention ne tsiatatienha tsini seniiatawenhonhatie ; askitenre aonkenikonhriiohake othenon nenionkiiere nonkwe, ok eken akhetenre tsi nise nisaieren naskwarihonnien.

Heren iret ne ronwannhaon N'ahosnienon ne Sonkwawenniio; li seken tewakatentson, li kati (2) katiasaketat. 


\section{V.-SIMON ROIENAWASE IESOS TSI ROIASAKEHTE.}

O Iesos Sewenniio, ise takwentenron nakwaienawase tsi sarontakehte : askitenre tiotkon akheienawaseheke ne satekonkwe nonwentsiake, aonkwatennitenraskonhake, tosa onka akhekenromniheke, ii tenhnon akatatkenronniheke.

Ka tonte tsini tsiatiiohne, Iesos, iah nonwa teskaieronni ; Ii ne ken kwaiataksaton, Ne tsini (2) iakionkwetaksen.

VI.-TSIAKOTHONWISEN WAHONWAKONSOKEWE NE IESOS.

O Iesos Sewenniio, shetenron naskonsarake nakoniataraa ne iesakonsokewaton; askitenre tiotkon askonsarake nakenikonhrakon, nakoniehiaraseke, nakonwennarakwake oni tsinenwe, tosa nonwenton akoniatanikonhren.

Tsini honwasentonkwa Iesos, Sekon enskat sahaiatienenne; Ok eken tehotisterihens losnore (2) ahrenheiate.

\section{VII.-Tekenihaton Iesos wailatienenne.}

O Iesos Sewenniio, ne tsini iokste nonkwariwanerahaksera, ne saiatienentha, askitenre nakswenseke onwe ne kariwaneren, tosa nonwenton aonsakonnikonhraksate, ok eken ii akatatkenrenseronniheke ne taonsakeriwaserako.

Tosa nii akeriwake Sewatstaren ne teskwakanere ;

Ise tenhnon sewatatitenr, Aseken (2) ise sewentent.

\section{VIII.-IESOS WaSaKoniKonhrahnirate NE KONTSTARANES.}

O Iesos Sewenniio, askitenre akonwennaierite tsi isen : ise sewariwake nok oni niletsiien okonha akoriwake sewatstaranesek; ise, Sewenniio, askon nokaseratokenti ne tokenske akatstarhakwake tsi wakeriwaneren nok tsi iakoriwaneren nakonkweta.

Tokenske onwe, Sewenniio, Ken tsiatienens, isese sesatste :

Ah ! onkwariwaneraksera Ehtake (2) saiatienenton.

\section{IX.-Asenhaton iesos Wallatieninnk.}

O Jesos Sewenniio ne iawet sennikonhroktakwa tsini iahte ionkwatsennonniatskon niakionkwe; askitenre tiotkon akeriwaientereseke tsini skitenranionhaties ne tiotkon takonnonweratontake naonkwateweientontieseke tsi konnhe.

Kasewe, swaronhiakehronon, Sewatkatho n'hetsiswaiatison : Ah ! hetsisewaiatakennha Ostonha (2) ahaiewentann. 


\section{X.-Iesos wahonwaiatawitiasionko.}

O Iesos Sewenniio, ne takwarihonniennitha tosa sotsi aionkwanikonhranentakonhake nonwentsiake ne kaientaon; askitenre tosa sotsi akennhaten nothenon akatkaronni, ise tenhnon akonwenniioste, akerheke : ise waskekwa nakwekon tisewenniio.

Oh ne ken niswanikonhroten. Iesos hetsisewaiesatanions ;

Ne ens ken sewatonraserons Tsinikon (2) sonkwentenranions.

\section{XI,-Iesos wahonwaiatanentakte.}

O Iesos Sewenniio, teiesasnonsaweheston, teiesasitaweheston oni tsi iesaiatanentakton tekaiasontne ii onkwariwa ; oronwaroskon tsiataniiontakwenne; askitenre akenonweseke nakeronhiaken nonwentsiake taonsakeriwaserako.

Sahrenheie, onterakwaton, Saiontonnheton iakowentaon,

Tsini hoson ne wakontrori : Raonha (2) Sonkwawenniio

\section{XII.-IESOS Wahrenheie teKaiasontwe.}

O Iesos Sewenniio, ethone senha saronhiakenhon saesaiatakaratate tekaiasonteke, aseken ne senheiaton tsi takwenheiase; askitenre akenonweseke saianerenseratohenti, ne aonkiatakaratate katke sonha karonhiake.

Wari onen wahiaiatonti Ne hetsienha hetsenoronkwakwe;

Iawet se ise waesario, Ne tsini (2) serientaksenhon,

\section{XIII.-IEsos wahon Watatanentasi.}

O lesos Sew neren, nonwa senheion, satskwekon, tesateronwekon, tesatskanenonte, iokahronton tsiatakwekon tsi teiesawehestanion; askitenre akatennikonhranentasi ne kariwaksenskwe ekon, naontsennonni ohnaken ne kieronke tsi nentsiontonnhete nonkwe.

Aiawen ens Iesos tsi siatat Iakwakwekon aiakweriheiate.

Tsi naonsaiakwatatswensek Iaiakwe (2) ne karonhiake.

\section{XIV.-Iisos wahon waiatata.}

O Iesos Sewenniı, satiatatas nonkweriane tsi takwatiennis okaristiakon ; askitenre tosa othenon aioiatoronhake nakwatonnhetston tsinikon nenkiatarane, nok oni nenkatonnhokten, aiawens katke sonha ii oni akonhiatienhase ne karonhiake. 
$-10 *$

Siaton, Iesos nonkweriasakon Tsini iesaronhiakenton nonkwe ; Takwentenr ise oni, Wari, Asteson (2) Aiakwehiarak.

TSI IEHASENTAKWA.

Takwaienha... Wari tekonnoronkwanions... 3 Shetenr Sewenniio... (sh. 90). 3 Shetenr Iesos... (sk. 11).

\section{ONEN.}






a

4

, 14

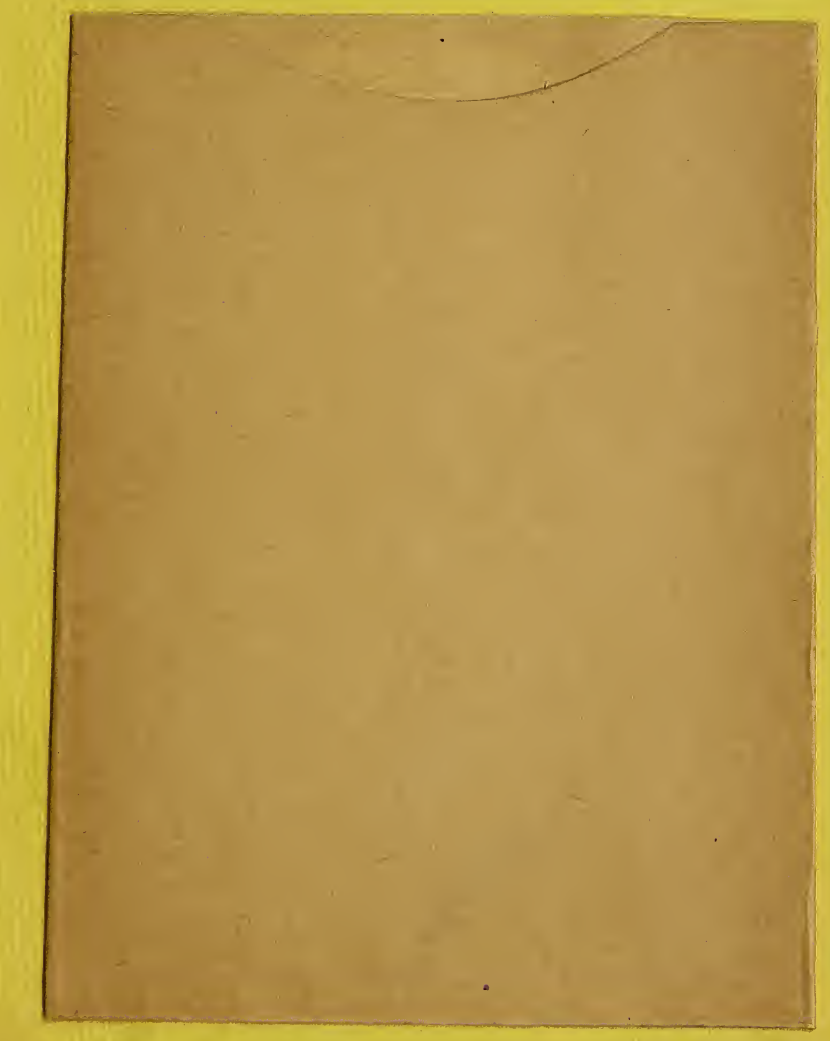


